\title{
PARAMETERS AND MICRO-PARAMETERS IN ARABIC SENTENCE STRUCTURE
}

By

Kemel Jouini

A thesis

submitted to the Victoria University of Wellington in fulfilment of the requirements for the degree of

Doctor of Philosophy in Linguistics

Victoria University of Wellington 


\section{ACKNOWLEGDMENTS}

I would like to thank Professor Elabbas Benmamoun, in particular, for accepting to be my adviser for the nine-month period I spent at the Department of Linguistics at the University of Illinois at Urbana-Champaign in the academic year 2004-2005 as a nondegree Fulbright grantee. During my stay at UIUC, Elabbas's lectures convinced me of the view that a proper understanding of the feature structure of functional categories could enlighten linguistic theory on how the structure of sentences could vary cross-linguistically.

Special thanks go to Dr. Elizabeth Pearce for willing to be my supervisor, for her patience to read through the so many drafts of chapters I was sending her, and for her advice on the numerous revisions I thereby had to make.

I also thank Professor Richard S. Kayne for the invaluable opportunity he offered me during his visit at VUW to discuss with him some aspects of microparametric syntax relevant to my analysis of verbal inflection.

Last, but not least, I thank my secondary supervisor Dr. Sasha Calhoun for her willingness to share ideas and offer suggestions, as well as Professor Laurie Bauer for his helpful comments on part of my thesis. I also want to thank Professor Tim Stowell, Professor Halldór Sigurðsson, Professor Henry Davis, Professor Jan Koster and Dr. Fred Hoyt for sending me documents I could not find elsewhere. 


\begin{abstract}
My thesis deals with dependency relations in the structure of sentences in Arabic and how properties of verbal morphology and associated lexical items dictate how sentences are derived. I adopt the probe-goal-Agree Minimalist view that variation between languages (even those that are closely related, such as Standard Arabic and Tunisian Arabic) is due to the 'feature structure' of functional elements that enter into the derivation.
\end{abstract}

In particular, the essential architecture of sentences expressing the dependency relations verbs and associated elements have with the 'functional' portion of sentences (i.e., tense/modality properties) is universal in that these dependency relations will be expressed on the basis of the same feature structure cross-linguistically. However, this architecture still allows for the kind of parametric variation that exists even between closely related languages.

In this context, I am interested in the status of subject-verb agreement configurations, in both VSO and SVO word orderings, and wh- and other A'dependencies in Standard Arabic (with comparisons to some modern spoken varieties of Arabic, where appropriate). The analysis is shown to extend to other V-raising languages of the Semitic/Celtic type with 'basic' VSO word ordering. A possible extension of the analysis to the V2 phenomenology is also discussed and the major role played by the raising of $\mathrm{V}-v$ to $\mathrm{T}$ and the raising of $\mathrm{T}$ to $\mathrm{Agr}_{\mathrm{S}}$ or $\mathrm{T}$ to Fin is highlighted.

An important aspect of my analysis is a proper understanding of the dependency relations involved in the derivation of the relevant sentences where the role of the $\mathrm{CP}$ domain projections, verb-movement, feature identification and/or feature valuation along with clause type is essential for interpretation at the interface at the output of syntax. In this feature-based analysis of parametric and microparametric variation, I show that variation between typologically similar and typologically different languages is minimal in that it is limited to the interaction of feature combinations in the derivation of sentences.

These feature combinations concern the feature structure of the T-node in relation to the position where $\mathrm{T}$ is spelled out at the interface. In particular, $\mathrm{T}$ raises to $\mathrm{Agr}_{\mathrm{S}}$ or to Fin in some languages and/or structures. Such raising processes are important in subject-verb agreement configurations cross-linguistically involving 
combinations of T-features and D-features, which would differ in interpretability (i.e., interpretable vs. uninterpretable) as the basis for feature valuation. Similar feature combinations also drive the raising processes in wh-dependencies with some F-feature (mainly related to 'focus') interacting with the T-features of Fin.

I propose that two modes of licensing of these feature combinations are at work. The first mode of licensing is the basic head-head agreement relation. This agreement relation is the basis for verb-movement to the functional field above $v \mathrm{P} / \mathrm{VP}$ in V-raising languages. The second mode of licensing is the Spec-head agreement relation, brought about by the Merge (internal or external) of $\mathrm{D}(\mathrm{P})$ elements in Adependencies and the Merge of wh-elements in A'-dependencies.

In dependency relations other than subject-verb agreement and whdependencies, I propose that the licensing of these feature combinations is strictly a question of 'identification' via head-head agreement whereby a feature on a functional head does not need to be valued, but it still needs to be 'identified' for the well-formedness of the $\mathrm{C}-\left(\mathrm{Agr}_{\mathrm{S}}\right)-\mathrm{T}$ dependency. This is the case of the interpretable D-feature of the Top node in Topic-comment structures and the interpretable Ffeature of the two functional head nodes, Mod(al) and Neg, in relation to the Tfeatures of Fin in a V-raising language like Standard Arabic. 


\section{ARABIC DATA}

\section{Sources of the Arabic Data}

I thank Dr. Hassan Makhad (Caddi Ayyad University, Morocco) for his help with judgments on the data from Standard Arabic (where no specific reference has been given). I also thank my fellow teachers Farhat Bessrour and Al-Hèdi Fezai in Tunisia for their willingness to corroborate my intuitions about the data from Tunisian Arabic.

\section{List of Transliteration Letters}

I follow the Qalam transliteration system of Romanization of Arabic for the transcription of the Arabic letters, which do not match correspondent letters in the English alphabet. As for the Arabic 'hamza' letter/sound I and the pharyngeal fricative sound $\varepsilon$, I adopted the International Phonetic Alphabet (IPA) transcription symbols ? and S, respectively. The Qalem transliteration letters fit more easily in terminal nodes in syntactic tree diagrams than the corresponding IPA transcription symbols, for which syntactic tree editor tools available on sourceforge.net do not provide a means to introduce them in syntactic trees.

$\begin{array}{ll}P & \text { Glottal stop } \\ d h & \text { voiced interdental fricative } \\ D & \text { voiceless pharyngealized/emphatic lateral fricative } \\ H & \text { voiceless pharyngeal fricative } \\ S & \text { voiceless pharyngealized/emphatic dental fricative } \\ Z & \text { voiced pharyngealized/emphatic interdental fricative } \\ T & \text { voiced pharyngealized/emphatic alveolar stop } \\ g h & \text { voiced uvular fricative } \\ G & \text { voiced pharyngeal fricative } \\ k h & \text { voiceless uvular fricative } \\ q & \text { voiceless uvular stop }\end{array}$

Long vowels are indicated by the doubling of the vowel rather than by a colon following the vowel - i.e., $\mathrm{VV}$ instead of $\mathrm{V}$ : 


\section{LIST OF ABBREVIATIONS}

\section{Technical abbreviations}

$1=$ first person of the verbal agreement system

$2=$ second person of the verbal agreement system

$3=$ third person of the verbal agreement system

$\mathrm{ACC}=$ accusative

$\mathrm{AGR} / \mathrm{Agr}=$ agreement

Asp $=$ aspect

$\mathrm{CL}=$ clitic

$\mathrm{D}=$ dual

$\mathrm{DEF}=$ defective

$\mathrm{DO}=$ Direct Object

EPP $=$ Extended Projection Principle

$\mathrm{EV}=$ epenthetic vowel

EXPL $=$ expletive

$\mathrm{F}=$ feminine

$\mathrm{FUT}=$ future

$\mathrm{GEN}=$ genitive

IMPERF $=$ imperfective

IND $=$ indicative mood

INDEF $=$ indefinite

JUSS $=$ jussive mood

LDA $=$ Long Distance Agreement

$\mathrm{LF}=$ Logical Form

$\mathrm{M}=$ masculine

MOD $=$ modal

NEG/Neg = negation

$\mathrm{NOM}=$ nominative

$\mathrm{P}=$ plural

PASS $=$ passive voice

$\mathrm{PERF}=$ perfective

$\mathrm{PF}=$ Phonetic Form

$\mathrm{Q}=$ question morpheme 
$\mathrm{S}=$ singular

SUBJ = subjunctive mood

VISH $=$ Verb-internal subject hypothesis

\section{Other abbreviations: Acronyms}

LA: Lebanese Arabic

MA: Moroccan Arabic

SA: Standard Arabic

TA: Tunisian Arabic 


\section{TABLE OF CONTENTS}

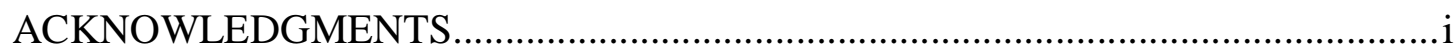

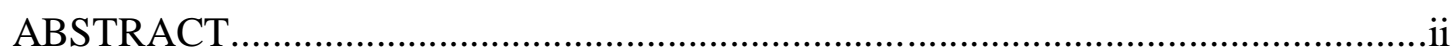

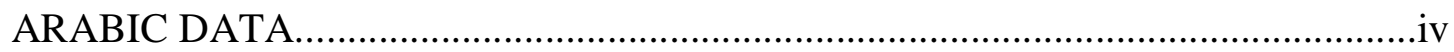

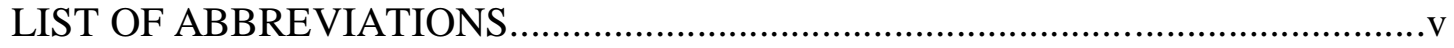

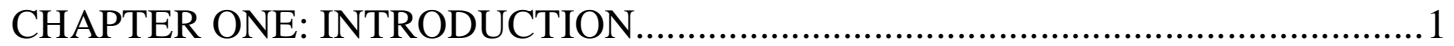

CHAPTER TWO: APPROACHES TO SENTENCE STRUCTURE AND PARAMETRIC VARIATION

2.0. Introduction. 4

2.1. The structure of IP and subject-verb agreement phenomena.............................

2.1.1 The structural subject position of the clause in null-subject languages and the EPP.

2.1.2. The derivation of VSO vs. SVO word orders and subject-verb agreement patterns in Arabic: micro-parametric differences.....................................9

2.1.2.1. Koopman \& Sportiche (1991) and Sportiche (1989/1998)................10

2.1.2.2. Arabic VSO word order: Benmamoun (1992a)................................ 14

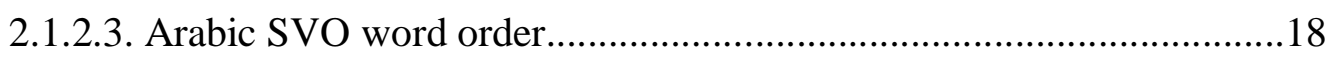

2.1.2.3.1. The Subject Theory (Mohammad 1989, Benmamoun 1992, 2000, Aoun et al. 2010)....

2.1.2.3.2. The Topic Theory (Akkal \& Gonegai 2000, Fassi Fehri 1993, 2000, Soltan 2006).

2.1.2.3.3. Morpho-syntactic and morpho-phonological evidence for the topicalized/clitic left-dislocate nature of subjects in SA.............26

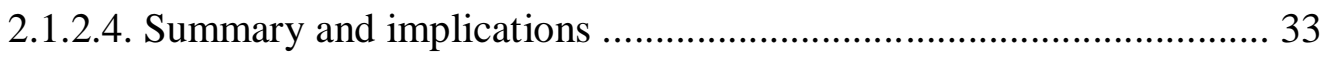

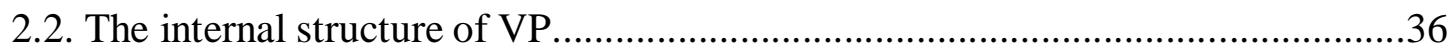

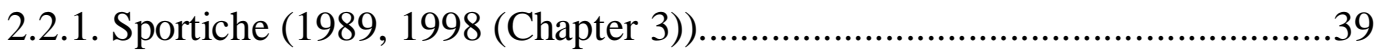

2.2.2. Chomsky (2008) on Agree-feature inheritance .......................................46

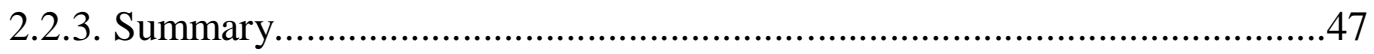

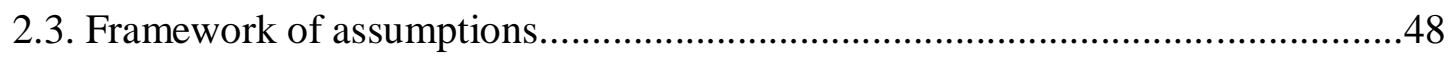

2.3.1. Early Minimalist assumptions on agreement relations..............................48

2.3.2. The Phase-theoretic framework of Chomsky $(2001,2004) \ldots \ldots \ldots \ldots \ldots \ldots \ldots \ldots . . . .57$

2.3.3. The agreement approach to the EPP (Miyagawa 2010)...........................65

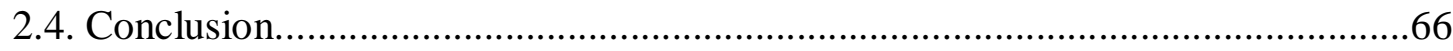




\section{CHAPTER THREE: FUNCTIONAL STRUCTURE AND CROSS-LINGUISTIC VARIATION IN ITS REPRESENTATION}

3.0. Introduction

3.1. Some Minimalist assumptions about functional structure and its derivation........68

3.1.1. Verbal inflection and the structure of IP and CP.

3.1.1.1. The structure of IP as $\operatorname{Agr}_{S} \mathrm{P}$ and parameters of variation in the realization of subject-verb agreement.

3.1.1.2. The nature of Tense in natural languages and its role in agreement in IP and CP.

3.1.1.3. Bobaljik \& Jonas (1996) and Bobaljik \& Carnie (1996) on the derivation of $\mathrm{Agr}_{S} \mathrm{P}$

3.1.2. The role of the Spec-head agreement relation and the Tense-dependency in the derivation of functional structure.

3.1.2.1. The central role of the Spec-head agreement relation:

Miyagawa (2010).

3.1.2.2. Roberts \& Roussou's (2002) T-criterion analysis:

The T-dependency and the distribution of expletive subjects

3.1.3. Summary. .86

3.2. The probe-goal-Agree system: Agreement features and structural Dependencies

3.2.1. EXPL-insertion in a probe-goal-Agree system of dependency relations....90

3.2.1.1. A typology of EXPL-insertion structures cross-linguistically .90

3.2.1.2. EXPL-insertion in Romance null-subject systems: The role of D- features (Roberts 2010a Biberauer et al. 2010). 104

3.2.2. Summary 110

3.3. The subject-verb agreement system of Arabic 110

3.3.1. Introduction.

3.3.2. Tense/aspect, mood and subject-verb agreement in the perfective vs. imperfective form of verbs in SA.

3.3.2.1. Tense/aspect, agreement and mood in SA

3.3.2.2. The imperfective in SA: the problem of the characterization of the suffixal morphology.

3.3.2.3. Benmamoun (2000b, 2008): A non-fusional analysis of SA verbal inflection 
3.3.3. Mood and modality as two distinct categories in the SA subject-verb agreement system.

3.3.4. On the absence of $\mathrm{Agr}_{\mathrm{S}}$ in the SA VSO word order................................141

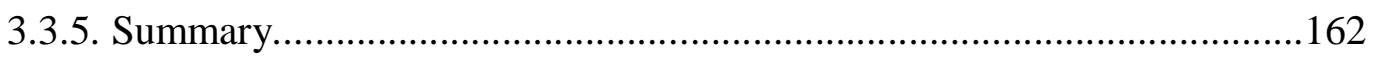

3.3.6. Different realizations of the C-( $\left.\mathrm{Agr}_{\mathrm{S}^{-}}\right) \mathrm{T}$ dependency in Arabic in VSO

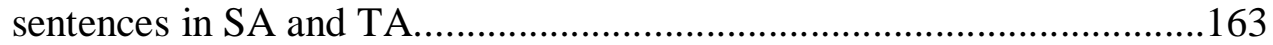

3.4. Conclusion.

CHAPTER FOUR: STRUCTURAL DEPENDENCIES IN THE HIGHER FUNCTIONAL FIELD OF ARABIC AND OTHER V-RAISING LANGUAGES

4.0. Introduction. 170

4.1. The derivation of the higher functional field and the split-CP domain of SA....172 4.1.1. Introduction. 172

4.1.2. The interaction of modality, negation, tense and topics in SA VSO and SVO sentences

4.1.2.1. The T-to-Fin raising process in SA VSO sentences.

4.1.2.2. The interaction of tense and modality in the derivation of SA VSO sentences.

4.1.2.3. The nature and function of $q a d$ in the Tense/modal system of SA...198

4.1.2.4. The feature structure of the split-CP in SA VSO configurations......206

4.1.2.5. A higher and a lower NegP projection in SA.................................220

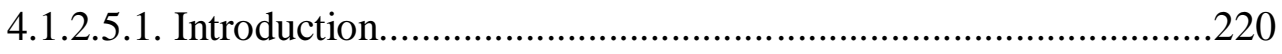

4.1.2.5.2. The derivation of the NEG elements laa/lam/lan in SA............223

4.1.2.5.3. The derivation of the NEG elements maa and laysa in SA......226

4.1.2.6. The interaction of tense and modality in the derivation of SA SVO sentences.

4.1.3. Summary. 261

4.2. The derivation of wh-dependencies in Arabic: SA vs. TA .262

4.2.1. Introduction. 262

4.2.2. wh-dependencies in SA and the strict Topic DP, wh-element word order.

4.2.3. The absence of word ordering restrictions on 'topicalized' DPs and wh-elements in wh-dependencies in TA 
4.3. Subject-verb agreement configurations in 'basic' VSO and V2 languages........289

4.3.1. A feature-based analysis of the V2/'weak’ V2 phenomenology...............290

4.3.2. Feature inheritance (Chomsky 2008) as $\varphi$-feature sharing......................298

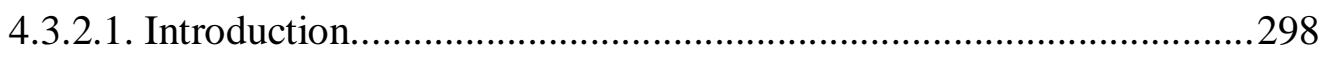

4.3.2.2. Kayne's (1989b, 2000) Agr-raising analysis revisited: A feature

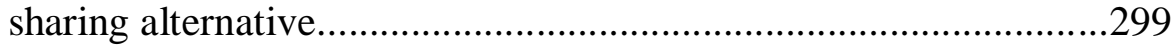

4.3.2.3. Complementizer Agreement in Dutch External Possessor

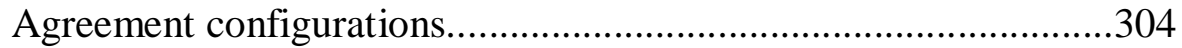

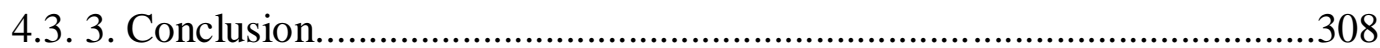

CHAPTER FIVE: SUMMARY AND CONCLUSIONS..........................................310

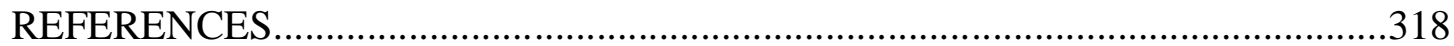




\section{CHAPTER ONE}

\section{INTRODUCTION}

This dissertation is an attempt to pin down the syntactic properties of elements within clause structure in terms of dependency relations that link functional projections (i.e., the functional layer of sentence structure) to thematic elements. It is via this close relationship between thematic and functional elements that 'functional relations' are established whereby agreement relations take shape out of the building up of derivations. The guiding principle that should emerge from the outset is that the crosslinguistic variation in the distributional properties of the elements 'merged' in sentence structure should be captured in terms of simple principles of syntactic computation in interaction with morpho-phonological properties of lexical items.

In this context, Chapter Two of this thesis introduces some preliminary remarks on verbal agreement phenomena (morpho-syntactic and morphophonological processes) in the grammar of V-raising/null-subject languages (Arabic, being the prototype) in particular, and, comparatively, languages of the English/Germanic type. Chapter Two also introduces the theoretical framework of the study and sets forth some general guidelines of what is going to be the focus of this dissertation. Of particular importance in the framework of assumptions developed in Chapter Two is the special derivational character of cyclic computations, or what Chomsky $(2001,2004)$ refers to as 'Derivation by Phase'. In this conception, syntactic computation is essentially 'derivational', but, at the same time, 'representational' in character in that the derived structures must satisfy some locality and licensing conditions at each 'Phase' of computation.

Chapter Three expands on the central role of cyclic computation in the derivational processes whereby structural dependencies and agreement relations obtain. As both Chapter Two and Chapter Three show, the derivational processes of the grammar conspire for the satisfaction of output conditions at the interface (the point in the derivation that Chomsky 2001, 2004 calls the 'strong Phase' or 'Spell Out'), at the point of which 'interpretability' arises. Both Miyagawa's (2010) 'EPPtriggered movement' analysis of agreement manifestations in natural language and Roberts \& Roussou's (2002) 'T(ense)-criterion’ approach show that the EPP remains 
(since its first inception in the framework of Chomsky 1981) a 'general' principle of the grammar as the main driving force of computations.

In the Phase-theoretic probe-goal-Agree system of Chomsky (2001, 2004, 2008), adopted in this thesis, 'Merge' and 'Move' form a composite operation along with the agreement processes (the operation 'Agree') which drive computations. This Phase-theoretic case-agreement framework supersedes the feature-checking account of syntactic derivation that was the hallmark of the 'Minimalist Program' of Chomsky (1995c). The syntactic Spec-head/head-head agreement configurations that arise from the cyclic application of Merge/Move processes in sentence structure seem to show a basic structural uniformity that, nonetheless, coheres with a certain amount of crosslinguistic parameterization. In these processes, 'parameterization' is conceived in terms of the feature structure of functional elements introduced in the computation to meet the requirement that 'probe' and 'goal' end up in a 'matching/Agree' relation that satisfies interpretability at the interface.

The process of 'expletive-insertion' cross-linguistically plays - in conjunction with the EPP - a fundamental role in, especially, subject-verb agreement processes. Expletives can be merged by base-generation in the so-called 'edge' positions (Chomsky 2001, 2004) of the 'strong' Phase heads - namely, [Spec, CP] and 'outer' [Spec, $\left.v^{*} \mathrm{P}\right]$ - and the Spec positions of the heads selected by these Phase heads namely, [Spec, IP/TP] and [Spec, VP].

In Chapter Four, I apply the probe-goal-Agree analysis of the case-agreement phenomena, which I have been mainly reviewing in Chapters Two and Three, to the derivation of the micro-parametric VSO/SVO word orders in Arabic (the standard variety vs. the modern spoken dialects). Syntactic computation is now largely driven by the satisfaction of some 'edge' property (in relation to the EPP as a requirement that has to be met in the derivation of sentence structure). Given this new emphasis on 'edge' properties of syntactic structure, the A/A-bar distinction in movement processes would be better understood in terms of the feature structure of functional elements that relate them to the elements they 'match/Agree' with.

This conception of syntactic computation puts more emphasis on the featurematching analysis of 'Agreeing' elements, where the core functional nodes C, T, and $v^{*}$ and (under the parameterized probe-goal-Agree conception of dependency formation I am advocating in this thesis) the core functional node $\mathrm{Agr}_{\mathrm{S}}$ are 
fundamental for an adequate understanding of the derivation of structural agreement configurations and their uniform representation at the interface for EPP satisfaction and interpretability at Spell Out.

It has been proposed (Rohrbacher 1994) that in V-raising languages, there is a cross-linguistic tendency for rich subject-verb agreement to correlate with the V-to-I raising process in the syntax proper since it is this movement process that licenses the elements involved in the very process. However, such a proposal does not seem to account for the case of subject-verb agreement in VSO constructions in SA, as a richly inflected language. Although SA shows evidence of V-to-I movement in VSO sentences, these sentences only show 'partial' (or 'weak') subject-verb agreement. Rather, the process of $\mathrm{V}$ to I seems to be a factor, among other possible factors, responsible for subject-verb agreement. The proposal developed here is that, in the derivation of SA VSO word order, verb-raising past the subject raised to the highest Spec position in the IP domain (i.e., the Spec of the T node, in the absence of an $\mathrm{Agr}_{\mathrm{S}}$ node, in SA VSO word order, as proposed here) can account for the absence of rich subject-verb agreement in such a derivation.

The central role of 'Tense' (Roberts \& Roussou's 2002 'T-criterion') in dependency formation is further upheld by the paramount role 'criteria' considerations (Rizzi 1997, 2006, Rizzi \& Shlonsky 2007) play in the derivation of the IP domain of clause structure in its relation to the CP domain. These across-theboard 'criteria' considerations reinforce the conception of Tense (or the tense properties of syntactic structure) as the main factor involved in defining both the 'deep' thematic and the 'surface' scope-discourse properties of elements. 


\section{CHAPTER TWO}

\section{APPROACHES TO SENTENCE STRUCTURE AND PARAMETRIC VARIATION}

\subsection{Introduction}

After introducing in this chapter the hypothesis I am putting forward as regards the close relationship that exists between subject-verb agreement and parametric variation in such closely related languages as Standard Arabic (SA) and Tunisian Arabic (TA) or Moroccan Arabic (MA), I deal in section 2.1 with some generalities about the structure of sentences in natural languages. In section 2.2, I have a look at the structure of the VP in relation to specifier (Spec) positions (including 'edge' positions in Chomsky's 2001, 2004 Phase-theoretic framework) and to possible affinities between subject-verb agreement and object agreement in relation to the caseagreement system of the grammar (as in Sportiche 1998). In section 2.3, I introduce the framework of assumptions that I will adopt, with particular reference to the Phasetheoretic conception of the derivation of sentence structure and the particular relevance of 'edge' positions in that framework. Section 2.4 is a conclusion to the chapter.

The main focus of the thesis is on subject-verb agreement phenomena and subject positions in Arabic (both SA and the modern spoken dialects such as TA and MA) in such VSO and SVO sentences, as in (1) and (2) below, where either the verb or the subject is in initial position. (The presence or absence of the object is not immediately relevant to the central hypotheses to be investigated in this thesis). ${ }^{1}$

\footnotetext{
${ }^{1}$ According to Mohammad (1989: Chapter 1), whereas the six possible word orders VSO, SVO, VOS, SOV, OSV and OVS between a subject DP, an object DP and a verb are all available in SA, only VSO, SVO and VOS word orders are available in the modern spoken dialects of Arabic. As Mohammad (1989: 9) mentions, this is due to the lack of overt phonological Case marking in the dialects.

As for the question of which word order is more 'preferable', Mohammad (1989: 2-4) argues that, although claims about 'pragmatic neutrality' in SA cannot be used to favour one word order over another in the ordering of a subject DP, an object DP and a verb, when Case marking fails to distinguish the subject and the object, VSO and SVO are the only acceptable word orders (examples adapted from Mohammad 1989: 4):

(i) a. Daraba musa Giisaa

PERF.hit-3MS Musa Isa

'Musa hit Isa.'

b. Musa Daraba Siisaa 'Musa hit Isa.'

c. * Musa Yiisaa Daraba 'Musa hit Isa.'

d. * Daraba Giisaa musa 'Musa hit Isa.'
} 
(1) VSO word order:
a. kataba
T-Tulaab-u
d-dars-a
wrote. $3 \mathrm{MS}$
the-students-NOM
the-lesson-ACC

(SA)

'The students wrote the lesson.'

b. kitb-uu T-Tulaab d-dars

wrote-3P the-students the-lesson

'The students wrote the lesson.'

\section{(2) SVO word order}

$\begin{array}{lll}\text { a. T-Tulaab-u } & \text { katab-uu } & \text { d-dars-a } \\ \text { the-students-NOM } & \text { wrote-3MP } & \text { the-lesson-ACC }\end{array}$

'The students wrote the lesson.'

$\begin{array}{lll}\text { b. T-Tulaab } & \text { kitb-uu } & \text { d-dars } \\ \text { the-students } & \text { wrote-3P } & \text { the-lesson }\end{array}$

'The students wrote the lesson.'

Apart from the fact that the modern spoken dialects of Arabic have lost the case morphology that marks the ending of nouns, such as Nominative Case and Accusative Case, the data in (1) is significant in that it points to a parametric difference in the realization of subject-verb agreement in such VSO sentences where SA (1a) does not show the same kind of subject-verb agreement we find in TA (1b). In contrast, the realization of subject-verb agreement in the SA sentence in (2a) and the TA sentence in (2b) is the same, as far as the agreement morphology on the verb katab-uu, in (2a), and kitb-uu, in (2b), is concerned. What is important in the data shown in (1) and (2) is that the agreement morphology on the TA verb does not show the kind of agreement asymmetry between the VSO and SVO word orderings, as in the SA equivalent sentences.

\footnotetext{
e. * Giisaa Daraba musa 'Musa hit Isa.'

f. * Giisaa musa Daraba 'Musa hit Isa.'
}

Mohammad (1989), Fassi Fehri (1993) and Akkal \& Gonegai (2000) claim that VSO word order is a 'basic' word order in Standard Arabic. As for the modern spoken dialects of Arabic, Ouhalla (1991) and Shlonsky (1997) claim that they tend to have SVO as a basic word order. Hoyt (2009: 654657) presents an overview of claims as to whether V-initial word order or subject/A(gent)-initial word order is more prominent in the standard variety and the dialects of Arabic. As far as the dialects are concerned, Hoyt (2009: 657) states that: "although both A-initial and V-initial word orders are asserted as being basic in various dialects, both are attested, and the preference for one or the other is affected by a variety of grammatical and pragmatic factors". 
In (1b) and (2b), the gloss under the TA verb kitbuu is 3P instead of 3MP (cf. example (2a) for SA) because TA does not have any feminine agreement marker on verbs in the plural - i.e., there is no masculine/feminine distinction in the agreement morphology on the verb in the plural in TA.

It should be noted here that, irrespective of whether the initial DP element is regarded as a 'subject' or a 'Topic' DP, this DP element has to be definite. Thus, in both SA and TA, an initial indefinite DP in SVO sentences yields an ungrammatical result at the interface, as in (3) below:

\section{(3) SVO word order}
a. *Tulaab-un
katab-uu
d-dars-a
students.INDEF-NOM wrote-3MP the-lesson-ACC

'Students wrote the lesson.'
b. *Tulaab kitb-uu d-dars students.INDEF wrote-3P the-lesson

'Students wrote the lesson.'

In contrast, an indefinite subject DP in VSO word order is perfectly grammatical in both SA and TA:

\section{(4) VSO word order:}
a. kataba
Tulaab-un
d-dars-a
wrote.3MS
students.INDEF-NOM
the-lesson-ACC

'Students wrote the lesson'
b. kitb-uu Tulaab d-dars
wrote-3P students.INDEF the-lesson
'Students wrote the lesson'

The sentences in (3) vs. (4) may seem to be the kind of data which support an analysis of initial 'subject' DPs as 'Topics' in null-subject languages (Fassi Fehri 1993, Akkal \& Gonegai 2000, Soltan 2006; see also Rizzi 2006). However, I will propose that, notwithstanding the fact that initial 'subject' DPs have to be definite in Arabic (as (3a) and (3b) show), the status of initial 'subject' DPs in SVO sentences as 'Topics' or mere 'subjects' is a question of micro-parameterization on the basis of which the derivation of subject-verb agreement configurations in the standard variety of Arabic differs from the derivation of subject-verb agreement configurations in the modern Arabic spoken dialects. Thus, I will propose that the 'subject' in such subject-initial 
sentences in SA (as in (2a)) is realized as a 'Topic' DP, the position of which is higher in the structure of sentences than the position occupied by subjects. In contrast, in TA SVO word order (as in (2b)), I will propose that the initial DP element is a 'subject' merged in the structural subject position in the IP domain.

\subsection{The Structure of IP and subject-verb agreement phenomena}

As is typical of VSO languages, Arabic (both the standard variety and the modern spoken dialects) is a null-subject language.

In section 2.1.1, I introduce the problem of the subject position in nullsubject/verb-raising languages. In section 2.1.2, I deal with the postulation of microparametric differences in the derivation of the VSO word order and the SVO word order in Arabic.

\subsubsection{The structural subject position of the clause in null-subject languages and the EPP}

Null-subject languages like Arabic and Italian exhibit some properties which set them apart from non-null-subject languages. ${ }^{2}$ As will be taken up presently (see (5) below), the most important property of null-subject languages is that they freely allow the subject of the sentence to be missing. The Italian sentences in (5a-b) (Belletti 2001) and the TA sentences in $(5 \mathrm{c}-\mathrm{d})$ are posited as having the respective partial structural

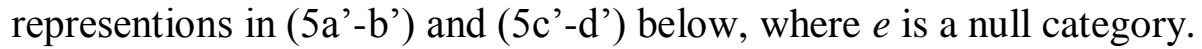
(5) a. Parla 'She/He speaks'
(Italian, Belletti 2001: 492)
b. Parlano 'They speak'
(Italian, Belletti 2001: 492)
c. yitkallam 'He speaks'
d. yitkalmuu 'They speak'
a'. [IP $e$ INFL [vp parla]] speak.3S
b'. [IP $e$ INFL [vp parlano]]
speak.3P

\footnotetext{
${ }^{2}$ TA and Italian pattern alike in contrast to SA in that the 'discourse neutral' word order in TA and Italian is SVO, not VSO as in Standard Arabic (see Mohammad 1989: 1, Fassi Fehri 1993: 39; Ouhalla 1991: 108, 114; Shlonsky 1997: 7).
} 


$$
\begin{aligned}
& \text { c'. [IP } e \text { INFL [vp yitkallam]] } \\
& \text { speak.3MS } \\
& \text { d'. [IP } e \text { INFL [vP yitkalmuu]] } \\
& \text { speak.3P }
\end{aligned}
$$

Although no element is phonologically realized as NP/DP (DP, henceforth) to the left of INFL in structures (5a'-d'), the subject of the clause is assumed to be present as a silent null element (or Empty Category (EC)) in the subject position of such structures (in this case, a referential null element in the absence of an overt argument DP in that position; ${ }^{3}$ cf. sentence (6) below for a non-referential counterpart).

The presence of an EC $e$ in the subject position is due to what Chomsky (1982: 10; 1986b: 116) calls the Extended Projection Principle or the EPP as "the requirement that clauses have subjects" (Chomsky 1982: 10). The EPP is a principle of the grammar in its own right as a 'formal' property of syntactic structure in natural languages linked to INFL (see also Holmberg 1999, Chomsky 2001, 2004, Roberts \& Roussou 2002, Roberts 2010a,b, Miyagawa 2010). The null element $e$ is now identified as pro, which can be a referential element as in (5a'-d') above, or a nonreferential pleonastic (or expletive) element (Chomsky 1982: 79, Chomsky, 1986a: 116, Chomsky \& Lasnik, 1993: 123). In either case, the important new proviso is that it needs to be case-marked (Rizzi 1986: 521-524 contra Chomsky 1981: 332-334). ${ }^{4}$ Chomsky (1982: 79) suggested that, in such examples as in (6) (Chomsky's (105b)), the EC (in this case as a non-referential expletive pro) "... functions essentially in the same way as the pleonastic element in the translation", as shown in the example Chomsky gives. This expletive pro, in null-subject languages, has essentially the same feature make-up as expletive there in non-null-subject languages: ${ }^{5}$

\footnotetext{
${ }^{3}$ As will be discussed in section 2.1.2.1 below, the referential subject (overt or null) raises out of [Spec, VP] - the VP-internal subject position.

${ }^{4}$ Rizzi (1986) discusses the licensing of pro (both referential and expletive pro in subject position and referential pro in object position) in Italian as a null-subject language.

${ }^{5}$ The translation of $e$ (or pro) as there in English is only available in some cases (unaccusatives and what Chomsky (1995a: 425-431) calls Transitive Expletive Constructions which are the counterpart of similar structures in Icelandic). For example, in the sentence in (ia), $e$ does not correspond to there in English:

(i) a. $e$ ha mangiato Giovanni has eaten Giovanni 'Giovanni ate.' b. '*There ate Giovanni'.

(Italian)
} 
(6) $e$ arriva un ragazzo

(Italian)

'There arrives a boy'

According to Chomsky (1981: 240), the clustering of properties that has given rise to the name of 'pro-drop'/null-subject parameter reduces to the first two of the five properties in (7): ${ }^{6}$

(7) (i) missing subject

(ii) free inversion in simple sentences

(iii) 'long wh-movement' of subject

(iv) empty resumptive pronouns in embedded clauses

(v) apparent violations of the $*$ [that-t] filter.

The property of 'missing subject' refers to the option of having an empty subject position where, nonetheless, a null category is inserted - namely, pro. The property of 'free inversion' refers to the option of having post-verbal subjects. These two properties are further reduced to one basic characteristic of null-subject languages - namely, the fact that they freely allow a pro element to occupy the structural subject position.

\subsubsection{The Derivation of VSO vs. SVO word orders and subject-verb agreement patterns in Arabic: micro-parametric differences}

After having reviewed in section 2.1.1 some basic assumptions about the derivation of the structure of sentences, I have a look, in section 2.1.2.1 below, at Koopman \& Sportiche's (1991) (cf. Sportiche 1998) view on case and agreement. Section 2.1.2.2 deals with the hypothesis developed in Benmamoun (1992a) (following Mohammad 1989) that a null expletive pro is responsible for explaining the different subject-verb agreement patterns in VSO sentences in SA as opposed to the modern spoken Arabic dialects. In relation to the SVO word order (section 2.1.2.3), I deal with the question of the topic-like nature of subjects in SA and the related problem of whether subject agreement markers on verbs are incorporated pronouns. Section 2.1.2.4 summarizes

As shown in (ib), the only grammatical output for the English counterpart of the Italian sentence in (ia) has the DP Giovanni itself in the subject position of the clause.

${ }^{6}$ The so-called Null Subject parameter (Rizzi 1982) is also known in the literature as the 'pro-drop' parameter (Chomsky 1982: 98, note 22; Jaeggli \& Safir 1989: 1). Since the 'null subject' option of this parameter is characteristic of a certain type of languages, the literature refers to these languages as null subject or 'pro-drop' languages. 
the main points reviewed in section 2.1.2 and suggests some implications for the proposal defended in this thesis as to the parametric character of the structural differences in question.

\subsubsection{Koopman \& Sportiche (1991) and Sportiche (1989/1998)}

Koopman \& Sportiche (1991: 212) hypothesize that, whereas the subject of a sentence may be overtly spelled out in [Spec, IP], it is inserted within the maximal projection of the verbal predicate (the VP-Internal Subject Hypothesis or the VISH) and then raises from [Spec, VP] to [Spec, IP] in "many (and perhaps all) languages". In addition to subject-raising, the verb - in many languages including Arabic - moves out of VP. In this raising process, it adjoins to INFL so that the structure of SVO clauses in the overt syntax, in these languages, is [IP Subject $t_{j} V_{i-I}-(N F L)\left[{ }_{v P} t_{j} t_{i j}\right]$, as represented in (8a) below (where $t_{i}$ is the trace left after V-movement to I and $t_{j}$ the trace of the raised subject). In VSO sentences, only verb-raising obtains so that the structure of the clause in the overt syntax, in these languages, is [IP $\mathrm{V}_{\mathrm{i}}-\mathrm{I}(\mathrm{NFL})$ [VP Subject $\left.\left.\mathrm{t}_{\mathrm{i}}\right]\right]$, as represented in $(8 \mathrm{~b})$ :

(8) a.

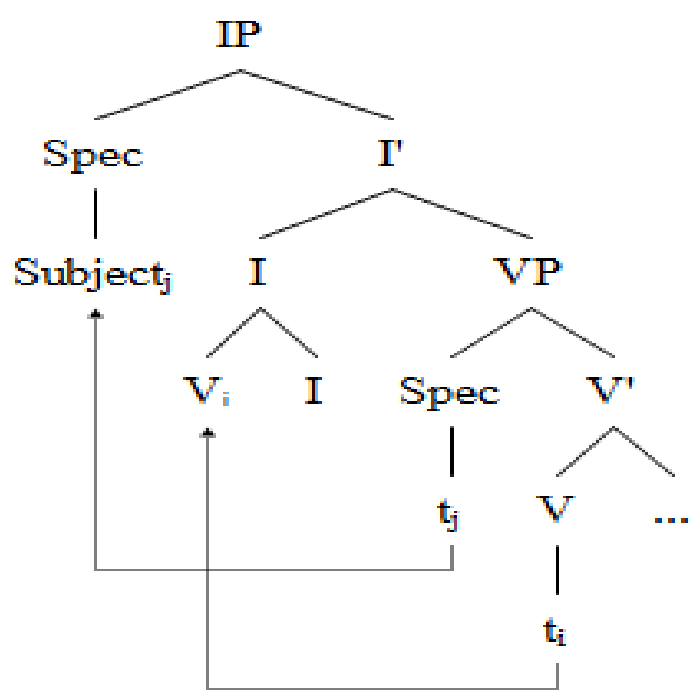


b.

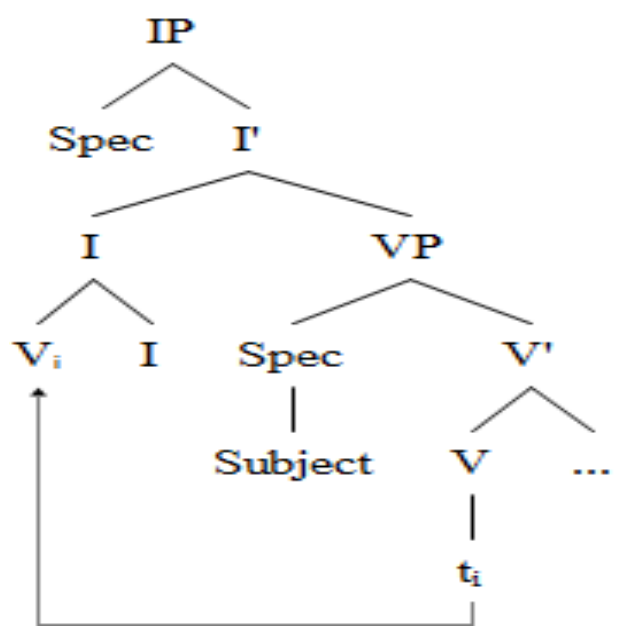

Subject-raising to the structural subject position of the clause would be driven by the need to satisfy some licensing condition on the derivation and representation of subject DPs in the grammar. The assignment of structural Case together with the satisfaction of the EPP justifies this raising process. Since the subject, raised to [Spec, IP] in (8a), enters into a Spec-head agreement relationship with INFL, Koopman \& Sportiche (1991: 228) take the Spec-head agreement pattern to be the structural configuration under which structural Case assignment takes place - namely, a tensed INFL assigns Case to its specifier, since "[a]greement is the morphological reflex of a relation between INFL and its specifier, or more generally between a head and its specifier" (Koopman \& Sportiche 1991: 221). ${ }^{7}$

\footnotetext{
${ }^{7}$ The Spec-head agreement pattern was formulated in the revised Government and Binding theoretical framework of Barriers (Chomsky 1986a) to explain the relation between a wh operator (or a trace) in [Spec, CP] and the head of CP. As Rizzi (1990: 51-52) notes, this agreement pattern was, by that time, generally assumed for the theory of abstract syntactic case. This Spec-head agreement pattern was viewed, in the literature, as an alternative view to claims about head-government, which operates in terms of m-command (Rizzi 1990: 6). For example, Rizzi (1990: 59) assumes that in a structure like the following (his (85)):

(i) L'homme que je crois [t Agr [ T être intelligent]]

"the Case feature originates in the main verb and is transmitted under government to Agr in Comp, which then assigns it to the subject trace". Nevertheless, Rizzi (1990: 93-94) maintains that no "actual transmission" is intended in the definition of agreement relations which follow the general agreement pattern of a head and a specifier (Rizzi, 1990: 51). Likewise, Chomsky's (1989: 159-160) definition of chain algorithms no longer refers to 'transmission' of case or $\theta$-role. The alternative explanation is formulated in terms of 'visibility' or what he calls the principle of Full Interpretation (FI). In Chomsky (1993, 1995c), visibility, the Chain Condition and FI are all subsumed under the morphological featurechecking account. In subsequent work (Chomsky 2001, 2004), it is assumed that agreement in case is only a by-product of the operation Agree and that no privileged Spec-head relation exists.
} 
With particular reference to SA, Koopman \& Sportiche (1991: 221) follow Mohammad (1989) in the assumption that when the subject, base-generated in [Spec, VP], fails to raise to the structural subject position [Spec, IP], as in (8b), a silent expletive is in [Spec, IP] ${ }^{8}$ In this case, [Spec, VP] becomes a NOM Case position, in which the subject kept in situ gets Case under government by INFL (Koopman \& Sportiche (1991: 227-228).

These ideas about the possibility of the co-existence of two different modes for the assignment of structural Case are developed in Sportiche (1998:162) by reference to the Strong Correlation Hypothesis (see to this effect the discussion of Sportiche's 1998 raising-to-object analysis in section 2.2.1 below). Sportiche (1998: 103) maintains that, although the assignment of structural Case (both NOM Case and ACC Case) via the Spec-head agreement relation and agreement in $\varphi$-features are assumed to be mutually related, the grammar of languages maintains the possibility of structural Case also being assigned under government.

However, Sportiche (1998: 172) differs from Koopman \& Sportiche (1991) in assuming that "when INFL is a Case assigner, it is a Case assigner by Agreement only". Thus, the structure of the clause in the VSO word order in SA, on these assumptions, is as in (9) below, where pro is a null expletive element that agrees with INFL (see section 2.1.1 for the legitimacy of positing such an element in sentence structure):

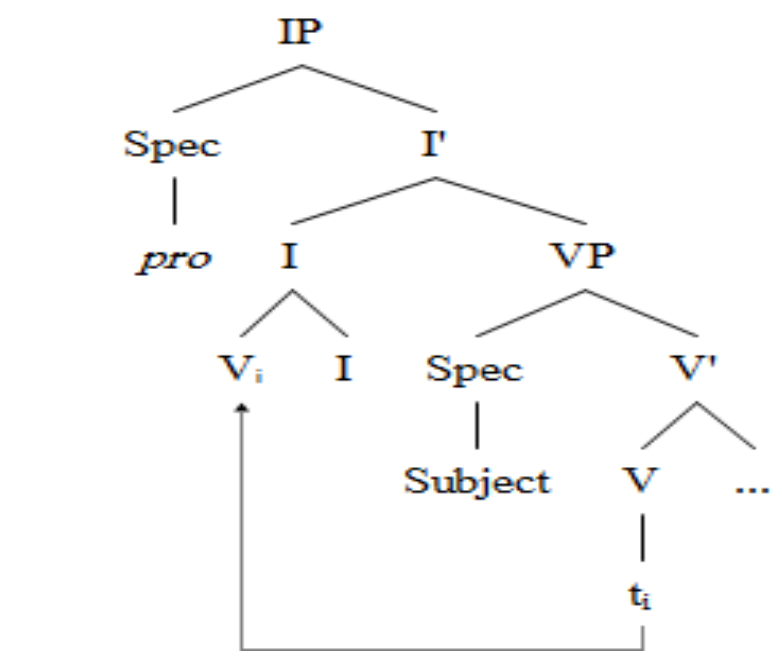

\footnotetext{
${ }^{8}$ I do not represent the expletive in structure (8b) above (in contrast to structure (9) under Sportiche's 1998 assumptions of 'Case inheritance', see below) because Koopman \& Sportiche (1991: 221) also allow [Spec, IP] to be missing altogether.
} 
In a structure like (9), Sportiche (1998) assumes that the VP-internally base-generated subject DP, in a VSO language like SA, is 'assigned' Case, not by INFL under government, but via a mechanism of 'Case transmission' which operates between the null expletive in [Spec, IP] - being Case-marked via Spec-head agreement with INFL to which V attaches - and the post-verbal subject kept in situ in [Spec, VP].

Koopman \& Sportiche's (1991) and Sportiche's (1989/1998) parameterization of the case-agreement system of languages in terms of Spec-head agreement and/or Case transmission is important in so far that it is the basis for Benmamoun's (1992a) analysis of subject-verb agreement in SA. Benmamoun (1992a) conceptualizes agreement in $\varphi$-features and Case between the subject and the verb, in SA, as exclusively obtaining under the Spec-head agreement relation. As already discussed in relation to the SA sentence (2a) above, such an agreement configuration realizing $\varphi$-features and Case obtains whenever there is an initial subject DP in the sentence in SA. In (10a) below, I provide a partially bracketed $\mathrm{SV}(\mathrm{O})$ structure contrasting subject-verb agreement in SVO sentences and subject-verb agreement in VSO sentences in SA, as shown in (10b):

$$
\begin{aligned}
& \text { (10) a. [IP l-Pawlaad- } \mathrm{u}_{\mathrm{j}}[\mathrm{V}-\mathrm{I}] \text { jaaP-uu } \mathrm{i}_{\mathrm{i}}\left[\mathrm{vP} \mathrm{t}_{\mathrm{j}} \mathrm{t}_{\mathrm{i}}\right] \text { ] } \\
& \text { the-boys-NOM came-3MP } \\
& \text { 'The boys came' }
\end{aligned}
$$

$$
\begin{array}{r}
\text { b. } \left.\left[\text { IIP }[\text { pro }]_{[\mathrm{V}-\mathrm{I}]} \text { jaa } \mathrm{Pa}_{\mathrm{i}} \text { [vP 1-Pawlaad-u } \mathrm{t}_{\mathrm{i}}\right]\right] \\
\text { came.3MS the-boys-NOM }
\end{array}
$$

'The boys came'

In (10a), full subject-verb agreement obtains since the fully inflected verb under I(NFL) - the head of IP - agrees in person and number with the raised subject in [Spec, IP]. As for the VS $(\mathrm{O})$ structure in $(10 \mathrm{~b})$, the verb fails to agree with the subject kept in situ. The result is that the verb bears a default set of $\varphi$-features $\left(3^{\text {rd }}\right.$ person singular). Thus, only in configuration (10a) does NOM Case assignment obtain simultaneously with agreement in $\varphi$-features under the Spec-head agreement relation. In (10b), however, the subject in [Spec, VP] fails to be assigned NOM case via the Spec-head agreement relation and, as a consequence, no agreement in $\varphi$-features arises. NOM Case is assigned to expletive pro in [Spec, IP] and is 'transferred' from 
pro to the subject in [Spec, VP]. Benmamoun (1992a) maintains that this 'transfer' does not depend on a government relation. ${ }^{9}$

In the following subsection, before proceeding to look at the controversy on the question of how the derivation of SVO structures arises in the grammar of Arabic, I discuss in more detail Benmamoun's (1992a) account of the differences in the subject-verb agreement configurations in VSO word order between the standard variety of Arabic and the modern spoken Arabic dialects.

\subsubsection{Arabic VSO word order: Benmamoun (1992a)}

As briefly reviewed in the introduction to this chapter, the SVO word order (exemplified by $(2 a, b)$ above) shows essentially the same agreement pattern in both SA and the modern spoken dialects, such as MA (Benmamoun 1992a: 20) and TA. In contrast, two different agreement patterns obtain in the VSO word order. The two VSO sentences in (11) and (13) - repeated from (1a,b)) -, and their corresponding syntactic structures in (12) and (14) (according to Benmamoun's (1992a) analysis) exemplify the proposed parametric difference between the standard variety of Arabic and the modern spoken dialects. Full subject-verb agreement in the modern spoken dialects obtains even when the subject fails to raise to the structural subject position in $\left[\right.$ Spec, IP]: $:^{10,11}$

(11) kataba

$$
\text { T-Tulaab-u d-dars-a }
$$

wrote.3MS the-students-NOM the-lesson-ACC

'The students wrote the lesson'

(12) [IP [pro] ${ }_{[\mathrm{V}-\mathrm{I}]} \mathrm{kataba}_{\mathrm{i}}$ [vP T-Tulaab-u t $\mathrm{t}_{\mathrm{i}} \mathrm{d}$-dars-a]]

(13) kitb-uu T-Tulaab d-dars $(\mathrm{TA}=(1 \mathrm{~b}))$ wrote-3P the-students the-lesson

'The students wrote the lesson'

\footnotetext{
${ }^{9}$ Later, in Benmamoun (1995), Benmamoun rejects this explanation in terms of 'Case transfer/transmission' in favour of expletive replacement at LF à la Chomsky (1989).

${ }^{10}$ The structure in (14) is based on the analysis of the agreement phenomena in SA and MA provided by Benmamoun (1992a). Benmamoun (1992a) does not provide a structure for the sentence he gives.

${ }^{11}$ Referring back to the comparative analysis of null-subject sentences in such languages as Arabic (SA and TA, for example) and Italian (see section 2.1.1 above), according to Rizzi (1990, note 26 to Chapter 2), there is an important word order difference with post-verbal subjects between Italian and Arabic (Bani Hassan Arabic in Rizzi's 1990 account) in that the unmarked word order with post-verbal subjects is VOS in Italian, whereas it is VSO in Arabic.
} 


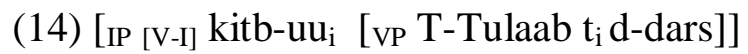

As discussed above, according to Benmamoun (1992a), the lack of full agreement in (11) follows from the assumption that the subject fails to overtly move to the structural subject position, thus allowing [Spec, IP] to be occupied by expletive pro, as represented in (12). In such a configuration, no Spec-head agreement relation is possible between the subject kept in [Spec, VP] and the verb which is marked with a 'default' number agreement. With reference to a sentence similar to (13), Benmamoun (1992a) assumes that expletive pro does not show up in [Spec, IP], as represented in structure (14). In such a structural configuration, agreement in $\varphi$ features (particularly, number agreement) and the assignment of NOM Case obtain under government by the inflectional head (to which the verb adjoins yielding the [VI] inflectional complex). Whereas in SA (structure (12)) NOM Case can be 'transferred' to the thematic subject in [Spec, VP] at S-structure, due to a Spec-head relationship between I (the head of IP) and a null expletive pro in [Spec, IP], no such 'transfer' is available in the TA structure in (14) since, as Benmamoun (1992a) argues, the expletive is absent. Instead, I functions as a governing head in the TA structure in (14), and as such it is able to Case-mark the subject DP in [Spec, VP]. ${ }^{12}$

The hallmark of Benmamoun's (1992a) analysis of the parametric difference between SA and the dialects is that, unlike in the dialects, number agreement in SA only obtains under the Spec-head agreement relationship. On this assumption, in the case where the subject is post-verbal in SA, agreement in number between expletive pro (assuming it to be $3^{\text {rd }}$ person) and the verb under I will fail since expletive pro lacks a number feature. By contrast, since expletive pro is precluded from being generated in the structural subject position in the dialects, the grammar compensates this lack by allowing both number agreement and NOM Case to obtain either via Spec-head agreement in the SVO word order, or under government in the VSO word order.

However, Benmamoun (1992a, footnote 10) notes that what remains unexplained on these assumptions is why the presence of a null expletive subject should be allowed in one null-subject language, but disallowed in another. ${ }^{13}$

\footnotetext{
${ }^{12}$ Recall that case morphology no longer shows on DPs in the Arabic dialects.

${ }^{13}$ Benmamoun (1997, footnote 4) considers the possibility of positing "a different type of expletive in the VSO word order in Moroccan Arabic and Standard Arabic", but rejects this possibility on the
} 
According to him, one possibility is that "in languages with the expletive, Case can only be assigned to Spec, IP and therefore the expletive is needed to discharge Case and transfer it to the post-verbal DP. In languages without expletives, Nominative can be assigned directly to Spec, VP”.

Benmamoun's (1992a) main standpoint in this analysis is that the difference in subject-verb agreement in VSO word order between the standard variety and the dialects cannot be elevated to the status of a parameter in its own right. Instead, he suggests analyzing it as an 'epiphenomenon' that has at its basis the general constraints of the grammar on referential dependency: if pro were allowed to be inserted in [Spec, IP] in the TA structure in (12) above, then it would have to be interpreted as a referential element not only agreeing with the verb, adjoined to I, via the Spec-head agreement relation, but also referentially dependent on the post-verbal subject in [Spec, VP]. ${ }^{14}$ A violation of Principle $\mathrm{C}$ of the binding theory ensues because of the binding relation between two referential elements, i.e., pro and the post-verbal subject, in the same local domain (i.e., the domain of verbal inflection proper). Therefore, Benmamoun (1992a) discounts viewing the differences in the realization of the subject position in SA and the modern spoken dialects in the VSO word order as emanating from a parameter of syntactic variation. By that token, Benmamoun (1992a) dismisses an agreement-based analysis of such phenomena and opts for considering case assignment/'transfer' and thematic considerations in sentence structure as the basis of the differences in question.

However, an agreement-based analysis of such phenomena could still be entertained whereby the problem of referential dependency in Benmamoun's account no longer arises. ${ }^{15}$ Miyagawa (2010) views the relation between the element in [Spec, IP] and [Spec, VP] as an agreement/movement relationship based on Agree. ${ }^{16}$ Agree

grounds that there is no independent motivation for such an expletive. Aoun et al. (1994: 200-1) also reject the assumption of there being a fully specified expletive pro with which the verb agrees in the modern colloquial Arabic dialects.

${ }^{14}$ Benmamoun (1992a) refers to Chomsky (1986b) where binding entails referential dependency.

${ }^{15}$ See also Roberts \& Roussou (2002) for a conceptually similar analysis based on the notion of Tensedependency (see Chapter Three).

${ }^{16}$ In Miyagawa's (2010) account, the relation of agreement/movement is between [Spec, TP] and [Spec, $v^{*} \mathrm{P}$ ] and their respective heads $\mathrm{T}$ and $\mathrm{v}^{*}$. T is a notational variant of I(NFL) under an account of sentence structure that does without the Split-IP hypothesis (Chomsky 1995b, 2001, 2004; see Chapter Three). Under the same account, the structure of VP is more complex with a lower V/ VP and a higher 
is a derivational operation built up into the Merge and Move components of the grammar (Chomsky 2001, 2004; see section 2.3.2. below). Agree establishes an agreement relation between a Probe and a Goal introduced into phrase structure irrespective of whether movement is involved or not. By reference to the 'KoopmanSportiche (1991) intuition' - i.e., moving the external argument DP into [Spec, TP] for case-agreement -, Miyagawa (2010: 9) states that: “... agreement requires a Spechead relation [in] that Agree takes place to establish functional relations". He adds:

The purpose of this movement is to keep a record of the functional relation beyond narrow syntax so that semantic interpretation and information structure make use of it. This, in effect, is Spec-head agreement but implemented as two independently motivated operations - Agree and Move.

This is what Miyagawa (2010: 4) calls the 'agreement approach to the EPP'.

According to this approach, agreement is responsible for the kind of movement that establishes functional dependency relations between the core functional heads projected in sentence structure and their respective Spec positions, or what Miyagawa (2010: 7) calls 'Probe-Goal Union' - namely, “a goal moves in order to unite with a probe". The only purpose of these dependency relations is the satisfaction of the EPP, i.e., the relation between a functional head and some XP. ${ }^{17}$ Thus, the relation between agreement and case, as in Koopman \& Sportiche (1991), could be revised in terms of a more fundamental relation between agreement and the checking of an EPP-feature (see also Lasnik 1995, 2001 and Rothstein's 1995 predication analysis of the EPP).

In light of this generalized Spec-head agreement and EPP-driven movement analysis of dependency relations obtaining in verb-initial structures in languages that interchangeably allow VSO and SVO word orders, I will now have a look at how

v/vP (see section 2.2. below for Chomsky's 1995a,b early assumptions about the 'light' verb $v$, and section 2.3.2. for the difference between 'light' verb $v$ and 'light' verb $v^{*}$ ).

${ }^{17}$ The null category pro (both referential and expletive) could, in principle, be an instance of this XP, which would agree with the functional head (INFL) and, by doing so, satisfy the EPP. A case in point is Miyagawa's (2010: 52) suggestion that Chinese 'pro-drop' instances where "small pro" (the term is Miyagawa's) is specified with a 'person' feature (accounting for lack of verbal agreement inflection in the language). The existence of these 'pro-drop' cases may be confirmation that the 'PGU' applies universally. A similar suggestion (Miyagawa 2010: 47) is presented for Romance and (Bani-Hassan) Arabic, where "person agreement at T licenses pro-drop". 
subject-initial structures in Arabic have been analysed in the literature according to two main approaches: the Subject Theory and the Topic Theory.

\subsubsection{Arabic SVO word order}

As noted in the introduction to this chapter in relation to sentences $(2 a, b)$ (repeated below as $(15 \mathrm{a}, \mathrm{b}))$, the SVO word order shows essentially the same agreement pattern in both SA and the Arabic dialects such as TA where subject-verb agreement fully obtains:

$\begin{array}{lll}\text { T-Tulaab-u } & \text { katab-uu } & \text { d-dars-a } \\ \text { the-students-NOM } & \text { wrote-3MP } & \text { the-lesson-ACC }\end{array}$

'The students wrote the lesson'

$\begin{array}{lll}\text { b. T-Tulaab } & \text { kitb-uu } & \text { d-dars } \\ \text { the-students } & \text { wrote-3P } & \text { the-lesson }\end{array}$

'The students wrote the lesson'

In the literature pertaining to the derivation of subject-initial sentences in SA, as exemplified by such an SVO sentence as in (15a) above, two approaches have been put forward as to the position of the subject: one is the Subject Theory (Mohammad 1989, Benmamoun 1992a, 2000b, Aoun et al. 2010) and the other is the Topic Theory (Akkal \& Gonegai 2000, Soltan 2006, and Fassi Fehri 1993, 2000). ${ }^{18}$

I first have a look, in section 2.1.2.3.1, at the Subject Theory. In section 2.1.2.3.2, I review the main morpho-syntactic arguments in support of the Topic Theory (mainly according to Akkal \& Gonegai 2000). Section 2.1.2.3.3 considers some morpho-phonological evidence (mainly from Fassi Fehri 1993, 2000) for subjects as Topics in SA and the status of subject-agreement affixes as incorporated pronouns.

\subsection{The Subject Theory (Mohammad 1989, Benmamoun 1992a, 2000b, Aoun et al. 2010)}

For Mohammad (1989) and Benmamoun (1992a, 2000b), Aoun et al. (2010), the 'externalized' DP T-Tulaab- $u$ in the SA sentence in (15a) is essentially a 'subject' in the usual subject position of the clause, i.e., [ Spec, IP]/[Spec, TP], as represented in (16):

\footnotetext{
${ }^{18}$ Fassi Fehri $(1993: 16,18)$ maintains that SVO structures with the subject moved to [Spec, IP] also exist independently of clitic left-dislocated structures where the initial, subject-related DP element, is considered to be a 'Topic'.
} 
(16) SA SVO (Mohammad 1989 and Benmamoun 1992a, 2000b, Aoun et al. 2010) [IP T-Tulaab- $u_{j[V-I]}$ katab-uu [vP $_{\mathrm{j}_{\mathrm{j}}} \mathrm{t}_{\mathrm{i}} \mathrm{d}$-dars-a]]

According to the Subject Theory, the 'subject' DP T-Tulaab- $u$, in (16), is raised out of its base position internal to VP (i.e., the VP-internal subject position), as discussed in 2.1.2.1 above.

As Aoun et al. (2010: 50) propose, in SVO sentences in Arabic, “... as far as the status of the preverbal subject is concerned, there is no difference between Arabic and English/French". Although Aoun et al. (2010: 52) acknowledge that in such SVO instances the subject could theoretically be supposed to be in a higher projection above the verb in TP/IP, they question the hypothesis that parametric variation has anything to do with the syntax of subjects. In particular, Aoun et al. (2010: 57-61) argue against the view that the position of preverbal subjects in Arabic could vary on the basis of the agreement asymmetry that shows up in VSO sentences vs. SVO sentences in SA, but not in the modern spoken dialects, as reviewed at the beginning of this chapter. Thus, Aoun et al. (2010) maintain that the representation of the basic structure for subject-initial sentences is essentially the same in Arabic (SA and the modern spoken dialects) as in other languages.

According to Aoun et al. (2010: 61), the main challenge to the hypothesis that initial 'subject' DPs are actually Topic or clitic-left dislocated elements comes from the context where an auxiliary verb and a lexical verb are used in the same sentence as in (17) below:

(17) kaan-at T-Taalibaat-u ya-drus-na PERF.be-3FS the-students(F)-NOM 3-IMPERF.study-FP 'The students were studying.'

In (17), the auxiliary verb kaanat 'was/were' is perfective/past and is marked for 'partial' agreement (i.e., third person feminine singular in (17)), but the lexical verb yadrusna 'study/studying' is imperfective and marked for full agreement (i.e., third person feminine plural). Aoun et al. (2010: 61) argue that the DP TTaalibaatu 'the female students' is presumably a subject DP in some Spec position between TP (the head position of which the auxiliary verb kaanat occupies) and the lexical verb yadrusna is in VP. However, this analysis is only tenable if we assume that the 
sentence in (17) is basically mono-clausal, i.e., that both the auxiliary verb and the lexical verb are in the same clause. ${ }^{19}$

In this thesis, extending on Akkal \& Gonegai's (2000) analysis of verbal inflection in subject-initial sentences in Arabic whereby the standard variety and the modern spoken dialects of Arabic differ parametrically as to the status of verbal inflection, I propose that the differences between the standard variety and the modern spoken dialects of Arabic are not confined to how the basic structure of subject-initial sentences is derived and/or how subject-verb agreement in SVO sentences arises in the syntax. Rather, I propose that there are parametric differences between SA and the modern spoken dialects, which also concern the derivation of $\mathrm{V}$-initial sentences.

The proposal I am putting forward here is that the subject DP is systematically represented in the IP domain in a spoken modern dialect like TA or MA (whether the derived word order is VSO or SVO, as the two main word order manifestations in Arabic). In contrast, the derivation of sentences in SA systematically involves the splitting-up of the CP domain (Rizzi 1997) in both VSO and SVO word orderings. This proposal will account for the differences in the derivation of 'subject' DPs in SA as opposed to the modern spoken Arabic varieties such as TA and MA in relation to the extent to which the $\mathrm{CP}$ domain is involved in the derivation of these sentences. In TA (as a sample example of a modern spoken Arabic dialect in this thesis), my hypothesis is that the CP domain in its split form, à la Rizzi (1997), is never involved. In this conception, TA systematically projects the structure of IP as $\operatorname{Agr}_{S} \mathrm{P}$ and both the subject and the verb are systematically bound to that domain, which would account for the absence of the agreement asymmetry in TA or MA. ${ }^{20}$

\subsection{The Topic Theory (Akkal \& Gonegai 2000, Fassi Fehri 1993,2000, Soltan 2006)}

The plausibility of treating subject-initial DPs as 'Topics' in SVO sentences in SA can be argued for on the basis of Akkal \& Gonegai's (2000) analysis of subjunctive

\footnotetext{
${ }^{19}$ I return to a different view maintaining a bi-clausal structure to such examples in SA in section 3.3.2.3 in my discussion of Benmamoun's $(2000 \mathrm{~b}, 2008)$ analysis of verbal inflection in SA. See also section 4.1.2.5.3 for Akkal \& Gonegai's (2000) argument for a bi-clausal structure for sentences similar to (17).

${ }^{20}$ See Chapter Three, mainly, for the hypothesis that the structure of IP projects as $\operatorname{Agr}_{S} \mathrm{P}$ in the relevant constructions/languages.
} 
clauses, as in (18) below, modal clauses, as in (19), and interrogative clauses, as in (21) (adapted from Akkal \& Gonegai's 2000: 8-9 sentences (24a-c)).

Sentence (18) below configures a lower clause embedded under a subjectinitial matrix clause:

(18) Par-rijaal-u Puriidu Pan y-akhruj-uu

the-men-NOM want(I) that 3-go.out-MP.SUBJ(unctive)

'The men, I want them to go out.'

In (18), the DP Par-rijaal- $u$ is topicalized across a clause embedded under the verb Puriidu 'I want'. The 'topicalized' DP is external to the embedded subjunctive clause ?an $y$-akhruj-uu '...that (the men) go out' to the left of the inflected verb ?uriidu 'I want' in the matrix clause. Still, the relationship between the inflected verb $y$-akhruj$u u$ in the embedded clause and the clitic left-dislocated DP Par-rijaal- $u$ obtains, signalling that this relationship is established on the basis of what Akkal \& Gonegai (2000) consider to be a resumptive pronoun, i.e., the subject agreement inflection - $-u и$ on the verb $y$-akhruj-uu. Thus, what is important for Akkal \& Gonegai (2000: 7-8) is the pronoun status of the morpheme [uu] in (18), or the morpheme [uuna] in (19) below, which they consider as 'a fully-fledged $\mathrm{Agr}_{\mathrm{S}}$-pronoun'.

In contrast to SA, which "presents a discrepant picture in the sense that the verbal agreement morphology seems to turn on the relative order of the subject and the verb at the surface level" (Akkal \& Gonegai 2000: 4), the relative order of the subject and the verb in a modern spoken Arabic dialect like MA or TA does not affect the morphological manifestation of subject-verb agreement (see examples (10a) vs. (10b) contrasting the $\mathrm{SV}(\mathrm{O})$ word ordering to $\mathrm{VS}(\mathrm{O})$ word ordering in $\mathrm{SA}$, and examples (11) vs. (13) contrasting the $\mathrm{VS}(\mathrm{O})$ word ordering in $\mathrm{SA}$ to the $\mathrm{VS}(\mathrm{O})$ word ordering in TA). As Akkal \& Gonegai (2000: 19) propose, this discrepancy in the representation of subject-verb agreement in SA vs. a modern spoken dialect such as MA is the basis for treating 'SA-type' languages (which would include Celtic languages as regards the Topic-like nature of subject-initial sentences) as different from 'MA-type' languages (including, for example, MA and Spanish). 
Sentence (19a) and its ungrammatical counterpart (19b) present another argument in favour of considering sentence-initial DPs as Topics: ${ }^{21}$

(19) a. ?ar-rijaal-u qad y-akhruj-uuna

the-men-NOM perhaps 3-go.out-MP.IND(icative)

'The men, they may go out.'

b. *qad Par-rijaal-u y-akhruj-uuna

The topicalized/clitic left-dislocated nature of the initial DP in (19a) and (19b) can be accounted for by reference to Rizzi's (1997) split-CP system, where there is a Finiteness (Fin) node expressing a specification of finiteness, ${ }^{22}$ generally reflecting "inflectional properties" linking it to Inflection in the IP domain (Rizzi 1997: 284; see Chapter Three). In Rizzi's (1997) system, there is a Topic node (Top) higher than Fin and another one higher than Focus (Foc), which is higher than the lower Top position (in Rizzi's 1997 system) above Fin, as represented in (20) below:

(20) [CP ... (Top) (Foc) (Top) (Fin) [IP ...]]

The Top node that is relevant in the account of topicalized/clitic left-dislocated initial DPs in SA SVO sentences in (19a) and (19b) is the Top node that is higher than Foc in $(20)$.

Thus, in (19a) (vs. the ungrammatical (19b)), the modal verb particle qad is presumably merged in a node to the left of the IP domain, on the assumption that the inflected verb raised out of $v^{*} \mathrm{P} / \mathrm{VP}$ is under $\mathrm{T}$ (itself raised to $\operatorname{Agr}_{\mathrm{S}}$ ). ${ }^{23} \mathrm{On}$ this

\footnotetext{
${ }^{21}$ I will follow Akkal \& Gonegai (2000), contra Fassi Fehri (2000), in not indicating the morpheme status of the indicative mood marker [na], in (19), which should be part of the agreement marker dominated by $\mathrm{Agr}_{\mathrm{S}}$ in sentence structure.

${ }^{22}$ Although Akkal \& Gonegai (2000) do not refer to the split-CP system, they do refer to a possible Modal Phrase projection higher than $\operatorname{Agr}_{S} \mathrm{P}$ (see section 4.1.2.2 on the position of ModalP in the splitCP system adopted in this thesis).

${ }^{23}$ Besides the respective positions of the verb and the modal particle qad to its left, another argument for the left-peripheral position of qad is Cinque's (1999: 78) account of modality. Although Cinque (1999) is only concerned with an extended IP domain, his suggestion that 'Mood' and 'Modal' could be expressed via the same category as moods in some languages (or language types), but not in others, is reflected in the fact that modals are "typically independent words", in contrast to moods "which are expressed in verbal morphology (Palmer 1986, 21)". As far as the derivation of the sentence structure of SA - as conceived here - is concerned, 'Mood' and 'Modal' are manifested as two distinct categories. Thus, it is plausible to propose that 'modals', as verbal particles such as SA qad, are not generated in the IP domain, but are merged in some node in the split-CP domain. In this connection, Rizzi's (1997) assumption that Fin is related to Inflection in the IP domain makes Fin a suitable candidate for hosting such verbal particles as SA qad. I return to a discussion of the possible Merge site of particle qad in section 4.1.2.1 in relation to the T-to-Fin raising process in SA VSO sentences.
} 
account, qad might be assumed to be merged in Fin (or, possibly, in a Modal node in between Fin and Top; see section 4.1.2.1) and it follows the DP ?ar-rijaal- $u$ in Top to the left of Foc.

The analysis of sentence (21) below in terms of sentence-initial DPs as Topics likewise relies on Rizzi’s split-CP system:

(21) Par-rijaal-u maadhaa Rakal-uu?

the-men-NOM what ate-MP

'The men, what did they eat?'

In (21), the same cartography of positions as shown in (20) applies to account for the topicalized/clitic left-dislocated position of the DP Par-rijaal-u under Top to the left of Foc, the Spec position of which the wh-word maadha moves to.

However, the advocates of the topic nature of the subject in initial position, in SA, do not uniformly agree as to the position of the Topic DP in sentence structure and the nature of the highest functional projection in the IP domain. For Akkal \& Gonegai (2000) and Fassi Fehri (1993, 2000), the subject is in a 'topicalized'/clitic leftdislocated position outside IP/TP, as represented in (22a) and (22b), respectively.

(22) SA SVO (Akkal \& Gonegai 2000 and Fassi Fehri 1993, 2000)

a. (Akkal \& Gonegai 2000)

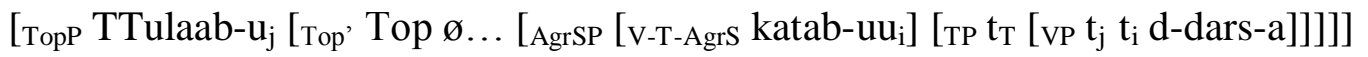

Cinque (1999: 78) further suggests that different types of moods and 'modals' could be distinguished, but the peculiarity of modals is that their interpretive distinction is correlated with a structural one in that "[e]pistemic modals are located higher in clausal structure than root modals, in fact higher than $\mathrm{T}$ (Past)/T(Future) (and negation), apparently". This hierarchical structural distinction between 'moods' and 'modals', and also between different kinds of modals, may be another justification for locating the Arabic verbal particle qad in the left periphery portion of sentence structure, as I suggest above. Still one additional argument for the high structural status of qad is that negation cannot precede this particle, but can only follow it, as suggested by the contrast in grammaticality in (i) and (ii) below:

(i) qad laa y-a?ti

perhaps not 3-come

(ii) *laa qad y-a?ti

not perhaps 3-come

'He is perhaps not coming'

As the grammatical (i) shows, not only is qad higher than the tensed verb, but it is also higher than negation. In view of this structural hierarchy, Fassi Fehri's (1993: 30) suggestion that Negation as a functional head selects IP as a complement in SA may be a further argument as to the left-peripheral position of the modal head qad in (19a) in the text above and in the sample examples mentioned in this footnote. 
b. Fassi Fehri $(1993,2000)$

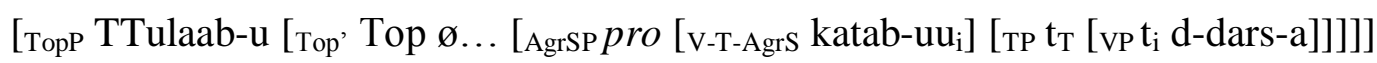
In a structure like (22a), Akkal \& Gonegai (2000) assume that the topic DP is moved outside the IP domain, projected as $\operatorname{Agr}_{S} \mathrm{P} .{ }^{24}$ In this relation, Akkal \& Gonegai (2000: 10) assert that: "What appears to be [in SA] an SV sequence on the surface must, therefore, be interpreted as a structure involving a topic". Akkal \& Gonegai (2000) also maintain that the $-u u$ subject morpheme/pronoun in (22a) cannot be reduplicated in $\left[\mathrm{Spec}, \operatorname{Agr}_{\mathrm{S}} \mathrm{P}\right]$ (which does not project) by the DP T-Tulaab- $u$, and that referential pro is not a necessary syntactic element in SVO structures in Arabic. By contrast, in a structure like (22b), Fassi Fehri (1993, 2000) assumes that the 'subject' DP is basegenerated as a 'topic', and that referential pro has to be generated in sentence structure.

The suggestion that the relevant Top head the Spec position of which the DP Par-rijaal- $u$, in a sentence like (21) above, occupies is the Top node that is higher than the Foc node has to do with the fact that a Topic can only precede a focalized element and cannot follow it, as the ungrammaticality of the sentence in (23) below testifies:

(23) *maadhaa Par-rijaal-u Pakal-uu? what the-men-NOM ate-3MP

'The men, what did they eat?'

The only way for the DP Par-rijaal-u to follow the wh-word maadhaa is to come in a final position in the sentence as in (24) below:

(24) maadhaa Pakala Par-rijaal-u?

what ate.3MS the-men-NOM

'what did the men eat?'

In (24), if the DP Par-rijaal- $u$ is in [Spec, TP] - the highest Spec position in SA VSO word order, as I will maintain later (see Chapter Three) -, then the morphologically 'impoverished' verb Pakala 'ate' should be in some node in the Split-CP domain, in Rizzi's (1997) system. This head node could either be Foc or Fin. I come back to the position of the verb in VSO sentences in section 4.1.2.1.

The examples in (18), (19) and (21) - and, to a certain extent, the grammaticality contrast in (19a) vs. (19b) in light of the evidence suggested in footnote 23 for such modal particles as qad and inflectional heads such as Negation

\footnotetext{
${ }^{24}$ Fassi Fehri's (2000: 89, note 20) analysis - based on the distribution of affixes - is neutral between the projection of sentence structure as IP/TP or as $\operatorname{Agr}_{S} \mathrm{P}$.
} 
laa - points to the plausibility of a Topic-based analysis of subjects in subject-initial sentences in SA. This is particularly interesting since, at first sight, verbal morphology in such SVO contexts, does not seem to help in arguing for either the Topic Theory or the Subject Theory (but see section 2.1.2.3.3. below).

A third advocate of the Topic Theory is Soltan (2006). Soltan (2006) assumes that an SA SVO sentence has the structure shown in (25) below:

(25) SA SVO (Soltan 2006)

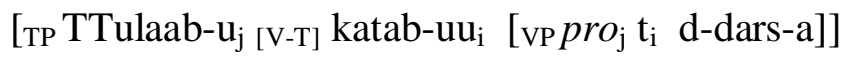

Soltan (2006) assumes that the structure of an SVO sentence in SA is essentially a TP, as represented in (25). He maintains that the 'subject' DP is a Topic base-generated in $[\mathrm{Spec}, \mathrm{TP}]$ co-referential with a referential pro in $[\mathrm{Spec}, \mathrm{VP}] .^{25}$

Thus, with the exception of Soltan (2006), who argues that the Topic DP is base-generated in the highest Spec position in the IP domain (i.e, it is base-generated in [Spec, TP], co-referential with a resumptive pro in [Spec, VP]), Fassi Fehri (1993, 2000) and Akkal \& Gonegai (2000) argue that 'topicalized' subject DPs are merged within a TopP projection in the CP domain, but according to different assumptions. One the one hand, under Fassi Fehri's (1993, 2000) approach to 'topicalized' DPs, allowing [Spec, IP/Agr $\mathrm{P}$ ] to be occupied by referential pro would account for the canonical subject position of the clause. On the other hand, Akkal \& Gonegai's (2000) contention is that the pronominal nature of $\operatorname{Agr}_{S}$ is sufficient to account for the canonical subject position and $\mathrm{Agr}_{\mathrm{S}}$ need not project a Spec position. For them, the existence of a resumptive pro in the IP domain in such SVO instances is dismissed. Under their analysis, as the Topic DP is moved out of $\operatorname{Agr}_{S} \mathrm{P}$ (in a Split-IP system), it A'-binds the trace it left behind in [Spec, VP].

I represent these different assumptions in Table 2.1 below (which also includes the assumptions of the Subject Theory (Mohammad 1989 and Benmamoun 1992a) for the sake of comparison):

\footnotetext{
${ }^{25}$ Ouhalla (1991) also assumes that, in the SA SVO word ordering, subjects are Topic DPs basegenerated in [Spec, TP]. I postpone my discussion of Ouhalla's (1991) assumptions on the derivation of SVO sentences vs. VSO sentences in SA to section 3.3.4.
} 
Table 2.1. Subject-initial sentences in SA:

\begin{tabular}{|c|c|c|c|c|}
\hline & & IP structure & $\begin{array}{l}\text { Nature of DP in } \\
\text { initial position }\end{array}$ & $\begin{array}{l}\text { Availabilty of } \\
\text { referential pro }\end{array}$ \\
\hline \multirow{3}{*}{$\begin{array}{l}\text { The Topic } \\
\text { Theory }\end{array}$} & $\begin{array}{c}\text { Fassi Fehri } \\
(1993,2000)\end{array}$ & $\begin{array}{l}\text { Either } \mathrm{Agr}_{S} \mathrm{P} \text { or } \\
\text { IP/TP (with no } \\
\text { theoretical } \\
\text { difference) }\end{array}$ & $\begin{array}{l}\text { Topic (base- } \\
\text { generated } \\
\text { outside the IP } \\
\text { domain) }\end{array}$ & $\begin{array}{c}\text { Pro base- } \\
\text { generated in } \\
{[\text { Spec, VP] and }} \\
\text { moved to }[\text { Spec, } \\
\text { IP/Agr } / \text { P }]\end{array}$ \\
\hline & $\begin{array}{c}\text { Akkal \& } \\
\text { Gonegai } \\
(2000)\end{array}$ & $\begin{array}{c}\operatorname{Agr}_{S} \mathrm{P} \\
\text { (Specifierless) }\end{array}$ & $\begin{array}{c}\text { Topic raised out } \\
\text { of [Spec, VP] to } \\
\text { a position } \\
\text { outside } \mathrm{Agr}_{S} \mathrm{P}\end{array}$ & $\begin{array}{c}\text { Unavailabilty } \\
\text { of pro }\end{array}$ \\
\hline & $\begin{array}{l}\text { Soltan } \\
(2006)\end{array}$ & $\mathrm{TP}$ & $\begin{array}{l}\text { Topic base- } \\
\text { generated in } \\
{[\text { Spec, TP }]}\end{array}$ & $\begin{array}{c}\text { Pro base- } \\
\text { generated in } \\
{[\text { Spec, VP], co- }} \\
\text { referential with } \\
\text { Topic in [Spec, } \\
\text { TP] }\end{array}$ \\
\hline $\begin{array}{l}\text { The Subject } \\
\text { Theory }\end{array}$ & $\begin{array}{c}\text { Mohammad } \\
(1989) \\
\& \\
\text { Benmamoun } \\
(1992 \mathrm{a})\end{array}$ & IP & $\begin{array}{c}\text { Basically, a } \\
\text { Subject }\end{array}$ & $\begin{array}{l}\text { Unavailability } \\
\text { of pro in simple } \\
\text { SVO sentences }\end{array}$ \\
\hline
\end{tabular}

\subsection{Morpho-syntactic and morpho-phonological evidence for the topicalized/clitic left-dislocated nature of subjects in SA}

Another kind of argument for subject-agreement affixes as 'incorporated pronouns' and for the topicalized/clitic left-dislocated nature of subjects in SA comes from morphosyntax.

In his morpho-syntactic analysis of subject-verb agreement configurations, Fassi Fehri (1993, 2000) argues for the pronominal nature of 'affixes' or 'agreement markers' on verbs in SA. Fassi Fehri $(1993,2000)$ takes it that these 'affixes' and other inflectional-morphemes (or i-morphemes, for short) represent designated positions in sentence structure to which the verb moves (for checking purposes, à la Chomsky 1995c, Chomsky \& Lasnik 1993) resulting in a bracketing structure that 
morpho-phonological rules at PF can handle. Fassi Fehri (1993, 2000) maintains that when verbs in SA are selected for the Numeration they are already derived with some form of 'inflection' - namely, Tense/Aspect and Voice. It is the role of the syntax to further manipulate verbs by linking them to the designated inflectional positions in syntactic structure.Thus, in the spirit of Chomsky's (1995c) and Chomsky \& Lasnik's (1993: 20) arguments against a strong version of 'lexicalism' in syntax (see also Lasnik 1999, Chapter 5), Fassi Fehri $(1993,2000)$ argues for a weak lexicalist view of the derivation of SA verbs.

The strong lexicalist view of inflectional processes maintains that there is no difference, in principle, between inflectional and derivational processes as both are carried out in the lexicon independently of the syntax. As for the weak lexicalist view of inflectional processes, it maintains that verbs are already fully inflected when they are selected for the Numeration, but it is the role of the syntax to associate the inflectional properties of verbs with appropriate 'hosts' or 'functional heads' that 'check' their own properties against the properties of the lexical items (mainly, verbs and argument DPs). This is the 'checking theory' of inflectional morphology (see section 2.3 below).

Under the checking account of inflection (Chomsky \& Lasnik 1993: 20), a lexical item like the verb walk, in English, could be syntactically derived according to two possibilities. One possibility is that the verb walk (as a root [walk]) and an inflectional feature of [tense] (one value of which is [past]) are taken from the lexicon as separate morphemes. Then, computational processes join the two items together. Another possibility is that "properties internal to the lexicon (...) form the word walked with the properties [walk] and [past] already specified", and then 'checking' applies to the feature [past]. Either way, the view that is adopted here is that inflectional properties of verbs such as [past] are "checked against abstract features of the functional heads (rather than acquired as affixes)" (Lasnik 1999: 102).

According to Fassi Fehri (1993: 76), the strong lexicalist view of inflectional morphology in Arabic has it that "there is no bare (stem) form of the verb with which $\mathrm{T}$ and other i-morphemes can concatenate to form a conjugated verb”. Arguing against this view, he maintains that Arabic verbs cannot be taken to be unconcatenated constituents formed wholesale out of the syntax and that a weak lexicalist, stem-based, analysis of verbal morphology is necessary. As in the checking theory of Chomsky (1995c), Fassi Fehri allows a necessary degree of lexicalism 
where the derivation of verbs and other inflected words is stem-based. According to this view, when lexically selected for the Numeration prior to syntactic derivation, Arabic verbs are already inflected for tense, aspect and voice. For example, for the Arabic verbal root $d x l$ 'enter/go in', the forms in (26) below would have to be inserted in the syntax with the inflectional information about aspect, tense and voice: (26) a. dakhal 'entered': past tense (PERF), active voice

b. y-adkhul '3-enters': non-past tense (IMPERF), active voice

A more convincing piece of evidence that Arabic verbal inflectional processes - in particular, and word formation in general - are only 'partially' concatenative' comes from such examples as in (27) below, where Arabic words include a number of operators and connectives, such as tenses, determiners, coordinators and also question and intensification markers:

(27) a. sa-y-aDrib-uu-na-hu

FUT-3-beat-MP-IND-him

'They will beat him'

b. la-sa-t-ajid-u-nna: 'indeed-will-you-find-IND-(emphatic)' (you will find indeed)

c. Pa-wa-jalas-ta?: 'Q-and-sit(PERF)-you?' (please, could you sit down)

d. fa-l-walad-u: 'and-the-boy-NOM' (and the boy)

e. bi-quluub-i-him: 'in-hearts-GEN-their' (in their hearts)

These examples could suggest that the formation of verbs/words is a concatenative, linear process done largely in the syntax. In particular, the examples in (27a-c) take the form of a word, but, syntactically, they are sentences.

Adopting a 'checking' view of verbal inflectional morphology, I follow Fassi Fehri $(1993,2000)$ in the assumption that the morphemes attached to the form [y$\mathrm{aDrib}$ ], in (27a), have to be derived in the syntax, each with its own inflectional projection. As for the form $y$-aDrib '3-beat', it could be assumed that this form represents a morphological merger inserted in the syntax as it is.

As Fassi Fehri (2000: 88, footnotes 18 and 19) notes, the person morpheme and the tense morpheme are fused in one single morpheme, which is part of the stem so that the hyphenated form $\boldsymbol{y}$-aDrib in (27a) - a combination of the root form [Drb], the person morpheme $y$ and the vocalic melody [...a...i...] - is morpho-phonologically handled by the syntax as an indivisible entity $\boldsymbol{y a D r i b}$ as evidenced from the vocalic 
alternation of the $/ a /$ and $/ u /$ sounds in the verbal stems in the examples in (29) below. The $/ a /$ and $/ u /$ sounds in the vocalic melody [...a......] (in $(24 \mathrm{a}, \mathrm{b}))$, or [ ...u...a/i...] (in $(29 c, d)$ ) differentiate, respectively, the active from the passive voice in both the imperfective and the perfective forms of the verb in SA. The tree diagram in (31) below schematically shows this process.

The sample sentences in (28) and (29), from English and Arabic respectively (adapted from Fassi Fehri (1993: 78)) show that, under the checking view of verbal inflectional morphology, there is no lexical equivalent of the English stem opens/opened in (28) as compared with the counterpart Arabic verbal stems fataH/futiH in (29a,c) and in aftaH/uftaH in (29b,d) below:

(28) a. The boy opened the door

b. The boy opens the door

c. The door was opened

d. The door is opened

(29) a. fataHa 1-walad-u 1-baab-a

opened.IND the-boy-NOM the-door-ACC

'The boy opened the door'

b. y-aftaHu l-walad-u l-baab-a 3-open.IND the-boy-NOM the-door-ACC

'The boy opens the door'

c. futiHa l-baab-u

opened.PASS.IND the-door-NOM

'The door was opened'

d. y-uftaHu 1-baab-u

3-open.PASS.IND the-door-NOM

'The door is opened'

The English verb forms in (28a-d) do not pattern like the Arabic stems in bold in (29a-d), which are already inflected for Tense/Aspect and Voice. The uniform stem opened (either active, as in (28a) or passive, as in $(28 \mathrm{c}, \mathrm{d})$ ) has as counterpart three different stems in Arabic: the stem fataH for the past morphology in (29a), the stem futiH for the past participle morphology in (29c), and the stem uftaH for the present participle morphology in (29d). As for the stem aftaH for the present morphology in (29b), it is likewise different from the other stems. 
Coming back to example (27a) above (repeated below as (30)) in connection with Fassi Fehri's (1993) argument for the 'partially' concatenative' nature of verbal inflectional processes in Arabic, Fassi Fehri (1993: 79-81) develops a featuredistribution model for the morpho-syntactic analysis of SA verbal inflection, where the i(nflectional)-morphemes Tense, Aspect, Voice, AGR and Mood are 'checked' against the inflectional properties of verbs via head-to-head movement processes involving V and the functional heads (or the i-morphemes, in Fassi Fehri's 1993 terminology) the verb adjoins to:

(30) sa-y-aDrib-uu-na-hu

FUT-3-beat-MP-IND-him

'They will beat him'

It is these adjunction processes that create a bracketing structure as that in (31) below for the partial expression $y$-aDribuu-na from the example in (30):

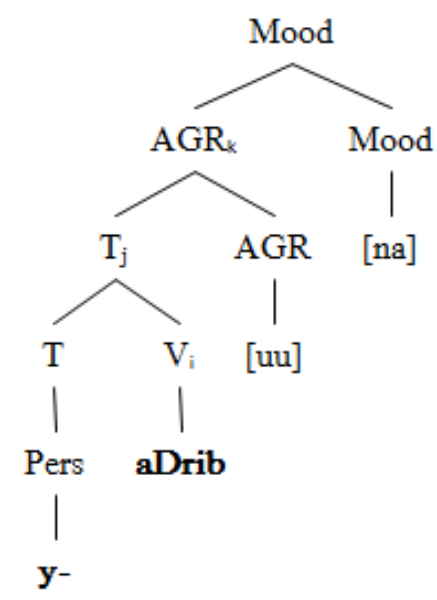

This structure is subsequently manipulated by morpho-phonological processes merging the morphemes together.

Leaving aside the future modal category $s a$ - in (30), according to Fassi Fehri (1993: 81), the sentence yaDribuuna 'They beat/are beating' would have a structural representation as in (32) below (adapted from Fassi Fehri's 1993: 81 structure (10)): 


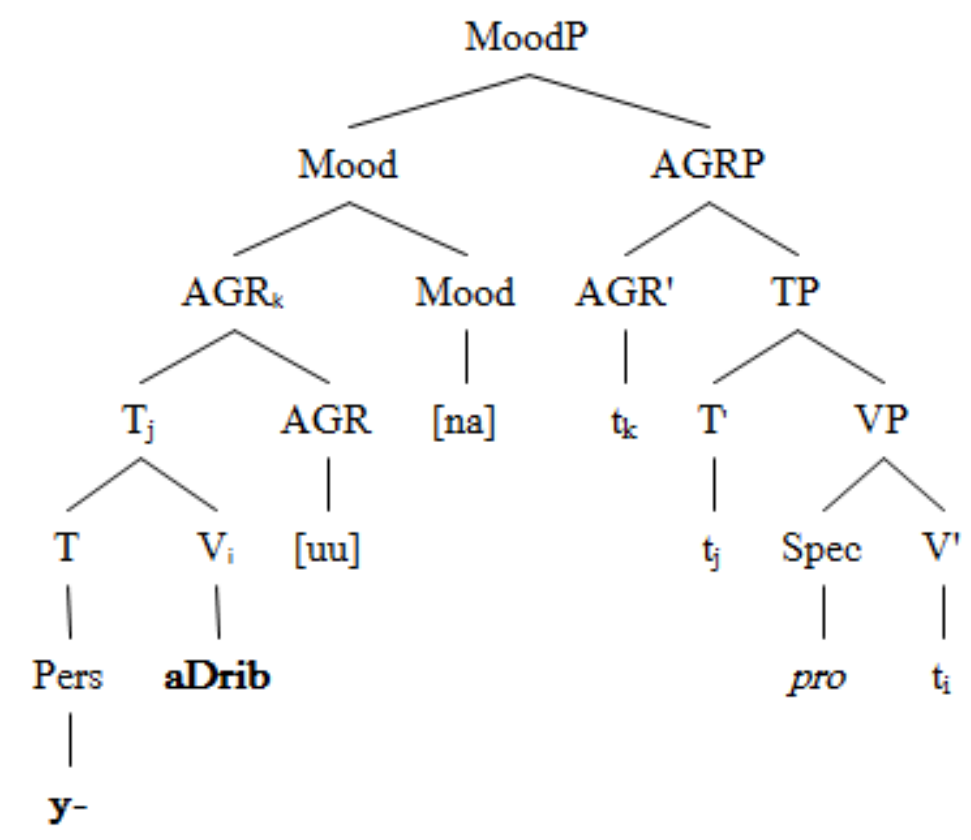

The process involving the derivation of (32) begins by raising the verb ( $a$ ) Drib (as a stem) from $\mathrm{V}$ to $\mathrm{T}$ forming the $3^{\text {rd }}$ person verb $y$-aDrib. After the complex $y a D r i b$ is formed, V-T raises to AGR and bracketing applies between yaDrib and the plural agreement morpheme $-u и$. Then V-T-AGR raises to Mood and bracketing applies between yaDribuu and the mood morpheme -na. When the derived structure is handed over to the morpho-phonological component of the grammar, the final form yaDribuuna is realized "deriving the right internal order of morphemes within the word, before phonological rules apply" (Fassi Fehri 1993: 84).

Fassi Fehri's (1993) underlying premise for the derivation of the structure in (27) is that the i-morphemes are already generated in their base positions and verb movement applies to bring these morphemes together so that they can be handled morpho-phonologically at Spell Out when the derived structure is handed over to PF. Presumably, a referential pro is base-generated in [Spec, VP] to account for theta-role assignment to the subject position inside VP prior to merging the functional structure above VP, at the point of which the subject first merged in [Spec, vP/VP] undergoes a movement process and is merged again in some higher functional Spec position (see sections 2.2. and 2.3 below).

According to Fassi Fehri (2000: 83), person agreement placement as prefix (e.g., the $3^{\text {rd }}$ person singular morpheme $y$ - in (32) above) or as suffix (e.g., plural morpheme -tum in (33) below) correlates with whether the derived verbal form is in 
the imperfective aspect or the perfective aspect, respectively. In this respect, the distinction in the placement of the person morpheme is significant in that it sheds light on the view that "imperfective verbs are Mood phrases (headed by Mood which is higher than T)", as represented in (31) and (32) above, whereas no Mood phrase is projected when the verb is in the perfective aspect. ${ }^{26}$

The structure of a perfective inflected verb such as katabtum-aa 'you (both of you) wrote' would be as follows:

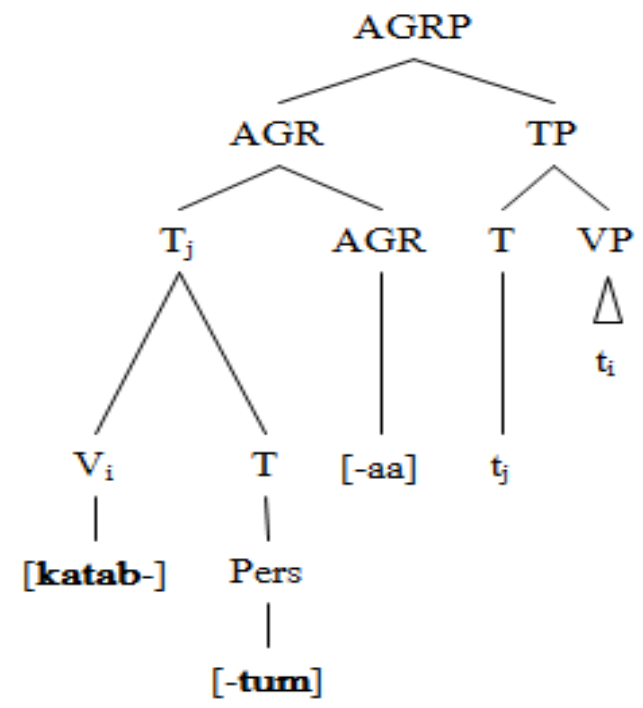

The derivation of such a structure goes as follows: Person is a pure expression of Tfeatures (Fassi Fehri 2000: 88, footnote 19) and it fuses with T forming a complex T which is realized as one node to which the verb raises. So bracketing in the syntax gives the morphological complex V-[Person-T] headed by T. Finally, the whole complex V-[Person-T] moves to AGR, which for Fassi Fehri (2000) is the realization of Number. The end product is the formation of the verbal inflectional complex V[Person-T]-AGR, which at the interface/Spell Out is V-Person-Number.

\footnotetext{
${ }^{26}$ What remains to be explained is why it is the case that the adjunction of the imperfective verb to the prefix in a structure like (32) involves a right-adjunction process, whereas the adjunction of the perfective verb to the suffix in a structure like (33) involves a left-adjunction process. Presumably, since these processes are head-to-head adjunction processes involving a combined morphological and syntactic distribution of the abstract features (representing the functional nodes projected in the syntax) verbs adjoin to in the process of the derivation, the syntax analyses them as 'substitution' rather than 'adjunction' processes, as Fassi Fehri $(1993,2000)$ conceives them. This would account for how the [Person-T] 'fused' morpheme happens to be an integral part of the verbal stem under T. If this line of argumentation can be maintained, the V-[Person-T] 'fused' verbal inflectional complex 'headsubstitutes' for the abstract tense 'affix'.
} 
I come back to a discussion of verbal morphology and its interaction with V-T movement in section 3.3.2, 3.3.3 and 3.3.4.

\subsubsection{Summary and implications}

In this section, I sum up the literature review I have been dealing with in subsections 2.1.2.1, 2.1.2.2, and 2.1.2.3 above. Of particular importance in this review is the conclusion I want to emphasize that the hypothesized analysis of dialectal differences between SA and the modern Arabic spoken dialects does not only concern the VSO word order. The SVO word order in Arabic would also be subject to a parametric analysis as regards the controversy over the projection of the IP domain in SVO sentences and the position of the preverbal DP in these sentences.

Irrespective of the specificities of the standpoints reviewed in section 2.1.2.3.2 above as regards Topic DPs in SVO sentences (schematically represented in Table 2.1 above), my hypothesis is that the parametric difference that sets the modern spoken dialects of Arabic apart from SA in the projection of the IP domain (in its relation to the CP domain) may prove to be a question of whether the initial 'subject' DP is in the IP domain (i.e., a subject DP moved to [Spec, IP] in the spirit of the VISH), as Mohammad (1989) and Benmamoun (1992a) argue, or a topicalized/left-dislocated position to the left of IP in [Spec, TopP] with a referential pro in [Spec, IP], as in Fassi Fehri (1993, 2000). In this conception, SA and the modern Arabic spoken dialects essentially differ in relation to where initial subjects in SVO sentences are representationally located in sentence structure -i.e., whether the subject is in [Spec, TopP] (which would be the case in SA) or within the IP domain (which would be the case in the modern spoken dialects).

In this light, as was the case for the derivation of the VSO word order in SA and the modern spoken dialects à la Benmamoun (1992a) (i.e., whether there is an expletive pro in these VSO instances: structure (12) vs. structure (14)), the derivation of SVO structures in SA, as opposed to the modern spoken dialects, could also prove to be a question of micro-parametric variation. In both cases (i.e., the derivation of VSO and SVO structures), the parametric difference would concern how the Phasetheoretic probe-goal-Agree system (Chomsky 2001, 2004, 2008) interacts with the feature structure of core functional heads projected in sentence structure in conjunction with the morpho-syntactic properties of lexical items in the 
language/dialect in question (cf. Roberts \& Roussou 2002, Roberts 2010a, Biberauer et al. 2010; see Chapter Three).

Table 2.2 below graphically represents the micro-parametric view that will be adopted in this thesis as it pertains to how the structure of IP projects in VSO and SVO word orders in SA as opposed to the modern spoken dialects:

Table 2.2. A Phase-theoretic probe-goal-Agree view of parametric variation in the derivation of VSO and SVO sentences in Arabic (SA vs. the modern spoken dialects, e.g., TA):

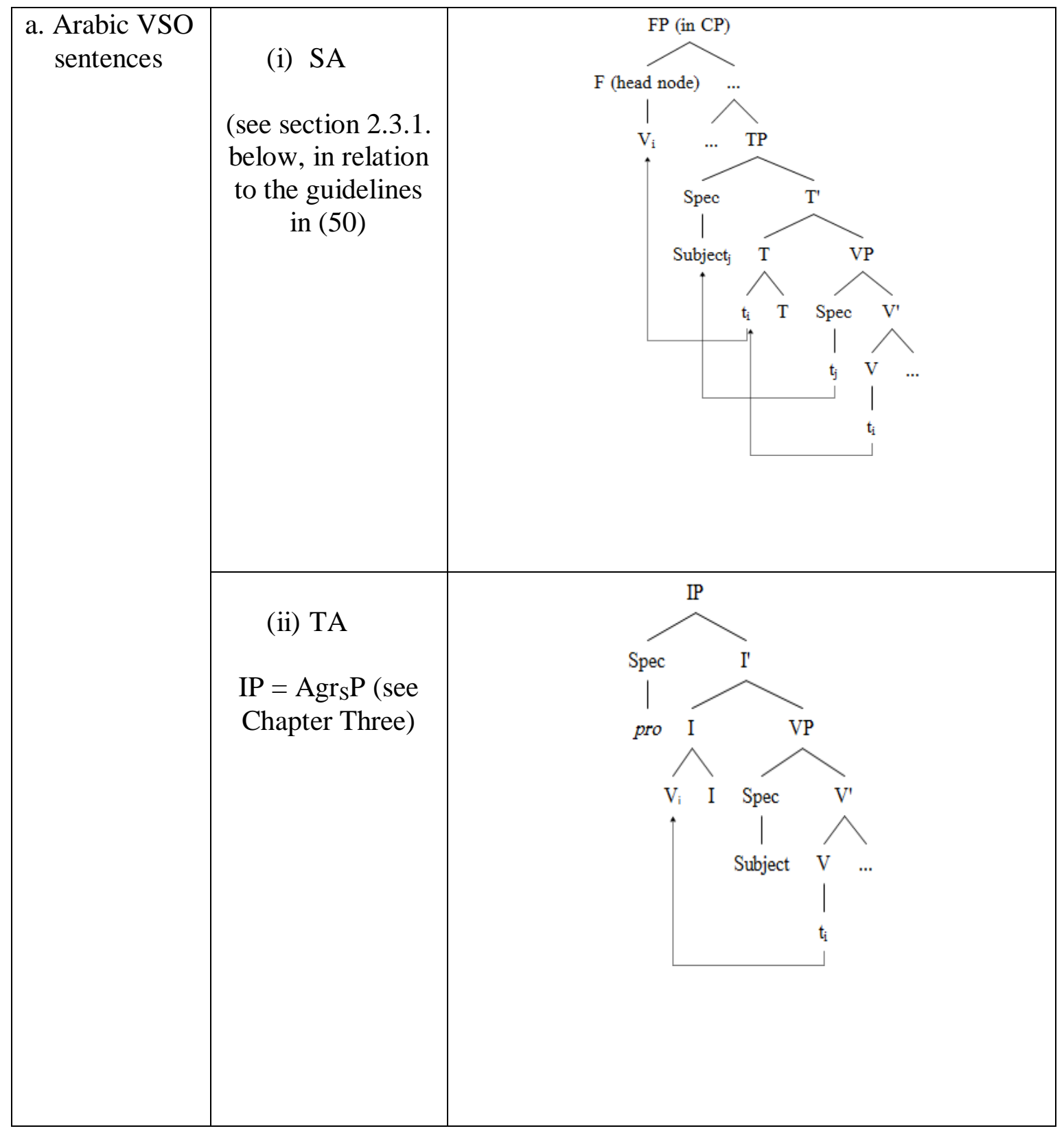




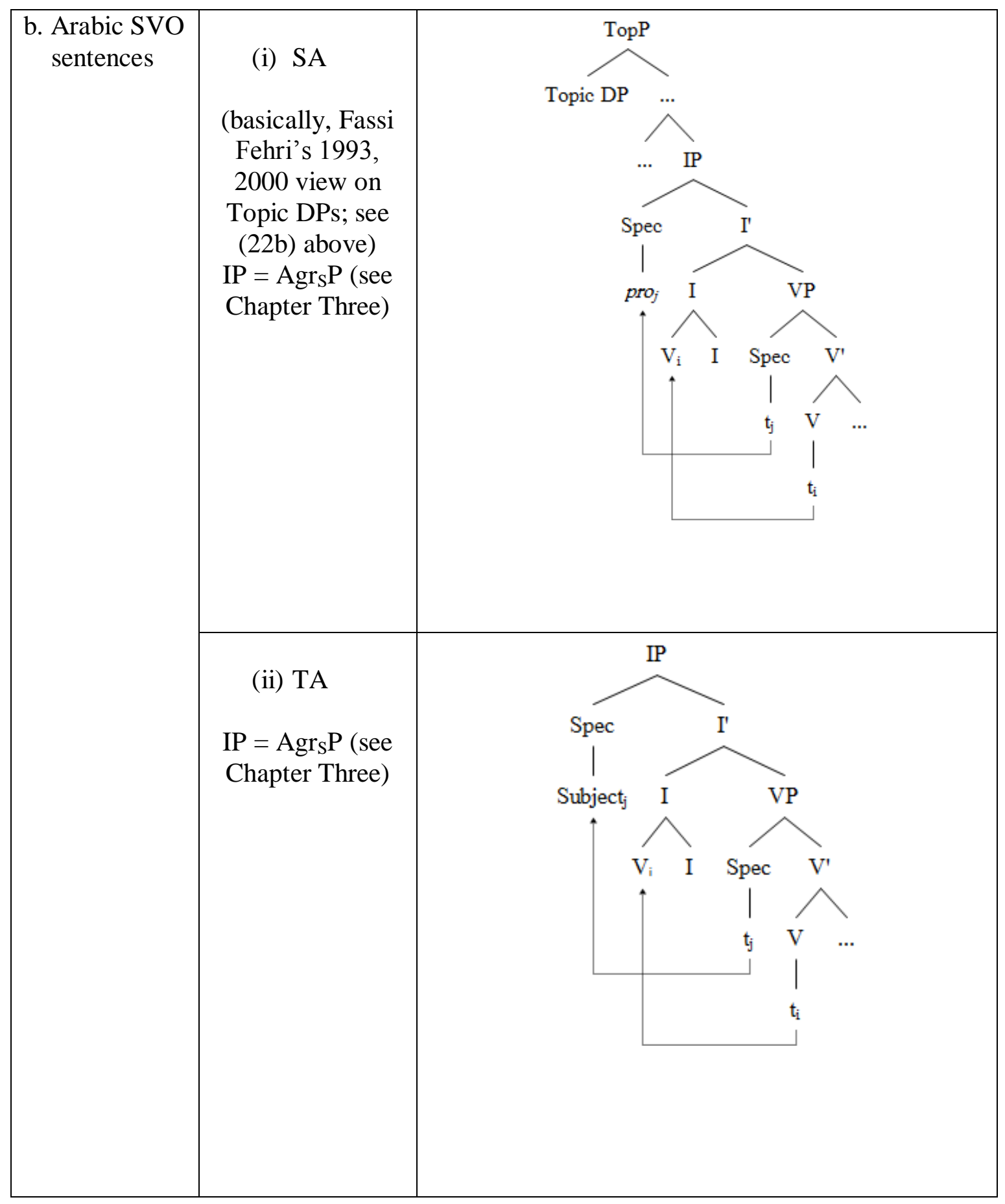

In a nutshell, the null expletive pro analysis of the subject position in the VSO word order in Arabic, as envisaged by Benmamoun (1992a), legitimizes the view that the differences between SA and the modern spoken dialects in the derivation and representation of sentence structure in VSO sentences could be parametric. The hypothesis I am putting forward in this thesis is that this could also be true of the derivation and representation of sentence structure in SVO sentences in SA and the modern spoken dialects as pertains, in particular, to where initial subjects are located 
in the derived structure. As can be seen from the preceding account of VSO and SVO word order alternatives in Arabic, the agreement analysis of these variations in terms of the EPP and generalized Spec-head agreement configurations, as in Miyagawa (2010) (cf. Roberts \& Roussou 2002), proved fruitful in that it opens the door for an alternative view of the agreement phenomena in question.

In relation to the reviewed morpho-syntactic and morpho-phonological evidence for the incorporated pronoun analysis of verbal inflection in sentences exhibiting an initial subject DP in the SVO word order in SA, the hypothesis that has been proposed in this chapter is in favour of the status of the initial subject DP in such instances as a Topic-like constituent, clitic left-dislocated and being resumed by the subject pronoun morpheme on the verb.

In section 2.2 below, I will be concerned with the structure of the thematic domain of languages, namely, the VP. The subject-verb agreement phenomena I have just described with reference to SA sentence structure may be replicated for such phenomena that arise in object participle agreement - for example, in French - and the controversial raising-to-object structures in English.

\subsection{The internal structure of $\mathrm{VP}$}

In this section, I will be dealing with the similarities between subject-verb agreement configurations like those reviewed in section 2.1 and agreement phenomena involving objects, as found in Romance languages like French. The section also deals with the controversy over object movement in English (Chomsky 1995b, Sportiche 1998). The similarities between these two cases of agreement/movement phenomena point to the possibility that the 'agreement approach to the EPP', as expounded mainly in Roberts \& Roussou 2002 and Miyagawa 2010, applies more generally in natural language.

I first discuss the internal structure of VP in particular reference to the VISH (see section 2.1.2.1). In section 2.2.1, I deal with Sportiche's (1998) raising-to-object analysis in English that parallels his treatment of raising-to-subject structures in such languages as SA, Welsh and Irish (see section 2.1.2.1 above). In section 2.2.2, I have a look at Chomsky's (2008) assumptions about raising-to-object structures in light of his latest reformulation of the Phase-theoretic framework (see section 2.3.2 below for some early assumptions about 'Phases' in the derivation of sentences). Section 2.2.3 is a summary. 
The hypothesis that subject DPs are base-generated in a Spec position within the verb phrase (within the spec position of the 'light' verb $v^{*}$ for verbs taking agentive subjects) brought into focus the importance of specifier/subject positions in the derivation of sentence structure. ${ }^{27}$ The Barriers framework (Chomsky 1986a) builds on such assumptions as pertains to the general requirement that some extraneous 'outer' position be targeted (called an 'edge' position in Chomsky 2001, 2004) in the process of deriving syntactic representations. A prototype example of such a requirement in Barriers is the VP-adjunction process that creates an escapehatch for wh-moved objects. In a structure like (34) below (where XP = VP and ZP = wh-moved object), XP further projects to $\mathrm{XP}^{\mathrm{max}}$, forming an adjunction structure. By reference to this structure, the derivation of object wh-extraction in an English structure like (35), for example, first targets an intermediate position whereby the whmoved object (ZP in (34)) is adjoined to VP (= XP in (34)) before it moves to the peripheral clausal position (i.e., [Spec, $\mathrm{CP}]$ in structure (35)):

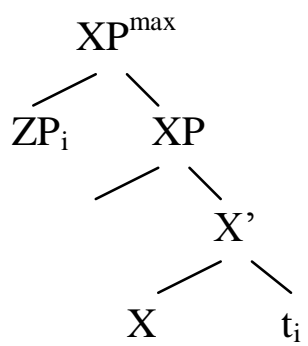

(35) $\left[\mathrm{CP}_{\text {whom }}\left[\mathrm{C}\right.\right.$ did [IP you [vP $\mathrm{t}_{\mathrm{i}}\left[\mathrm{vP}\right.$ see $\left.\left.\left.\left.\left.\mathrm{t}_{\mathrm{i}}\right]\right]\right]\right]\right]$ ?

The targeting of 'edge' positions in the derivation of sentences is the hallmark of recent developments in the conception of structural dependencies including movement and agreement processes particularly in connection with the local character of the movement of maximal and head constituents, mainly DPs and verbs, respectively, and the agreement configurations thereby generated in sentence structure. In such a conception, the only condition on the derivation and representation of sentences is that interpretability be satisfied (see section 2.3 below and Chapter Three).

\footnotetext{
${ }^{27}$ [Spec, $\left.\nu \mathrm{P}\right]$ is absent in the $\nu \mathrm{P} / \mathrm{VP}$ structure of raising/unaccusative verbs. See structure (36) below for early minimalist assumptions on 'light' verbs (Chomsky 1995c). I come back below to the Phasetheoretic distinction between the agentive light verb $v^{*}$ and the unaccusative/raising light verb $v$ (Chomsky 2001, 2004, 2008).
} 
Along with the expansion of the structure of IP which has been taken to show evidence of an abstract AGR element that may interact with the tense properties of derived structural representations (Pollock 1989; Chomsky 1989), the internal structure of the VP has also been proved to be complex (Larson, 1988; Chomsky, 1995a, b). According to Chomsky (1995a: 422; 1995b: 331, 352), VP structures include two verb projections: an outer VP-shell ( $v \mathrm{P}$ in (36) below) and an inner VP core. The structure of $v \mathrm{P} / \mathrm{VP}$ includes at least the following:

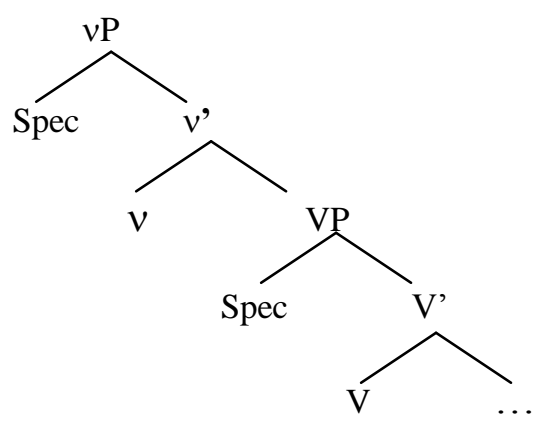

In this structure, $\mathrm{V}$ is a lexical category at the lower part of the $v \mathrm{P} / \mathrm{VP}$. It maximally projects to VP with a possible Spec position. $v$ is a 'light verb' at the higher part of the $v \mathrm{P} / \mathrm{VP}$. It maximally projects to $v \mathrm{P}$ with an obligatory Spec position (except for unaccusative/raising constructions). An argument DP, base-generated in [Spec, $v \mathrm{P}]$, is assigned the theta-role associated with the 'external' argument (according to the VISH) and undergoes DP movement to [Spec, $\left.\operatorname{Agr}_{S} \mathrm{P} / \mathrm{TP}\right]$ for Case (alternatively, EPP) licensing.

Chomsky (1995b: 321) assumes that lexical verbs, inserted under the V node, obligatorily move to $v$ (V-to- $v$ raising) since $v$, selected in the initial Numeration, is a "light verb requiring a verbal affix". In a V-raising language like French, V-to- $v$ raising is a necessary precondition for tense feature licensing in particular (Pollock 1989; Biberauer \& Roberts 2010: 265-268). In this case, the lexical verb will be as high as $v$ to satisfy further movement of $\mathrm{V}$ to $\mathrm{T}$. Nevertheless, whether the language is V-raising or not, the requirement of tense interpretation, within the verbal inflectional complex at LF must be satisfied; thus V-v will be as high as $\mathrm{T}$ in the end product at LF (cf. Roberts 2010a: 166-167). 


\subsubsection{Sportiche (1989, 1998 (Chapter 3))}

In parallel to the raising (or non-raising) of the subject outlined in section 2.1.2 above, Sportiche (1998) argues for a raising-to-object analysis in English, as opposed to other languages such as French. To account for the cross-linguistic variation in the realization of object participle agreement (in the languages where such an agreement phenomenon occurs, e.g., in French), Sportiche (1998) assumes that the object is in a structural relationship of movement to, and/or agreement with, a higher Spec position in the lower part of $v \mathrm{P} / \mathrm{VP}$.

Sportiche (1998) shows on the basis of small clauses complement-selected by verbs like consider in English that objects, generally, raise from their base-generated position as complements of $\mathrm{V}$ to $[\mathrm{Spec}, \mathrm{VP}]$ in a structure like (36) above (or, equivalently, in NP** in (37) below). ${ }^{28}$ For Sportiche (1998: 95) the structure corresponding to $\mathrm{vP} / \mathrm{VP}$ in (36) is as in (37), with $\mathrm{NP} * *$ representing the position where objects are assigned Accusative Case after raising from $\mathrm{NP}^{* * *}$ (here $\mathrm{V}^{\max }$ is the same as $v \mathrm{P}$ except that the intermediate verbal category $v^{\prime}$, above the lower VP, is not represented): $:^{29}$

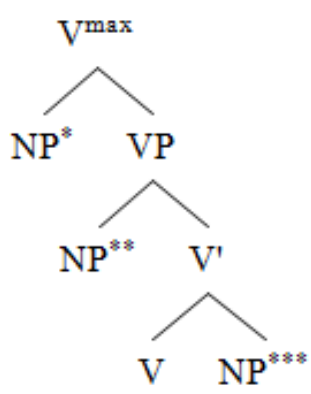

Sportiche (1998) provides the following evidence from small clauses for raising to object in English (the ungrammatical sentences (39a) and (39b) are Sportiche's 1998: 99 examples (22a-b); sentences (38a) and (38b) are provided for the sake of comparison ( $\mathrm{W}=\mathrm{AP}$ small clause $)$ ):

\footnotetext{
${ }^{28}$ I keep here to Sportiche's terminology for identifying the Spec and complement syntactic positions in $\mathrm{V}^{\max }(\mathrm{vP})$, namely $\mathrm{NP}^{*}, \mathrm{NP}^{* *}$ and $\mathrm{NP} * * *$.

${ }^{29}$ Structure (37) is to be compared to structure (40) below where VP* is the maximal projection of the higher $\mathrm{VP}\left(=v \mathrm{P}\right.$ or $\mathrm{V}^{\mathrm{max}}$ in (37)) projected from the intermediate projection $\mathrm{V}$ ' after verb-raising internally within the structure of $\nu \mathrm{P} / \mathrm{VP})$.
} 
(38) a. They consider $\mathrm{John}_{\mathrm{i}}\left[\mathrm{W} \mathrm{t}_{\mathrm{i}}\right.$ [proud of himself]]

b. [ $\mathrm{W} \mathrm{t}_{\mathrm{i}}$ [How proud of himself]] do they consider $\mathrm{John}_{\mathrm{i}}$

(39) a. *They consider $\mathrm{John}_{\mathrm{i}}$ [W t $\mathrm{T}_{\mathrm{i}}$ [proud of each other]]

b. $*\left[\mathrm{~W} \mathrm{t}_{\mathrm{i}}\right.$ [How proud of each other] $]$ do they consider $\mathrm{John}_{\mathrm{i}}$

In the ungrammatical sentences (39a) and (39b) (vs. the corresponding grammatical sentences (38a) and (38b)), the lexical anaphor cannot take the matrix subject as antecedent. This grammaticality contrast suggests that AP preposing in (38b) carries along the trace of John as subject of the AP. Raising of the DP John out of the small clause leaves the trace $t_{i}$ in AP, which undergoes preposing. This allows the reflexive pronoun himself to be locally bound by the trace. Thus, according to Sportiche (1998), John moves to a Spec position outside the small clause, but still within $\mathrm{V}^{\max }\left(=\mathrm{VP}^{*}\right.$ in (40) below).

In support of his raising-to-object analysis whereby John raises to a specifier position in the internal structure of the lower VP (i.e., [NP**, VP] where, as Sportiche 1998 argues, Accusative Case is assigned), Sportiche (1998) follows Larson's (1988: 342-343) proposal that lexical verbs move internally to VP* (i.e., the higher VP in (40) below) from a lower $\mathrm{V}$ position to a higher empty $\mathrm{V}$ position. This assumption would account for why the lexical verb consider raised from its base position inside the lower VP remains higher than the DP John raised to [NP**, VP]. Thus, in (38a) and (38b), the preposing of the W constituent cannot pied-pipe John since, if John were pied-piped it would fail to receive Accusative Case. The structure in (40) is the derived representation resulting from object-raising in (38a) whereby John moves from its base-generated position NP*** to the Spec position NP**: 30

\footnotetext{
${ }^{30}$ As will be shown below, in relation to French object participle agreement structures (see examples (43) and (44) below), Sportiche takes it that, contrary to English, French requires that an object DP stay in situ, and by that token, licenses the existence of a silent expletive pro in [NP**, VP]. As for English, it requires objects DPs to surface in NP** - i.e., the Accusative position - because the language does not license silent expletives. Note that, since French does not allow object DPs to raise to [NP**, VP], the raising-to-object structures in (38)/(39) cannot be reproduced in French.
} 
(40)

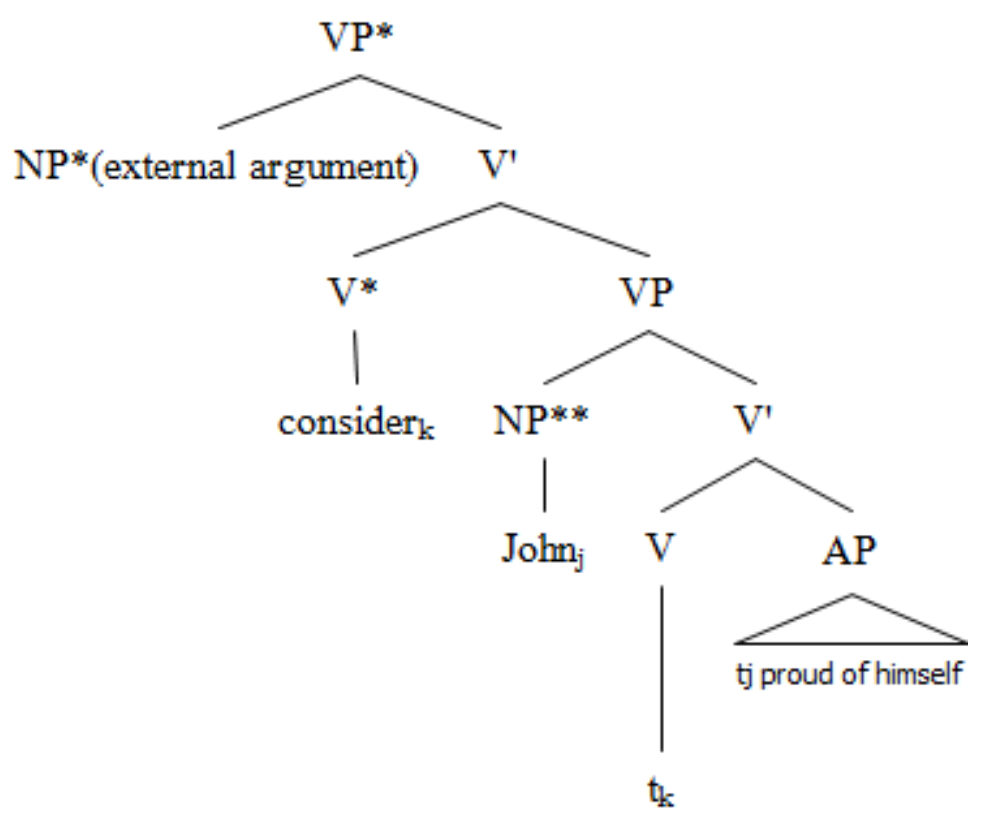

Likewise, in a simple sentence like 'I will see John', the object DP is assumed to raise to $[\mathrm{NP} * *, \mathrm{VP}]$.

Further upholding his analysis of object-raising structures in English, Sportiche (1998:162) argues that the direct link that exists between the assignment of Accusative Case and movement to (and/or agreement with) a higher Spec position in the lower part of $v \mathrm{P} / \mathrm{VP}$ (as in English raising-to-object structures such as those in (38) or in French participle agreement structures, as in (43) and (44) below), generalizes to Nominative Case assignment.

Sportiche (1998:162) refers to this generalization as the Strong Correlation Hypothesis whereby the assignment of structural Case (Accusative and Nominative) correlates with the agreement configurations that arise in different structural word orders (mainly SVO vs. VSO) in different languages. According to Sportiche (1998: 173) subjects receiving Nominative Case in languages such as French, English and Arabic (with SVO word order) behave like objects receiving Accusative Case in languages such as Dutch (and, possibly, English). Sportiche (1998) argues that, in these instances, subjects and objects are in a derived Spec position at S-structure (i.e., the Nominative position $\mathrm{NP}^{\wedge}$ (i.e., [Spec, IP]) in a structure like (41) below, for raised subjects, and the Accusative position NP** (i.e., [Spec, VP]) in a structure like (40) above, for raised objects). It is in these Spec positions that - parametrically - Case is assigned and agreement is triggered: 


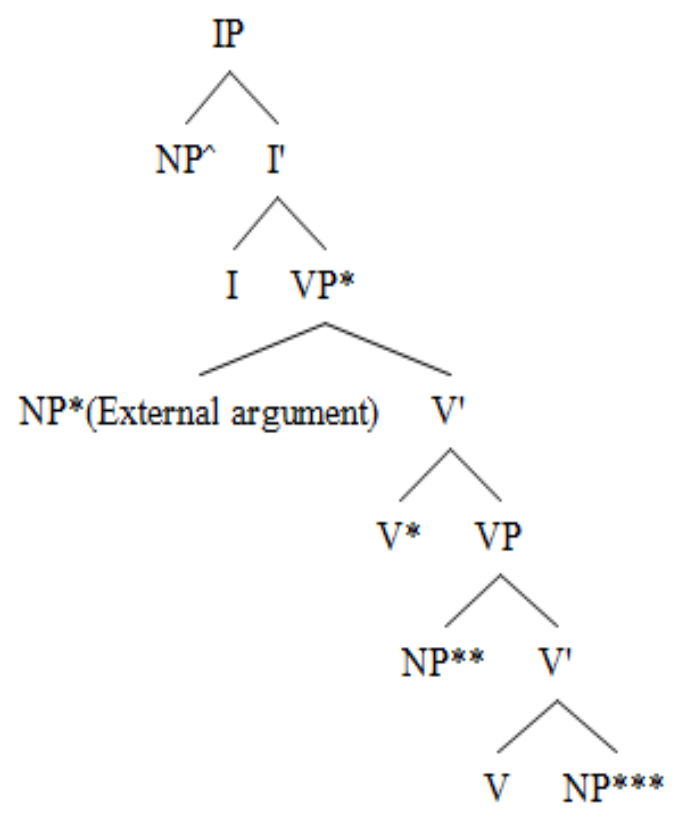

In contrast, subjects in Welsh, Irish or Arabic (with VSO word order) are not in the derived Nominative position $\mathrm{NP}^{\wedge}$ even though they receive Nominative Case. Sportiche's (1998) argument is that since in Welsh, Irish or Arabic (with VSO word order) subjects are not in the Nominative position, they do not trigger agreement. In this respect, they behave like French object DPs which are precluded from raising to the Accusative position [ NP**] (as opposed to sentences where French direct objects move as clitics or as wh-elements showing participle agreement). ${ }^{31}$ In what follows, I exemplify these affinities between object agreement and subject-verb agreement by reference to object participle agreement in French clitic structures (in contrast to the absence of such agreement with object DPs) and also by reference to subjects in the SA VSO word ordering (Sportiche 1998: 142-145; 168-173). ${ }^{32}$

According to Sportiche (1998: 143), the properties of object participle agreement that make it comparable to subject-verb agreement in VSO languages like SA are as follows:

\footnotetext{
${ }^{31}$ It might be the case that NP** in [Spec, VP] systematically 'transmits' Case to the object base position NP***. This would account for why Accusative Case is uniformly assigned even in structures where a clitic does not move through the Accusative position, as in structure (45) below corresponding to sentence (44a). However, the problem of how Case is 'transmitted' would not pose itself in a Phasetheoretic Minimalist framework where Case is not a property that is 'assigned', but is a by-product of the Merge/Move processes involved in Agree relations (whether actual subject-verb and/or object-verb agreement is morphologically manifested or not).

${ }^{32}$ I come to a discussion of Sportiche's (1998) view on subjects in the SA VSO word ordering in relation to sentences like (48) below.
} 
(42) The properties unifying subject-verb agreement in VSO languages and object participle agreement in languages such as French and Dutch:

- Object participle agreement is only possible with direct objects in the form of clitics or wh-words (moved dative indirect objects and locatives cannot trigger participle agreement)

- The extracted direct object must precede the participle

- Object participle agreement is optional (according to how movement proceeds)

- As in subject-verb agreement, participle agreement is a Spec-head agreement relation. In structures where subject DPs in the SA VSO word ordering are kept in situ, a process of 'Case transmission'/'Case inheritance' between an EXPL pro and the in situ DP accounts for how Case is assigned in the absence of Spec-head agreement. The same 'Case transmission' process applies in the derivation of French object DPs, which are necessarily kept in situ.

Structure (45) below (Sportiche's 1998: 144 structure (102)) exemplifies the case (corresponding to example (44a) below; cf. Sportiche 1998: 143) where object participle agreement in feminine gender in French is not necessarily morphologically marked on the participle. ${ }^{33}$ As the minimal contrast between (44a) and (44b) shows, object participle agreement with clitics is optional. The optionality of such agreement is due to the fact that the clitic can skip the NP** Spec position in its movement to attach to the auxiliary verb higher up in the VP structure. As Sportiche (1998: 145) recognizes, this is essentially Kayne's (1989a) analysis of such object participle agreement structures in French:

(43) ... avoir décrit/(*décrite) la robe (French) to have described $/(*$ described.F) the dress
(44) a. ... l' (= la $)_{\mathrm{i}}$ avoir décrit
$\mathrm{t}_{\mathrm{i}}$
it to have described
b. ... l' $(=\text { la })_{\mathrm{i}}$ avoir décrite
$\mathrm{t}_{\mathrm{i}}$ it to have described.F

\footnotetext{
${ }^{33}$ Clitic movement is a combination of head-movement, wh-movement (adjunction to VP) and DPmovement (movement to [NP**, $\left.\mathrm{VP}_{\mathrm{k}}\right]$ in (45)) (Sportiche 1998: 143); thus, in structure (45), $\mathrm{NP}_{\mathrm{j}}$ is whmoved and adjoins to $\mathrm{VP}_{\mathrm{h}}$ (skipping NP**). In $\left[\mathrm{NP}_{\mathrm{j}}, \mathrm{VP}\right]$, it is it is realized as both a maximal and a minimal category $\left(\mathrm{NP}_{\mathrm{j}}\right.$ and $\mathrm{N}$ in (45)) (see also Chomsky 1995b: 249) for the suggestion that clitics share XP and $\mathrm{X}^{\mathrm{O}}$ properties).
} 
(45)

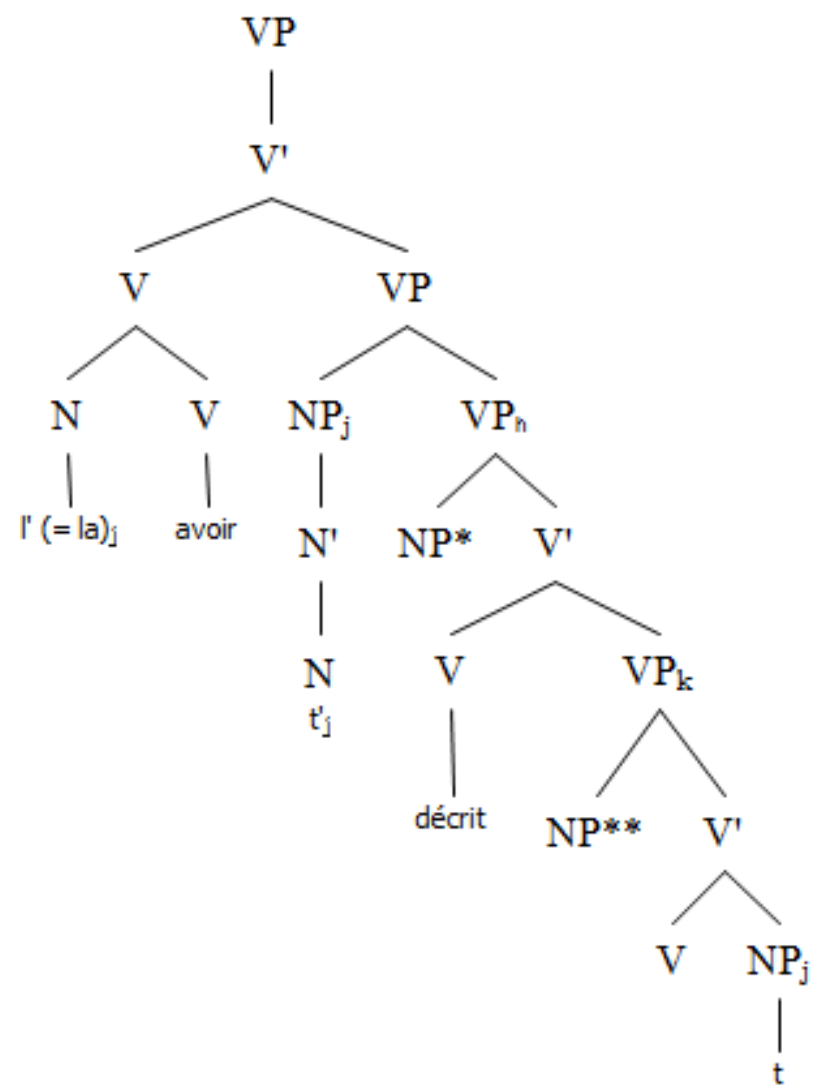

Thus, in structure (45), $\mathrm{NP}_{\mathrm{j}}$ skips the position $\mathrm{NP} * *$ and, as a consequence, participle agreement does not obtain. Adjunction to $\mathrm{VP}_{\mathrm{h}}$ in (45) cannot trigger agreement because participles cannot agree with adjoined positions. In contrast, in (44b), the clitic presumably moves first to $\left[\mathrm{NP}^{* *}, \mathrm{VP}_{\mathrm{k}}\right]$, triggering agreement between $\mathrm{NP}^{* * *}$ and the raised participle. On this account, clitic movement of indirect objects and locatives does not trigger agreement on participles in French because they are barred from transiting through $\left[\mathrm{NP}^{* *}, \mathrm{VP}_{\mathrm{k}}\right]$, and can only move by adjoining to $\mathrm{VP}_{\mathrm{h}}$.

As for accusative DPs, they cannot trigger participle agreement as exemplified by the ungrammaticality of décrite in $(46 \mathrm{~b})(=(43)$ vs. (46a) with the structural representation (47) below:

(46) a. Jean a décrit la robe (French)

John has described the dress

b. *Jean a décrite la robe

John has described.F the dress 


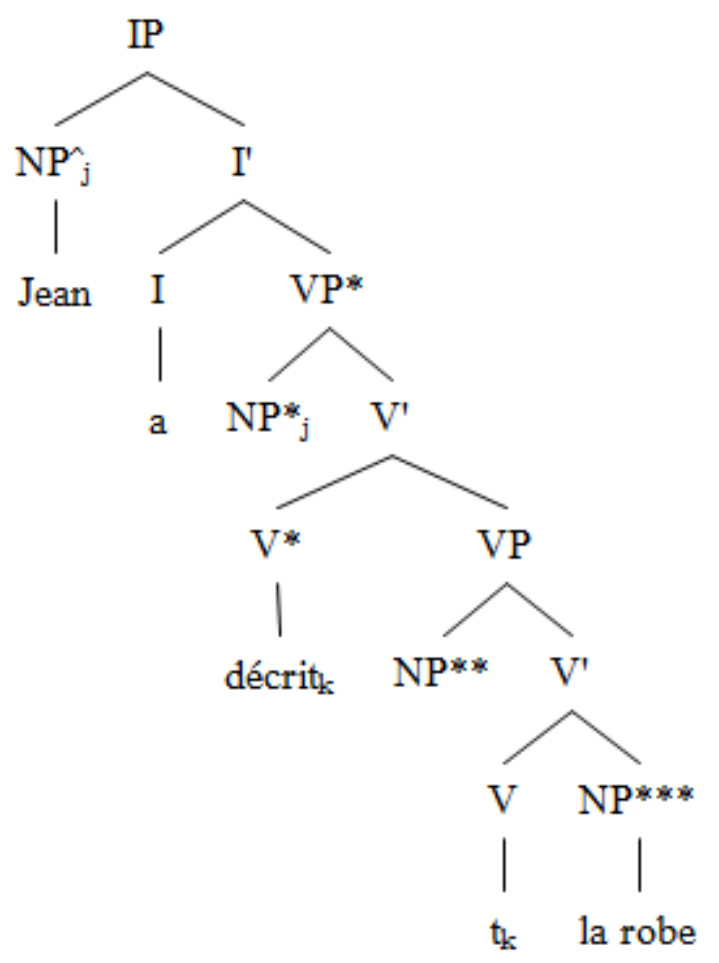

According to Sportiche (1998), the absence of participle agreement in (46a/47) shows that the direct object la robe 'the dress' cannot be in NP**. If it were, it would have triggered object agreement since $[\mathrm{NP} * *, \mathrm{VP}]$ is the Accusative position where object participle agreement applies. Sportiche (1998: 168) argues that NP*** gets Accusative Case without being in the Accusative position (i.e., by inheritance from NP**). Since in structure (47) NP*** inherits Case from position NP**, Sportiche (1998: 168) assumes that a silent expletive pro is merged in NP**. According to Sportiche, silent expletives are not licensed in English and accusative objects in that language must surface in NP**, i.e., the Accusative position (see Sportiche's argument in relation to structure (37) above for such a claim).

With reference to the same basic IP structure as that in (47) or (41) above, Sportiche (1998:172-173) points to the affinity between object participle agreement in French and the subject-verb agreement pattern in VSO word order in SA. Thus, Nominative Case could be assumed to be assigned to subjects or inherited by them via the same process that Accusative Case assignment/inheritance applies to objects. In this respect, the subject, in English or French, cannot surface as NP* in (47/41) (i.e., the Spec position of $v \mathrm{P}$ in structure (36) above). In Sportiche's (1998) analysis, this is construed as meaning that NP* receives no Case in this position in these languages. Rather, the subject has to surface as $\mathrm{NP}^{\wedge}$ exhibiting agreement with INFL (in a fairly 
impoverished way in English). This means that when INFL is a Case assigner, it is a Case assigner by Agreement only.

In contrast, the external argument in SA (as reviewed in section 2.1.2.1 above) can surface in either position NP* or NP^ (examples from Mohammad, 1989; Sportiche's (146)):
(48) a. iddaia r-rižaal-u Panna... claimed.3MS the men-NOM that ...
'The men claimed that ...'
b. al-Pawlaad-u katab-uu risaalat-an the-boys-NOM wrote-3MP letter-ACC 'The boys wrote a letter'

In (48a), the verb gets a default third person singular agreement. Sportiche (1998) follows Mohammad (1989) in positing a null expletive responsible for the default agreement on the verb iddaSa 'claimed' in (48a). Sportiche (1998: 173) argues that in such a VSO sentence in SA, subjects somehow inherit Nominative Case from $\mathrm{NP}^{\wedge}$ and that some Case inheritance mechanism must be invoked here, in a similar way to the French examples of inheritance of Accusative Case, as seen above in relation to (46) and (47).

According to Sportiche (1998), the subject, in (48b), is in $\mathrm{NP}^{\wedge}$. In that position, agreement/Case is assigned as a consequence of Spec-head agreement between the fully-inflected verb katab-uu 'wrote' raised to INFL and the DP alPawlaad- $u$ in the specifier position of INFL (see section 2.1.2.1 above). In such a configuration, the verb obligatorily agrees in Person and Number.

\subsubsection{Chomsky (2008) on Agree-feature inheritance}

The latest Phase-theoretic framework (Chomsky 2008) also relies on the idea that agreement configurations in sentence structure are derived via a process of feature transmission or feature inheritance. The mechanism of Agree-feature inheritance in Chomsky (2008: 144-149) is conceived of in terms of the selectional relation between $\mathrm{C}$ and $\mathrm{T}$ and between the 'light' verb $v^{*}$ (as a 'strong' Phase-head different from 'light' verb $v$ of unaccusative structures; see section 2.3.2 below) and V. Feature inheritance (which replaces the notion of $\varphi$-completeness; see section 2.3.2) takes it that $\varphi$-features/Agree features are generated on Phase heads $C$ and $v^{*}$ (also, possibly, D) and inherited by the head they select as complement in sentence structure. $\mathrm{T}$ 
inherits its $\varphi$-features from $\mathrm{C}$ and $\mathrm{V}$ inherits them from $v^{*}$. This process is universal and it reinforces the uniformity of computational processes in the architecture of the grammar. Thus subject-verb agreement configurations and object agreement are natural phenomena that need only be linked to Phase heads. In Chomsky's (2008: 148) own words:

... transmission of the Agree feature should be a property of Phase-heads in general, not just of C. Hence $v^{*}$, should transmit its Agree features to V, and probe of an object with structural Case by $v^{*}$ should be able to raise it to Spec$\mathrm{V}$, as a step-by-step analogue to raising to Spec-T by C. That would yield the intriguing but puzzling conclusions about raising to Spec-V, particularly in ECM constructions, but perhaps generally.

\subsubsection{Summary}

In a nutshell, the cross-linguistic evidence reviewed above points to a wide array of parametric choices languages from various typological types choose from. The agreement analysis of these parametric choices shows promise in narrowing them down to a single parameter, namely, the EPP or what Bobaljik \& Jonas (1996: 211) call 'the [Spec, TP] parameter'. ${ }^{34}$ In this connection, the analysis has been helpful in pointing out the similarities between subject-verb agreement configurations - at the level of IP - and the agreement phenomena involving objects in French - at the level of $\nu \mathrm{P} / \mathrm{VP}$ - in terms of movement processes or the Merge of EXPL elements (overt or null). The importance of these similarities has also been highlighted in relation to Chomsky's (2008) Agree-feature inheritance theory, briefly introduced in section 2.2.2 above, whereby agreement configurations are derived on the basis of probe-goal structural relationships (see section 2.3.2).

In the context of the proposal I am advancing in this thesis (see section 2.3 below and, in particular, Chapter Three), the projection of an $\mathrm{Agr}_{\mathrm{S}}$ node for the derivation of subject agreement - maximally projecting to $\operatorname{Agr}_{S} \mathrm{P}$ - remains a parametrically free 'choice'. The projection of the structure of IP as $\mathrm{Agr}_{S} \mathrm{P}$ would be the consequence of the free interaction of morphological requirements of elements

\footnotetext{
${ }^{34}$ In Chapter Three and Chapter Four, I propose that the parameter in question is not whether the EPP applies in a given language or not. Rather, the parameterized probe-goal-Agree system, adopted here, links parameter variation to some uninterpretable 'edge' D-feature on functional heads at the highest level of the derivation of sentence structure.
} 
selected for the derivation and the feature structure of functional elements projected into the syntax to meet these requirements. In addition to parameterizing the projection of $\mathrm{Agr}_{\mathrm{S}}$ in clause structure, I will also provide a feature-checking/featurevaluation account of a variety of structural configurations that supports the thesis that the EPP remains a universal principle of the grammar whereby the surface positions of DPs are derived in the syntax either by Merge (mainly, the case of expletive DPs) or Move (mainly the case of argument DPs), in some edge position (either Spec or 'outer Spec' position, cf. Chomsky 1995b, 2001, 2004).

\section{3. Framework of Assumptions}

\subsubsection{Early Minimalist Assumptions on Agreement Relations}

In the Minimalist guidelines of Chomsky (1995c), agreement is a question of checking via a Spec-head/head-head structural relation. The overwhelming concern with this general agreement mechanism in the generative theories of agreement and case advocated by such linguists as Koopman, Sportiche, Belletti and Rizzi was concomitant with the split-up of the IP domain into different functional projections on top of which was the Agr(s) node (cf. Chomsky's 1989 reformulation of Pollock 1989; see section 2.1.2. above).

These guidelines have generally kept intact the insight about the morphological properties of elements involved in the derivation of sentence structure. Lexical elements (verbs and associated arguments) projected into the syntax during the derivation of sentences rarely occur in their base position where they are first merged. In most cases, such elements appear in a position to which they necessarily move ('overtly' in the syntax itself or 'covertly' after the syntax) for morphological licensing purposes and to be 'fully interpretable' (i.e. to satisfy the principle of Full Interpretation or FI) at the interface levels PF and LF. What is more, languages are now assumed to 'minimally' vary in the syntax proper and abide by what Chomsky (1989) variably calls the 'least effort' or 'economy' principle/condition. This general principle is an even more overarching condition of UG than FI in that it legislates against 'superfluous elements' in both derivations and representations. In this conception, the 'economy principles' of the grammar allow only those derivations that involve no superfluous steps and lead to representations that contain no superfluous elements. The essential characteristic of the economy framework is that convergent 
derivations involve only the 'least costly' or 'shortest' moves and yield those structural representations that could find a language-invariant interpretation at LF.

The effect of this revision of the theoretical apparatus of the grammar not only led to the dismissal of the notion of 'government' (as a 'derivative', but nonetheless, unifying structural notion in the Government and Binding theory, Chomsky 1981, $1986 \mathrm{a}, \mathrm{b})$ from the grammar, but also to the elimination of the two levels of representation D-structure and S-structure, leaving LF and PF as the only levels of representation. Nevertheless, considerable attention has been devoted to the question of whether subject-verb and/or object-verb agreement relations (including the checking of Case) could find the right characterization in terms of an Agr node being projected into the syntax as an independent syntactic head to set the right Spec-head agreement relations in clause structure in conjunction with the tense properties of natural languages.

Chomsky (1995b: 267-268) reformulates these earlier Minimalist assumptions by postulating that agreement features (the uninterpretable $\varphi$-features of $T$ and its uninterpretable Case-feature, and the uninterpretable Case-features of object and subject DPs) are involved in movement operations only as 'free riders', i.e., they are only 'derivatively' involved in the checking procedure. This revision meant that computations are only driven by the uninterpretable features of the target (i.e., the Dfeature of $\mathrm{T}$ in subject-verb agreement configurations or the $\mathrm{Q}$-feature of $\mathrm{C}$ in the movement of wh-elements) in relation to 'strength' (i.e., the assumption that, in English, for example, the D-feature of T - which Chomsky (1995b) equates with the EPP - and the Q-feature of C are 'strong' and must be checked as quickly as possible).

As far as subject-verb agreement in English is concerned, Chomsky (1995b: 279-289) seeks to reduce both overt movement of DPs to the specifier of an inflectional position ([Spec, TP] in Chomsky 1995b, vs. [Spec, $\left.\operatorname{Agr}_{S} \mathrm{P}\right]$ in Chomsky 1995a: 425-426) and the direct insertion of expletives via Merge to the need to check a 'strong' (EPP) D-feature of the target. According to this view, Nominative Case on the raised subject DP and the $\varphi$-features on T, in relation to the interpretable $\varphi$ features of the subject DP, are checked as 'free riders' in the same checking configuration (Chomsky 1995b: 282). In there-constructions, the expletive element there has an uninterpretable D-feature directly involved in the checking procedure 
since it checks the 'strong' (EPP) D-feature of T, in contrast to Case on the subject DP, which is only checked covertly as a 'free rider' (Chomsky 1995b: 287).

In relation to the postulation of Agr projections in the sentence structure of languages, Chomsky (1995b: 388, note 81; see also pp. 333, 351-352) argues that Agr is absent from the grammar of English. In particular, English does not present conclusive evidence of the presence of a 'strong' Agr node in sentence structure for the assignment of Accusative Case. It is on such a premise that Chomsky (1995b: 330, 333) questions the relevance of object-raising to some Spec position higher than the base position of objects in English in the absence of an Agr node for the purpose of Accusative Case assignment.

Chomsky's main thrust in this reformulation is that the fundamental property [ \pm interpretable], "determined by bare output conditions" at the interface (Chomsky 1995b: 280), is the driving force for the feature-checking procedure. With respect to this property, the $\varphi$-features of argument DPs need not be checked because they are interpretable at LF (Chomsky 1995b: 285). Case, however, is always uninterpretable, and so are the $\varphi$-features of the T-node. The categorial D-feature of nouns is interpretable and, like their $\varphi$-features, need not be checked. Nevertheless, since a categorial 'strong' feature within the sublabels of the target (a D-feature for T and a Q-feature for C) must be checked (Chomsky 1995b: 278, 289), the D-feature of DPs and the wh-/Q-feature of Wh-elements will necessarily be involved in eliminating the 'strong' feature of the target. Due to the importance of [ \pm interpretable] features in the checking procedure, the interpretable features of argument DPs (and those of whmoved elements) are always accessible to the $\mathrm{N} \rightarrow \lambda$ computation (i. e., from the Numeration to LF), and can be involved in the multiple checking of targets, for example in raising structures (Chomsky 1995b: 284) and structures involving successive-cyclic wh-movement (Chomsky 1995b: 295).

In relation to the multiple checking of targets, Chomsky (1995b: 385, note 49) seeks to accommodate Watanabe's (1991) layered case theory to his revised checking account. For Watanabe (1991), a Case-feature can be 'validated' when the target (T or V) overtly raises, thus allowing Case to be checked again. Chomsky's (1995b) alternative account that does not involve case, is to allow the uninterpretable Dfeature of $\mathrm{T}$, as the target of movement (along with the uninterpretable D-feature of EXPL in embedded EXPL-constructions) to be exempt from 'erasure' - i.e., not being 
eliminated entirely (Chomsky 1995b: 280) - when deleted after being checked by the interpretable categorial D-features of DPs. Much to the same effect, the wh-/Q-feature of moved operators in the case of successive-cyclic wh-movement in embedded interrogatives is involved in the multiple checking of the target. In such instances, the target $\mathrm{C}$ has a 'strong' Q-feature which must be 'erased', but can escape being eliminated when checked. As for the wh-/Q-feature of operators, it does not delete since it is interpretable, being a variant of D on DPs, and remains accessible to the $\mathrm{N} \rightarrow \lambda$ computation (Chomsky, 1995b: 289-290).

In relation to subject-verb agreement in VSO word order in SA (as discussed in section 2.1.2.2), there have been two influential approaches to subject-verb agreement relying on PF-operations. The first approach within this trend is Aoun \& Benmamoun's (1999) and Benmamoun's (2000a, b) 'PF merger' analysis. The second approach is Ackema \& Neeleman's (2003) 'PF agreement weakening'. In the following few paragraphs, I will first discuss Aoun \& Benmamoun (1999) and Benmamoun (2000a, b) and then turn to Ackema \& Neeleman (2003).

Aoun \& Benmamoun (1999) and Benmamoun (2000a, b) argue for an analysis of the absence of number agreement between the verb and the subject DP that is not tied up with deletion of uninterpretable features on the target of movement, i.e., I/T à la Chomsky (1995b). Instead, what is relevant in subject-verb agreement in such a context is a post-syntactic process of 'PF merger' between the verb and the 'postverbal' subject DP. Aoun \& Benmamoun (1999: 181) explain that in the process of raising higher than the subject in $\left[\mathrm{Spec}, \mathrm{IP} / \mathrm{Agr}_{\mathrm{S}} \mathrm{P}\right]$, the verb - being already generated with full agreement - cannot retain the number features due to the fact that "the inherent features on the noun spell out the relational agreement features on the verb. This, in turn, makes the number suffix redundant". According to this analysis, the verb itself becomes the target since, in the process of being merged to the left of the subject - itself raised to [Spec, IP] -, it 'loses' its number features that fail to be 'spelt out' on it and "are not overtly realized by an affix" (Aoun \& Benmamoun 1999: 181).

This approach to the problem of the VSO word order in Arabic and the accompanying agreement asymmetry that shows up in SA as against the modern spoken dialects (as reviewed in section 2.1.2.2 above) differs from the parametric approach to these differences adopted in this thesis in that it seeks an account of the absence of number agreement on the verb that entirely relies on PF phonological 
processes involving the relation between the subject in [Spec, IP] and the verb raised past it. Through this emphasis on phonological properties of verbal inflection, Aoun \& Benmamoun's (1999) 'PF merger' analysis of the absence of number agreement on verbs in VSO word order in SA seems to discard the possibility that expletive proinsertion is a defining factor in agreement facts in Arabic - the standard variety and the modern spoken dialects - (see also Aoun et al. 1994: 200).

Notwithstanding the fact that their approach to the derivation of subject-verb agreement in the VSO word order in SA does not rely on the central role played by the feature structure of the target of verb movement and the 'checking' of uninterpretable features that follow from the raising of verbs to T, Aoun \& Benmamoun (1999: 186) still allow some form of morphological feature checking à la Chomsky (1995b) in that they suggest that "movement of the subject outside the VP in the VSO order is driven by some feature on I", i.e., the EPP.

Apart from Aoun \& Benmamoun's (1999) 'PF merger' claim for analyzing the absence of number agreement on verbs in VSO word order in SA, the suggestion that the derivation of VSO structures in SA, in part, involves a raising process of the subject DP to the structural subject position (i.e., [Spec, IP/TP]) is interesting enough in the context of the parametric account of the differences between SA and the modern spoken Arabic dialects adopted in this thesis. In fact, the structure I am adopting for the derivation of VSO sentences, in SA, which ties it up to the projection of the IP domain in its relation to the $\mathrm{CP}$ domain (in the context of the Phase-theoretic probe-goal-Agree system; see Table 2.2 at the end of section 2.1), is the same structure Aoun \& Benmamoun (1999) adopt to uphold their 'PF merger' analysis. The derivation of a VSO sentence in SA is as in (49) below (repeated from Table 2.2): 


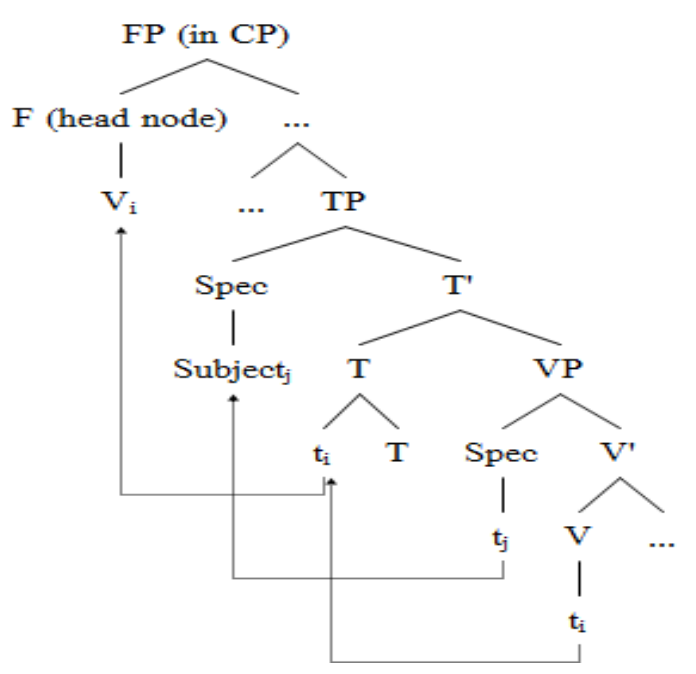

However, neither the raising of the verb per se higher than $\mathrm{T}$ to a projection in the left periphery of the IP domain (as in Aoun \& Benmamoun 1999), nor the availability or unavailability of expletive pro in the specifier of the highest projection in IP (as in Benmamoun 1992a), seems to help pin down the minimal unit of parametric variation opposing the standard variety to the modern spoken dialects of Arabic in the derivation of VSO sentences.

As for Ackema \& Neeleman (2003: 699-703), they adopt a theory of agreement weakening in $\mathrm{SA}$, where a context-sensitive rule of feature deletion at $\mathrm{PF}$ targets the $[\mathrm{Pl}]$ feature in VSO word order configurations where agreement is restricted to person and gender to the exclusion of number (i.e, weak subject-verb agreement as opposed to full agreement between the verb and the subject in person, number and gender in SVO word order configurations). In their account of the agreement asymmetry in SA, the agreement weakening in subject-verb agreement configurations in VSO sentences is a context-sensitive rule of feature deletion that operates according to the mapping rule in (1) below in conjunction with the first of two general sets of post-syntactic allomorphy rules (i.e., the rule given in (51) below).

To begin with, Ackema and Neeleman (2003: 684) adopt the mapping rule in (50) which operates in the morphological module of the grammar at the phonologysyntax interface along with a set of post-syntactic allomorphy rules (as in (51) and (53) below):

(50) The right edge of syntactic XPs corresponds to the right edge of prosodic phrases $(\varphi s)$. 
Ackema and Neeleman (2003: 686-688) also adopt two types of post-syntactic allomorphy rules. First, Ackema and Neeleman (2003: 686) adopt a type of rules like the rule in (51) below:

$$
\begin{gathered}
(51)\left\{\ldots\left[{ }_{\mathrm{A}} \mathrm{F}_{1} \mathrm{~F}_{2}\right] \ldots\left[{ }_{\mathrm{B}} \mathrm{F}_{1} \mathrm{~F}_{3}\right] \ldots\right\} \rightarrow \\
\left\{\ldots\left[\mathrm{A} \mathrm{F}_{2}\right] \ldots\left[{ }_{\mathrm{B}} \mathrm{F}_{1} \mathrm{~F}_{3}\right] \ldots\right\}
\end{gathered}
$$

An allomorphy rule like (51) deletes a morpho-syntactic feature, where 'recoverability' of deleted features operates under agreement on the basis of the premise that "... the terminal whose features are suppressed must be in a relation of agreement with a terminal whose features are not suppressed" (p. 688).

The import of this rule is that features of a terminal A can delete iff they are contained in the same local prosodic domain as a particular other terminal B. As a consequence of the application of this rule, the phonological realization of A in (51) is changed, according to the generalization in (52):

(52) " [I]f a particular terminal finds itself in the same prosodic domain as some other terminal, its phonological realization is altered" (p. 688).

However, it is not always the case that a morpho-syntactic feature in the target of the rule is deleted when the change in phonological realization of the target occurs. This is the second type of allomorphy rules in Ackema and Neeleman's (2003: 688) theory, as given in (53) below:

$$
\left\{\ldots \mathrm{A} \ldots\left[\text { в } \mathrm{F}_{1} \mathrm{~F}_{3}\right] \ldots\right\} \rightarrow\left\{\ldots<\mathrm{A} \ldots\left[\text { в } \mathrm{F}_{1} \mathrm{~F}_{3}\right]>\ldots\right\}
$$

According to Ackema and Neeleman (2003: 688), such allomorphy rules as in (53) "can lead to an alternative spell-out of a terminal namely if the usual form of B is itself a phonological word", but "does not delete a morpho-syntactic feature in their target". An example of this type of rule is the phonetic realization of a pronoun as a clitic in the presence of another terminal in the same prosodic domain. In this case, the clitic forms a phonological word with its host (indicated by angled brackets in (53) above).

In Ackema and Neeleman's (2003: 699-703) account of agreement weakening in SA, only in VSO word order is the verb contained within the same prosodic domain as the subject (in conformity with the mapping rule in (50) above). Ackema and 
Neeleman (2003: 702) give the PF representations in (54a') and (54b') for both, respectively, VSO and SVO word orders (54a) and (54b) in SA:

(54) a. [FP [F V] [IP subject $t_{V}\left[v_{P} t_{V}\right.$ object]]]

$$
\begin{aligned}
& \text { a'. }\{\text { V subject }\} \text { object\} } \\
& \text { b. [FP subject [F V] [IP } t_{\text {subject }} t_{V}\left[{ }_{V P} t_{V}\right. \text { object]]] } \\
& \text { b'. \{subject }\} \text { V object }\}
\end{aligned}
$$

In (54a') and (54b'), a verb ' $V$ ' represents a terminal and is either spelled out in the same local prosodic domain as a particular other terminal - namely, 'subject' - or not. Thus, only in the PF representation in (54a'), representing the VSO word order in SA, is the verb contained in the same prosodic domain as the verb being raised to some $\mathrm{F}$ head position to the left of IP (cf. Table 2 in Chapter Two of this thesis and section 4.1.2.1). In contrast, in the PF representation in (54b'), representing the SVO word order in SA, the subject and the verb are contained in two different prosodic domains.

Thus, according to the feature-deletion allomorphy rule in (51) above, and the premise of 'recoverability' of deleted features under agreement, the verb in the VSO word order in SA is allowed to show a 'weak' form of subject-verb agreement as it is spelled out in the same prosodic domain of the terminal it is agreeing with, i.e., the subject. However, since in the SVO word order the verb and the subject do not share the same prosodic domain, the verb can only be spelled out with full subject-verb agreement features.

As Ackema and Neeleman (2003: 702) acknowledge, their theory based on post-syntactic 'weakening' of subject-verb agreement is similar to Benmamoun's (2000b) account of the post-syntactic process of 'PF merger' (see also Aoun and Benmamoun 1999 above for the same approach), in which the verb 'loses' its number features after it raises past the subject left behind in [Spec, IP/TP].

Although I agree with Ackema and Neeleman's (2003) claim that adjacency might not be the right syntactic condition under which the 'weakening' of subjectverb agreement features in VSO word order in SA operates, I do not share their assumption that "Locality is an effect of prosodic domain formation" (p. 690) within the grammar of a language. It is certainly the case that there are locality conditions that apply at the PF side of the grammar, as there certainly are adjacency rules also applying at that interface, but locality conditions on the derivation and representation of sentences in natural language are first and foremost 'syntactic' and have an effect 
on both LF and PF. Under such a premise, the derivation of VSO and SVO sentences in SA operates on the basis of the feature structure of functional elements within the grammar of the language and the conditions this feature structure imposes on (subjectverb) agreement relations.

As will be argued in Chapter Three and Chapter Four, it is in terms of the general principle of $\mathrm{T}$-dependency that the derivation of the agreement configurations is achieved cross-linguistically. As far as the varieties of Arabic are concerned, the account I provide as to the availability of the agreement asymmetry in SA, but its absence in such modern Arabic dialects as TA and MA (among other microparametric differences between these varieties of the same language), is based on the parameterization of the probe-goal-Agree relations in the derivation of the IP domain of sentence structure and its relation to the derivation of the $\mathrm{CP}$ domain.

The line of reasoning I will follow (see Chapter Three) in analyzing differences in agreement configurations in Arabic abides by the following general guidelines:

(55) a. Rich agreement on V in VSO sentences is tied up with the presence of an $\mathrm{Agr}_{\mathrm{S}}$ node, the feature content of which requires the insertion of expletive pro in [Spec, $\left.\operatorname{Agr}_{S} \mathrm{P}\right]$ (a 'strong Phase', in the terminology of Chomsky 2001, 2004; see below). The feature content of $\mathrm{Agr}_{S}$ is determined by the Spechead/head-head dependency relations that are built up incrementally in the process of the derivation of sentence structure (see section 3.1.2).

b. SA exhibits poor agreement in VSO sentences due to the non-projection of $\mathrm{Agr}_{S}$. In this case, as the verb raises past the subject in [Spec, TP] expletive pro cannot be merged in the IP domain, but it can be phonologically realized as a pronominal clitic - $h u$ in the CP domain (in both matrix and embedded sentences). This process depends on the presence of complementizer Pinna 'that' merged in some node to the left of the raised verb in a split-CP system à la Rizzi (1997). The clitic - $h u$ cliticizes to the complementizer head Rinna (see section 3.3.4).

The strong Phase, in this case, is the position where the verb ends up attached to - a functional head $F$ in a split CP-domain à la Rizzi (1997) - irrespective of the landing site of the subject DP, which remains in the IP-domain after having been 'frozen' in [Spec, TP] after Case has been checked/valued in relation to $\varphi$-feature 
valuation and EPP satisfaction (cf. Chomsky 2001, 2004 for the valuation of Case on DPs in English).

The following section deals with the basic assumptions of the Phase-theoretic framework.

\subsubsection{The Phase-theoretic framework of Chomsky (2001, 2004)}

Chomsky's $(2001,2004)$ new agreement framework narrows down the scope of agreement configurations and functional projections to the bare minimum in that Spec-head agreement relations do not exist and are in reality just head-head relations operating under c-command in terms of identity (or, more accurately, non-distinctness of features). However, in light of the insights the Cartographic approach (see, among others, Guasti \& Rizzi 2002, Rizzi, 2004a,b, 2006) has brought to syntactic structure, Chomsky (2004: 126, note 44) does not deny that Spec-head agreement may play an important role in the realization of "surface phrase structure" (Chomsky 2004: 114). ${ }^{35}$

The Cartographic approach to syntactic structure has put forward the working hypothesis that 'interpretability' in the grammar does not merely arise as the outcome of the checking/valuation of uninterpretable features on functional heads (as in Chomsky's 1995c, 2001, 2004 Minimalist agreement framework), but could also be the outcome of 'criterial' conditions, whereby agreement configurations arise from the necessity of 'criterial satisfaction' (Rizzi 2006: 97-98) between a head and a category moved to the Spec of that head. It is this conception of how feature licensing works in syntactic structure that presented compelling evidence that Spec-head agreement relations do indeed exist (see Guasti \& Rizzi 2002).

However, what remains important in Chomsky's $(2001,2004)$ featurevaluation framework is that the property of features as either being interpretable or uninterpretable in natural languages is dissociated from the strong/weak feature dichotomy that has been the hallmark of the feature-checking account of earlier frameworks (see section 2.3.1. above). What is more, lexical items have no categorial features and are inserted in phrase-markers as roots. In this framework, verbs differ from the nouns they semantically select in argument structure in not having an intrinsic set of $\varphi$-features of their own. It is the role of the functional categories

\footnotetext{
${ }^{35}$ See also Chomsky (2004: 126, note 45) in relation to McCloskey's (2001) work on the distribution of subject properties in Irish and the possibility that expletive pro could be 'externally merged' in [Spec, CP].
} 
projected into syntactic structure to mediate the 'functional relation' that exists between verbs (and other predicates) and the DPs they associate with. Agreement in $\varphi$-features is the prototype manifestation of this functional relation between argument DPs and verbal predicates. The functional node $\mathrm{T}$, for example, is allowed to have a certain set of uninterpretable $\varphi$-features, accounting for subject-verb agreement at the interface (cf. Chomsky 1995b: 377). Chomsky's (2001, 2004) Phase-theoretic framework makes it a requirement that all functional heads, including $\mathrm{C}$, have a certain set of these features.

In view of the paramount role 'functional relations' play in the syntax of movement and agreement processes (cf. Miyagawa: 2010: 9), the Phase-theoretic case-agreement system establishes a direct relation between the core system of formal features ( $\varphi$-features, structural case, the EPP and A-movement, as interrelated notions) and the system of core functional categories T, $\mathrm{C}$ and $v$. In this caseagreement system, Agree operates to link the label LB of a 'probe' K (= LB (K)) with a 'matching goal' within the local 'search' domain of the probe (i.e., the head the probe selects as its complement and the Spec of that head). This 'matching' relation is what induces the uninterpretable features of the probe to be 'checked' or 'valued' by the goal. Probe and goal match if features are 'valued' for the goal and 'unvalued' for the probe. Matching between probe and goal is governed by the following premises (adapted from Chomsky (2001: 6)):

(56) (i) Probe and goal must both be 'active' for Agree to be able to match between them.

(ii) The probe (for example, T) must have a complete set of $\varphi$-features (it must be $\varphi$-complete, i.e., $\left.\mathrm{T}_{\text {COMPLETE }}\right)$ to have its uninterpretable $\varphi$-features valued by the paired matching element (the goal).

(iii) Case is the feature that activates DPs to enter a matching relation with the probe. This Case-feature on the goal is not matched (i.e., the probe does not carry a Case-feature), but it deletes under matching of $\varphi$-features. After valuation of Case on them, DPs are 'frozen' in place'.

The thrust of Chomsky's (2001, 2004) matching/valuation mechanism, via which Agree operates, is that if uninterpretable features entered the derivation already valued, there would be no motivation for movement to apply to bring the probe and 
the matching goal into an Agree structural relation. The values themselves would be redundant and there would be no justification as to why they should delete.

Example (57) below (Chomsky 2001: 7, example (4)) illustrates how Chomsky's $(2001,2004)$ case-agreement system works. In (57a), the 'probe' T (= P in $(57 \mathrm{a})$ ) in the matrix clause $(=\beta)$ is a raising construction. $\alpha$ is an infinitival, YP is VP, and SUBJ is the DP raised from the infinitival embedded clause as in (57b) and (57c). (57b) exemplifies the case where the embedded clause is a there-construction, with there being SUBJ raised out of the infinitival embedded clause. (57c) involves the raising of a DP (the direct object several prizes) to subject position. Chomsky notes that "awarded several prizes" in (57b) surfaces more naturally as "several prizes awarded":

(57) a. ${ }_{\beta} \mathrm{P}[\alpha[\mathrm{SUBJ}[\mathrm{H}$ YP $\left.]]]\right]^{36}$

b. There are likely [ $\mathrm{T}_{\mathrm{DEF}}$ to be awarded several prizes].

c. Several prizes are likely to be awarded.

In (57a), $\mathrm{T}$ is a defective head $\left(=\mathrm{T}_{\mathrm{DEF}}\right)$. It is a 'weak Phase' which is unable to determine case-agreement, but has an EPP-feature and a [person] feature, both of which must be valued. ${ }^{37}$ The Phase-theoretic framework retains the assumption of earlier frameworks within the Generative tradition in connection with the EPP. ${ }^{38}$ In a case like the raising structure in (57a), the EPP can either be satisfied by movement of a DP (the object several prizes in (57c)) to [Spec, IP/TP] in the matrix clause, or by insertion of expletive there into the embedded [Spec, TP] and subsequently raising there to matrix [Spec, TP]. According to this assumption, valuation of the EPP feature (or OCC(urrence)-feature) in the embedded clause, in (57b), is satisfied by there merged in [Spec, $\mathrm{T}_{\mathrm{DEF}}$ ]. As Chomsky (2004: 114) explains: "EXPL[etive] externally merged in Spec-T must delete the OCC-feature of $\mathrm{T}$ and lose its own uninterpretable features (if T is complete)".

\footnotetext{
${ }^{36}$ Chomsky (2001: 7) does not discuss the relevance of $\mathrm{H}$ in such a structure, but he later (p. 13) refers to $\mathrm{H}$ as the head of HP in his definition of the Phase-Impenetrability Condition in relation to the Phasetheoretic notion 'edge' (i.e., specifiers and adjoined elements).

${ }^{37}$ I return to the discussion of how the [person] feature of $\mathrm{T}_{\mathrm{DEF}}$ is valued in relation to the discussion of $(59 \mathrm{~b}, \mathrm{c})$ below.

${ }^{38}$ See section 2.1.1 for frameworks that predate the Minimalist Program, and section 2.3.1 for Minimalist theories that predate the Phase-theoretic framework.
} 
In relation to the raising process EXPL there undergoes, Chomsky's (2004: 114) assumption is that there in (57b) need only have a [person] feature to raise to the matrix subject position and value the $\varphi$-features of matrix $\mathrm{T}$. This suggestion follows from the fact that after satisfying the EPP in embedded subject position, there still has its own [person] feature which could not be valued by the [person] feature of $\mathrm{T}_{\mathrm{DEF}}$ in embedded subject position. Since $\mathrm{T}_{\mathrm{DEF}}$ is not $\varphi$-complete and cannot determine agreement, [person] on EXPL there survives 'deletion'/'erasure' (see section 2.3.1 for Chomsky's 1995b conception of deletion processes in agreement relations). On this account, the relevant feature for raising there would be its [person] feature rather than the EPP.

However, the EPP feature associated with $\mathrm{T}$ in the matrix clause in (57b) could still be the motivating factor for the raising process of the EXPL (i.e., 'internally' merging EXPL in matrix [Spec, TP]) the same way as the EPP feature of the embedded clause has been the motivating factor for the 'insertion' process in the embedded clause (EXPL 'externally' merged in [Spec, TP]). In this connection, Chomsky (2004: 114) discusses two possibilities for how [person] on EXPL is valued, the second of which he seems to adopt. One possibility is that [person] on there in matrix $[\mathrm{Spec}, \mathrm{TP}]$ is valued by the uninterpretable $\varphi$-features of T. Then the EPP feature on $\mathrm{T}$ in the matrix clause gets a value as a consequence of its Spec position being occupied after raising there to that position. Another possibility is that EXPL there in matrix [Spec, TP] is a head, and as such it probes and agrees with the $\varphi$ complete goal $\mathrm{T}$ (i.e., $\mathrm{T}_{\text {COMPLETE }}$ selected by $\mathrm{C}$, the latter being a strong Phase determining agreement). In Chomsky's (2004: 114) own terms: "Suppose EXPL is a simple head, not formed by Merge. In a label-free system, EXPL is accessible without search as a probe and can match and agree with the goal T". 39

\footnotetext{
${ }^{39}$ These two possibilities are theoretically similar in that the matching/Agree relation between there in matrix [Spec, TP] and matrix T in (57b) can only apply after the DP several prizes kept in situ values the $\varphi$-features of matrix T via Long Distance Agreement (see the next paragraph for the discussion of such a process). Under Phase-theoretic assumptions, this matching relation could happen only if we assume that matrix T gets a value for its uninterpretable $\varphi$-features from the interpretable $\varphi$-features of the DP several prizes only after EXPL there is raised to matrix [Spec, TP]. It is only at this stage of the derivation that both the $\varphi$-features of matrix $\mathrm{T}$ and the [person] feature of there both get a value and are erased.
}

Chomsky's $(2001,2004)$ Phase-theoretic assumption that the $T_{\mathrm{DEF}}$ has a [person] feature, which $T_{\text {DEF }}$ shares with EXPL, suits well Chomsky's (2004: 114) assumption that EXPL is a head rather than a phrase category. 
The question that remains is that, since the matrix [Spec, TP] in (57b) has to be occupied by further raising there to the matrix subject position, how could the DP several prizes - which is kept in situ since it cannot raise over there (see the discussion of $(59 \mathrm{~b}, \mathrm{c})$ below $)$ - have its Case-feature valued? The assumption is that if there is selected for the Numeration, the interpretable $\varphi$-features of the DP several prizes can only match and value the uninterpretable $\varphi$-features of matrix $\mathrm{T}$ by a process of Long Distance Agreement (LDA) without raising (Chomsky 2004: 114). Case on several prizes is valued as a consequence of the matching/Agree relation. Presumably, LDA also accounts for how the [person] feature of $\mathrm{T}_{\mathrm{DEF}}$ is valued. ${ }^{40}$ In $(57 \mathrm{c})$, in the absence of there in the Numeration, matching of several prizes and $\mathrm{T}_{\mathrm{DEF}}$ of the embedded infinitival clause in the feature [person] induces raising of $\mathrm{DP}$ to $\left[\mathrm{Spec}, \mathrm{T}_{\mathrm{DEF}}\right.$, and as a consequence of DP raising, the EPP in the embedded [Spec, TP] position is satisfied. The DP several prizes is further raised to the matrix subject position where it values the full $\varphi$-set of the T node. As a result, Case on DP gets a value because matrix $\mathrm{T}$ is $\varphi$-complete, and the EPP is satisfied as a result of the raising process. As stated in (56iii) above, the uninterpretable Case-feature of a DP, not being a feature of the target, is assumed to be valued as a by-product of the matching/Agree relation. It is obvious that, in the Phase-theoretic framework, Case is no longer assumed to be a trigger of movement and agreement processes.

In an unaccusative structure like (58) below, the DP associated with the verb and $\mathrm{T}$ is a direct object (DO) (according to Chomsky 2001), which is selected by the 'light' verb $v$ (which is different from the 'strong' Phase head 'light' verb v*) which makes up the $v \mathrm{P}$ above VP is a weak Phase only, and does not project a Spec position. As in the raising example in (59) with EXPL there, agreement between DP and the verb is determined by (matrix) $\mathrm{T}$ :

\footnotetext{
${ }^{40}$ What is interesting in this account in relation to the nature of $\mathrm{T}$ (or Tense features) in natural languages (see section 3.1.1.2) is the assumption that, when T is defective in English, it enters the derivation with an unvalued [person] feature that seems to be intrinsic to it since it does not come from a $\mathrm{C}$ node selecting $\mathrm{T}$ as a complement in the embedded clause in a there-construction like (57b) above. If this is the right conclusion to draw for $\mathrm{T}_{\mathrm{DEF}}$ in English, then the [person] feature of $\mathrm{T}_{\mathrm{DEF}}$ is, in fact, engaged in some sort of agreement albeit 'defective' since it is not reflected in the subject-verb agreement configuration that obtains at Spell Out. This defective Agree relation would obtain at the intermediate 'weak' Phase in the embedded clause between $T_{\mathrm{DEF}}$ and EXPL, and no valuation of the person feature of there applies at this stage. As already discussed above, EXPL awaits the next 'strong' Phase to Agree with $\varphi$-complete $T$ in the matrix clause, thus getting a value for its uninterpretable [person] feature at the 'strong' Phase level where LDA applies between the subject DP kept in situ in the embedded clause and $\mathrm{T}_{\text {COMPLETE }}$ in the matrix clause.
} 
(58) ?There arrived three men. (Chomsky's 2001: 21, example (29a))

In (58), the interpretable $\varphi$-features of DO value both the uninterpretable $\varphi$-features of $\mathrm{T}$ and those of the 'light' verb $v$. The uninterpretable Case-feature of DO is valued as a by-product of the matching/Agree relation between $\varphi$-complete $\mathrm{T}$ and the DP, as was the case in the raising example (57b). The similarity between the two examples is further suggested by the Case value the in situ DP receives in both sentences, i.e., NOM Case. Due to its uninterpretable [person] feature, EXPL there raises to [Spec, $\mathrm{TP}$ ] and its [person] feature gets a value because $\mathrm{T}$ is $\varphi$-complete. The EPP is satisfied as a consequence of the raising process.

Embedding (58) under a raising verb yields the structure in (59a), where $\mathrm{EXPL}=$ 'expletive' (adapted from Chomsky 2001: 19, examples (21a-c)):

(59) a. [C [T be likely [EXPL to arrive three men]]]

b. There are likely to arrive three men.

c. *Three men are likely there to arrive.

In (59b), EXPL values the EPP feature of $\mathrm{T}_{\mathrm{DEF}}$ and then EXPL is raised, triggered by its [person] feature which gets a value because $\mathrm{T}$, in the matrix clause is $\varphi$-complete. Since matrix [Spec, TP] is now occupied by EXPL, the EPP is satisfied. Both the [person] feature of $\mathrm{T}_{\mathrm{DEF}}$ in the embedded clause and the $\varphi$-features of the tense node in the matrix clause are uninterpretable and, presumably, have to be valued by the interpretable $\varphi$-features of the in situ DP three men via the process of LDA.

As discussed in relation to the valuation of the Case-feature of the DP several prizes by LDA in (57b) above, the DP three men in (59b) is precluded from raising over EXPL which bars the application of Move. EXPL is raised instead, since it is closer to the target. LDA applies between $\varphi$-complete matrix T and the DP three men. The matching relation that results from this pairing values the $\varphi$-features of the matrix T node and, in turn, the Case-feature of the DP three men gets a value (i.e., NOM Case).

The movement and Agree processes (59b) and (57b) undergo suggest that what the derivation seeks to achieve is to minimize both the 'cost' and 'search' of computations: EXPL is 'closer' to the probe and its movement satisfies the EPP of the matrix clause with no so great a cost as would have resulted if three men were raised 
instead. This, in turn, reinforces the suggestion discussed above as to the motivating factor in the raising of there, which might still be the EPP rather than the mere fact that EXPL has a [person] feature in need of valuation. In this case, [person] on the EXPL - a simple D category - would be like the Case-feature on full lexical DPs since the function of these features on such elements selected for the Numeration is to render the element to be raised 'active' as a trigger for movement (see (56i) above). The motivating factor of the raising process itself is to satisfy the EPP.

On this account, the derivation of (59c) cannot converge because raising three men over there in the embedded clause to satisfy the EPP in the matrix clause is not necessary and raising there minimally meets that requirement. The fact that the DP three men matches the matrix $\mathrm{T}$ in all features and, in principle, could be favoured as a goal over the EXPL (with a [person] feature only) is irrelevant for convergence of the derivation at the interface. In other words, movement (Move) is strictly local and has to apply successive-cyclically - or, in Phase-theoretic terms, by Phases - from one position in the derived structure to the next available position in the local 'search' domain of the probe (Chomsky's 2001 Phase Impenetrability Condition). In contrast, Agree can apply by long distance and can apply across clause boundaries. Thus, in (59b) - as in (57b) - LDA is responsible for the Agree relation that obtains between the DP kept in situ and the $\varphi$-complete matrix T. LDA, thus, accounts not only for subject-verb agreement, but also for how the EPP is satisfied.

To sum up the assumptions of the Minimalist Phase-theoretic framework (as compared to the earlier Minimalist assumptions of Chomsky 1995a,b; see section 2.3.1) in the context of verbal inflection as conceived in this thesis, an important prerequisite of the analysis of raising processes, which Chomsky $(2001,2004)$ entertains for the case-agreement system in Phase-theoretic terms, is that elements undergoing raising have some feature (case on DPs or [person] on EXPL) that makes them 'active' in relation to the probe that seeks them.

Another important characteristic of the Phase-theoretic case-agreement framework is the assumption that EXPL in English has a [person] feature that has to be erased at the end of the process that raises EXPL in embedded contexts in satisfaction of the EPP in both embedded and matrix clauses. On this assumption, not only can the EPP be paired with another formal feature or features for convergence (i.e., the uninterpretable [person] feature of EXPL there (in Chomsky 2001, 2004), the 
uninterpretable Case-feature of T and that of EXPL there (in Chomsky 1995a), or the uninterpretable D-feature of T and that of EXPL there (in Chomsky 1995b)), but the paired feature(s) can also survive 'erasure', after EXPL there satisfies the EPP in the embedded clause and raises to [Spec, TP] in the matrix clause to check the EPP again. $^{41}$

Thus, the 'checking' and 'deletion' of uninterpretable features on EXPL elements in relation to $\mathrm{T}$ (i.e., Case on embedded $\mathrm{T}$ and on EXPL in the framework of Chomsky 1995a, the D-feature of embedded T and of EXPL in Chomsky 1995b, or [person] on EXPL in Chomsky 2001, 2004 in relation to $\mathrm{T}_{\mathrm{DEF}}$ in embedded clauses), in the sample examples involving there constructions, does not necessarily 'erase' these features before Spell-Out (alternatively at the strong Phase). Since these features can escape being 'erased', they can still be 'visible' at the next strong Phase satisfying the EPP for interpretability of the structure at LF, and for any morphological 'reflexes' the valuation procedure yields at PF. In this connection, Chomsky (2004: note 45 , p. 126) - in the context of his discussion of whether the Spec-head relation has a role to play in the grammar (Chomsky 2004: 114) - comments on the problem of accounting for the distribution of EXPL pro in Irish (citing McCloskey 2001). In particular, EXPL could be externally merged in the specifier position of the three core functional heads the Phase-theoretic framework of Chomsky $(2001,2004)$ is concerned with, i.e. $v^{*}, \mathrm{~T}$ and $\mathrm{C}$ to satisfy some EPP feature of these heads. ${ }^{42}$

\footnotetext{
${ }^{41}$ In Chomsky (2001, 2004), [person] on EXPL survives 'erasure' to be able to further raise to matrix [Spec, TP], thus satisfying the EPP again in the matrix clause. In Chomsky (1995a: 431) the 'intrinsic' Case-feature of $\mathrm{T}$ and that of EXPL in there-constructions, involving an embedded clause, survive 'erasure' once the EPP is satisfied in the embedded clause. In the latter case, the consequence of Case not being 'erased' on EXPL, first merged in [Spec, TP] in the embedded clause, allows EXPL to raise to matrix [Spec, TP] (the specifier of TP in the embedded clause not being a Case position) not only to satisfy the EPP again in the matrix clause, but also to have its Case-feature 'erased' by matrix T. Similarly, the consequence of Case not being 'erased' on embedded T allows Case on the subject DP kept in situ to be checked 'covertly' against the Case-feature of embedded T. At the interface, all Casefeatures are appropriately checked and 'erased'.

${ }^{42}$ Taking the structure [XP [(EA) H YP]], Chomsky (1998: 102), states: "If H is $v / C$, XP is not introduced by pure Merge". He explains this by tying external Merge of EXPL to non-Phase heads, i.e. $H$ must be $T$, when $T$ is not selected by a $\varphi$-complete $C$, i.e., when $T$ is $T_{\text {DEF }}$. Nevertheless, the existence of V2-expletives which function as 'expletive topics' merged directly (by external Merge) into spec-CP when no other XP raises to the initial position before the verb (Holmberg \& Platzack 1995, Bobaljik 2002) undermined this assumption.
} 


\subsubsection{The Agreement Approach to the EPP (Miyagawa 2010)}

In the preface to his book Why Agree? Why Move? Unifying Agreement-Based and Discourse Configurational Language, Miyagawa (2010: $\mathrm{x}$ ) introduces the notion of 'EPP-triggered movement' as follows:

'EPP-triggered movement' ... is the type of movement that ... refer[s] to a broader range of movement than just movement of the subject to Spec, TP. Included in this 'general' type of 'last resort' movement are certain head movements, which I discuss in conjunction with pro-drop, and movement of the A' variety such as wh-movement.

In this move, Miyagawa (2010) is inspired both by Chomsky's Phase-theoretic Minimalist framework $(2001,2004,2008)$ and the kind of agreement framework expounded in Kiss (1995) where 'agreement' takes on a wider meaning by taking grammatical features such as Focus and Topic into account as features that trigger movement in the first place. This agreement approach to the EPP and to movement processes in general abides by the following premises (Miyagawa 2010: 4):

(60) - The occurrence of agreement always correlates with movement

- Number agreement is responsible for movement

- Agreement, not case, triggers movement

The last premise in (60) is already familiar from Chomsky's Phase-theoretic framework $(2001,2004)$ as reviewed in section 2.3.2, above. As for the two first premises, they point to the necessity of an agreement trigger for the kind of movement Miyagawa (2010) is interested in - namely, 'EPP-triggered' movement.

Nevertheless, what differentiates Chomsky's $(2001,2004)$ approach to the EPP and movement from Miyagawa's (2010) is Chomsky's $(2001,2004)$ view that the relation Agree is independent of movement and is only involved in head-head agreement relationships rather than head-head and Spec-head agreement relationships. As for Miyagawa (2010: 8-9), he contends that “ ... this intuition that agreement emerges as a specifier-head (Spec-head) relation is correct...” and that "... agreement requires a Spec-head relation by showing that Agree takes place to establish functional relations". 
The thrust of Miyagawa's (2010: 7-8) EPP-triggered movement approach to agreement is that the grammar of languages establishes functional relations between features on elements in the lexical layer and matching features on elements in the functional layer of sentence structure giving rise to such notions as "topic-comment", "subject of a clause", "focus", and content questions among many other modes of expression. According to Miyagawa (2010: 9), this result can only be implemented by the combination of the two independently motivated operations of the grammar namely, Agree and Move.

\subsection{Conclusion}

The Phase-theoretic, parameterized probe-goal-Agree approach to the derivation of the VSO vs. SVO word orders in SA and the modern spoken Arabic dialects, as expounded in this chapter, will be significant in the two following Chapters (Chapter Three and Chapter Four) in pinning down the units of parametric variation in the derivation (and representation) of the functional domain - both IP and CP - crosslinguistically.

This approach primarily relies on the EPP-triggered movement analysis of the functional structure of sentences in natural language (Roberts \& Roussou 2002, Miyagawa 2010, Roberts 2010a, Biberauer et al. 2010) and the import of the 'edge' feature Chomsky (2008) posits on C and $v^{*}$ as independent of both the Agree-features and the EPP-feature in allowing free 'internal Merge' (i.e., Merge via Move) to any head. It is the processes involved in the valuation of these features, which ultimately bring the goal closer to the probe via movement within a feature-based probe-goalAgree system of dependency relations within agreement configurations. 


\section{CHAPTER THREE}

\section{FUNCTIONAL STRUCTURE AND CROSS-LINGUISTIC VARIATION IN ITS REPRESENTATION}

\subsection{Introduction}

In this chapter, I propose the working hypothesis that at least some languages project sentence structure as $\operatorname{Agr}_{S} \mathrm{P}$ rather than as IP/TP. This hypothesis finds support in a system that postulates a direct relation between Move and Agree, and the Spec-head agreement relation as important ingredients in the derivation of IP and CP in sentence structure.

In addition to the important role $\mathrm{Agr}_{\mathrm{S}}$ parametrically plays in the derivation of sentence structure, natural languages rely on the T-node in establishing dependency relations between the lexical layer of sentence structure (i.e., the domain of 'thematic relations' involving the verb and its arguments) and the functional layer (i.e., the domain of 'functional relations').

Section 3.1 of this chapter deals in more detail with the working hypothesis I have put forward in sections 2.2 and 2.3.3 - namely, the fundamental role of Spechead/head-head Agree relations (in particular, Miyagawa's 2010 'EPP-triggered movement' analysis of agreement) and that of an $\operatorname{Agr}_{S}$ node in setting the right configurational dependency relations in sentence structure for subject-verb agreement. The cross-linguistic variation in the formal means by which subject-verb agreement finds its expression in sentence structure is further upheld in sections 3.2, 3.3 and 3.4 in a system of probe-goal Agree structural configurations that govern the dependency relations between the elements projected in sentence structure. In section 3.2, I address the problem of EXPL-insertion crosslinguistically. In section 3.3, I deal with the expression of tense/aspect, mood, and modality in the SA verbal agreement system. I also discuss the hypothesis of the non-projection of $\operatorname{Agr}_{S}(\mathrm{P})$ in the sentence structure of SA in the VSO word order and pin down the main difference between SA and TA in terms of this hypothesis. Section 3.4 is a conclusion to the chapter. 


\subsection{Some Minimalist assumptions about functional structure in derivations}

In section 3.1.1 below, I have a look at how subject-verb agreement is conditioned, in particular, by the dependency relations that bind the projection of the IP domain to the $\mathrm{CP}$ domain. In addition to this close relationship between the projection of the two fundamental domains of functional structure cross-linguistically, the parametric availability of an $\mathrm{Agr}_{\mathrm{S}}$ node and its interaction with the formal properties of Tense set further structural restrictions on word order properties in V-raising/null-subject languages, particularly in relation to the availability of Spec positions (Bobaljik \& Jonas's 1996: 211 '[Spec, TP] parameter').

In section 3.1.2, I further discuss the central role of the Spec-head agreement relation in relation to Miyagawa's (2010) 'agreement approach' to the EPP and Roberts \& Roussou's (2002) 'Tense-criterion' hypothesis in the derivation of functional structure in natural language. Section 3.1.3 is a summary.

\subsubsection{Verbal Inflection and the Structure of IP and CP}

The early Minimalist approach to the cross-linguistic analysis of the agreement facts obtaining in the inflectional domain of sentences (Chomsky 1995c) highlighted the notion of 'feature checking'. According to Chomsky (1993: 178), the checking domain is "'heterogeneous', an 'elsewhere' set". The idea was that the domain of feature checking under Agr projections (specifically projected for this purpose in the higher functional layer of sentence structure) and that of $\theta$-role assignment are disparate though essentially related for the well-formedness of structural representations (cf. Chomsky, 1995a: 403-4). Later (in Chomsky 1995b), the role of autonomous agreement heads for subject $\left(\mathrm{Agr}_{\mathrm{S}}\right)$ as well as object $\left(\mathrm{Agr}_{\mathrm{O}}\right)$ agreement was restricted. A further step (in Chomsky 2001, 2004) was the restriction on the role

of the Spec-head agreement relation. Chomsky $(2001,2004)$ argued that agreement is essentially a head-head structural relation (see Chapter Two, section 2.3.2).

However, according to the Cartographic approach to syntactic structure (Cinque 1999, Rizzi 2006, Guasti \& Rizzi, 2002; see also Kayne 2000), the restriction applying to the Spec-head agreement relation is perhaps best looked at as a theoretical guideline or a notational variant of the basic structure that has been adopted for the study of parametric and micro-parametric variation ever since Pollock (1989). In this light, Chomsky's (1995b, 2001, 2004) revision in the Minimalist framework of case- 
agreement relations, where the $\mathrm{Agr}_{\mathrm{S}}$ head no longer figures as a core functional category, is not a dismissal of the possibility that languages could still, parametrically, project the structure of sentences as $\operatorname{Agr}_{S} \mathrm{P}$. Chomsky (1995b: 351), for example, explains that $\operatorname{Agr}_{S}$ could still be allowed to occur in sentence structure in some languages or even some constructions in some languages (for example, in multiplesubject constructions (MSCs)):

It [i.e., $\operatorname{Agr}_{S}$ ] appears in MSCs, but lacks independent motivation elsewhere, as matters now stand. For languages of the French-English type, then, Agr is not in the lexicon (unless MSCs appear marginally, with extraposition). Agr therefore occurs in highly restricted ways.

Opting for that parameter (i.e., projecting the sentence as $\operatorname{Agr}_{S} \mathrm{P}$ instead of simply as IP/TP) would trigger the right setting for Spec-head agreement configurations - at least at the IP level - in the languages that could be postulated to have an $\mathrm{Agr}_{\mathrm{S}}$ node above the T node.

Section 3.1.1.1 below is a discussion of how the structure of IP could be posited to project as $\operatorname{Agr}_{S} \mathrm{P}$. As part of the view that an Agr node is projected in the syntax of some languages, I have a look at Kayne's (1989b, 2000) idea that an abstract AGR element is responsible for establishing a special case of subject-verb agreement in (a variety of) English. Section 3.1.1.2 highlights the central role the tense properties of sentences play in the derivation of IP and CP. Section 3.1.1.3 deals with Bobaljik \& Jonas's (1996) and Bobaljik \& Carnie's (1996) parametric approaches to the derivation of $\operatorname{Agr}_{S} \mathrm{P}$.

\subsubsection{The Structure of IP as $A g r_{S} P$ and Parameters of Variation in the Realization of Subject-verb Agreement}

Allowing $\mathrm{Agr}_{\mathrm{S}}$ to be, parametrically, projected as a functional head in the syntax (linked via a dependency relation to the projection of the $\mathrm{T}$ node in IP and the $\mathrm{C}$ node in $\mathrm{CP}$ ), the structure in (1) would be normally derived in a pro-drop (not necessarily V-initial) language like Italian or TA: 
(1)

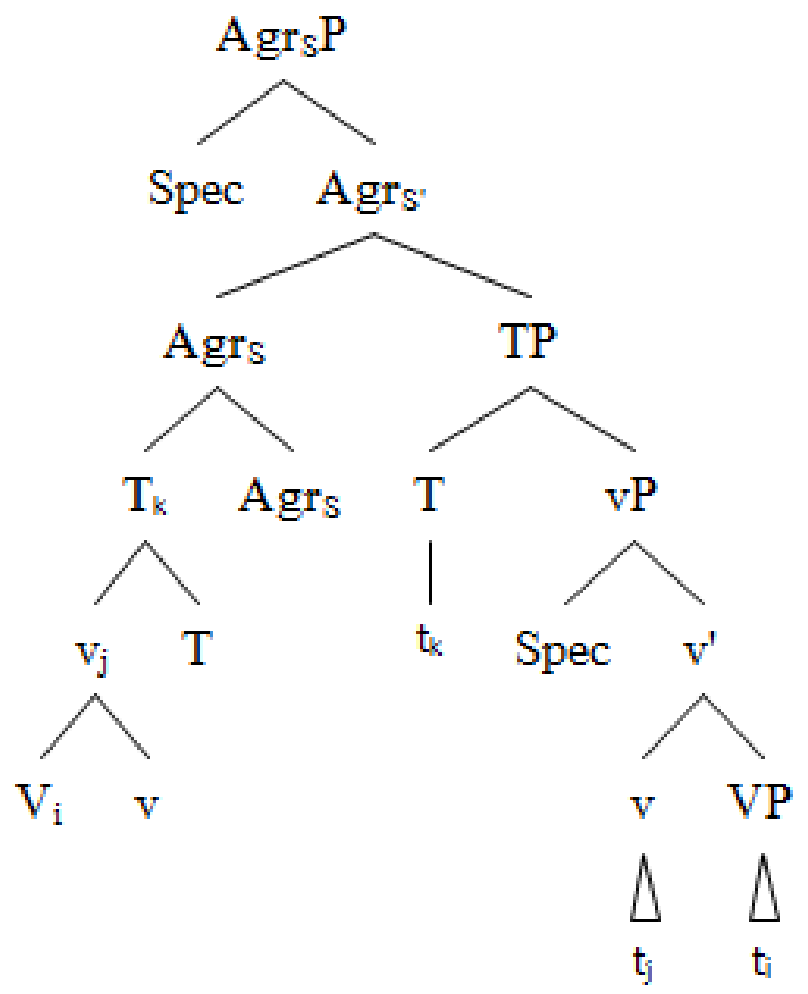

In (1), the lexical verb first attaches to the 'light' verb $\mathrm{v}$, and then the $\mathrm{V}-v$ complex moves to $\mathrm{T}$, which also raises to attach to the $\mathrm{Agr}_{\mathrm{S}}$ node. These adjunction processes ultimately yield the composite complex $\mathrm{V}-v$-T-Agrs. I will come back below, in this section, to Bobaljik \& Carnie's (1996) cross-linguistic characterization of these processes, particularly in relation to the theoretical possibility that [Spec, TP] does not project in some languages, (as the representation in (1) shows). In section 3.3 below, I put forward the proposal that $\mathrm{Agr}_{S}$ is the minimal unit of parametric variation in Arabic.

Looking first at the sentence structure of English, Van Gelderen's (1993) suggestion that the loss of the V2 effect in declarative sentences in English was concomitant with the relocation of Tense features from $\mathrm{C}$ to I may point to the possibility that sentences in English simply project as IP/TP directly complementselected by C. I come back below to the importance of Tense (and the features associated with the T-node in sentence structure) in establishing dependency relations in the derivation of sentences cross-linguistically. The features associated with the Tnode of sentence structure are either interpretable or uninterpretable. In addition to this fundamental dichotomy, which has survived the major revisions Chomsky's (1995b) Minimalist program has undergone, these features differ on the Phase- 
theoretic assumption - in Chomsky's (2008) revised probe-goal-Agree system - that some of these features are inherent to $\mathrm{T}$, but others are inherited by $\mathrm{T}$ from $\mathrm{C}$. Table 3.1 below shows the breakdown of the feature composition of $\mathrm{T}$ in the IP domain according to a conception of feature valuation as found in Chomsky's (2008) featureinheritance theory, and, in particular, in Roberts' (2010a,b) and Biberauer and Roberts' (2010) copy deletion theory:

Table 3.1. The feature composition of Tense in the IP domain of functional structure

\begin{tabular}{|c|c|c|}
\hline Features on $\mathrm{T}$ & Inherent to $\mathrm{T}$ & $\begin{array}{c}\text { Inherited by } \mathrm{T} \text { in the IP } \\
\text { domain from } \mathrm{C}\end{array}$ \\
\hline a. [+interpretable] & $\mathrm{T}($ ense)-features $(=[\boldsymbol{i} \mathbf{T}])$ & - \\
\hline \multirow{4}{*}{ b. [-interpretable] } & \multicolumn{2}{|c|}{ Matched Features } \\
\hline & $\begin{array}{l}\text { V-features } \\
\text { a D-feature }(= \\
\\
[\boldsymbol{u D D}])\end{array}$ & $\begin{array}{ll}- & \varphi \text {-/Agree features } \\
& (=[\boldsymbol{u} \varphi])\end{array}$ \\
\hline & \multicolumn{2}{|c|}{ Non-matched Features } \\
\hline & \multicolumn{2}{|c|}{$\bullet \quad$ EPP feature } \\
\hline
\end{tabular}

As Table 3.1 above shows, only the T-features on $\mathrm{T}$ in the IP domain are [+interpretable] (i.e., $[\boldsymbol{i T}]$ on $\mathrm{T}$ ), and there are no [+interpretable] features that $\mathrm{T}$ inherits from C. As for [-interpretable] features, these are either inherent to $\mathrm{T}-$ namely, T's unvalued V-features (Biberauer and Roberts 2010: 265) and the D-feature that Roberts (2010a: 108, 113) posits on $\mathrm{T}$ to account for the parametric availability of pro in [Spec, TP] in some null-subject systems. T also inherits [-interpretable] features from C - namely, $\varphi$-/Agree-features (Chomsky 2008: 144-149) and, possibly, its EPP-feature (Chomsky 2008: 157).

However, as Table 3.1 also shows, the [-interpretable] EPP-feature is different form the other [-interpretable] features of the T-node in that only the EPP-feature is a non-matched feature. By contrast, the categorical V-and D-features and the $\varphi$-/Agree features have to match 'non-distinct' features on agreeing elements in the process of subject-verb agreement. This difference might be an indication that the EPP-feature is 
actually an intrinsic property of the T-node, which plays the essential role that used to be attributed to NOM Case in earlier Minimalist theories (Chomsky 1993, 1995a,b) - namely, providing the necessary trigger for displacement processes in the overt syntax.

As I will be dealing with later (see section 3.3 and 4.3, in particular), the uninterpretable D-feature, which Roberts $(2010 \mathrm{a}, \mathrm{b})$ posits to be an intrinsic property of the T-node in null-subject languages, may be a more widespread feature in the grammar of languages cross-linguistically. In the probe-goal-Agree system I am adopting, the $[u \mathrm{D}]$-feature is posited to be a characteristic property of the higher functional domain of clause structure in a system where the INFL node splits into T and $\mathrm{Agr}_{\mathrm{S}}$ (see section 3.1.1.3) and the $\mathrm{C}$ node into Force and Finiteness (see section 3.3.4).

The dependency relation between $\mathrm{C}$ and $\mathrm{I} / \mathrm{T}$ in English might have triggered another aspect of feature association or feature sharing of the kind Rizzi (1990) describes in terms of Agr-in-Comp that establishes Spec-head/head-head agreement configurations in CP in relation to IP. The sample examples Rizzi (1990: 51-53) discusses in this context involve wh-extraction of subjects both in matrix and embedded contexts, as in (2) and (3) below:

(2) a. Who do you think [t' $\varnothing[\mathrm{t}$ left $]]$

b. * Who do you think [t' that $[\mathrm{t}$ left $]]$

(3) a. Who Ø [t left]

b. * Who did [t leave]

Rizzi (1990) proposes that in both (2a) and (3a) an abstract Agr element (represented by $\varnothing$ in (2a) and (3a)) is present in C triggering the necessary Spec-head agreement configuration in $\mathrm{CP}$ (between $\emptyset$ and $t^{\prime}$ in (2a), and between $\emptyset$ and who in (3a)) for the licensing of the subject wh-trace $t$ in the Spec position of the head C selects as complement in sentence structure - namely, I/T. Thus, in (2a) and (3a) the licensing of $t$ in the subject position [Spec, IP/TP] follows from both the Spec-head agreement relation in C and the head-head agreement relation between C and I/T. Rizzi (1990: 57) explains that in the grammatical cases (2a) and (3a) "by transitivity $\mathrm{C}$ agrees with the maximal projection of I, its complement IP", thus allowing I to agree with its own Spec for the licensing of the subject trace in [Spec, IP]. 
In contrast, in (2b) and (3b) $\mathrm{C}$ is already occupied by some lexical element (the lexical complementizer that in (2b) and the auxiliary did in (3b)). Thus, Rizzi's (1990) assumption is that there is no Agr element in C in (2b) and (3b), and that Spechead agreement in CP cannot be triggered. Similarly, no head-head agreement relation can ever be established between $\mathrm{C}$ and $\mathrm{I} / \mathrm{T}$ in these subject wh-extraction cases, and Spec-head agreement in IP fails to arise. The subject trace $t$ in [Spec, IP/TP] in these wh-constructions remains unlicensed ruling (2b) and (3b) out.

Thus, it is in terms of the mechanism of Agr-in-Comp that the parallel in the grammaticality/ungrammaticality of the wh-constructions in (2) and (3) finds a plausible explanation suggesting that a certain dependency of functional relations is operative in sentence structure in both the IP and the CP domains.

Similar to Rizzi's (1990) Agr-in-Comp analysis is Kayne's (1989b, in Kayne 2000: 190-3) proposal of a manifestation of an AGR element in the sentence structure of a variety of English previously studied by Kimball \&Aissen (1971). Kayne (1989b, 2000) proposed that the postulation of the parametric option of an abstract AGR element in $\mathrm{C}$ could explain the contrast in agreement configurations exemplified by (4a) vs. (4b) below:

(4) a. Which girls do the boy think should be invited? (Kayne's 2000 (18): 193)

b. *Which girl does the boys think should be invited? (Kayne's 2000 (19): 193)

As Kayne (1989b, 2000) puts it, since the abstract AGR element does not dominate an affix in the IP domain, in (4a), "we take the bare form of English present tense verbs not to bear any suffix" (Kayne 2000: 191). Kayne proposes that, in a sentence like (4a), AGR is free to raise to C. In contrast, in (4b), AGR dominates an affix in the IP domain, i.e., the $-s$ of the auxiliary does. Thus, in (4b), AGR cannot raise independently to the CP domain. As Kayne (2000: 194) maintains, the raising of the abstract AGR element in (4a) is not the same as the raising of the auxiliary do in that sentence (similarly for the auxiliary does in (4b)). Therefore, besides the assumption that AGR raises to C in the grammatical (4a), Kayne (1989b, 2000) maintains that raised auxiliaries - such as do and does (4a) and (4b), respectively - occupy "a head position below AGR". ${ }^{1}$

\footnotetext{
${ }^{1}$ Building on Pollock's (1989) assumptions of a Split-IP domain, Kayne (2000: 194-197) maintains contra Pollock (1989) - that, in English, auxiliary verbs (do, have - in some of its uses - and be) do not end up in the highest functional position $\mathrm{F}_{1}$, in the IP domain below $\mathrm{C}$, but only raise as far as $\mathrm{F}_{2}$, as a
} 
According to Kayne's $(1989 b, 2000)$ assumptions, the raising process of AGR to $\mathrm{C}$ in (4a) has the effect of coindexing the abstract AGR element raised to $\mathrm{C}$ and the wh-phrase which girls. As pointed out in the previous paragraph, this agreement configuration does not obtain in (4b) where the abstract AGR element is assumed not to be in C. In this case, AGR fails to be coindexed with the appropriate XP element. In Kayne's (2000: 192) own terms: "coindexing $-s$ with the wh-phrase will never hold”. (Kayne 2000: 193).

It may be the case that the abstract AGR that Kayne (1989b, 2000) postulates in the variety of English in question is an additional feature associated with $\mathrm{C}$ that enters into the characterization of the C-T dependency. More generally, Kayne's (1989b, 2000) analysis is reminiscent of the kind of analysis that Rizzi (1990) refers to as Agr-in-Comp, which relates to the licensing of the traces of wh-moved subjects (as the discussion of (2) and (3) above shows) and/or the licensing of a certain array of specifier and head positions linking the IP domain to the left periphery (Rizzi 1997). As already discussed (see, in particular, sections 2.2.3 and 2.3.2), a similar trend of investigation has been proposed by the Phase-theoretic framework in Generative Grammar (Chomsky 2001, 2004) and more recently by Chomsky (2008) in relation to the mechanism of 'feature inheritance'.

\subsubsection{The nature of Tense in natural languages and its role in agreement in IP and $C P$}

In the cross-linguistic characterization of functional structure, not less important than the projection of $\mathrm{Agr}_{\mathrm{S}}$ is the role of Tense in establishing the right dependency relations in IP and CP. As far as the tense properties of sentence structure are concerned, there have been two different, but reconcilable, claims about the nature of

\footnotetext{
lower functional head position in the IP domain. Taking into account the positioning of adverbs (as adjoined to maximal positions, and never to nonmaximal positions ) and Negation (as a head Neg), Kayne (2000:195-196) assumes that an adverb like probably, in (i) below, is adjoined to the maximal projection of $\mathrm{F}_{2}$, and that when Neg is present (as in (i)), the adverb is adjoined to NegP:

(i) John probably wasn't there

To account for why the auxiliary verb was under $\mathrm{F}_{2}$ in (i), ends up to the left of Neg, Kayne (2000) assumes (following Pollock 1989) that Neg occurs below $F_{1}$ but above $F_{2}$, and that (contrary to Pollock 1989) was adjoins only to Neg rather than moving on from Neg to $F_{1}$.

${ }^{2}$ In section 4.3.2, I return to a reconsideration of Kayne's analysis in terms of Agree-feature inheritance.
} 
Tense in natural language. The first view has it that Tense (i.e., the abstract functional T-node of sentence structure) is 'referential'/'anaphoric' in nature. This is the view held by most practitioners in the linguistic tradition of Transformational Generative Grammar since its first inception in the nineteen fifties. The second approach to the nature of Tense in natural language has been predominant ever since Stowell's (1981) investigation of the interaction of argument/thematic structure and predication. This approach has focused on the question of syntactic predication that bears on agreement phenomena (Stowell 1996).

However, both views (i.e, the 'referential'/'anaphoric' view of Tense and the 'predication' view) can be reconciled in the sense that they seem to square with Chomsky's (2001: 12) conjecture that T may be a substantive category paralleling the projection of the verbal element in the thematic domain of clause structure. ${ }^{3}$ In fact, the claim that $\mathrm{T}$ is a syntactic predicate linked in some way to the (verbal) predicate in clause structure was proposed in Rothstein's (1983: 19) syntactic predication analysis of IP structure, whereby a predicative XP - essentially the VP - needs 'saturation' or 'closure' by an argument XP, its 'syntactic subject'(i.e., the 'external' argument in [Spec, IP/TP]). Rothstein (1983: 130) thus derives the EPP property of T from syntactic predication.

A similar claim about the connectedness between $\mathrm{V}$ and $\mathrm{T}-$ and by 'extension' V, T and C - was proposed by Grimshaw (2000: 116) in her view that the core functional categories I and C share the feature complex [verbal] with the lexical category $\mathrm{V}$, since I and $\mathrm{C}$ are 'extended projections' of V bearing the same categorial specification for purposes of structure dependency formation.

In this connection, Biberauer and Roberts' (2010: 265) assumption that two kinds of structural connectedness are established in sentence structure "distinguishing T's tense (i.e., 'verbal' or V-related) properties from its agreement $\varphi$ - (i.e. 'nominal' or D-related) properties" directly links the suggestions about the connectedness between the thematic domain and the functional domain of sentence structure

\footnotetext{
${ }^{3}$ The same claim has recently been made in Biberauer \& Roberts (2010) under a parametric theory of syntactic variation much like the one proposed in this thesis, where feature-valuing in the probe-goalAgree system varies parametrically. As already discussed several paragraphs above, Biberauer \& Roberts (2010: 265) claim that T comes to the derivation with an unvalued V-feature. In their conception, $\mathrm{T}$ is inherently verbal but it lacks argument structure. They adopt a version of the Minimalist program similar to Chomsky's (1995b) - albeit in a different guise - where T is generated not only with T-features and V-features, but also - parametrically - a D-feature. I return to such issues in section 3.2 below.
} 
discussed above to the parametric, probe-goal-Agree account Biberauer and Roberts (2010) entertain for the feature-valuation model they propose. Thus, depending on whether structural dependency relations are based on V-related properties or on Drelated properties of the functional nodes $\mathrm{T}$ (and, possibly, $\mathrm{C}$ ) in relation to $\mathrm{V}$ in sentence structure, the grammar will set different parametric restrictions and licensing conditions on what could be derived and what could not be derived in the language.

Granting that this much is reasonable enough, it seems that the complex of $\varphi$ features to be checked/valued in the functional structure of clauses, in a V-raising language like TA, are more plausibly associated with $\mathrm{Agr}_{S}$, which, according to the structure of sentences proposed in (1) above, is the highest functional projection in the IP domain that needs to be saturated as in Rothstein's (1983) proposal for the relation that binds $\mathrm{V}$ to $\mathrm{T}$. Nevertheless, I am putting forward the proposal that $\mathrm{Agr}_{\mathrm{S}}$ does not project in VSO word order in SA, as a closely-related language to TA. Not projecting $\mathrm{Agr}_{\mathrm{S}}$ is, then, a parametric choice SA opts for (in contrast to TA which systematically projects $\mathrm{Agr}_{\mathrm{S}}$ whether the derived structure surfaces as $\mathrm{SVO}$ or as VSO).

Due to the absence of $\mathrm{Agr}_{\mathrm{S}}$ in the derivation of SA VSO sentences (and, in turn, the unavailability of EXPL pro in the IP domain due to the non-projection of $\mathrm{Agr}_{S}$ ), the projection of [Spec, TP] becomes a requirement for EPP satisfaction at the IP level, whereby the subject is raised out of $\left[\mathrm{Spec}, \mathrm{v}^{* \mathrm{P}}\right]$ to fulfill that requirement in [Spec, TP]. The verb is then raised further up past the subject. Presumably, this raising process is triggered by some feature (or feature complex) associated with a functional head position $F$ in the split-CP domain (Rizzi 1997). Postponing the discussion of the structural status of the functional head $F$ to section 3.3, projecting $F$ in the sentence structure of VSO instances in SA triggers the right setting for the kind of agreement configuration SA VSO word order exhibits.

\subsubsection{Bobaljik \& Jonas (1996) and Bobaljik \& Carnie (1996) on the Derivation of $\mathrm{Agr}_{S} P$}

Bobaljik \& Jonas (1996) propose the idea that agreement, the EPP and the Spec-head agreement relation are structurally related in the derivation of sentences (see section 2.3.3, and section 3.1.2 below, for a more recent Minimalist analysis of this idea).

In an analysis primarily interested in the properties of Transitive Expletive Constructions cross-linguistically, Bobaljik \& Jonas (1996: 200; see also footnote 6 on the same page) argue that $\operatorname{Agr}_{S}\left(\right.$ or $\operatorname{Agr}_{O}$ ) cannot project in the syntax if it is 
specifierless since the specifier of such a projection is a 'potential' target for movement of the subject. According to Bobaljik \& Jonas (1996) not only does [Spec, $\left.\mathrm{Agr}_{\mathrm{S}} \mathrm{P}\right]$ project in a language like Icelandic, but the language also allows $\mathrm{T}$ to have a specifier position (the so-called '[Spec, TP] parameter') in the so-called Transitive Expletive Constructions (see also Chomsky 1995a: 425-431).

Bobaljik \& Carnie (1996) develop a similar parametric analysis to that of Bobaljik \& Jonas (1996), particularly in relation to the importance of [Spec, TP] in word order differences in V-raising languages. Bobaljik \& Carnie (1996) explain the main difference in the derivation of the relevant structures in Irish (a VSO language) and French (an SVO language), both of which exhibit overt verb movement, in relation to the T-node in overt syntax. Their analysis is grounded in the Minimalist assumptions of Chomsky (1993) of the strength of category features on functional nodes. Bobaljik \& Carnie (1996: 225-229) argue against both a verb-to-Comp and a subject-and-object-in-situ analysis of Irish VSO word order. Instead, they (Bobaljik \& Carnie 1996: 230-233) argue that the derivation of VSO word order in Irish results from the raising of the lexical subject DP to the projected specifier position of the Tnode due to a strong $\mathrm{N}$-feature on $\mathrm{T}$. The subject DP checks its features (including case) in that position. The verb (actually the $\left[\mathrm{V}+\mathrm{Agr}_{\mathrm{O}}\right]$ complex]) moves to $\mathrm{T}$ and then the whole $\left[\left[\mathrm{V}+\mathrm{Agr}_{\mathrm{O}}\right] \mathrm{T}\right]$ complex moves to $\mathrm{Agr}_{\mathrm{S}}$ deriving the VSO word order. ${ }^{4}$ The tree structure in (5) below represents this process:

\footnotetext{
${ }^{4}$ In the representation in (5), I ignore Bobaljik \& Carnie's (1996) discussion of object raising to [Spec, $\left.\mathrm{Agr}_{\mathrm{O}} \mathrm{P}\right]$ and verb movement to $\mathrm{Agr}_{\mathrm{O}}$ since these processes do not directly bear on the discussion.
} 
(5) Irish VSO:

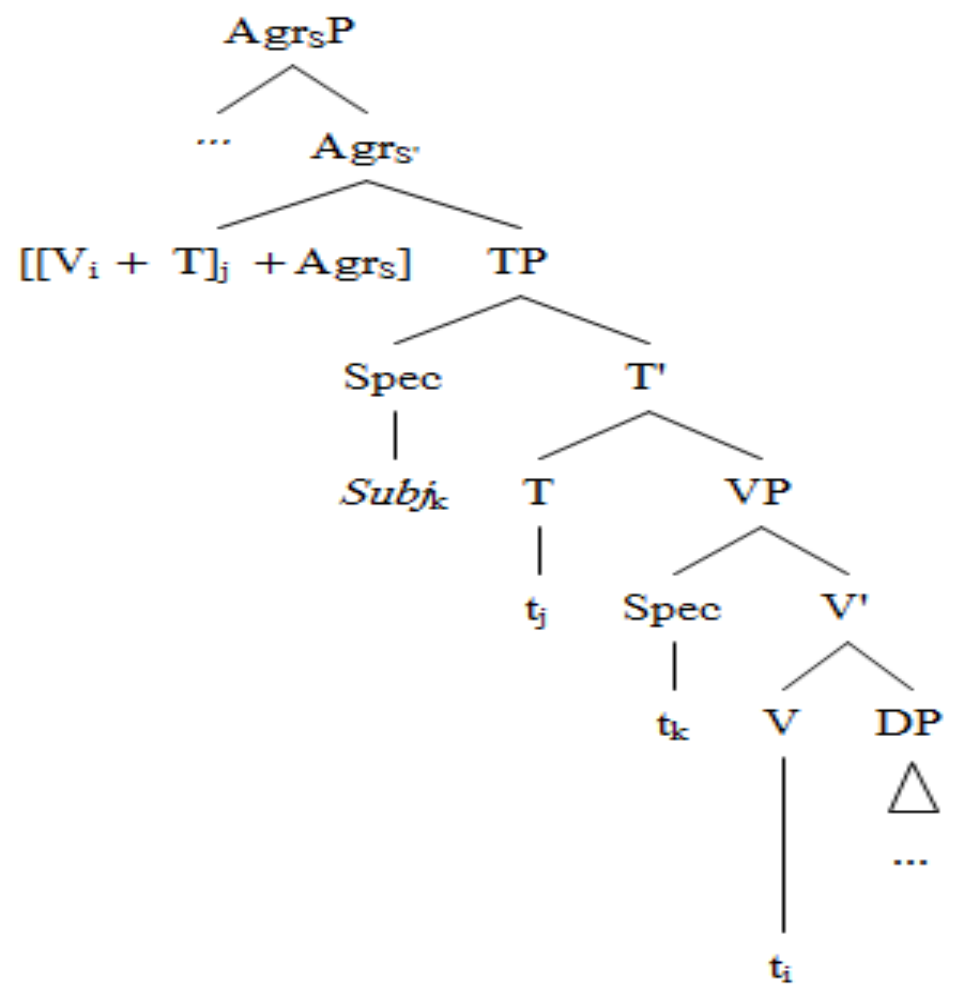

By contrast, the specifier position of the $\mathrm{T}$-node, in a V-raising language like French, is not projected. The T-node raises up to attach to $\mathrm{Agr}_{\mathrm{S}}$ and the verb raises to attach to the inflectional complex [Agrs T-Agr $]$ ]. In Bobaljik \& Carnie's (1996: 234) account of SVO word order in French, T-to-Agr $\mathrm{S}_{\mathrm{S}}$ is explained in terms not only of strong V-features on Tense that have to be checked in the overt syntax, but also in terms of strong N-features of Tense (whereby the subject DP is inserted in [Spec, T-Agr $r_{S}$. The subject DP moves to the specifier of this complex (essentially a verbal complex at LF) yielding the tree structure in (6) below: 
(6) French SVO:

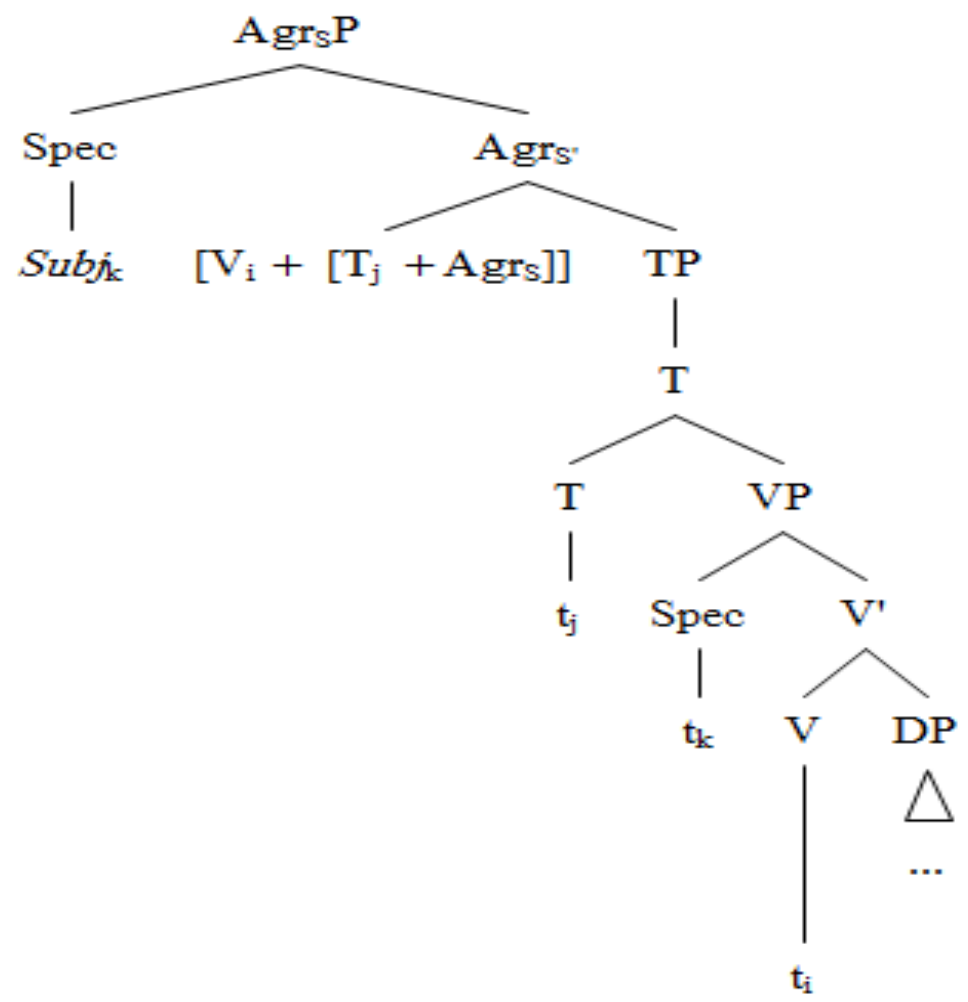

On this account, the difference between Irish and French concerns the V-features of Tense, which are strong in French necessitating $\mathrm{T}$ to raise to $\mathrm{Agr}_{\mathrm{S}}$, but weak in Irish disallowing T-to-Agr $\mathrm{S}$ as an independent raising process (Bobaljik \& Carnie 1996: 234-235). In Bobaljik \& Carnie's (1996: 235) own terms:

By hypothesis, Irish has weak V-features and thus T need not (and so cannot) raise independently to $\mathrm{Agr}_{\mathrm{S}}$.... Note that in Irish T does, in effect, raise overtly to $\mathrm{Agr}_{\mathrm{S}}$, but only as a step in the sequence of head movements $\mathrm{V} \rightarrow$ $\operatorname{Agr}_{\mathrm{O}} \rightarrow \mathrm{T} \rightarrow$ Agr $_{\mathrm{S}}$. This difference correlates with the possibility of checking the N-features of Tense in the specifier of TP (Irish) as opposed to in the specifier of $\operatorname{Agr}_{S} \mathrm{P}$ (with the complex head [T $\left.+\mathrm{Agr}_{\mathrm{S}}\right]($ French)).

Although Bobaljik \& Carnie's (1996) analysis of VSO word order in V-raising languages is important in that it binds the parametric difference between the Irish-type languages and the French-type languages to the availability or unavailability of [Spec, $\mathrm{TP}]$ in the derivation of the relevant structures, it runs counter to Bobaljik \& Jonas's (1996) assumption that $\operatorname{Agr}_{S}$ cannot project unless it has a specifier position. More generally, this analysis does not take into account the kind of 'last resort' movement 
Miyagawa (2010) bases on the premise of an EPP-triggered analysis of agreement that relies on the central role of Spec-head agreement under the highest functional projection in the IP domain, as mentioned earlier in this section (see also section 3.1.2.2 in relation to Roberts \& Roussou's 2002 guideline in (7) below).

Bobaljik \& Carnie (1996: 232) further assume that, in a structure like (5), for Irish, covert movement occurs at LF, whereby the subject raises from [Spec, TP] to a newly projected $\left[\mathrm{Spec}, \mathrm{Agr}_{\mathrm{S}} \mathrm{P}\right]$ to check agreement features and Case against $\mathrm{Agr}_{\mathrm{S}}$. The problem with this assumption is that, on Chomsky's (1993) Minimalist assumptions, 'covert movement' (or 'feature-attraction') applies to 'weak' features on a head that could not be 'checked' via Spec-head agreement in the syntax proper. In this case, the Spec position of $\mathrm{Agr}_{\mathrm{S}}$ would be superfluous at LF; and so would be the head $\mathrm{Agr}_{S}$ since both functional positions - i.e., [Spec, $\left.\operatorname{Agr}_{S} \mathrm{P}\right]$ and $\mathrm{Agr}_{\mathrm{S}}-$ would be associated with 'weak' features that play no role at all in the derivational process and/or in interpretation, which is overruled on the Minimalist assumptions of ‘economy of derivation' Chomsky’s (1993) framework abides by.

To sum up, the cross-linguistic characterization of word order and agreement configurations in terms of the availability or unavailability of functional projections and associated specifier positions is significant in that it points to an available generative paradigm that could serve as a framework for an adequate account of parametric variation in terms of the potential availability of an $\operatorname{Agr}_{S}$ node, the specifier position of which would be fundamental in accounting for subjecthood and varying subject properties in a variety of closely-related languages.

In the following section, I argue that, over and above the potential availability of an $\mathrm{Agr}_{\mathrm{S}}$ node accounting for parametric variation between closely related languages of the Arabic/Semitic or Celtic kind, parametric variation also concerns the Spechead/head-head agreement relations that are established via movement and the properties of the functional node the verb raises to in the IP domain - namely, Tense or the node that embeds Tense features in the syntax - namely, Agr or $_{\text {C }}$ (Roberts \& Roussou 2002).

\subsubsection{The role of the Spec-head agreement relation and the Tense-dependency in the derivation of functional structure}

This section highlights the role of $\mathrm{T}$ (tense features) in relation to the possible projection of an $\mathrm{Agr}_{\mathrm{S}}$ node in the IP domain in establishing the Spec-head/head-head 
dependency relations that would account for parametric and micro-parametric differences in subject-verb agreement configurations. In section, 3.1.2.1, I further review Miyagawa's (2010) agreement approach to movement and the EPP (see also section 2.3.3) in relation to the fundamental role Spec-head agreement plays in the derivation of sentences. Section 3.1.2.2 is a discussion of Roberts \& Roussou's (2002) T-criterion analysis that makes similar predictions to those of Miyagawa (2010) about sentence structure and the 'functional relations' incrementally built up in it.

\subsubsection{The central role of the Spec-head agreement relation (Miyagawa 2010)}

The account of the derivation of functional structure I am proposing essentially relies on a characterization of sentence structure in terms of a 'last resort' analysis of movement processes of the kind Miyagawa (2010: $\mathrm{x}$ ) links to an EPP-triggered analysis of agreement. In this approach, movement and agreement are associated with the availability of specifier positions that establish 'functional relations' between lexical items (essentially verbs and their associated arguments) and functional heads in terms of "Spec-head agreement [...] implemented as two independently motivated operations - Agree and Move" (Miyagawa 2010: 9). As in Chomsky (2001, 2004), the Agree relation guarantees that agreement features are checked/valued at each 'strong Phase' ( $v^{*} \mathrm{P}$ and CP) and that the EPP is satisfied, whereby interpretability at 'Spell Out' follows.

According to Miyagawa (2010: 9), the Spec-head agreement relation between a functional head and some XP is what underlies both agreement and movement, since movement only plays the role of bringing the goal of the Agree relation close to the probe that seeks that goal, i.e., establishing a Spec-head agreement configuration between the two elements. In doing so, Agree and Move help breach the gap between narrow syntax and information structure so that semantic interpretation is available at each Phase of the derivational process. Miyagawa (2010: xi) justifies this line of argumentation in that:

Without agreement and movement, human language would be a shadow of itself for expressing human thought, impoverished to the degree that it would not be able to express such common notions as topic-comment, subject of a clause, focus, and content questions. 
In relation to Miyagawa's (2010: 4) assertion that agreement and movement always co-occur, one important corollary of an adequate account of parametric variation in subject-verb agreement configurations in V-raising/null-subject languages is that such variation cannot be the result of verb movement per se. The feature structure of functional elements projected in the process of the derivation and the Spec-head/head-head agreement configurations that verb movement helps build up are important ingredients in this parametric account. ${ }^{5}$ An approach to subject-verb agreement configurations that only partially takes into account the feature structure of functional elements would lack in generality since it limits itself to properties of sentence structure that do not rely on the fundamental role 'functional relations' play, in the syntax proper, in the derivation and representation of sentences. This is the case with Aoun \& Benmamoun's (1999) account of the agreement asymmetry in terms of the PF phonological properties of verb movement in the derivation of VSO structures in a language like SA (see section 2.3.1). Such an account misses out the importance of the 'functional relations' Move and Agree processes create in the syntax proper and the Cartography of functional head and specifier positions that could shed new light on parametric variation in such configurations.

As I mentioned in section 3.1.1 above, the derivation of VSO sentences in Arabic finds a plausible parametric account in terms of an $\mathrm{Agr}_{S}$ node - along with the base-generation of expletive pro in $\left[\mathrm{Spec}, \mathrm{Agr}_{\mathrm{S}} \mathrm{P}\right]$ in the modern spoken dialects of Arabic - that would mediate the Spec-head/head-head Agree dependency relations in the functional structure incrementally derived via Merge/Move.

This section has focused on the argument that satisfying the EPP at the highest level of sentence structure at the 'strong Phase' is the trigger for Move and Agree, in the first place. Spec-head agreement - over and above Move and Agree - plays a central role in producing such a result at the relevant Phase. In the next subsection, I have a look at Roberts \& Roussou's (2002) T-criterion and the role the T-dependency plays in accounting for the distribution of expletive subjects cross-linguistically.

\footnotetext{
${ }^{5}$ The sample examples Miyagawa (2010: 47, 52) mentions are those of languages that lack 'overt' verbal agreement inflection but still manifest 'person agreement'. This is the case of Chinese 'prodrop' and the kind of 'pro-drop' and 'person agreement' that is concomitant with V-raising processes in Romance and (Bani Hassan) Arabic.
} 


\subsubsection{Roberts \& Roussou's (2002) T-criterion analysis: The T-dependency and the distribution of expletive subjects}

Miyagawa's (2010) account of movement and agreement in terms of EPP-based Spechead/head-head agreement dependency relations is reminiscent of Roberts \& Roussou's (2002; henceforth, R\&R) 'T(ense)-criterion' and its relation to parametric variation in the derivation and representation of sentences. The T-criterion is an attempt to point to the paramount role of both $\mathrm{Agr}_{\mathrm{S}}$ and $\mathrm{C}$ in relation to the $\mathrm{T}$ node in unifying two seemingly unrelated structural phenomena: the EPP (Chomsky 1982, 1995c), and the V2 effect. R\&R (p. 127) state the T-criterion (in their conception, the EPP) as follows:

(7) The head containing T must have a filled specifier.

The guideline in (7) has the effect of reducing the EPP - the subject requirement, in R\&R's terminology - and the V2 effect to a property of $\mathrm{T}$ in connection with the functional position where $\mathrm{T}$ is spelled out after the derivation is handed over to the PF component of the grammar. In other words, “ ... the 'subject requirement' arises when $\mathrm{T}$ is realized in the IP domain, while the 'V2 requirement' arises when T is spelled out in the CP domain" (R\&R: 125). In the first case, it is [Spec, IP/Agr $\mathrm{P}]$ that has to be occupied by some DP element. In the second case, it is [Spec, CP] that has to be occupied by some XP element (not necessarily a DP). This formulation of the T-criterion in terms of a basic Spec-head agreement relationship between a head and some XP element makes the EPP similar to 'criteria' of the kind proposed by Rizzi (1996: 64) in his formulation of the Wh-criterion (see also Rizzi 2006). ${ }^{6}$

Following Chomsky's (1995b) conception of the EPP as the necessity of checking the D-feature of $\mathrm{T}$ by the subject (as an XP category ), $\mathrm{R} \& \mathrm{R}$ argue that the focus should not be on the simple restatement of the EPP as a 'subject requirement', but rather on an explanation of why it is the case that both the EPP and V2 involve T. Relying on the 'T-criterion' as a kind of "generalized EPP”, R\&R argue that a more satisfactory explanatory theory of the EPP would be to link the two requirements as

\footnotetext{
${ }^{6}$ Rizzi (2006: 98) dubs the same principle (i.e., the T-criterion) the 'Subject Criterion' which arises in A-movement involving the checking/valuation of Case at the interface. In A'-movement involving whmoved elements - hence, the name 'Wh-criterion' -, however, no Case-checking is assumed to be involved, and the trigger of wh-movement is the structural requirement that wh-elements reach their final landing site in the CP domain - by definition, a 'criterial position' (the term is Rizzi's 2006: 98).
} 
both involving Spec positions, i.e., [Spec, $\mathrm{CP}]$ as far as the 'V2 requirement' is concerned and [Spec, IP] as far as the 'subject requirement' is concerned.

Similarly to Miyagawa's (2010) EPP-triggered analysis of agreement, the 'Tcriterion' analysis argues for parametric - and micro-parametric - variation in terms of a Spec-head relation between the head containing $\mathrm{T}$ (or, the head that is related to $\mathrm{T}$ in the IP domain - namely, $\operatorname{Agr}_{S}$ ) and the Spec position of the functional head that $\mathrm{T}$ attaches to. Interestingly enough, the conception of the Spec-head agreement relation in the Phase-theoretic framework (Chomsky 2001, 2004), as only being relevant at the 'strong Phase' (i.e., the interface, which obtains cyclically at the $v^{*} \mathrm{P}$ and CP levels), may be an argument (pace Chomsky 2004) in favour of the role the Spec-head agreement relation plays in dependency and agreement configurations. Spec-head agreement would be the result of the application of the Agree relation as it obtains at the strong Phase after all Merge/Move processes have applied. At the strong Phase, all features end up being valued for convergence at the Interface.

An even more compelling argument for the T-criterion, as formulated in (7) above, is that both the 'subject requirement' and the 'V2 requirement' can be satisfied by an expletive, as (8) shows (R\&R: 126):

(8) a. Es wurde getrunken It was drunk (German) 'People were drinking'

b. There's a fly in my soup.

In (8a), the auxiliary verb wurde occupies the $\mathrm{C}$ node, and $\mathrm{T}$ is therefore realized in that position. The expletive es has to be inserted in [Spec, $\mathrm{CP}]$ for the satisfaction of the V2 requirement for the lack of any other element that can occupy that position. In (8b), likewise, Tense is realized in the IP domain, but, due to the failure of the subject argument DP to raise to [Spec, IP], expletive there is inserted in that position, instead. According to (R\&R: 131), the features that realize the 'T-dependency' (or the 'C- $\mathrm{Agr}_{\mathrm{S}}-\mathrm{T}$ dependency') are those associated with $\mathrm{Agr}_{\mathrm{S}}$. In [-Finite] embedded raising clauses in English (as in the embedded contexts in there-constructions reviewed in 2.3.2), $\operatorname{Agr}_{S}$ may be absent in the lower clause ( $\left.R \& R: 136\right) . R \& R$ argue that, in this case, identification of the T dependency in the lower clause operates with $\operatorname{Agr}_{S}$ in the matrix clause. 
Similar to R\&R's 'T-dependency' analysis, Alexiadou \& Anagnostopoulou (1998) develop an approach to EPP satisfaction in clause structure where $\mathrm{Agr}_{\mathrm{S}}$ figures as the highest functional node in the IP domain. In Alexiadou \& Anagnostopoulou's (1998) account, EPP feature-checking is achieved either via the realization of the features of $\mathrm{Agr}_{S}$ as a head (i.e., via V-raising to $\mathrm{T}$ and $\mathrm{Agr}_{\mathrm{S}}$ in VSO languages) or via the realization of those features as a specifier (i.e., via DP-raising to [Spec, $\left.\operatorname{Agr}_{S} \mathrm{P}\right]$ ). Nevertheless, this approach to EPP satisfaction at the highest level of functional structure in IP runs counter to Roberts' (2010a: 166-167) argument that satisfaction of the EPP (and, as a side effect, agreement in $\varphi$-features/case) obtains independently of $\mathrm{V}$-movement per se. According to Roberts (2010a), head movement (i.e., movement of $\mathrm{V}$ to $\mathrm{T}$ ) is dissociated from EPP-driven movement since "EPP-features of $\mathrm{T}$ do not attract V-elements" (Roberts 2010a:167). The explanation Roberts (2010a:107-109) gives for this dissociation is that, in the case of V-to-T movement, the goal of the Agree relation is 'defective' in that the features in which the probe P and the goal G agree are a subset of the features of P (i.e., V- $v$ and $\mathrm{T}$ agree in $\varphi$-features and these are a subset of T). As Roberts (2010a: 166 ) also notes, there is " a general incompatibility between incorporation and EPP features".

As will be discussed in section 3.2.1.2 below, Roberts (2010a) argues that satisfaction of the EPP, in this instance of probe-goal-Agree relation, is by filling up [Spec, TP] with a D element that not only values the EPP-feature of $\mathrm{T}$, but it also satisfies the requirement that a 'defective' goal in [Spec, TP] matches and values the uninterpretable D-feature on T (i.e., $[u \mathrm{D}]$ on T) in V-raising/null-subject languages. Thus, in Roberts' (2010a) parametric account of the differences between null-subject and non-null-subject systems in the derivation of subject-verb agreement configurations, over and above the $\varphi$-features - which T inherits from $\mathrm{C}$ (possibly, together with the EPP feature, (Chomsky, 2008: 157)) -, the feature on T that specifically enters into the definition of 'defective' goal in null-subject languages is an additional intrinsic D-feature. In this case, T's D-feature matches the D-feature of a subject D - namely, pro - necessitating it to raise to [Spec, TP] (or be inserted in [Spec, TP] via Merge in the case of EXPL pro) for 'checking' purposes. According to Roberts (2010a: 108), in a null-subject language, "in order to license a null subject 
(which involves deletion of a null pronoun in SpecTP), T must have $\varphi$-features, Dfeatures, and an EPP-feature". 7

In other cases - as in the derivation of VSO structural representations in SA vs. TA -, the process of pro-insertion in [Spec, TP] may not be the right process for EPP satisfaction and $\varphi$-feature valuation at the IP level (in this connection see footnote 7 above). This might be the case where the availability of the intrinsic Dfeature, which Roberts $(2010 \mathrm{a}, \mathrm{b})$ associates with $\mathrm{T}$, is parametrically missing in such typologically closely-related languages (i.e., in SA vs. in TA).

\subsubsection{Summary}

In this section, I have highlighted the central role of $\mathrm{T}$ - and its features, whether inherited or not, à la Chomsky (2008) - in establishing the kind of functional Spechead/head-head dependency relations involved in the valuation of agreement features. These $\mathrm{C}-\mathrm{Agr}_{\mathrm{S}}-\mathrm{T}$ dependency relations - and the specifier positions they involve - are argued to account for the parametric and micro-parametric structural agreement configurations arising from differentiations in the feature structure of functional elements that is responsible for the differences in agreement configurations at Spell Out.

Thus, in light of the postulated feature composition of Tense in the IP domain (see Table 3.1 above), the availability or unavailability of features (or combination of features) on functional heads lies at the centre of the differentiations in question. In this respect, the parametrically available option of projecting an $\operatorname{Agr}_{S}$ node (maximally projecting as $\operatorname{Agr}_{S} \mathrm{P}$ ), and whatever feature, or features, $\mathrm{Agr}_{\mathrm{S}}$ is generated with would be the trigger for the T-to-Agr ${ }_{S}$ raising process discussed in section

\footnotetext{
${ }^{7}$ Roberts (2010b: 67, footnote 10) assumes that the condition for copy (pronoun) deletion is that one set of features has to delete. Since, in the case of pro, as a 'defective' goal, pro's features are a subset of the probe T, it is the goal's feature(s) that delete. On this account, the deletion of the features of pro ( $\varphi$-features and D for referential pro, and a D-feature only for EXPL pro) entails the deletion of pro itself at $\mathrm{PF}$ after the uninterpretable $\varphi$-features on $\mathrm{T}$ - along with its additional uninterpretable $\mathrm{D}$ feature, in the case at hand - get a value, and thus, survive deletion. In this valuation procedure, the interpretable D-feature on pro links $[u \mathrm{D}]$ on $\mathrm{T}$ to $\varphi$-feature specification. Since all features are valued, pro deletes after having satisfied the EPP at Spell-Out in [Spec, TP]. As I will discuss later (see section 3.3.4 and Chapter Four), in SA VSO word order, pro fails to be instantiated in the IP domain and, instead of being deleted at $\mathrm{PF}$, it is morphologically realized as a clitic pronominal element in the $\mathrm{CP}$ domain in case the assertive clause-type particle element Pinna 'that' is merged under a declarative $\mathrm{C}$ node. In this case, since [Spec, TP] still has to be occupied for the satisfaction of the EPP in the IP domain, the subject raises from [Spec, $\mathrm{v}^{*} \mathrm{P}$ ] to meet that requirement. After raising to $\mathrm{T}$, the verb further raises to the $\mathrm{CP}$ domain to attach to some functional node $F$ in a split-CP system à la Rizzi (1997).
} 
3.1.1.3. In particular, T-to-Agr (with $\mathrm{V}-v$ attached to $\mathrm{T}$ ) would, independently, account for the combination of features Roberts (2010a: 108) attributes to $\mathrm{T}$ in the IP domain (i.e., a D-feature in addition to $\varphi$-features and an EPP feature, in the relevant languages) and could shed new light on a possible account of parametric variation in agreement configurations cross-linguistically (see, in particular, section 3.3 below).

In the next section, I expand on the theoretical significance of the dependency-based analysis of agreement configurations for expletive-constructions in light of Chomsky's Phase-theoretic framework.

\subsection{The probe-goal-Agree system: Agreement features and structural dependencies}

The Phase-theoretic framework of assumptions (Chomsky 2001, 2004; see section 2.3.2) claims that feature strength on $\mathrm{T}$ and $\mathrm{C}$ is not the real trigger of Merge/Move processes. In Chomsky (2001, 2004) - and likewise in Chomsky (2008) - parametric variation is no longer accounted for in terms of a 'strong' D-feature on the core functional categories of sentence structure (see section 2.3.1 for how 'feature strength' was conceived in earlier Minimalist frameworks). Nevertheless, the [ \pm interpretable] dimension remains a core prerequisite of the case-agreement system in conjunction with the EPP.

In addition to this major revision of the architecture of the functional structure of natural languages, Chomsky (2004) argues that the Spec-head agreement relation can no longer figure as the basis of the procedure whereby uninterpretable features are eliminated in the course of the derivation for convergence at the interface, and that the very process of merging elements in the derived structures is the only factor responsible for valuing any uninterpretable features in the sublabels of functional heads. Nevertheless, as Miyagawa's (2010) movement analysis of agreement and the EPP directs attention to, when considering how both Agree and Move concur to achieve the satisfaction of the EPP, there are good reasons to suppose that the Spechead agreement relation is responsible for that result at the 'strong Phase' level when the derived structure is transferred to the interface for interpretation.

By making a difference between Agree-features ( $=\varphi$-features) and a certain Edge feature (EF), the framework of assumptions of Chomsky (2008: 148) brings another dimension into the account of Agree relations and the EPP. Since $\varphi$-feature valuation and satisfaction of the EPP in clause structure in the C-T Phase are 
interrelated in the Phase-theoretic approach (Chomsky 2001, 2004, 2008), Chomsky (2008: 157) assumes that in addition to the essential dissociation between the EF and $\varphi$-features, a similar dissociation could be entertained between the EF and the EPP. Thus, as represented in Table 3.1 above, the formal features that enter into the kind of subject-verb agreement operating at the IP/TP level are actually features that are associated with $\mathrm{C}$ in the first place. Thus, T's role in such subject-verb agreement configurations is only 'derivatively' obtained via the essential C-T structural dependency relation that binds the two functional nodes, $\mathrm{T}$ and $\mathrm{C}$, together.

In Chomsky's (2008) feature-inheritance conception of the dependency relation that links $\mathrm{T}$ to $\mathrm{C}$, the dissociation between the $\mathrm{EF}$ and the other formal features that $\mathrm{T}$ and $\mathrm{C}$ share is also significant in that singling out the $\mathrm{EF}$ as an exclusive property of $\mathrm{C}$ that is not shared by $\mathrm{T}$ accounts for why subject wh-elements are attracted to $\mathrm{C}$, rather than to $\mathrm{T}^{8}$

In a similar approach to the feature structure of functional categories that enter into the probe-goal-Agree system to that of Chomsky (2008), Roberts (2010a: 108, 113; see section 3.1.2.2 above and section 3.2.1.2) postulates a $\mathrm{D}$-feature on $\mathrm{T}$ that accounts for why [Spec, TP] has to be occupied by some element by PF in the relevant languages. Nevertheless, unlike the EF on C, T's D-feature would somehow be associated with the EPP, particularly so, since - as Chomsky (2008: 157) suggests - C does not keep its EPP-feature, but transmits it to T. The association that I am proposing between the parametrically available D-feature and the EPP makes the socalled 'subject requirement' (R\&R: 125) an essential ingredient in any account attempting to shed light on differences in the realization of subject-verb agreement dependency relations cross-linguistically. I take up the role of D-features in the derivation of the T-dependency in section 3.2.1.2 below.

As far as the postulated D-feature on $\mathrm{T}$ is concerned, this line of argumentation seems plausible since, as I have discussed in section 3.1 in relation to

\footnotetext{
${ }^{8}$ This is not quite true, because in Chomsky's (2008) system two copies are generated in the process of wh-moving a subject in English matrix clauses: one copy lands in [Spec, TP] and the other lands in [Spec, CP]. For reasons of linearization at the PF side of the grammar, only the highest copy is spelled out. I come back to a discussion of such Merge processes in section 3.2.1.1 below, in the context of the parallelisms that exist between $\mathrm{C}$ and $\mathrm{T}$ in terms of features and the Merge/insertion processes driven by these features. Again, this parametric account will show that the feature structure of the functional elements involved in these processes may trigger different requirements as to how the T-dependency is realized in different languages (as, for example, in English wh-moved subjects vs. TA wh-moved subjects), and perhaps also in the same language (as for example, subject wh-extraction, as studied by Chomsky 2008 vs. subject wh-extraction from an embedded clause involving a there-construction; see section 3.2.1.1).
} 
Miyagawa 2010 and R\&R, the EPP only applies at the highest level of functional structure (i.e., $\mathrm{T}$ selected by $\mathrm{C}$ in English) before the derivation is handed over to $\mathrm{PF}$ and LF at the 'strong Phase'. At the strong Phase, Move applies to bring the matching elements (i.e., $\mathrm{T}$ and whatever element happens to be in its Spec position) close to each other so that some form of agreement can obtain. Thus, it seems that at the IP/TP level, Agree-features and the EPP always coincide, and that the D-feature (in conjunction with V movement in VSO sentences) could be argued to be generated higher than the first Merge site of the T-node before T 'remerges' in sentence structure (as in Rouveret's 2010 system of feature 'fission'; see right below). According to the cross-linguistic account of the derivation of sentences that parametrically vary due to differing probe-goal functional relations in terms of Move and Agree, the tense features and, parametrically, the postulated D-feature on $\mathrm{T}$ (in Roberts' 2010a: 108, 113 analysis) would rather reside either in $\mathrm{Agr}_{\mathrm{S}}$ or in $\mathrm{C}$ than on $\mathrm{T}$. In the account provided here, $\mathrm{T}-$ in the relevant languages/structures - is associated with $\mathrm{T}$ because of the raising process of $\mathrm{T}$ to either $\operatorname{Agr}_{\mathrm{S}}$ or to $\mathrm{C}$.

In a different cross-linguistic typology of V1 (and V2) structures from the typology adopted in this thesis, Rouveret (2010: 240-243), proposes that tense features, which originate on $\mathrm{T}$ in the IP domain, are passed on (by a process of 'Fission') to the immediate superordinate category in sentence structure. In Germanic languages, with the exception of English, tense features are passed on to C. In typologically different languages in the Germanic paradigm (including Icelandic, Yiddish), T 'remerges' and projects a 'proxy' T category with a second Spec position for the satisfaction of the EPP under Rouveret's (2010: 244) assumption that "the EPP position can never coincide with the one dedicated to the valuing of T's [phi]". 9 In Rouveret's (2010) account, although the [EPP] feature is interpretable on T, it still has to be satisfied - in the languages that allow T to 'remerge' - and to meet the EPP requirement for convergence at the Interface.

Thus, in the probe-goal-Agree approach to parametric variation adopted in this chapter, it seems that the Spec-head/head-head dependency relations would play a

\footnotetext{
${ }^{9}$ According to Rouveret (2010: 245, footnote 32), English is different from all the other Germanic languages in that there is no independent [tense] feature on T in English. In Rouveret's (2010) system of the 'scattering' of tense and agreement features, the consequence is that the English T-node in the IP domain does not need to 'remerge', and the EPP, in English, is not a requirement of sentence structure as it is in the other Germanic languages. A further consequence is that English lacks both the 'Remerge' option and the 'fission' on C option (see, in that respect, Rouveret's 2010: 246 table summarizing his parametric variation account of T's features).
} 
central role in the kind of parametric variation I am arguing for in terms of feature complexes at the highest level of sentence structure. In particular, this is where the role of $\mathrm{Agr}_{\mathrm{S}}$ becomes paramount. In connection with this, I will propose in section 3.3 that $\mathrm{Agr}_{\mathrm{S}}$ is the minimal unit of parametric variation in Arabic accounting for the micro-parametric difference in the manifestation of subject-verb agreement. Of particular importance in this respect is that the projection (or non-projection) of an $\mathrm{Agr}_{\mathrm{S}}$ node in the overt syntax in V-raising languages - with VSO and SVO as alternative word orders - is dictated by the feature structure of core functional categories and the dependency relations thereby generated in sentence structure.

Before capitalizing on the parametric role Agr slays in Arabic (and perhaps more generally in similar language types), I discuss in section 3.2.1 below the relevance of the 'probe-goal-Agree' system in light of the importance of EXPLinsertion in an account of subject-verb agreement manifestations relying on the Spechead/head-head agreement relations underlying the $\mathrm{C}-\mathrm{Agr}_{\mathrm{S}}$-T dependency (which, as reviewed in section 3.1.2.2 above, is the cornerstone of the T-criterion-i.e., the EPP). Section 3.2.2 is a summary.

\subsubsection{EXPL-insertion in a probe-goal-Agree system of dependency relations}

Section 3.2.1.1 reviews the process of EXPL-insertion cross-linguistically. By reference to Romance null-subject languages, section 3.2.1.2 deals with EXPLinsertion in the context of the probe-goal-Agree system of dependency relations and the role of D-features on agreeing elements in setting the right conditions on the Spechead/head-head agreement configurations responsible for interpretability of the structures at the interface at Spell Out.

\subsubsection{A typology of EXPL-insertion structures cross-linguistically}

In Chomsky $(2001,2004)$, the locus of $\varphi$-agreement in simple sentences - in a strict SVO language like English - is T, complement-selected by a $\varphi$-complete C. In Chomsky (2008), $\varphi$-completeness is replaced by a theory of Agree-feature 'inheritance' whereby $T$ 'inherits' its $\varphi$-features from $C$, thus highlighting the essential dependency relation $\mathrm{T}$ and $\mathrm{C}$ engage in in structural terms. As far as $\mathrm{T}$ is concerned, it seems that a system like that of Roberts (2010a), discussed in section 3.1.2.2 (see also section 3.2.1.2, below), in connection with expletive subjects in a 
variety of languages, makes stronger assumptions than Chomsky's $(2001,2004)$ in that it posits a D-feature accounting for parametric variation at the IP level crosslinguistically.

As I will discuss in this section and the next, the satisfaction of the EPP at the highest level of sentence structure, in the probe-goal-Agree system, is concomitant with the valuation of Agree-features. Satisfaction of the EPP also coincides with the Merge sites of some elements, such as EXPL DPs (like there in English or es in German) or wh-words (expletive or 'contentful', which is the specific case of German-type languages; see the examples in (9) and (10) below). These elements are inserted in the process of the derivation either via 'internal Merge' as in the process of wh-movement to [Spec, CP] or via 'external Merge' whereby EXPL is inserted at the highest level of sentence structure at the strong Phase in the C-T system. Depending on the properties of the language in question, the Merge site could be either [Spec, $\mathrm{CP}]$ or $[\mathrm{Spec}, \mathrm{IP}]$.

As reviewed above, the probe-goal-Agree system and R\&R's T-criterion approach make similar assumptions as to the role that EXPL and other elements, such as wh-words, play in the derivation of dependency relations in sentence structure both at the level of IP and that of CP due to the special relationship that links T to C. According to both approaches, EXPL could be merged directly into [Spec, CP], as in (9) or in [Spec, TP], as in (10):

(9) EXPL-insertion in [Spec, CP]:

a. V2 or topic expletives

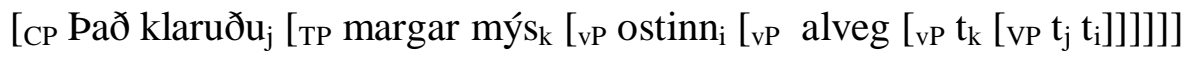
there finished many mice the-cheese completely 'Many mice completely finished the cheese'

(Icelandic, adapted from Alexiadou \& Anagnostopoulou 2001: 199; their (17)) b. Wh-expletive pronouns

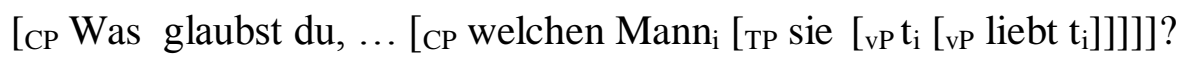
what believe you which man she loves 'Which man do you believe that she loves'

(German, adapted from Felser 2003: 52; her (17)) 
(10) EXPL-insertion in [Spec, TP] - subject expletives:

a. [ ${ }_{\mathrm{CP}} \mathrm{C}\left[{ }_{\mathrm{TP}}\right.$ There [т [vP arrived a man] $\left.\left.]\right]\right]$

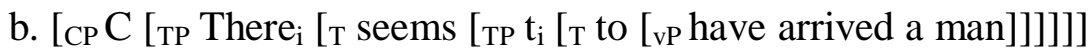

In connection with the Icelandic Transitive Expletive Construction in (9a), Alexiadou \& Anagnostopoulou (2001: 209-212) argue that the EPP and Case are 'checked' in two different positions in relation to $\mathrm{T}$ in a multiple-specifier/multiple-adjunction approach like that of Chomsky (1995b) or an approach similar to Bobaljik \& Jonas's (1996), briefly discussed in section 3.1.1.3 above. Alexiadou \& Anagnostopoulou (2001) assume that the subject DP margar mýs in (9a) overtly moves to a Spec position - namely [Spec, TP] - different from that occupied by EXPL $P a ð$. Thus, EXPL position could either be [Spec, CP] or [Spec, $\left.\operatorname{Agr}_{S} \mathrm{P}\right]$. Nevertheless, on Alexiadou \& Anagnostopoulou's (2001: 212, footnote 23) assumption that the projection of an $\operatorname{Agr}_{(S)}$ node just provides a landing site for the verb in Icelandic Transitive Expletive constructions (thus, not projecting a Spec position), ${ }^{10}$ the position of EXPL-insertion would be [Spec, CP]. Alexiadou \& Anagnostopoulou (2001) argue that the subject in [Spec, TP] checks Case. ${ }^{11}$

Alexiadou \& Anagnostopoulou (2001: 209) contend that the same conclusion applies to the derivation of VSO word order in Arabic, Celtic and the kind of Romance languages that allow VSO word order, where the EPP is also checked in a higher position than that of the subject (presumably, remaining in $[\mathrm{Spec}, v \mathrm{P}]$ in these languages; cf. Koopman \& Sportiche 1991). On their assumptions, the EPP in these languages is checked not via phrasal movement, but via V-raising to $\mathrm{I} \operatorname{Agr}_{(\mathrm{S})}$ because of a D-feature on V that allows it to satisfy the 'strong' D-feature of T, i.e. the EPP according to Chomsky's (1995b) Minimalist approach (see also Alexiadou \&

\footnotetext{
${ }^{10}$ In Alexiadou \& Anagnostopoulou's (2001: 212, footnote 23) own terms: “... an Agr is inserted as a proxy, in order to create a landing site for the verb, without playing any other role in the checking relations".

${ }^{11}$ According to Alexiadou \& Anagnostopoulou (2001: 211-212), the Case-feature of T could be checked in either of two ways according to the specificities of the language in question. One possibility is that the lexical subject moves to [Spec, TP] to check T's Case-feature leaving EXPL to be merged higher (presumably, in [Spec, CP]). The second possibility is to allow Case to be checked covertly without phrasal movement. In this case, the subject remains in situ in [Spec, $v \mathrm{P}]$. Under their approach, these two Case-checking possibilities coincide with the two options of EPP-feature checking - namely, by Merging EXPL in [Spec, CP] (as in Icelandic) or by raising V to I/Agr (as in the VSO languages they discuss).
} 
Anagnostopoulou 1998). In this case, VSO sentences in V-raising languages will have a structure where the Spec position of the highest projection in the IP domain (i.e. $\mathrm{I} / \mathrm{Agr}_{\mathrm{S}}$ ) is not necessarily projected in the syntax (see section 3.1.1.3 for Bobaljik \& Carnie's 1996 similar assumptions about the derivation of VSO structures in Irish).

Notwithstanding Alexiadou \& Anagnostopoulou's (2001) contention that the EPP can be checked by V in VSO languages (or the languages allowing VSO word order), the position where EXPL is merged for EPP satisfaction, is consistently assumed to be higher than any other position in clause structure. The EPP-related Spec position could be [Spec, TP] or $\left[\mathrm{Spec}, \mathrm{Agr}_{S} \mathrm{P}\right]$ - under the assumption that $\mathrm{Agr}_{\mathrm{S}}$ projects a Spec position as soon as it is introduced in sentence structure -, or [Spec, $\mathrm{CP}$ - under the assumption that $\mathrm{C}$ is always associated with $\mathrm{T}$ (or T-features). ${ }^{12}$

In the German construction (9b), the EXPL wh-pronoun was 'what' is merged in the $[\mathrm{Spec}, \mathrm{CP}]$ position of the matrix clause. As for the 'contentful' wh-phrase welchen Mann, it partially moves to the $[\mathrm{Spec}, \mathrm{CP}]$ position in the lower clause, landing first in the 'outer Spec' of $v \mathrm{P}$ ('outer Spec' of $v * \mathrm{P}$ in Chomsky 2001, 2004). As in the Icelandic example in (9a), the EXPL in (9b) is directly merged in a higher Spec position associated with a $\varphi$-complete T (in Chomsky's 2001, 2004 Phasetheoretic terminology).

The English unaccusative there-constructions in (10) show a similar insertion process. As already pointed out in Chapter Two, the ultimate purpose of having there inserted in English there-constructions is to satisfy the EPP-feature of T, whether T is selected by $\mathrm{C}$ (as in (10a)) or not (as in the embedded clause in (10b)).

In all the cases just reviewed in connection with the Merge processes involved in the derivation of (9) and (10), the EPP is valued after the Merge processes conspire with Agree to bring the goal of the matching/Agree relation closer to the probe seeking that goal, and merge it - by a raising process or else by a pure Merge process - in the highest Spec position the derivation has introduced in conformity with the Spec-head/head-head structural dependency relations resulting from the Merge

\footnotetext{
${ }^{12}$ This is actually Rouveret's (2010: 244-245) conclusion in his system that allows T to 'Remerge' and project a second Spec position or to 'fission' onto C. In Rouveret's (2010) system - discussed above the tense features of T and its EPP feature could coincide in the languages where the EPP is a requirement. This possible correlation between the valuation of tense and the EPP on T obtains, in the relevant languages, because the 'Remerge' or 'fission' of T for the satisfaction of T's tense features will coincide with the projection of a higher Spec position - namely, second Spec of TP or Spec of CP - for the satisfaction of the EPP, which "can never coincide with the one dedicated to the valuing of T's [phi]" (Rouveret 2010: 244).
} 
processes themselves and the feature makeup of the functional category or categories that triggers these Merge processes in the first place.

In this connection, if Roberts (2010a: 167) is right in his assumption that Velements are not EPP-checkers, then the element responsible for checking the EPP will also be responsible - under Roberts' (2010a,b) approach - for checking a Dfeature of the T-node in [Spec, TP] in the relevant languages. ${ }^{13}$ This is indeed Roberts' (2010a) assumption in relation to the null-subject languages he is concerned with (see, in particular, the next section). In this case, the D-feature that Alexiadou \& Anagnostopoulou (1998) argue to be the trigger of V-raising in the VSO languages they discuss is actually not a property of $\mathrm{V}$, but a feature of the probe $\mathrm{T}$ itself different from the EPP - that requires an element to raise to (or be inserted in) its Spec position.

As discussed earlier, at the stage in the derivation where $\mathrm{T}$ is introduced at the IP level (in a language like English or Arabic) and seeks a matching goal to value its EPP-feature, only a D-element can be merged in syntactic structure to check that feature. In this respect, the EPP is a universal of syntactic structure that is not parameterized. What is parameterized, according to Roberts (2010a: 108-113), is the additional D-feature on T that Roberts $(2010 \mathrm{a}, \mathrm{b})$ links to the null-subject parameter (see section 2.1.1). The parameterization of T's D-feature explains Roberts' (2010a,b) assumption that the D-feature on $\mathrm{T}$ in a null-subject system like the Milanese variety among the Northern Italian dialects (in Roberts 2010a; see section 3.2.1.2 below) or TA (see the discussion in relation to example (13) below) is checked by a null element pro merged in [Spec, TP/IP]. If this assumption is correct, then the D-feature of $\mathrm{T}$ should be absent in a language like English.

However, the sample examples of EXPL constructions discussed above in this section (see also section 3.1.2.2) and in section 2.3.2 suggest that Roberts' (2010a,b) parametric characterization of T's additional nominal D-feature and its effect in triggering particular Merge and probe-goal Agree processes probably applies more

\footnotetext{
${ }^{13}$ In the case of Niuean, as studied by Massam (2000: 111), where VSO is argued to be derived by remnant VP-fronting to [Spec, TP], the process by which VP is raised to [Spec, TP] is dissociated from the type of V-to-T movement process Roberts (2010a) is interested in. V-to-T movement depends on rich $\mathrm{T}$-features in $\mathrm{T}$ that attract $\mathrm{V}$ to raise to $\mathrm{T}$. Since Niuean does not show evidence of rich inflection at the level of IP, then Roberts' (2010a) conclusion is that the kind of VP-fronting Niuean has is actually not movement of a V-element. As such, VP-fronting in Niuean could still be a process that values the EPP feature of T, and would not be a counter-example to Roberts' (2010a) generalization that V-elements are not EPP-checkers.
} 
generally cross-linguistically. What I am proposing here is that Roberts' $(2010 \mathrm{a}, \mathrm{b})$ postulation of a $\mathrm{D}$-feature as a defining property of $\mathrm{T}$ in null-subject languages should be equated with Chomsky's $(2001,2004)$ [person] feature on $\mathrm{T}_{\mathrm{DEF}}$ in a language like English in that both coincide with the satisfaction of the EPP in the relevant examples. Thus, the T-node in an English-type language could also be assumed to be endowed with an intrinsic D-feature in those instances where a D-element is merged (externally, by first Merge only, or internally, by first and then second Merge, as in English embedded there-constructions) in [Spec, TP]. This is the assumption I am making in relation to there-insertion in finite matrix contexts or infinitive embedded contexts since there is the right D-element that could be assumed to be the counterpart of the null EXPL element pro.

The very existence of EXPL-constructions cross-linguistically accounts for why EXPL elements (for example, there in English; es in German, and pro in nullsubject languages) could be merged in the dedicated Spec positions that actually coincide with the EPP cross-linguistically - namely, [Spec, IP/TP] and [Spec, CP]. In my view, the two features on T (i.e., T's EPP feature and its 'parameterized' Dfeature) remain different, however, in that they only co-exist in certain contexts namely, in sentences where T raises to $\mathrm{Agr}_{\mathrm{S}}$ or to $\mathrm{C}$ as in EXPL-constructions in the relevant languages, where a $\mathrm{D}$-feature of the relevant functional node $\left(\mathrm{Agr}_{\mathrm{S}}\right.$ or $\left.\mathrm{C}\right)$ is involved. $^{14}$

Coming back to the movement processes that are involved in the derivation of wh-constructions per se, such instances show how Roberts' (2010a) parameterized characterization of the feature composition of $\mathrm{T}$ can again be viewed as a more general aspect of the functional categories that the probe-goal-Agree system considers to be the Phase heads $\left(\mathrm{C}, \mathrm{T}\right.$, and $\left.v^{*}\right)$ that drive the computation from the Numeration to LF. Again, the relevant structures are generalizable across language types (in particular, whether the language is a null- or a non-null-subject language). In this respect, I will have a look at the English wh-construction in (11) below (Chomsky’s

\footnotetext{
${ }^{14}$ In passing, this parametric characterization of T's additional nominal D-feature could be given as a new interpretation of Bobaljik \& Jonas's (1996: 211) '[Spec, TP] parameter' in that the D-feature - as an 'edge' feature in the system of parametric variation I am proposing - and T's EPP feature will be satisfied in the same Spec position in some languages (TA vs. SA VSO structural representations), or in certain contexts in a given language (as, for example, in English there-constructions), due to T-to$\mathrm{Agr}_{\mathrm{S}}$ (which, in some way, corresponds to Rouveret's 2010 assumption about T's 'Remerge' option).
} 
2008: 149 example (10)), comparing it later to the kind of wh-constructions TA exemplifies:

(11) a. C [T [who [ $\mathrm{v}^{*}[$ see John] $\left.]\right]$

b. $\mathrm{Who}_{\mathrm{i}}\left[\mathrm{C}\left[\mathrm{who}_{\mathrm{j}}\left[\mathrm{T}\left[\mathrm{who}_{\mathrm{k}} v^{*}[\mathrm{see} \mathrm{John}]\right]\right]\right]\right]$

c. Who saw John?

Chomsky (2008) contends that movement of a wh-phrase out of the subject position in $v^{*} \mathrm{P}$ that yields the wh-question in (11c) forms two different chains: (a) an $\mathrm{A}^{\prime}-\mathrm{A}$ chain $\left(\right.$ chain $\left._{1}\right)$, and (b) an A-chain $\left(\right.$ chain $\left._{2}\right)$. Chain 1 links $w h o_{k}$ in $\left[S p e c, v^{*} \mathrm{P}\right]$ to $w h o_{i}$ in [Spec, CP] - a typical A'-position. Chain 2 links who $o_{k}$ in $\left[\mathrm{Spec}, v^{*} \mathrm{P}\right]$ to $w_{h} o_{j}$ in $[\mathrm{Spec}$, TP] - a typical A-position. Since the two copies of the wh-phrase target two different positions in the derived wh-question, it is plausible to argue that two different features are valued in each chain. If $\mathrm{Chain}_{2}$, linking [Spec, $v^{* \mathrm{P}}$ ] to [Spec, TP], is arguably responsible for valuing T's Agree-features and its EPP feature (both of which, presumably, are transmitted from C to T; cf. Chomsky 2008: 144-149, 157), chain values a certain Edge feature $(\mathrm{EF})$ that $\mathrm{C}$ withholds from transmitting to $\mathrm{T}$. For reasons of linearization processes in the PF component, only the first copy (involved in Chain ${ }_{1}<w h o_{\mathrm{i}}, w h o_{\mathrm{k}}>$ ) gets a phonological matrix. The second copy (involved in Chain $_{2}<w h o_{\mathrm{j}}, w h o_{\mathrm{k}}>$ ) remains silent.

Chomsky's (2008: 149, example (12)) evidence for two different copies who and $w h o_{j}$ in (11b)) in subject wh-extractions comes from such contrasts as in (12):

(12) a. Who was [who] never seen?

b. *Who was there never seen?

In (12a), the square-bracketed lower wh-moved copy who is silent. If, prior to the deletion of the wh-copy who, at PF (as argued above), no other element can occupy the landing site of this lower wh-copy, then the ungrammaticality of the wh-extraction case in (12b) is explained: there in (12b) cannot be inserted in the position where the lower copy of who is supposed to land.

In TA wh-constructions, as in (13a) with the structural representation in (13b), the process of wh-movement relies on the presence of an EXPL pro in subject position at the IP /Agr $\mathrm{P}$ level: 
(13) a. Shkuun illi pro shaaf-ik?

who that saw-you

'Who saw you?'

b.

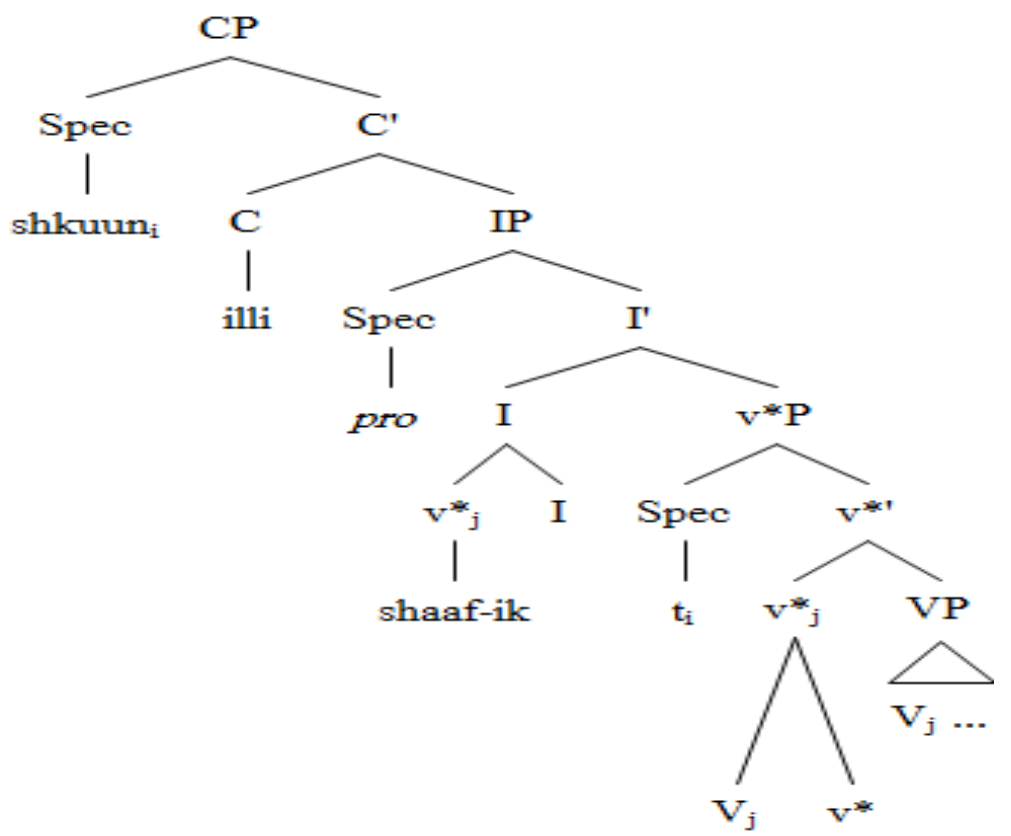

The TA wh-question in (13) shows a familiar process peculiar to null-subject languages. According to Rizzi (1982), due to the possibility of having EXPL pro occupy [Spec, IP/Agr $\mathrm{P}$ ] in null-subject languages, subject wh-extraction in these languages operates directly from the post-verbal position (namely, [Spec, $\left.\mathrm{v}^{* \mathrm{P}}\right]$ in (13b)). If Biberauer (2010: 195) is right in claiming that “... only English, MSc [Main Scandinavian] and, on the deletion analysis, canonical NSLs [Null-Subject Languages] have a canonical subject position in the sense of the original EPP, i.e. a position which must always be occupied either by a subject or a subject-related expletive", then we can directly link the availability of pro in the TA subject whquestion in (13), and the availability of the wh-copy who in the corresponding English subject wh-question in (11) to the necessity of valuing the EPP in [Spec,IP/TP].

However, the question that comes to mind is the following: since the process of wh-moving a subject question-word out of a Spec position lower than IP (i.e., $\left[\right.$ Spec, $\left.v^{*} \mathrm{P}\right]$ in the case at hand) is no longer related to the null-subject parameter (as Rizzi 1982 originally proposed it to be), why is it the case that English subject whextraction, as in (11), and TA subject wh-extraction, as in (13), involve different processes for satisfying the EPP in [Spec, IP/TP]? The natural answer to that question 
- in the context of the parameterization approach to the feature structure of the core Phase heads in the syntax we have been dealing with in this section - is that English and TA differ in some property of T (present on T in TA, but absent in English), which is different from the EPP-feature, although somewhat related to it since both features are valued in the same position- i.e., [Spec, IP/Agr $\mathrm{P}$ ].

As discussed above, the relevant parameterized property that allows a null pro D-element to be merged in $[\mathrm{Spec}, \mathrm{IP} / \mathrm{TP}]$ is a $[u \mathrm{D}]$ feature of the probe that is valued by an interpretable D-feature on the goal. The effect this feature has on structural representations (and dependency relations in derivational terms) is to relate $\mathrm{D}$ on $\mathrm{T}$ to $\varphi$-feature specification, and by that token, pronominalizing the structural subject position of clauses for EPP satisfaction. As Roberts (2010a: 80) puts it, “... pro is just like an overt pronoun; its non-overtness is purely a PF matter".

If that much is reasonable enough, the specific feature $\mathrm{D}$ on $\mathrm{I} / \mathrm{T}$ in those examples involving a silent element, in a null-subject language like TA, could only be an instantiation of a null EXPL pro element, the D-feature of which licenses the uninterpretable counterpart D-feature on I/T. Presumably, a silent copy of the whmoved subject in [Spec, IP/TP] could not satisfy such an inherent feature in TA and similar null-subject languages.

Further evidence from EXPL-associate pairs in English and TA suggests that the EXPL analysis of subject wh-extractions is also available in English. This evidence involves there-associate pairs in such contrasts as the following (Chomsky 1995c: 158, example (50), which Chomsky assigns to Safir 1985):

(14) a. [wh How many men] did John say that there were $t_{w h}$ in the room?

b. $*\left[{ }_{w h}\right.$ How many men] did John say that $\mathrm{t}_{\mathrm{wh}}$ were in the room?

(14b) is a common that- $t$ violation that is obviated in (14a) by virtue of thereinsertion. As Chomsky (1995c: 158) notes, this is essentially Rizzi's (1982) analysis of wh-extraction of subjects in Italian, with the only difference that the EXPL in English-type languages must be overt, whereas it has to be null in null-subject languages (namely, pro).

The TA examples in (15) are the counterpart of the English examples in (14):

(15) a. [wh Qaddash min raažəl] qul-t (illi) pro kaan-uu $\mathrm{t}_{\mathrm{wh}}$ fii l-biit? how many of man say-2S that be-3MP in the-room 'How many men did you say were in the room?' 
b. *[wh Qaddash min raažəl] qul-t (illi) humə kaan-uu $t_{w h}$ fii l-biit? how many of man say-2S that they be-3MP in the-room 'How many men did you say were in the room?'

(15a) is the TA grammatical counterpart of the English EXPL-associate pair in (14a). Over and above valuing the EPP-feature of T in (15a), EXPL pro presumably also values T's intrinsic D-feature. On this account, EPP satisfaction in [Spec, IP/TP] and valuing the $[u \mathrm{D}]$ on $\mathrm{I} / \mathrm{T}$ coincide in null-subject languages allowing a null EXPL pro to be merged in that position in subject wh-extraction cases so that the subject in [Spec, $\left.v^{*} \mathrm{P}\right]$ could be wh-moved directly from a post-verbal position - assuming V-vto-T raising applies. The wh-question (15b) shows that, in TA, nothing other than a silent EXPL pro can occupy [Spec, IP]; hence, the ungrammaticality of spelling out the EXPL as a pronominal (humə in (15b)) in that position. The fact that English shows evidence of the involvement of EXPL-associate pairs in some instances of subject wh-extraction (as in (14)) lends credence to the EXPL pro analysis of the counterpart subject wh-extraction cases in a null-subject language like TA (see Table 3.2 below).

Thus, this parallelism between English and TA in EXPL-associate pairs may point to the conclusion that, in an embedded context in English like (14a), the Dfeature of $\mathrm{T}$ may be available even if $\mathrm{T}$ in such an embedded context is not $\mathrm{T}_{\mathrm{DEF}}$, and normally would not be instantiating the [person] feature of Chomsky $(2001,2004) .{ }^{15}$ The hypothesis that $\mathrm{T}$ in such a context does indeed instantiate a D-feature is suggested by the observation that the wh-phrase how many men in (14a) wh-moves over there in the embedded subject position of a $\varphi$-complete $\mathrm{T}$ (as a complement of the embedded C, which is a 'strong' Phase head) in apparent violation of Chomsky's (1995c) Minimal Link Condition or Chomsky’s (2001) Phase Impenetrability Condition. ${ }^{16}$

\footnotetext{
${ }^{15}$ In relation to my discussion of the examples in (9) and (10) above, I have suggested that the Dfeature as a defining property of T in null-subject languages should be equated with Chomsky's (2001, 2004) [person] feature on $T_{D E F}$ in a language like English in that both features coincide with the satisfaction of the EPP in the relevant examples, and that perhaps the [person] feature on $\mathrm{T}_{\mathrm{DEF}}$ in English is the manifestation of the D-feature that Roberts (2010a,b) and Biberauer et al. (2010) argue is a defining property of $\mathrm{T}$ in null-subject languages.

${ }^{16}$ The same conclusion seems to be true of movement of a wh-phrase out of a matrix clause involving a there-construction, with the exception that there-insertion is not obligatory in this case, as exemplified in (ia,b) below:

(i) a. [ст [How many men $]_{i}$ were $\left[\right.$ TP $_{\text {there }}\left[\mathrm{t}_{\mathrm{i}}\right.$ in the room $\left.\left.]\right]\right]$

b. [CP [How many men $]_{i}$ were $\left[{ }_{T P} t^{\prime}{ }_{i}\left[t_{i}\right.\right.$ in the room $\left.\left.]\right]\right]$
} 
A possible explanation (in the framework of Chomsky 2001, 2004) for why EXPL does not violate any licensing or locality conditions of the grammar is that the Case-feature of the wh-trace $t_{w h}$ in (14a) is valued by LDA and, in turn, $\varphi$-features on embedded T and [person] on there are valued and delete, as well. EXPL is 'frozen in place' and rendered inactive for the probe-goal-Agree system, which no longer 'sees' EXPL as a goal to be raised, and the wh-phrase how many men is free to wh-move over EXPL. In any case, this derivational scenario is actually the same as that involved in the derivation of the TA interrogative construction in (15a): the wh-phrase qaddash min raažal is wh-moved over EXPL pro, and this raising process does not violate any licensing or locality conditions of the grammar.

What I am suggesting here is that there in such an embedded context as in (14a), which is comparable to the context where EXPL pro in used in the TA example (15a), might have an interpretable [person]/D-feature. As Biberauer (2010) argues for the null pronominal D-element pro, as discussed above, the D-feature of there in English and pro in a null-subject language like TA has the effect of relating D on T to $\varphi$-feature specification necessitating some element to be merged in the highest subject position in the clause being derived for EPP satisfaction. If this suggestion about the interpretability of the [person]/D-feature of there in the embedded clause of (14a) could be maintained, it would generalize to all instances of merging there in clause structure, i.e., whether $\mathrm{T}$ is $\mathrm{T}_{\mathrm{DEF}}$ (as in certain embedded clauses) or $\mathrm{T}_{\text {COMPLETE (as in }}$ matrix clauses). Table 3.2 below, provides a summary of the different contexts,

In this case, the postulated D-feature would be available in both (ia) and (ib). The suggestion that a Dfeature may be available in the wh-questions in (ia) and (ib) may point to the conclusion that in such wh-questions where $b e$ is used as the auxiliary verb undergoing subject-verb inversion, the postulated D-feature - as an 'edge' feature in the system of assumptions I am proposing - may coincide with the 'edge' feature (EF) Chomsky (2008) assumes is generated on C (cf. Chomsky 1995b: 289-290 for the assumption that Q-features on elements in CP are variants of the D-features of DPs and the T-node). In this case, the D-feature I am positing for both (ia) and (ib) need not be generated in the IP domain, but need only obtain in the $\mathrm{CP}$ domain. This assumption coincides with the assumption that $\operatorname{Agr}_{\mathrm{S}}$ may be missing in such cases of wh-movement irrespective of the presence of there in the Numeration in the wh-question in (ia). Due to the absence of $\mathrm{Agr}_{\mathrm{S}}$ in (ia) and (ib), the T node, under which the auxiliary verb be is merged in the IP domain, raises to $\mathrm{C}$ with which $\mathrm{T}$ is associated via the C-T dependency. At the same time, the Wh-criterion of Rizzi (1996) requires that [Spec, CP] be overtly realized in these wh-questions. In (ia), the D-feature I am postulating to be an intrinsic feature of EXPL (there, in (ia)) is interpretable and need not be valued. In this case, the insertion of there is entirely in response to the requirement that the EPP be satisfied in [Spec, TP] in English, but not the checking of a D-feature on T. Thus, the derivation of (ia) is assimilated to that of (ib) in terms of an 'edge' feature generated at the highest head position in sentence structure - namely $\mathrm{C}$. 
exemplified above, in which the D-feature of $\mathrm{T}-\mathrm{Agr}_{\mathrm{S}}$ in relation to EXPL-associate pairs in English and TA could be parametrically available:

Table 3.2. The parametric availability of an interpretable D-feature on $\mathrm{T}-\mathrm{Agr}_{\mathrm{S}}$ in English and TA

\begin{tabular}{|c|c|c|}
\hline $\begin{array}{l}\text { Type of EXPL- } \\
\text { associate } \\
\text { construction } \\
\text { involving a D- } \\
\text { feature on } \mathrm{T}-\mathrm{Agr}_{\mathrm{S}}\end{array}$ & English & TA \\
\hline $\begin{array}{l}\text { EXPL-associate } \\
\text { pairs in simple } \\
\text { sentences }\end{array}$ & $\begin{array}{l}\text { e.g.: There arrived three } \\
\text { men }\end{array}$ & $\begin{array}{l}\text { e.g.: pro jaw thlaatha } \\
\text { EXPL came.3P three } \\
\text { rjaal } \\
\text { men } \\
\text { 'There arrived three } \\
\text { men' }\end{array}$ \\
\hline $\begin{array}{l}\text { EXPL-associate } \\
\text { pairs in infinitival } \\
\text { raising contexts }\end{array}$ & $\begin{array}{l}\text { e.g.: } \text { There seems to have } \\
\text { arrived a man }\end{array}$ & - \\
\hline $\begin{array}{l}\text { EXPL-associate } \\
\text { pairs in subject wh- } \\
\text { extraction in simple } \\
\text { sentences }\end{array}$ & - & $\begin{array}{l}\text { e.g.: Shkuun illi pro jaa? } \\
\text { who that EXPL came } \\
\text { 'Who came?' }\end{array}$ \\
\hline $\begin{array}{l}\text { EXPL-associate } \\
\text { pairs in subject wh- } \\
\text { extraction in } \\
\text { embedded contexts }\end{array}$ & $\begin{array}{l}\text { e.g.: [ }{ }_{\text {wh How many men] }} \\
\text { did John say that } \\
\text { there were } t_{\mathrm{wh}} \text { in the } \\
\text { room? }\end{array}$ & $\begin{array}{l}\text { e.g.: }[\text { wh Qaddash min } \\
\text { how many of } \\
\text { raajol qul-t (illi) } \\
\text { man say-2S (that) } \\
\text { pro kaan-uu fii l-biit? } \\
\text { be-3MP in the-room } \\
\text { 'How many men did } \\
\text { you say were in the } \\
\text { room?' }\end{array}$ \\
\hline
\end{tabular}

Maintaining that [person] on there is actually an interpretable D-feature linked - quite like EXPL pro in a null-subject language like TA - to an intrinsic unvalued Dfeature of T in the examples discussed above, I come back to Chomsky's $(2001,2004)$ characterization of DP-movement processes in raising constructions in embedded contexts where both a DP subject and there are selected for the Numeration (see 
section 2.3.2). Of particular importance is the contrast in structures (16b) and (17b) below (which are the structural representations for sentences (54b) and (54c), respectively, in section 2.3.2, repeated below as (16a) and (17a); adapted from Chomsky 2001: 19, examples (21b,c)):

(16) a. There are likely to arrive three men.

b. [CР C [TP There $_{\mathrm{i}}$ are likely [TP $\mathrm{t}_{\mathrm{i}}\left[\mathrm{TDEF}_{\mathrm{T}}\right.$ to arrive three men]]]] (17) a. *Three men are likely there to arrive.

b. *[ СР C [TP Three men i $_{\mathrm{i}}$ are likely [TP there [TDEF to arrive $\left.\left.\left.\left.\mathrm{t}_{\mathrm{i}}\right]\right]\right]\right]$ According to Chomsky's $(2001,2004)$ Phase-theoretic framework, the structure in (16b) converges at the interface not because the derivation seeks the most appropriate candidate to be raised to the matrix [Spec, TP] position in terms of $\varphi$-feature specification, but because the derivation is only interested in raising an element close enough to matrix T for EPP satisfaction, which, in (16b), happens to be there - first inserted in embedded [Spec, TP] in satisfaction of the EPP, as well. LDA operates to guarantee that whatever feature $\mathrm{T}_{\mathrm{DEF}}$ has (i.e., the [person] feature of Chomsky 2001, 2004, or, the intrinsic D-feature in the present account) in the embedded clause and the $\varphi$-features of $\mathrm{T}_{\text {COMPLETE }}$ in the matrix clause are appropriately valued and deleted. The same logic works pretty well for an explanatory account of the ungrammaticality of (17b). Although both the lexical subject three men and there are equally able to satisfy the EPP, the derivation seeks to raise the closest element to matrix [Spec, TP]. In this case, raising three men over there violates the licensing and locality conditions of the grammar.

As I have discussed in relation to (52b) in Chapter Two (repeated below as (18), Chomsky's $(2001,2004)$ assumption is that the [person] feature of there survives deletion at the embedded IP level after checking the EPP in [Spec, $\mathrm{T}_{\mathrm{DEF}}$ ] and is raised to matrix [Spec, TP], not only to check a second instance of the EPP, but also - supposedly - to have its own uninterpretable [person] feature 'erased' and valued by the $\varphi$-features of $\mathrm{T}_{\text {COMPLETE, }}$ in the matrix clause:

(18) There are likely [ $\mathrm{T}_{\mathrm{DEF}}$ to be awarded several prizes].

However, as I suggested in passing in footnote 40 in Chapter Two, the uninterpretable feature $T_{D E F}$ has seems to be intrinsic to it since $T_{D E F}$ in such raising 
contexts is not selected in sentence structure by a $\mathrm{C}$ node. If this conclusion about the feature structure of $\mathrm{T}_{\mathrm{DEF}}$ could be maintained, the parametric difference between English and TA in the feature structure of T - in relation to EXPL elements in such wh-movement contexts as (14) and (15) - is no longer relevant. As discussed above, English and TA seem to be fairly similar in the derivation of EXPL-associate pairs. EXPL elements, in this conception, would enter the derivation with an interpretable intrinsic D-feature that matches the intrinsic uninterpretable D-feature of the T node that awaits valuation and deletion at Spell Out at the highest level of the derivation of sentence structure, which also coincides with the satisfaction of the EPP.

This suggestion would account for why [person] on EXPL in Chomsky's (2001, 2004) account survives deletion and cannot be 'erased' in the embedded clause in such an example as (16b) - namely, it is not because [person]/D on EXPL is not valued and 'erased' that EXPL is able to further raise to matrix [Spec, TP], but because this feature does not need valuation in the first place. ${ }^{17}$ What is important in this account - as in Biberauer's (2010) and Roberts' (2010a,b) accounts - is that, besides the D-feature on T that EXPL values, the ultimate purpose of the insertion and/or raising process is to satisfy the EPP at the 'strong' Phase in the derivation. It is perhaps this close relationship that exists between the D-feature on EXPL (matching the D-feature of T) and the EPP cross-linguistically that makes the status of EXPL elements quite peculiar among other D-categories. The Merge of these elements does not seem to be driven by their own feature structure. Rather, it is the feature structure of the target that requires their presence - namely, the satisfaction of the EPP at the level where T (or T's feature complex) is introduced. This is indeed the conclusion that such an approach as Miyagawa's (2010) EPP-triggered movement analysis of agreement or that of R\&R's T-criterion in relation to the T-dependency would also have us reach.

What remains to be determined is whether the relation between EXPLinsertion and $\mathrm{T}$ in embedded contexts in raising structures could generalize to all contexts involving there in English, as I suggest above.

Before looking in more detail at EXPL-insertion structures in null-subject systems in the next section in relation to the theoretical import of D-features on EXPL

\footnotetext{
${ }^{17}$ Recall that in Chomsky $(2001,2004)$, valuation of Case on DPs and [person] on EXPL is what renders them active in the process of valuing the EPP on T. Reinterpreting [person] on EXPL as [D], the role of $[\mathrm{D}]$ on EXPL is to link $[u \mathrm{D}]$ on $\mathrm{T}-\mathrm{Agr}_{S}$ to $\varphi$-feature specification for the EPP satisfaction.
} 
elements and associated functional heads, I would like to comment on the typology of EXPL-insertion structures cross-linguistically in light of the discussion of the merge site of EXPL elements in this section. If Chomsky $(2008$ : 148-149, 157) is right in his suggestion that not only $\varphi$-features, but also the EPP-feature are 'transmitted' by the 'strong' Phase heads $\mathrm{C}$ and $v^{*}$ to the heads they select as complements in sentence structure (i.e., respectively, $\mathrm{T}$ and $\mathrm{V}$ - with which $\mathrm{C}$ and $v^{*}$ share the features they transmit), then EXPL-insertion should theoretically be allowed in the Spec position of any of these heads.

Apart from the fact that this is not true for $\left[\mathrm{Spec}, v^{*} \mathrm{P}\right]$ (the position where referential DP subjects receiving the 'agentive'/'experiencer' $\theta$-role are inserted; see section 2.3.2), EXPL-insertion in [Spec, $\mathrm{CP}$ ] has been argued to be a possible process in a number of languages, as for example, the evidence from Germanic languages reviewed above, and also McCloskey's (2001) suggestion that EXPL pro could be inserted in [Spec, CP]. Likewise, EXPL pro-insertion in [Spec, VP] has been argued by Sportiche (1998) to take place in relation to object DPs in French (see section 2.2.1 for Sportiche's 1998 analysis).

\subsubsection{EXPL-insertion in Romance null-subject systems: The role of D-features} (Roberts 2010a, Biberauer et al. 2010)

In this section, I discuss the assumption that, at least in some languages, the role of $\mathrm{T}$ in agreement configurations not only depends on the availability of the Spechead/head-head agreement relation, but also on the availability of EXPL elements that set specific requirements on the T-node and the realization of the subject position in sentence structure. Under a T-to-Agr analysis, as assumed in the parameterized probe-goal-Agree approach adopted here, the fundamental relation in question is

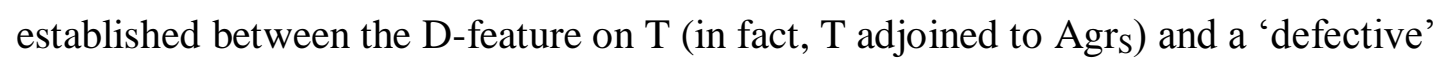
goal - i.e., a pro element as assumed in Roberts' (2010a) analysis of some varieties of null-subject languages such as the Northern Italian dialects. As Roberts (2010a: 102) argues, in some of these varieties, subject clitics are weak pronouns rather than subject agreement markers, and as such, may also play the role of 'defective' goals.

According to Roberts (2010a: 110), in some Northern Italian dialects "where the subject is null, there is a pro, which, following Cardinaletti \& Starke 1999, I take to be a weak pronoun in SpecTP licensed by the features of T'. Building on Poletto's (2000a) study of subject clitics in the northern Italian dialects, Roberts (2010a) shows 
how the characterization of the feature structure of the core functional category $\mathrm{T}$ in terms of a defining D-feature relates to the null-subject parameter and to parametric variation in general. Following Poletto (2000a), Roberts (2010a: 106-107) suggests that in the Northern Italian dialects (with such characteristics as in (19a-c) below) “... the subject clitics are seen as 'raddoppiamento dell'accordo' (agreement doubling), ... as 'extra' subject agreement markers", or else, “ ... manifestations of unvalued $\varphi$ features associated with $\mathrm{T}$ in some way".

In this respect, Roberts (2010a: 106) assumes that null-subject systems could be characterized by the following typology in relation to subject clitics (SCLs) and verbal inflection (the verbal agreement marking paradigm), as in (19) below:

(19) a. SCL [-agr], V [+agr] (a non-redundant null-subject system)

b. SCL [+agr], V [+agr] (a fully redundant null-subject system)

c. SCL [-agr], V [-agr] ((usually) a complementary system)

d. SCL [+agr], V [-agr] (a non-null-subject system)

As I will be dealing with presently, the description of languages in terms of a binary [ \pm Agr] typology applied to the subject pronoun system and to the verbal agreement system is meant to show the extent to which these systems interact for a possible characterization of the null-subject parameter. According to this typology, the language types in (19a-c) have a positive setting for the null-subject parameter thus allowing for null-subjects. In contrast, the language system in (19d) has a negative setting for the null-subject parameter.

To take the simplest case (19b) first, this null-subject system (exemplified in Table 3.3 below for the Fiorentino variety) has, at least, five different agreement markings on verbs. Thus, these markings count as [+agr]. Likewise, the subject pronoun system distinguishes at least five persons. The null-subject system in (19c) (exemplified in Table 3.4 below for the Carrara variety) is characterized by [-agr] for both the subject pronoun system and verbal agreement markings. This null-subject system has fewer agreement markings on verbs than the variety in (19b), but still it distinguishes, at least, five persons if the verbal agreement markings and subject pronouns are taken together. The variety in (19a) (exemplified in Table 3.5 below for the Como dialect) is characterized by [+agr] for the verbal agreement markings, but as 
[-agr] for the subject pronoun system. In this variety, the verbal endings are fully differentiated, but there are fewer than five distinct subject pronouns. Finally, in the non-null-subject system in (19d) (exemplified in Table 3.6 below for French), the subject pronoun system is characterized as [+agr] given the total differentiation of these pronouns. In contrast, the verbal agreement endings do not provide enough distinctions as the subject pronoun system does, and as such these verbal endings are characterized as [-agr].

In addition to the assumption that the subject clitics and the verbal agreement markings, combined, have to distinguish at least five persons, Roberts' (2010a) analysis of the typological types in $(19 \mathrm{a}-\mathrm{c})$ as null-subject systems relies on the primary assumption, mentioned above, that subject clitics in such varieties are instances of 'agreement doubling'. In this light, Roberts (2010a: 109) differentiates null-subject varieties of Northern Italian dialects with characteristic (19c) from other null-subject varieties with characteristics $(19 a, b)$, and non-null subject systems with characteristic (19d).

According to the typology in (19) above, the varieties classified under (19c) are characterized as '(usually) complementary systems', which makes them similar to type (19b). This typological similarity concerns the extent to which subject clitics and verbal inflection covary and instantiate T's unvalued $\varphi$-features in relation to T's Dfeature. Taking the Carrara variety and the Fiorentino variety as sample examples of similar agreement-doubling languages, Roberts (2010a) provides the following subject-verb agreement paradigms: ${ }^{18}$

Table 3.3 (Type (19b)): A fully redundant null-subject system; e.g., the Fiorentino dialect (Roberts 2010a: 106)

\begin{tabular}{|l|l|ll|}
\hline $\mathrm{S}$ & 1 & (E) parlo \\
& 2 & Tu & parli \\
& $3(\mathrm{M})$ & $\mathrm{E}$ & parli \\
& $3(\mathrm{~F})$ & La & parla \\
\hline $\mathrm{p}$ & 1 & $\mathrm{Si}$ & parla \\
& 2 & Vu parlate \\
& $3(\mathrm{M})$ & E & parlano \\
& $3(\mathrm{~F})$ & Le & parlano \\
\hline
\end{tabular}

\section{('I speak', etc.)}

\footnotetext{
${ }^{18}$ See also, below, the discussion of the Milanese variety, of type (19c), in relation to how D-features relate to the null-subject parameter.
} 
Table 3.4 (Type (19c)): (Usually) a complementary system; e.g., the Carrara dialect (Roberts 2010a: 108-109)

\begin{tabular}{|c|c|c|}
\hline $\mathrm{S}$ & $\begin{array}{l}1 \\
2 \\
3(\mathrm{M} / \mathrm{F})\end{array}$ & $\begin{array}{ll}\text { a } & \text { dormə } \\
\text { tə } & \text { dərmə } \\
\text { i/al dormə }\end{array}$ \\
\hline $\mathrm{p}$ & $\begin{array}{l}1 \\
2 \\
3(\mathrm{M} / \mathrm{F})\end{array}$ & $\begin{array}{l}\text { a durmin } \\
\text { durmitə } \\
\text { i/al dormənə }\end{array}$ \\
\hline
\end{tabular}

('I sleep', etc.)

In Roberts' (2010a) description of the subject-verb agreement paradigm in Table 3.3, at least five persons are distinguished. In this system, both subject agreement and verbal agreement markers count as [+agr], and in this respect, "the clitics and the verbal endings covary, both indicating the person and number of the subject" (Roberts 2010a: 106). Similarly, in the subject-verb agreement paradigm in Table 3.4, Roberts (2010a: 106-107) mentions that the subject clitics and the verbal agreement markers (combined together) distinguish at least five persons. Thus, in a (usually) complementary system, although both subject agreement and verbal agreement markers count as [-agr], they provide distinct agreement marking for each person in case they are taken together. As Roberts' (2010a: 107-108) description of such subject-verb agreement paradigms as in Table 3.3 and Table 3.4 indicates, notwithstanding the different morphological manifestations of 'covariation' between the subject clitics and the agreement markings in these two closely-related dialects, both subject clitics and agreement markings play a role in " ... somehow instantiating T's $\varphi$-set ... as uninterpretable features" (Roberts 2010a: 108), thus allowing null subjects in relation to D-features to co-occur with overt subject DPs in the IP domain, both of which are relevant for the valuation of T's features (namely, T's $\varphi$-set, its Dfeature and its EPP feature; see Roberts 2010a: 108).

As for type (19a), it is exemplified by such varieties as the Como variety with the subject-verb agreement paradigm in Table 3.5 below: 
Table 3.5 (Type (19a)): A non-redundant null-subject system; e.g., the Como dialect (Roberts 2010a: 108)

\begin{tabular}{|l|l|ll|}
\hline $\mathrm{S}$ & 1 & \multicolumn{2}{r|}{ dormi } \\
& 2 & ta & dormat \\
& $3(\mathrm{M} / \mathrm{F})$ & al/la & dorma \\
\hline $\mathrm{P}$ & 1 & & ('I sleep', etc.) \\
& 2 & & durmum \\
& $3(\mathrm{M} / \mathrm{F})$ & i/al & dorman \\
\hline
\end{tabular}

As Table 3.5 shows, the Como dialect's verbal inflection system is fully

differentiated. However, the subject-clitic system, counting as [-agr], does not covary with, or complement, the different agreement markings on the verb, counting as [+agr]. Thus, this system provides only a 'partial' indication of a doubling style of analysis for subject clitics. ${ }^{19}$

In contrast to the null-subject systems in (19a-c) is the non-null-subject system exemplified by French, with characteristic (19d). In this non-null-subject system, verbal agreement markers count as [-agr] in that these markers do not covary with the subject pronominal system, counting as [+agr]. Roberts (2010a) concludes that French subjects are not clitics but weak pronouns in [Spec, TP]. The French subject-verb agreement paradigm is exemplified in Table 3.6 below:

Table 3.6 (Type (19d)): A non-null-subject system: e.g., French (Roberts 2010a: 107)

\begin{tabular}{|c|c|c|}
\hline $\mathrm{S}$ & 1 & Je dors $[\mathrm{d} \supset \mathrm{R}]$ \\
\hline & 2 & $\mathrm{Tu}$ dors $[\mathrm{d}\lrcorner \mathrm{R}]$ \\
\hline & 3 & Il/elle dort $[\mathrm{d}\lrcorner \mathrm{R}]$ \\
\hline \multirow[t]{3}{*}{$\mathrm{P}$} & 1 & Nous dormons \\
\hline & 2 & Vous dormez \\
\hline & 3 & Ils/elles dorment \\
\hline
\end{tabular}

\footnotetext{
${ }^{19}$ With reference to the subject-clitic system in (19a-c), Roberts (2010a: 109) maintains that this system may be an indication of "the possibility of a 'doubling' style of analysis for subject clitics, according to which features of the D of the subject DP may be realized in the probe $\mathrm{T}$ as a consequence of the Agree relation between the two elements". In this connection, Roberts (2010a: 114) makes reference to a 'doubling typology', whereby various kinds of doubling could account for the differences between the three varieties of null-subject systems mentioned in (19a-c). According to Roberts (2010a: 114), this typology may ultimately be linked to how "the features of the subject DP are copied onto T in relation to T's D-feature". Roberts (2010a: 114) further assumes that this variation in feature copying may involve what he refers to as 'full copying' as opposed to 'partial copying'. He, nevertheless, leaves the question of this variation open. The copy (pronoun) deletion theory of Roberts (2010a,b) and Biberauer (2010), mentioned earlier in this section may also be helpful in pinning down the minimal units of parametric variation in other null-subject systems such as SA vs. TA/MA.
} 
In Table 3.6, judging from the fact that the singular forms dors (for $1^{\text {st }}$ person singular and $2^{\text {nd }}$ person singular) and dort (for $3^{\text {rd }}$ person singular) are homophonous, these three singular forms do not provide a reliable distinction in terms of the morphological feature of person. In this case, the subject-verb agreement system of French does not provide enough distinctions in terms of person agreement markings, and the speaker/learner has to have recourse to the pronominal system to make the appropriate distinctions. Thus, on the basis of such an example, Roberts (2010a) concludes that the difference in spelling for the singular persons is not a determining factor for the type of distinction that sets null-subject systems apart from non-nullsubject systems.

Coming back to the null-subject systems in (19a-c), Roberts (2010a: 114) assumes that in these language types, "features of the subject DP are copied onto T as a consequence of the agree relation; all of these are null-subject systems, where $\mathrm{T}$ has a $\mathrm{D}$-feature, thereby making the subject $\mathrm{D}$ a defective goal. This gives rise to clitic doubling involving the subject D and the subject agreement on the verb". Of particular importance in the context of how D-features relate to the null-subject parameter is the null-subject system of type (19c) exemplified by the Milanese variety (sentences (20a,b); Robert's 2010a: 112 examples (119a,b)):

(20) a. El fio el mangia l pom

(Milanese) the boy he eats the apple 'The boy eats the apple'
b. Un quidum el riverà in ritart a somebody he will-arrive in late 'Somebody will arrive late'

As shown in (20), the subject clitic el co-occurs with a 'subject' DP - an instance of a Clitic Left Dislocation, i.e., a 'topicalized' subject outside the IP domain proper, which el resumes. If Roberts (2010a: 110) is right in characterizing subject elements like $e l$ in (20a) as clitics cliticized to the verb under the inflectional node $\mathrm{T}$ in this null-subject variety, then a null subject pro in the structural subject position of the clause (i.e., [Spec, TP] in Roberts' 2010a account) is obligatorily present realizing subject D-features in conjunction with T. According to Roberts (2010a: 112) the assumption that pro is merged in the subject position of null-subject sentences "is also necessary if we are to maintain our assumption that T always has an EPP-feature in these dialects". 
Under such assumptions, the characterization of the null-subject property in terms of the parametric availability of an additional D-feature on the highest functional head in the IP domain opens up the possibility of the potential association between D-features, Agree features, and the EPP for the expression of agreement at the highest level of functional structure at the IP level.

\subsubsection{Summary}

In this section, I have been concerned with the role of EXPL elements in accounting for the parametric variation in the realization of the subject position and the valuation of T's morphological/agreement features. Different accounts of how languages (including null-subject systems) parametrically vary in this respect have been discussed. I first reviewed some earlier accounts about EXPL-insertion processes in the grammar of Germanic and null-subject languages. Then, in a comparison of English and TA subject wh-extraction cases, I have tried to show that the parametric availability of a D-feature on $\mathrm{T} / \mathrm{Agr}_{\mathrm{S}}$ accounts for the differences in the realization of the subject position in these cases. Nevertheless, although English and TA differ somewhat in this respect, the availability of EXPL-associate pairs cross-linguistically shows that the two languages (i.e., English and TA) may resort to similar processes in extraction strategies - namely, by merging an EXPL element in subject position. The role of D-features and D/DP elements is further discussed in relation to the copy pronoun theory (Roberts 2010a and Biberauer et al. 2010).

In the next section, I discuss the role of $\mathrm{Agr}_{\mathrm{S}}$ in accounting for the differences between typologically similar languages such as SA and TA, as two varieties of Arabic, in the derivation of the $\mathrm{C}-\left(\mathrm{Agr}_{\mathrm{S}}\right)-\mathrm{T}$ dependency, arguing that $\mathrm{Agr}_{\mathrm{S}}$ could be a minimal unit of parametric variation in Arabic.

\subsection{The subject-verb agreement system of Arabic}

\subsubsection{Introduction}

In light of the parametric account I have been entertaining in the previous sections in terms of differences in the projection and derivation of sentence structure as $\operatorname{Agr}_{S} \mathrm{P}$ or as TP, the hypothesis according to which an $\mathrm{Agr}_{S}$ node projects in the clause structure of a null-subject language like TA, or, in a typologically different language like English (in EXPL-associate pairs, see Table 3.2), has been fruitful in delimiting the 
parametric boundaries that make typologically different languages look similar, and typologically similar languages look different.

In this context, the account I have proposed above shows that the insertion processes resulting in EXPL-associate pairs (such as the insertion of pro as a referential null element or null expletive, according to the structure being derived, or the insertion of there in English) are not merely in response to the requirement that the subject position of sentence structure be occupied by some element at the interface as in the traditional account (Chomsky 1982: 79; Chomsky \& Lasnik 1993: 123). EXPL-insertion processes may also be in response to the feature structure of the core functional nodes of sentence structure including the T-node in both the IP domain and the $\mathrm{CP}$ domain in their interaction with syntactic predication.

Thus, in terms of the feature-valuation mechanism and the probe-goal-Agree system (Chomsky 2001, 2004, 2008; Roberts 2010a, Biberauer and Roberts 2010), valuation of the EPP feature on $\mathrm{T}$ in the IP domain may not be the driving force of these insertion processes. In this respect, the morphological feature that has been the centre of interest, in my parametric account of the relevant structures, is a certain Dfeature that (contra Roberts 2010a and Biberauer et al. 2010) I have proposed to be an intrinsic and defining feature of the $\mathrm{Agr}_{\mathrm{S}}$ node projected higher than the $\mathrm{T}$ node in sentence structure. Nevertheless, since - in the EXPL-associate constructions I have been concerned with - the T node necessarily raises to the $\operatorname{Agr}_{S}$ node, my proposal has been that the interpretable D-feature on pro (in TA) and on there (in English) is responsible for the valuation of $[u \mathrm{D}]$ on $\mathrm{Agr}_{\mathrm{S}}$, and, by that token, linking $[u \mathrm{D}]$ on $\operatorname{Agr}_{S}$ to $\varphi$-feature specification for EPP satisfaction in [Spec, T-Agr $\left.\mathrm{P}\right]{ }^{20}$

Similar considerations apply in relation to the different realization of subjectverb agreement configurations in SA and TA. The parameterized probe-goal-Agree system of structural dependency relations would set different requirements in terms of

\footnotetext{
${ }^{20}$ The same is true in non-finite raising structures, in English, involving the process of there-insertion in the Spec position of infinitival clauses, as discussed in section 3.2.1.1. The T node in such an embedded context is $\mathrm{T}_{\mathrm{DEF}}$, and, as such, it is presumably not endowed with the relevant $\mathrm{D}$-feature. Nevertheless, the assumption that $\mathrm{T}_{\mathrm{DEF}}$ is, in fact, endowed with a $\mathrm{D}$-feature could be maintained since there-insertion in Spec of $\mathrm{T}_{\mathrm{DEF}}$, in English, links T's D-feature in the embedded clause to T merged at the highest level of sentence structure where Spell Out operates. In terms of the probe-goal-Agree system and the $\mathrm{C},\left(\mathrm{Agr}_{\mathrm{S}}\right), \mathrm{T}$ dependency approach to subject-verb agreement configurations, the valuation of $[u \mathrm{D}]$, in this case, operates on the inflectional complex $\mathrm{T}-\mathrm{Agr}_{\mathrm{S}}$ in the matrix clause to which $\mathrm{T}$ in the embedded clause is anaphorically related (cf. R\&R's 'clause union' notion (p. 136)), and both $[u \mathrm{D}]$ features are safisfied. At the level of Spell Out, where the complex T-Agr is formed in the matrix clause, there inserted in the Spec of $\mathrm{T}_{\mathrm{DEF}}$ is raised to [Spec, $\mathrm{T}-\mathrm{Agr}_{\mathrm{S}}$ ], thus satisfying the Dfeature at that level resulting in EPP satisfaction in the matrix clause.
} 
the parametric availability of $\mathrm{Agr}_{\mathrm{S}}$ and its intrinsic D-feature in VSO word order configurations in the relevant language.

In this section, I first discuss some assumptions about subject agreement morphology (including 'mood' morphology), verbal inflection and modality that are held by most linguists in the Arabic linguistics tradition. Thus, in section 3.3.2, I consider the contrasts that show up in the perfective and the imperfective forms of verbs in SA in order to identify how tense, aspect and mood are encoded on verbal forms. In section 3.3.3, I deal with the distinction between agreement $/$ mood and modality (including negation) in subject-verb agreement configurations in SA. This distinction mainly concerns the encoding of person and tense in the perfective and the imperfective and how this difference in tense encoding interacts with modality.

Then, in section 3.3.4 below, I deal with my proposal for the main parametric difference that sets SA apart from the modern Arabic dialects as regards the representation of elements (phonetically realized at $\mathrm{PF}$, or left null at the interface) in subject position in VSO structural representations. Section 3.3.5 is a summary of the main points dealt with in section 3.3.4. Section 3.3.6 deals with implications as to the nature of $\mathrm{T}$ (namely, the feature-makeup of tense) in relation to the different structural representations SA and TA exhibit in the probe-goal-Agree system discussed in section 3.2.

\subsubsection{Tenselaspect, mood and subject-verb agreement in the perfective vs. imperfective form of verbs in $S A$}

Apart from the two distinct stem forms and the accompanying vocalic melody that differentiates the perfective from the imperfective in SA, the difference between the two verbal forms is further manifested in the encoding of subject agreement - namely, it is encoded in the form of both a prefix and a suffix in the imperfective, but merely as a suffix in the perfective. In section 3.3.2.1, on the basis of the suffixal nature of number subject agreement in the imperfective, I will propose that the suffixal morphology in the imperfective represents a single morpheme in each paradigm cell that encodes both number subject agreement and mood. In contrast, in the perfective, there are no mood distinctions in the suffixal morphology. Thus, the difference between the two verbal inflectional paradigms in SA is shown to be simply in how tense/aspect information is encoded on the verb: whereas, in the perfective, the agreement morphology is purely suffixal and encodes both subject agreement and 
tense/aspect information, it is the prefixal morphology of the verb, in the imperfective, that encodes tense/aspect information. ${ }^{21}$

In section 3.3.2.2, I will discuss the difference in the encoding of subject agreement in the perfective and the imperfective. This difference is simply a difference in the encoding of subject agreement and not a difference in how sentence structure projects in terms of an additional Mood Phrase (= MoodP) that projects higher than IP in the imperfective (contra Fassi Fehri 1993 as reviewed in 2.1.2.3.3). In section 3.3.2.3, I deal with my proposal that this difference does not correlate with a difference in V-to-T movement either (contra Benmamoun 2000b, 2008). ${ }^{22}$ Section 3.3.2.4 is a summary.

\subsubsection{Tense/aspect, agreement and mood in $S A$}

Table 3.7 below gives the inflectional paradigm of the verb kataba 'write' in both the perfective aspect and the imperfective aspect (cf. Benmamoun 2000b: 20 and Aoun et al. 2010 who provide inventories of the perfect and imperfect paradigms in SA similar to what is being described in this section):

\footnotetext{
${ }^{21}$ In this respect, aspect and tense on the SA verb are not only encoded on stems by also on the morphology affixed to the verb. Recall from section 2.1.2.3.3 the discussion of Fassi Fehri's (1993, 2000) contention that, in SA, tense on the verb is an integral part of the verb itself as a lexical property, not only in the imperfective (the prefixal morpheme/tense) but also in the perfective (the suffixal morphology/tense).

${ }^{22}$ Benmamoun $(2000 \mathrm{~b}, 2008)$ argues that present tense T in Arabic lacks the categorial feature [+V], which is also a characteristic of verbless sentences. In these instances, Benmamoun (2000b, 2008) argues that V-to-T movement does not occur. I come back to a discussion of this issue in section 4.1.1.2.
} 
Table 3.7. The perfective and imperfective conjugation of the verb kataba ${ }^{23}$

\begin{tabular}{|c|c|c|c|c|c|c|}
\hline & & Subject & Perfective & & rfective aspe & \\
\hline & & System & & $\begin{array}{l}\text { Indicative } \\
\text { mood }\end{array}$ & $\begin{array}{l}\text { Subjunctive } \\
\text { mood }\end{array}$ & $\begin{array}{c}\text { Jussive } \\
\text { mood }\end{array}$ \\
\hline $\mathrm{S}$ & $1 \mathrm{M} / \mathrm{F}$ & anaa & katab-tu & Pa-ktub-u & Pa-ktub-a & Pa-ktub \\
\hline & $2 \mathrm{M}$ & anta & katab-ta & ta-ktub-u & ta-ktub-a & ta-ktub \\
\hline & $2 \mathrm{~F}$ & anti & katab-ti & ta-ktub-iina & ta-ktub-ii & ta-ktub-ii \\
\hline & $3 \mathrm{M}$ & huwa & katab-a & ya-ktub-u & ya-ktub-a & ya-ktub \\
\hline & $3 \mathrm{~F}$ & hiya & katab-at & ta-ktub-u & ta-ktub-a & ta-ktub \\
\hline $\mathrm{D}^{24}$ & $2 \mathrm{M} / \mathrm{F}$ & antumaa & katab-tumaa & ta-ktub-aani & ta-ktub-aa & ta-ktub-aa \\
\hline & $3 \mathrm{M}$ & humaa & katab-aa & ya-ktub-aani & ya-ktub-aa & ya-ktub-aa \\
\hline & $3 \mathrm{~F}$ & humaa & katab-ataa & ta-ktub-aani & ta-ktub-aa & ta-ktub-aa \\
\hline $\mathrm{P}$ & $1 \mathrm{M} / \mathrm{F}$ & naHnu & katab-naa & na-ktub-u & na-ktub-a & na-ktub \\
\hline & $2 \mathrm{M}$ & antum & katab-tum & ta-ktub-uuna & ta-ktub-uu & ta-ktub-uu \\
\hline & $2 \mathrm{~F}$ & antunna & katab-tunna & ta-ktub-na & ta-ktub-na & ta-ktub-na \\
\hline & $3 \mathrm{M}$ & hum & katab-uu & ya-ktub-uuna & ya-ktub-uu & ya-ktub-uu \\
\hline & $3 \mathrm{~F}$ & hunna & katab-na & ya-ktub-na & ya-ktub-na & ya-ktub-na \\
\hline
\end{tabular}

The inflectional/temporal system of SA is characterized by an aspectual dichotomy that differentiates the perfective and the imperfective. The perfective form of the verb

\footnotetext{
${ }^{23}$ This conjugation is characteristic of verbs in the SVO word ordering where $\mathrm{S}$ can be a phonologically realized or non-realized Topic DP. In the VSO word ordering, only the third person singular (feminine or masculine, depending on the gender agreement feature of the post-verbal DP) shows up on the verb (see section 2.1.2 for a discussion of the derivation of SVO and VSO word orders in SA vs. modern spoken Arabic dialects like TA and MA).

${ }^{24}$ There is no first person dual in Arabic. For the expression of the involvement of two people in an event or situation, the first plural form is used in such cases.
} 
is used in the expression of past events. ${ }^{25}$ As for the imperfective form of the verb, it is used in the expression of non-past events. ${ }^{26}$ Table 3.7 shows that this aspectual difference is not only manifested in a distinction of stems, but also in the different affixation patterns the agreement morphology is associated with in the perfective vs. the imperfective. Whereas the agreement inflection on the imperfective verb takes the form of both a prefix (which encodes person in addition to tense) and a suffix (which encodes number/gender and mood), the agreement inflection on the perfective verb is uniquely suffixal (encoding subject-verb agreement in $\varphi$-features and, on Fassi Fehri's 1993, 2000, 2004 view, tense).

As can be seen in Table 3.7, the subject pronominal system shows some cases of partial correspondence between the form of the pronoun and the suffixes of the perfective form, as in the second person dual antumaa katab-tumaa and in the second person feminine plural antunna katab-tunna. As Givón (1976: 183) mentions in relation to the Semitic perfect conjugation, "the suffixal conjugation of the perfect ... bear[s] some unmistakable relationship to some unmistakable pronoun series" as evidence that, at some historical point, the VS syntax became a dominant feature of the language (on a par with the SV word order, which, as already mentioned in Chapter Two, is a close alternative to the VS order). Benmamoun (2000b, 2008) proposes that person agreement in Semitic languages evolved out of a pronominal clitic taking the form of a suffix in the perfective aspect/past tense and the form of a

\footnotetext{
${ }^{25}$ Dahl (1985: 79) notes that the perfect(ive) tends to be used for past events. Bahloul (2008: 47-66) enunciates the contexts in which the perfective is tied up with a past tense interpretation, a present tense interpretation, a future tense interpretation or a gnomic interpretation.

${ }^{26}$ In negative sentences, the imperfective can also be used in the expression of a past event (see section 4.1.2). The past tense interpretation of the imperfective verb can also occur in another context - namely, in embedded clauses selected by a matrix perfective verb - as in (i) (to be compared to (ii)) (examples from Fassi Fehri 2012: 15):
}

(i) qaal-a l-ii Pinna-hu ya-ktub-u r-risaalat-a

PERF.say-3MS to-me that-him 3-IMPERF.write-MS.IND the-letter-ACC

'He said to me that he was writing the letter'

(ii) qaal-a l-ii Pinna-hu katab-a r-risaalat-a

PERF.say-3MS to-me that-him PERF.write-3MS the-letter-ACC

'He said to me that he wrote/had written the letter'

In (i), the imperfective verb yaktubu'he is writing' has the past interpretation 'he was writing', which is dependent on, and simultaneous (or 'anaphoric') with, the act of saying in the past as given by the matrix verb qaala 'he said'. In contrast, in (ii), the event described by the verb kataba 'he wrote' is anterior to the saying. 
prefix in the imperfective aspect/present tense. However, the historical relationship between the subject pronominal system and the so-called pronominal clitics is more transparent in the case of the suffixal morphology of the perfective than in the case of the prefixal morphology of the imperfective (see, in particular, the second person masculine/feminine dual and the second person masculine and feminine plural).

\subsubsection{The imperfective in SA: the problem of the characterization of the suffixal morphology}

According to Fassi Fehri (2000: 81-82; 1993: 79-81), the derivation of agreement inflection on the imperfective verb involves fusion of morphemes for the encoding of gender and number as one suffix, and the encoding of person and tense through prefixation (Fassi Fehri 2000: 88, footnote 19). These processes of suffixation and prefixation are also assumed to represent an instance of fission of subject agreement morphemes and their discontinuity in the encoding of person (by use of prefixation) and of number/gender (by use of suffixation). However, the so-called 'mood suffixes' of the imperfective form of the SA verb may turn out to be unconcatenatively encoded together with the agreement morphology (encoding number/gender). In order to test this hypothesis, I first begin with a description of such suffixes in the singular and the first person plural. Then, I deal with the suffixal morphology of the other forms of the imperfective verb.

In the singular (except for the second singular feminine) and the first person plural forms of SA imperfective subject-verb agreement paradigms, a two-way contrast holds distinguishing the indicative and the subjunctive uses of the imperfective verb from its jussive use. Whereas an $-u$ suffix is added to the root of the verb kataba in the imperfective in the indicative use, and an - $a$ suffix in the subjunctive use, no jussive suffixal morphology is phonetically realized on the imperfective verb in SA. ${ }^{27}$ Thus, in the first person masculine/feminine singular and the second person masculine singular, the $3^{\text {rd }}$ person of the singular, and the $1^{\text {st }}$ person

\footnotetext{
${ }^{27}$ An epenthesis process applies to the imperfective form of the SA verb in the jussive in the context of 'the linking hamza' $(=1)$ that necessarily appears with the definite article $/ l /$ at the beginning of the word. In such contexts, a suffix $-i$ is phonetically realized on the non-suffixed verb in the jussive as an epenthetic vowel (thus different from the $-u$ and $-a$ suffixes that show up, respectively, in the indicative and in the subjunctive uses of the imperfective verb. The /l/ sound undergoes a process of assimilation when followed by a vowel (apenthetic or not), as in ya-ktub-u r-risaalat-a 'he writes the letter' and in lam ya-ktub-i d-dars- $a$ 'he did not write the letter', where the $/ l /$ sound is assimilated to a $/ r /$ sound and to a $/ d /$ sound, respectively.
} 
masculine/feminine plural of the SA subject agreement system, the subject agreement suffix $-u$ also encodes the indicative mood, and the suffix $-a$ also encodes the subjunctive mood. For example, a $3^{\text {rd }}$ person singular masculine verbal form like ya$k t u b-u$ 'he writes/ he is writing' is an indicative form by virtue of carrying a subject agreement suffix $-u$. Similarly, a $3^{\text {rd }}$ person singular masculine verbal form like $y a$ $k t u b-a$ is a subjunctive form by virtue of carrying the subject agreement suffix $-a$.

In contrast to the realization or non-realization of the suffixal agreement morphology in the singular and the first person plural in the contexts discussed above, there is consistently some suffixal agreement morphology phonetically realized in all the remaining forms of the imperfective paradigm in the indicative, the subjunctive and the jussive. ${ }^{28}$ Nevertheless, in this respect, the subjunctive and the jussive pattern alike as against the indicative in losing a part of the suffixal agreement morphology namely, the /na/ ending form of the -uuna suffix in the second and third person plural masculine, and the /ni/ ending form of the of the -aani suffix in the second and third person dual masculine and feminine. Leaving aside for the moment the discussion of the encoding of mood on the imperfective verb in the plural and the dual, I will focus first on the encoding of subject agreement on the imperfective verb in SA. Taking the verb in the indicative as a representative form as far as the suffixal morphology of the imperfective verb is concerned, Table 3.8 below gives a breakdown of the morphosyntactic composition of the plural forms of the verb kataba' write' taking into account both suffixes and prefixes:

Table 3.8. Morpho-syntactic composition of the second and third person plural forms $(\mathrm{M} / \mathrm{F})$ of the verb kataba in the indicative use of the imperfective:

\begin{tabular}{|l|c|l|l|c|}
\hline \multirow{3}{*}{ Plural masculine } & 2 & ta- & ktub & -uuna \\
\cline { 2 - 5 } & 3 & ya- & ktub & -uuna \\
\hline \multirow{2}{*}{ Plural feminine } & 2 & ta- & ktub & -na \\
\cline { 2 - 5 } & 3 & ya- & ktub & -na \\
\hline
\end{tabular}

\footnotetext{
${ }^{28}$ This is also the case for the suffixal agreement morphology -iina of the second person singular feminine.
} 
In Table 3.8, the prefixed subject agreement system of the plural forms consistently distinguishes second person $t a$ - from third person $y a$-. This distinction does not, however, encode gender since each of these prefixes is used for both the masculine and the feminine. As for the suffixal morphology of these plural forms, it is consistent in making the distinction between masculine and feminine irrespective of person: -uuna is used for both second and third person masculine, whereas -na is used for both second and third person feminine. The present account of these plural forms goes against Bulos's (1965: 4-7) claim that, in the imperfective (indicative) form yaktubuuna, the encoding of gender (masculine) in the suffix -uu(na)is redundant since, as Bulos (1965) assumes, the prefix $y a$-already expresses gender (in addition to person). However, as my discussion of Table 3.8 above shows, the suffixal morphology of the imperfective only encodes number/gender subject-verb agreement, whereas the prefix encodes person agreement and tense as one fused entity (Fassi Fehri 2000: 88, footnote 19). Thus, there does not seem to be a redundant encoding of gender morphology in a form like yaktubuuna.

A similar breakdown of the morpho-syntactic composition of the verb kataba can be given in relation to the suffixes and prefixes of the dual, as inTable 3.9:

Table 3.9. Morpho-syntactic composition of the second and third person dual forms $(\mathrm{M} / \mathrm{F})$ of the verb kataba in the indicative use of the imperfective:

\begin{tabular}{|l|c|l|l|c|}
\hline \multirow{2}{*}{ Dual masculine } & 2 & ta- & ktub & -aani \\
\cline { 2 - 5 } & 3 & ya- & ktub & -aani \\
\hline \multirow{2}{*}{ Dual feminine } & 2 & ta- & ktub & -aani \\
\cline { 2 - 5 } & 3 & ta- & ktub & -aani \\
\hline
\end{tabular}

Table 3.9 shows that, in contrast to the morpho-syntactic composition of the plural forms, the prefixal morphology of the dual does not consistently distinguish person since $t a$ - is employed for both second plural masculine and for second and third plural feminine. Furthermore, the suffixal morphology of the dual masculine and feminine does not make any distinction in terms of person and gender since it is -aani for all forms. 
As far as the expression of both subject agreement and mood in the suffixal morphology of the imperfective verb is concerned, Bulos (1965: 37) suggests that the morpheme-iina, -uuna or -aani represents a composition of a number/gender morpheme $-i i-,-u u-$ or $-a a$ - and a mood morpheme $-n a$ or $-n i$. Under Bulos's (1965: 37) analysis, the difference between the imperfective verb used in the indicative and its use in the subjunctive and jussive in SA is that-na and -ni are dropped out in the subjunctive and the jussive.

However, there is evidence in the grammar of SA that the suffixal plural masculine morphology, taken as a whole (i.e., -иuna), may be an instantiation of a number/gender agreement morpheme rather than of the composition of two separate morphemes - namely, an agreement morpheme (i.e., - - uu) and a 'mood' suffix (i.e., -na) (contra Bulos 1965). Benmamoun (2000b: 30-31) and Pesetsky and Torrego (2001: 336) point out that the so-called 'mood suffixes' of the imperfective indicative verb across all three numbers of the subject-verb agreement system correspond to the agreement/case suffixes found on NOM Case-marked DPs, as Table 3.10 below shows (adapted from Pesetsky and Torrego 2001: 336, example (17)):

Table 3.10. Suffixal morphology on NOM Case-marked DPs and imperfective verbs in $\mathrm{SA}$

\begin{tabular}{lll}
\hline Singular & Dual & Plural \\
\hline $\begin{array}{l}\text { PaT-Taalib-u } \\
\text { the-students-MS.NOM }\end{array}$ & $\begin{array}{l}\text { PaT-Taalib-aan(i) } \\
\text { the-students-MD.NOM }\end{array}$ & $\begin{array}{l}\text { Pal-muSallim-uun(a) } \\
\text { the-teachers-MP.NOM }\end{array}$ \\
$\begin{array}{ll}\text { ya-ktub-u } \\
\text { 3-IMPERF.write-MS.IND }\end{array}$ & $\begin{array}{l}\text { 3a-ktub-IMPERF.write-MD.IND } \\
\text { 3-IM-Gallim-uun(a) }\end{array}$ \\
\hline
\end{tabular}

The occurrence of these 'mood suffixes' both on verbs and NOM Casemarked DPs may be an indication that this 'mood' morphology is actually a reflection of subject-verb agreement at Spell Out and cannot be dissociated from the inflectional morphology expressing number/gender subject agreement in SA. In this connection, Bulos (1965: 6-7) also considers the fact that since the prefix ya-can occur without the -uun(a) suffix as in ya-ktubu 'he writes', in Table 3.10, or in $y u$-Sallimu 'he teaches', and since the -uun(a) suffix can occur without the $y a$-prefix as in muSallimuun(a) 'teachers', the prefixal and suffixal morphology of the imperfective 
form of the SA verb cannot be taken to make up a "one verb-subject affix" (contra Harris 1947: 285 who entertains that view).

In relation to the -na suffix of the two feminine plural forms of the SA imperfective indicative verb (i.e., $2^{\text {nd }}$ plural feminine and $3^{\text {rd }}$ plural feminine as in Table 3.8 above) taktubna and yaktubna, Bulos (1965: 39) suggests that, since this suffix is preserved on the imperfective verb used in the subjunctive and jussive, it not only expresses aspect but also gender and number (in the absence of 'mood' distinctions in these forms in the subjunctive and the jussive). Although in this case no correspondence similar to the correspondence that exists in the plural masculine morphology can be established between the agreement suffix - $n a$ of feminine plural forms (as in yuSallimna 'they(F) teach') and the agreement/case suffix of the corresponding DP (i.e., ?al-muSallimaat- $u$ 'the-female teachers-NOM'), it still holds that the -na feminine plural suffix is the counterpart of the -uuna masculine plural suffix of the subject-verb agreement system of the imperfective indicative verb. In this case, the fact that the feminine plural suffix -na is preserved in the subjunctive and the jussive may have to do with the possibility that if it were the case that this suffix was dropped there would be no indication of any encoding of number and gender agreement on the plural form of the imperfective verb.

An analysis of mood and agreement in SA (as expressed by the suffixal morphology of the imperfective verb) similar to Bulos's (1965) morphological analysis has been undertaken by Fassi Fehri (1993) in his morpho-syntactic analysis of verbal inflection in SA (see section 2.1.2.3.3). As in Bulos's (1965) morphological analysis of the plural suffixes on the imperfective verb, Fassi Fehri (1993: 79-81) considers the so-called 'mood suffix' of the imperfective form of the SA verb as a separate entity. Nevertheless, although mood suffixes may be considered to project in the syntax as a head Mood above the AGR node harbouring number/gender morphemes, they are basically unconcatenatively represented with these number/gender morphemes. In the morpho-syntactic terms of Fassi Fehri's (1993, 2000) analysis, as discussed above, both the agreement morpheme and the 'mood' morpheme are 'fused' in one single suffix on the imperfective verb. ${ }^{29}$

\footnotetext{
${ }^{29}$ Shlonsky's (1997: 101) view on the imperfective paradigm in SA is similar to Fassi Fehri's (1993) in that mood and agreement $\varphi$-features on verbs in the inflectional domain of sentences are 'fused', but he does not represent 'mood' as a separate syntactic node in sentence structure as Fassi Fehri (1993) does.
} 


\subsubsection{Benmamoun (2000b, 2008): A non-fusional analysis of SA verbal inflection}

In this section, I discuss Benmamoun's $(2000 \mathrm{~b}, 2008)$ approach to the derivation of verbal inflection, which Benmamoun (2000b, 2008) argues rests on the presence or absence of a $[+\mathrm{V}]$ feature on $\mathrm{T}-$ namely, the difference between a perfective/past tense interpretation of sentences vs. an imperfective/present tense interpretation of sentences, respectively. This difference is also the basis of Benmamoun's (2000b, 2008) analysis of small clause structure where either a past tense or a present tense interpretation arises in the derivation bound to the presence or absence, respectively, of a $\mathrm{V}$ position in the small clause selected by the $\mathrm{T}$ node in sentence structure. The alternative I will be proposing is based on the head-head dependency relations, which are established in terms of T-features associated with $\mathrm{V}-v$ and $\mathrm{T}$ (along with $\mathrm{C}$ (= Fin)). Thus, this proposal here mainly rests on a $[u \mathrm{~T}]$ feature on $v$, which gets valued/identified in the process of deriving verbal inflection in SA - i.e., the raising of $\mathrm{V}$ to $\mathrm{T}$.

In Benmamoun (2008), the problem of characterizing the different affixation patterns in the imperfective/present tense form of the SA verb and its perfective/past tense form in the syntax is accounted for in derivational terms rather than in terms of a difference in how sentence structure projects in the derivation of the different forms of the verb as in Fassi Fehri $(1993,2000) .{ }^{30}$ According to Benmamoun (2008: 122123), whereas the perfective/past tense verb raises to T in order to 'check' a verbal feature on the T node, the imperfective/present tense verb does not raise to $\mathrm{T}$ because, in this case, $T$ lacks the verbal feature. Benmamoun (2008: 122-123) claims that this derivational, feature-checking, difference between the perfective/past tense and the imperfective/present tense forms of the SA verb can account for the affixation patterns for person agreement that differentiate the two forms. ${ }^{31}$ Taking the verb kataba in the second person feminine singular as a representative example, Benmamoun (2008: 122-123) differentiates the suffixation pattern of the perfective/past tense verb katab-ti 'you(FS) wrote' with the prefixation pattern of the

\footnotetext{
${ }^{30}$ In section 2.1.2.3, I have proposed, following Fassi Fehri (1993, 2000) and Akkal \& Gonegai (2000), that agreement on the verb in SA is a case of pronominal cliticization in morpho-syntactic terms (see section 2.1.2.3.3 in particular).

${ }^{31}$ Although Benmamoun (2000b, 2008) refers to person agreement as being 'pronominal', this only follows from the fact that, historically, "person agreement evolved out of a pronominal clitic" (Benmamoun 2000: 122).
} 
imperfective/present tense verb ta-ktub(-iina)'you(FS) write/are writing', as represented in (21a) vs. (21b) below: ${ }^{32}$

(21) a. The derivation of the perfective/past tense prefixation pattern in SA (Benmamoun 2008)

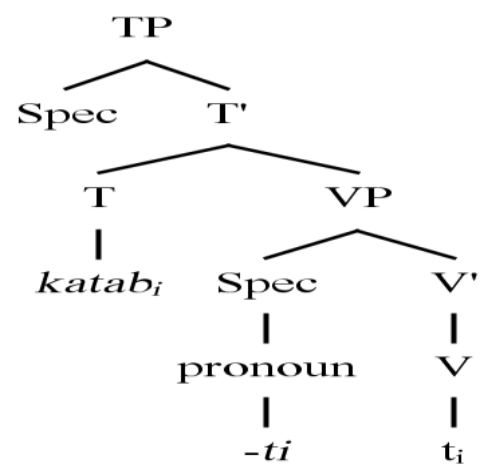

b. The derivation of the imperfective/present tense suffixation pattern in SA (Benmamoun 2008)

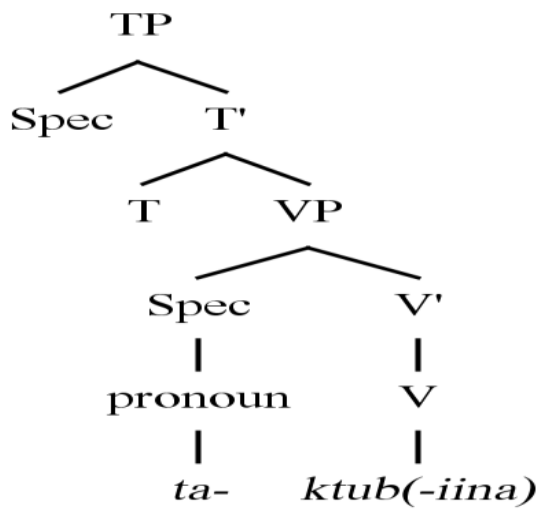

In (21a), the root of the perfective verb katab is merged first under the V node within the VP. Then, on Benmamoun's (2008) assumption that the T node associated with a perfective/past tense verb, is specified for a $[+\mathrm{V}]$ feature, the lexical verb raises to $\mathrm{T}$ for feature-checking purposes. The affixation pattern arises as a person and number/gender suffix phonologically realized in the second person feminine singular as katabti. In (21b), the root of the imperfective verb ktub is also merged under the $\mathrm{V}$ node within the VP, but, unlike the case of the root of the perfective verb katab, the T node associated with $k t u b$ is not specified for a [+V] feature, and, as such, it does not

\footnotetext{
${ }^{32}$ Benmamoun (2008) does not represent the so-called 'mood' morphology -iina on the root ktub of the imperfective verb in (21b).
} 
trigger the movement of the imperfective verb, which remains lower than the T node in the structure of the sentence and gets the phonological matrix taktub(iina) at Spell Out. Thus, according to Benmamoun (2008: 123), the prefixation versus the suffixation patterns of verbal inflection in SA are to be accounted for in terms of "a syntactically grounded dependency involving categorial features". This difference is exclusively defined in terms of the categorial feature $[+\mathrm{V}]$ (present on $\mathrm{T}$ when the verb is perfective, but absent on $\mathrm{T}$ when the verb is imperfective).

However, contrary to the assumption of the present analysis of verbal inflection in SA that the T-feature of tense is also a feature of verbs (i.e., $[u \mathrm{~T}]$, as the unvalued uninterpretable counterpart of $[i \mathrm{~T}]$ on $\mathrm{T}$ ) in the syntax of languages, Benmamoun's (2008) analysis is not compatible with the view that, in SA, the Tfeature of tense would be the driving force for verb movement in the syntax irrespective of the perfective/imperfective dichotomy. ${ }^{33}$ In addition, on the assumption that the tense morphology in verb-raising languages is 'rich' enough (Roberts 2010a: 162-164; Biberauer \& Roberts 2010: 265), the [uT] feature of the fuctional $v$ element - to which the verb merged in $\mathrm{V}$ attaches - would systematically be triggered to raise to T. Thus, according to this view, even if T does not have a [V] feature that needs to be valued in the imperfective paradigm in SA, on Benmamoun's (2008) assumptions, the verb (either perfective or imperfective) would still have to raise because of the 'rich' $[u \mathrm{~T}]$ feature of $v$ it is associated with, which is triggered to raise to $T^{34}$

As far as the so-called verbless sentences in SA are concerned (sentence 22a), Benmamoun (2000b, 2008) assumes that they lack the VP lexical structure above the

\footnotetext{
${ }^{33}$ As far as the perfective/past tense paradigm is concerned, Benmamoun (2000b: 25) acknowledges that "the exclusively suffixal agreement pattern on verbs always indicates that the verb is in the past tense", and that “... [T] he perfective verb carries past tense features". However, Benmamoun (2000b) considers these features to be different from the categorial $[+\mathrm{V}]$ and $[+\mathrm{D}]$ features that drive derivations.

${ }^{34}$ If this account of the dependency relation that exists between $\mathrm{T}$ and $\mathrm{V}$ in syntactic structure is reasonable enough, then it would also be true that $[u \mathrm{~V}]$ on $\mathrm{T}$ could be universally absent on $\mathrm{T}$. The raising process of $\mathrm{V}$-to- $\mathrm{T}$ (actually, $\mathrm{V}$ adjoined to $v$ and then raised to $\mathrm{T}$ on the assumptions adopted in this thesis) would exclusively be accounted for on the basis of the $v / \mathrm{V}$ 's 'rich' $[u \mathrm{~T}]$ feature (corresponding to the rich $[i \mathrm{~T}]$ feature on $\mathrm{T}$ ) that the raising of a verb to $\mathrm{T}$ is possible in $\mathrm{V}$-raising languages. I will leave this issue open. Nothing of what follows hinges on whether $[u \mathrm{~V}]$ exists on $\mathrm{T}$ or not. I return below in this section - in relation to my discussion of sentences with auxiliary kaana 'be' in SA - to Roberts' (2010a: 162-164) view about the pre-syntactic formation of verbal inflection as $[\mathrm{V}+\mathrm{T}]$ compouds inserted as such in the syntax.
} 
small clause predicative structure (i.e., AP/PP/NP in (22b) below, adapted from Benmamoun 2008: 115): ${ }^{35}$

(22) The feature structure of T in 'verbless' sentences in SA (Benmamoun 2008)

a. Pal-walad-u dhakiyy-un/fii l-bayt-i/tilmiidh-un the-boy-NOM intelligent-NOM/in the-house-GEN/pupil.INDEF-NOM 'The boy is intelligent/in the house/ a pupil.'

b.

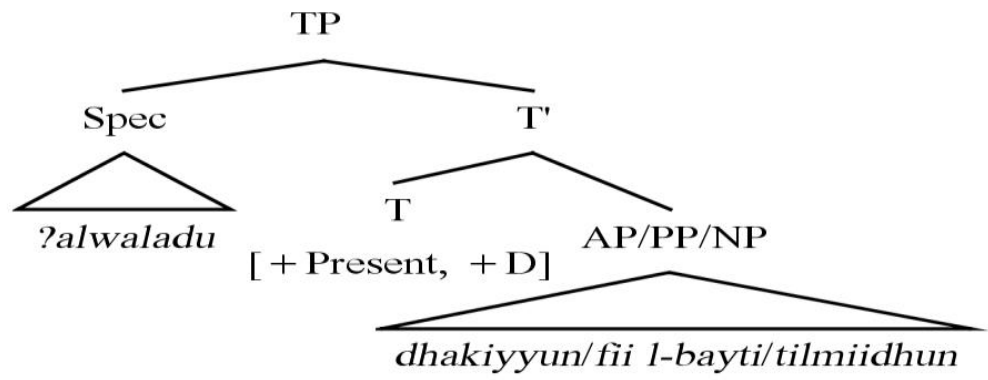

In (22b), Benmamoun (2000b, 2008) assumes that there is no V projection and that the T-node, bearing the present-tense feature, lacks the categorial $[+\mathrm{V}]$-feature, but is specified for the categorial $[+\mathrm{D}]$-feature. According to the discussion above, Benmamoun $(2000 b, 2008)$ claims that the absence of the $[+\mathrm{V}]$-feature on $\mathrm{T}$ is characteristic of the contexts where the present tense interpretation of a sentence arises in the syntax, as the verbless sentences in (22a) show.

As discussed above, although Benmamoun's (2000b, 2008) theory allows $\mathrm{V}(\mathrm{P})$ to project for the Merge process of imperfective/present tense verbs, the T-node in such a derivation is not different from the T-node in a structure like (22b) for verbless sentences. Thus, the feature structure of a T-node selecting as complement an imperfective/present tense verb is as represented in (23b) for sentence (23a):

(23) The feature structure of T with imperfective/present tense verbs in SA (Benmamoun 2008)

a. ?al-walad-u ya-?kul-u the-boy-NOM 3-IMPERF.eat-MS.IND 'The boy eats/is eating.'

\footnotetext{
${ }^{35}$ In the structures in (22b), (23b), (24b) and (25b), the subject DP in [Spec, TP] originates from the specifier position of the small clause AP/PP/NP.
} 
b.

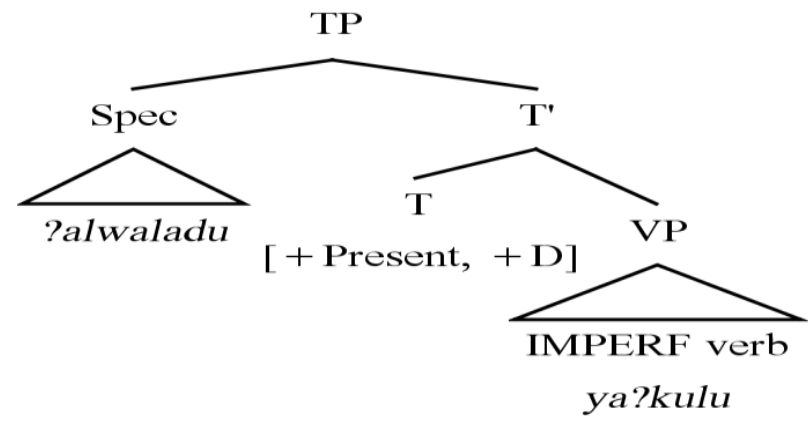

In (23b), the imperfective verb ya?kulu 'eats/is eating' remains within the VP, as there is no $[+\mathrm{V}]$ feature on $\mathrm{T}$ to 'check'.

By contrast, whenever $\mathrm{T}$ is interpreted as [+Past], it is specified for both [+D] and $[+\mathrm{V}]$, as represented in $(24 b)$ for sentence $(24 a)$ below:

(24) The feature structure of T with perfective/past tense verbs (Benmamoun 2008) in SA

a. ?al-walad-u ?akal-a the-boy-NOM PERF.eat-3MS

'The boy ate.'

b.

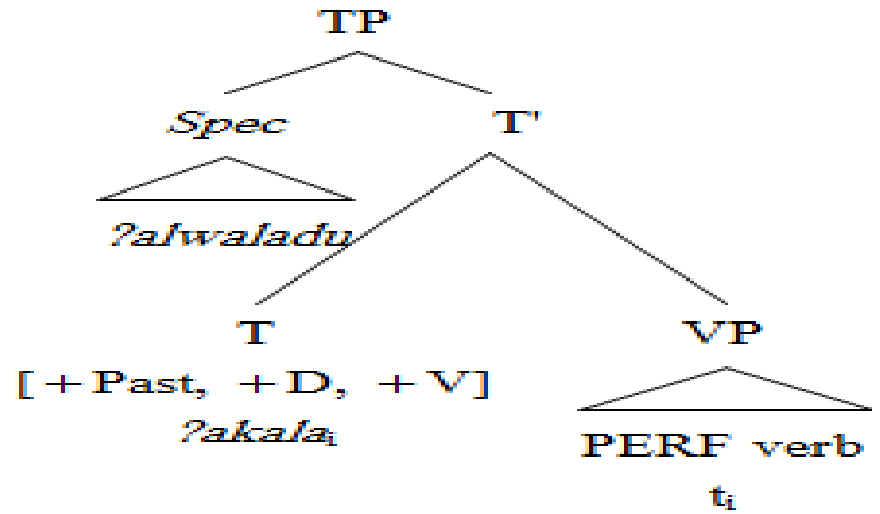

In $(24 b)$, the $[+\mathrm{V}]-$ feature is available because a perfective/past tense verb is merged under $\mathrm{V}$ whereby the [+Past] interpretation of the sentence arises in the syntax. The perfective verb Pakala raises to T to 'check' the $[+\mathrm{V}]$ feature on $\mathrm{T}$. Benmamoun (2000b, 2008) claims that the structural configuration in (24b) also obtains in the contexts where the auxiliary verb kaana is used to refer to a past situation, as represented in $(25 \mathrm{~b})$ for sentence $(25 \mathrm{a})$ : 
(25) The feature structure of T with the auxiliary verb kaana in SA

a. Pal-walad-u kaana dhakiyy-an/fii l-bayt-i/tilmiidh-an the-boy-NOM PERF.be.3MS intelligent-ACC/in the-house-GEN/pupil.INDEF-ACC 'The boy was intelligent/in the house/ a pupil.',

b.

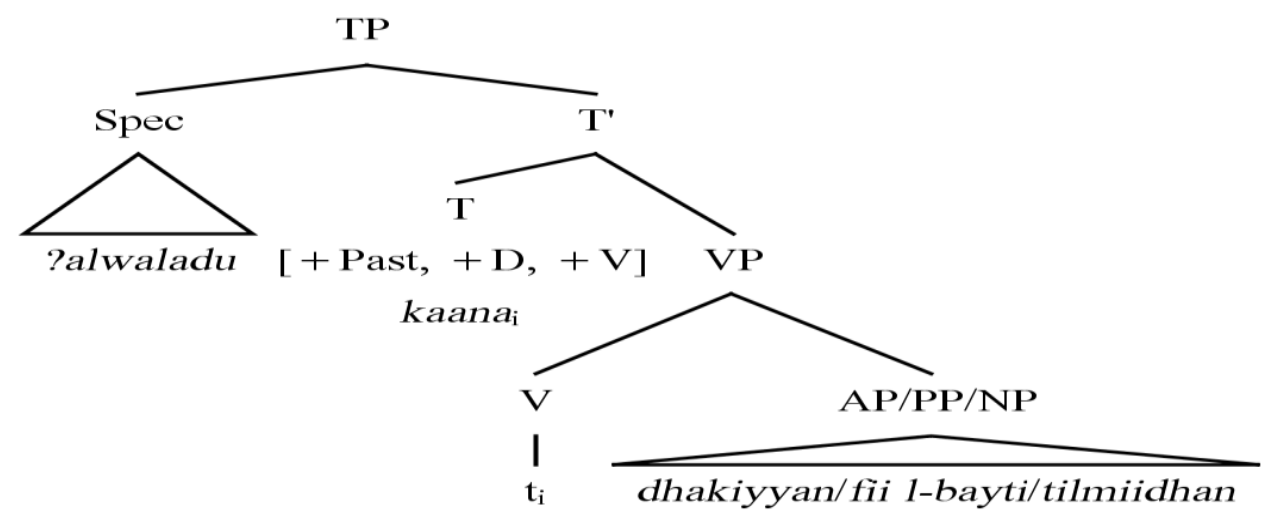

In (25b), Benmamoun (2000b, 2008) assumes that the auxiliary verb kaana is merged under $\mathrm{V}$ and is raised to $\mathrm{T}$ to 'check' the $\mathrm{V}$-feature of [+Past] $\mathrm{T}$ to check the $[+\mathrm{V}]$ feature of $\mathrm{T}$.

Under the assumptions adopted in this thesis in relation to clause structure formation, whether the auxiliary verb kaana is merged in the so-called predicational sentences in Arabic (the standard variety and the dialects) or not, the V position is assumed to project along with the functional portion of vP/VP headed by the light verb $v{ }^{36}$ The $\mathrm{V}$ head selects a clausal constituent - namely, either a SC complement, as in (26), or a CP (= ForceP) complement in a biclausal structure (see structure (28) below):

\footnotetext{
${ }^{36}$ In this respect, I differ from Benmamoun (2000b, 2008) in the assumption that the null copula V position, which Benmamoun (2000b, 2008) argues against, is not just $\mathrm{V}$, but a complex of a null copula $\mathrm{V}$ raised to the functional $v$ position. The complexity of the structure selected by the $\mathrm{T}$ node in 'verbless' sentences is also a cornerstone of Aarts's (1992) analysis of small clauses in English. However, Aarts (1992: 180) argues that the internal structure of the SC contains an I-node (marked [-tense]/[+Agr], which depends on the T-node of the higher tensed clause for its interpretation) and a VP constituent headed by a null copular verb $B E$ selecting an NP, AP or PP complement. As for Sportiche's (1998, Chapter 3) conception of small clauses (see section 2.2.1), a SC, complementselected by a lexical verb like consider in English for example, has two $\mathrm{V}$ positions. The lexical verb moves internally to VP from the lower V position to the higher empty V position. In Chomsky (1995b: 321), this internal movement of verbs is conceived of in terms of V-to- $v$ raising whereby a verb inserted under the $\mathrm{V}$ node obligatorily moves to a functional $v$ position, as assumed here (see also the beginning of section 2.2).
} 


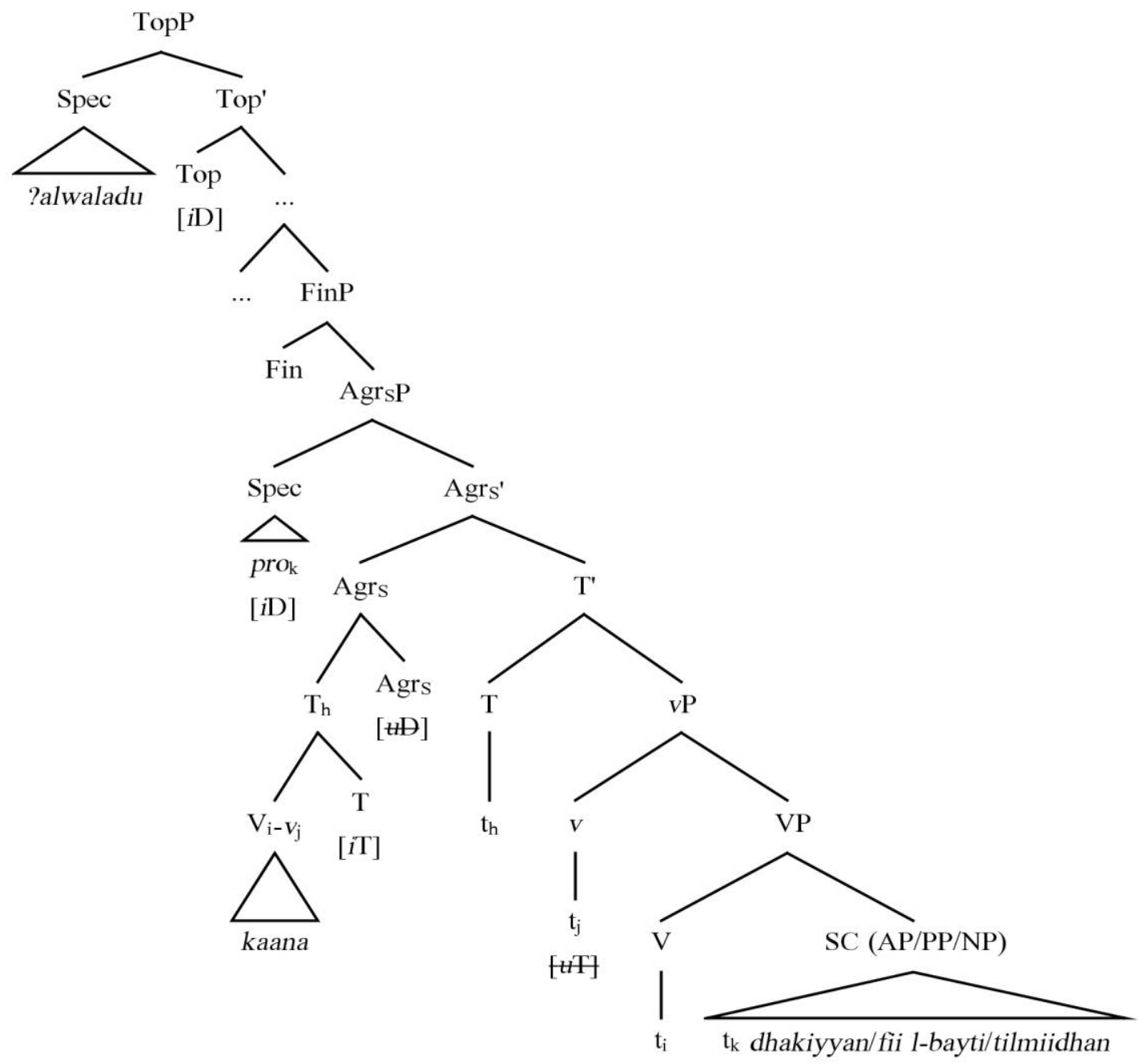

In (26), the 'empty' $v$ position of the $v \mathrm{P}$ selected by the $\mathrm{T}$ node of the matrix clause has uninterpretable T-features (i.e., $[u \mathrm{~T}]$ on $v$ ) linking it to T. V moves to $v$ irrespective of whether the auxiliary verb kaana 'be' is merged in $\mathrm{V}$ or not. The last step represented in (26) is the raising of $\mathrm{V}-v$ to $\mathrm{T}$. This movement process is characteristic of structures where the $\mathrm{V}$ position is lexicalized by merging a verb (either a copula or a lexical verb) in that position. Thus, in (26), the auxiliary verb kaana is merged under $\mathrm{V}$, and is raised to $v$. Then the complex [ $\mathrm{V}-v]$ moves further up to $\mathrm{T}$. $[u \mathrm{~T}]$ on $v$ is valued as a consequence of Agree. ${ }^{37}$ Since the structure exhibits the SVO word ordering, $\operatorname{Agr}_{S}(\mathrm{P})$ projects above T. a Referential pro is merged in [Spec, $\left.\mathrm{Agr}_{S} \mathrm{P}\right]$ raised out of the Spec position of the small clause. The DP Pal-walad- $u$ is

\footnotetext{
${ }^{37}$ I come back below to a discussion of the case where the auxiliary verb kaana is not in the Numeration.
} 
realized as a Topic merged in $[\mathrm{Spec}, \mathrm{TopP}]$ in the split-CP domain (see section 2.1.2.3.2, for the assumptions of the Topic Theory adopted in this thesis). ${ }^{38}$

In the instances where both the auxiliary verb kaana and a lexical verb are present in the Numeration, the structure of these sentences can, theoretically, be assumed to be either mono-clausal or bi-clausal. Aoun et al. (2010: 61) assume that such sentences are mono-clausal. Thus, under Aoun et al.'s (2010: 61) assumption, sentence (27a) below would have structure $(27 \mathrm{~b}):^{39}$
kann-at
T-Taalibaat-u
ya-drus-na
PERF.be-3FS the-students(F)-NOM 3-study-FP
'The students were studying.'

b.

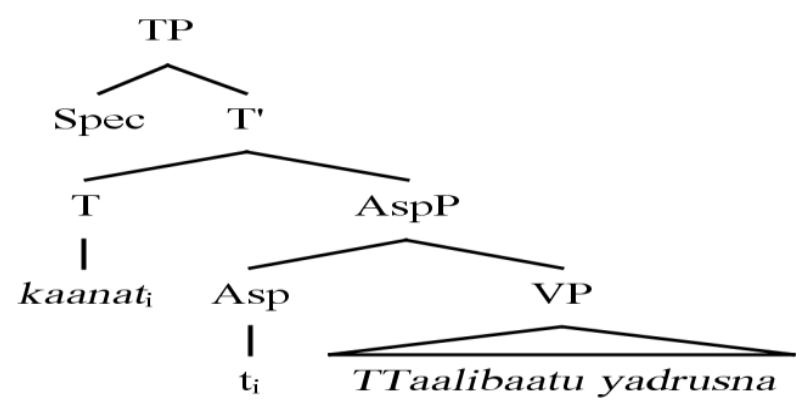

In (27b), the imperfective verb yadrusna 'they(F) study' is used in conjunction with the auxiliary verb kaana. On Benmamoun's (2000b, 2008) assumption that the imperfective verb is merged in $\mathrm{V}$ and remains in that position throughout the derivation, the auxiliary verb kaanat would be merged in a position higher than $\mathrm{V}$, which, as represented in (27b), could be an Aspect head (Asp) position projecting above VP. Then the auxiliary verb is raised to 'check' the V-feature of [+Past] T.

The alternative analysis adopted here is that such sentences, where both the auxiliary verb kaana and a lexical imperfective verb are selected for the Numeration,

\footnotetext{
${ }^{38}$ The Top node projects in the split-CP domain à la Rizzi (1997) (see section 2.1.2.3.2). As shown in (26) above, I assume that Top is endowed with an $[i \mathrm{D}]$ feature that is essential for its interpretation as part of the licensing of the T-dependency at the interface (see section 4.1.2.4 for a discussion of the feature structure of functional elements in sentence structure).

${ }^{39}$ Aoun et al. (2010: 61) do not provide a structure or a detailed analysis for this sentence.
} 
have a bi-clausal structure (cf. Akkal \& Gonegai 2000: 13-14). On this assumption, the structure of (27a) would rather be as in (28): $:^{40}$

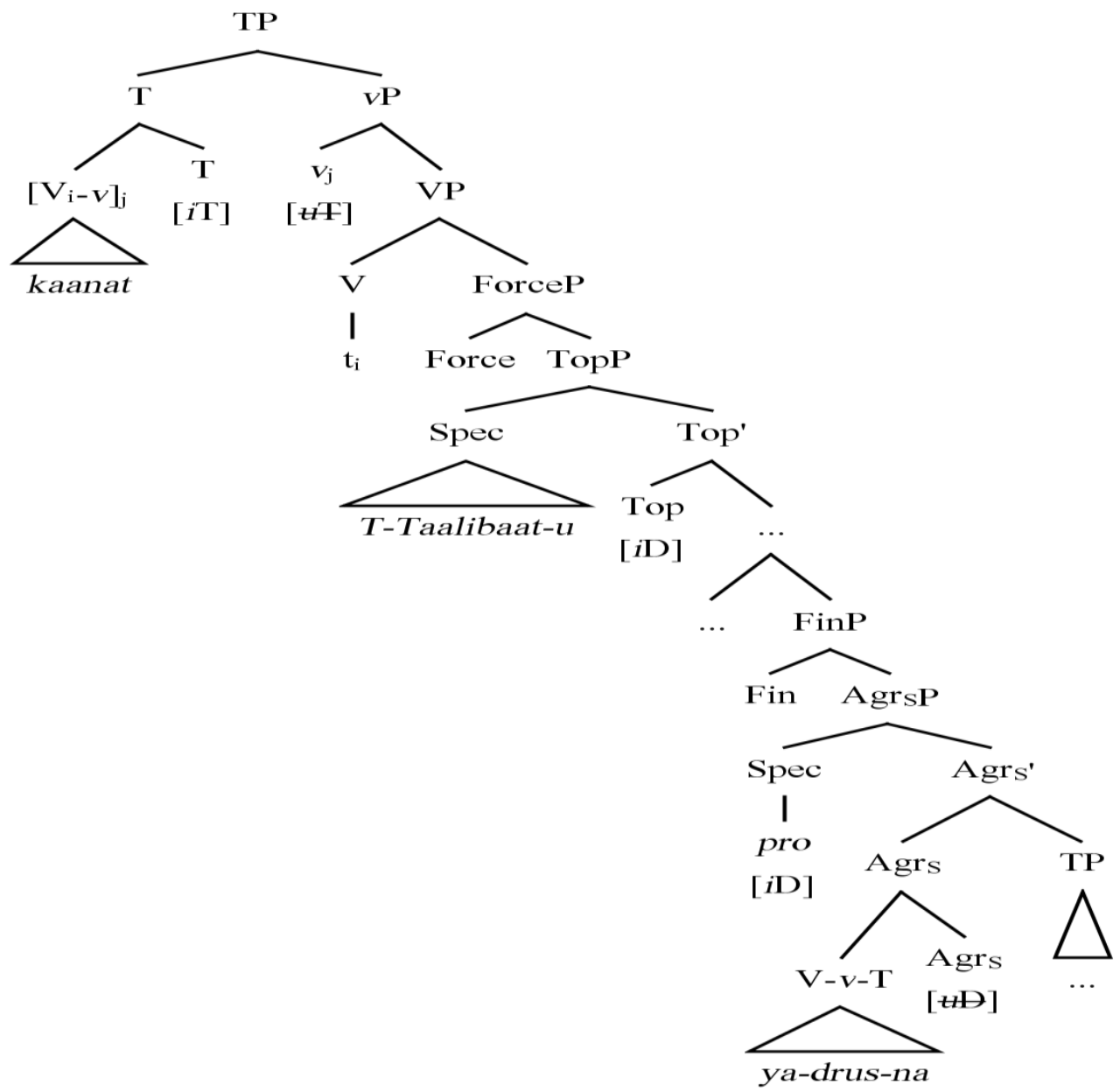

Contrary to Aoun et al.'s (2010) analysis where, as shown in (27b), the DP

TTaalibaatu 'the female students' is a 'subject' DP merged in (or raised to) a Spec position below T, the bi-clausal analysis of such sentences, as represented in (28), takes the DP TTaalibaatu to be a 'Topic' DP externally merged in [Spec, TopP] in the left periphery of the lower clause projecting as $\operatorname{Agr}_{S} \mathrm{P}$. The lexical verb (i.e., $\mathrm{V}$ adjoined to $v$ ) is triggered to raise to $\mathrm{T}$ in the lower clause as a consequence of the

\footnotetext{
${ }^{40}$ In section 3.3.4 below, I come back to the structure of IP in VSO word ordering and the assumption that the verb is in some low position in a split-CP system à la Rizzi (1997) where the highest head projection is Force. As Aoun et al. (2010: 70-71) acknowledge, the position of the auxiliary verb kaana in (28) is higher up to the left of TP/IP (see section 4.1.2.1). The same should be true of the negative auxiliary verb laysa inserted under Neg and raised to T, contrary to assumptions made in Benmammoun (2000b: 55) (see section 4.1.2.1). I return to a detailed analysis of such SA VSO biclausal sentences and their SVO counterparts in section 4.1.2.5.
} 
Agree/matching relation linking $v$ 's 'strong' $[u \mathrm{~T}]$ feature (not represented in (28)) to T's $[i \mathrm{~T}]$ feature. ${ }^{41}$ Similarly, the auxiliary verb kaanat in the higher clause of the biclausal structure (28) is merged in V. V moves to attach to $v$, and the complex V-v further raises up to T. As structure (28) shows, [Spec, TP] does not project in the absence of a subject raised out of $v \mathrm{P} / \mathrm{VP}$ of the matrix clause. Thus, irrespective of whether $[u \mathrm{~V}]$ is present on $\mathrm{T}$ or could be universally absent on $\mathrm{T}$ (see footnote 34 ), lexical (perfective and imperfective), verbs and auxiliary verbs would share the property of having a $[\mathrm{T}]$ feature. In both cases, this feature has to meet some licensing requirement.

As discussed earlier in this section, the complex $[\mathrm{V}-v]$ is phonetically empty in 'verbless' predicational sentences in SA where the auxiliary verb kaana is not in the Numeration. In this case, the V position is occupied by a null copula V. Thus, the SA sentence in (29a) below would have the representation in (29b):

(29) a. Ral-walad-u fii l-bayt-i

the-boy-NOM in the-house-GEN

'The boy is in the house.'

b.

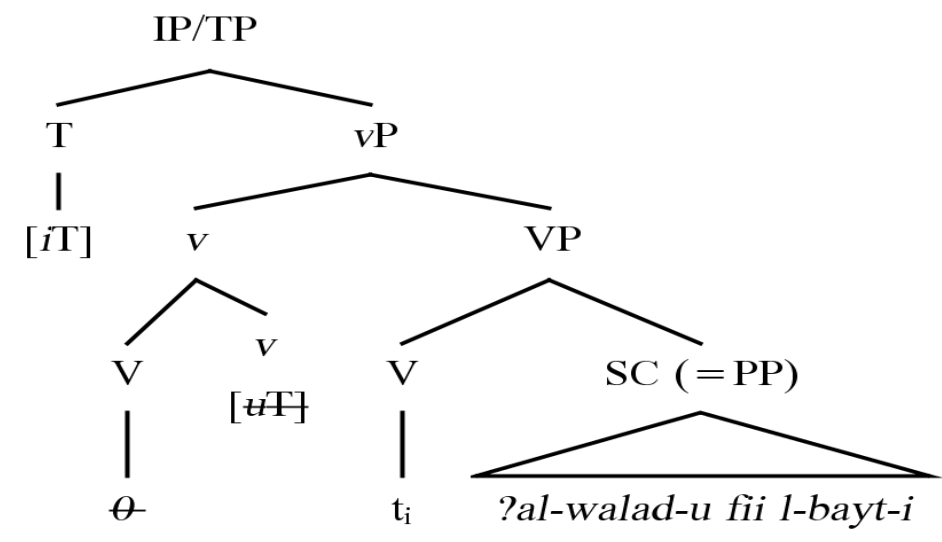

Although the auxiliary verb kaana is not in the Numeration (thus, yielding the present interpretation of (29a)), V still needs to adjoin to $v$ in (29b). Presumably, the [uT]feature of $v$ is valued as a consequence of a head-head agreement relation between $\mathrm{T}$ and $v$, as $[i \mathrm{~T}]$ on $\mathrm{T}$ matches and Agrees with $[u \mathrm{~T}]$ on $v$. However, as in (26) above,

\footnotetext{
${ }^{41}$ In view of the raising process of $\mathrm{V}$ to $v$, the $[u \mathrm{~T}]$-feature would actually be a feature of the functional element $v$ in accordance with Chomsky's $(2001,2004,2008)$ probe-goal-Agree system.
} 
$\operatorname{Agr}_{S}(\mathrm{P})$ projects above $\mathrm{T}$ in the derivation of (29a) and the DP Pal-walad- $u$ is realized as a Topic merged in [Spec, TopP] in the split-CP domain. A Referential pro is merged in $\left[\mathrm{Spec}, \operatorname{Agr}_{\mathrm{S}} \mathrm{P}\right]$ raised out of the Spec position of the small clause, as represented in (30) below:

(30)

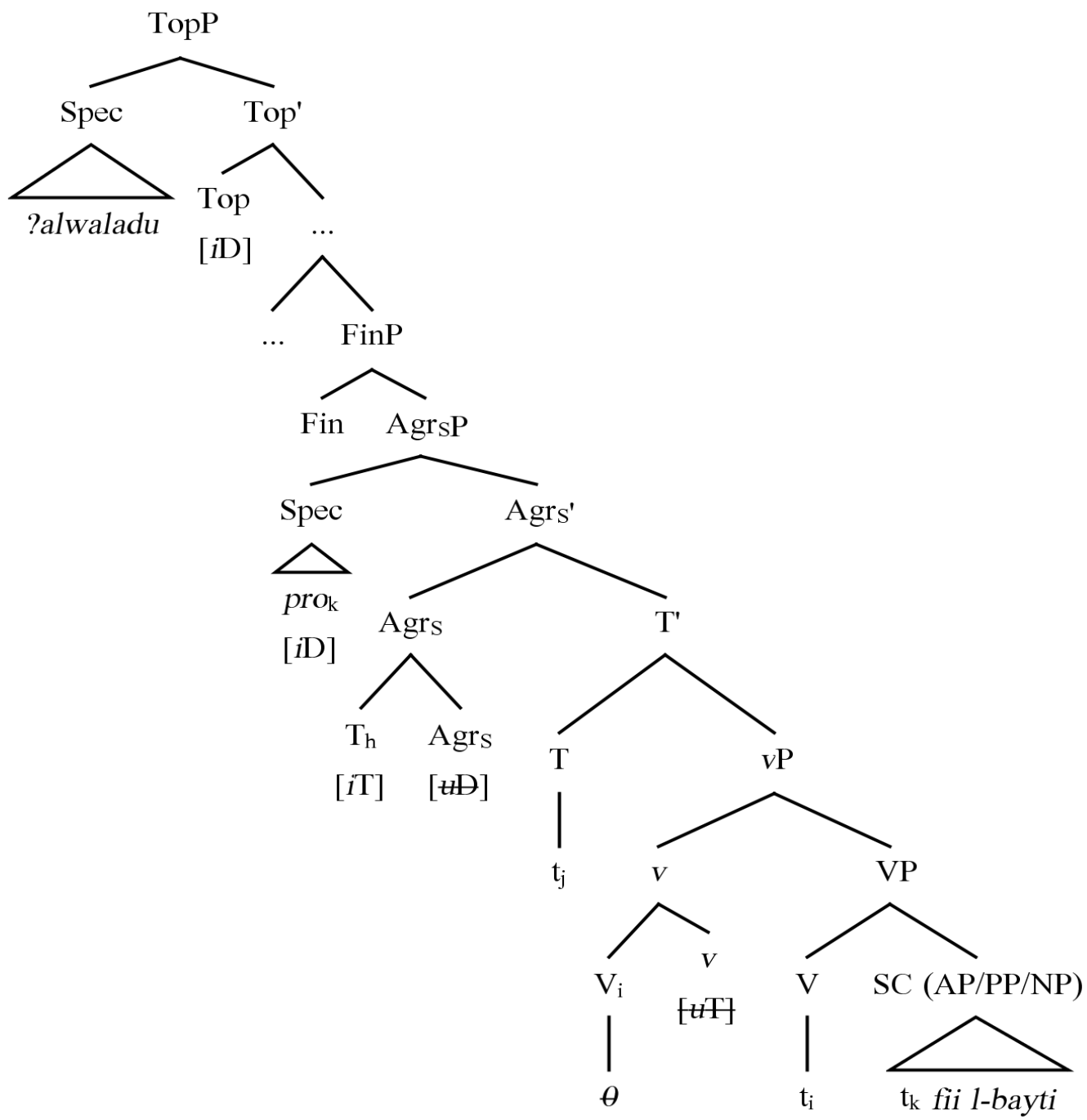

In contrast to (29a), the auxiliary verb kaana is included in the Numeration in the derivation of (31a) below:

(31) a. kaan-a Ral-walad-u fii l-bayt-i

PERF.be-3MS the-boy-NOM in the-house-GEN

'The boy is/was in the house.' 
b.

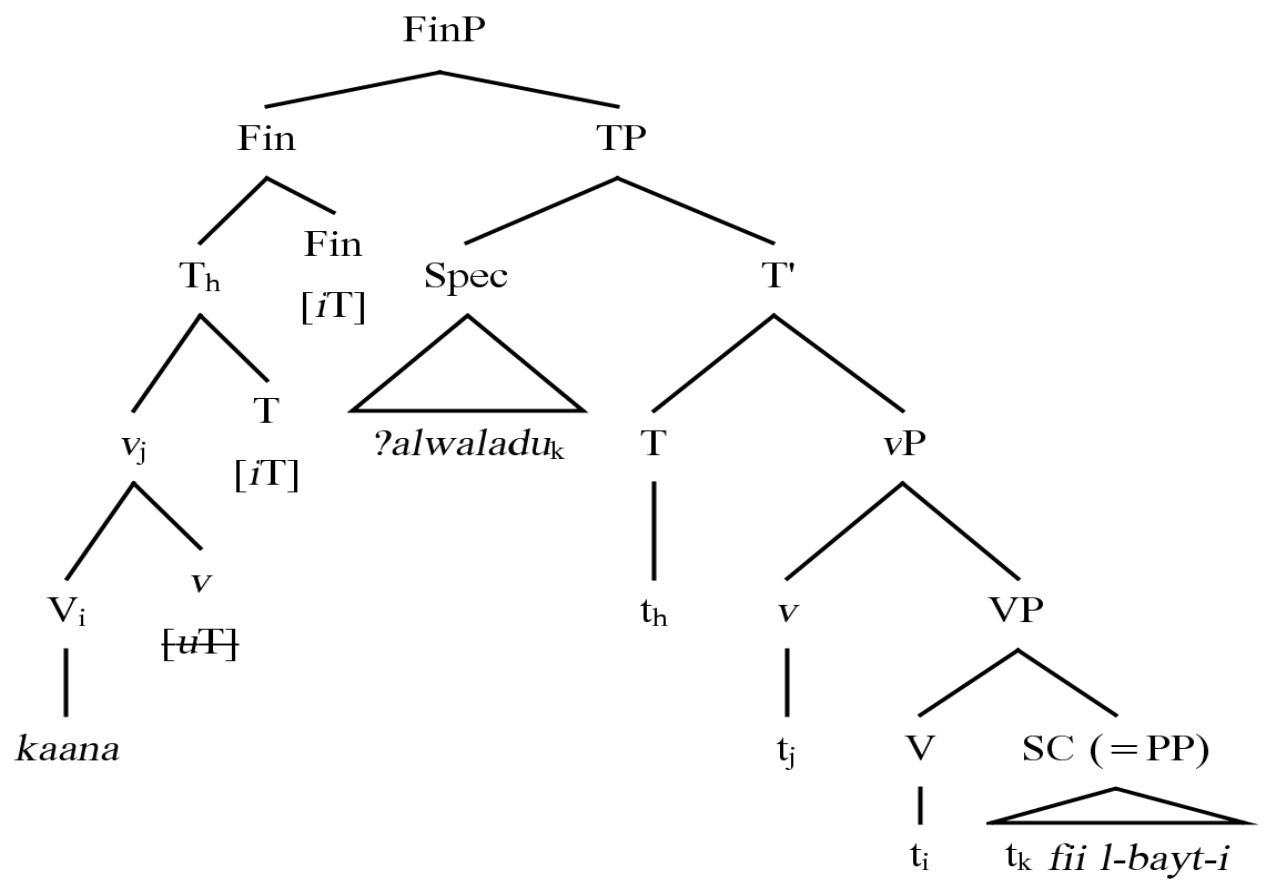

In (31b), the auxiliary verb kaana is merged in $\mathrm{V}$ and raised to $v$. The complex $\mathrm{V}-v$ raises further up to $\mathrm{T}$ (thus, yielding the past interpretation of (31a)). As in (29b), the $[u \mathrm{~T}]$-feature of $v$, in (31b), is presumably valued as a consequence of a head-head agreement relation between $\mathrm{T}$ and $v$, irrespective of the raising of $\mathrm{V}-v$ to $\mathrm{T}$. Since structure (31b) exhibits the VSO word ordering, the subject DP ?al-walad-u raises to [Spec, TP], and the complex V-v-T raises to Fin.

As already discussed above, Benmamoun (2000b, 2008) argues against a null copula V analysis of 'verbless' predicational sentences in SA. Benmamoun (2000b, 2008) uses evidence based on the syntactic properties of the auxiliary verb kaana, as in (33a), and other similar head elements (i.e., the Neg element laysa 'not', as in (33b), and the aspectual head element laazaala 'still', as in (33c)) to argue against the existence of a null copula $\mathrm{V}$ position in the so-called 'verbless' sentences. Thus, according to Benmamoun (2008), contrary to the 'verbless' sentence in (32) where no 'verbal' element is phonetically realized, the sentences in (33a-c) show evidence of a verbal (or verb-like) element that has the same syntactic behaviour in each case (adapted from Benmamoun 2008: 113-114): 
(32) r-rajul-u muSallim-un

the-man-NOM teacher-NOM

'The man is a teacher.'
(33) a. kaan-a r-rajul-u muSallim-an
PERF.be-3MS the-man-NOM teacher-ACC
'The man was a teacher.'

b. lays-a r-rajul-u muSallim-an

NEG-3MS the-man-NOM teacher-ACC

'The man is not a teacher.'
c. laazaal-a r-rajul-u muSallim-an
still-3MS teacher-NOM teacher-ACC
'The man is still a teacher.'

In contrast to (32), where the nominal predicate muSallim-un 'teacher-NOM' is marked for NOM Case, the nominal predicate muSallim-an 'teacher-ACC' in (33a-c) is marked for ACC Case. Benmamoun's (2000b, 2008) assumption is that the auxiliary verb kaana, the Neg element laysa, and the aspectual head laazaala in (33ac) 'assign' ACC Case to the nominal predicate muSallim-an within the SC.

However, in the probe-goal-Agree system adopted in this thesis, Case is actually not a property that is 'assigned' to nominal categories by verbs or by the functional elements verbs relate to in derivations (i.e., $v$ and T). Rather, Case is a byproduct of the Agree relations that obtain in the derivation of sentences. Thus, in (32) and (33a-c), as a by product of valuing $v$ 's $[u \mathrm{~T}]$-feature and T's $\varphi$-features, the NOM Case feature of the subject DP is also valued. As for the nominal predicate in (32), in the absence of a lexicalized $\mathrm{V}$ position that would lexicalize the relation between $\mathrm{T}$ and $v$ (as the two syntactic predication head nodes in IP (Rothstein 1983)), the nominal predicate gets a 'default' NOM Case. In (33a-c), the ACC Case feature of the nominal predicate muSallim-an might not actually be a Case feature at all, but some $[+\mathrm{F}]$ feature of the kind argued for by Shlonsky (2000) in relation to the ACC Case feature that linguists working in the Arabic linguistic tradition claim to be 'assigned' by the complementizer Pinna/Panna. As will be discussed in section 3.3.4 below, the complementizer Pinna/Panna is traditionally claimed to 'assign' ACC Case to the nominal DP that follows it in sentence structure. However, Shlonsky (2000) claims that the relevant feature that is a property of complementizer Pinna/Panna in SA is 
actually not an ACC Case feature, but a $[+\mathrm{F}]$ feature that has the "same phonetic realization as accusative Case" (p. 333).

Evidence that the feature that is relevant in the licensing of the nominal predicate in the sentences in (33) is not Case-related comes from grammaticality contrasts as in (34a) vs. (34b) (adapted from Alharbi 2000: 53) and in (35a) vs. (35b):

(34) a. Panta las-ta huwwa

you.MS NEG-2MS he

b. *Panta las-ta -hu you.MS NEG-2MS -him(CL)

'You are not him'

(35) a. Panta kun-ta huwwa

you.MS PERF.be-2MS he

b. *Panta kun-ta -hu

you.MS PERF.be-2MS -him(CL)

'You were him'

In (34a), as the English gloss shows, the pronominal predicate huwwa 'he' is a nominative pronoun. In (34b), the ACC Case-marked counterpart - $h u$ (phonetically realized as a clitic element) to the pronominal predicate huwwa in (34a) is ungrammatical. The same is true of the sentences in $(35 \mathrm{a}, \mathrm{b})$ where the copular verb used is kaana. To account for the ungrammaticality of (34b) (likewise for (35b)), Alharbi (2000: 54) assumes that: “... [A] predicate, either nominal or pronominal, does not receive/check case, but copy [sic] the features of the subject ... in Arabic". In a probe-goal-Agree system à la Chomsky (2001, 2004, 2008), this assumption amounts to saying that some essential Agree relation links the nominal/pronominal predicate to the subject of predication.

Having argued against the claim that the head elements kaana, laysa, laazaala and Pinna/Panna in SA have the property of 'assigning' Case, how can we characterize the $[+\mathrm{F}]$ feature that enters into the relation between the (null) copula $\mathrm{V}$ and the nominal/pronominal predicate in (32), (33a-c), (34a) and (35a)? Sticking to the cases in (33a-c) above and the apparent phonetic realization of the $[+\mathrm{F}]$ feature as an ACC Case feature, the nature of the $[+\mathrm{F}]$ feature appears to be distinct in the case of complementizer Pinna/Panna versus the case of the copular elements kaana, laysa and laazaala. I first discuss the nature the $[+\mathrm{F}]$ feature in relation to complementizer 
Pinna/Panna. Then, I discuss the nature the $[+\mathrm{F}]$ feature in sentences with the auxiliary kaana, the negation marker laysa and the aspectual head laazaala.

As far as the complementizer Pinna/Panna is concerned, the $[+\mathrm{F}]$ feature may turn out to be akin to some 'tense' property that interacts with the tense/modal properties of the sentences (namely, the T-features that are shared by Fin and T) as Pinna/Panna being merged in Force selects Fin, which itself selects T. A similar assumption would apply in relation to the auxiliary verb kaana, the negation marker laysa and the aspectual head laazaala, as these elments relate to $\mathrm{T}$ and the functional predicate head $v$ in terms of $\mathrm{T}$-features.

However, unlike the case of the $[+\mathrm{F}]$ feature of Pinna/ianna, the $[+\mathrm{F}]$ feature of the copular verbal elements kaana, laysa and laazaala has a bearing on syntactic predication, as it relates to the two syntactic predication head nodes $\mathrm{T}$ and $v$. Thus, irrespective of the phonetic realization of the $[+\mathrm{F}]$ feature of the copular verbal elements kaana, laysa and laazaala as ACC Case on the nominal predicate of the SC, I assume that this feature might be involved in dependency relations and in predication linking them to $\mathrm{T}$ for interpretation at the interface. ${ }^{42}$

Such an analysis would conform to Roberts' (2010a: 162) assumption that auxiliaries lack V-features but bear interpretable $\mathrm{T}$ features, (i.e., [iT] features). Roberts (2010a: 162) claims that $[i \mathrm{~T}]$ features trigger auxiliaries to incorporate into $\mathrm{T}$. In the probe-goal-Agree system proposed here, the auxiliary verb (e.g., kaana in structure (29b) and structure (31b) above) would first attach to $v$ and then raise further up to T. The same applies to the copular elements laysa and laazaala in the derivation of (33b) and (33c), respectively.

Roberts (2010a: 166) also proposes that it is on the basis of partial reprojection of $\mathrm{T}$ that the $\mathrm{T}$-features in $\mathrm{T}$ (and $\mathrm{C}$ ) attract verbs to raise in $\mathrm{V}$-raising configurations in Celtic languages and Germanic V2 languages. The same would be true of Semitic languages such as SA. In such V-raising languages, verbs are morpho-phonologically handled by the syntax as a compound element $[\mathrm{V}+\mathrm{T}]$ (V and a fully specified $\mathrm{T}$ ) (Roberts 2010a: 163-164). ${ }^{43}$ Such [V+T] compounds are formed in the Numeration

\footnotetext{
${ }^{42}$ The $[+\mathrm{F}]$ feature of laysa is presumably more a question of modality and 'focus' properties of NEG elements in SA, but it interacts with 'tense' as a consequence of dependency relations linking the $[+\mathrm{F}]$ feature to $\mathrm{V}-v$ and to $\mathrm{T}$ (and, ultimately, to $\mathrm{C}(=$ Fin)) (see section 4.1.2.4). I deal with a more detailed discussion of the feature structure of NEG elements in section 4.1.2.5.

${ }^{43}$ Roberts (2010a) does not mention infinitives in his account of V-to-T movement processes in a language like French.
} 
pre-syntactically and are inserted in the syntax as such (cf. the particular Distributed Morphology view of inflectional morphology as elaborated in Müller 2006). An important assumption of such a framework is that it is the T-features of the $[\mathrm{V}+\mathrm{T}]$ compound element that determine the formation of $\mathrm{TP}$, and that this formation does not depend on V-features (Roberts 2010a: 164). On this account, a form like yaktub(u) 'he writes' in SA (as discussed in section 2.1.2.3.3), is morphophonologically handled by the syntax as a compound element $[\mathrm{V}+\mathrm{T}]$ ( $\mathrm{V}$ and a fully specified T) where person, tense/aspect and the vocalic melody [...a........], expressing active voice in yaktub(u) 'he writes' (vs. [...u.......] as in yuktabu 'it is written' for the passive), are 'fused' (Fassi Fehri 1993, 2000).

Benmamoun's $(2000 \mathrm{~b}, 2008)$ analysis of verbal inflection rests on the main difference between the two forms of the verb in SA in that, whereas the imperfective has a basic prefixed tense morphology, the perfective has a basic suffixed tense morphology. ${ }^{44}$ This salient distinction between the two opposite affixation patterns as a representation of tense verb morphology in SA is the cornerstone of the difference between perfectivity/past tense and imperfectivity/present tense in Arabic. However, under the assumptions of the present analysis, whether the verb takes the perfective/past tense morphology or the imperfective/present tense morphology in SA is independent of the [V-v]-to-T raising process. Thus, the raising of $\mathrm{V}-v$ to $\mathrm{T}$ is necessarily involved in the derivation of verbal inflection in a $\mathrm{V}$-raising language like SA. ${ }^{45}$

\subsubsection{Summary}

As far as verbal inflection is concerned, it seems that the suffixal morphology of the imperfective form of SA verbs cannot be dissociated from the expression of the socalled 'mood morphology' in the subject agreement system of SA. In this conception, the suffixal morphology of the imperfective (encoding number/gender) is also an expression of 'mood' in the SA imperfective conjugation. As for the prefixal

\footnotetext{
${ }^{44}$ As the title of this subsection indicates, I have characterized Benmamoun's $(2000 \mathrm{~b}, 2008)$ analysis of verbal inflection as 'non-fusional' as opposed to Fassi Fehri's (1993, 2000, 2004, 2012) claims about the fusional charater of verbal inflection in SA where the imperfective is characterized as a 'Prefixed Tense' (PT), as against the perfective which is characterized as a 'Suffixed Tense' (ST).

${ }^{45}$ The problem of delimiting boundaries between morphemes (agreement, aspect/tense, mood) has also been observed in relation to other languages. For example, Tallerman (1997: 627) asserts that, in Welsh, “... there are no uniquely identifiable tense and agreement morphemes".
} 
conjugation of the imperfective, not only does it express imperfective aspect (which fuses with 'tense' according to Fassi Fehri 1993, 2000, 2004), but also person.

As for the salient distinction between the prefixation and suffixation patterns on SA verbs, it is a morpho-syntactic expression of how the perfective/past tense differs from the imperfective/present tense in SA. I have assumed that the suffixal (basically person, but also number/gender) tense agreement morphology of the perfective is not correlated with any differences in the derivation and representation of sentences when compared to the prefixal tense morphology (encoding person to the exclusion of number/gender) of the imperfective (contra Benmamoun 2000b, 2008). The derivation of both the perfective and imperfective forms of the verb in SA would involve the $\mathrm{V}$-to-T raising process.

\subsubsection{Mood and modality as two distinct categories in the SA subject-verb agreement system}

In relation to the representation of mood and modality in the grammar of SA, I will be arguing that modality is a separate category that projects its own node in SA sentence structure. ${ }^{46}$ Nevertheless, as R\&R (p. 130) point out, mood and modality are both tied up with the temporal status of an event and, by that token, are linked to the inflectional node I/T as the basis of the dependency relations that link the IP domain to the $\mathrm{CP}$ domain. In the presence of an $\mathrm{Agr}_{\mathrm{S}}$ node in clause structure, the C-T dependency has to be mediated by $\mathrm{Agr}_{\mathrm{S}}$ for 'interpretive' reasons (R\&R: 131), which have to do with the T-criterion and identification of the relevant features (namely, $\varphi$ features and/or D-features and the EPP) involved in the agreement relations established in the structure being derived (see section 3.1.2.2).

In Stowell's (2007) theory of tense, the role syntax plays in establishing such dependency relations on the basis of temporal/modal properties of syntactic structure and the temporal status of an event determines the semantics of tense. ${ }^{47}$ According to

\footnotetext{
${ }^{46}$ As already noted in Chapter Two (see footnote 23), Cinque (1999: 78) observes that there is crosslinguistic variation in the expression of mood and modality, either by the same category or by two different categories. The analysis of modality I will be defending is similar to Jouitteau's (2005) view that there is a Modal projection in the lower part of the split-CP domain above FinP (see section 4.2.2).

${ }^{47}$ According to R\&R (p. 142), the same is true for the tense/mood system of the clause that relates to the 'binding' relation that exists between $\mathrm{C}(=$ Fin) and $\mathrm{T}$ in a split-C system where Fin and T agree in some 'temporal properties' (Rizzi 1997: 283-284). In the system of probe-goal-Agree dependency relations adopted in this thesis, whether $\mathrm{Agr}_{\mathrm{S}}$ projects or not, the C-T dependency relation in the derivation of sentence structure is systematically a relation that binds $\mathrm{T}$ to $\mathrm{C}$ (= Fin) since in the cases
} 
Stowell (2007), the clause - i.e., TP - is headed by T(ense) "as the highest predicate in the clause" (Stowell 2007: 440). T takes VP as complement in syntactic structure. Semantically, the VP expresses the 'event time' (ET) in the clause. As R\&R point out, ET actually follows from the 'speech event time', which is given by C. Tense (i.e., the T node) provides the 'reference time' (RT). RT essentially mediates the relationship between $\mathrm{V}(\mathrm{P})$ and $\mathrm{C}$. In other terms, the temporal properties of a clause are derived from the interaction of $\mathrm{C}, \mathrm{T}$, and $\mathrm{V}$.

Given these assumptions about the 'interpretive' characteristics of the dependency relations that link verbs to the functional projections that syntactically (as much as semantically) bind them, the tense and agreement properties of verbs, including mood and aspect, interact with modality and negation markers for the expression of [+Past] or [-Past] events (see the examples in (39)-(41) below). ${ }^{48}$ Nevertheless, when no modality and/or negation markers are used in sentence structure in matrix clauses, the imperfective form of the verb can only express a [-Past] event, as in (37), in contrast to the perfective form of the verb which is used for [+Past] events, as in (36):

(36) a. ?aT-Taalib-u katab-a d-dars-a

the-student-NOM PERF.write-3MS the-lesson-ACC 'The student wrote the lesson.'

b. RaT-Tullaab-u katab-uu d-dars-a the-students-NOM PERF.write-3MP the-lesson-ACC 'The students wrote the lesson.'

(37) a. RaT-Taalib-u ya-ktub-u d-dars-a the-student-NOM 3-IMPERF.write-MS.IND the-lesson-ACC 'The student writes/is writing the lesson.'
b. RaT-Tullaab-u
ya-ktub-uuna
d-dars-a

the-students-NOM 3-IMPERF.write-MP.IND the-lesson-ACC

'The students write/are writing the lesson.'

\footnotetext{
where T-to- $\mathrm{Agr}_{\mathrm{S}}$ applies, $\mathrm{T}$ remains in a direct binding relation with the next higher head in sentence structure, i.e. Fin.

${ }^{48}$ In (36)-(42), I use the SVO word order since only in this case is subject-verb agreement marked for the plural when the subject DP is plural. In these examples, I only discuss the general characteristics of the data. I return to a more detailed analysis in the subsequent sections in this chapter for both the VSO and the SVO word orderings in Arabic.
} 
In (36a) and (36b), the sentences express an event in the past. The verb katabalkatabuu is used in the perfective aspect. In (37a) and (37b), the sentences express an event in the present. The verb kataba is used in the imperfective form namely, yaktubu/yaktubuuna.

In the presence of a modality or a negation marker in the sentence, the tense properties of the sentence depend on the relation between the modal or the negation marker and the verb in its perfective or imperfective form. To begin with, recall that Fassi Fehri $(1993,2004)$ points out that the future tense is not instantiated by an inflectional marker in the tense system of SA. In this respect, the expression of the future in SA is generally made by the use of the modal particle $s a$ - or its non-affixal variant sawfa in affirmative contexts as in (38) where the imperfective form of the verb must be used: ${ }^{49}$

(38) a. PaT-Taalib-u sa-ya-ktub-u

d-dars-a

the-student-NOM MOD(FUT)-3-IMPERF.write-MS.IND the-lesson-ACC 'The student will write the lesson.'

a'. ?aT-Taalib-u sawfa ya-ktub-u d-dars-a the-student-NOM MOD(FUT) 3-IMPERF.write-MS.IND the-lesson-ACC 'The student will write the lesson.'

b. PaT-Tullaab-u sa-ya-ktub-uuna d-dars-a the-students-NOM MOD(FUT)-3-IMPERF.write-MP.IND the-lesson-ACC 'The students will write the lesson.'

b'. PaT-Tullaab-u sawfa ya-ktub-uuna d-dars-a the-students-NOM MOD(FUT) 3-IMPERF.write-MP.IND the-lesson-ACC 'The students will write the lesson.'

The sentences in (38) assert that the subject of the sentence (i.e. the singular DP ?aTTaalib- $u$ 'the student' in (38a,a') and the plural DP ?aT-Tullaab- $u$ 'the students' in $\left.\left(38 b, b^{\prime}\right)\right)$ is certainly writing the lesson in the future.

Similarly to the modal particle $s a$-, the negation markers lam and lan are also exclusively used with the imperfective form of the SA verb. However, unlike the particle $s a$ - (and the modal marker qad, see sentences (41a,b) and (42a,b) below), lam and lan inflect for tense. As shown in examples $(39 a, b)$ and $(40 a, b)$ below, the use of negation as a preverbal marker - namely, lam for [+Past] events and lan for [-Past] events - determines the tense properties of the sentences:

\footnotetext{
${ }^{49}$ The expression of a future event can also be made by the use of other modal/tense markers as in the use of the negation marker lan (see examples $(40 \mathrm{a}, \mathrm{b})$ ) or in the use of the modal qad (see examples $(42 a, b))$.
} 
(39) a. PaT-Taalib-u lam yaktub-i d-dars-a (SA) the-student-NOM NEG.PAST 3.IMPERF.write-MS.JUSS the-lesson-ACC 'The student did not write the lesson.'

b. PaT-Tullaab-u lam yaktub-uu d-dars-a the-students-NOM NEG.PAST 3.IMPERF.write-MP.JUSS the-lesson-ACC 'The students did not write the lesson.'

(40) a. PaT-Taalib-u lan yaktub-a d-dars-a $(\mathrm{SA})$ the-student-NOM NEG.FUT 3.IMPERF.write.MS.SUBJ the-lesson-ACC 'The student will not write the lesson.'

b. PaT-Tullaab-u lan yaktub-uu d-dars-a the-students-NOM NEG.FUT 3.IMPERF.write-MP.SUBJ the-lesson-ACC 'The students will not write the lesson.'

In $(39 a, b)$, the negation marker lam encodes past tense. In this dependency relation between Neg and the tense/finiteness properties of sentences in SA, the imperfective verb that is selected by $\mathrm{T}$ in such an instance of negation has to be in the jussive. In $(40 \mathrm{a}, \mathrm{b})$, the negation marker lan encodes future tense. The imperfective verb related to such an instance of negation has to be in the subjunctive. Thus, the expression of tense in these sentences is the outcome of the interaction between the tense/aspect properties of the verb and the tense/modality properties of negative markers. This interaction is the basis via which the C-T dependency in sentence structure is interpreted at the interface.

In contrast to the modal particle $s a$ - and the negation markers lam and lan, the modal qad can be used with a perfective/[+Past] verbal event and with an imperfective/[-Past] verbal event, as exemplified in sentences (41a,b) and (42a,b), respectively:

(41) a. 2aT-Taalib-u qad katab-a d-dars-a the-student-NOM MOD PERF.write-3MS the-lesson-ACC 'The student has, indeed, written the lesson.'

b. RaT-Tullaab-u qad katab-uu d-dars-a the-students-NOM MOD PERF.write-3MP the-lesson-ACC 'The students have, indeed, written the lesson.'

(42) a. RaT-Taalib-u qad ya-ktub-u d-dars-a (SA) the-student-NOM MOD 3-IMPERF.write-MS.IND the-lesson-ACC 'The student might write the lesson.' 
b. ?aT-Tullaab-u qad ya-ktub-uuna d-dars-a the-students-NOM MOD 3.IMPERF.write-MP.IND the-lesson-ACC 'The students might write the lesson.'

Whereas in $(41 \mathrm{a}, \mathrm{b})$, the modal element qad is used in conjunction with the perfective form of the verb kataba, in $(42 \mathrm{a}, \mathrm{b})$ qad is used in conjunction with the imperfective form of the verb. The combination of modal qad with either perfectivity, as in $(41 \mathrm{a}, \mathrm{b})$, or imperfectivity, as in $(42 a, b)$, is correlated with a meaning difference in each pair of sentences depending on the aspect/tense of the verb. In $(41 \mathrm{a}, \mathrm{b})$, the modal qad is used with the perfective verb kataba/katab-uи to affirm that an event/action has taken place. In $(42 a, b)$, the modal qad is used with the imperfective verb yaktubu/yaktubuuna to express possibility of an event/action in the future. Thus, these particular examples are witness to the semantic information that tense/modal categories, such as qad in SA (in combination with the aspectual information encoded on verbal stems), contribute to how the C-T dependency is interpreted at the interface.

The sentences in (38)-(42) are important in showing that tense properties are not exclusive to verbs, but verbs share these properties with the items they are related to in sentence structure via the $\mathrm{C}-\mathrm{T}$ dependency. This sharing of tense/modality properties in the derivation of sentences in SA is a straightforward indication of the feature-sharing mechanism on the basis of which the C-T-dependency is identified. However, the interaction between pre-verbal modality and/or negation markers with tense is not always morphologically encoded on the items in question. Thus, whereas the negation markers lam and lan, in (39) and (40), carry a morphological indication that some tense property is encoded on them, the modal elements $s a$ - (and its variant sawfa), in (38), and qad, in (41) and (42), do not show any indication of such an encoding.

In the next section, I discuss the parametric character of the derivation of subject-verb agreement configurations in VSO structural representations in SA vs. such modern spoken Arabic dialects as TA, MA, LA in terms of the presence or absence of an $\mathrm{Agr}_{\mathrm{S}}$ node in IP.

\subsubsection{On the absence of Agrs in the SA VSO word order}

In light of Roberts' (2010a) typology of null-subject systems and the hypothesis that some languages would allow pro to be merged in [Spec, TP], I propose, in this section, that the process of pro-insertion in $\left[\mathrm{Spec}, \operatorname{Agr}_{S} \mathrm{P}\right]$ is absent in the derivation of 
VSO structural representations in SA. Thus, in SA VSO sentences, the $\mathrm{Agr}_{\mathrm{S}}$ node does not project, and the highest Spec position in the IP domain is [Spec, TP] to which the lexical subject is raised out of [Spec, $v \mathrm{P}]$ for the satisfaction of the EPP requirement. In this respect, the $\mathrm{C},\left(\mathrm{Agr}_{\mathrm{S}}\right)$, $\mathrm{T}$ structural dependency may be differently involved in the derivation of the relevant structures in the two languages of main concern (i.e., SA and TA).

It is in terms of the parametric availability of the intrinsic D-feature of $\mathrm{T}\left(-\mathrm{Agr}_{\mathrm{S}}\right)$ in SA and TA that a possible characterization of this difference in structural dependency relations in these typologically closely-related languages could be provided.

In this context, Ouhalla (1991) pointed out that the variation that exists between languages could be captured in terms of a theory of parameterization that involves the role functional categories play in movement processes (see also Roberts \& Holmberg 2010). Ouhalla's (1991: 4) aim in such an approach to parametric variation is to develop "a theory of typology which bases itself exclusively on the properties of functional categories". Thus, for Ouhalla (1991: 57), the I-elements TNS and AGR (which, Ouhalla 1991: 49 conceives as 'affixes' to which the verb - in Vraising languages - raises) are derived differently in sentence structure "along typological lines" and in response to the morphological properties of the affixes in question such as the property of 'NOM Case assignment'.

Ouhalla (1991: 57) takes it that, in Berber and Arabic, Tense (TNS) is higher than AGR. Ouhalla (1991: 117) further assumes that, in V-raising languages of the Berber/Arabic type, the VSO word order is derived by V-raising to AGR and then to TNS, leaving the subject behind in [Spec, VP]. Ouhalla (1991: 29) claims that a nominal pleonastic element (i.e., EXPL-pro) is inserted in [Spec, AGRP] to satisfy what he calls the Predication Requirement. Again, as reviewed in relation to Rothstein's (1983) syntactic predication analysis in section 3.1.1.2., Ouhalla's (1991) account of EXPL pro-insertion in the derivation of VSO structures in Berber and Arabic is in essence similar to Chomsky's (1982) EPP by virtue of which the structural subject position has to be occupied by some element in the syntax (see also section 2.1.1). In this case, the insertion of EXPL pro in the structural subject position (here, [Spec, AGRP]) is necessary for the licensing of the predicate (Ouhalla: 1991: 30). On this account, according to Ouhalla (1991: 126), the derivation of a VSO 
structure in Arabic/Berber looks like the tree structure in (43) below (adapted from Ouhalla's (35)):

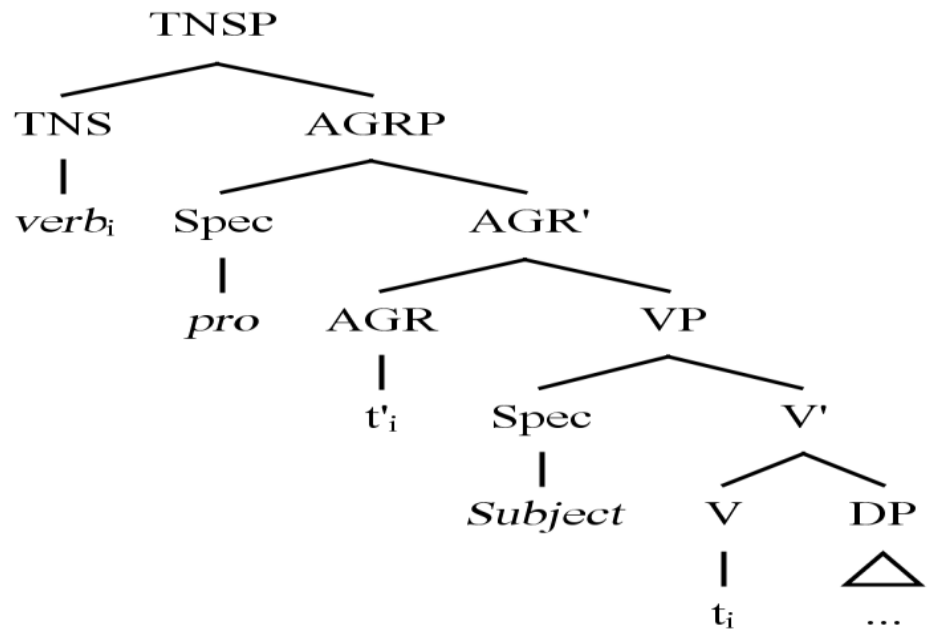

As for the derivation of the SVO word order in languages of the Berber/Arabic type, Ouhalla (1991: 117) assumes that "preverbal subjects are essentially topics basegenerated in Spec of TNSP and are coindexed with a resumptive pro which occupies the Spec of AGRP and acts as the subject of the sentence", as represented in (44) (adapted from Ouhalla's (22)):

(44)

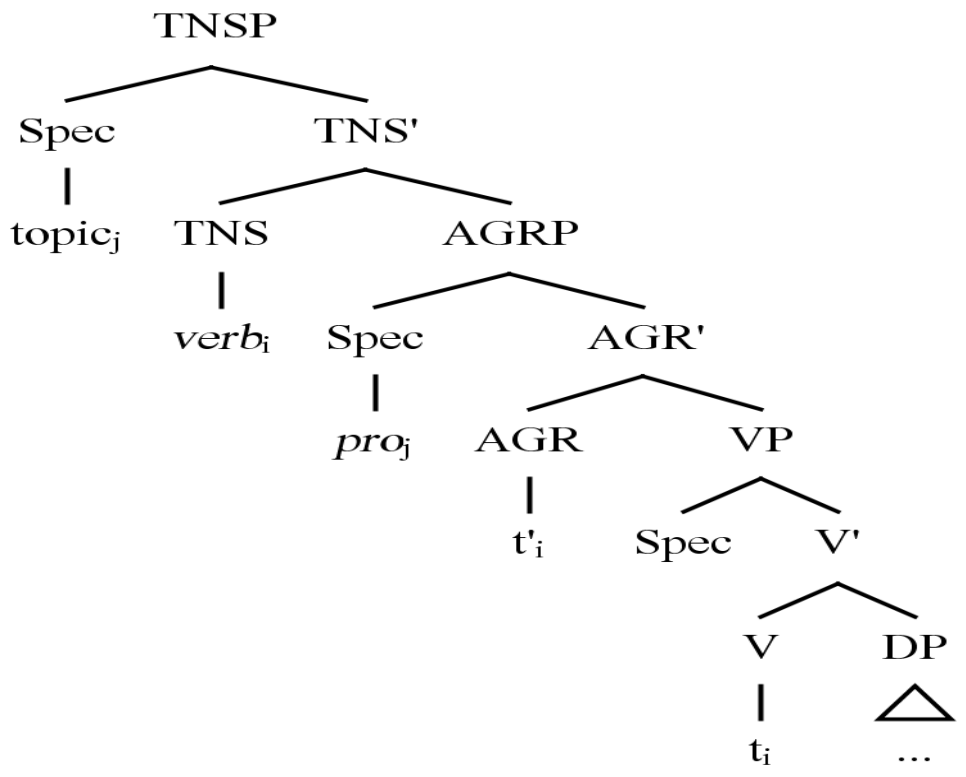


However, the assumption that T (TNS in Ouhalla's 1991 account) is higher in sentence structure than $\operatorname{Agr}_{S}$ (AGR in Ouhalla's 1991 account), in Berber/Arabic-type languages, is no longer necessary if the $\mathrm{T}$ node is assumed to raise overtly in the syntax to $\mathrm{Agr}_{\mathrm{S}}$ (Chomsky 1993: 195; Bobaljik \& Carnie 1996: 234, see section 3.1.1.3). On this account, V-raising targets the T node within the inflectional complex [T-Agr $]$ deriving the right order of inflectional morphemes. Thus, in a language like $\mathrm{SA}$, the agreement morpheme is clearly outside other morphemes forming the verb as exemplified by the imperfective aspect in SA (Fassi Fehri 1993: 84; see section 3.3.2.2 above for a morphological analysis of the affixes of imperfective verbs).

The assumption that the verb moves to the T node, which attaches to the $\operatorname{Agr}_{S}$ node that projects higher than the T node, is necessary in view of Fassi Fehri's (1993: 79-81) account of the adjunction structures verbs help create as they raise to the inflectional nodes projected higher in sentence structure (see footnote 26 in Chapter Two). The relevant structures are repeated below as (45b), for the imperfective verb in (45a), and (46b), for the perfective verb in (46a):

(45) a. y-aDrib-uuna

IMPERF.3-beat-MP.IND

'They beat/hit'

b.

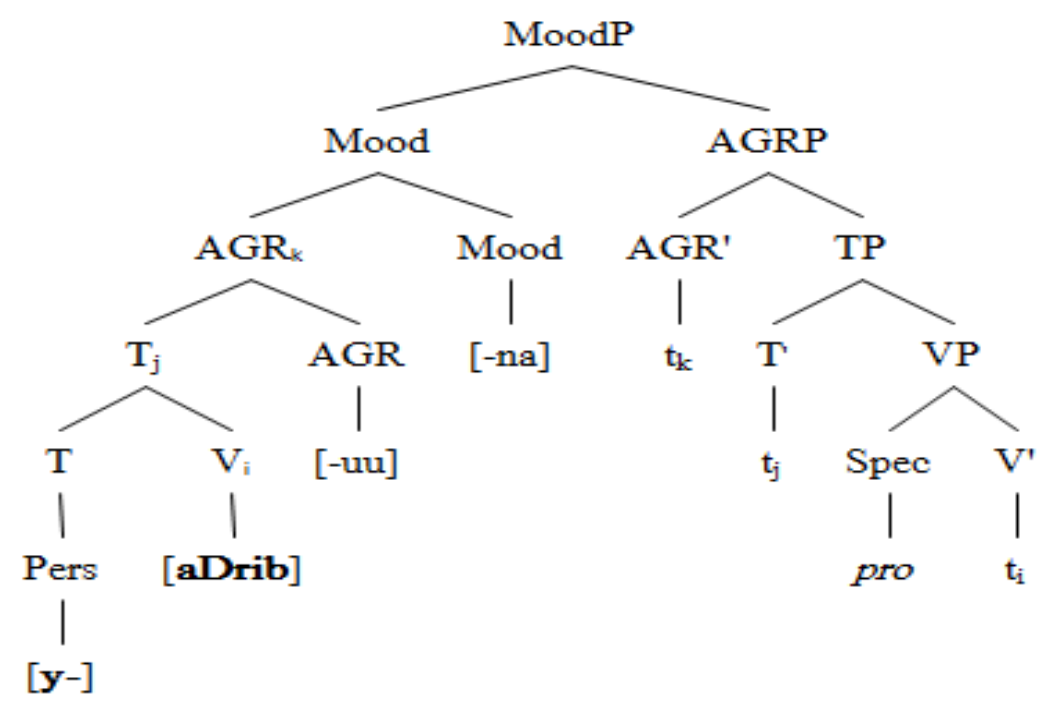


(46) a. katabt-umaa

PERF.write-2.M/FD

(SA)

'you (both of you) wrote'

b.

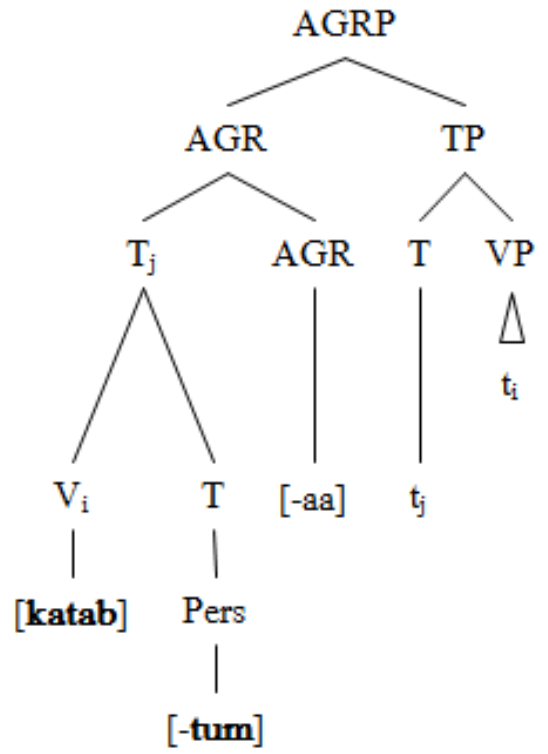

In these structures, the adjunction of the imperfective verb to the 'tense' prefix, for an expression like $y$-aDribuu-na 'they beat him' in (45b), involves a right-adjunction process, whereas the adjunction of the perfective verb to the 'agreement' suffix, for an expression like katabtum-aa 'you (both of you) wrote' in (46b), involves a leftadjunction process.

My assumption has been that since these head-to-head adjunction processes involve a combined morphological and syntactic distribution of the abstract features (representing the functional nodes projected in the syntax), the grammar analyses the raising of the verb as a 'substitution' rather than an 'adjunction' process. Thus, assuming that $\mathrm{T}$-to-Agr $\mathrm{A}$ is undeniably an adjunction process, $\mathrm{V}$-raising to $\mathrm{T}$, in the [T-Agrs] inflectional complex, head-substitutes for the abstract tense 'affix' rather than adjoins to it. This account is also in keeping with the assumption, made earlier, that the [Person-T] 'fused' morpheme is an integral part of the verbal stem raised to $\mathrm{T}^{50}$. Under this interpretation, structures $(45 \mathrm{~b})$ and (46b) would have representations as in (47) and (48), respectively:

\footnotetext{
${ }^{50}$ As briefly discussed in section 3.3.2.3, in Roberts' (2010a: 163-164) theory of the partial reprojection of $\mathrm{T}$ in $\mathrm{V}$-raising languages, $\mathrm{T}$ and $\mathrm{V}$ form a compound element in VP formed presyntactically. Roberts (2010a: 164) further assumes that V-to-T movement is actually "internal merger of $\mathrm{V}+\mathrm{T}$ with $\nu \mathrm{P}$ rather than ... external merger of $\mathrm{T}$ combined with internal merger of $\mathrm{V}$ to $\mathrm{T}$ ". On this view, $\mathrm{V}$ and $\mathrm{T}$ seem to be morphologically inseparable entities in $\mathrm{V}$-raising languages of the Celtic and Semitic type.
} 
(47)

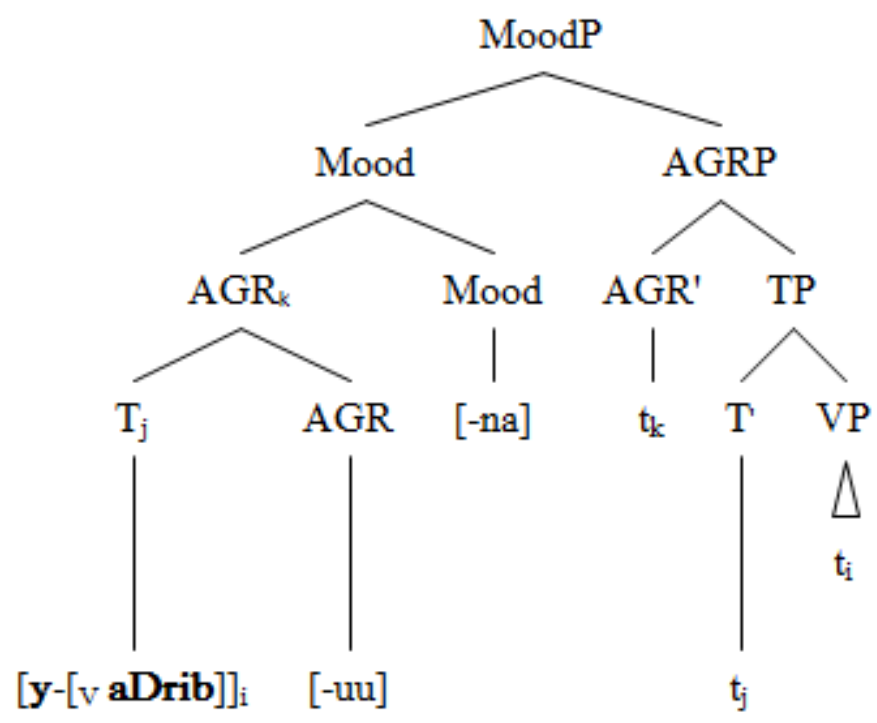

$(48)$

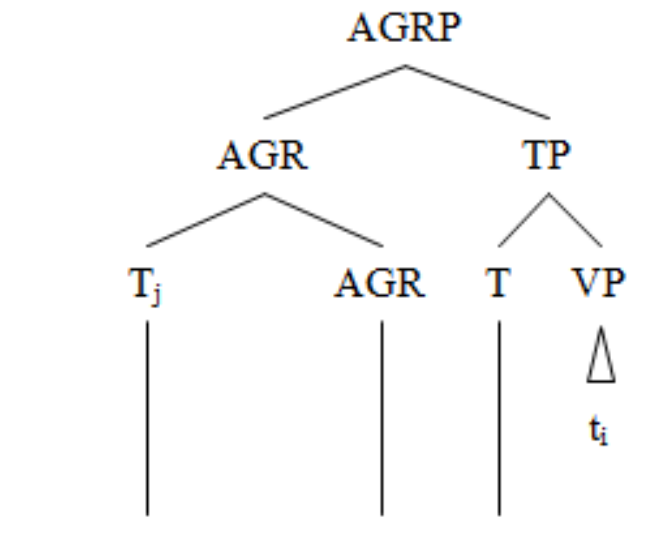

$[[\mathrm{v} \text { katab }]-\text { tum }]_{\mathrm{i}} \quad[-\mathrm{aa}] \quad \mathrm{t}_{\mathrm{j}}$

Irrespective of Ouhalla's (1991) parametric account of the different ordering possibilities of the inflectional morphemes in the IP domain that languages opt for, Ouhalla's $(117,126)$ assumption that Tense, in Berber/Arabic-type languages, is the highest node in the IP domain may, after all, be correct as far as the derivation of SA VSO word order is concerned. In this context, if the right order of the two inflectional nodes $\mathrm{T}$ and $\mathrm{Agr}_{\mathrm{S}}$ - in the cases where both of them project - is universally determined by the grammar of languages, opting for not projecting $\operatorname{Agr}_{\mathrm{S}}$ in VSO word order in SA (see section 2.3.1, in particular) means that the projection of the IP level of sentences proceeds first by merging the structure of $v \mathrm{P}$ with $\mathrm{T}^{51}$ The next step in the derivation of the VSO structure involves the application of the $\mathrm{V}-\mathrm{v} \rightarrow \mathrm{T}$ raising

\footnotetext{
${ }^{51} \mathrm{I}$ will continue to represent the raising of $\mathrm{V}$ (to which $v$ attaches) to $\mathrm{T}$ as an adjunction structure for the sake of convenience.
} 
process, and the addition of a Spec position to T', which then maximally projects as TP. In view of the option of not projecting an $\mathrm{Agr}_{\mathrm{S}}$ node above $\mathrm{T}$ in VSO structural configurations in SA, $\mathrm{T}$ is the highest functional projection in the IP domain that fulfils the requirement of syntactic predication as in Rothstein (1983). Thus, the computation proceeds by applying DP raising to [Spec, TP] to satisfy the EPP. These processes are represented in intermediate structure (49) below:

(49) The SA VSO word order (TP Phase)

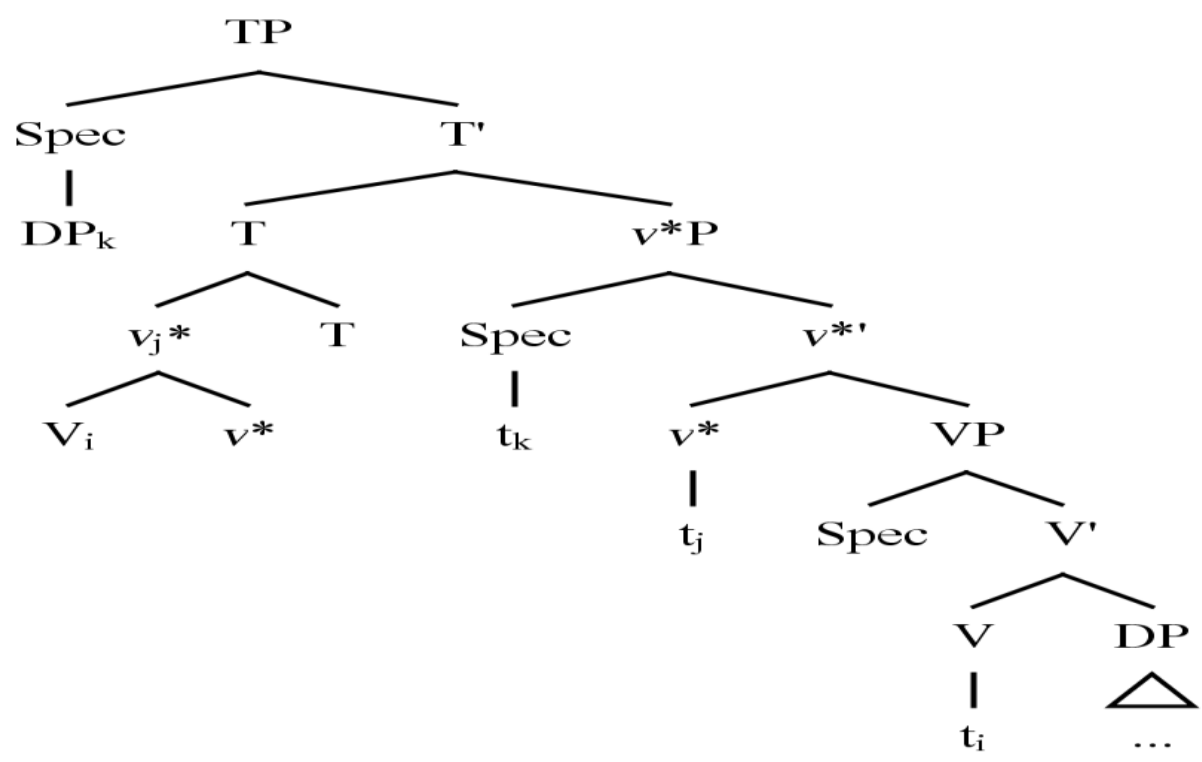

As already discussed (see section 2.3.1), the verb (actually, the [V-v-T] inflectional complex) raises further up to attach to a node $F$ in the split $\mathrm{CP}$ domain, thus bypassing the subject in [Spec, TP], as represented in (50) below: 
(50) The SA VSO word order (CP Phase)

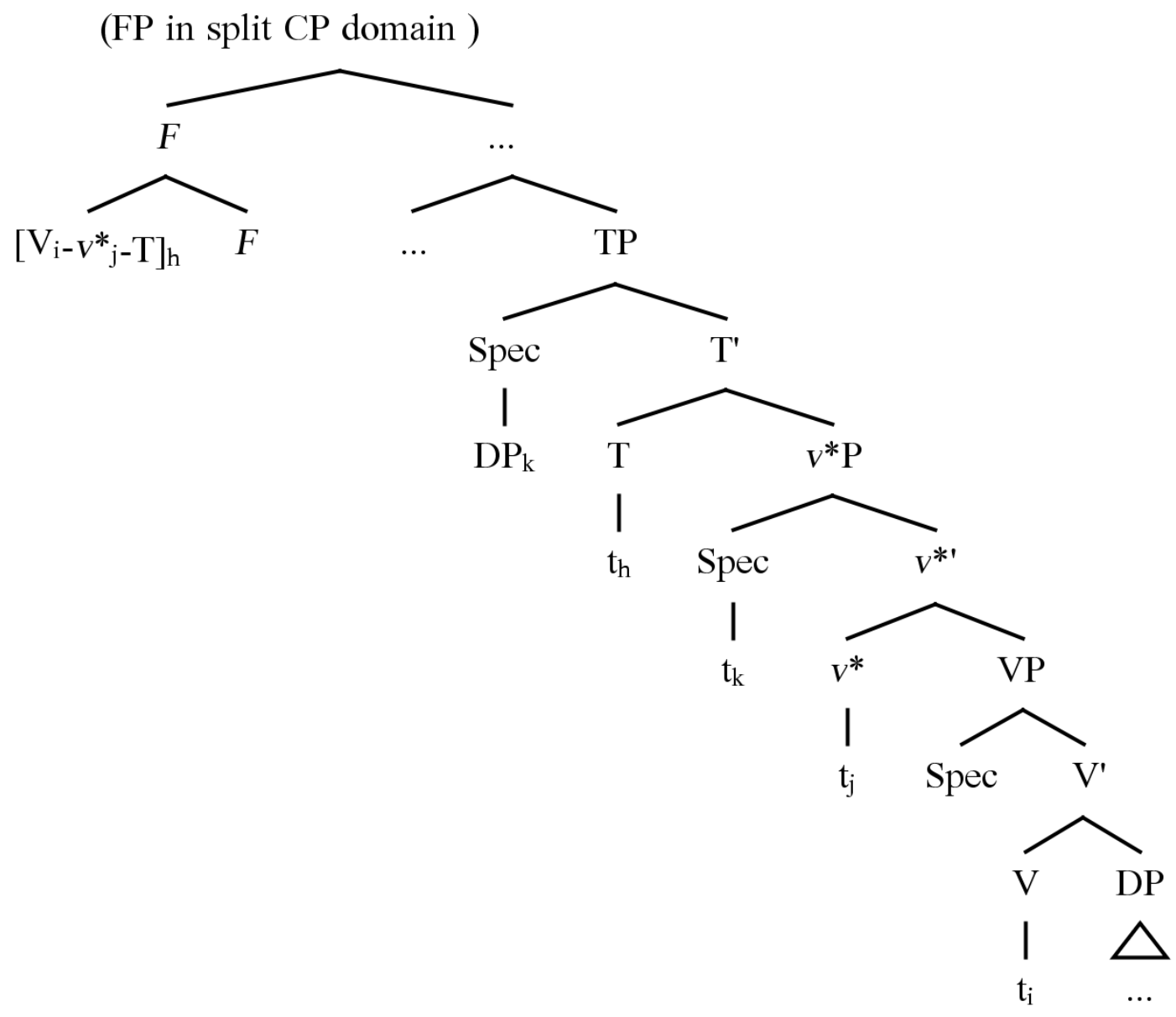

In contrast, in the derivation of a VSO structure in TA, when T is introduced in sentence structure it raises to $\mathrm{Agr}_{\mathrm{S}}$ forming the inflectional complex [Agrs $\mathrm{T}-\mathrm{Agr}_{\mathrm{S}}$ ]. As discussed in section 3.1.1.3, Agr $_{S}$ cannot project in the syntax unless it also projects a Spec position. Thus, to derive the VSO word order in TA two head-raising processes have to be posited to occur separately, after which a Spec position is added to the highest functional projection at the IP level - i.e., as T raises to $\mathrm{Agr}_{\mathrm{S}}, \mathrm{V}-\mathrm{v}$ raises to $\mathrm{T}$. In this case, since it is the complex $\mathrm{T}-\mathrm{Agr}_{\mathrm{S}}$ that realizes the highest functional projection in the derived VSO structure, the addition of a Spec position to T' does not apply. Thus, after the head raising $\mathrm{V}-\mathrm{v} \rightarrow \mathrm{T}$ and $\mathrm{T} \rightarrow \mathrm{Agr}_{\mathrm{s}}$ processes have applied (see Bobaljik \& Carnie 1996), the computation proceeds by merging a Spec position to $\operatorname{Agr}_{S} \mathrm{P}$ to derive the highest level of IP. To finally derive the VSO structure in TA, the computation proceeds by merging an EXPL pro in $\left[\mathrm{Spec}, \operatorname{Agr}_{\mathrm{S}} \mathrm{P}\right]$, as represented in (51) below: 


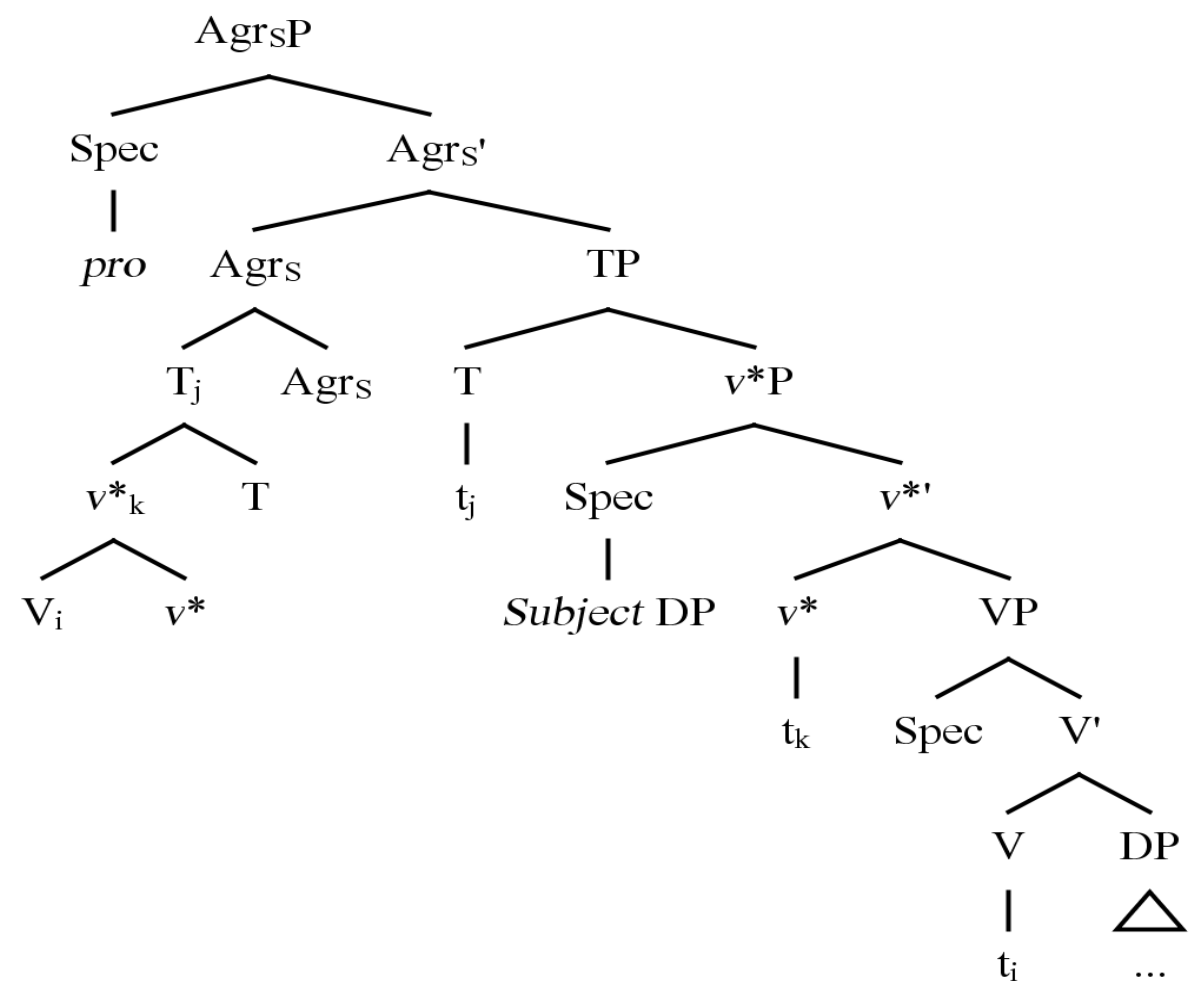

The raising processes discussed in relation to structures (49-51) show that the [AgrS T-Agr ] inflectional complex could be conceived as the 'locus' of agreement relations and parametric variation in the null-subject languages exhibiting the SVO word order as a possible alternative to the VSO word order. In this respect, raising the verb to $\mathrm{T}-\mathrm{Agr}_{\mathrm{S}}$ and EXPL pro-insertion in $\left[\mathrm{Spec}, \mathrm{Agr}_{\mathrm{S}} \mathrm{P}\right]$, in TA, is a plausible parametric account of the essential difference in the representation of the IP domain in TA vs. SA. The assumption that the projection of $\mathrm{Agr}_{\mathrm{S}}$ in clause structure requires merging a Spec position to form $\operatorname{Agr}_{S} \mathrm{P}$ in the IP domain conforms to Rizzi's (1982) assumptions about the 'strength' of Agr in the IP domain. In this connection, it is due to some defining property of the Agr node (i.e., Agrs's D-feature under the assumptions of the present analysis) that both the head raising processes $\mathrm{V}-v \rightarrow \mathrm{T}$ and $\mathrm{T} \rightarrow \mathrm{Agr}_{\mathrm{S}}$, along with the EXPL-pro insertion process, define the strong Phase in the IP domain in TA and, by that token, 'rich' subject-verb agreement arises in such VSO configurations. The same is true for the kind of subject-verb agreement that obtains in there-constructions in English in the different contexts reviewed above; (see the summary of these contexts in Table 3.2). The parallels between EXPL-associate pairs 
in English and their counterparts in TA, as reviewed in section 3.2.1.1, are evidence that these pairs do indeed exist and that TA VSO sentences are parameterically different from their counterpart VSO sentences in SA.

Thus, with respect to the process of EXPL-insertion in the IP domain - as discussed earlier in relation to the derivation of subject wh-questions in TA (see section 3.2.1.1) -, TA is closer to English than to SA. Both TA and English have recourse to EXPL-insertion, which operates in the IP domain to derive EXPLassociate pairs. In these constructions, the EXPL is finally merged - via first or second Merge - in $\left[\mathrm{Spec}, \operatorname{Agr}_{S} \mathrm{P}\right]$ to value a $[u \mathrm{D}]$ feature on the inflectional complex [T-Agr $]$, and to satisfy the EPP at the highest level of sentence structure in the IP domain where Spell Out applies. ${ }^{52}$

In relation to the feature-valuation procedure in general, the parametric choice of not projecting $\mathrm{Agr}_{\mathrm{S}}$ in SA VSO word order (where 'rich' subject-verb agreement fails to obtain) points to a major difference in the subject-verb agreement configurations at the IP level in SA and TA. Since in SA VSO sentences subject-verb agreement operates with $\mathrm{T}, \mathrm{T}$ would lack the right feature complex for determining the full set of the agreement features involved in the Agree relation at the IP level - by definition, a 'weak' Phase' since (rich) agreement cannot be determined at that Phase. Nevertheless, similar to the case of $\mathrm{T}_{\mathrm{DEF}}$ in English embedded infinitives in raising contexts, some kind of subject-verb agreement already obtains at this stage of the derivation (i.e., $\left(3^{\text {rd }}\right)$ person agreement, which is not morphologically manifested in English). Thus, similar to the case of $\mathrm{T}_{\mathrm{DEF}}$ in English embedded raising contexts, $\mathrm{I}$ propose that the parametric option of not projecting $\mathrm{Agr}_{\mathrm{S}}$, in SA, sets the right agreement configuration for a 'defective' $\mathrm{T}$ in that language, whereby $\mathrm{T}$ is no longer selected by $\mathrm{C}$ in conjunction with an $\mathrm{Agr}_{\mathrm{S}}$ node, as it is in TA. Nevertheless, as already mentioned, EPP satisfaction is operative at this 'weak' Phase of the derivation just as it is in English embedded contexts in the there-constructions discussed in section 3.2.1.1 (see also section 2.3.2).

As a consequence of the non-projection of $\mathrm{Agr}_{\mathrm{S}}$ in VSO word order, SA exhibits a 'poor' subject-verb agreement pattern, and EXPL pro fails to be represented in the IP domain. Instead, pro can be merged in the $\mathrm{CP}$ domain where it

\footnotetext{
${ }^{52}$ In Jouini (2012), I further analyze the structure of dependency relations in TA, not only within IP, but also within DP, and show that the derivation of both domains is subject to the same dependency relations.
} 
necessarily gets a phonological matrix whereby it is realized as an EXPL clitic pronoun - $h u$. EXPL - $h u$ cliticizes to the matrix declarative complementizer head Pinna 'that' (as shown in (52) below) in the cases where matrix Pinna is merged in sentence structure. I assume that the EXPL clitic $-h u$ is first merged in the Spec position of a Top node projected to the left of the verb under some $F$ node in the split CP à la Rizzi (1997). Then the EXPL clitic cliticizes to the complementizer head Pinna. These processes are represented in the structure in (53) for the sentence in (52):

(52) Pinna-hu Rakala l-Rawalaad-u khubz-an

that-EXPL(ACC) ate.3MS the-boys-NOM bread-ACC

Lit: 'The boys, indeed, ate some bread /It is indeed the case that the boys ate some bread'

(53) The SA VSO word order: V-raising to CP

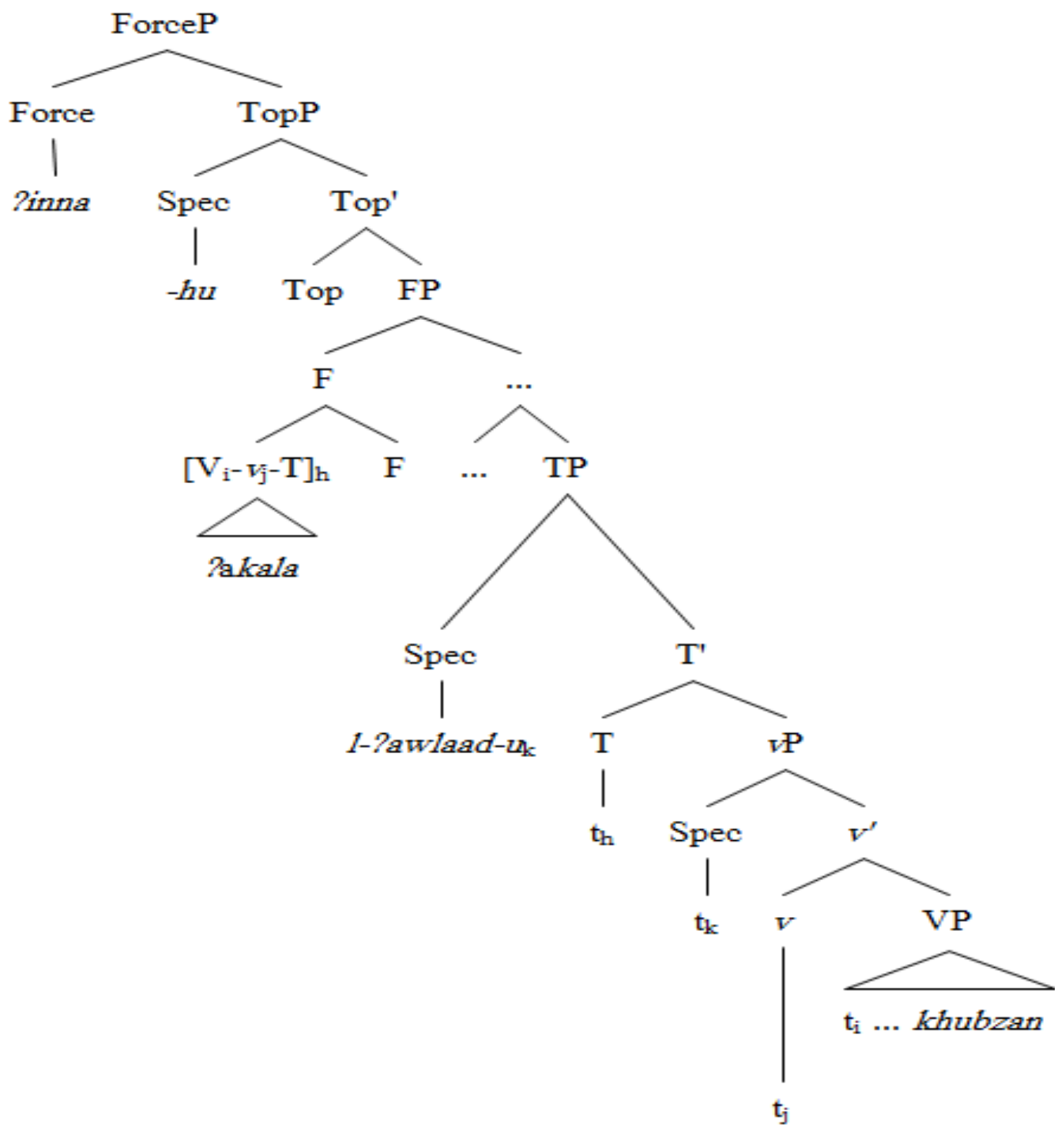


In (53), the process whereby EXPL - $h u$ is phonetically realized in [Spec, TopP] is bound to the realization of the C node as Pinna 'that' signalling that the sentence is declarative in clausal type. In the VSO structure (53), the position to which the verb ends up attached denotes the strong Phase where features get spelled out after being 'identified' at the interface. At that stage of the derivation, Agree relations apply and link the highest $\mathrm{C}$ node (Force, under which Pinna is inserted), to Top (from which Spec - $h u$ cliticizes to Pinna), and to the functional node $F$ (to which the inflectional complex $[\mathrm{V}-v-\mathrm{T}]$ is raised). ${ }^{53}$

Thus, in the derivation of the SA VSO sentence (52), as represented in (53), the subject DP raised to [Spec, TP] defines a lower 'weak' Phase. Nevertheless, the fact that the DP $l$-Pawlaad- $u$ is marked for NOM Case is confirmation of the assumption that some kind of subject-verb agreement has already taken place at the level of IP before the inflectional complex [V-v-T] raises to CP. Presumably, the raising of $[\mathrm{V}-v-\mathrm{T}]$ to the functional node $F$ in $\mathrm{CP}$ could be tied up to the feature structure of functional categories in the $\mathrm{CP}$ domain in SA and the probe-goal-Agree system of dependency relationships the raising of $[\mathrm{V}-v-\mathrm{T}]$ to $F$ helps create (see section 4.1.2.1).

I first introduced the internal structure of the split CP (Rizzi 1997) in section 2.1.2.3.2 in connection with the assumption that initial DPs in SA are Topics basegenerated in the Spec position of a TopP maximal projection in the CP domain. I further consider here the split-CP system in relation to the nature of the $F$ node the inflectional complex [V-v-T] raises to. According to Rizzi (1997), the CP domain is more elaborate than was previously thought in that it takes the form of a ForceFiniteness system in interaction with a Topic-Focus system (although some languages do not seem to make use of all the available positions, e.g., French (Rizzi 1997: 286)). The structure of CP in this conception takes the form of a Topic-Focus system sandwiched in between ForceP and FinitenessP (=FinP), as represented in (54) below:

\footnotetext{
${ }^{53}$ Rizzi (1997: 286) alludes to the analogous status of the Top node, in the CP domain, and the Agr node, in the IP domain, in relation to predication. I leave the status of the node(s) the inflectional complex [V-v-T] raises to open for the moment returning to a detailed discussion of the different raising processes that link the CP domain to the IP domain, à la Rizzi (1997), in Chapter Four.
} 


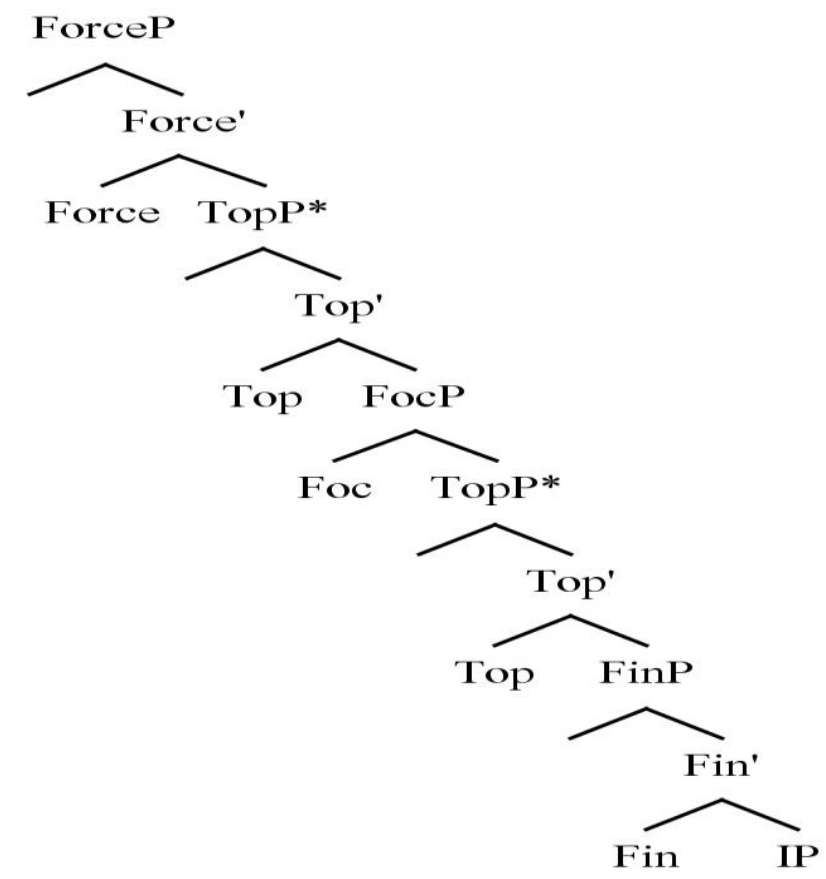

Structure (54) shows that not only can topicalized elements, merged in Top(ic)P, precede or follow focused elements, merged in Foc(us)P, but the Topic projections can be recursive as indicated by the asterisk on both TopP* projections in (54). Another characteristic of this structure is that, according to the different kinds of leftperipheral elements that each functional node in the split-CP domain harbours, each maximal projection may have a potential Spec position. ${ }^{54}$

Rizzi (1997: 284) assumes that languages vary in the extent to which they 'replicate' IP information in the CP domain, particularly in relation to mood, modal and finiteness elements. As already discussed above in connection with the lexicalized C element Pinna in structure (53), the Force node in the split-CP domain bears the function of signalling 'clausal type' (Rizzi 1997: 283). In SA, the complementizer Pinna (or its variants ?an and Panna) would naturally be located under Force indicating that the derived sentence is declarative in clause type.

As also discussed in relation to structure (53), I assume that the so-called 'lexicalized expletive' (Mohammad 1989; Shlonsky 2000) or 'expletive topic' (Fassi Fehri 1993), which cliticizes onto its host to the left, is located in [Spec, TopP]. If the parallelism Rizzi (1997: 286) suggests exists between Top (in the CP domain) and

\footnotetext{
${ }^{54} \mathrm{I}$ return to a revised system of the split-CP domain in section 4.1.2.1.
} 
$\operatorname{Agr}_{S}$ (in the IP domain) is correct, then the claim that the EXPL clitic - $h u$ is located in [Spec, TopP] finds further support in that both the lexicalized version of EXPL pro and the null EXPL itself are merged in the Spec position of a node that is related to the argument DP of the predication relation - i.e., to the subject DP of the clause in subject-verb agreement configurations.

The lexicalized EXPL element not only encodes person but could also, optionally, encode gender. Ouhalla (1991: 125-126) and Fassi Fehri (1993: 38-39) cite the following examples:
(55) a. Pinna-hu zaara-nii thalath-u shaa@iraat-in that-EXPL.3MS visited.3MS-me three.M-NOM poets.F-GEN Literally: 'It visited me three poets'
b. Pinna-ha zaarat-nii thalath-u shaafiraat-in that-EXPL.3FS visited.3FS-me three.M-NOM poets.F-GEN Literally: 'It visited me three poets'

In $(55 a, b)$, the subject is feminine notwithstanding the fact that the numeral thalath $u$ is marked for masculine gender (as opposed to thalathat- $u$ in which the $-t$ morpheme expresses feminine gender). Thus, although the subject is feminine, the gender expression on the DP thalath- $u$ shaaSiraat-in does not seem to be a determining factor in subject-verb agreement at the IP level. This agreement configuration seems to point to the fact that the verb (which I am assuming is in the CP domain) seems to obligatorily agree with the EXPL clitic (i.e., $-h u$ in (55a) and $-h a$ in (55b)) rather than with the lexical subject. ${ }^{55}$ Nevertheless, due to the absence of a fully realized $\mathrm{Agr}_{\mathrm{S}}$ node in the derivation of SA VSO sentences (as represented, for example, in (53) above), this agreement configuration is, presumably, not an instance of $\varphi$-feature agreement that is directly linked to the satisfaction of the EPP in the IP domain. Rather, as I will discuss in Chapter Four, this agreement configuration seems to be an instance of the identification of a D-feature on the Top node in the CP domain in relation to the T-features of the Fin and T. In such instances, feature licensing is more a question of 'identification' rather than feature valuation (cf. R\&R, Ouhalla 1993).

\footnotetext{
${ }^{55}$ See, however, the sentences in (59) below for some seemingly recalcitrant cases.
} 
The same is true of cases where the subject is a simple singular feminine DP, as shown in (56) below:
(56) a. Pinna-hu zaara-nii shaalirat-un that-EXPL.3MS visited.3MS-me poet.F-NOM
Literally: 'It visited me a poet'
b. Pinna-ha zaarat-nii shaaYiraat-un that-EXPL.3FS visited.3FS-me poet.F-NOM Literally: 'It visited me a poet'

As was the case in (55), the lexicalized EXPL cliticized onto Pinna, in (56), again optionally encodes gender. Again, the verb seems to obligatorily agree with the clitic rather than with the subject DP. As discussed in the previous paragraph, I assume that this agreement configuration is not an instance of $\varphi$-feature agreement related to the satisfaction of the EPP in the IP domain.

Fassi Fehri (1992: 134-135) provides other examples of optional gender agreement in SA with [- HUMAN] DPs (the sentences in (57) below) and with, the so-called, 'broken' plural nominals that are formed by altering the structure of the singular DP (the sentences in (58) and (59)). These plural DPs are feminine in gender: ${ }^{56}$
(57) a. dhahaba
saaYat-un mina 1-layl-i has passed.3MS hour.INDEF-NOM of the-night-GEN 'An hour of the night has passed'
b. dhahaba-t saaYat-un mina l-layl-i has passed.3FS hour.INDEF-NOM of the-night-GEN 'An hour of the night has passed'
(58) a. jaa?a l-kilaab-u came.3MS the-dogs-NOM 'The dogs came'
b. jaa?a-t l-kilaab-u came.3FS the-dogs-NOM 'The dogs came'

\footnotetext{
${ }^{56}$ According to Roman (1990: 46-48) this instance of 'feminine Gender' marking, which gives rise to 'feminine pseudo-agreement' between the verb and the subject DP, has "mistakenly" been analysed as 'third person feminine' (see footnote 89, p. 46). This observation is relevant in the current context in that it dissociates the kind of agreement in sentences (55)-(59) from $\varphi$-feature agreement linked to EPP satisfaction, as I am proposing.
} 
(59) a. Pinna-hu jaa?a 1-kilaab-u

that-EXPL.3MS came.3MS the-dogs-NOM

Lit: 'It is indeed the case that the dogs came'

b. Pinna-ha jaa?a-t 1-kilaab-u that-EXPL.3FS came.3FS the-dogs-NOM

Lit: 'It is indeed the case that the dogs came'

c. Pinna-hu jaa?a-t l-kilaab-u

that-EXPL.3MS came.3FS the-dogs-NOM

Lit: 'It is indeed the case that the dogs came'

The sentences in (59a) and (59c) are of particular interest in the context of my contention that such instances of apparent subject-verb agreement in the IP domain are actually instances of 'Criteria' that are different from the processes involved in the valuation of uninterpretable features in the case-agreement system of languages. In (59c), the clitic - $h u$, marked for $3^{\text {rd }}$ masculine singular agreement morphology does not agree with the verb marked for $3^{\text {rd }}$ feminine singular agreement (apparently agreeing with the 'broken' plural subject DP al-kilaab- $u$ 'the dogs'). In (59a), the reverse is true: the clitic - $h u$ agrees with the verb, and the verb does not seem to agree with the subject DP. Nevertheless, as I have discussed in relation to the sentences in (55) above, the agreement configuration in question seems to be dissociated from the kind of 'rich' $\varphi$-feature agreement, which - at least, in Arabic - obtains under an $\operatorname{Agr}_{S} \mathrm{P}$ projection in the IP domain, as repeatedly discussed in this chapter.

Fassi Fehri (1993: 92, note 28) cites the sentences in (60) below from Syrian Arabic (from Barlow \& Ferguson 1988). These sentences pattern like the sentences in (58) and (59), and, as such, seem to present an argument for an SA-type of the derivation of VSO sentences in Syrian Arabic:

$$
\begin{aligned}
& \text { (60) a. Pijaa-ni makatiib } \\
& \text { came.3MS-me letters } \\
& \text { 'I got some letters' } \\
& \text { b. Pijit-ni makatiib } \\
& \text { came.3FS-me letters } \\
& \text { 'I got some letters' }
\end{aligned}
$$

(Syrian Arabic)

In (60a), subject-verb agreement in $3^{\text {rd }}$ singular masculine agreement features does not seem to be with the feminine subject makatiib 'letters' (a 'broken' plural nominal). According to Fassi Fehri (1993: 92, note 28), subject-verb agreement in (61a) is 'null' 
and agreement between the verb and the subject makatiib in (61b) is exclusively in the agreement feature of (feminine) gender. ${ }^{57}$ Such an example of absence of number agreement with 'broken' plural nominals (together with the possibility of gender agreement) makes Syrian Arabic similar to SA in only showing 'partial' subject-verb agreement in the VSO word ordering and not the full set of $\varphi$-agreement as in TA and MA. However, other sample examples from Syrian Arabic show that the language is after all not so different from the other modern spoken dialects of Arabic in allowing subject-verb agreement configurations in VSO sentences where the verb inflects for number. Cowell (1964: 421) provides evidence from the Syrian Arabic dialect spoken in Damascus showing that a verb followed by a feminine and/or plural DP like banaat 'girls', as in (61), does not necessarily agree with that subject:
(61) a. wuSəl banaat
arrived.3MS girls
'Some girls arrived'
b. wuSl-uu banaat
arrived.3P girls
'Some girls arrived'

In (61a), the verb wuSal inflects for $3^{\text {rd }}$ person masculine singular. In contrast, in (61b), the verb wuSl-uu is marked for $3^{\text {rd }}$ person plural morphology. This evidence suggests that although Syrian Arabic shares some characteristics of SA subject-verb agreement (i.e., 'partial' agreement of a verb with a post-verbal plural subject), Syrian Arabic also allows the subject-verb agreement configuration characteristic of the modern Arabic spoken dialects in having full subject-verb agreement in VSO sentences. On this account, the Syrian Arabic dialect freely allows subject-verb agreement characteristics in VSO sentences that are not normally co-existent in one and the same dialect.

Returning to the sample sentences in (55)-(59) above, the optionality in the realization of gender on both the verb and the lexicalized clitic pronoun suggests that the gender feature obtaining in the $\mathrm{CP}$ domain where the verb and the lexicalized EXPL 'agree' - under the assumptions I am entertaining for the derivation of VSO

\footnotetext{
${ }^{57}$ Fassi Fehri's (1993) account of subject-verb agreement in SA VSO sentences differs from that of Benmamoun (1992a) (see section 2.1.1.2) Aoun \& Benmamoun (1999) (see section 2.3.1), and Aoun $e t$ al. (1994) (see below) in that Fassi Fehri denies that there is a kind of 'partial' or 'default' number agreement in these structures. My guess is that in both (60a) and (60b), there is some kind of subject agreement, but that verb agreement with the subject in the gender feature in the IP domain, in such a dialect, can remain 'null'.
} 
structures in SA - is not an instance of $\varphi$-feature agreement that is linked to the satisfaction of the EPP in the IP domain.

In this connection, Fassi Fehri (1993: 34) states that: “... phi-feature or AGR specifications can only be licensed by argumental (or referential) subjects", and that expletive subjects "... are not sufficient licensers of these features". Fassi Fehri (1993: 34, 38-39) further maintains that although expletives do not play a direct role in licensing AGR feature specifications in VSO structural representations in SA, VSO sentences may exhibit an instance of an expletive topic, as in (62) below (Fassi Fehri’s 1993: 39 example (76)) or (55), (56) and (59) above: ${ }^{58}$

(62) Pinna-ha laa t-aSma 1-PabSar-u

that-her NEG 3F-be blind the-eyesight.PL-NOM

Lit: 'Eyesights do not become blind'

Fassi Fehri (1993: 34, 38-39) claims that this is also true of Arabic dialects other than SA as in $(63 \mathrm{a}-\mathrm{c})$ from MA: ${ }^{59}$

(63) a. *ra-h ja l-ulaad

EXPL-MS came.3MS the-boys

Lit: 'There came the boys'

b. ra-h jaw l-ulaad

EXPL-MS came.3P the-boys

Lit: 'There came the boys'

c. ra-hum jaw 1-ulaad

EXPL-3P came.3P the-boys

Lit: 'There came the boys'

Fassi Fehri (1993) interprets ra-h/ra-hum in (63a,b) (see also the sentences in (65) and (66) below) literally as 'see-him(it)/see-them', since these elements can be decomposed into $r a$ - a non-inflected form of the Arabic verb $r a$ Pa 'see' - and a clitic pronoun -h/-hum. Fassi Fehri (1992: 130; 1993: 41) asssumes that $r a-h$ and its variants $r a$-ha and ra-hum are expletive and topic-like in nature and that they occur in Spec of an AGR node in satisfaction of the EPP. Fassi Fehri (1993: 41) further assumes that: "AGR on the predicate does not necessarily agree with the form of the

\footnotetext{
${ }^{58}$ As already reviewed in section 2.1.1, Fassi Fehri (1993: 39) takes SA to have a basic VSO word order.

${ }^{59}$ The same sentences in $(63 a-c)$ (as well as in $(64 a, b)$ below) can be reproduced in TA.
} 
expletive (which is in its Spec), while it does so with the postverbal argumental subject".

However, judging from the ungrammaticality of the verb $j a$ 'came' in (63a) vs. the grammatical form jaw '(they) came' in $(63 b, c)$, subject-verb agreement seems to exclusively obtain in the IP domain irrespective of the form the so-called expletive topic element takes - namely, $r a-h$ in (63b) and ra-hum in (63c). Thus, these expletive topic elements do not seem to occupy the structural subject position in the IP domain - namely [Spec, IP/Agr $\mathrm{P}]$. Rather, these elements seem to be located in some peripheral Spec position, which I propose to be an adjoined position to IP/Agr $\mathrm{P}$ in the derivation of such MA sentences.

Another argument for the suggestion that the 'expletive topic' elements $r a-h$, ra-ha, ra-hum are presumably in a peripheral position to the left of the element occupying the structural subject position (and that they are not the elements with which the inflected verb in $(63 \mathrm{~b}, \mathrm{c})$ agrees) comes from such SVO sentences in MA as (64a,b) below (Fassi Fehri 1993: 41):

(64) a. ra-ha Hlima waqfa EXPL-FS Hlima standing.FS

'It is Hlima standing up.'

b. ra-h Hlima jaa-t

EXPL-MS Hlima came-FS

'It is Hlima (who) has come.'

As noted earlier in relation to ra-h/ra-hum in $(63 b, c)$, Fassi Fehri $(1992,1993)$ assumes that these elements are in the IP domain. Nevertheless, assuming that the DP Hlima in both (64a) and (64b) occupies the Spec of AGR ( = Agr $)$, as the highest Spec position in the IP domain determining subject-verb agreement at Spell Out in such SVO sentences, positioning $r a-h$ and its variants in the IP domain seems difficult to maintain.

My guess is that such sentences introduced by the 'expletive topic' element $r a-h$, or one of its variants $r a-h a, r a-h u m$, represent a 'residue' of SA VSO sentences introduced by the matrix complementizer Pinna to which an EXPL clitic pronoun 
-hu/-ha gets attached, as in sentences (55)-(59) above. ${ }^{60}$ Both elements involve some peripheral position to the left of the subject DP, which remains in the IP domain. The difference between SA VSO sentences and VSO sentences in such modern spoken dialects as TA and MA is that agreement configurations in these VSO instances are parameterized according to the feature structure of the functional nodes that participate in agreement relations and according to how the $\mathrm{C},\left(\mathrm{Agr}_{\mathrm{S}}\right), \mathrm{T}$ dependency is established via these configurations.

On this account, the agreement configurations obtaining in such sentences as $(63 \mathrm{~b}, \mathrm{c})$ and $(64 \mathrm{a}, \mathrm{b})$ (likewise for the sample sentences in (65) and (66) below) do not represent counterevidence to the analysis I am proposing in relation to the derivation of the VSO word order in TA or MA as modern spoken dialects of Arabic. As discussed in relation to structure (51) above, EXPL pro-insertion in [Spec, $\mathrm{Agr}_{S} \mathrm{P}$ ] remains a legitimate process in the modern spoken dialects of Arabic, whereby full subject-verb agreement under an $\mathrm{Agr}_{S} \mathrm{P}$ projection in VSO sentences finds ample justification.

Makhoukh (1998) and Aoun et al. (1994) entertain similar assumptions to those made by Fassi Fehri $(1992,1993)$ in relation to the absence of an agreement relation between the verb and an EXPL element in SA and the other Arabic varieties. According to them, the agreement relation in VSO sentences in Arabic holds directly between the verb and the post-verbal argument subject, and not between the verb and an EXPL element in the Spec of IP/Agr(s)P. The sentences in (65) and (66) below are from MA and Lebanese Arabic (LA):

(65) a. ra-ha nejHat meryem EXPL-FS succeeded-3FS Meriem 'Meriem has succeeded.'

b. ra-h Radi takhud meryem minHa EXPL-MS will get.3FS Meriem a grant 'Meriem will get a grant'

(Makhoukh 1998: 246)

\footnotetext{
${ }^{60}$ Talmudi (1981: 24) assumes that the verb $r a$ 'see' has a demonstrative function. In this respect, this verb may have undergone a process of 'grammaticalization' whereby it has assumed the function of a presentative particle that in a way asserts the truthfulness of the proposition being introduced by the particle, very much like Pinna/Panna does in SA. Under such an interpretation, a possible alternative translation to the 'grammaticalized' verb $r a$ 'see' at the beginning of such sentences as in (63) and (64) (as well as (65) below) would be something like 'I assert that ..." or "It is indeed the case that...".
} 
(66) a. fakkar Pinno raaHo l-baneet

thought.3MS that.3MS left.3P the-girls

'He thought that the girls left.'

$$
\begin{aligned}
& \text { a'. *fakkar Pinnun raaHo l-baneet } \\
& \text { thought.3MS that.3P left.3P the-girls } \\
& \text { 'He thought that the girls left.' }
\end{aligned}
$$

$$
\begin{aligned}
& \text { b. fakkar Pinno raaHit zeena. } \\
& \text { thought.3MS that.3MS left.3FS Zeena } \\
& \text { 'He thought that Zeena left' }
\end{aligned}
$$

$$
\begin{aligned}
& \text { b'. *fakkar Pinna raaHit zeena. } \\
& \text { thought.3MS that.3F left.3FS Zeena } \\
& \text { 'He thought that Zeena left' }
\end{aligned}
$$

(Aoun et al. 1994: 202)

The sentences in (65) pattern like the sentences in (63) above. Again, the assumption that the topicalized elements $r a-h$ and $r a-h a$ occupy the structural subject position is dubious. Rather, an EXPL pro can be assumed to be merged in [Spec, $\operatorname{Agr}_{S} \mathrm{P}$ ] determining subject-verb agreement at Spell-Out. As discussed in relation to (63), I assume that $r a-h$ and $r a-h a$ in (65) are left-adjoined to $\operatorname{Agr}_{S} \mathrm{P}$.

The same assumption about an EXPL pro seems to apply to the sentences in (66). Subject-verb agreement at Spell Out obtains in number/person (sentence (66a)) and number/person and gender (sentence (66b)). ${ }^{61}$ According to the assumptions about agreement features in syntactic structure made earlier (see, in particular section 2.1.2, and sections 2.3.1 and 2.3.3), number agreement will only arise if the right Spec-head agreement relation is available configurationally. Although Aoun et al. (1994: 200-203) acknowledge the role of Spec-head agreement relations in determining subject-verb agreement configurations and that "there may very well be an expletive element preverbally in the VS cases", in the varieties of Arabic they are interested in - namely, SA, MA and LA -, they maintain that the agreement relation is not mediated by expletive pro.

As I have discussed in relation to structures (50) and (51) above, I am proposing that the derivation of VSO structural representations in SA differs from the derivation of VSO sentences in such spoken dialects of Arabic as TA, MA and LA in

\footnotetext{
${ }^{61}$ As in TA, LA and MA do not have any feminine agreement marker on verbs in the plural and the verb morphology for the plural is the same for the masculine and feminine plural. On this account, subject-verb agreement in (66a) is actually an instance of agreement in all $\varphi$-features, irrespective of whether gender is phonetically realized on verbs in the plural or not.
} 
terms of the presence or absence of an $\operatorname{Agr}_{S}$ node and, thereby, the presence or absence of an EXPL pro in [Spec, $\left.\operatorname{Agr}_{S} \mathrm{P}\right]$. On this account, the assumption that the verb in VSO sentences in SA, is not under an $\mathrm{Agr}_{S}$ node (or a complex T-Agr ${ }_{S}$ node) already discounts an analysis whereby an EXPL pro is in the highest Spec position in the IP domain in SA that would mediate the subject-verb agreement relation at that level. Rather, besides the probe-goal-Agree relation that holds between the verb and the post-verbal subject raised to [Spec, TP] (as a 'weak' Phase at the highest Spec position in the IP domain in SA) the EXPL clitic pronoun phonetically realized in a position to the left of, and adjacent to, the verb in the split-CP domain determines a strong Phase in terms of which the T-dependency in SA VSO sentences is ultimately established.

Under such assumptions, the agreement configurations, as pertaining to verb morphology and the relation between the IP domain and the CP domain in SA and the other dialects of Arabic (such as MA, TA, Syrian Arabic, and LA), as discussed in relation to the sample sentences in (63)-(66) above, again shows that there is some variation as to how these agreement configurations are manifested in clause structure. In this light, Aoun et al.'s (1994) analysis of subject-verb agreement in Arabic misses the very issue the authors set out to address in the first place - namely, the parametric character of structural agreement relations and representations. The important point in this connection is that Aoun et al.'s (1994: 200-203) analysis does not exclude altogether the availability of EXPL pro in the structural subject position of the varieties they are concerned with. The authors simply exclude the possibility that the EXPL has anything to do with subject-verb agreement. On the assumptions I have been entertaining thus far, the nonexistence of the EXPL in the Spec position of the highest inflectional node (i.e., [Spec, TP]) in SA VSO word order may happen to be the answer to the parametric character of the structural agreement relations in question.

\subsubsection{Summary}

In this section, I have been concerned with the parametric character of the derivation of subject-verb agreement configurations in VSO structural representations in SA vs. such modern spoken Arabic dialects as TA, MA and LA. I have proposed that the variation in question concerns an $\mathrm{Agr}_{\mathrm{S}}$ node in the specifier position of which an EXPL pro is merged at the level where Spell Out applies. Thus, whereas TA, MA 
and LA - as modern spoken dialects of Arabic - project $\mathrm{Agr}_{\mathrm{S}}$ in VSO sentences, SA does not.

In SA VSO sentences, the subject is assumed to raise to [Spec, TP] where some form of third person singular subject-verb agreement defines a 'weak' TP Phase. The T-dependency is thus not determined by subject-verb agreement at the level of IP. After having first raised to T in the IP domain, the verb moves further up to some $F$ functional head position in the split $\mathrm{CP}$ domain. I return to a feature-based account of the raising of $\mathrm{V}$ (actually, $\mathrm{V}-\mathrm{v}$ ) to $\mathrm{T}$ and to $F$ in Chapter Four.

In the following subsection, I have a look at how the assumption of different realizations of the T-dependency in the IP domain accounts for the difference in subject-agreement configurations in SA and TA.

\subsubsection{Different realizations of the $C$-( $\left(A g r_{S^{-}}\right) T$ dependency in VSO sentences in SA and $T A$}

Biberauer and Roberts (2010: 265) point to the necessity of “distinguishing T's tense (i.e., 'verbal' or V-related) properties from its agreement $\varphi$ - (i.e. 'nominal' or Drelated) properties ...". Abiding by the feature-valuation system such a distinction is linked to, the process of verb-raising to the inflectional domain of sentence structure concerns primarily the relation between T's unvalued V-features and V's unvalued $\mathrm{T}$ (ense)-features. ${ }^{62}$ Nevertheless, this process, whereby tense inflection is appropriately interpreted at the interface, arises concomitantly with the process whereby $\varphi$-agreement between the subject DP and the verb arises in the IP domain (as discussed in section 3.3.4. in relation to Berber and Arabic).

In the probe-goal-Agree system of structural dependency relations that parametrically vary according to the feature structure of functional categories, the interrelatedness of these, nonetheless, distinguishable processes has been primarily presented in this chapter as an argument against a V-raising style of EPP-feature checking/valuation. V-raising per se does not determine the kind of subject-verb

\footnotetext{
${ }^{62}$ I have assumed in my discussion of Benmamoun's (2000b, 2008) analysis of SA verbal inflection in section 3.3.2.3, that $[u \mathrm{~V}]$ on $\mathrm{T}$ could be universally absent on $\mathrm{T}$ and that the raising process of $\mathrm{V}$-to- $\mathrm{T}$ would exclusively be accounted for on the basis of the $v / V$ 's 'rich' $[u \mathrm{~T}]$ feature that triggers its raising to the T node in V-raising languages (see footnote 34 above).
} 
agreement we find in richly-inflected V-raising languages. ${ }^{63}$ Such a system also goes against Chomsky's (1995b) suggestion that verbs 'overtly' move only as a 'phonological' process, whereby V-raising may be categorically different from XPmovement processes in the syntax. As discussed in this chapter in relation to Roberts' (2010a) and Roberts \& Biberauer's (2010) arguments for a parameterized probe-goalAgree system, raised verbs will still be involved in the 'valuation' procedure at Spell Out whereby the derived structure is transferred to both LF and PF for interpretation. This is so because such a raising process is an integral part of the process by which the $\mathrm{C},\left(\mathrm{Agr}_{\mathrm{S}}\right), \mathrm{T}$ dependency is derived in sentence structure. By that token, any raising process in the grammar will have to be involved in some valuation procedure and will have to have a bearing on interpretation at LF.

As discussed in section 3.3.4, Berber and SA/TA are languages where the VSO word order alternates with the SVO word order. Nevertheless, in Berber and TA, the VSO word order exhibits full subject-verb agreement (sentences (67) and (68), respectively), whereas SA does not (sentence (69)):

(67) sqad-n timgharin lbrat. (Berber) sent-they the women the letter 'The women sent the letter' (Ouhalla, 1991: 124) $)^{64}$

(68) b९ath-u n-nsaa l-jwaab sent-3P the women the-letter 'The women sent the letter'

(69) baSatha-t n-nisaa?-u r-risaalat-a sent-3FS the women-NOM the-letter-ACC 'The women sent the letter'

In the TA and Berber sentences in (67) and (68), according to my analysis, the T-node is directly associated with the $\operatorname{Agr}_{S}$ node thus directly linking $\varphi$-feature agreement specification (person, number and gender) on the raised verb and EPP-feature valuation - whereby [Spec, $\left.\operatorname{Agr}_{S} \mathrm{P}\right]$ has to be occupied - to tense interpretation at Spell Out. As for the SA sentence in (69), I have adopted the hypothesis whereby

\footnotetext{
${ }^{63}$ The claim I am making here in relation to V-raising languages of the Semitic/Celtic type may also be true of matrix V2 sentences in a language like German (see section 4.1.2.1), and the importance of the uninterpretable $\mathrm{T}$-feature of $\mathrm{V}-v$ as the trigger for $\mathrm{V}$-raising in such languages (see section 3.3.2.3).

${ }^{64}$ I kept Ouhalla's (1991) glosses where the agreement inflection on the verb is interpreted as a pronominal affix, i.e., as a nominal argument of the verb, making Berber's agreement inflection similar to SA's (see section 2.1.2.3.3).
} 
$\mathrm{Agr}_{\mathrm{S}}$ is not projected in sentence structure in the SA VSO word order. Since T is not directly associated with an $\mathrm{Agr}_{S}$ node, the highest Spec position for $\varphi$-feature and EPP-feature valuation in the IP domain is [Spec, TP].

Table 3.11 below provides a breakdown of the feature structure of functional categories in the IP domain in TA and SA, as discussed in the previous sections:

Table 3.11. The $\mathrm{C}-\left(\mathrm{Agr}_{\mathrm{S}^{-}}\right) \mathrm{T}$ dependency and the feature-structure representation of the IP domain in VSO sentences in TA and SA

\begin{tabular}{|c|c|c|}
\hline Feature structure & TA VSO sentences & SA VSO sentences \\
\hline $\begin{aligned} \text { Agrs: }_{\text {S }} & \text { D-feature (as an } \\
& \text { edge feature in } \\
& \text { the IP domain) }\end{aligned}$ & 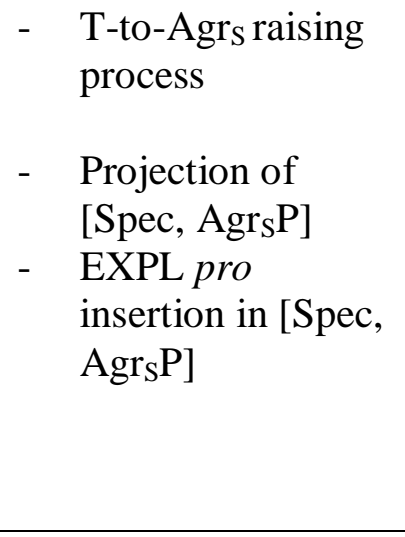 & 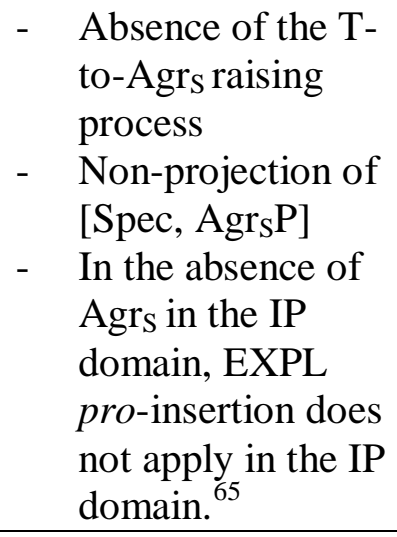 \\
\hline $\begin{array}{l}\mathrm{T}: \\
\quad \text { EPP feature } \\
-\quad \varphi \text {-features }\end{array}$ & $\begin{array}{ll}\text { - } & \text { EPP valuation in } \\
& {\left[\mathrm{Spec}, \mathrm{Agr}_{\mathrm{S}} \mathrm{P}\right]} \\
- & \varphi \text {-feature valuation } \\
\text { in }\left[\mathrm{Spec}, \mathrm{Agr}_{S} \mathrm{P}\right] \\
\text { associated with the } \\
\text { EPP and D-feature } \\
\text { valuation. }\end{array}$ & $\begin{array}{l}\text { - } \quad \text { EPP valuation in } \\
{[\text { Spec, TP }]} \\
\text { - } \quad \text {-feature } \\
\text { valuation in [Spec, } \\
\text { TP] associated } \\
\text { with the EPP. }\end{array}$ \\
\hline
\end{tabular}

Recall my proposal in section 3.3.4 that the projection of Agr $_{S}$ in sentence structure determines a strong Phase in Arabic. In TA, the process by which T-to-Agrs applies, coupled with the process via which $\mathrm{V}-v$ raises to $\mathrm{T}$, will determine subjectverb agreement. In SA VSO sentences, the T-to-Agr process does not apply because

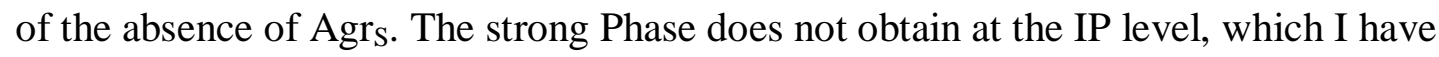
assumed to be a 'weak' Phase in the derivation of these sentences.

\footnotetext{
${ }^{65}$ As discussed above, SA VSO sentences allow the possibility of lexically realizing EXPL pro as an EXPL clitic pronoun - $h u /-h a$ in the event that the complementizer Pinna/Panna is used (both in matrix and embedded contexts). In this case, the D-feature that (in TA VSO sentences) obtains in the IP domain is associated, in SA VSO sentences, with the functional projection in the split-CP domain (namely, Top) that parallels $\mathrm{Agr}_{S}$ in the IP domain (see footnote 53).
} 
I have also proposed that the [V-v]-to-T raising process in SA may prove to be a substitution process rather than an adjunction process. Thus, in the first step of the derivation of VSO sentences in SA, the verb already inflected for person and tense raises to T, as a 'substitution' rather than 'adjunction' process. Thus, T, in SA, necessarily projects a Spec position for EPP satisfaction. In this respect, the difference between the SA VSO word order and the TA VSO word order concerns the nature of the highest inflectional node the verb raises to in conjunction with the dependency relations established within the derived structure for the valuation and/or identification of features including the satisfaction of the EPP within the IP domain.

In this conception, it is due to the different character of the Spec-head/headhead structural agreement relations - fundamental for the representation of subjects at the IP level - that the derivation of VSO structural representations in TA and in SA proceeds via different paths. In TA, the $\left[\mathrm{V}-v-\mathrm{T}-\mathrm{Agr}_{\mathrm{S}}\right]$ inflectional complex is under an $\mathrm{Agr}_{S} \mathrm{P}$ maximal projection whereby, in the VSO word ordering, Spec-head agreement applies with an EXPL pro in [Spec, $\left.\mathrm{Agr}_{S} \mathrm{P}\right]$ for EPP satisfaction. In SA, my assumption has been that EXPL pro-insertion does not apply at the IP level in VSO sentences. In this case, $\mathrm{Agr}_{S}$ does not project above TP, and Spec-head agreement for EPP satisfaction applies between the $[\mathrm{V}-v-\mathrm{T}]$ inflectional complex and the subject DP in $[$ Spec, TP].

As discussed in section 3.2.1.1, EXPL-insertion processes, as in a null-subject language like TA, or as in English there -associate pairs, not only involve an additional D-feature that requires merging an EXPL element under the highest maximal projection in the IP domain (i.e. $\operatorname{Agr}_{S} \mathrm{P}$ ) for EPP satisfaction, but also the process whereby $\mathrm{T}$ raises to adjoin to $\mathrm{Agr}_{\mathrm{S}}$ and EXPL is inserted in [Spec, $\left.\mathrm{Agr}_{S} \mathrm{P}\right]$ to value $[u \mathrm{D}]$ on $\mathrm{Agr}_{\mathrm{S}}$. This probe-goal-Agree system of dependency relations establishes the desired subject-verb agreement configuration whereby valuation of $[u \mathrm{D}]$ on $\mathrm{Agr}_{\mathrm{S}}$ is ultimately linked to $\varphi$-feature specification and EPP satisfaction. Thus, in a null-subject language like TA, which exhibits full subject-verb agreement in the VSO word order, agreement in the IP domain is concomitant with the [V-v]-to$\mathrm{T}$ and T-to-Agr $\mathrm{S}$ raising processes, and with EXPL pro-insertion in [Spec, [v-V-T-AgrS] $\left.\mathrm{Agr}_{\mathrm{S}} \mathrm{P}\right]$ rather than with $\mathrm{V}$-raising per se.

V-raising in the IP domain also applies in SA, but the adjunction of T to $\mathrm{Agr}_{\mathrm{S}}$ does not apply in this language in the derivation VSO sentences. Rather, - as I will discuss in more detail in Chapter Four - the V-raising process by which the verb, 
raised to T raises further up past the subject DP in $[\mathrm{Spec}, \mathrm{TP}]$ is linked to some identification process in the split-CP domain involving the verbal inflectional [V- $v-\mathrm{T}]$ complex raised to some $F$ head position.

Under such assumptions as to the differences in the realization of the $\mathrm{C}$ ($\mathrm{Agr}_{\mathrm{S}}$ )-T dependency in Arabic, an account of the parametrically different subject-verb agreement configurations that arise in the derivation of VSO sentences in SA and the corresponding sentences in TA at the IP level in terms of a parameterized probe-goalAgree system of feature-valuation finds a justifiable explanation. The following sentences in $(70 a, b)$ and $(72 a)$, from SA, corresponding to those in $(71 a, b)$ and $(72 b)$, from TA, show such differences in agreement configurations:
(70) a. kharaj-a/*kharaj-uu 1-Pawlaad-u went out-3MS/went out-3MP the- boys-NOM 'The boys went out'
b. kharaj-at/*kharaj-na l-banaat-u. went out-3FS/went out-3FP the-girls-NOM 'The girls went out'

(71) a. kharj-uu/*khraj l-ulaad went out-3P/went out-3MS the-boys 'The boys went out'
b. kharj-uu/*kharj-t 1-bnaat. went out-3P/went out-3FS the-girls 'The girls went out'
(72) a. man qaala Pinna-hu žaa?a 1-Pawlaad-u? who said that-CL(ACC) came.3MS the-boys-NOM 'Who said that the boys came?'
b. shkuun qaal illi jaa-w l-ulaad? who said that came-3P the-boys 'Who said that the boys came?'

In the absence of $\operatorname{Agr}_{S}$, in the SA sentences in $(70 \mathrm{a}, \mathrm{b})$, the full set of $\varphi$-feature specification on the [V-v-T] inflectional complex does not appear (kharaj-a vs. *kharaj-uu in (70a) and kharaj-at vs.*kharaj-na in (70b)). As proposed in section 3.3.4, the [V-v]-to-T raising process is necessarily involved in some subject-verb agreement which is linked to the satisfaction of the EPP at the highest level the derivation reaches in the IP domain, i.e., [Spec, TP] where the raised subject DP values the uninterpretable $\varphi$-features of the T node giving rise to the so-called, $3^{\text {rd }}$ 
person singular 'default' agreement configuration. In the TA sentences in $(71 \mathrm{a}, \mathrm{b})$, the inflected verb is under an $\operatorname{Agr}_{S} \mathrm{P}$ projection, which is responsible for the full agreement pattern (kharj-uu vs. *khraj in (71a) and kharj-uu vs. kharj-t in (71b)).

In the wh-questions in (72), the same subject-verb agreement relations apply. In the SA wh-question in (72a), the verb (or, the $[\mathrm{V}-v]$ complex) first raised to $\mathrm{T}$ agrees with the subject DP raised to [Spec, TP], where valuation of the uninterpretable $\varphi$-features of T by the subject DP applies. This probe-goal Agree relation only gives rise to 'partial' subject-verb agreement (jaa?a). The EPP is, nonetheless, satisfied as a reflection of the probe-goal-Agree relation. Since in the derivation of such VSO sentences as in (70) (and, likewise, in the wh-construction in (72a)) SA opts for expanding the CP domain into Fin and Force (à la Rizzi 1997, see section 3.3.4) - where the Top-Foc system is activated -, the inflected verb further raises to some $F$ node in the spilt-CP domain.

In the TA wh-question in (72b), however, the raising of $\mathrm{T}$ to $\operatorname{Agr}_{\mathrm{S}}$ applies, and the raised verb agrees with an EXPL pro merged in $\left[S p e c, \operatorname{Agr}_{S} \mathrm{P}\right]$ giving rise to full subject-verb agreement (jaaw) at Spell Out.

As in Roberts' (2010a: 114) typology of 'clitic doubling' configurations in the Romance null-subject systems that Roberts (2010a) is interested in (see section 3.2.1.2), the differences in subject-verb agreement configurations in SA and TA may ultimately be linked to how the process of feature-valuation applies at the highest level of the derivation of the IP domain in relation to T's D-feature. ${ }^{66}$ Presumably, it is due to the absence of T's D-feature (and the accompanying absence of $\mathrm{Agr}_{\mathrm{S}}$ in the projection of the IP domain in SA VSO word order) that feature valuation (assuming that it involves some 'feature copying' mechanism) may involve 'partial copying' of the subject agreement features, in SA, as opposed to 'full copying' of those features in

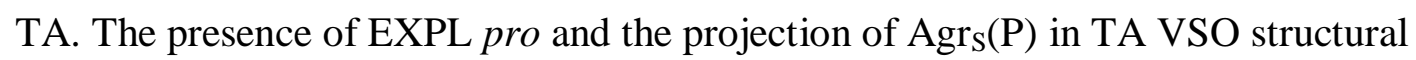
representations would account for this difference in the feature-valuation mechanism in subject-verb agreement configurations.

\footnotetext{
${ }^{66}$ In section 3.2, I have proposed that the D-feature, which Roberts (2010a) and Biberauer et al. (2010) assume to be an additional feature of $\mathrm{T}$, could rather be conceptualized as an 'edge' feature that is the property of an $\mathrm{Agr}_{S}$ node that parametrically projects higher than T, in the IP domain. The assumption of the existence of an additional D-feature at the IP level has been fruitful in accounting for such subject-verb agreement configurations as those that obtain in EXPL-associate pairs in English and TA.
} 


\subsection{Conclusion}

In this chapter, I have proposed an analysis of sentence structure cross-linguistically in terms of a parameterized probe-goal-Agree system of subject-verb agreement configurations. The cornerstone of such an approach has been the proposal that sentence structure parametrically projects as $\operatorname{Agr}_{S} \mathrm{P}$ in some languages (e.g., TA vs. SA in the VSO word order) or in some constructions in a particular language (e.g., there-constructions in English). The role of EXPL-insertion (in such instances of $\operatorname{Agr}_{S}(\mathrm{P})$ projection) has been linked to the availability of D-features that not only represent a defining property of the null pronominal element pro (both referential and EXPL), but also of the functional head that such D-elements (pro in null-subject languages, or there in English) are associated with in terms of the valuation process. The probe-goal-Agree framework of feature-licensing that has been the cornerstone of the parametric account of the so-called EXPL-associate pairs reviewed and further analysed in this chapter helped show that EXPL elements like pro and there share the property of entering the derivation with an intrinsic interpretable Dfeature, which matches an uninterpretable D-feature on the probe they are related to, thus satisfying licensing conditions and Tense interpretation at the interface. In these terms, the feature-valuation processes that underlie the probe-goal-Agree dependency relations in sentence structure represent the only licensing conditions via which subject-verb agreement configurations ultimately obtain cross-linguistically. 


\section{CHAPTER FOUR}

\section{STRUCTURAL DEPENDENCIES IN THE HIGHER FUNCTIONAL FIELD OF ARABIC AND OTHER V-RAISING LANGUAGES}

\subsection{Introduction}

As proposed in the previous chapters, the status of verbal inflection, in the derivation of sentence structure in a richly inflected language like Arabic, depends on the raising process of $\mathrm{V}$ to $\mathrm{I}$. There is a strong correlation between the course the derivational process takes and the agreement patterns that emerge in sentence structure at the output of formal syntax. In this respect, it has been proposed (Rohrbacher 1994) that there is a tendencyfor rich subject-verb agreement to correlate, cross-linguistically, with the V-to-I raising process in the syntax proper since it is this movement process that licenses the elements involved in the very process. However, such a proposal does not seem to account for the case of subjectverb agreement in VSO constructions in SA, as a richly inflected language. Although SA shows evidence of V-to-I movement in VSO sentences, these sentences only show 'partial' (or 'weak') subject-verb agreement. Rather, the process of V to I seems to be a factor, among other possible factors, responsible for subject-verb agreement. The proposal developed here is that, in the derivation of SA VSO word order, verb-raising past the subject raised to the highest Spec position in the IP domain (i.e., the Spec of the T node, in the absence of an $\mathrm{Agr}_{\mathrm{S}}$ node, in SA VSO word order, as proposed here) can account for the absence of rich subject-verb agreement in such a derivation.

In this perspective, the sentence structure of SA and the morpho-syntactic processes involved in the derivation of verbal inflection (including subject-verb agreement configurations) would allow for instantiations of categories that would normally lie outside the domain of inflection proper in the structure of sentences with some discourse-related interpretation (Rizzi 1997, 2004a, Holmberg 1999, Chomsky 2001). ${ }^{1}$ In SA, such categories not only include the Topic and Focus heads familiar

\footnotetext{
${ }^{1}$ The reference here is to the IP-CP continuum where $\mathrm{C}$ complement-selects IP (and where feature valuation for subject-verb agreement obtains at the strong Phase). When the $\mathrm{CP}$ system splits à la Rizzi (1997), it expresses a specification of finiteness, generally reflecting 'inflectional properties' (Rizzi 1997: 284) though not encoded in terms of verbal inflectional affixes that must be valued in the process of syntactic computation. To anticipate much of the discussion that follows, the assumption that not all these 'finite'/'temporal' elements need be involved in some checking/valuation procedure at Spell Out (Rizzi 2006, Holmberg 1999 for Wh-/Focus structures; cf. R\&R for Topic-comment structures) may
} 
from Rizzi’s (1997) split-CP approach, but also Negation and Modal(ity) nodes. ${ }^{2}$ For example, Fassi Fehri $(1993,2004)$ points out that the future tense is not an instantiation of an inflectional marker in the tense system of SA. Rather, it is a modal category. Granting that this much is correct, this tense/modal category would be linked to the articulated system of structural relationships in the CP layer of sentences that relates to the C-Tdependency. Such a category and other similar temporal/finite elements would be instantiated by a Modal node in the CP domain and would be linked to the agreement manifestation at the IP level.

In this chapter, I will focus on the tense properties of functional projections in IP and CP and how these properties interact with modality and negation within a feature-based approach to probe-goal-Agree structural relations and Rizzi's (1997) split-CP system. By reference to this same probe-goal-Agree system, the chapter also revisits earlier assumptions introduced in Chapter Two about the topic properties of initial DPs in SVO word order configurations in SA and highlights the role of a Topic projection (i.e. TopP) in the expression of a 'higher' predication relation in the derivation of SVO structures and relative clauses. As for the derivation of VSO structural configurations in SA, the chapter shows the importance of the tense/modal properties of SA sentence structure in the projection of the articulated left-periphery of IP including TopP.

In light of Rizzi's (1997) split-CP analysis introduced in Chapter Three, section 4.1 deals with the interaction of tense and modality properties of the sentence structure of SA in the derivation of VSO and SVO structural configurations and the respective Focus and Topic interpretation of sentences at Spell Out. In section 4.1, I also look at the position of the NegP projection in SA. I propose that there is evidence for two NegP projections in the grammar of SA, and that the Merge of SA negation elements is driven by the functional feature(s) these elements are endowed with. In section 4.2, I discuss the derivation of wh-dependencies in Arabic showing how SA

\footnotetext{
turn out to be crucial for an understanding of the phenomena that arise in the derivation of V-raising structures. These elements are, nonetheless, subject to the satisfaction of criteria that abound in A'dependencies/Operator-variable binding relations where Spec-head and head-head agreement relationships are equally paramount.

${ }^{2}$ In this connection, I adopt later (see section 4.1.2.1) a reformulated architecture of the Split-CP domain adapted from Poletto (2000b) and Jouitteau (2005) in relation to modal and negation elements above FinP.
} 
and TA differ in that respect. In section 4.3, a possible extension of the feature-based analysis of the left periphery as analysed in sections 4.1 and 4.2 to V2 languages (such as German and Dutch) or residual V2 structural configurations in English is discussed in relation to a feature-sharing conception of Chomsky's (2008) notion of feature inheritance.

\subsection{The derivation of the higher functional field and the split-CP domain of SA}

\subsubsection{Introduction}

As discussed in section 2.1.2. (see, in particular, section 2.1.2.4 which summarizes that discussion), I have proposed a micro-parametric analysis of both VSO and SVO word orderings in Arabic whereby SA differs from a modern Arabic spoken dialect like TA in the representation of the subject position at the interface in both of these constituent orderings. SA VSO word order seems to be 'basic' (Mohammad 1989, Fassi Fehri 1993, Akkal \& Gonegai 2000). TA and the other modern Arabic spoken dialects tend to have SVO as a 'basic' and more 'discourse neutral' word ordering (Ouhalla 1991, Shlonsky 1997). This difference in the prominence of one word order over another in SA vs. the modern Arabic spoken dialects may be related to a difference in how VSO and SVO sentences in SA and the modern Arabic spoken dialects are syntactically derived and, as far as the derivation of VSO sentences are concerned, the availability of the agreement asymmetry in SA as opposed to TA.

As proposed in Chapter Two and further discussed in Chapter Three, SA VSO word order differs from the VSO word order in a modern Arabic spoken dialect like TA in that only in SA is the subject raised out of $v \mathrm{P} / \mathrm{VP}$. The post-verbal status of the subject in SA VSO word order is derived by first raising the verb to T. Then the subject raises to [Spec, TP] for EPP satisfaction. Finally, the verb is further raised (together with $v$ and $\mathrm{T}$, to which the verb has attached) to some node in the split-CP domain of Rizzi (1997), thus deriving the post-verbal position of the subject in SA VSO word order. These processes in SA are represented as in (1) below (reproduced from section 3.3.4): 
(1) SA VSO word order

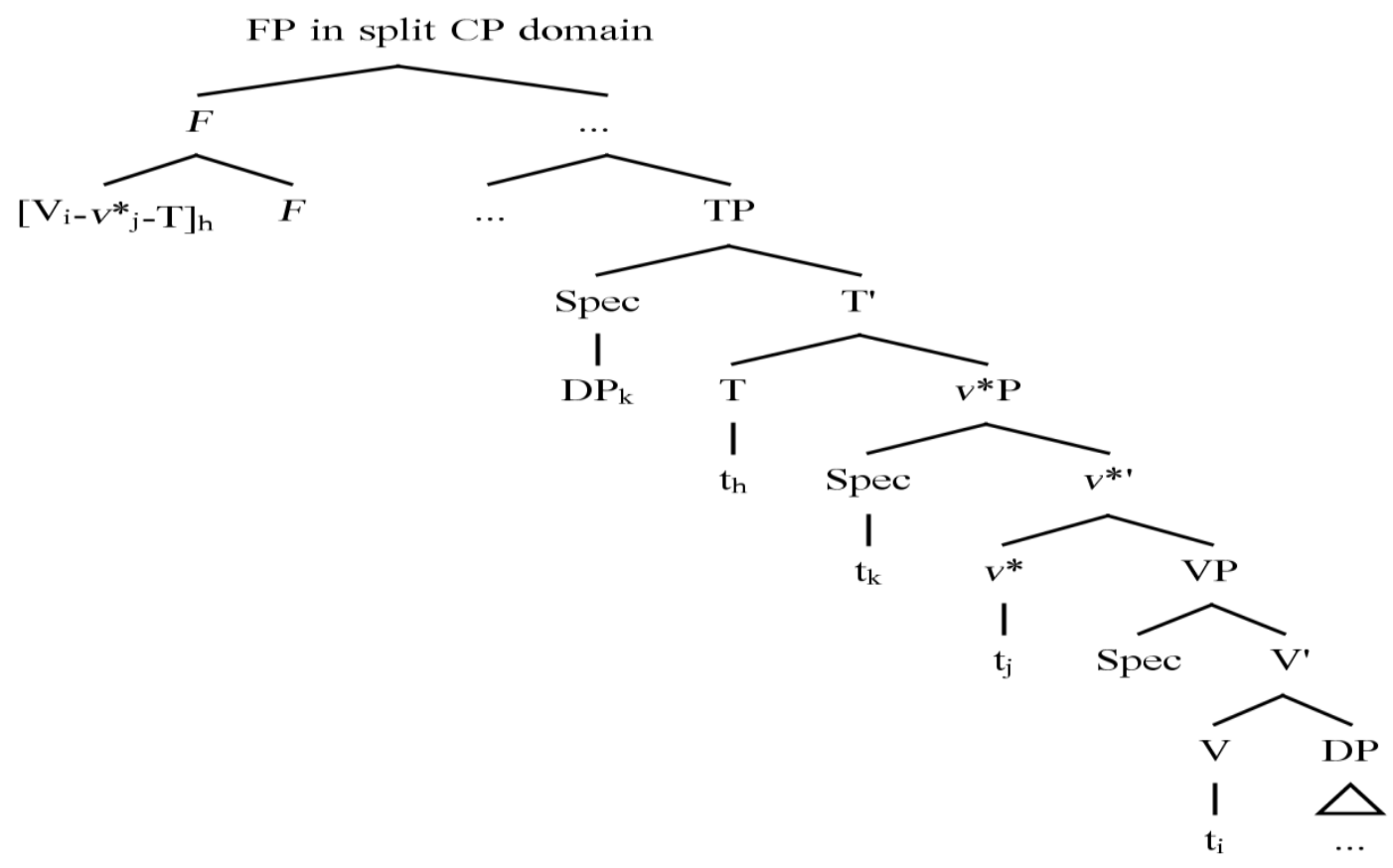

In (1), $F$ designates the Functional projection in $\mathrm{CP}$ to which the $\mathrm{V}-v$-T complex raises (see section 4.1.2.1).

In contrast, in the TA VSO word ordering, whereas the verb is raised to the inflectional domain (projecting as $\operatorname{Agr}_{S} \mathrm{P}$ ), the subject DP remains in situ in [Spec, $\nu \mathrm{P} / \mathrm{VP}]$ in a post-verbal position, as represented in (2): 
(2) TA VSO word order

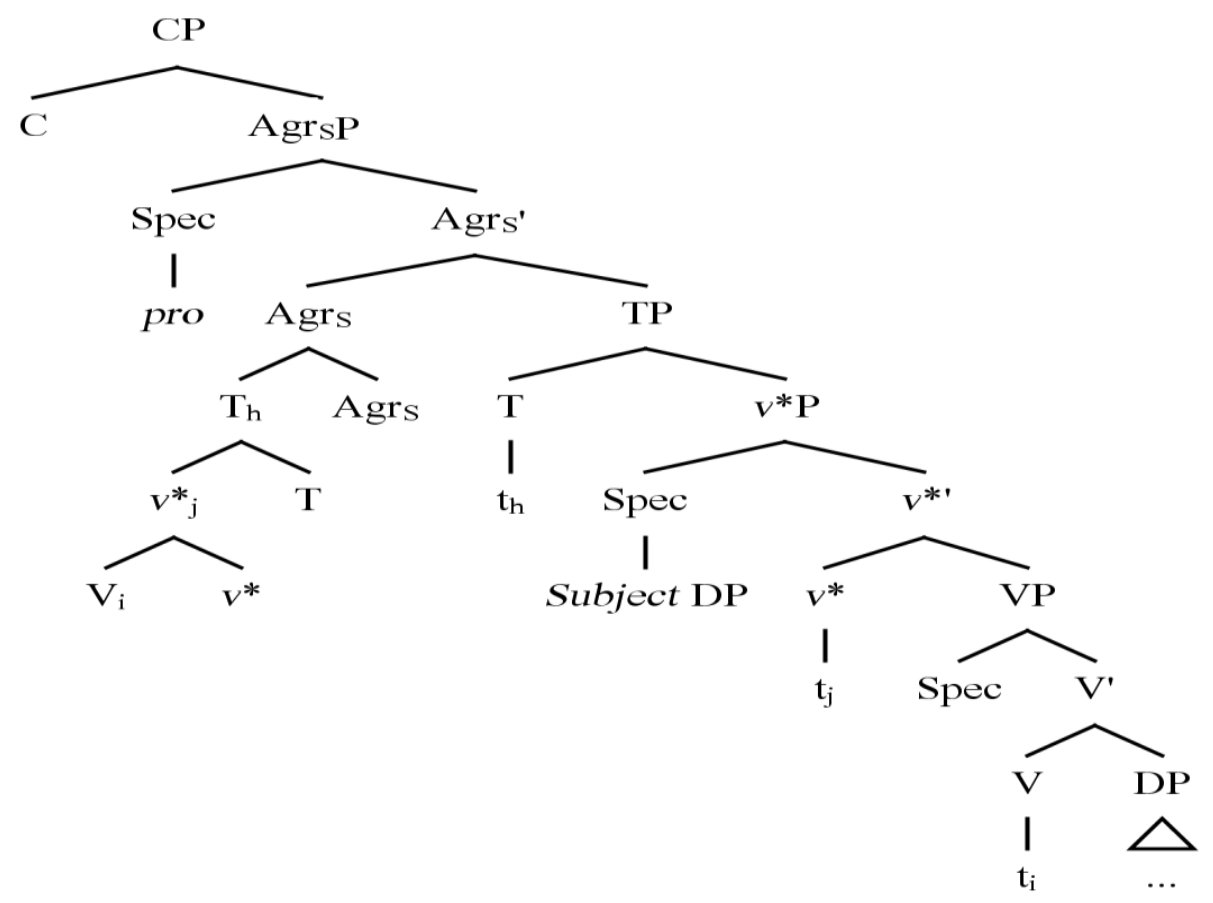

In relation to the TA VSO structure (2), my assumption has been that, whereas in SA VSO sentences expletive pro is not instantiated in the highest Spec position in the IP domain for EPP satisfaction (i.e., [Spec, TP] in (1) above), TA VSO sentences require the projection of $\operatorname{Agr}_{S} \mathrm{P}$ and its Spec position for expletive pro insertion and EPP satisfaction. As represented in (2), the verbal complex $\mathrm{V}-v$-T is raised to the $\mathrm{Agr}_{S}$ node and does not raise further up.

As for the derivation of SVO sentences in Arabic, the tree structures in (3) and (4) illustrate the difference in the derivation of SVO sentences between SA and TA, respectively: 
(3) SA SVO word order

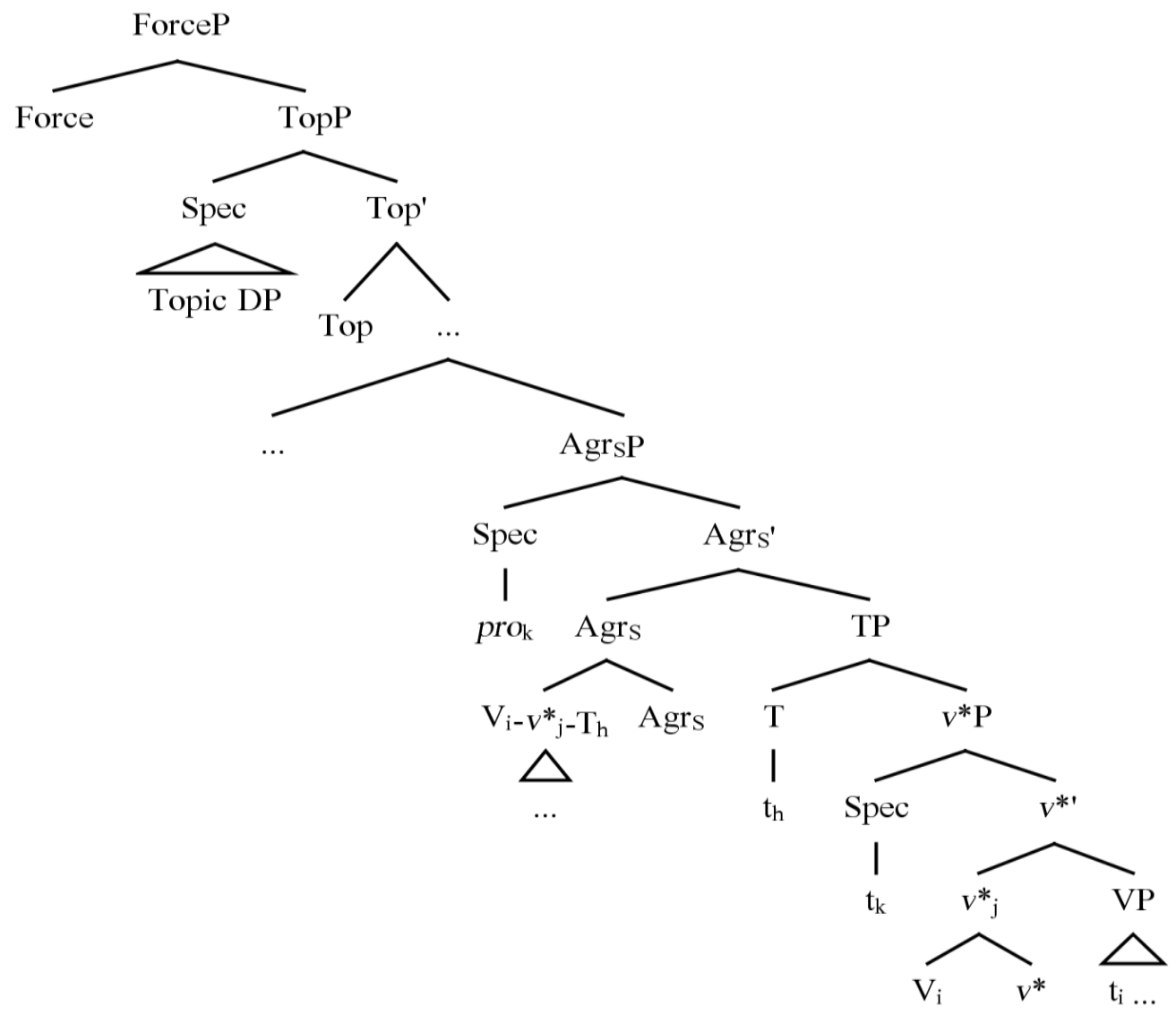

(4) TA SVO word order

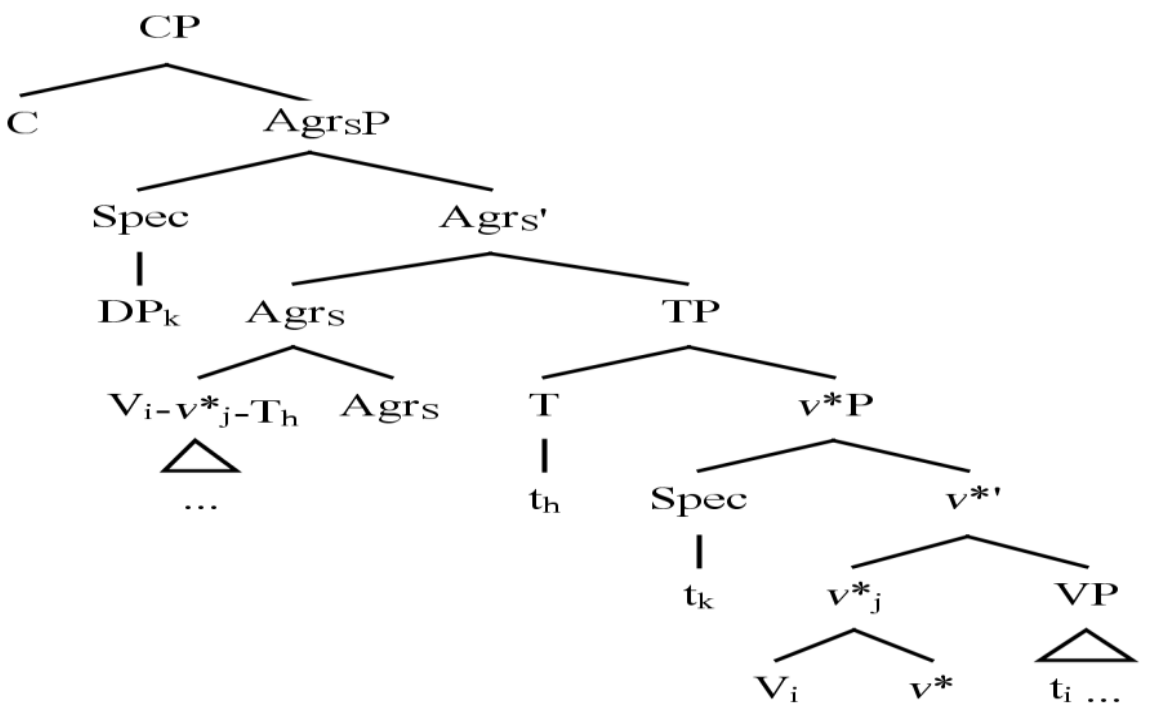


According to these tree representations, SA micro-parametrically differs from a modern spoken dialect like TA. In the SA SVO structure (3), the preverbal subject DP is base-generated in a 'topicalized', left-dislocated position (i.e., in the [Spec, TopP] position of Rizzi's 1997 split-CP system). ${ }^{3}$ In contrast, a subject DP in TA SVO word order, as represented in (4), would be systematically raised out of [Spec, $v \mathrm{P} / \mathrm{VP}]$ and internally merged in $\left[\mathrm{Spec}, \mathrm{Agr}_{\mathrm{S}} \mathrm{P}\right]$. However, both SA and TA SVO sentences have $\mathrm{Agr}_{\mathrm{S}} \mathrm{P}$ as the highest maximal projection at the IP level (with $\mathrm{T}$ raised to $\mathrm{Agr}_{\mathrm{S}}$ forming the $\mathrm{V}-v$-T-Agrs complex).

In section 4.1 .2 below, I discuss the interaction of the tense and modal properties of sentences (including modal and negation markers) in the derivation of SA VSO and SVO word orderings.

\subsubsection{The interaction of modality, negation, tense and topics in SA VSO and SVO sentences}

In this section, I develop my proposal for the derivation of the VSO and SVO word orderings in SA.

Beginning with VSO sentences, the verbal complex $\mathrm{V}-v$ raised out of $v \mathrm{P} / \mathrm{VP}$ to the inflectional domain attaches to $\mathrm{T}$ and the verbal inflectional complex $\mathrm{V}-v$ - $\mathrm{T}$ thus formed is further raised past the subject DP itself raised to [Spec, TP], as seen above in (1). The second raising process of the inflected verb involves some node (or nodes) in the split-CP domain (i.e., ForceP ... FinitenessP (FinP), possibly with a TopicP (TopP) projection, a NegationP (NegP) projection - in negative sentences - and a ModalP (ModP) projection located in between ForceP and FinP).

I assume that both the ForceP and the FinP projections are represented in the structure of matrix sentences in SA. That the ForceP projection is potentially represented in sentence structure allows for the possibility that a lexical complementizer like Pinna functions as a matrix complementizer (irrespective of whether the sentence is VSO or SVO). In 4.1.2.1, I first propose that, in the SA VSO word order, the raised verbal inflectional complex V-v-T attaches to Fin, but does not go further than that head position in the split-CP system. In 4.1.2.2, I deal with some basic assumptions about the derivation of SA VSO sentences, including my assumption concerning the projection of a potential Topic Phrase in such VSO

\footnotetext{
${ }^{3}$ The ellipsis ' ...' in (3) is meant to point to the existence of other functional nodes above IP/Agr ${ }_{S} \mathrm{P}$, and below TopP, as in Rizzi (1997), Poletto (2000b) and Jouitteau (2005).
} 
sentences in matrix contexts. In sections 4.1.2.3, 4.1.2.4 and 4.1.2.5, I have a look at the modal/tense properties encoded by verbs and the particles or markers (including negation markers) verbs interact with in the derivation of SA VSO sentences. In section 4.1.2.6, I deal with the interaction of these modal/tense properties in the derivation of SA SVO sentences where the initial DP figures as a Topic DP.

\subsubsection{The T-to-Fin raising process in SA VSO sentences}

In this section, I attempt to show that a uniform analysis of the derivation of VSO sentences in Celtic/Semitic languages with 'basic' VSO word ordering is a possible alternative to analyses that claim that inflected verbs (i.e., the $\mathrm{V}-v$-T complex) in such languages do not systematically raise to a designated position outside the IP domain. Thus, in this section, the discussion will focus on a comparative analysis of VSO structural configurations in Celtic/Semitic languages with 'basic' VSO word ordering (namely, in Irish and SA) not only in terms of the distribution of tense/modality properties (i.e., preverbal particles, such as modality and negation markers), but also in terms of the distribution of subject properties in relation to agreement on verbs. The same analysis will be claimed to extend to matrix declarative V2 sentences in a V2 language like German.

Such an account based on the distribution of subject agreement properties will allow a prima facie characterization of the similarities (and differences) in the relevant formal properties encoded by verbs or somehow expressed by preverbal particles and/or the placement of subject DPs in Celtic/Semitic and V2 languages. The next step will be to show how this account can be translated in terms of the featurebased account I have been developing in Chapter Three and the beginning of this chapter - in particular, the role of T-features and D-features in the derivation of sentences.

In the aim of configuring the structural underpinnings upon which such an account can be envisaged, I introduce the extended analysis of the architecture of the split-CP domain as conceived of in Poletto (2000b), Benincà \& Poletto (2004) and Jouitteau (2005). Whereas Rizzi (1997), in his split-CP analysis (as reviewed in section 2.1.2.3.2 and section 3.3.4), designates two TopP projections (one below FocP, and another above FocP), Poletto (2000b) and Benincà \& Poletto (2004), argue that the lower TopP is not instantiated in the architecture of the split-CP domain cross-linguistically. Instead, this lower part of the split-CP domain above FinP is 
argued to be a more articulated portion of the split-CP system for merging elements bearing some kind of focus (namely, what Poletto 2000b and Benincà \& Poletto 2004 designate as the 'Focus sublayer'). Thus, according to Poletto (2000b: 217) “there is no Topic position lower than the Focus layer". ${ }^{4}$

Jouitteau (2005) adopts Poletto's (2000b) approach as to the projection of the split-CP domain in Celtic and Semitic languages. The structure in (5) below is a representation of the split-CP portion below ForceP (which Jouitteau 2005: 126 adapts from Poletto 2000b: 236-237) in the languages Jouitteau (2005) is primarily interested in (the labels for the layers composing the split-CP system in between ForceP and FinP are based on Poletto's 2000b conception of the split CP-domain):

(5) [ForceP $\underbrace{\text { Hanging TopicP }[\text { Scene setting }}_{\text {'higher Topic sublayer' }}[$ Force

Neg [FinP... ]]]]]]]] $\underbrace{\left[\begin{array}{c}\text { FocPFocus }[\operatorname{Mod}(\mathrm{al}) \mathrm{P} \\ \text { [ower Topic }\end{array}\right.}_{\begin{array}{c}\text { TopP Topic } \\ \text { sublayer' }\end{array}}$

As (5) shows, the Focus sublayer projects in between ForceP and FinP and it contains a FocP projection and a ModP projection. The possibility of the interaction between negation markers and the tense/modality properties of sentences is suggested by having Neg (i.e., the structural position of negation markers in the split-CP domain) occupy the head position of the ModP projection. Then the 'lower Topic sublayer' projects above the Focus field. This lower Topic field is reserved for elements that can be resumed only by a resumptive clitic in the IP domain. As for the 'higher Topic sublayer', projecting above both the lower Topic field and the Focus field as represented in (5), it is reserved for Hanging Topics and 'scene setting' elements such as circumstantial adverbs.

As far as the head position the raised verb in VSO sentences in SA attaches to in the split-CP domain is concerned, Aoun et al. (1994: 204, footnote 8, see also Aoun et al. 2010) suggest that this head node is similar to Laka's (1990: 100) $\Sigma$ (accommodating both negation and emphatic affirmation) or a head F "whose effect is

\footnotetext{
${ }^{4}$ On this account, the non-existence of a lower TopP in SA, as suggested by Shlonsky (2000), would be in response to a constraint that states that Focus phrases need to be adjacent to the verb, as in the case of subject-verb inversion in wh-questions. As I suggest below, this constraint may be reformulated in terms of the C-T dependency and the requirement that 'finiteness' features (R\&R) associated with the verb have to be 'identified' in a process involving Criterial satisfaction where no real 'checking' or 'valuation' (in the sense of Chomsky 1995b, 2001, 2004) is at work. A low TopP projection will block that identification process. I return to a discussion of Topic DPs and focalized elements in the following sections.
} 
to focalize the verb" - namely, Focus. The evidence Aoun et al. (2010: 70-71) provide for the raising process of (auxiliary) verbs in SA to a higher node than the highest functional head in the IP domain - i.e., T - comes from existential constructions involving the locative pro-form hunaaka 'there', as in (6a), with a representation as in (6b) (Aoun et al.'s 2010: 71 (63)):
(6) a. kaan-a hunaaka Taalib-un fii l-Hadiiqat-i
PERF.be-3MS there student.INDEF-NOM in the-garden-GEN 'There was a student in the garden"

b.

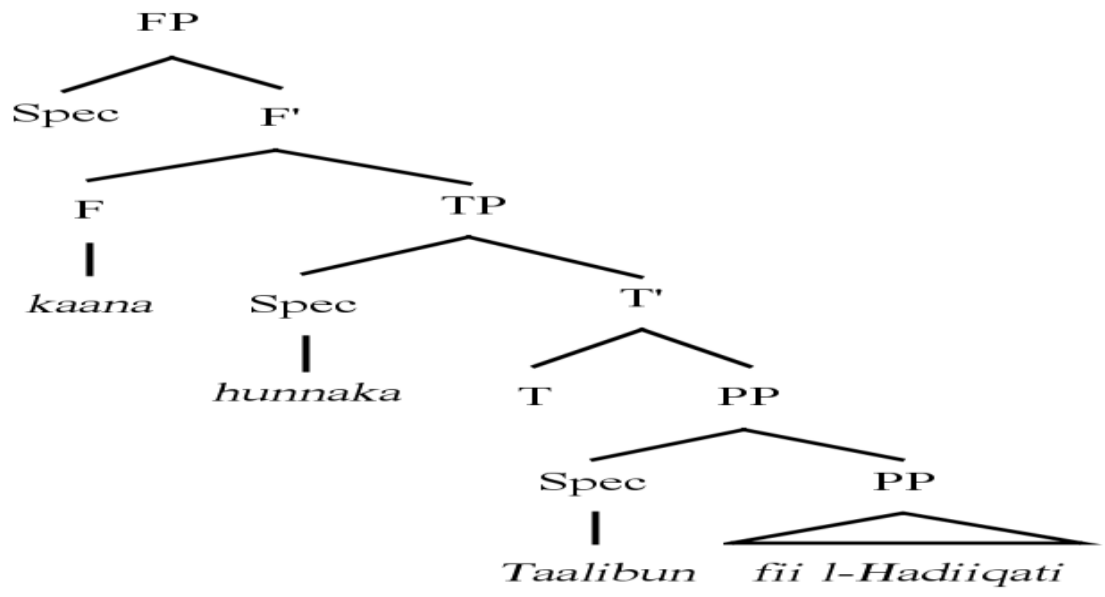

As (6b) shows, the functional head position the SA auxiliary verb kaana 'be.Past' in VSO word order occupies is higher than TP, the latter being for Aoun et al. (2010) the highest projection in the IP domain in such VSO structural configurations. The auxiliary verb kaana appears in $\mathrm{F}$ to the left of the locative pro-form hunnaka which is merged in [Spec, TP].

Aoun et al. (2010: 215-216) assume that FP, in SA, can be designated as Rizzi's (1997) FocusP. Aoun et al.'s (2010: 215-216) conception of the architecture of the split-CP domain differs from Rizzi's (1997) in that "FinP stands for TP; it is where the tense features are projected" (p. 215). Thus, in Aoun et al.'s (2010) conception of the architecture of the split-CP domain, the only position that projects above TP and Rizzi's (1997) lower TopP projection is the Focus head, which, for 
Aoun et al. (2010), is the position where the verb in SA VSO structural representations is spelled out. ${ }^{5}$

However, as reviewed below in connection with German V2 declarative clauses and Old Irish V-to-C configurations (Carnie et al. 1994, R\&R), the raising of $\mathrm{T}$ to a low head position in the $\mathrm{CP}$ domain preceded by other $\mathrm{X}(\mathrm{P})$ elements, such as raised subject DPs in V2 contexts in German and negation particles in VSO structural configurations in Old Irish, is possibly an indication that the verb raises to a position that is lower than Laka's (1990: 100) $\Sigma$ head node or Rizzi's (1997) Focus head position. ${ }^{6}$ As far as SA VSO configurations are concerned, in light of an extended cartographic analysis of the split-CP domain (as represented in (5) above, where a Modal head node projects higher than TP (and FinP) but below FocP), the verb does not move higher than modal particles and/or negation markers like qad and lam/lan (see the sentences in (15) and (16) below). ${ }^{7}$ Given this cartography, if verbs in SA move higher than T in VSO configurations as Aoun et al. (2010) propose, then the raised verb would still be in a lower head position than Focus.

On this account, I propose that the head the verb (auxiliary or lexical) raises to in SA VSO structural configurations is Fin. The representation for (6a) should thus be as in (7) below:

\footnotetext{
${ }^{5}$ In Aoun et al.'s (2010: 215-216) system, Rizzi's (1997) lower and higher TopP projections are reserved for Clitic Left Dislocated elements. Except for the structure in (6b) above, Aoun et al. (2010) do not provide an articulated structural representation of the split-CP system they adopt in its relation to the structure of IP.

${ }^{6}$ According to Laka (1990: 98-99), there is variation in the projection of $\Sigma \mathrm{P}$ cross-linguistically. Whereas, $\Sigma$ projects below $\mathrm{T}$ in English-type languages, it projects higher than $\mathrm{T}$ in Basque. As discussed above, Laka (1990) claims that $\Sigma$ houses both the head Neg and whatever head plays the role of 'emphatic affirmation' in syntactic structure. Note that this is also Jouitteau's (2005) standpoint on negation and modal markers as represented by structure (5) above. However, as discussed below, SA presents evidence that the Neg head (for negation markers like lam and lan) and the Mod head (for 'emphatic' modal markers like qad/laqad) should have separate projections in the split-CP system of SA.

${ }^{7}$ I discuss such VSO configurations in the sentence structure of SA in section 4.1.2.2 below.
} 


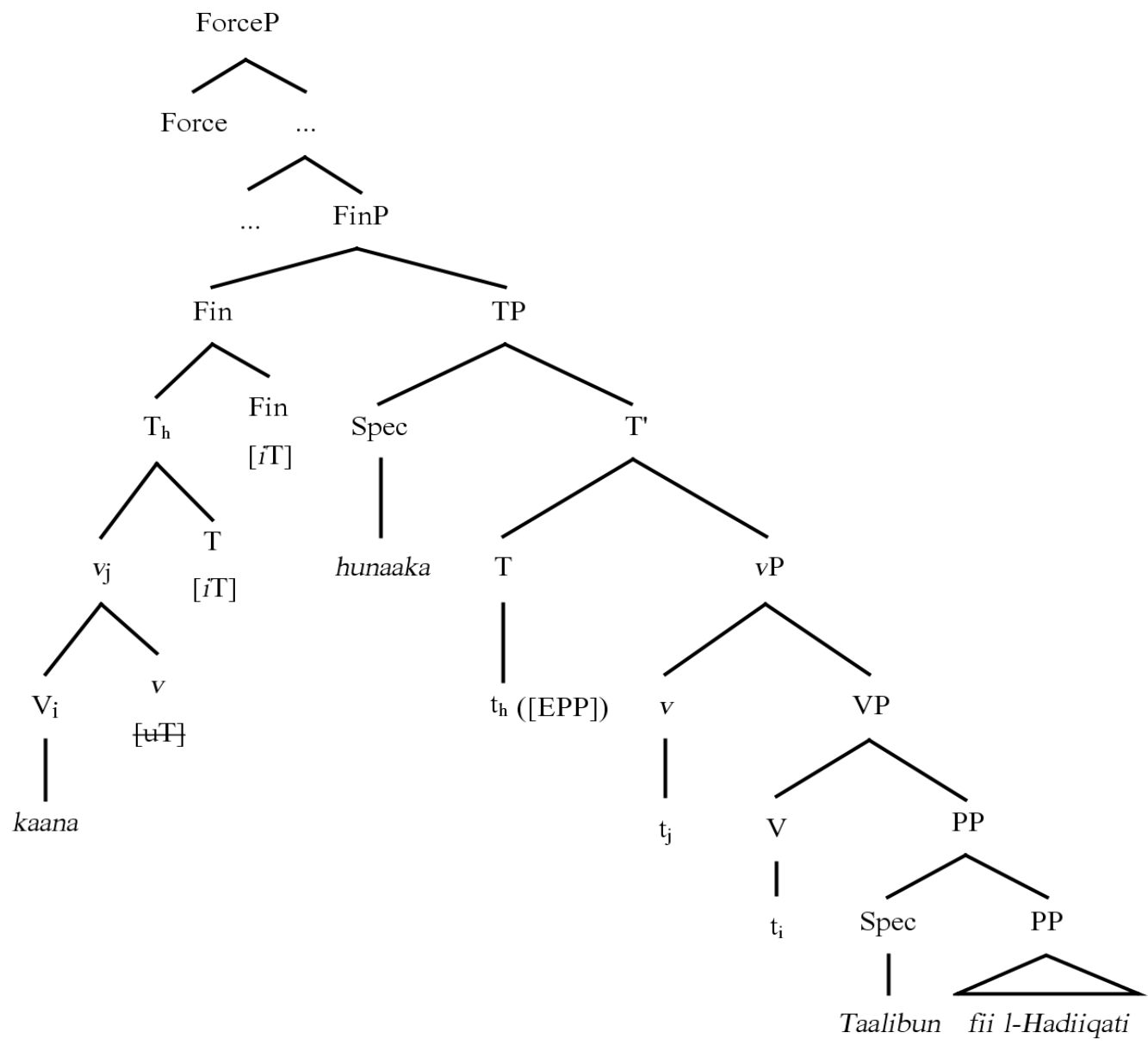

In (7), CP splits into ForceP and FinP as the two main projections at the opposite sides of the split-CP domain (what Rizzi 1997: 296-297 calls the ForceFiniteness system). Disregarding, for the moment, the Topic-Focus system (designated by the ellipsis '...' in (7)), the auxiliary verb kaana 'be', first merged under $\mathrm{V}$ in the SA structure in (7), subsequently adjoins to an empty $v$ position. Then the verbal complex $\mathrm{V}-v$ raises to $\mathrm{T}$ before being raised to Fin. As discussed in section 3.3.2.3, the relevant feature that governs the dependency relation that exists between verbs - auxiliary or lexical - and T would be a T-feature (contra Benmamoun's 2000b, 2008 assumptions about the relevance of some [+V] feature on T). Thus, the auxiliary verb kaana, being merged directly under $\mathrm{T}$, would be endowed with an $[i \mathrm{~T}]$ feature matching the $[i T]$ feature of $\mathrm{T}$, and thus, satisfying the condition on the identification of tense features in a sentence like (6a) with representation (7). As for the raising of $\mathrm{T}$ (with $\mathrm{V}-v$ attached to $\mathrm{T}$ ), assuming that whatever features $\mathrm{T}$ has are 
passed down from Fin (Chomsky 2008) to T, it might be the case that the $[i \mathrm{~T}]$ feature on Fin, shared with T, is 'rich' enough to cause $[\mathrm{T}-v-\mathrm{V}]$ to raise to Fin in SA (cf. 'richness' of agreement features in Roberts' 2010a: 164 account of V-to-T movement in V-raising languages).

The analysis of Aoun et al. (2010: 70-71) as to the position of auxiliary kaana in $(6) /(7)$ in some F position higher up to the left of TP/IP should carry over to the position of the negative auxiliary laysa at Spell Out as represented in (8b) below for sentence $(8 \mathrm{a}):^{8}$

(8) a. lays-a hunaaka Taalib-un fii 1-Hadiiqat-i

NEG-3MS there student-NOM in the-garden-GEN

"There is not a student in the garden."

b.

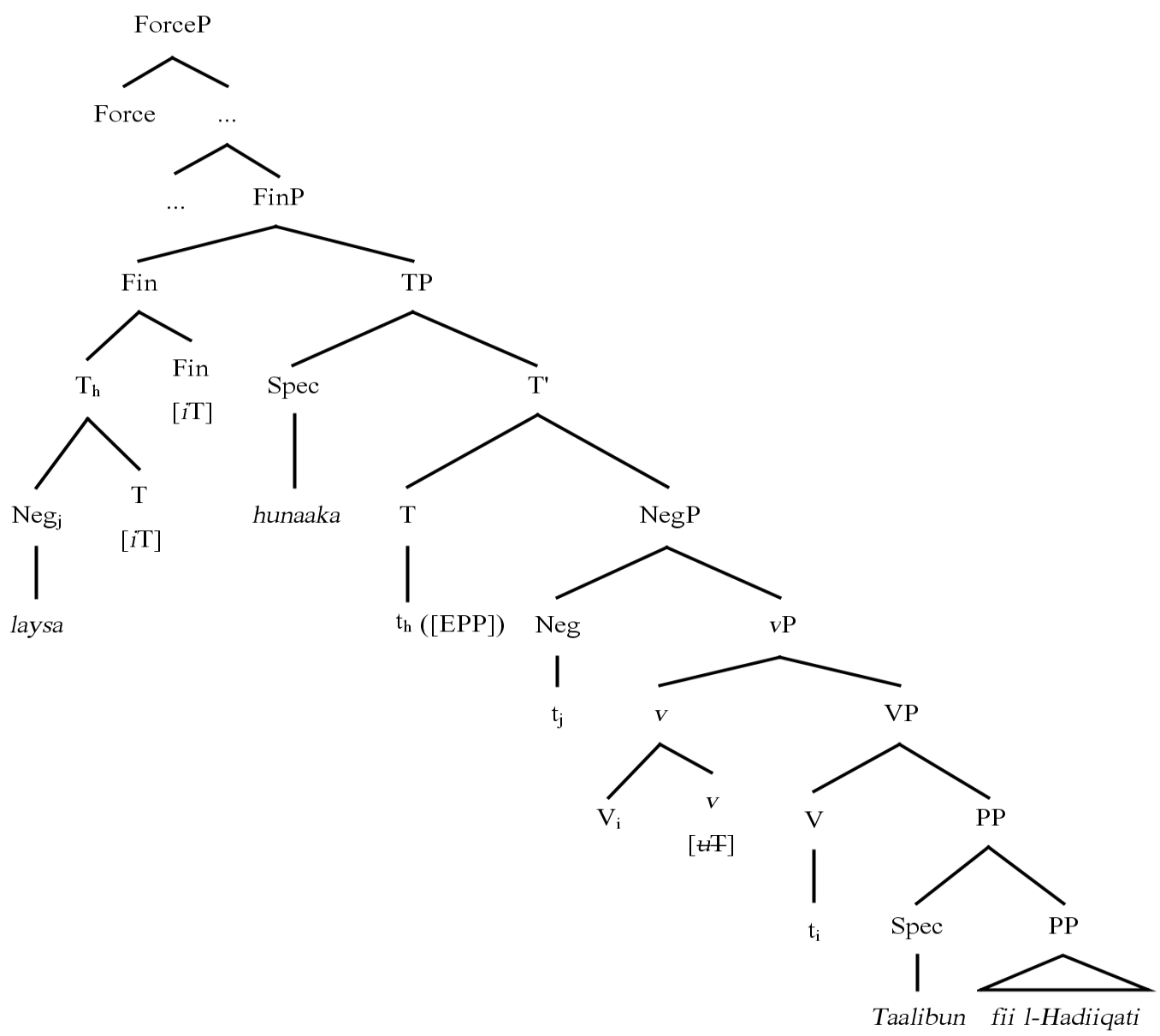

\footnotetext{
${ }^{8}$ I come back to the feature structure of such NEG elements as laysa, maa, and the 'tensed' NEG markers laa/lam/lan in section 4.1.2.5.
} 
In (8b), laysa is inserted under Neg and raised to T. Then the [Neg-T] complex is raised to Fin above TP. As structure (8b) shows, the structural position of laysa to the left of the locative pro-form hunaaka 'there' in subject position in [Spec, TP] is the same as the position of auxiliary kaana to the left of the the locative pro-form hunaaka 'there' in subject position in [Spec, TP] in (6)/(7). As discussed in section in section 3.3.2.3, the adjunction of $\mathrm{V}$ to the empty $v$ position systematically occurs in the derivation of copular sentences irrespective of the presence or absence of auxiliary kaana in the Numeration.

This analysis, based on the relevance of $\mathrm{T}$-features on functional projections as the driving force of computations, goes against assumptions made in Benmamoun (2000b: 55) as regards the derivation of negative sentences with laysa, which are based on the importance of $[+\mathrm{V}]$ features in the derivation of verbal inflection in SA, and perhaps, universally (Benmamoun 2000b: 160, footnote 13; see section 3.3.2.3). Benmamoun's (2000b: 55) argument goes as follows: The Neg element laysa, being marked for 'past tense' morphology (which is, nonetheless, not related to a past tense interpretation of the situation described, as the English gloss in (8a) shows), cannot move to $\mathrm{T}$ because $\mathrm{T}$ (being [+Present]) is not marked as [+V]. Thus, laysa "does not need to move to tense given that there is no $[+\mathrm{V}]$ feature that would drive this movement" (p. 56). Benmamoun (2000b: 56) concludes that the Neg element laysa remains in between $\mathrm{T}$ and $\mathrm{V}$ - namely, under the Neg head projected in the IP domain. However, as (8b) shows, the Neg element laysa is clearly above the highest functional projection within the IP domain - namely, TP.

Thus, after being raised from $\mathrm{V}-v$ to $\mathrm{T}$, kaana in (7b) raises to Fin. A similar raising process to Fin applies in the case of laysa in (8b) subsequent to the raising of laysa from Neg to T. The raising of kaana, in (7b), and laysa, in (8b), to Fin satisfies the identification requirement on Fin's $[i T]$ feature shared by the Finiteness head in the split-CP domain and the Tense node in the IP domain. On this account, the dependency relation between kaana and laysa and the functional heads $\mathrm{T}$ and Fin in $\mathrm{SA}$ is tied up with the identification of $[i \mathrm{~T}]$ features for the proper representation of the C-T dependency at the interface.

The T-to-Fin raising process, via which (auxiliary) verbs move to $\mathrm{C}$, is also assumed to be operative in other languages. $R \& R$ propose that the derivation of $V 2$ declarative sentences in German and VSO sentences in Old Irish involves the raising 
of inflected verbs or auxiliaries to Fin. In R\&R's (p. 140) proposal, this raising process is triggered by a [+Fin] feature on the host node.

As far as VSO sentences in Irish are concerned, Carnie et al. (1994: 90-92) discuss such examples as in $(9 a, b)$, with the bracketed representations in $(10 a, b)$ (adapted from Carnie et al.'s 1994: 92 examples (25) and (26)):

(9) a. Beirid in fer in claideb carries(ABSOLUTE) the man the sword

(Old Irish) 'The man carries the sword.'

b. Ní beir in fer in claideb.

NEG carries(CONJUNCT) the man the sword

'The man does not carry the sword.'
(10) a. [CP [C Beiridi
b. [CP [C Ní [IP beir ${ }_{i}+$ INFL [vp in fer [vp $t_{i}$ in claideb]]]]]
NEG carries(CONJUNCT) the man the sword

According to Carnie et al. (1994: 92), the verb moves to the C position in (10a). In this case, the verb is inflected for the absolute morphology beirid. In (10b), the C position is occupied by the negative particle ní. In this case, the verb is assumed to be in a lower position than C "at the edge of IP" and the inflection on the verb is a more basic conjunct morphology (e.g., beir in (10b)).

On the basis of such sentences (9a,b), Carnie et al. (1994: 86-87), argue that in Old Irish two alternative hypotheses - namely, the Weak V2 Hypothesis and the Left Edge of Inflection Hypothesis - could be put forward for the derivation of Irish VSO sentences. ${ }^{9}$ The first hypothesis is spelled out as in (11):

(11) The Weak V2 Hypothesis $\left(\mathrm{V} \rightarrow \mathrm{C}^{\circ}\right)$

VSO order is derived via head movement of the verb to $\mathrm{C}^{\circ}$. There is a requirement that $\mathrm{C}^{\circ} \mathrm{S}$ in VSO languages be filled, but the specifier of $\mathrm{C}$ need not be filled.

According to the hypothesis in (11), a possible derivation of a VSO sentence in a VSO language would be as in (12) below:

\footnotetext{
${ }^{9}$ Carnie et al. (1994) argue that the raising of V-to-C is only attested in Old Irish (see example (9a) above). Modern Irish VSO structural configurations may be different (but see the discussion in relation to (18) below).
} 
(12) [V-T/INFL] to $\mathrm{C}$ in Irish

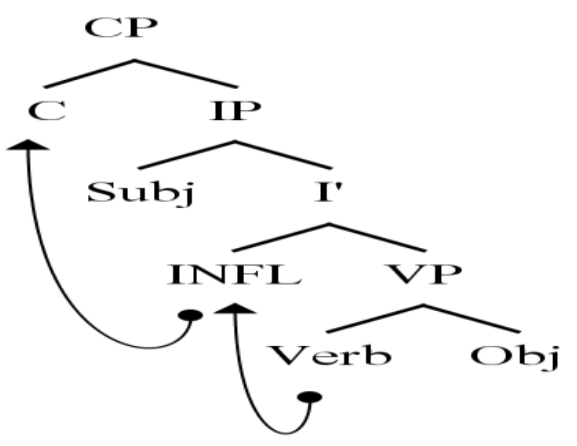

In (12), the verb moves to INFL in its way to C, leaving the subject behind in [Spec, IP]. Although Carnie et al. (1994: 86-87) propose that (12) is a possible derivation for sentence (9a), they note that they remain agnostic as to the actual placement of the subject (i.e., either in [Spec, VP] or in [Spec, IP]). As for the evidence Carnie et al. (1994: 90-92) propose for the so-called 'filled $C^{\circ}$ requirement', it comes from the placement of enclitic pronouns (object clitics and relative markers) in the conjunct form of the verb as opposed to their absence when the verb is in the absolute form. In Carnie et al.'s (1994: 92) own words: "when the verb has raised to $\mathrm{C}^{\circ}$ it takes the absolute morphology. When the verb is in any other position ..., it takes the more basic conjunct form". Carnie et al. (1994: 92) also assume that the raising of V to C is actually an incorporating process whereby $[\mathrm{INFL}+\mathrm{V}]$ incorporates into a null $\mathrm{C}^{\circ}$ that is morphologically realized differently from the conjunct form of the verb (see the examples in (9)/(10) above). They argue that, in absolute first position, the verb forms "vary depending upon what type of complementizer is present in the clause ...

lend[ing] support to the theory that these verbs are in fact in $\mathrm{C}^{\circ \%}$.

The second hypothesis Carnie et al. (1994: 87) put forward is the Left Edge of Inflection Hypothesis, as spelled out in (13):

(13) The Left Edge of Inflection Hypothesis (V $\rightarrow$ INFL)

VSO order is derived via head movement of the verb to the highest inflectional head $\left(\mathrm{Agr}_{\mathrm{S}}\right)$. Arguments appear in surface positions lower than this head. There is no (overt) raising to $\mathrm{C}^{\circ}$

According to this second hypothesis, the derivation of a VSO sentence in a VSO language could be as in (14) below: 
(14) V to T/INFL in Irish

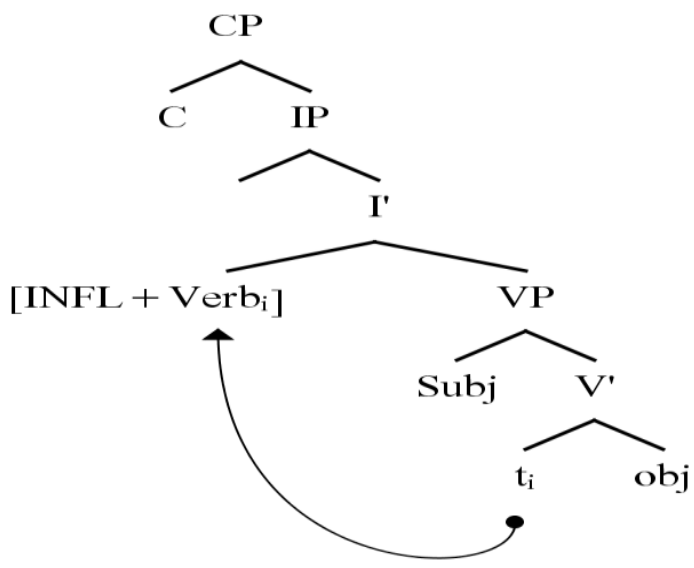

In (14), the verb remains lower than C - namely, under INFL. Although Carnie et al. (1994) refer to $\mathrm{Agr}_{\mathrm{S}}$ in (13) as the highest inflectional head in the IP domain, they use INFL in (14) as a shorthand to represent the highest inflectional node in the IP domain. According to Carnie et al. (1994), this structure accounts for the negative sentence $(9 b)$ where what occupies $\mathrm{C}$ is the negation marker ní.

In such a negation context, R\&R (p. 143) argue that the Old Irish Neg element $n i ́$ is directly merged in the $\mathrm{C}$ position where "what is merged are inflectional features (these features might be thought of as comparable to the marked clause-type features)". R\&R's (p. 143) reference to 'inflectional features' is significant in pointing out that, although these features are essential in the identification of the C-T dependency, they remain different from the features involved in clause typing. ${ }^{10}$

However, such an analysis of negation contexts in Irish does not, in itself, rule out the possibility that verbs are still able to raise to $\mathrm{C}$ in such negation contexts as (9b). On such an account, negation markers such as ní, in Old Irish, would be merged in a separate projection to the left of the verb raised to $\mathrm{C}$.

Such an assumption about the feature structure of negative sentences in relation to a VSO language like Old Irish is also relevant to the derivation of VSO sentences in SA, as in (15) and (16) below:
(15) a. lam
yaktub-i
T-Taalib-u
d-dars-a
NEG.PAST 3.IMPERF.write-MS.JUSS the-student-NOM the-lesson-ACC
'The student did not write the lesson.'

\footnotetext{
${ }^{10}$ In section 4.1.2.4, I return to a detailed characterization of the feature structure of the head nodes that project in the split-CP domain of SA VSO configurations in relation to clause type and to the 'inflectional features' R\&R (p. 143) link to Fin and NEG elements, in particular.
} 

b. lam
yaktub-i
T-Tullaab-u
d-dars-a

NEG.PAST 3.IMPERF.write-MS.JUSS the-students-NOM the-lesson-ACC

'The students did not write the lesson.'
(16) a. lan
yaktub-a
T-Taalib-u
d-dars-a

(SA)

NEG.FUT 3.IMPERF.write.MS.SUBJ the-student-NOM the-lesson-ACC

'The student will not write the lesson.'
b. lan
yaktub-a
T-Tullaab-u
d-dars-a

NEG.FUT 3.IMPERF.write-MS.SUBJ the-students-NOM the-lesson-ACC

'The students will not write the lesson.'

As already discussed in section 3.3.3, the negation marker lam encodes past tense, which cooccurs with the imperfective verb in the jussive as in (15a,b). As for the negation marker lan, it encodes future tense, which cooccurs with the imperfective verb in the subjunctive as in $(16 a, b)$. In terms of the tree structure in (7) above, the Neg node would project in between ForceP and FinP in Rizzi’s (1997) architecture for the split-CP domain. Thus, the structure of a negative sentence like (15a), for example, would be as in (17): ${ }^{11}$

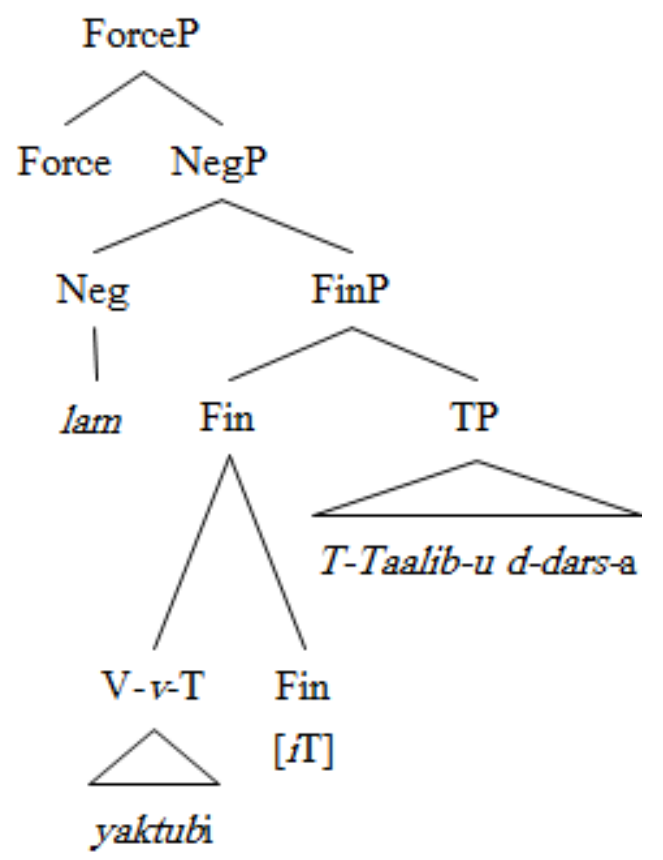

\footnotetext{
${ }^{11}$ In contrast to laysa, which starts out below T (see the dicussion in relation to (8) above), laa and its 'temporal' variants lam and lan are assumed here to be merged higher than T in a second Neg head projection (see section 4.1.2.5).
} 
On this account, in the VSO sentences (15) and (16), the feature that seems to be involved in the 'identification' of the Neg elements lam and lan is a tense feature - namely, the $[i \mathrm{~T}]$-feature of the Fin head projection in (17) with which negation, as a head that projects in sentence structure in its own right, interacts. The same is true of the relation of the Neg elements lam and lan and the T node. Under such assumptions, the identification of $[i \mathrm{~T}]$-features on Fin and $\mathrm{T}$ is essential for tense interpretation at the interface. The same raising and identification processes apply in the derivation of sentences where a Mod(ality) (and/or Foc) head projects in the split-CP domain. ${ }^{12}$

In a split-CP system of the kind argued for by Poletto (2000b), Benincà \& Poletto (2004) and Jouitteau (2005), as discussed above, both the raised verb and the Neg element, in a negative context such as Old Irish (10b), could be assumed to be in the CP domain at Spell Out where the Neg element $n i$ would be merged in a higher head position than the position of the raised verb in Fin. This conception of the splitCP domain could provide an alternative view to Carnie et al.'s (1994: 92) proposal that the verb exhibiting the conjunct form, in Irish, is not in C (= Fin).

On this account, the plausibility of an analysis of VSO structural configurations whereby the T-to-Fin raising process would apply uniformly in VSO languages of the Celtic and Semitic type (cf. Jouitteau 2005) and in languages exhibiting the V2 phenomenon (cf. Schwartz \& Vikner 1996, R\&R) could still be maintained.

An argument for such a [V-T]-to-C analysis in Modern Irish could also be based on McCloskey's (2001: 159) conception of sentence structure where three XP positions are clearly outside of VP, as represented in (18):

\footnotetext{
${ }^{12}$ Mod projects in the Focus sublayer above Fin, as repesented in structure (5) above (repeated here for convenience):

(i) [ForceP [Hanging TopicP [Scene setting [Force [TopP Topic [FocP Focus [Mod(al)P Neg [FinP $\ldots$ ] ] ] ] ] ] $]$ In SA, structure (i) would incorporate two separate functional projections above FinP: Neg(P) projecting above FinP, and $\mathrm{Mod}(\mathrm{P})$ projecting above NegP, as represented in (ii):

(ii) [Forcep [Force [TopP Topic [FocP Focus [ModP Mod [negP Neg [FinP ... ] ] ]]]]] the split-CP domain as far as the distribution of modal and negation elements in SA is concerned.
} 


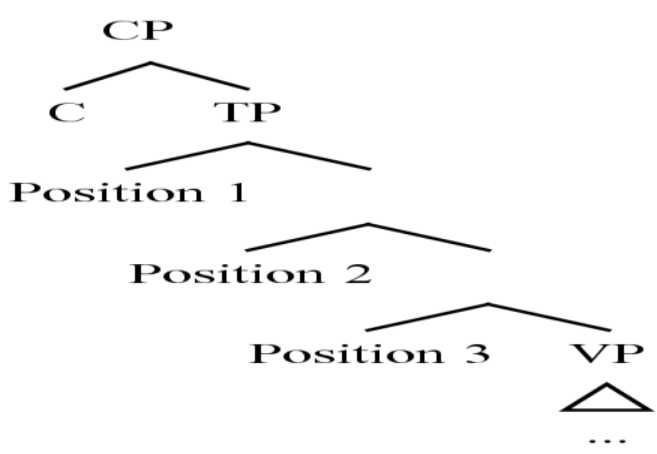

Position 1 of structure (18) is the canonical position for expletives crosslinguistically. ${ }^{13}$ Position 2 harbours NOM Case-marked DPs. Position 3 harbours ACC Case-marked DPs. McCloskey (2001: 159) suggests that, in Irish, "the finite verb occupies a position between Position One and Position Two". However, if the position of an expletive like $P a ð$ 'there' in Icelandic, for example (see footnote 13), could be assumed to be higher than position 1 in (18), McCloskey's (2001) cartography of the inflectional domain could still support an analysis whereby Irish verbs, in VSO sentences, raise above position 1 and position 2 to $\mathrm{C}$ (= Fin, in the split CP system considered here) yielding the VSO word ordering.

In light of such a cartography, Carnie et al.'s arguments for both a V-to-I/T and a V-to-C analysis of VSO sentences in Irish, discussed above, could be reformulated in terms of an account whereby verbs in VSO sentences in Irish

\footnotetext{
${ }^{13}$ McCloskey (2001: 159) gives the example of transitive expletive constructions in a V2 language like Icelandic, as in (i):

(i) Pað klaruðu margar mýs ostinn alveg (Icelandic) there finished many mice the-cheese completely 'Many mice completely finished the cheese'

According to McCloskey (2001: 159), the expletive $P a ð$ 'there' is in Position 1 of structure (18). Position 2 is occupied by the raised NOM subject margar mýs 'many mice', and Position 3 by the raised object ostinn 'the-cheese'. However, another account of such sentences is Alexiadou \& Anagnostopoulou's (2001: 209-212) account, reviewed in section 3.2.1.1, whereby expletives in transitive expletive constructions in Icelandic are higher than TP (i.e., in [Spec, CP]), as represented in (ii):

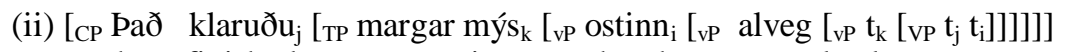
there finished many mice the-cheese completely 'Many mice completely finished the cheese'
}

Thus, in the event that $\mathrm{Agr}_{\mathrm{S}} \mathrm{P}$ does not project in such V2 constructions in Icelandic, both the expletive and the verb would be higher than TP, i.e., in the CP domain as structure (ii) shows. 
(Modern or Old Irish) raise to a position similar to the position of their counterpart in SA. In the event that Irish VSO word ordering patterns like SA VSO word ordering as far as the absence of expletive pro in the IP domain is concerned, ${ }^{14}$ verbs in both VSO types (i.e., Celtic and Semitic) could be in Fin in the split-CP domain.

On this account, the claim I am putting forward for a uniform cartography of the structure of VSO sentences in Celtic/Semitic languages with 'basic' VSO word ordering (possibly, extending to matrix V2 declarative contexts) would be based on the requirement of $[i T]$-feature identification at the interface in such structural contexts. This uniform cartography would configure the verb, first raised to T, in Fin at Spell Out.

In the subsequent sections, I will revisit the expanded structure of the split-CP domain, as conceptualized by Poletto (2000b), Benincà \& Poletto (2004) and Jouitteau (2005) (see (5) above). In this respect, I will show that the Neg node that projects above FinP in SA is just one instantiation of a number of similar nodes forming the lower Focus sublayer for elements bearing some modal and/or focus properties in relation to the tense/finiteness properties of sentences. However, unlike for Jouitteau (2005), NegP would be yet another instantiation of a Focus projection separate from ModP in SA (see section 4.1.2.5).

In particular, in sections 4.1.2.2 and 4.1.2.3, I show how Jouitteau's (2005) Modal head should be reserved, in SA, to such emphatic elements as the modal element $q a d$ and the presentative assertive element $l a$, which can co-occur with qad forming one morphologically realized lexical item - namely, 'laqad'. ${ }^{15}$ In section 4.1.2.4, I assume that the assertive elements $l a$ and laqad are merged under the Foc head in the split-CP domain, which would project, in affirmative contexts, as the

\footnotetext{
${ }^{14}$ See Jouitteau (2005: 387) for an argument that satisfaction of the EPP via expletive pro insertion could still be assumed to be operative in Modern Irish, even in the 'salient unaccusatives' on the basis of which McCloskey (1997) argued against the existence of the EPP in Irish. If Jouitteau (2005) is right in linking VSO word ordering to expletive pro insertion in Modern Irish, then the derivation of Vinitial sentences in Modern Irish would constitute a simple case of V-to-T movement with no further raising to $\mathrm{C}$ in contrast to the SA VSO word ordering and to the V2 phenomenon with subject DPs in German. I have previously assumed that, in SA VSO word order, expletive pro is not merged in the structure at the IP level due to the absence of $\operatorname{Agr}_{S}(\mathrm{P})$ in such a configuration. Nevertheless, the EPP remains a universal principle in the analysis advocated here, which can either be satisfied in [Spec, TP], as in the VSO configurations discussed above, or in [Spec, $\mathrm{CP} / \mathrm{FinP}]$, as in expletive transitive constructions in Icelandic and other matrix V2 contexts with expletives or raised subject DPs in [Spec, FinP]. In section 4.3 below, I return to such matrix declarative V2 contexts involving a subject DP referential or expletive - in German.

${ }^{15}$ See structures (37b) and (38b) in section 4.1.2.4.
} 
counterpart of the negation particle maa merged under the Foc head in negative contexts. I also assume that the head of ModP and the head of NegP would both interact with verbal inflection (mainly, tense represented as $[i \mathrm{~T}]$-features on both $\mathrm{T}$ and Fin, in terms of the C-T dependency and the feature structure of functional elements adopted in this thesis; see section 4.1.2.4). ${ }^{16}$

In addition to the head-head agreement processes involved in the 'identification' of the inflectional properties of sentences in VSO languages in relation to the placement of negation and other modal elements with respect to verb movement, the feature-based account adopted here also relies on a Spec-head agreement relationship for the derivation of wh-dependencies and/or operator-variable relationships involved in Focus and Negation preposing.

In this respect, as dealt with in section 4.2 below, I adopt an analysis of the licensing conditions under which wh-dependencies are represented at the interface in terms of an additional [WH]/Focus feature, different from clause-type features on Force and Fin's $[i \mathrm{~T}]$ feature(s), which will account for the XP raising process involved in the derivation of these dependencies. In these instances, my assumption is that two feature-matching processes seem to be at work. In addition to the identification of $[i \mathrm{~T}]$ features for the well-formedness of the C-T dependency at the interface, the relationship between the wh-element and the Focus head is established in terms of the morpho-syntactic 'checking'/valuation of the relevant features namely, interpretable vs. uninterpretable [WH]-features. The valuation of these features establishes head-head and Spec-head agreement relations in terms of which the C-T dependency is identified at Spell Out.

\subsubsection{The interaction of tense and modality in the derivation of SA VSO sentences}

In the aim of pinning down the structural distribution and function of preverbal particles and markers in relation to raised verbs in SA VSO sentences, in this section, we first have a look at the head (= Force) that bears the function of signalling "clausal type' in the split-CP system (Rizzi 1997: 283). The derivation of VSO sentences will be viewed as the outcome of the interaction of these particles and markers with the

\footnotetext{
${ }^{16}$ In section 4.1.2.5, I also develop my proposal for the position of NegP within the sentence structure of SA where two different structural positions seem to be justified in accordance with the parameterized probe-goal-Agree system of dependency relations assumed in this thesis.
} 
modal/tense properties encoded by verbs. The potential projection of a Topic Phrase in VSO sentences in matrix contexts will also be discussed.

In SA, Force can be lexically realized even in a matrix context, whereby the complementizer Pinna is externally merged under Force. ${ }^{17}$ In matrix declarative contexts, Pinna is optional irrespective of word ordering possibilities (i.e., whether the sentence is VSO or, alternatively, SVO). In embedded contexts, Pinna (or one of its variants, see footnote 17) has to be merged in the structure of the sentence to introduce the embedded clause.

As far as matrix contexts without complementizer Pinna in the VSO word ordering are concerned, the split-CP domain of the VSO sentence (19a) would include the projection of FinP and ForceP above TP, as structurally represented in (19b):

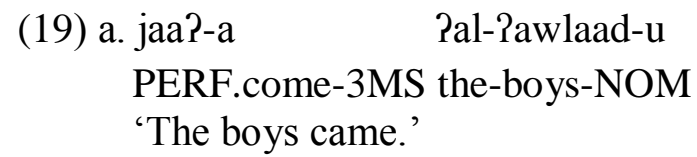

\begin{abstract}
${ }^{17}$ In embedded contexts, the form of the declarative complementizer differs according to the type of verb introducing the embedded clause. Verbs of request or command such as sa?ala 'ask' or ?araada 'want' take as complement a clause introduced by the complementizer ?an. The imperfective verb of the complement clause selected by verbs of request/command takes the subjunctive form as in (ia,b) below:
\end{abstract}
(i) a. ?amar-a-hu
Pan ya-fial-a
haadhaa
PERF.order-3MS-him that 3-IMPERF.do-MS.SUBJ this
'He ordered him to do this.'
b. Pamar-a-hu Pan laa ya-fial-a haadhaa
PERF.order-3MS-him that NEG 3-IMPERF.do-MS.SUBJ this
'He ordered him not to do this.'

When used in embedded contexts, the complementizer Pinna is selected by the verb qaala 'say':

(ii) qaal-a RaHmad-u Pinna l-walad-a kaan-a fii 1-Hadiiqat-i

PERF-say-3MS Ahmad-NOM that the-boy-ACC PERF-be-3MS in the-garden-GEN

'Ahmad said that the boy was in the garden.'

All other verbs (with the exception of verbs of request/command, as mentioned above) select a clause introduced by the complementizer Panna. ECM verbs like PiStaqada 'believe' and Hasaba 'think, believe' can take a complement clause introduced by ?anna or a clause which is the counterpart of a 'raising to object' structure in English. Thus, sentences with ECM verbs in SA are as in (iiia) or (iiib):

(iii) a. PiStaqad-tu Panna r-rajul-a qad jaa?-a Pamsi

PERF.think-1S that the-man-ACC MOD PERF.come-3MS yesterday 'I thought that the man came yesterday.'
b. Pistaqad-tu
r-rajul-a
qad jaa?-a
Pamsi
PERF.think-1S the-man-ACC MOD PERF.come-3MS yesterday
'I thought that the man came yesterday.' 
b.

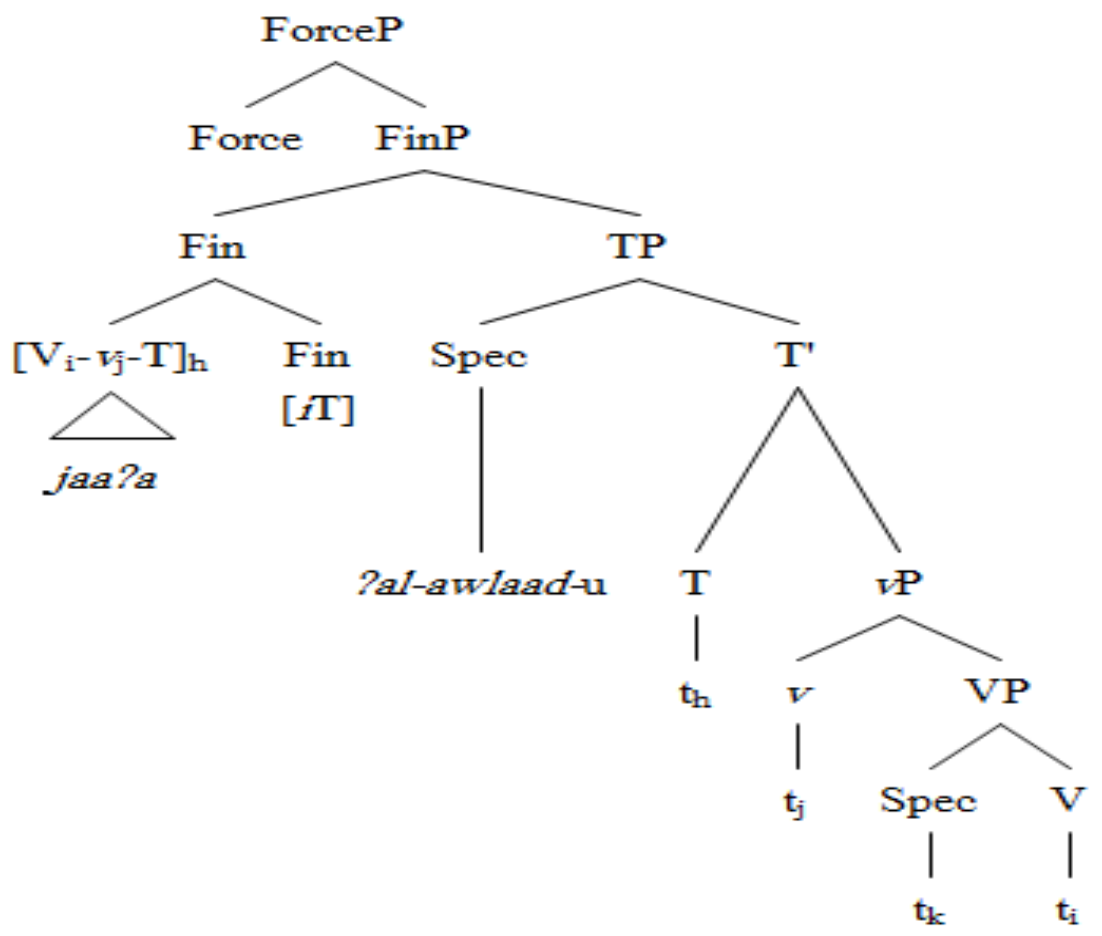

Although in (19b) complementizer Pinna is not part of the structure, the assumption is that the $\mathrm{CP}$ domain still splits into Force $(\mathrm{P})$ and Fin $(\mathrm{P})$. However, no other functional head is required to project in such a structure, and the Force head remains empty. The verbal inflectional complex V- $v$-T, realized as the 'partially' inflected verb jaa? $a$ 'came', raises to Fin.

Another matrix VSO word order configuration is a structure where the Force node is lexically realized by Pinna, as represented in (20b) for sentence (20a):

(20) a. Pinna-hu jaa?-a Pal-Pawlaad-u that-it PERF.come-3MS the-boys-NOM 'The boys came." 
b.

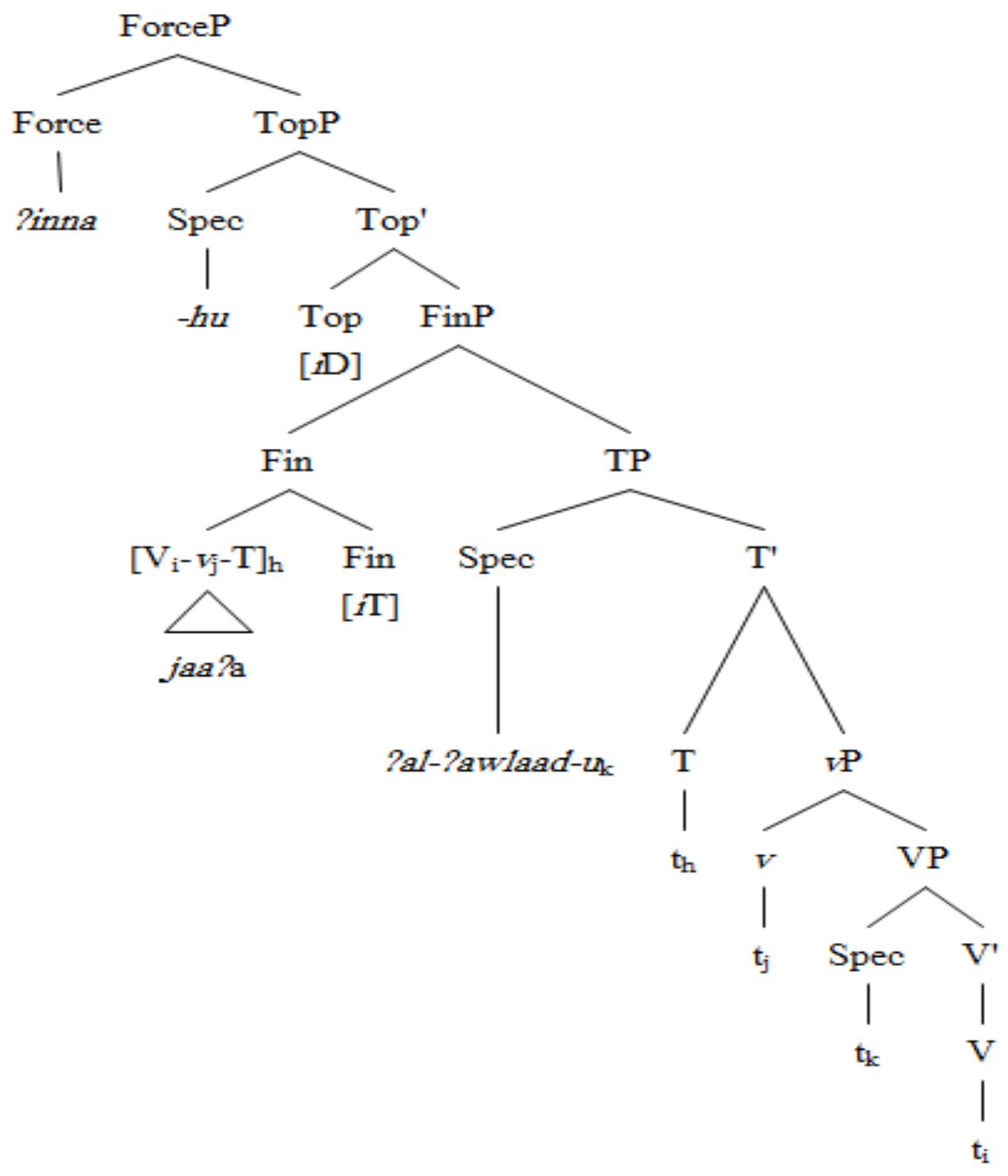

In (20b) (similarly for (21b) below), in case the matrix complementizer ?inna is merged under the Force head an EXPL pronominal clitic $-h u$ has to be inserted into the structure and a TopP is necessarily projected. Recall from section 3.3.6 my proposal that such an EXPL element is base-generated in [Spec, TopP] and that it phonologically cliticizes to the complementizer Pinna to its left. In terms of the feature structure adopted here, the insertion process of $-h u$ in [Spec, TopP] could be conceived of as the realization of a 'higher predication' in the split-CP domain (Rizzi 1997 ) whereby a D-feature (namely, $[i \mathrm{D}]$ on Top) is involved in a Spec-head agreement relation in association with the subject DP of the clause (cf. Rothstein 


\title{
1983, 1995 conception of syntactic predication) ${ }^{18}$ In structure (19b), however, no
}

complementizer is lexically realized, and the cliticization process cannot be realized.

\begin{abstract}
${ }^{18}$ The role Top plays as a 'higher predication' node in the split-CP domain is comparable to syntactic predication as expressed by TP and VP in the inflectional domain of sentence structure (Rothstein 1983, 1995). Thus, elements inserted in [Spec, TopP] are assumed to be resumed by some pronominal resumptive element in the IP domain. These pronominal elements, resuming subject and object DPs or other XP elements, are clitic-like (see section 2.1.2.3 for the view, adopted in this thesis, that subject agreement morphology on SA verbs may be an instance of a pronominal clitic).

According to Benincà \& Poletto (2004: 64-65), the two Topic positions that project higher than the Focus field (Hanging Topics, or HT, and Dislocated Topics, or LD) have different properties. HT, which projects higher than complementizer elements, allows the resumptive element to be a tonic pronoun or a full DP. This is not the case for LD, which projects lower than complementizer elements. However, it seems that, in SA, the interpretation of a Topic element (either LD or HT) higher than a complementizer is not distinct from a Topic element lower than the complementizer, as there seems to be no significant difference in interpretation between the position of these elements to the right or to the left of the complementizer in that language:
\end{abstract}
(i) a. Pinna haadhaa r-rajul-a
laa Pa-Srif-u-hu
that this the-man-ACC NEG 1-PERF.know-MS.IND-him
'The fact is that, this man, I do not know him.'
b. haadhaa r-rajul-u ?inna-nii laa ?a-Srif-u-hu
this the-man-NOM that-I.ACC NEG 1-PERF.know-MS.IND-him
'This man, the fact is, I do not know him.'

In both (ia) and (ib), the object Topic DP haadhaa r-rajul-u/-a 'this man' is resumed by an object resumptive pronoun - $h u$ on the verb ?a-Srif- $u$ 'I know'. The ordering between the Topic DP and the complementizer Rinna appears to be free. When the Topic element is an adverbial co-occurring with the object Topic, the same free ordering possibilities obtain:
(ii) a. haadhaa r-rajul-u
fii 1-Hadiiqat-i
Pinna-nii qaabal-tu-hu
(fii-ha) (SA)
this the-man-NOM in the-garden-GEN that-I-ACC PERF.meet-1S-him (in-it)
'This man, in the garden, the fact is, I met him there/in it'
b. fii l-Hadiiqat-i haadhaa r-rajul-u ?inna-nii qaabal-tu-hu (fii-ha)
c. Pinna haadhaa r-rajul-a fii l-Hadiiqat-i qaabal-tu-hu (*?fii-ha)
d. Rinna-nii fii l-Hadiiqat-i haadhaa r-rajul-u qaabal-tu-hu (*?fii-ha)

In (ii), the ordering possibilities between the object Topic, the complementizer Rinna, and the adverbial appear to be free. These 'free' ordering possibilities are possibly due to the non-distinctness of Topic elements in the split-CP domain of SA. However, a difference in acceptability can be detected between the resumption of these topicalized elements within the sentence. Whereas, an object Topic has to be resumed by a resumptive pronoun on the verb ( $-h u$ in the examples above), this is not the case with adverbials. In (iia,b), the inflected preposition fii-ha 'in-it', resuming the prepositional adverbial fii $l$ Hadiiqat $-i$ 'in the garden', higher than the complementizer Rinna, can be left out. In (iic,d), the presence of the inflected preposition, resuming the prepositional adverbial fii l-Hadiiqat $-i$ ' in the garden', seems to render the sentences less acceptable. This minimal contrast between (iia,b) and (iic,d) is interesting in so far that it constitutes a parallel distinction to Benincà \& Poletto's (2004) differentiation between the properties of $\mathrm{HT}$ and $\mathrm{LD}$ in terms of the nature of the resumptive element $\mathrm{HT}$ and LD allow.

Irrespective of the presence or absence of a resumptive process in structures similar to (iid), Ayoub (1981: note 43 to Chapter 1; quoted in Shlonsky 2000: 333, footnote 9) observes that whenever an adverbial PP follows Rinna/ Ranna, as in (iii) below, the sentence becomes "peu productive" or less acceptable: 
As already introduced in the previous section, there is also the option, in SA, of merging the verb along with the modal element qad as in (21a) below. Merging qad into the sentence structure of (20a) above requires the projection of the Mod head, as represented in (21b) for sentence (21a):

(21) SA VSO with matrix complementizer ?inna and modal qad

a. Pinna-hu qad jaa?-a Pal-Pawlaad-u that-it MOD PERF.come-3MS the-boys-NOM 'Indeed, the boys came."

(iii) Rinna Sinda-ka ya-naam-u zayd-un

that at-you 3-IMPERF.sleep-MS.IND Zayd-NOM

'(the fact is) that Zayd sleeps at your place.'

However, Fassi Fehri (1982: 45; quoted in Shlonsky 2000: 333, footnote 9) observes that “...PPs peuvent être topicalisés dans les déclaratives enchâssées sans problèmes (... PPs can be topicalized in declarative embedded clauses without problem)". Fassi Fehri's example is as in (iv) below:

(iv) Ra-Zunn-u Ranna fii baghdaad-a HaSal-a 1-Pittifaaq-u

1.think-S that in Bagdad-ACC happened the-agreement-NOM

'I think that, in Bagdad, the agreement took place.'

Further testing would be appropriate to spell out the right conditions under which a prepositional adverbial can follow the complementizer Rinna/Panna and whether it could be resumed. I leave this issue for future research. 
b.

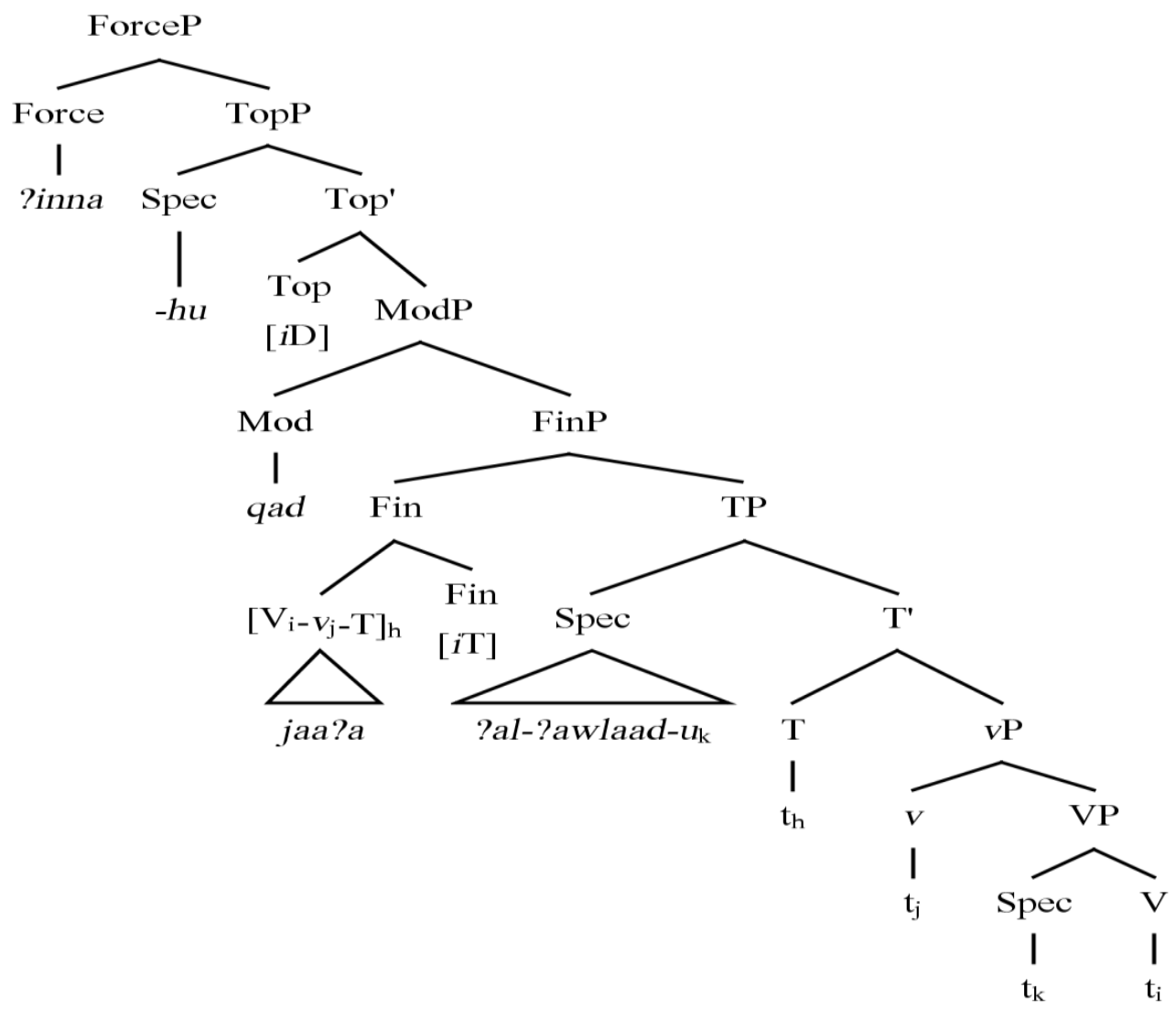

In (21b), both a TopP and a ModP project in between Force(P), realized as Pinna, and Fin $(\mathrm{P})$ where the verbal inflectional complex $\mathrm{V}-v$-T is phonetically realized (i.e., the 'partially' inflected verb jaa?a 'came'. Unlike in (19b) and (20b), the Mod node that projects in the structure of (21b) allows an element like qad to be merged in that position and be construed in conjunction with the verb jaa? a 'came'.

Under these assumptions, a VSO sentence not introduced by Pinna differs from a similar VSO sentence introduced by Pinna in that the Force head position at the highest level of the CP layer (as with $\mathrm{C}$ in a non-split CP layer) is not lexically realized and remains empty. Nevertheless, in all the three structures (19b), (20b) and (21b) discussed above, the verbal inflectional complex $\mathrm{V}-v-\mathrm{T}$ is assumed to raise to Fin.

Sentence (21a), where Pinna and qad co-occur, is an alternative to sentence (20a), where only Pinna is merged in the split-CP domain. As Ouhalla (1993: 280) points out, the role the elements Pinna and qad play in sentence structure is "to assert the truthfulness of the proposition expressed by the sentence". In this respect, the 
assertive interpretation of the proposition in which these elements appear is the outcome of the interaction of the features Pinna and qad are associated with and the temporal/modal properties of the sentence. Nevertheless, as I will discuss in relation to examples $(27 \mathrm{a}, \mathrm{b})$ in section 4.1.2.3 below, the assertive role played by Pinna in sentence structure may be different from the assertive role palyed by $q a d .{ }^{19}$ It seems that the difference in the assertive function of Pinna and qad in SA boils down to the presence of a certain 'focus' feature that is an inherent property of the head nodes that project in the 'Focus sublayer' in the lower part of the split-CP domain above Fin. This feature, being interpretable, would be an essential ingredient in the relation between these head nodes and the $[i \mathrm{~T}]$ feature(s) of Fin and $\mathrm{T}^{20}$

What is true of modal qad seems also to be true of other modal elements that contribute to the assertive interpretation of the proposition. Thus, the NEG markers lam and lan, seen in such examples as (15) and (16) above, contribute to the assertiveness of the proposition expressed by the verbal event (see also section 4.1.2.5 below).

In section 4.1.2.3 below, I return to the problem of characterizing the nature and function of modal qad in sentence structure building on the suggestions made in Bahloul (2008) in his study of qad/laqad and the interaction of this modal with other modality/assertion markers in the sentence structure of SA.

\subsubsection{The nature and function of qad in the tense/modal system of SA}

In relation to the main function a modal like qad plays in the sentence structure of SA, Bahloul (2008: 77) notes that: “... there seems to be some dispute in the literature regarding the function of QAD. The question remains whether this verbal particle interacts with the temporal, aspectual, or modal (emphatic) interpretation of the verbal event". These different properties of qad are reviewed and discussed under the headings in (i)-(iii) below:

\footnotetext{
${ }^{19}$ Section 4.1.2.3 reviews and extends on Bahloul's (2008) analysis of modal qad and the confirmatory kind of emphasis this modal brings about in the sentence where it is used (irrespective of whether Pinna is merged under Force or not).

${ }^{20}$ The properties of the FocP projection involved in wh-dependencies seem to be somewhat different in terms of interpretability. As will be discussed in section 4.2, the 'focus' feature involved in such dependencies, within the split-CP domain, is subject to a valuation process similar to the valuation of $\varphi$-features, within the verbal inflectional complex, in subject-verb agreement configurations at the level of IP.
} 
(i) Qad as an aspect-related element

An aspectual analysis of the verbal complex [ $q a d+$ perfective verb] can be found in Wright (1996, Vol. II: 3) who notes that the combination of qad and the perfective verb "implies that the act is really finished and completed just at the moment of speaking". Al-Aswad (1983) also adopts an aspectual analysis of [ $\mathrm{qad}+$ perfective verb] in which he proposes that the SA perfective verb (as in kataba ar-risaalata 'He wrote the letter') is the equivalent of the English past tense. As for the instances where the perfective verb is preceded by qad (as in qad kataba ar-risaalata ' $\mathrm{He}$ has written the letter'), Al-aswad (1983) proposes that the verbal form [ qad + perfective verb] is the equivalent of the English present perfect.

According to Bahloul (2008), Al-Aswad's (1983) proposal does not adequately account for the data. Bahloul (2008: 67) points to the contrast in (22a) between the expected English gloss '*The boy has come yesterday' (on Al-Aswad's 1983 assumptions) and the correct English gloss ‘The boy came yesterday'. Similar contrasts to that mentioned by Bahloul (2008) can be provided as in the expected English gloss ' $*$ This scientist has died in 1995 ' for example (22b) and the correct English gloss 'This scientist died in 1995':

(22) a. qad jaa?-a Pal-walad-u Pamsi qad come.PERF-3MS the-boy-NOM yesterday 'The boy came yesterday.'/*‘The boy has come yesterday.'
b. qad maat-a
haadha 1-Yaalim-u
sanat-a 1995
qad die.PERF-3MS this the-scientist-NOM year-ACC 1995
'This scientist died in 1995.'/*'This scientist has died in 1995.'

In $(22 \mathrm{a}, \mathrm{b})$, although it is the verbal complex [ $q a d+$ perfective verb] that is used, the correct English equivalent of the combination of qad and the perfective verb is the English simple past, and not the English present perfect as the semantically incorrect gloss 'has come', in (22a) and 'has died', in (22b), shows.

\section{(ii) Qad as a temporal marker}

Unlike Wright's (1996) and Al-Aswad's (1983) aspectual analysis of the verbal complex [qad + perfective verb], other linguists have focused on the function of modal qad as a temporal indicator of a recent past (Ibn-Hisham 1359, Er-Rayyan 
1986). According to Ibn-Hishaam (1359, Vol. I: 294), the difference between the verbal forms $[\varnothing$ perfective verb] and [qad + perfective verb] is that the verbal form without qad does not include a specific temporal indication of the verbal event except for the fact that its occurrence precedes the speech time. In contrast, the [qad + Perfective Verb] form includes a specific temporal indicator - namely qad signalling the occurrence of a recent event in the past. On this account, Ibn Hishaam (1359, Vol. I: 294) contrasts sentence (23a) to sentence (23b) (examples quoted in Bahloul 2008: 73):

(23) a. qaam-a zayd-un

PERF.stand up-3MS Zayd-NOM

'Zayd stood up.'

b. qad qaam-a zayd-un

qad PERF.stand up-3MS Zayd-NOM

'Zayd (has) just stood up.'

In (23b), the closest English equivalent to the verbal form [ $q a d+$ perfective verb] is the gloss where the English adverb 'just' or 'just ...now' is assumed to play the same role as qad in the SA sentence.

As for Er-Rayyan (1986: 149; quoted in Bahloul 2008: 74-75), he mentions that modal qad "denotes the factual completion of a situation", and justifies the role of qad as a temporal indicator of the verbal event in the sentence structure of SA by reference to such sentences as in (24) below, where (24a) has to be interpreted differently from sentence $(24 b)$ :

(24) a. kaan-a dhakar-a khaalid-un maa Hadath-a (SA) PERF.be-3MS mention.PERF-3MS Khalid-NOM what PERF.happen-3MS 'Khalid (has) mentioned what happened'

b. kaan-a qad dhakar-a khaalid-un maa Hadath-a PERF.be-3MS qad PERF.mention-3MS Khalid-NOM what PERF.happen -3MS 'Khalid had mentioned what happened'

In sentence (24b), Er-Rayyan's (1986) proposal is that the combination of auxiliary kaana 'was', the particle qad and the perfective verb derives the equivalent of the English past perfect. On this account, the insertion of qad in the structure of a sentence referring to a past event has the effect of associating the verbal event with a specific time frame in which it is interpreted somewhat differently from a sentence where qad is absent. 
According to this analysis, the temporal function of qad is not only linked to some aspectual interpretation of the verbal event being described, but also to the modal (emphatic/assertive) function that qad basically encodes. I discuss the basic modal function of qad under heading (iii) below.

\section{(iii) Qad as an emphatic/assertive element}

The use of modal qad for emphasis has been the main concern of Dahl \& Talmoudhi (1979), Ryding (2005) and Bahloul (2008).

As Bahloul (2008: 93) notes, "[the] modal function, which is assertive in nature, seems to be central to the basic meaning of the verbal particle QAD". ${ }^{21}$ To this effect, Dahl \& Talmoudhi (1979) and Ryding (2005) maintain that qad/laqad is used along with the perfective verb to express a degree of certainty, a function which is conveyed by emphatic DID in English as the examples in (25) suggest (adapted from Bahloul 2008: 76):

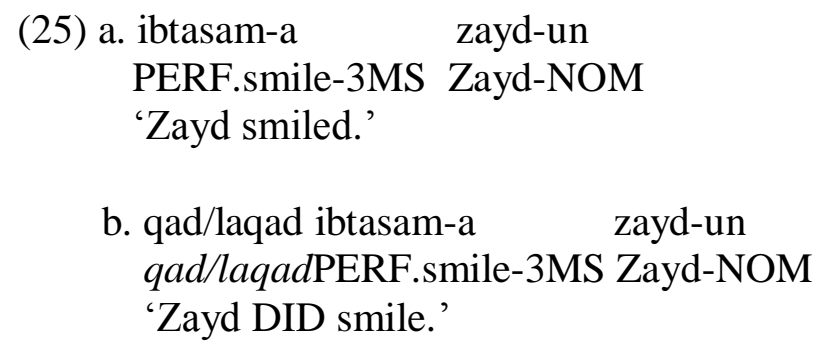

In (25b), the insertion of qad/laqad before the perfective verb adds emphasis to the verbal event described by the sentence. As Ryding (2005: 450) points out: "The use of qad ... serves to confirm the meaning of the past tense by emphasizing that the action did indeed happen".

However, as Bahloul (2008: 96) notes: “[T] he verbal particle QAD is only a part of a larger system which includes other particles such as Pinna and LA-. However, unlike other particles, QAD seems to interact with a broad range of grammatical categories, such as Tense, Aspect and Modality." On this account, whatever other interpretations the particle $q a d$ may express in the sentences where it occurs in conjunction with the perfective verb seem to be subsumed under one

\footnotetext{
${ }^{21}$ Actually, this assumption about the modal function of qad in the sentence structure of SA confirms the analysis outlined above whereby qad is merged under a Mod head that projects as a functional node in its own right within the 'Focus sublayer' in the split-CP domain, as exemplified by (21b) above.
} 
'invariant function' which is basically emphatic or 'assertive' in nature (Bahloul 2008: 93, 101).

This analysis of the role a modal element like qad plays in SA, not just as an element that has some temporal and/or aspectual properties, but also as an element that is used for emphasis, seems to have some counterpart validity in other languages. Thus, according to Bahloul (2008: 75), similar to the situation in SA where "the aspectual function of $Q A D$ does not appear to be central in several other analyses" (but it is nevertheless important in conjunction with qad's modal function), in English the use of the so-called 'Experiential Perfect' in such sentences as (26) (Hassan 1990: 127-9, quoted in Bahloul 2008: 74-75) is important for adding emphasis to the occurrence of an event:

(26) I've seen it as well.

In (26), the use of the 'Experiential Perfect' is intended by the interlocutor to confirm his/her having seen a flash of lightning.

According to this analysis, whether qad plays a role similar to the one played by an aspectual marker for very recent events or incidents or is used in relation to some temporal interpretation of the event, it still holds that the basic function of modal qad is the one that confirms the truthfulness of the verbal event expressed by the sentence. As suggested at the end of section 4.1.2.2 above, this basically 'emphatic' function that modal qad expresses seems to follow from the presence of a certain 'focus' feature that is an inherent property of the head nodes that project in the 'Focus sublayer' in the lower part of the split-CP domain above Fin in relation to the $[i \mathrm{~T}]$ feature(s) of Fin and T. ${ }^{22}$

In his frequency study of qad, Bahloul (2008) examines the functions of qad in relation to its invariant contextual meaning within the broader semantico-pragmatic context of 'TMA categories' (i.e., categories of Tense Mood/modality and Aspect as in Dahl 1985). According to Bahloul (2008: 101): "Although it has temporal, aspectual, and modal variants, we would like to suggest that the use of $Q A D$ invariantly asserts the occurrence of the verbal process, and as such, it is inherently

\footnotetext{
${ }^{22}$ A similar reference to the importance of the 'Focus sublayer' in the determination of the interpretation of verbal events and the subject-verb agreement configurations thereby expressed crosslinguistically is the theory developed by Haegeman \& Koppen (2012), whereby the distribution of subject properties in External Possessor Agreement constructions in West Flemish is related to some head-head agreement relation at the higher level of the split-CP domain in relation to temporal adverbials such as toen just 'just then' merged under the Focus projection (see section 4.3.2.3).
} 
assertorial". Bahloul (2008: 96-97) examines the occurrence of modal qad in samples of newspaper and magazine articles, as exemplified by such passages as in $(27 a, b)$ from an article in Al-Quds Al-Sarabi 'The Arabic Quds' dated 02/20/92 (adapted from Bahloul 2008: 96):

(27) a. wa qad qaal-a raadiyu Pisraa?iil (...) Pinna Sadad-an

and qad PERF.say-3MS radio Israel (..) that number-ACC

kabiir-an mina s-sukkan-i fii shammal-i Pisraa?iil

big-ACC from the-residents-GEN in north-GEN Isreal

?amD-aw al-layl-a fii 1-malaaji?-i

PERF.spend-3MP the-hight-ACC in the-shelters-GEN

'Radio Israel did announce that a large number of residents in North

Israel spent the night in shelters.'

b. wa $\varnothing$ qaal-a maSdar-un siyaasiyy-un muwaal-in

and PERF.say-3MS source-NOM political-NOM associated-GEN

li-Piiraan li-ruytir ?inna r-radd-a ya-jib-u Pan

with-Iran to-Reuters that the-reply-ACC 3-(is).necessary-MS that

ya-kuun-a hujuum-an fii mustawaa Rightiyaal-i

3-IMPERF.be-SUBJ attack-ACC in level assassination

sh-shaykh-i 1-musawii

the-Sheikh-GEN Al-Musawii

'Some political source linked to Iran said to Reuters that the retaliation should be an attack as serious as the assassination of Sheikh Al-

Musawii.'

As can be seen in $(27 a, b)$, the speaker/writer (journalist Ali Thamer) switches from the use of the verbal complex [ $\mathrm{qad}+$ perfective verb] to the use of a simpler form [ $\varnothing$ perfective verb]. As Bahloul's (2008) study on the use of qad in everyday life situations in the contemporary Arab world shows, the trigger for the use of qad is not to be found in the syntactic context per se. Rather, the use of qad along with the verb qaala 'said' reporting the major event in the embedded clause introduced by complementizer Pinna 'that', in (27a), Pinna Sadadan kabiiran ... 'that a large number ...' seems to be conditioned by the constraints of the discourse/textual context where the utterance takes place. Thus, in (27a), by choosing to include qad along with the reporting verb, the writer/speaker seems to confirm the already established fact, reported by Radio Israel, that the ongoing conflict between Israel and the fighters in South Lebanon has, indeed, destabilized the day-to-day mode of life of people in North Israel. In doing so, the writer/speaker directs our attention to the fact that one 
major aspect of this destabilization, in the present situation at the time of the utterance, is the confinement of people to living in shelters.

As for the absence of the use of qad along with the reporting verb qaala in (27b), the writer/speaker may be less willing (than he is in the context of uttering (27a)) to accord too much importance to the possible event reported by Reuters and described in the embedded clause. As in (27a), the embedded clause is introduced by complementizer Pinna 'that' Pinna ar-radd-a ya-jib-u Pan ya-kuun-a ... 'that the retaliation should be ...' describing the event reported to us. Nevertheless, in (27b), although the writer/speaker informs us of the fact, reported by Reuters, that there will most probably be some form of retaliation on the opponent, he chooses to use a simpler form of the perfective verb to report the event in the embedded clause. i.e., the verb qaala 'said' not preceded by modal qad. This choice may be an indication of the writer/speaker's mere informative attempt at conveying to us the news surrounding 'the retaliation' being reported without committing himself to practically 'asserting' that the retaliation and/or attack will actually take place.

The difference between either using modal qad along with the verb, as in (27a), or leaving qad out, as in (27b), might also be linked to the evidentiality of the verbal event. Whereas in (27a) the speaker/writer refers to a definite entity as the source of the reported event (i.e., raadiyu Pisraa?iil 'radio Israel'), in (27b) the referent used as a source for the reported event is an indefinite DP maSdar-un siyaasiyy-un muwaal-in li-Piiraan 'some political source linked to Iran'. Thus, unlike in (27a), the speaker/writer is less willing to assert the truthfulness of the event being reported in (27b), and he translates his mere 'informative' attempt at reporting the facts by opting not to use modal qad along with the reporting verb in such an instance.

Coming back to the basic assertive function of $q a d$ in the syntactic structure of SA, the framework of assumptions we have been concerned with in section 4.1.1 and at the beginning of section 4.1.2 links the syntactic role played by qad to some dedicated node, which, not only relates to $[i T]$-features on $\mathrm{T}$ and Fin, but also to some 'focal' property that qad expresses by virtue of being merged in the 'focus sublayer' of the split-CP domain. For Bahloul (2008: 163-165), the node that qad occupies in sentence structure is a functional head projection, which he labels as Assertion $\left(=\mathrm{Asrt}^{\circ}\right)$ maximally projecting as AsrtP above TP, as represented in (28): 


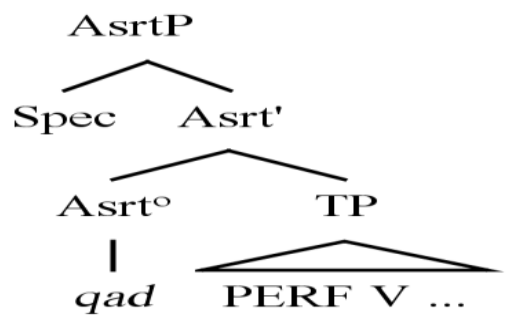

Bahloul (2008: 87-88) also proposes that, since Pinna and qad both contribute to the assertive interpretation of the proposition expressed by the sentence where they occur (see also Ouhalla 1993: 280), Pinna occupies the same structural position as $q a d,-$ namely, $\mathrm{Asrt}^{\circ}$, as represented in (29) below (where XP may range over TP, PP and $\mathrm{AP}):^{23}$

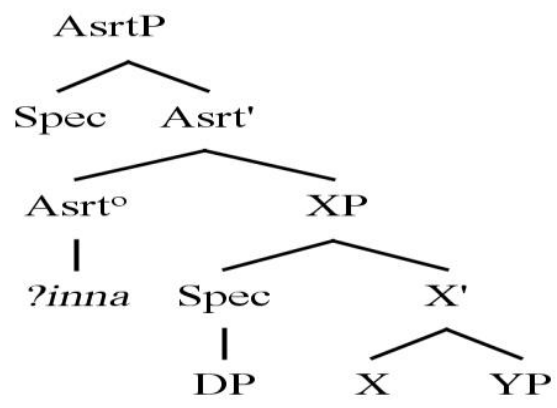

However, as Bahloul (2008: 88) acknowledges, Pinna and qad can co-occur in the same sentence as in (21a) above, repeated as (30):
(30) Pinna-hu qad jaa?-a Pal-Pawlaad-u that-it MOD PERF.come-3MS the-boys-NOM 'Indeed, the boys came.'

In (30), Pinna clearly occupies a node that is higher than the head node occupied by the modal particle qad. As discussed at the beginning of section 4.1.2.2 in light of the revised split-CP system I have been considering in section 4.1.2.1, although both

\footnotetext{
${ }^{23}$ Bahloul (2008: 164-165) also proposes that the Neg head maa is likewise inserted under Asrt ${ }^{\circ}$ in SA. I return to Bahloul's (2008) proposal in section 4.1.2.4 below and to a discussion of negation in SA in section 4.1.2.5.
} 
Pinna and qad seem to contribute to the assertive interpretation of the proposition, the complementizer Pinna, as a clause type marker, would primarily serve the function of signalling the type of clause being derived. Then, Pinna would interact, together with modal qad and other CP preverbal particles, with the tense/modality properties of the sentence in which these particles are derived by virtue of the C-T dependency. The purpose of the following section is to properly characterize such an interaction within the feature structure of VSO configurations.

\subsubsection{The feature structure of the split-CP in SA VSO configurations}

I have assumed in section 4.1.2.2 that, in the derivation of an SA sentence like (19a) (as structurally represented in (19b) repeated below as (31b)), the Force head projects but can remain empty:
(31) a. jaa?-a
Pal-?awlaad-u
PERF.come-3MS the-boys-NOM
'The boys came.'

b.

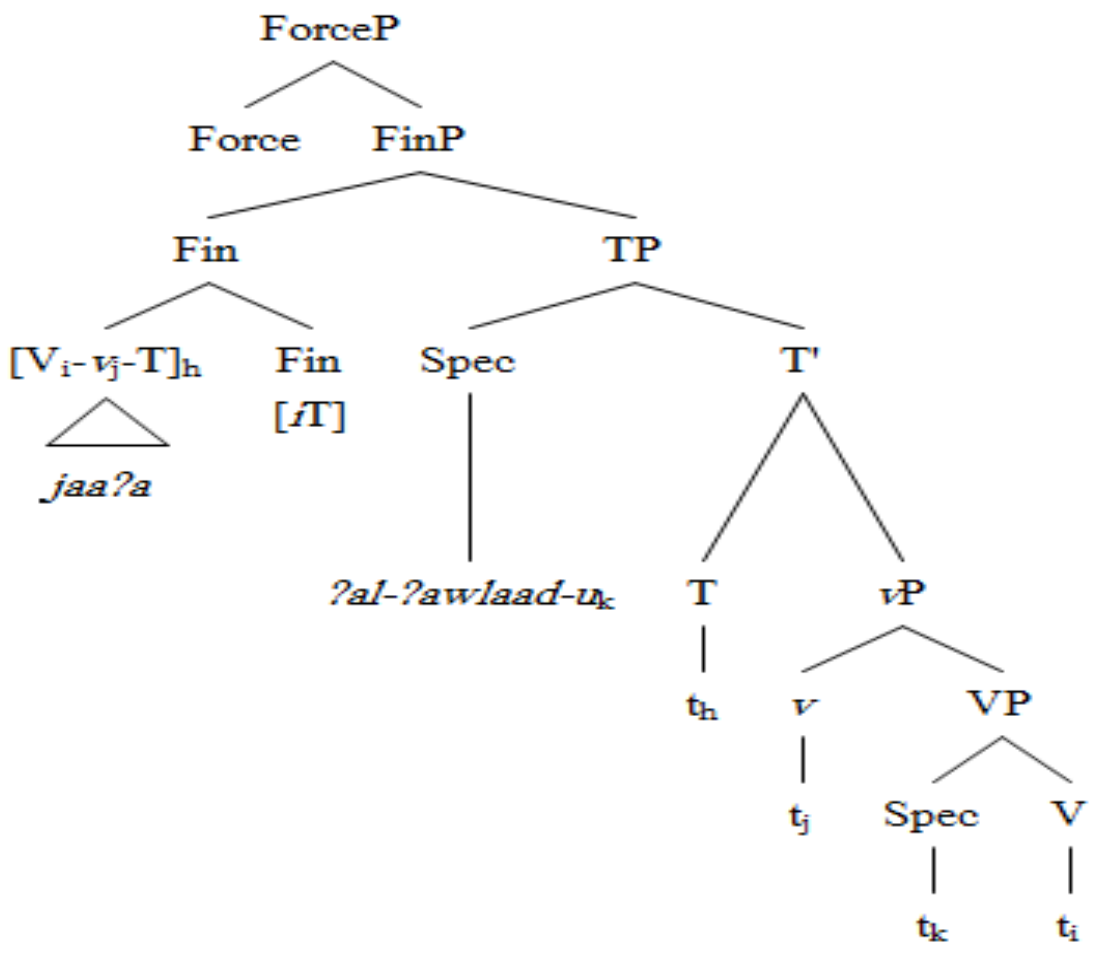

In (31b), the Force head projects in addition to Fin, but remains empty in the absence of a phonetically realized lexical complementizer. This assumption can be maintained 
by reference to the derivation of a matrix interrogative sentence in SA as in (32a), structurally represented as in (32b) below:

\section{(32) a. ?a jaa?-a Ral-Pawlaad-u? \\ Q PERF.come-3MS the-boys-NOM}

'Did the boys come?'

b.

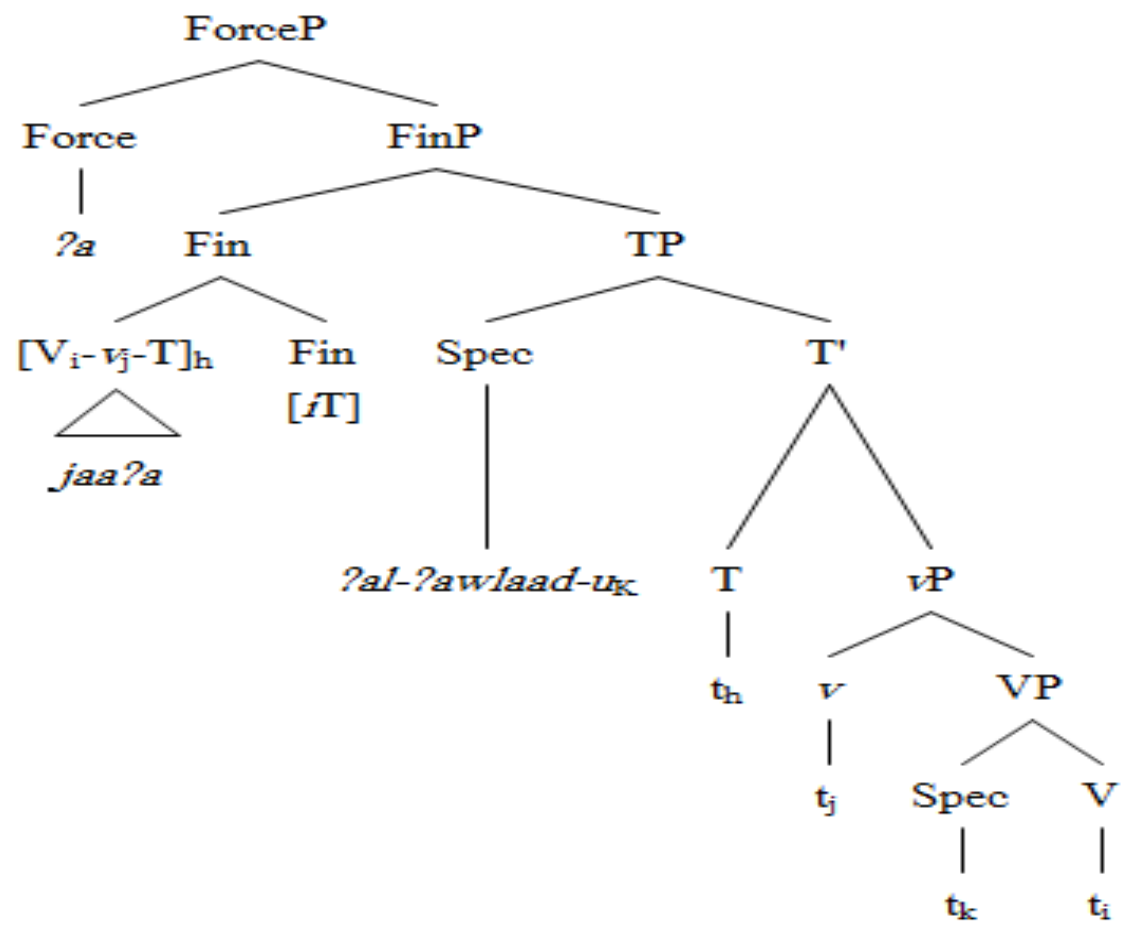

Thus, the derivation of the interrogative sentence (32a) requires the phonetic realization of the complementizer head left empty in structure (31b). The derivation of (32a) involves the use of the interrogative particle $? a$, which is merged under Force as the highest structural head projection. ${ }^{24}$ In this case, the realization of the Force head in (32b) as $? a$ signals that the sentence is interpreted as interrogative at the interface.

In such structural configurations as in (32b), the C-T dependency is established at the interface between the interrogative particle $? a$ realizing the Force head and the verbal inflectional complex V- $v$-T raised to Fin. This dependency relation between the higher and the lower $\mathrm{C}$ projections at the two opposite sides of the split-CP system ensures that all elements involved in the representation of the sentence at the interface are properly identified. According to such assumptions, feature identification does not exclusively apply to interrogative particles like $? a$ in

\footnotetext{
${ }^{24}$ Alternatively, the question marker $P a$ is raised from a lower position in the split-CP domain. I discuss this alternative analysis in relation to sentences $(35 \mathrm{a}, \mathrm{b})$ below.
} 
SA in relation to the inflectional properties of the Fin head. Rather, this feature identification process generalizes to any element moved to or directly 'merged' in some position in the split-CP domain (cf. Ouhalla 1993, 1994, R\&R and Vangsnes 2002). Thus, feature identification applies not only to focused/assertive and interrogative elements but also to negation elements either directly merged in or moved to the position where they are spelled out at PF (see section 4.1.2.5). Although Ouhalla (1993, 1994), Vangsnes (2002) and R\&R differ in their formulation of the identification requirement on features such as $[\mathrm{wh}] /[$ focus $]$ in the case of question formation or focalization processes or (for R\&R) the [+Fin] feature involved in V2 contexts in German, this requirement seems to be, first and foremost, a licensing process akin to the cartographic notion of criterial satisfaction applying to the head nodes projected within the split-CP domain, and not a 'feature-checking' requirement. $^{25}$

In this connection, Aoun et al. (2010: 209-210) refer to Ouhalla's (1994) "morphological identification" requirement pointing out that a parallel can be drawn between Focus fronting and question formation in SA. Aoun et al. (2010: 209-210) suggest that the parallel between Focus and interrogative structures does not uniquely concern the projection of a designated functional projection in the $\mathrm{CP}$ domain, but also designated particles that can be merged in their respective positions to morphologically identify the sentence as a sentence with some 'focus' property or an interrogative sentence in terms of "an abstract head F, bearing the $[+\mathrm{F}]$ feature, which on a par with the $\left[+\mathrm{Q}(\right.$ uestion) $]$ feature on $\mathrm{C}$, needs to be identified". ${ }^{26}$

In the system of assumptions adopted here, in an interrogative sentence as (32a) above, the feature identification process applies in terms of $[i \mathrm{~T}]$ features $(=$ the

\footnotetext{
${ }^{25}$ However, as I will discuss in section 4.2.2, in relation to the derivation of wh-dependencies in SA involving an $\mathrm{XP}_{\mathrm{Foc}}$ and Foc, the identification process involved in the derivation of wh-dependencies seems to involve the 'checking' or valuation of an uninterpretable [WH]-feature on Foc both in relation to the interpretable wh-feature of the raised wh-element (which values it), and in relation to the finiteness/tense features of the Fin head projecting in the lower part of the split-CP domain (cf. R\&R p. 141).

${ }^{26}$ Under a different conception of the licensing of focus features in the syntax, Horvarth (1995) adopts a 'feature-checking' analysis of focus structures in Hungarian à la Chomsky (1995b). Horvath (1995) assumes that the derivation of focus structures in Hungarian involves some focus feature (originating on V) 'checked' in a Spec-head agreement relation between V, raised to T, and an XP in [Spec, T], quite similar to the checking of Case on DPs via the same structural configuration. On similar grounds, Benmamoun (2000b) assumes that the feature involved in the formal licensing of NEG elements in Arabic is a [+D] feature, which he assumes is subject to a 'feature-checking' requirement quite similar to the checking of $[+\mathrm{D}]$ on T for EPP satisfaction in the framework of Chomsky (1995b).
} 
[+Fin] feature of R\&R) on the Fin head in the lower part of the split-CP domain. As the structure builds up, the feature identification process carries on to the other node(s) introduced into the derived structure. Thus, in the derivation of (32a), the identification of $[i \mathrm{~T}]$ features interacts with the identification of clause-type features for singling out the sentence as interrogative, as opposed to negative in negation contexts or, simply, declarative in [+declarative] contexts.

As my discussion in relation to the insertion of modal/assertive markers and NEG elements shows, derivations involving the 'Focus sublayer' of Poletto (2000b) and Benincà \& Poletto (2004) seem to involve an additional 'focus' feature (= the [+F] feature of Ouhalla 1993 and Aoun et al. 2010) that interacts with both clause type at the higher part of the split-CP domain (i.e., clause type features on Force) and with the features encoded on Fin at the lower part of the split-CP domain (i.e., $[i \mathrm{~T}]$ features or the [+Fin] feature of R\&R: 140 on Fin). Thus, the system of assumptions adopted here, in which the identification procedure is more than a question of morphological identification at Spell Out, views identification in terms of the structural relations determining the realization of the $\mathrm{C}-\left(\mathrm{Agr}_{\mathrm{S}}\right)-\mathrm{T}$ dependency at the interface.

Thus, taking 'identification' to be an interface licensing condition on the representation of elements merged into the structure of such SA VSO sentences as (19a), (21a), and (32a) above, the T-to-Fin raising process establishes head-head agreement relations that relate to the $\mathrm{C}-\mathrm{T}$ dependency. Within the $\mathrm{T}$-dependency established in both the IP and CP domains, V-v to T and V-v-T to Fin apply for tense interpretation at $\mathrm{LF}^{27}$ Thus, depending on whether verb movement applies in the syntax or not, the verbal inflectional complex $\mathrm{V}-v$ is either directly or indirectly linked to $\mathrm{T}$ and Fin. The trigger for these raising processes is probably an unvalued [T]-feature on V. On the assumption discussed earlier (see section 3.3.2.3) that, in case $\mathrm{T}$ has $\mathrm{V}$-features (lexical verbs vs. auxiliary verbs), these features on $\mathrm{T}$ are valued as a result of the dependency relation that exists between $\mathrm{T}$ and $\mathrm{V}$ in sentence

\footnotetext{
${ }^{27}$ English-type languages lack the V-to-T raising process in the syntax. Recall from section 3.2.1.1, that Roberts (2010a) links V-to-T movement to rich $\mathrm{T}$-features enabling $\mathrm{T}$ to attract $\mathrm{V}$ to raise to it. Presumably, in English-type languages where no overt verb-movement applies, it may be the case that the licensing conditions under which the dependency relation between $\mathrm{V}$ and $\mathrm{T}$ is established at LF involve some form of covert verb movement. Alternatively, Bobaljik (1994) assumes that, in English, the only condition that applies in relation to the dependency between $\mathrm{T}$ and the verb is that, at the interface, T must be adjacent to the VP it selects as complement in the syntax. Adverbs will not intervene in this dependency relation since they are not relevant to syntactic adjacency.
} 
structure. In SA, and similar V-raising languages, after $\mathrm{V}-v$ raises to attach to $\mathrm{T}$, the whole V-v-T complex is attracted to Fin. Thus, the dependency relation that relates V$v$ to $\mathrm{T}$ and $\mathrm{V}-v$-T to Fin is established in terms of interpretable tense features (i.e. an $[i \mathrm{~T}]$ on $\mathrm{T}$ and on Fin) matching and Agreeing with $[u \mathrm{~T}]$ on $\mathrm{V} / v^{28}$

As for the contexts where the 'Focus sublayer' is involved, it seems that some 'focus' feature identification is an additional prerequisite licensing condition for the well-formedness of the C-T dependency at the interface. This may be understood in terms of Criterial satisfaction (as conceptualized in R\&R). In contrast to Topic elements merged in the split-CP domain, focused elements have to satisfy a Criterion. One relevant example of this difference is R\&R' s (pp. 138-141) argument that XP fronting, in German V2 structures involving topicalization of some XP in the left periphery of IP, is derived by neither a feature-checking process nor a criterion satisfaction requirement. $R \& R$ (p. 138) state that:

[T] he Topic Criterion seems problematic to us. The various criteria that have been proposed in the literature (wh-, Focus, Negative Criterion) are basically formulated to account for the movement of phrases with quantificational properties, that is, XPs that need to satisfy scope requirements and receive a special interpretation. Topics, however, do not seem to function like quantifiers/operators (see the papers in E. Kiss 1995 and more recently Rizzi 1997 for detailed argumentation to this effect). If this is correct, then the idea that they are subject to a Topic Criterion, which has the same properties as the other criteria, turns out to be dubious.

On R\&R's assumptions, the 'topic interpretation' of XP fronting in V2 declarative contexts is provided by both " $\mathrm{C}$ and $\mathrm{SpecCP}$... as together specifying contextually given information" (p. 140). R\&R (pp. 140-141) add: "Note that our analysis goes through even if we assume that the XP moves into a separate Topic projection along

\footnotetext{
${ }^{28}$ Chomsky (2008: 144) suggests that the Tense features of T, in addition to T's $\varphi$-features, are derivative from $\mathrm{C}$ (i.e., the node that selects $\mathrm{T}$ as its complement in sentence structure - Fin if the Csystem is split as in Rizzi 1997). Extending on claims made in Haegeman \& Koppen (2012), I reinterpret Chomsky's (2008) feature-inheritance mechanism between $\mathrm{C}(=\mathrm{Fin})$ and $\mathrm{T}$ as a 'featuresharing' relationship between the two functional nodes whereby the $\mathrm{C}$-T dependency is first and foremost conceived of in terms of head-head agreement configurations on the basis of discrete sets of features (T-features and $\varphi$-features) shared by the respective nodes (i.e., Fin and T; see section 4.3).
} 
the lines proposed by Müller \& Sternefeld (1993). Our alternative requires a topic feature, but no checking of that feature, and so no Topic Criterion". ${ }^{29}$

However building on Rizzi and Shlonsky's (2007) revision of the mechanisms involved in Criterial satisfaction, as not only involving Spec-head agreement relations, but also head-head agreement relations, it may be the case that whenever an additional 'focus' feature is involved in the derivation of the split-CP domain, the mode of licensing such a feature would vary according to the properties of the functional projection being involved in such licensing, in the spirit of the parameterized probe-goal-Agree system adopted here. In this conception, both a Focus head and a Topic head would be involved in some kind of Criterial satisfaction. The only difference between the two would be in the mode of licensing of these two Criterial head projections. Whereas Focus could be licensed via a Spec-head agreement relation involving an $\mathrm{XP}_{\mathrm{Foc}}$ and the Focus head or via a strict head-head agreement relation between Focus and Finiteness or Focus and Tense, Topic could only be licensed via a strict head-head agreement relation. This head-head agreement relation would depend on the connection that exists between the the $[i \mathrm{D}]$ feature on the Topic head and the V-v-T-Agrs complex in IP where the valuation of $[u \mathrm{D}]$ on $\mathrm{Agr}_{\mathrm{S}}$ via pro-insertion satisfies the C- $\mathrm{Agr}_{\mathrm{S}} \mathrm{T}$ dependency at Spell Out. As already discussed in section 3.2 (see, in particular section 3.2.1.2), D-features play an essential role in the licensing of null subjects and the satisfaction of the EPP at the level of IP $\left(=\operatorname{Agr}_{S} \mathrm{P}\right.$, in the present analysis). Thus, it is in terms of the valuation and/or identification of D-features that both Top and the T-Agr inflectional complex configurationally connect a DP to syntactic predication.

This difference in the additional 'focus' property and how it is satisfied in sentence structure may account for the absence of scope whenever a Topic element is involved in the derivation of sentence structure, as R\&R (p. 138) direct our attention to.

In my discussion of sentence (32a), I have assumed that interrogative $? a$ is merged under ForceP to signal the introduction of a yes/no interrogative sentence. In SA, yes/no interrogative sentences are also formed by the use of the question marker

\footnotetext{
${ }^{29} \mathrm{R} \& \mathrm{R}$ do not make a difference between the insertion of an expletive, the preposing of a subject DP, or the preposing of any other XP constituent under the assumption that all these constituents could satisfy the EPP (cf. Holmberg 2000). In the probe-goal-Agree system adopted here, the derivation of V2 configurations involving subject DPs (including EXPL es) is proposed to be fundamentally different from the process of deriving a 'topicalized' XP in such V2 configurations, as will be discussed in section 4.3 .
} 
hal. However, according to Mohammad (1989: 57), hal differs from ?a in that hal seems to require the verb to follow it, while $? a$ can be followed by either the subject or the verb. The sample examples in (33) and (34), illustrating this difference, are adapted from Mohammad (1989: 58-60) (EV= Epenthetic Vowel):

(33) a. Ra Saliyy-un Darab-a khaliil-an?

Q Ali-NOM PERF.hit-3MS Khalil-ACC

'Was it Ali that hit Khalil?'

b. Pa Darab-a Saliyy-un khaliil-an?

Q PERF.hit-3MS Ali-NOM Khalil-ACC

'Was it Ali that hit Khalil?'

(34) a. *? hal-i l-walad-u Pakal-a Pat-tuffaHat-a?

Q-EV the-boy-NOM PERF.eat-3MS the-apple-ACC

'Did the boy eat the apple?'

b. hal Rakal-a l-walad-u Pat-tuffaHat-a?

Q PERF.eat-3MS the-boy-NOM the-apple-ACC

'Did the boy eat the apple?'

Mohammad (1989) does not dicuss examples where the complementizer Pinna is used in such interrogatives. Sentences $(35 a, b)$ and $(36 a, b)$, where Pinna is merged in Force, are parallel sentences to (35a,b) and (36a,b),

(35) a. Ra-Pinna Saliyy-an Darab-a khaliil-an?

Q-that Ali-ACC PERF.hit-3MS khalil-ACC

'Was it Ali that hit Khalil?'

b. Pa-Pinna-hu Darab-a $\quad$ Saliyy-un khaliil-an?

Q-that-it PERF.hit-3MS Ali-NOM khalil-ACC

'Was it Ali that hit Khalil?'

(36) a. *? hal Pinna l-walad-a Pakal-a Pat-tuffaHat-a?

$\mathrm{Q}$ that the-boy-ACC PERF.eat-3MS the-apple-ACC

'Did the boy eat the apple?'

b. hal Pinna-hu Rakal-a l-walad-u Rat-tuffaHat-a?

$Q$ that-it PERF.eat-3MS the-boy-NOM the-apple-ACC

'Did the boy eat the apple?'

In (35b) and (36b), having complementizer Pinna follow the interrogative elements $? a$ and hal seems to be as acceptable as having the verb follow them as in (33b) and 
(34b). On this account, the grammaticality of the sentences in (35) and (36b) shows that the verb does not have to immediately follow the interrogative elements $? a$ and hal since ?inna can be merged in Force. Thus, in both (35b) and (36b), it is ?inna that precedes the verb and not hal. Merging interrogative elements under ForceP in sentences (32)-(36) can account for the process whereby ?a is phonetically realized together with Pinna in Force in $(35 a, b){ }^{30}$

On the assumption that the yes/no question marker hal is a Wh-phrase basegenerated in [Spec, CP] much like the Wh-phrase limaadha 'why' in SA (Bahloul 2008: 181-182, following Rizzi 1990: 46-47 for the wh-phrases why in English and pourquoi in French), hal merged in [Spec, CP] (possibly corresponding to [Spec, ForceP] in the present analysis) would be considered as a Focused element and not just as a Q-marker. By that token, the order of constituents in (34a) and (36a) would configure a Focused element or wh-word preceding a Topic DP, which, as noted earlier (see footnote 4) leads to an ungrammatical output at the interface in SA. This is not the case for the yes/no question marker $? a$, which would be considered as a pure Q-head marker merged in Force and not in the specifier position of ForceP. I further discuss the question of word order possibilities in wh-dependencies in section 4.3.1.1.

On the assumption that the yes/no interrogative marker $? a$ is merged along with Pinna in Force, a yes/no interrogative sentence like (37a) would have the structural represention in $(37 b)$ :

(37) a. Pa-Pinna-ka la-Panta yusuf?

Q-that-you FOC-you Joseph

'Are you really Joseph?'

\footnotetext{
${ }^{30}$ Nothing hinges on the assumption that the interrogative particle $? a$ is merged in Force or in some other position in the higher field of the split-CP domain above FocP. Thus, ?a could, alternatively, be assumed to be merged in some Interrogative head position $\alpha$ higher or lower than ForceP. On the assumption that $\alpha$ is merged lower than ForceP, the raising process of the interrogative particle $? a$ to Force, in which position the interrogative particle adjoins to Pinna, might be for the sake of providing the [+interrogative] interpretation to the Force head, and by that token, replacing the default [+declarative] interpretation of the clause.
} 
b.

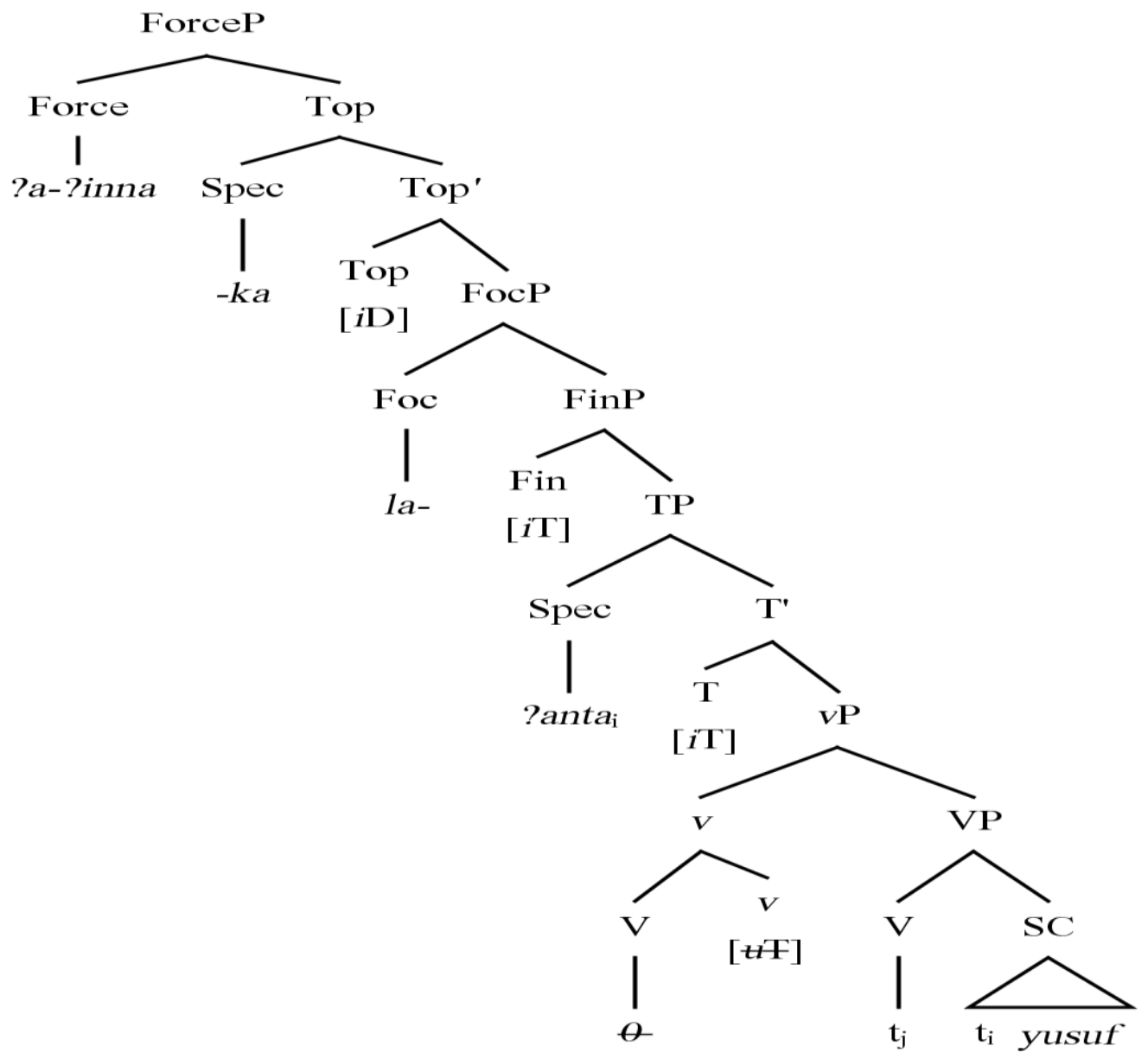

In (37), the interrogative interpretation is brought about by the use of the interrogative particle ? $a$ in connection with two other particles (namely, the complementizer Pinna and the affirmative particle $l a$ ), both of which are assertive in nature. As discussed in section 4.1.2.2, the assertive element Pinna is merged under $\mathrm{C}$ in matrix or embedded contexts. When used by itself, Pinna serves to signal declarative force as a default clause type (Rizzi 1997, R\&R). However, in (37), Pinna is used in conjunction with the yes/no interrogative marker $? a$. By that token, it is the interrogative clause type that prevails, and the structure is interpreted as [-declarative] at the interface.

As for the assertive element $l a$ in (37), my assumption is that it is inserted under the Foc head projection above FinP. The optional presence of the particle $l a$ adds a contrastive focus interpretation of the event or situation described by the sentence. By that token, the sentence in (37) is actually the affirmative counterpart sentence to a negative sentence where the contrastively focused NEG element maa is 
used (see example (38) below). ${ }^{31}$ A similar assumption carries over to the modal element laqad. As discussed in relation to footnote 21 above, the assertive element $l a$ can co-occur with the modal (emphatic) element qad as a phonetically realized single element laqad. Citing Moutaouakil (1989), Ouhalla (1994: 75) points out that sentences involving the modal element laqad "convey information which contradicts the information possessed by the addressee, and, therefore, are instances of contrastive focus". Thus, on a similar basis as the particle la, laqad would be merged under Focus.

Since (37a) is a verbless sentence where no lexical verb is merged in the $\mathrm{V}$ position, the raising of $\mathrm{V}-v$ to $\mathrm{T}$ does not apply. Under the assumptions of the derivation of (null) copular sentences discussed in section 3.3.2.3, a head-head Agree relation links $[u \mathrm{~T}]$ on $v$ to the $[i \mathrm{~T}]$ feature on $\mathrm{T}$ (which, in turn, links to the $[i \mathrm{~T}]$ feature on Fin) for 'identification' at the interface. The subject of the sentence is the pronominal Panta 'you', internally merged in [Spec, TP] after raising out of the small clause, which is sister to the light verb $v$. The function of ?anta in (37a) is to co-refer with, or resume, the Topic pronominal clitic $-k a$ 'you'.

In the negative counterpart of (37a), as in (38a) below, the negative marker maa is merged under the Foc node as represented in (38b):
(38) a. Pinna-ka maa ?anta yusuf that-you NEG you Joseph 'You are NOT Joseph.'

\footnotetext{
${ }^{31}$ Sibawayhi (796: Vol. I: 460) points out that, in Classical Arabic, affirmative sentences with a verb by itself or with the verbal complex [qad + Perfect] have, as a negative counterpart, sentences with lam or lamma, respectively. As for affirmative sentences with the verbal complex [laqad + Perfect], they have sentences with maa as a negative counterpart.
} 
b.

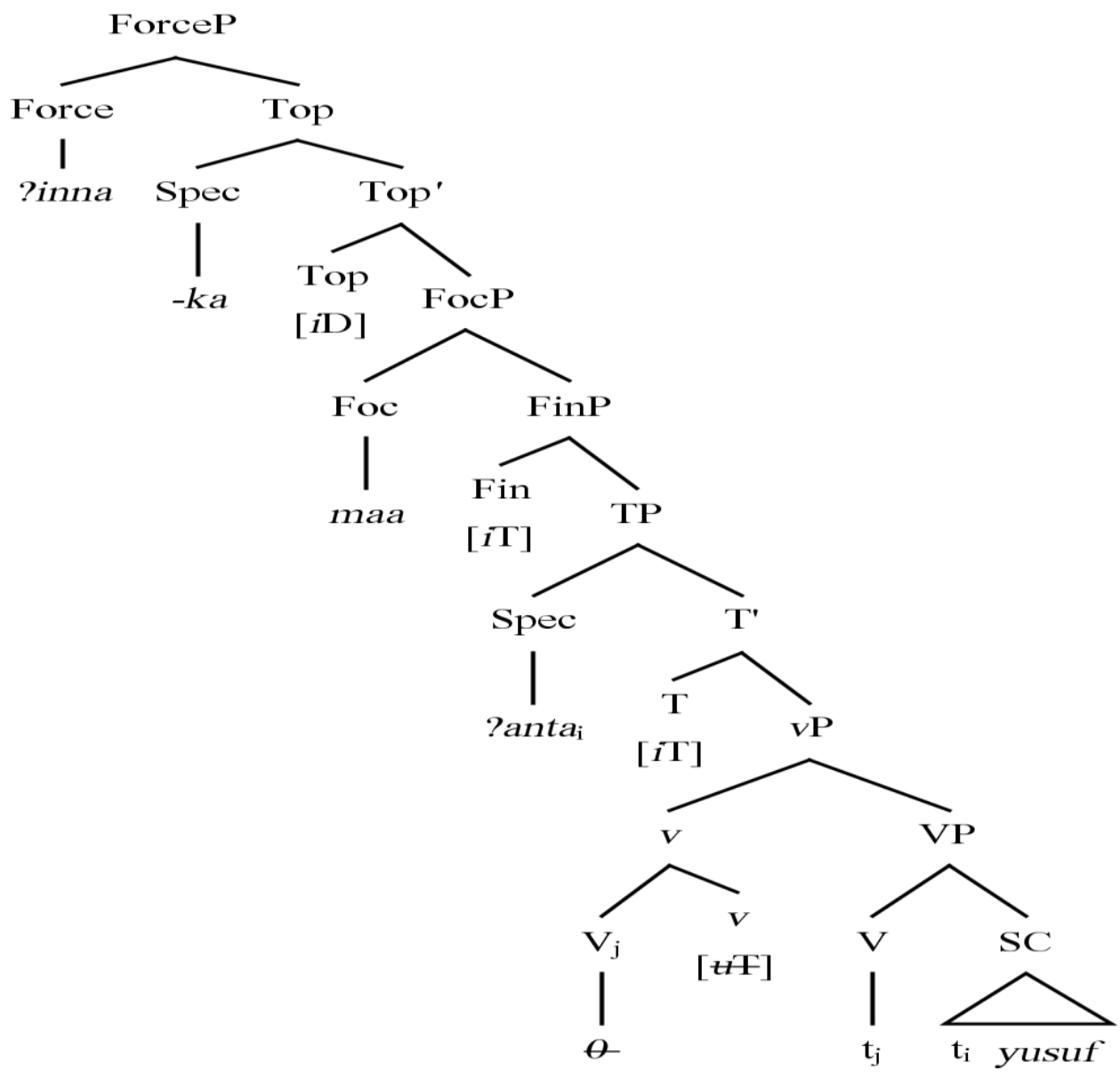

As was the case in (37), the pronominal Panta 'you' is internally merged in [Spec, TP] as the subject of the sentence in (38a). The negative interpretation of the sentence is brought about by inserting the negative particle maa into the structure of the sentence.

I follow Bahloul (2008: 164-165) in the assumption that maa does not head a NegP projection in SA. As the structural representation (38b) shows, I assume that $m a a$ is merged in Foc above Fin. In this respect, maa patterns like the assertive emphatic element $l a$ in (37b). As already discussed in section 4.1.2.1, the idea that negation and emphatic affirmation should be accommodated in the same structural position was first suggested by Laka (1990: 100) who locates both negative and affirmative elements in a head node she calls $\Sigma$.

At the interface, the negative interpretation of the sentence arises out of the identification of $[i \mathrm{~F}]$ features on maa and Foc in conjunction with $[i \mathrm{~T}]$ features of Fin in the lower part of the split-CP domain and in relation to the $[i \mathrm{~T}]$ feature of $\mathrm{T}$ in the IP domain (see section 4.1.2.5). In this respect, the 'identification' of NEG elements 
operates by combining $[i \mathrm{~F}]$ features and $[i \mathrm{~T}]$ features irrespective of whether $[i \mathrm{~F}]$ is a contrastive focus feature, as in the case of maa in (38) and laysa (see (39) below) or not, as in the case of the 'temporal' NEG elements laa/lam/lan. ${ }^{32}$ As for the function of the matrix complementizer Pinna under Force in (38b), it is purely assertive. It denotes negative assertiveness as Force links to Fin and Foc via the C-T dependency. An alternative to (38a) is (39a) below where the subject-inflected NEG element lasta 'you (are) not' is used instead of maa, as represented in (39b): ${ }^{33}$

\author{
(39) a. Pinna-ka las-ta (Panta) yusuf \\ that-you NEG-you (you) Joseph \\ 'You are NOT Joseph.'
}

\footnotetext{
${ }^{32}$ Ouhalla (1993: 277) and Benmamoun (2000b: 109) assume that, unlike laa/lam/lan, maa is a contrastively focused negative element in contrast to laa/lam/lan. (see section 4.1.2.5).

${ }^{33}$ The 'verbal' behaviour of laysa as a negative auxiliary has already been discussed in section 4.1.2.1 above with reference to Aoun et al. (2010) and in section 3.3.2.3 with reference to Benmamoun (2008: 113-114).). Laysa inflects for $\varphi$-features in relation to initial subjects in negative sentences. In this case, laysa inflects for subject person, number and gender agreement features of the imperfective/past tense paradigm (i.e., (anna) las-tu 'I am not', (anta) las-ta 'you.MS are not', (anti) las-ti 'you.FS are not' (huwa) laysa 'he is not', (hiya) laysa-t 'she is not', (naHnu) las-naa 'we are not', (antum) las-tum 'you.MP are not', (antunna) las-tunna 'you.FP are not' (hum) lays-uu 'they.M are not' (hunna) las-na 'they.F are not'). In this respect, laysa is different from maa, which does not inflect for either subjectverb agreement nor for tense (which is a property of NEG elements lam/lan). I return to an analysis of negative elements in SA in section 4.1.2.5.2 below.
} 
b.

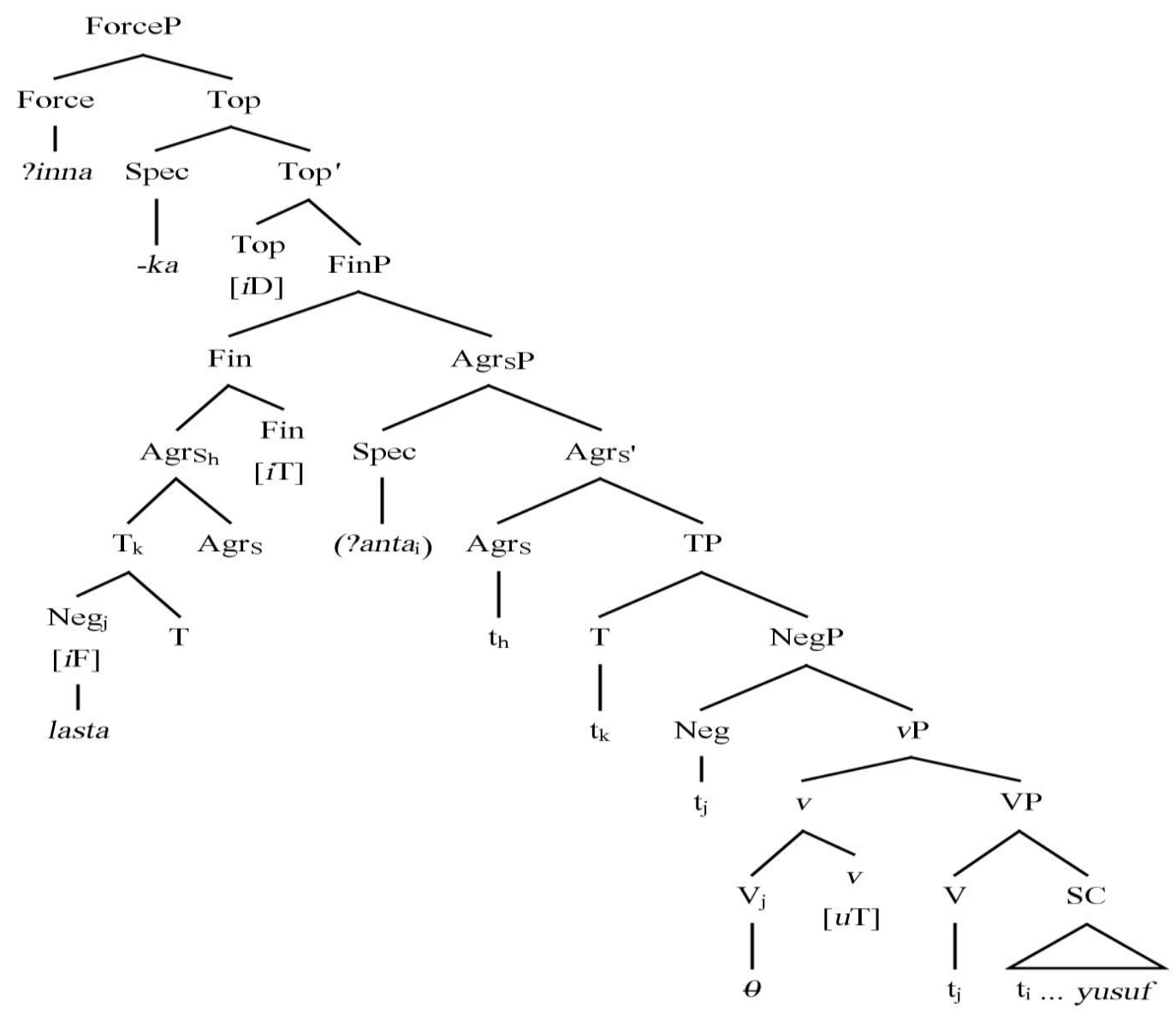

The sentence in (39a) projects as an $\operatorname{Agr}_{S} \mathrm{P}$ at the IP level, as structurally represented in (39b). As Benmamoun (2000b: 103) proposes, the SA NEG element laysa projects as a Neg node heading NegP in the IP domain. In structure (39b), the projection of $\mathrm{Agr}_{\mathrm{S}}$ above TP follows from the verb-like behaviour of laysa in relation to its subject agreement properties in that this SA negation marker behaves just like a verb in showing the agreement asymmetry in the derivation of VSO sentences as opposed to the derivation of SVO sentences in SA discussed at length in Chapter Two. The NEG elements maa, lam and lan do not show such a characteristic.

The raising of Neg-T-Agr to Fin, as shown in (39b), is a necessary step for identification of the sentence as [+declarative], [+negative] at the level where Fin and Force are introduced into sentence structure. As in (38b), the function of the matrix complementizer Pinna under Force, in (39b), is to link the assertive interpretation of the proposition expressed by the sentence to negation (i.e., via the head-head dependency relation linking Force to Fin, to which Neg-T-Agr ${ }_{S}$ is attached at the 
interface). Apart from the initial Merge location of the NEG elements maa and laysa, the only other difference between the two negative structures is that the derivation of (39a) requires the projection of a subject-agreement node (i.e., $\operatorname{Agr}_{S}$ ) accounting for the agreement in person/number/gender features on the negative element laysa (spelled out as lasta 'you (are) not' in (39a)). Since a Topic pronominal clitic is merged in [Spec, TopP] in structure (39b), the phonetically realized pronominal Panta 'you' can alternatively remain silent. In this case, a referential pro would occupy [Spec, $\left.\operatorname{Agr}_{S} \mathrm{P}\right]$ yielding the sentence Pinna-ka las-ta yusuf 'You are NOT Joseph".

In sum, the insertion of complementizer Pinna in the matrix sentences I have discussed in this section is not exclusively driven by the necessity of signalling clause-type, but it also contributes to the assertive interpretation these sentences get at the interface. In this respect, in the sample sentence given in (23a), both complementizer Pinna and modal qad share the property of asserting the truthfulness of the proposition expressed by the sentence.

Apart from the insertion of complementizer Pinna, in the derivation of the SA VSO sentences (19a), (20a), (21a) and (32a), and, by extension, the verbless sentences (37a) and (38a) where no verb movement applies and the T-to-Fin raising process is not operative, the proposal I have maintained is that the $\mathrm{Agr}_{\mathrm{S}}$ node does not project in these constructions. In the verbless negative sentence (38a), the negation marker maa is merged in Foc, in which respect it patterns like the affirmative emphatic element $l a$ in (37a). In contrast to maa, the verb-like NEG element laysa in sentence (39a) is merged in the IP domain where the Agr node projects accounting for overt subjectverb agreement between laysa and the subject pronominal in [Spec, $\operatorname{Agr}_{\mathrm{S}} \mathrm{P}$ ].

As the tree representations of (19a), (20a), (21a) and (32a) have shown, the IP domain takes the form of a TP selected as complement by the Fin head in the split-CP domain. As discussed in Chapter Three, subject-verb agreement in terms of $\varphi$-feature valuation and EPP satisfaction, in such VSO configurations in SA, applies at the level where TP is introduced, with the subject raised to [Spec, TP]. Since the inflectional complex is further raised to the split-CP domain, Spell Out, at the strong Phase, only obtains when all the head-head merging or raising processes, in the derivation of the relevant sentences, have applied for feature identification at the interface.

As far as the verbless negative sentence in (38a) (with the negation marker maa) and the negative sentence in (39a) (with the negation marker laysa) are concerned, the sentences differ minimally in that maa is a negation marker that does 
not inflect for either tense or subject agreement. This fact not only makes it different from the negation marker laysa in relation to subject agreement but also from lam and lan, which inflect for tense. Thus, the logical expectation that follows from the verblike behaviour of laysa in relation to its subject agreement properties is that this negation marker would behave just like a verb, in SA, in showing the agreement asymmetry between VSO and SVO structural configurations, as in VSO (40a) vs. SVO (40b) below (EV = Epenthetic Vowel):

(40) a. laysa-t-i l-banaat-u fii l-bayt-i

NEG-3FS-EV the-girls-NOM in the-house-GEN

'The girls are not in the house.'

b. 1-banaat-u las-na fii l-bayt-i the-girls-NOM NEG-3FP in the-house-GEN 'The girls are not in the house.'

I come back to some sample examples of negation sentences in SA (including sentences with laysa) in section 4.1.2.5.

\subsubsection{A higher and a lower NegP projection in $S A$}

\subsection{Introduction}

As shown in Table 4.1 below, two Neg head nodes are assumed to project in the sentence structure of SA: the first Neg head selects FinP within the split-CP domain. The second Neg head position projects within IP: 
Table 4.1. The distribution of NEG elements in SA sentence structure

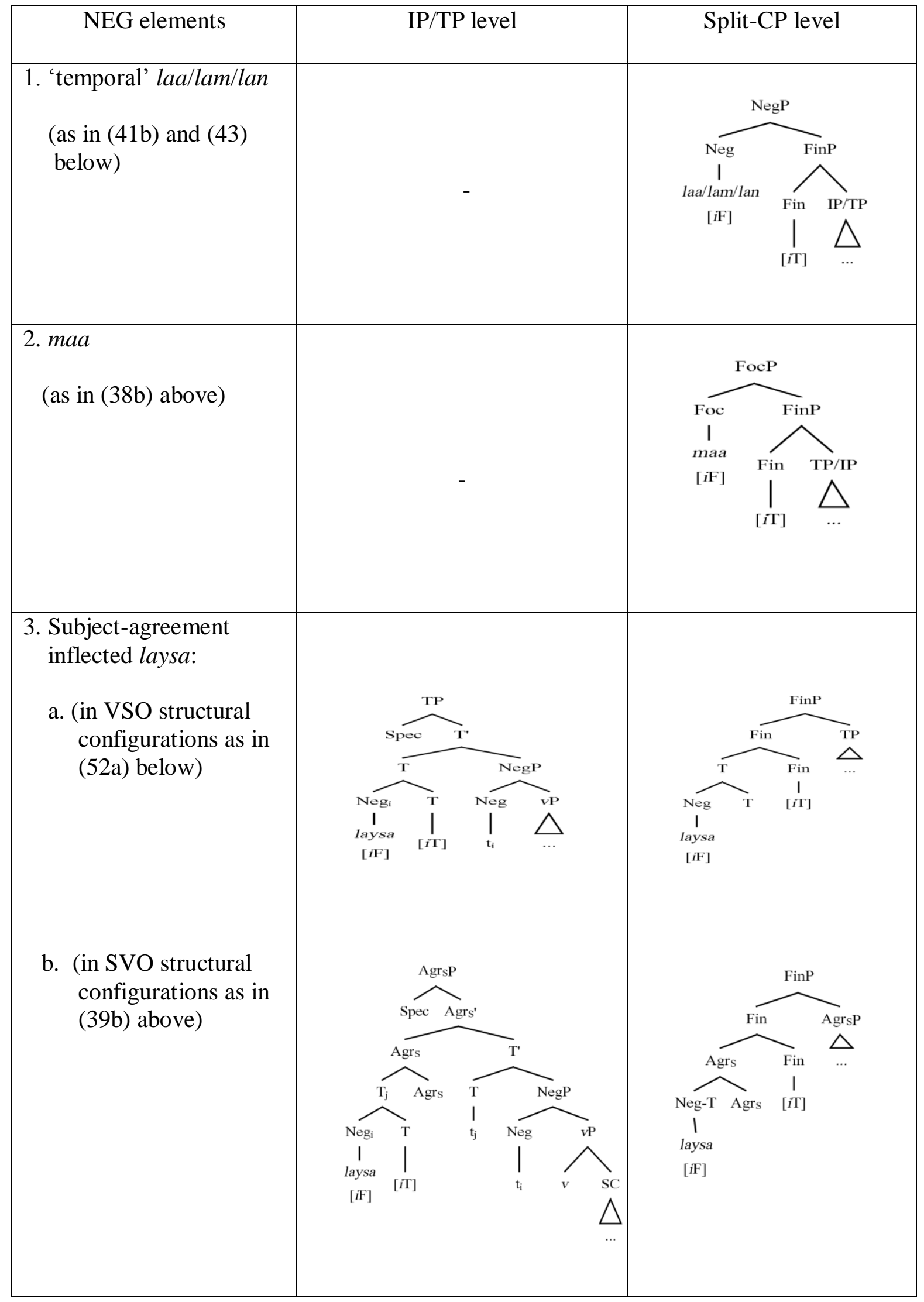


Table 4.1 summarizes the distribution of NEG elements in the sentence structure of SA on the basis of their location and their feature properties. In section 4.1.2.1, I followed Jouitteau (2005) in the assumption that, in the split-CP analysis of languages with 'basic' VSO word ordering, there is a Neg head projection (maximally projecting as NegP) projecting higher than the head Fin node that Rizzi (1997) posits to be the head that selects the inflectional projection of sentences (i.e., TP/IP). As shown in (1) in Table 4.1, this higher Neg node is the Merge site for the 'temporal' negation elements lam and lan (as 'temporal' variants of the NEG element laa, Ouhalla 1993: 276).

The second Neg(P) projects in between TP and $v \mathrm{P} / \mathrm{VP}{ }^{34}$ This lower Neg head is the Merge site for the NEG subject-agreement inflected negation marker laysa (as shown in (3) in Table 4.1). The NEG element laysa can either be phonetically realized in IP or higher in the sentence depending on whether it is used in a sentence where the 'subject' DP is a Topic or in a sentence where some element is under negative contrastive focus.

As for the NEG element maa, I have already discussed the assumption that it does not project as a Neg node, but has the same distribution as the affirmative emphatic element $l a$ in verbless sentences.

According to the assumptions adopted in sections 4.1.2 and 4.1.2.1, the Merge of NEG elements either within the split-CP domain or the IP domain depends on the inflectional features they morphologically encode - namely, either 'tense', as in the case of the 'tensed' negation markers lam and lan, or subject agreement as in the case of the NEG element laysa. As for maa, it does not morphologically inflect for either tense or subject agreement, but it behaves rather like the subject-inflected agreement NEG element laysa in sentence structure in relation to negative contrastive focus.

On this account, both Neg head positions interact with the tense/modal properties of sentences, and their projection in the respective domains where they are first merged (i.e., the split-CP domain and the IP domain) is essential for the proper

\footnotetext{
${ }^{34}$ Ouhalla (1991, 1993) and Bemamoun (2000b) assume that, in SA, Neg is projected lower than TP. As for Fassi Fehri (1993), he assumes that NegP is higher than TP/Agr ${ }_{S} \mathrm{P}$ in sentence structure. The account provided here differs from these previous analyses of negation in that I assume that there are two Neg(P) projections in the sentence structure of SA. As already noted, Bahloul (2008), also allows two structural positions for the Merge of NEG elements (i.e., the Merge of maa in Asert ${ }^{\circ}$ in CP and the Merge of laa/lam/lan in a Neg position within IP). Bahloul (2008) does not provide a particular analysis for the subject-agreement inflected NEG element laysa.
} 
representation of the $\mathrm{C}-\left(\mathrm{Agr}_{S}\right)-\mathrm{T}$ dependency at the interface. Thus, the different structural positions NEG elements occupy in the sentence structure of SA may not entirely depend on the Merge point where these elements are first merged (via external Merge) - namely within the IP domain or within the CP domain. Rather, SA NEG element laysa may end up being spelled out in the split-CP domain according to the structure being derived whereby one or more head-raising processes would be needed for the satisfaction of the $\mathrm{C}-\left(\mathrm{Agr}_{\mathrm{S}}\right)-\mathrm{T}$ dependency at the interface.

In section 4.1.2.5.2 below, I first deal with the derivation of the NEG elements laa/lam/lan in SA (taking the NEG marker of past tense lam, as a representative example). In section 4.1.2.5.3, I deal with the derivation of the NEG elements maa and laysa.

\subsection{The derivation of the NEG elements laa/lam/lan in $S A$}

As Ouhalla (1993: 277, 280) notes, 'assertive' elements (both affirmative like SA modal qad or negative like SA NEG elements lam and lan) interact with Focus as a consequence of the essential relation they have with the temporal/modal properties of the sentence where they are used. In this respect, negation patterns much the same as modality in the split-CP domain as both are represented above the position of the partially inflected verb raised to Fin in SA VSO configurations. Thus, in (41) below, the NEG element lam is merged in the NEG head node maximally projecting as NEGP just above the FinP projection, the head of which harbours the verb ya?ti 'come': (41) a. Pinna-hu lam ya-Pt-i
that-it NEG.past 3-IMPERF.come-MS.JUSS the-boys-NOM
'Indeed, the boys did not come.' (SA) 
b.

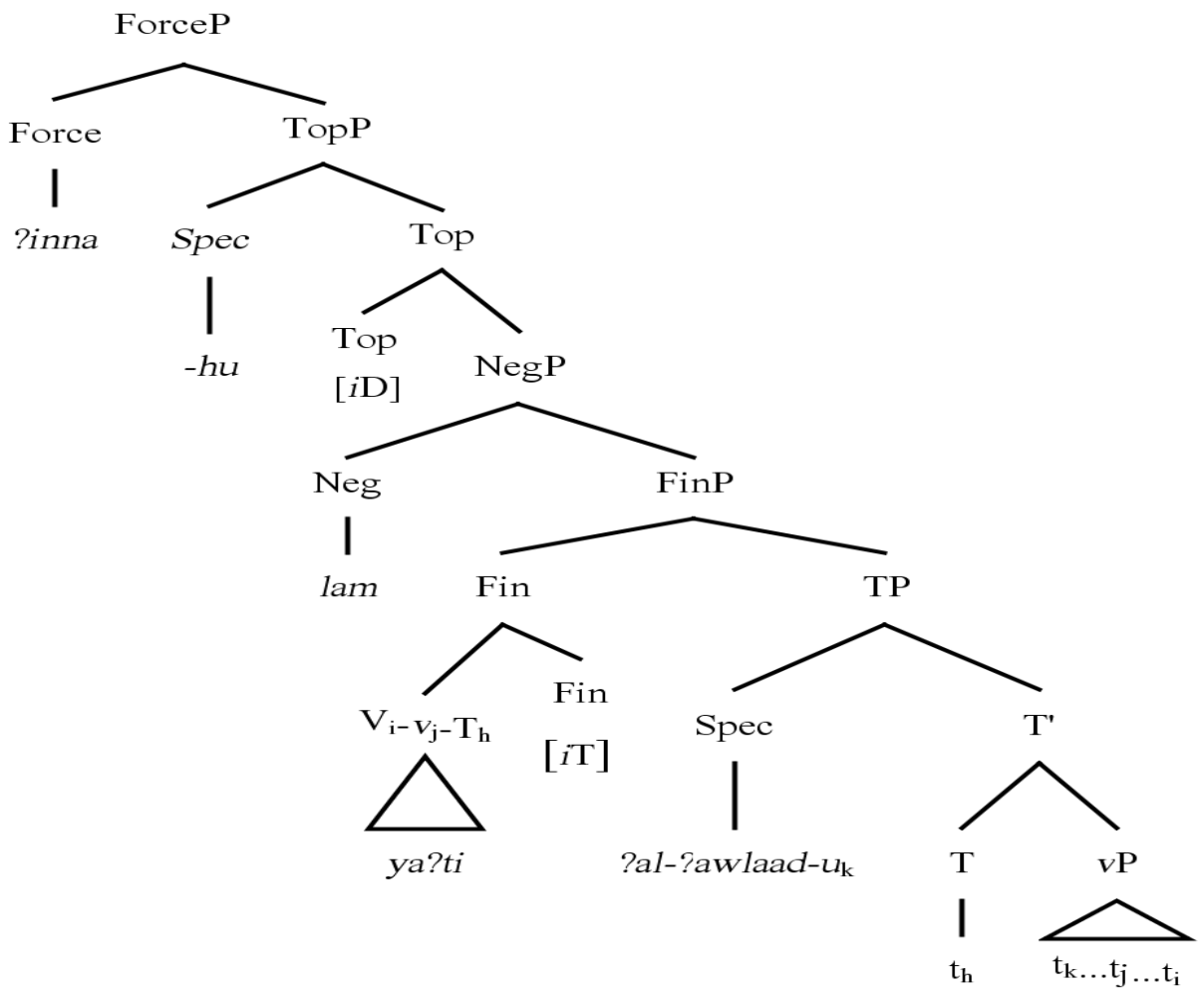

As the VSO structure in (41b) shows, the higher NEG projection selects FinP as its complement in sentence structure. It may be the case that the temporal properties of such SA NEG elements as lam, in (41a) (where the verb ya?ti 'come' is in the jussive), and lan (merged in a clause where the verb is in the subjunctive) is a consequence of the close relationship that such elements have with Finiteness as a head projecting in its own right in the split-CP domain of SA. Whether lam and lan in SA are generated with T-features (i.e. $[i \mathrm{~T}]$ on $\mathrm{Neg}$ ) of their own remains questionable. ${ }^{35}$ The tense properties of such elements may simply be the outcome of their interaction with the Fin projection, which is itself generated with such features in addition to $\varphi$-features, and shared between Fin and T. Ultimately, a NEG element like SA lam in structure (41b) has to satisfy the C-T dependency at the interface.

\footnotetext{
${ }^{35}$ This is also the case for the modal element $q a d$. Thus, whatever temporal properties $q a d$ has (cf. Bahloul 2008; see section 4.1.2.3), they may simply be the outcome of the interaction of such a modal element with the temporal properties encoded on Fin and the head Fin selects as complement in sentence structure i.e., T.
} 
In a system abiding by different assumptions from the analysis adopted here about the projection of sentence structure in SA, Shlonsky (1997: 97-98, following Benmamoun 1992b), argues that Neg, in SA, projects lower than TP but higher than $\mathrm{Agr}_{S} \mathrm{P}$ in the IP domain. Under such an analysis, Neg moves to T independently of the verb (both perfective and imperfective). As Shlonsky (1997) argues, movement of NEG laa and its 'temporal' variants lam and lan to T independently of the imperfective verb raised to the Agr node accounts for why lam and lan carry 'tense'. This raising process prevents the verb from further moving to the T node.

However, if the NEG elements lam and lan are actually merged higher up in the structure of SA negative sentences outside the IP domain as discussed above in relation to structure (41b), the raised verb will still be in a lower position than the Neg head position in which lam/lan are merged. As the VSO structure (41b) shows, the landing site of the raised verb in such negative sentences is the Fin node. In the SVO structure in (43) for sentence (42a) below, the verb is raised to T, which is itself raised to the $\mathrm{Agr}_{\mathrm{S}}$ node on the assumptions adopted in this thesis as to the derivation of SVO sentences in SA.

As Ouhalla (1993) points out, what is important in the derivation of such negative sentences is the system of structural dependency relations that NEG elements establish with the elements they are associated with (namely, verbs, focus elements and/or modal elements) in terms of the system of identification of features discussed above. Ouhalla (1993: 297) proposes an analysis of the different movement and dependency relations involved in derivations that has much in common with the analysis developed here:

...laa and its temporal variants head a NegP which interacts with other functional categories in terms of head-movement processes. The system of identification ... on the basis of the distribution of maa and focus elements in general also seems to account for the distribution and properties of laa and the way it interacts with $\mathrm{V}$-movement. The underlying idea is that the Identification Requirement motivates movement of the verb complex in much the same way ... [it] motivate[s] movement of $f$-/wh-phrases to Spec-FP.

As sentences (42a) (with the structural representation in (43)) and (42b) below show, Topic DPs have to precede 'tensed' NEG elements such as lam: 
(42) a. Pal-?awlaad-u lam ya-Pt-uu the-boys-NOM NEG.Past 3-IMPERF.come-3MP.IND

'The boys did not come.'

b. Pal-Pawlaad-u Pinna-hum lam ya-?t-uu the-boys-NOM that-they NEG.Past 3-IMPERF.come-3MP.IND Lit.: 'As for the boys, they indeed did not come.'

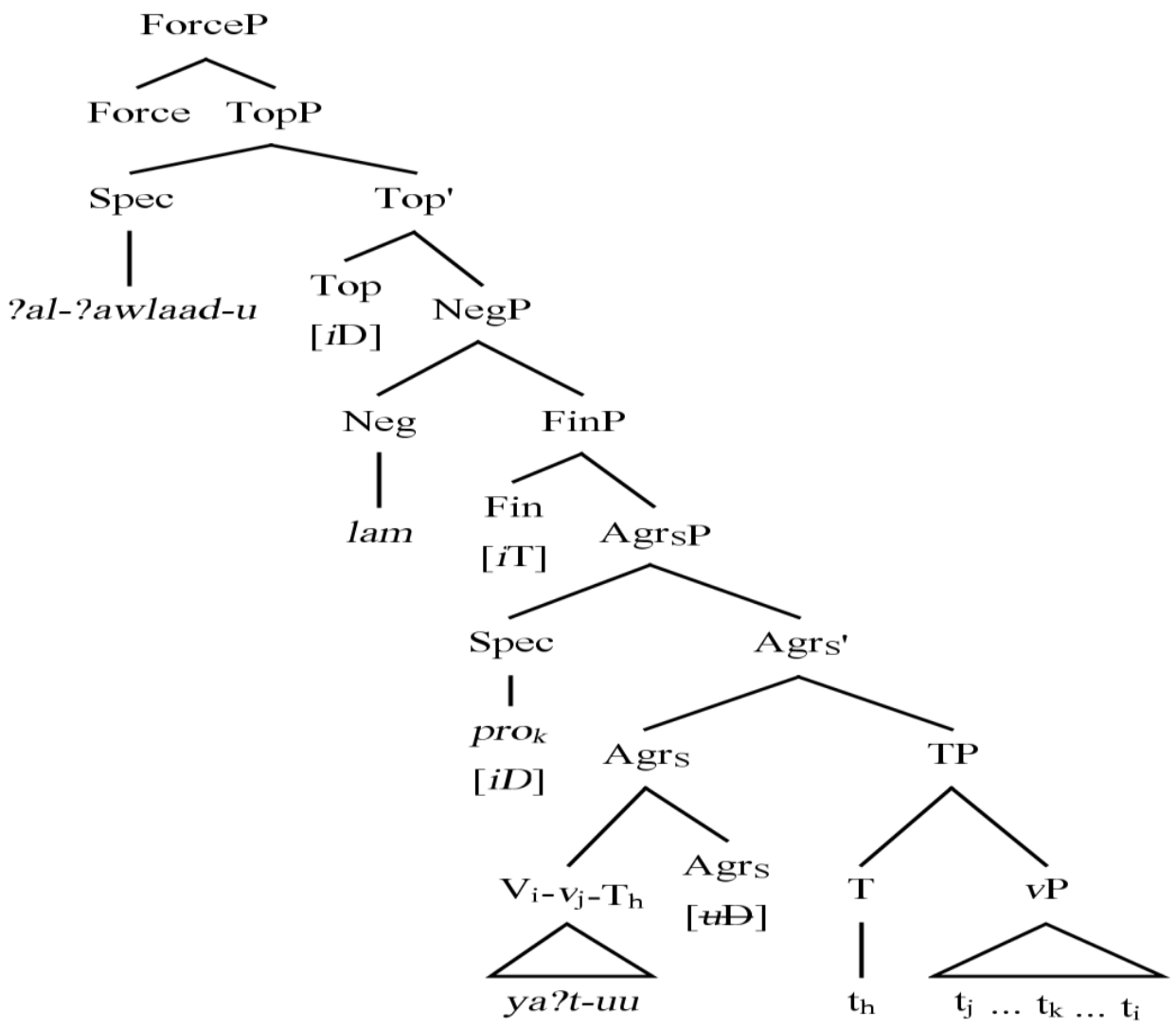

As the structure in (43) proposes, the verb stops under the Agrs projection in the IP domain where full subject-verb agreement obtains. As before, where a Topic DP is base-generated in the split-CP domain in SA, the D-feature of the referential pro raised to $\left[\mathrm{Spec}, \mathrm{Agr}_{S} \mathrm{P}\right]$ is essential in establishing the relation between $\mathrm{D}$ on $\mathrm{Agr}_{\mathrm{S}}$ to $\varphi$-feature specification of the V-v-T-Agr ${ }_{S}$ complex thus determining full subject-verb agreement on the verb ya?t-uu.

\subsection{The derivation of the NEG elements maa and laysa in $S A$}

The NEG element laysa patterns rather like the NEG element maa in its interaction with focus in the 'focus' field within the CP domain in the derivation of VSO 
sentences (see, for example structure (52a) for sentence (51a) below in comparison to structure (52b) for sentence (51b) with the same meaning).

I have discussed the derivation of negative sentences where maa is used in a context where no lexical verb is generated (see section 4.1.2.4). In the presence of a verb in a negative sentence with maa, different assumptions have been developed by linguists in the Arabic linguistics tradition for accounting for the merger of the raised verb with the NEG element maa (and with the enclitic -sh in such modern spoken dialects as MA and TA). For example, both Benmamoun (2000b) and Halila (1992) assume that maa projects as a Neg node in between TP and vP/VP in MA and TA, respectively. Thus, when the verb moves to $\mathrm{T}$ it has to go through Neg first and merge with maa. According to Bemamoun (2000b) and Halila (1992), this is the only available option to derive negative sentences in such modern spoken dialects as MA and TA, which make use of the discontinuous Neg element maa ... sh.

However, Bemamoun's (2000b: 72) analysis of the discontinuous Neg element maa ... sh differs from Halila's (1992: 35) analysis as to how the morphemes maa and -sh end up merged with the verb. Thus, Halila (1992: 35) assumes that negative sentences in TA are derived as represented in (44b) for sentence (44a) (adapted from Halila 1992: 35):

(44) a. nawaal ma-jaa-t-sh Nawal NEG PERF.come-3MS-NEG

'Nawal did not come.'

b.

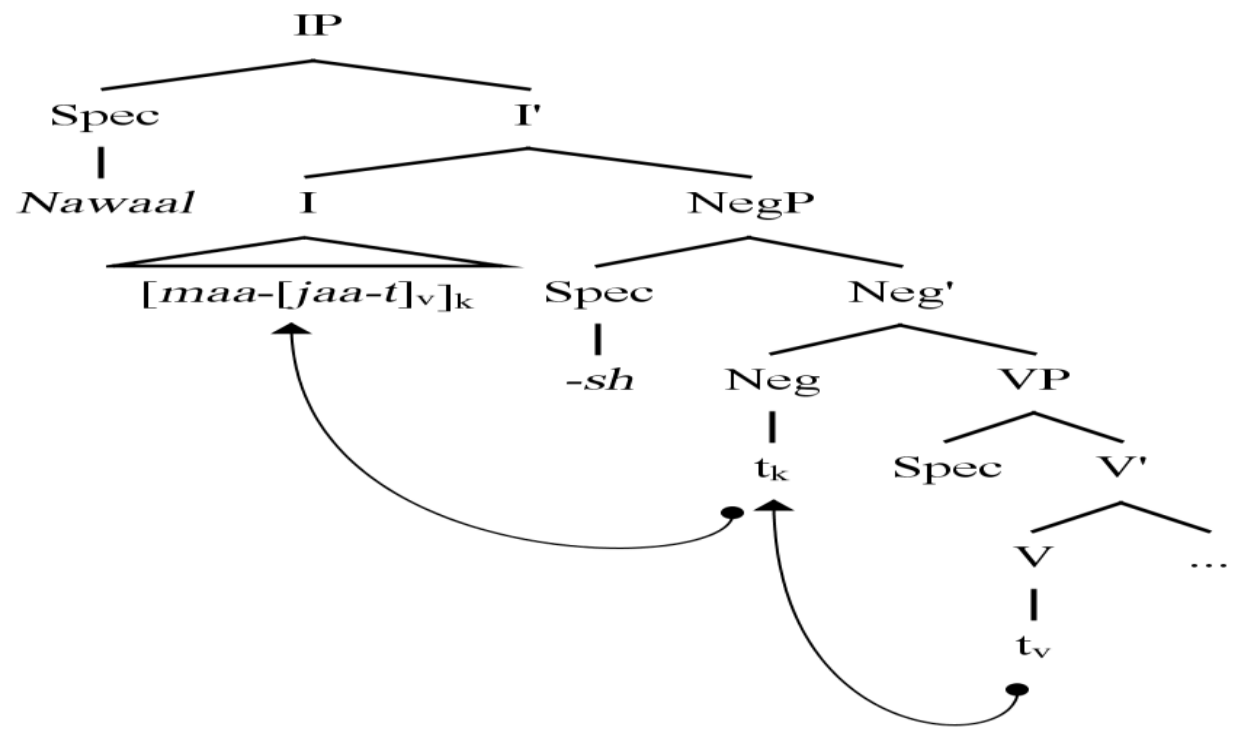


In (44a), for verb movement to be cyclic, the verb jaa-t raises to Neg, where it merges with $m a a$, and then the complex [maa + jaa-t] moves up to $\mathrm{T}$ where a process of encliticization of $-s h$ in $[\mathrm{Spec}, \mathrm{NegP}]$ to $[\mathrm{maa}+$ jaa-t] applies “... in PF by simple merger between the verbal complex in tense and the Spec of NegP that follows it" (Benmamoun 2000b: 73).

Benmamoun (2000b: 76-78), while assuming that this "simple merger" is theoretically possible for the derivation of negative sentences in a spoken Arabic dialect like MA, contends that the so-called discontinuous Neg element maa ... sh is actually not discontinuous. Rather, he assumes that maa and sh are parts of a complex head of NegP maa-sh, an assumption which, according to Benmammoun (2000b) "adequately deals with the interaction between negation and head movement" (p. 76).

In SA, however, Benmamoun (2000b: 108) assumes that maa is in [Spec, $\mathrm{NegP}$. Since Benmamoun (2000b) assumes that the feature involved in the formal licensing of NEG elements is a $[+\mathrm{D}]$ feature (see section 4.1.2.4, in relation to footnote 26), he assumes that maa is subject to a 'feature-checking' requirement quite similar to the checking of $[+\mathrm{D}]$ on $\mathrm{T}$ for EPP satisfaction in the framework of Chomsky (1995b). On such an account, the verb moves to Neg to check the [+D] feature of that node, and in the process of doing so merges with maa in [Spec, NegP]. With past tense verbs, Benmamoun assumes that the verb moves further up to $\mathrm{T}$ to check the V-feature of [+Past] $\mathrm{T}$ (see section 3.3.2.3).

Bahloul (2008) provides a different analysis from that of Benmamoun (2000b) for the derivation of negative sentences with maa in SA. As briefly discussed in section 4.1.2.1, Bahloul (2008: 164-165) assumes that among the elements that are merged in some $\mathrm{Asrt}^{\circ}$ head position that selects TP is the NEG element maa. According to Bahloul (2008), the same $\mathrm{Asrt}^{\circ}$ head is also the Merge position for such elements as the modal element qad and the complementizer element Pinna. As for the 'temporal' NEG elements laa/lam/lan, Bahloul (2008: 155) assumes that such elements are base-generated in some Neg head position below $\mathrm{T}$ and are then raised to T.

It seems that Bahloul's (2008) assumption in relation to the licensing conditions applying to a functional element like maa adequately accounts for the fact that this negation element patterns like the assertive emphatic element $l a$, both of which would be merged in a head node Asrt $^{\circ}$ above IP (Bahloul 2008: 164-165). In the framework of assumptions about the split-CP in SA adopted here, la and maa 
would be merged in the Foc head projection at the highest level of the 'focus field' in Poletto's (2000b) and Benincà \& Poletto's (2004) terms (see section 4.1.2.1). In such a position, the $[i \mathrm{~F}]$ feature of maa or the $[i \mathrm{~F}]$ feature of an assertive element like $l a$ would be structurally identified in relation to the $[i T]$ feature of Fin in the lower part of the split-CP domain, and in relation to the $[i \mathrm{~T}]$ feature of $\mathrm{T}$ in the IP domain.

For example, the representation in (45b) below shows how verb movement in the VSO word ordering in SA interacts with maa in the derivation of sentence (45a):

(45) a. Pinna-hu maa jaa?-a yusuf that-it NEG PERF.come-3MS Joseph 'Joseph did NOT come.'

b.

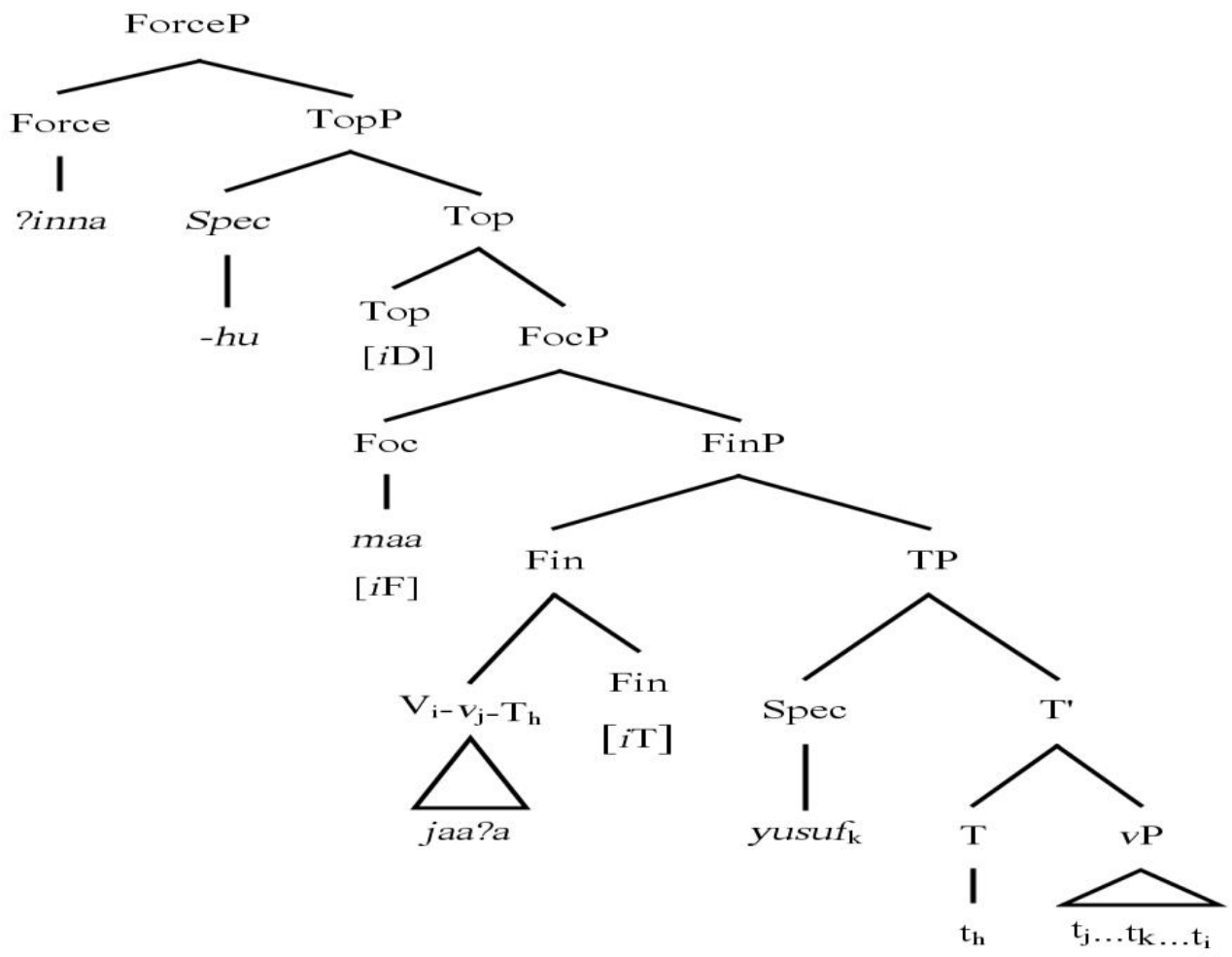

The VSO structure (45b) shows that the complex V-v raises to T in its way to Fin. The NEG element maa is merged in Foc where the identification of its $[i F]$ feature operates in relation to the inflectional verbal complex $\mathrm{V}-v$-T under the Fin node specified for interpretable $[i \mathrm{~T}]$ features. Thus, it is possible that the Agree relation between maa under Foc and T raised to Fin along with $\mathrm{V}-v$ is actually not a relation of feature valuation à la Chomsky $(2001,2004)$. Rather, this Agree relation could be 
understood in terms of Criterial satisfaction à la Rizzi (1996, 1997, 2006), whereby the features that enter into the 'matching' relation are the $[i \mathrm{~F}]$ feature of $\mathrm{Neg}$ maa and the $[i T]$ feature of $T$. These features have to be 'identified' for the satisfaction of the C-T dependency at the interface.

As far as is the $[i \mathrm{~F}]$ feature of maa is concerned, the probe-goal-Agree system adopted here is more in line with Ouhalla's (1993), R\&R's, Vangsnes (2002) and Aoun et al.'s (2010) views on feature licensing in that maa in SA has to satisfy some feature identification requirement in terms of some 'focus' feature which is a characteristic of quantifier elements (cf. R\&R). In section 4.1.2.4 above, I assumed that this feature on the functional nodes that project in the 'focus' field of split-CP domain is an interpretable $[i \mathrm{~F}]$ feature that does not need checking/valuation (contra Benmamoun 2000b).

As for the subject-agreement inflected NEG element laysa in comparison to the NEG element maa in SA, laysa can interchangeably be used instead of maa in sentences implying a contrastively focused reading, as in $(47 \mathrm{~b})$ for sentence $(46 \mathrm{~b}):^{36}$

(46) a. maa ?allafa-t zaynab-u riwaayat-an

NEG PERF.write.3FS Zaynab-NOM novel-ACC

'Zaynab did NOT write a novel.'

b. maa riwaayat-an Pallafa-t

zaynab-u

NEG novel-ACC PERF.write.3FS Zaynab-NOM

'It is NOT a novel that Zaynab wrote.'

(47) a. *laysa-t Pallafa-t zaynab-u riwayat-an

NEG.3FS PERF.write.3FS Zaynab-NOM novel-ACC

'Zaynab did NOT write a novel.'

b. laysa-t riwaayat-an Pallafa-t zayanb-u

NEG.3FS novel-ACC PERF.write.3FS Zaynab-NOM

'It is NOT a novel that Zaynab wrote.'

\footnotetext{
${ }^{36}$ Sentences $(46 a, b)$ are adapted from Ouhalla (1993: 287). The NEG marker laysa-t $(47 \mathrm{~b})$ can be used instead of maa in (46b). In contrast, in (47a), laysa-t cannot replace maa in (46a). This is accounted for on the assumption that laysa is incompatible with verbs used in the past tense because laysa and its subject-agreement inflected variants (as listed in footnote 33 above) already carry a 'past-tense' morpheme which would encode a pure agreement relation. Thus, in (47a), laysa cannot negate the verbal event ?allafa-t zaynab-u riwaayat-an 'Zaynab wrote a novel'. In terms of syntactic structure, the co-occurrence of a 'past-tense' inflected verb and laysa would be ruled out on the basis that both elements would occupy the T node (or would be merged with it as a result of raising laysa, merged in Neg, to T).
} 
In $(46 a, b)$, the scope of maa in sentence structure varies depending on what element maa contrastively focuses. Thus, according to Ouhalla (1993: 287), the NEG marker maa in (46a) has scope on the whole sentence Pallafa-t zaynab-u riwaayat-an denying that 'Zaynab wrote a novel'. The implication is that Zaynab did something else other than write a novel. As for (46b), the NEG marker maa has scope only on the preposed object DP riwaayat-an 'novel'. The implication is that Zaynab could have written something, but certainly not a novel. The same applies to laysa, save for the ungrammaticality of using laysa when it is the verbal event that is contrastively focused (see footnote 36).

On this account, merging maa under Foc in (46b) and raising laysa to $\mathrm{T}$ and then to Fin in (47b) contribute to the scope properties these two sentences encode. By virtue of the contrastive focus function the Merge of maa and laysa in (46b) and (47b) brings about in sentence structure, the identification requirement (which modal and negation elements in the 'focus field' above Fin have to meet) is satisfied (see also the discussion of (45b) above).

As Benmamoun (2000b: 105) points out, laysa is not compatible with future tense, as in (48a). This is also true of maa as exemplified in sentence (48b) (example (48a) is adapted from Benmamoun 2000b: 109):
(48) a. *laysa sa-yu-saafir-u
NEG.3MS MOD(FUT)-3-IMPERF.travel-MS.IND Zayd-NOM
zayd-un
'Zayd is not going to travel'
b. *maa sa-yu-saafir-u
zayd-un
NEG MOD(FUT)-3-IMPERF.travel-MS.IND Zayd-NOM
'Zayd is not going to travel'

As sentences (48a,b) exemplify, laysa and maa cannot co-occur with the modal marker $s a$ - which is used for the expression of future tense.

The incompatibility of these NEG elements with future tense may have to do with the fact that the future tense is not an instantiation of an inflectional/agreement marker that would be encoded on the verb in the tense system of SA, but is a modal category (Fassi Fehri 1993, 2004). Similarly, the laysa and maa NEG elements cannot 
be used in conjunction with qad, which when co-occurring with an imperfective verb expresses the future tense, as in $(49 \mathrm{a}, \mathrm{b}):{ }^{37}$

$$
\begin{aligned}
& \text { (49) a. *qad laysa yu-saafir-u } \quad \text { zayd-un } \\
& \text { MOD NEG.3MS 3-IMPERF.travel-MS.IND Zayd-NOM } \\
& \text { 'Zayd will probably not travel.' }
\end{aligned}
$$

b. *qad maa yu-saafir-u zayd-un

MOD NEG 3-IMPERF.travel-MS.IND Zayd-NOM

'Zayd will probably not travel.'

In (49a,b), the NEG elements laysa and maa are not compatible with the modal element qad for the expression of the non-occurrence of a future event. The only grammatical output is to use modal qad with the NEG element laa as in (50a), to express the probable non-completion of a future event, or the use of the morphologically future tense inflected NEG element lan, for the expression of the impossibility of the occurrence of a future event (see section 4.1.2.5.2), as in (50b):

$$
\begin{aligned}
& \text { (50) a. qad laa yu-saafir-u } \\
& \text { MOD NEG.Fut 3-IMPERF.travel-MS.IND Zayd-NOM } \\
& \text { 'Zayd will probably not travel.' }
\end{aligned}
$$

$$
\text { b. lan yu-saafir-a zayd-un }
$$

NEG.Fut 3-IMPERF.travel-MS.SUBJ Zayd-NOM

'Zayd will not travel.'

I will attempt in what follows to give an account for the difference between the behaviour of the NEG elements maa and laysa as against the behaviour of the NEG element laa and its 'temporal' variants. As Bahloul (2008: 137-139) observes, unlike the 'temporal' negators laa, lam, and lan, the NEG element maa "does not control the temporal interpretation" of the sentences where it occurs. Thus, unlike laa/lam/lan, the NEG element maa can be used in sentences where the verb is perfective or imperfective.

My assumption has been that, in contrast to maa and laysa, the 'temporal' negators laa, lam, and lan project as $\mathrm{Neg}(\mathrm{P})$ above FinP and encode some tense properties (only morphologically realized on lam, and lan, but not on laa) by virtue of selecting FinP, the head node of which is the repository of 'tense' and agreement $\varphi$ -

\footnotetext{
${ }^{37}$ As discussed in section 4.1.2, the combination of modal qad with the imperfective verb expresses a future tense, although this property is not morphologically encoded on qad itself.
} 
features (according to Chomsky 2008) shared by Fin and T in sentence structure (as discussed, for example, in relation to (41) above). By that token, laa, lam, and lan do control the temporal interpretation of the sentences where they are used. As for the negation markers maa and laysa, although the assumption has been that they are not linked to any tense property of the sentence where they are merged, they ultimately have to meet Ouhalla's (1993) Identification Requirement by of virtue of being linked to the $[i \mathrm{~T}]$ features of Fin and $\mathrm{T}$.

Coming back to the Merge (and raising) processes involved in the derivation of negative sentences with laysa and maa, (52a) and (52b) are the tree representations for the first conjunct of sentences $(51 \mathrm{a})$ and $(51 \mathrm{~b})$, respectively $(\mathrm{EV}=$ Epenthetic Vowel): ${ }^{38}$
a. Pinna-hu laysa-t-i 1-Pumahaat-u (Pallaatii) ya-Drib-na that-it NEG-3FS-EV the-mothers-NOM that.3FP 3-IMPERF.hit-FP Pawlaad-a-hunna bal-i 1-Pabaa?-u boys-ACC-their but-EV the-fathers-NOM
Lit.:'The fact is that it is NOT mothers that hit their sons/children but it is fathers that do'
$\begin{array}{lll}\text { b. Pinna-hu maa 1-Pumahaat-u (Pallaatii) } & \text { ya-Drib-na } \\ \text { that-it NEG the-mothers-NOM that.3FP } & \text { 3-IMPERF.hit-FP }\end{array}$
Pawlaad-a-hunna bal-i l-Pabaa?-u boys-ACC-their but-EV the-fathers-NOM
Lit.:'The fact is that it is NOT mothers that hit their sons/children but it is fathers that do'

\footnotetext{
${ }^{38}$ Assuming that laysa is a 'negative auxiliary' (Ouhalla 1993: 277-278) similar to auxiliary kaana in SA (see the VSO structure (66b) below; cf. Akkal \& Gonegai 2000: 13-14), an argument for a biclausal structure of (51a) can be maintained. For a different view maintaining a mono-clausal structure to such examples in SA, see Aoun et al. (2010: 61). I come back to Akkal \& Gonegai's (2000) argument below in relation to the bi-clausal VSO structure (66b) and the bi-clausal SVO structures (67b) and (68b).
} 
(52). a

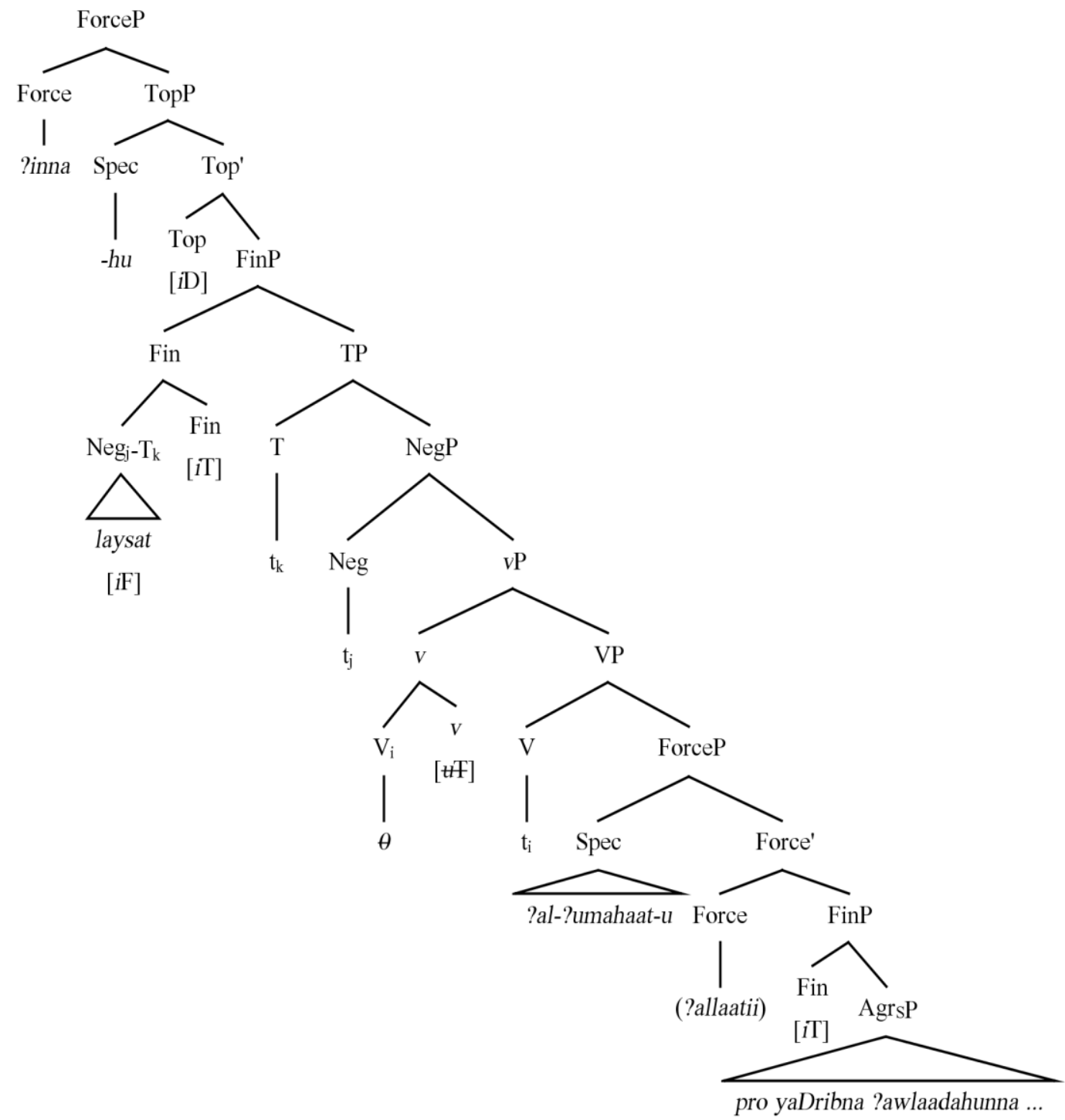


b.

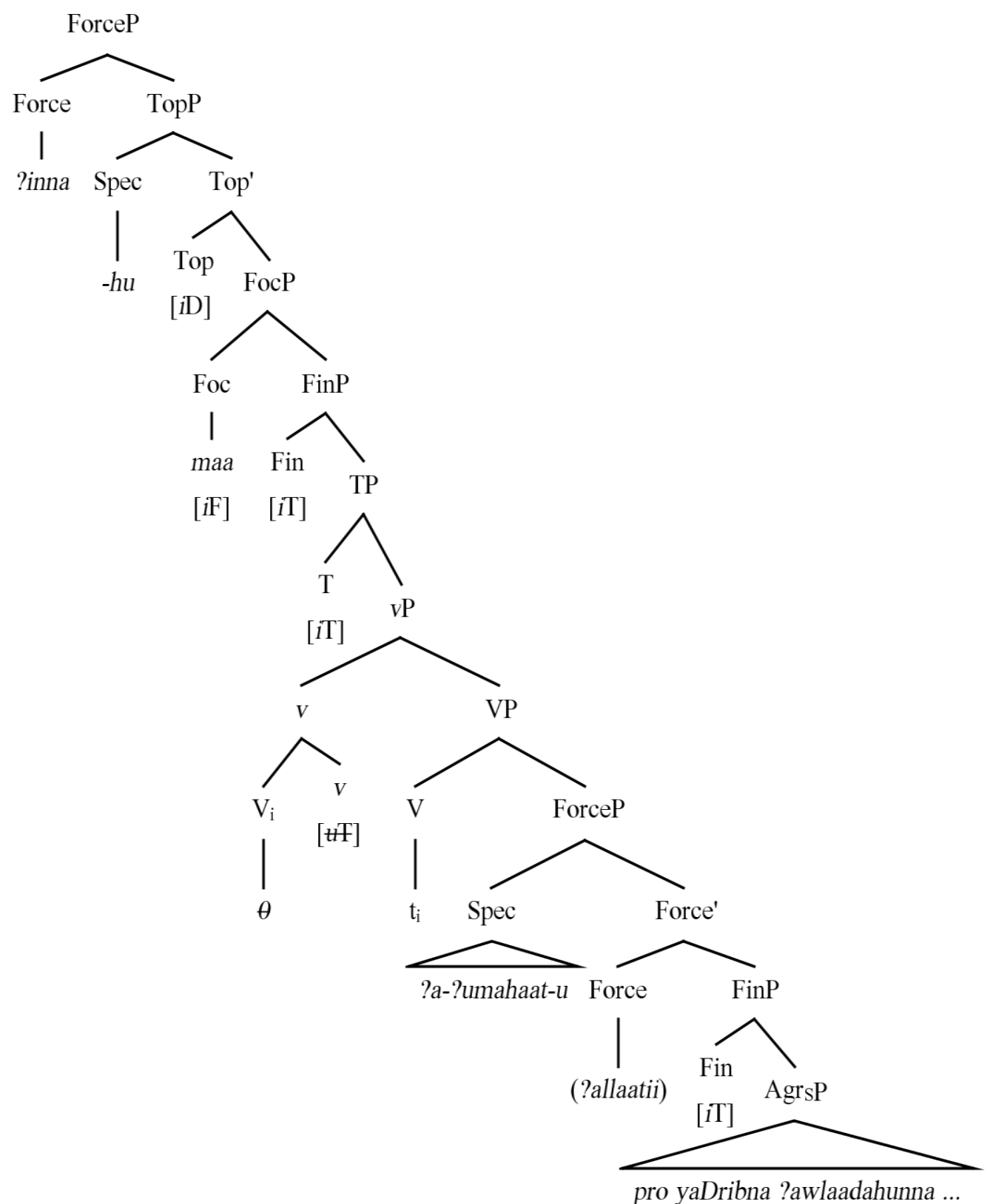

The structures in (52a) and (52b) are bi-clausal with two separate IP domains. These bi-clausal structures involve a lower IP domain, which projects as $\operatorname{Agr}_{S} \mathrm{P}$ (with a leftperiphery involving the projection of FinP and ForceP), and a higher IP domain, which projects as TP. Since no lexical verb is generated in the higher clause, the V head of the VP projection is not lexicalized and is left empty as a null V. V raises to $v$ for the dependency relations between $\mathrm{T}$ and $\mathrm{V}-v$ to apply in terms of the head-head agreement configurations linking $[u \mathrm{~T}]$ on $v$ to $[i \mathrm{~T}]$ on $\mathrm{T}$. As discussed in section 
3.3.2.3, the relation between $v$ and $\mathrm{T}$, in terms of the identification of $\mathrm{T}$-features, remains essential within IP for the satisfaction of the C-T dependency at the interface.

In (52a), the higher IP domain involves the raising of NEG to T and the NEGT complex to the left periphery where the Top-Foc system is displayed. The NEG-T complex moves to Fin, just like 'partially' inflected verbs do in SA VSO sentences (see section 4.1.2.1). In (52b), maa is merged under the Foc head. In both structures, interpretability of the $[i \mathrm{~F}]$ focus feature of laysa and maa operates with the interpretable $[i \mathrm{~T}]$ features on Fin and $\mathrm{T}$.

As for the DP Pal-Pumahaat-u 'the mothers' in (52a) and (52b), it has the status of a focused DP merged in [Spec, ForceP] where it is base-generated (see section 4.2 below on wh-dependencies in SA). Base-generating the DP Pal-Pumahaat$u$ in the Spec of Force in these structures is supported by the optionality of having the relative complementizer ?allaatii merged in Force and agreeing in gender and number with the DP in [Spec, ForceP]. Thus, being base-generated to the left of IP (i.e., the lower clause represented as $\operatorname{Agr}_{S} \mathrm{P}$ in (52a) and (52b)), the DP Pal-Pumahaat- $u$ is coreferential with a resumptive pro in the lower clause. In the lower clause, subject-verb agreement is established at the level of $\operatorname{Agr}_{S} \mathrm{P}$ (i.e., the strong Phase, at the level where T-to-Agr applies in the lower clause) accounting for the fully realized agreement inflection -na on the verb yaDrib-na 'they hit'.

Given the anaphoric relation between $\mathrm{T}$ in the lower clause and $\mathrm{T}$ in the higher clause, (Akkal \& Gonegai 2000; see right below), the subject-agreement inflected NEG element laysa- $t$ 'partially' agrees in $3^{\text {rd }}$ person and feminine gender features with the feminine plural noun Pal-Pumahaat- $u$ to its right in [Spec, ForceP] in the higher part of the split-CP of the lower clause. As discussed above, the DP PalPumahaat- $u$ is itself resumed in the lower clause by the resumptive pro in the Spec position of the inflectional complex V-v-T-Agrs.

This 'partial' agreement configuration between a 'verbal' element (i.e., the subject-agreement inflected NEG element laysa- $t$ in (51a)) and a DP to its right is reminiscent of the agreement configuration that obtains between the verb raised to Fin and a subject DP in [Spec, TP] in SA VSO sentences, as in (53) below:

(53) Rinna-hu jaa?a-t ?al-bannat-u

that-it PERF.come-3FS the-girls-NOM

'The girls came.' 
In (53), the verb jaa?a-t 'came' raised to Fin 'partially' agrees with the feminine plural noun Pal-bannat- $u$ 'the girls' in [Spec, TP], in the VSO sentence

Thus, in (51a) and (53), both the 'partially' subject-agreement inflected NEG particle laysat and the subject-agreement inflected verb jaa?at 'came' agree with their respective DP elements to their right (i.e. Pal-Pumahaat- $u$ in the higher part of the split-CP domain of the lower clause in (51a) and the 'post-verbal' subject DP Palbannat- $u$ 'the girls' in (53)).

The bi-clausal structure of such sentences, as in (1) below, where the auxiliary kaana is used in combination with a thematic verb to form complex tenses is also defended in Demirdache (1989/2013: 294-307): $:^{39}$

(54) kaana y-aktub-u

PERF.be-3MS 3-IMPERF.write-MS.IND

'He was writing.'

Like Akkal \& Gonegai (2000), Demirdache (2013: 294-295) underlines the fact that what is important in such bi-clausal constructions are the tense properties of the auxiliary verb kaana used in the higher clause of (54). In Demirdache's (2013: 294) own terms: “... the copula verb kaana is merely a tense carrier". Quite similar to Akkal \& Gonegai's (2000) assumptions, Demirdache (2013: 297-298) points to the fact that the auxiliary verb kaana in the higher clause has the mere function of referring to a certain point in time, whereas the thematic verb in the lower clause has the function of encoding the degree of realization of an event.

However, the main difference between Akkal \& Gonegai's (2000) and Demirdache's (2013) accounts of the derivation of subject-verb agreement configurations within these constructions is that Akkal \& Gonegai (2000) reject the hypothesis of the existence of null pro elements in the syntax of SA. As reviewed in Chapter Two, Akkal and Gonegai (2000) argue for a movement analysis of the derivation of Topic DPs. According to Akkal \& Gonegai's (2000) analysis, there is no referential pro (nor, for that matter, an EXPL pro) in the structure of the sentences in question.

In the analysis of these bi-clausal sentences in this chapter, I partially agree with both the analysis of Akkal \& Gonegai (2000) and Demirdache (2013) in that,

\footnotetext{
${ }^{39}$ For convenience, I will henceforth be referring to Demirdache (2013).
} 
like Akkal \& Gonegai (2000), I assume that EXPL pro is not merged in the IP domain in the higher clause of such constructions, but, like Demirdache (2013), I assume that there is necessarily a referential pro in the Spec position of the $\mathrm{Agr}_{\mathrm{S}}$ node in the lower clause and that Topic DPs are base-generated in position (contra Akkal \& Gonegai 2000).

Demirdache's (2013: 296-297) set of assumptions can be summarized as follows. First, both the auxiliary kaana and a thematic verb, like yaktubu 'he writes' in (54) above, are morphologically ‘main' verbs since they are fully inflected for tense. Demirdache (2013) assumes that both the auxiliary (merged in an AUX node and raising to an AGR node selecting it) and the thematic verb (merged in a V node and likewise raising to an AGR node selecting it) must both be dominated by a Tnode (which selects both AGR nodes). Next, Demirdache (2013: 298) assumes that the Spec position of the AGR node in the higher clause is occupied by an EXPL pro element raised out of [Spec, AUXP] whenever cases of subject-verb agreement mismatches between the thematic verb in the lower clause and the auxiliary verb kaana in the higher clause occur. I come back to examples of such cases below. Thus, in such a system, to derive the VSO word order in the higher clause in such SA biclausal constructions where 'weak' subject-verb agreement arises, auxiliary kaana first moves to AGR (for Case-licensing) and then to T leaving EXPL pro behind in [Spec, AGRP].

Adopting such an architecture for the clause structure of sentences with complex tenses, Demirdache (2013: 299) is able to account for subject-verb agreement between a nominative topic in [Spec, TP] and the auxiliary verb kaana in AGR in the higher clause, as for example in (55b) and (55c), which differ from (55a) in the distribution (and status) of the 'subject' DP hindun 'Hind' (i.e., being either a 'real' subject as in (55a), or a Topic DP as in (55b-c)):

(55) a. kaan-at t-aktub-u hind-un li-xaalid-in (SA) PERF.be-3FS 3-IMPERF.write-FS.IND Hind-NOM to-Khalid-GEN 'Hind was writing to Khalid.'

b. kaan-at hind-un t-aktub-u li-xaalid-in

c. hind-un kaan-at t-aktub-u li-xaalid-in 
Returning, later on, to a structural representation of sentence (55c) (for which Demirdache 2013 does not provide a structure), the representations of (55a) and (55b) are, respectively, as in (56a) and (56b) below (Demirdache 2013: 300):

(56) a.

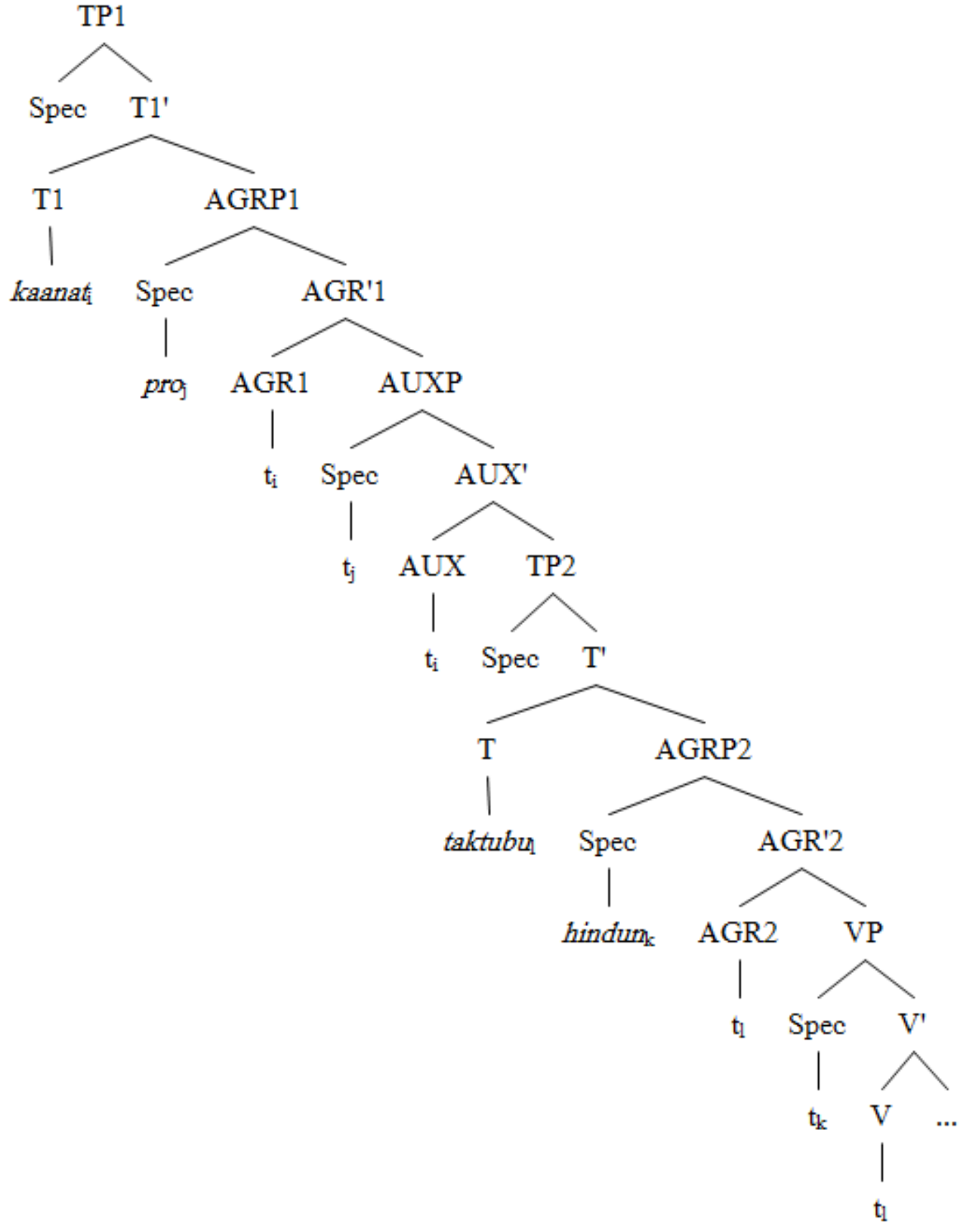


b.

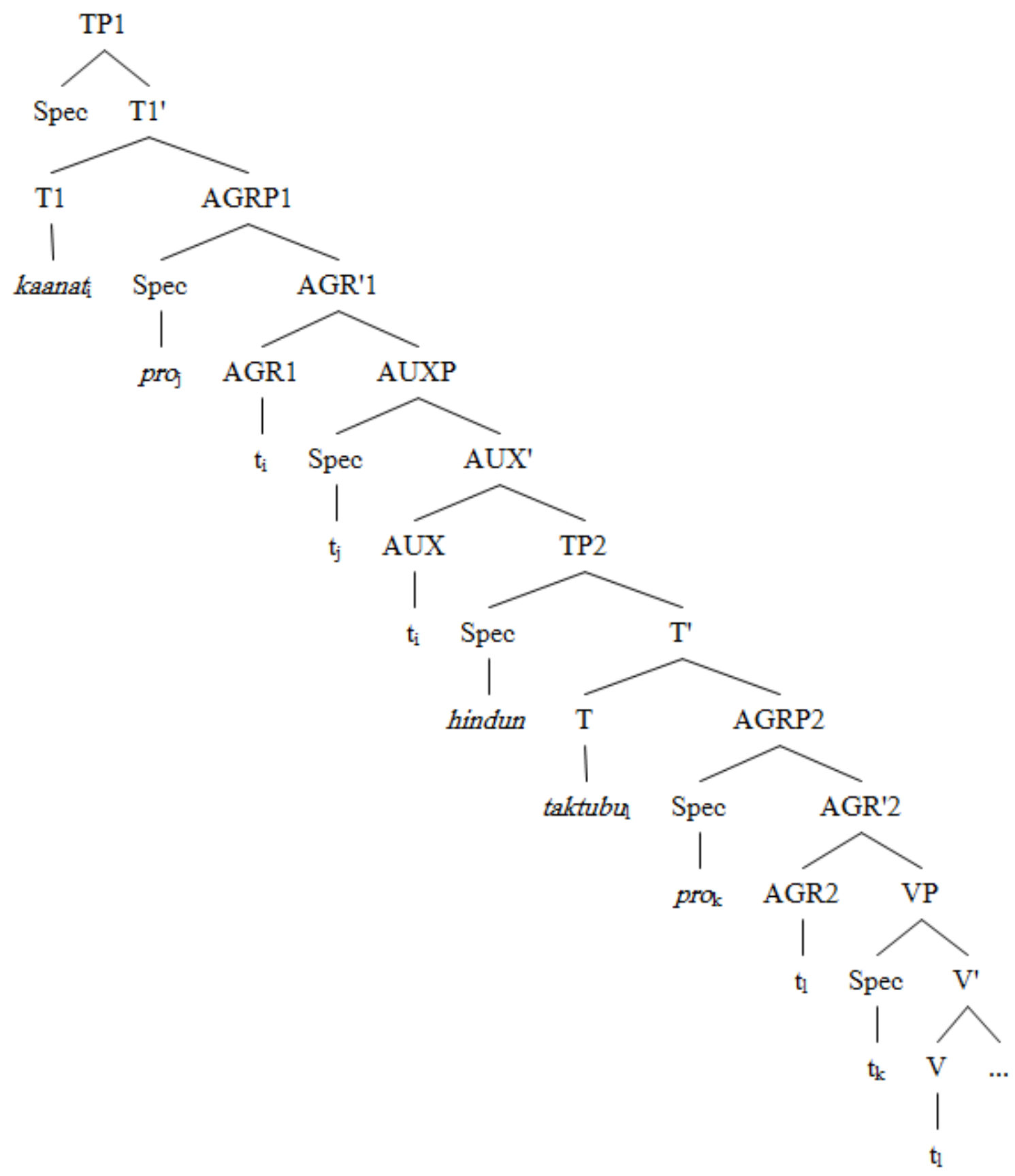

Apart from the difference in the status of the DP hindun in (56a) and (56b) - i.e., as a 'subject' DP in (56a) and as a Topic DP in (56b) -, the representation in (56a) is similar to the representation in (56b) in the Merge process of an EXPL pro in [Spec, AuxP] in the higher clause, and the subsequent movement of this EXPL to [Spec, AGRP2] for Case-licensing. 
According to my understanding of Demirdache's (2013) system of assumptions, the structural representation of (55c) would be as in (57):

(57)

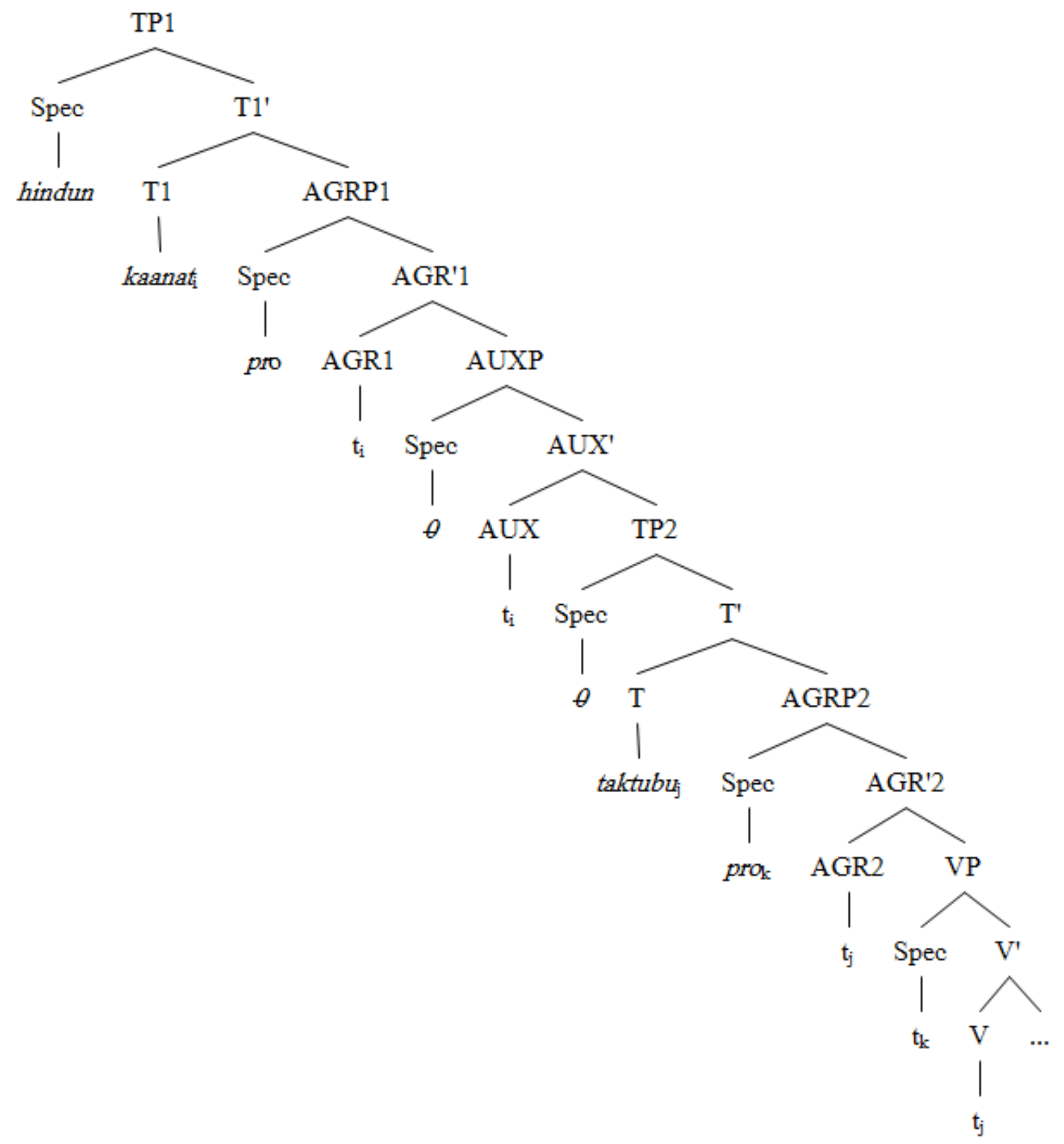

Adopting a non-movement analysis of Topic DPs in the sentence structure of SA (as in Demirdache's 2013 system of assumptions), the DP hindun in (57) is presumably base-generated in the higher clause in [Spec, TP1]. As can be seen in the representation provided in (57), my guess is that both [Spec, TP2] and [Spec, AUXP] remain empty (and are presumably not projected since they are not landing sites for the movement of DPs). The reason for such a derivation is that the DP hindun in (57), 
being a Topic DP, is base-generated in the position where it is spelled out at PF. Thus, in (56c), the relation of hindun in [Spec, TP1] to the auxiliary verb kaanat in T1 is similar to the relation of hindun in [Spec, TP2] to the thematic verb taktubu 'she writes' in T2. Necessarily, [Spec, AUXP] remains empty (or unprojected, as I have assumed here) since the auxiliary verb kaana does not assign an external theta role (cf. Demirdache 2013: 300). If the representation in (57) is correct, then both the pro in [Spec, AGRP1] and the pro in [Spec, AGRP2] are referential, but differ with respect to the raising process undergone by pro in the lower clause from [Spec, VP] to [Spec, AGRP2], and the absence of such a raising process for pro in the higher clause, which would be base-generated in [Spec, AGRP1] due to the non-projection of [Spec, AUXP] in such a structure.

The subject-verb agreement configurations in the lower clause and the higher clause of a structure like (57) in terms of two referential pro elements, as analyzed above, can also involve a 'rich' morphological realization of number (appearing as a plural agreement morpheme on the verb agreeing with a plural noun) in addition to person and gender (i.e., the phonetically realized morphology on verbs agreeing with a singular noun) in such configurations. Since the framework of assumptions about functional projections within the structure of sentences in SA developed in this thesis also adopts an incorporation analysis of agreement in subject-verb agreement configurations in SA similar, in some respects, to that adopted in Demirdache (2013), I will be providing a structural representation for the sentence, in (58a) below, in terms of the split-CP domain where the Force node selects the FinP projection, which, in turn, selects the IP domain in the form of an $\operatorname{Agr}_{S} \mathrm{P}$ projection (for ease of exposition, I omit the representation of the Force $(\mathrm{P})$ above the Topic DP in the higher clause. I also omit the representation of the feature structure - T-features in combination with D-features - of the functional head projections and functional elements involved in the derivation of such a sentence):

(58) a. Pal-muSallimuuna kaan-uu yu-darris-uuna n-naHw-a the-teachers-NOM PERF.be-3MP 3-IMPERF.teach-MP the-syntax-ACC 'The teachers were teaching syntax.' 
b.
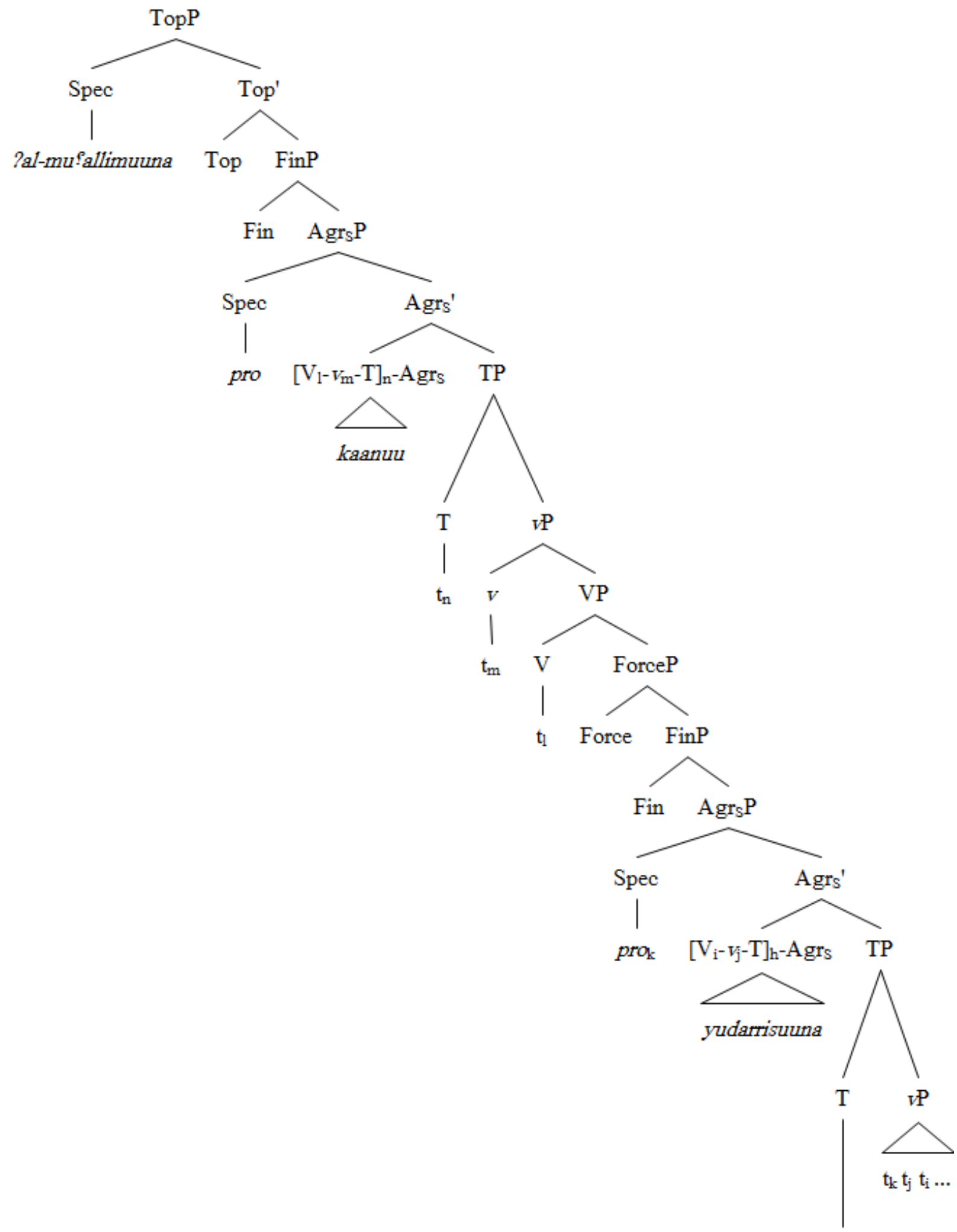

$t_{h}$

The representation in (58b) shows that the two hypothesized thematic pro elements (i.e., pro and $p r o_{\mathrm{k}}$ ) occupy the Spec position of the two $\operatorname{Agr}_{\mathrm{S}} \mathrm{P}$ projections in the two respective domains of subject-verb agreement within this bi-clausal structure. Thus, 
subject-verb agreement occurs between pro and the auxiliary verb kaanuu in the higher clause, and between pro $_{\mathrm{k}}$ and the thematic verb yudarrisuuna 'they teach' in the lower clause. The two subject-verb agreement relations are structurally related in that they are both anaphorically relating a referential pro element, within their respective IP/Agr $\mathrm{P}$ domains, to the Topic DP PalmuSallimuuna 'the teachers' in the split-CP domain of the higer clause.

Within the framework of assumptions adopted in this thesis, as well as the framework of assumptions Demirdache (2013) adopts, this anaphoric relation is the direct consequence of the tense properties involved in the subject-verb agreement configurations established within this bi-clausal structure. Ultimately, the analysis presented here supports the basic thesis I have been developing in this and the previous chapters - namely, that it is by virtue of the T-dependency and the feature structure of functional elements that the derivation and representation of sentences is achieved.

Now, there is a special subject-verb agreement configuration where Demirdache's (2013: 301) EXPL-pro replacement at LF (which she assumes is the counterpart of there-insertion constructions in English) and the accompanying assumption about the presence of an AGR node the Spec position of which is occupied by the EXPL pro seems to be problematic. The bi-clausal sentence in (59a) below, represented as in (59b), cannot be dealt with in a framework of assumptions where EXPL-pro replacement is assumed to be operative:

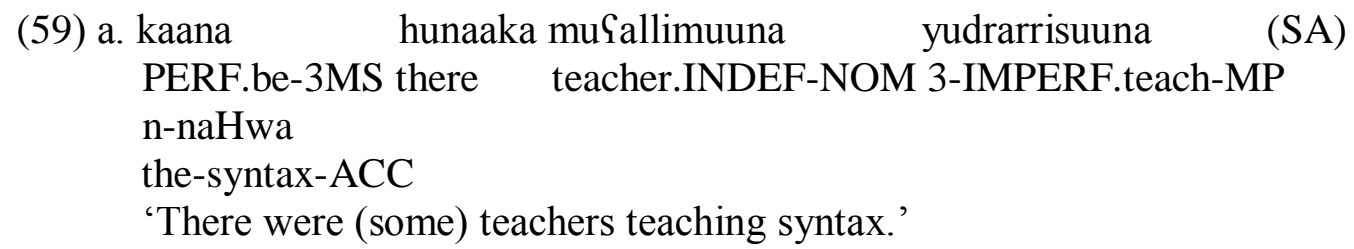


b.
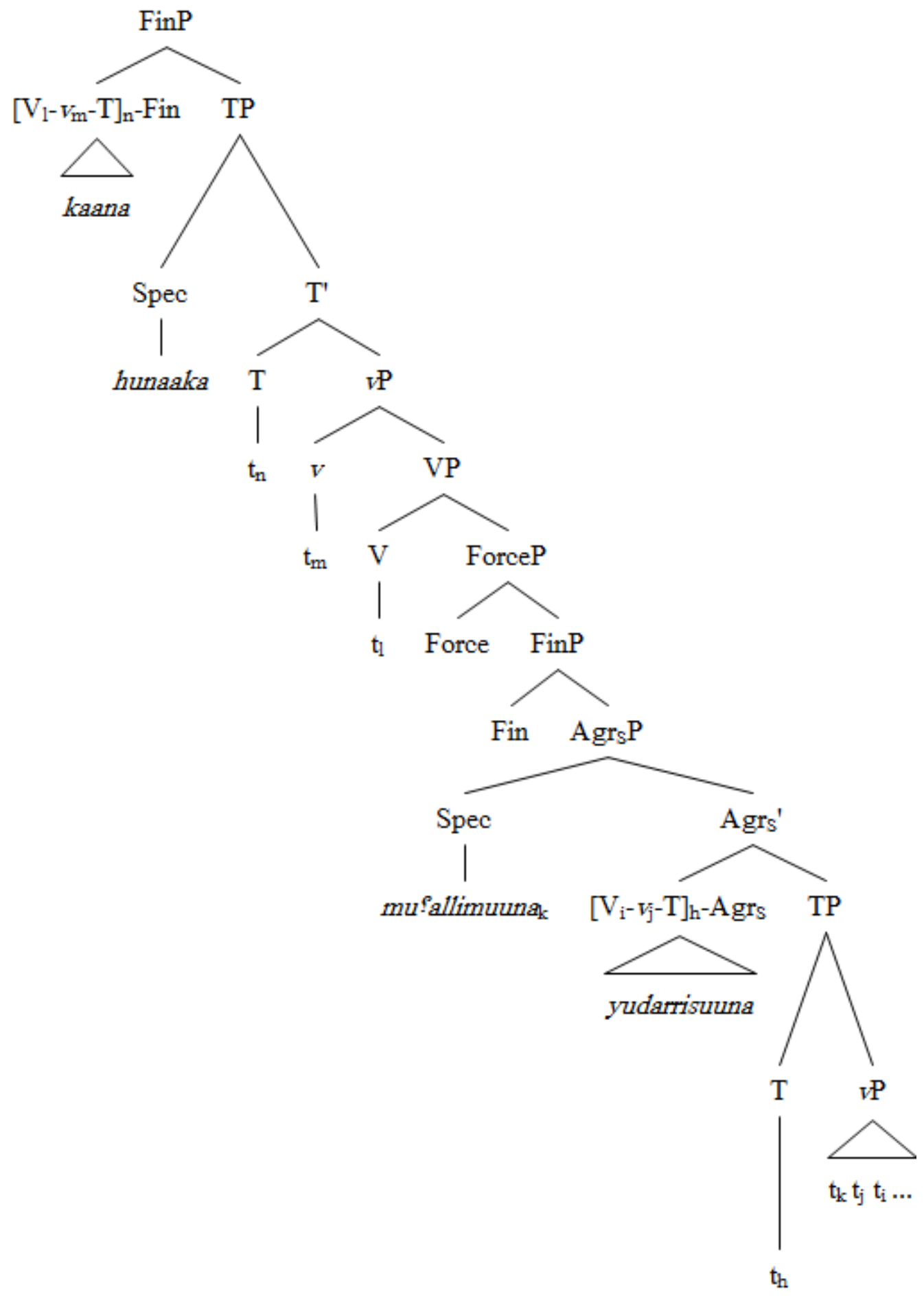

Before accounting for why the bi-clausal structural representation in (59b) is problematic for an EXPL-replacement account in SA, let us come back to structure (7) in section 4.1.2.1 (repeated below, with some omissions, as (60b)), which was provided as an alternative representation to Aoun et al.'s (2010: 71) structure for a sentence like (60a) below: 
(60) a. kaan-a hunaaka Taalib-un fii l-Hadiiqat-i

PERF.be-3MS there student.INDEF-NOM in the-garden-GEN

'There was a student in the garden".

b.

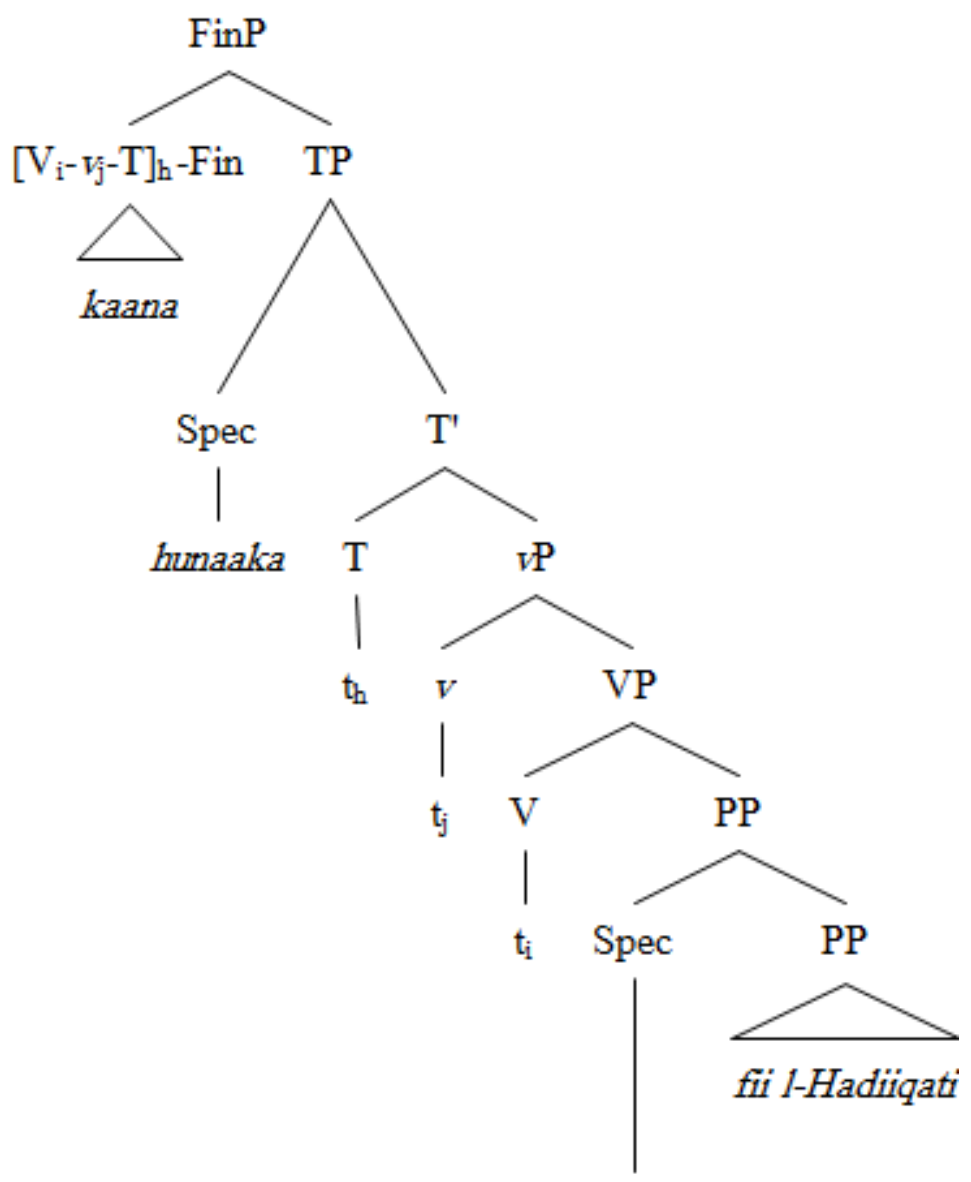

Taalibun

According to structure (60b) for sentence (60a), the locative pro-form hunnaka 'there' is merged in [Spec, TP] to satisfy the EPP while the thematic subject (i.e., the DP Taalibun 'a student') remains lower in the Spec position of the PP small clause selected by $\mathrm{V}$.

Granting that the representation in (60b) is correct, if EXPL pro can be merged as the subject with which auxiliary kaana is 'partially' agreeing in $3^{\text {rd }}$ person masculine agreement features, the Spec position EXPL pro would occupy would be [Spec, FinP], the head position of which the auxiliary verb adjoins to (since, as (60b) shows, the Spec of the highest functional projection within the IP domain - i.e., [Spec, TP] - is already occupied). However, contrary to assumptions developed in section 4.1.2.1 (see also section 4.3), this configuration would give rise to a case of a V2 configuration as in the case of German matrix V2 constructions with lexical or 
EXPL subjects phonetically realizing the Spec position of FinP. Since the VSO phenomenology of a V-raising language like SA only exemplifies a weak version of the V2 configuration (cf. Carnie et al. 1994; see section 4.1.2.1 above), [Spec, FinP] need not - hence, must not - be occupied. In addition, the assumption of filling up [Spec, FinP] of a structure like (60b), as the highest specifier position in the structure of a matrix VSO sentence in SA under this alternative analysis, would go against the premise adopted in this thesis that the EPP, in SA, can only be satisfied within the IP domain - i.e., by the raising of a lexical subject to [Spec, TP], or the Merge of EXPL hunaaka 'there' in such a VSO configuration, and in [Spec, $\left.\operatorname{Agr}_{\mathrm{S}} \mathrm{P}\right]$ in $\mathrm{SVO}$ configurations by the raising of a referential pro out of [Spec, $v \mathrm{P} / \mathrm{VP}]$ to that position.

Given that the structure provided in (59b) above is bi-clausal, the lower clause projects as ForceP, where Force selects FinP and the Fin head selects IP (realized as $\left.\operatorname{Agr}_{S} \mathrm{P}\right)$. Assuming, for the sake of argumentation, that the bi-clausal sentence in (59a) can be derived within a structure like (56b) above (i.e., according to Demirdache's 2013 conception of the derivation of such bi-clausal constructions with EXPL pro sitting in [Spec, AGRP1]), then we could allow for hunaaka to be base-generated in [Spec, TP2]. In such a structural representation for sentence (59a), the verb yudarrisuuna 'they teach' will raise up to AGR2 and stop in that position. In such a case, the verb would get its tense features - located in T2 - by some head-head agreement relationship, which would operate whenever V-to-T does not operate, as is common in languages with strict SVO word order (like English). This derivational scenario is necessarily correct since the DP muSallimuuna 'teachers', being INDEF cannot be a Topic. Thus, the DP muSallimuuna in (59a) is raised out of [Spec, VP] (or $[\mathrm{Spec}, v \mathrm{P}]$ in the corresponding structural position in the tree structure I have provided in (59b)) to [Spec, AGRP2] (corresponding to [Spec, $\left.\operatorname{Agr}_{S} \mathrm{P}\right]$ in (59b)) as the structural subject position in the lower clause, which $\mathrm{V}$ in the higher clause of the biclausal construction (59a), selects.

If this reasoning about the derivation of a sentence like (59a) within the framework of assumptions adopted by Demirdache (2013) is correct, then we would have to account for the problematic nature of EXPL-pro replacement within the structure of such a sentence. In that framework, the 'closest' Spec position to the DP muSallimuuna 'teachers' in [Spec, AGRP2] is the next higher Spec position above 
[Spec, AGRP2], i.e. (according to the system of the formation of A-dependencies in the grammar of languages and the strict local character of DP-movement), [Spec, TP2]. Thus, in the derivation of such subject-verb agreement configurations - within Demirdache's 2013 framework of assumptions - what seems to be 'replaced' is the locative pro-form hunaaka 'there' in [Spec, TP2] and not the hypothesized EXPL pro sitting in [Spec, AGRP1] in a structure like (56b) above (and in the corresponding representation of the bi-clausal sentence (59a) in Demirdache's 2013 system of assumptions for the derivation of such a sentence, which would allow for the Merge of EXPL pro).

The analysis I have provided for sentence (59a), as represented in (59b), as an alternative account of the derivation of subject-verb agreement configurations in the structure of bi-clausal sentences in SA (where the DP muSallimuuna 'teachers', being INDEF cannot be a Topic, but only a 'subject') is also interesting in another respect in that it seems to confirm Fassi Fehri's $(1993: 16,18)$ intuition about SVO structural configurations in which there are instances where an initial, subject-related DP can be hypothesized to have been moved to [Spec, IP] (or [Spec, $\left.\operatorname{Agr}_{S} \mathrm{P}\right]$ ) instead of being considered as a Topic DP within a clitic left-dislocated structure (see footnote 18 in Chapter Two).

However, the cases of subject-verb agreement discussed in Demirdache (2013: 299-304) seem to be evidence of some kind for EXPL-pro replacement in the cases where a DP other than the subject-related DP, base-generated in the Topic position [Spec, TP2] (in Demirdache's 2013 system of assumptions), intervenes between auxiliary kaana and the subject-related DP, as in (61a-b) (from Demirdache 2013: 301-304):

$\begin{array}{lll}\text { (61) a. kaana/*kaan-at } & \text { xaalid-un } & \text { t-aktub-u } \\ \text { PERF be-3MS/PERF be-3FS Khalid-NOM 3-IMPERF }\end{array}$

PERF.be-3MS/PERF.be-3FS Khalid-NOM 3-IMPERF.write-FS.IND

la-hu (hind-un) to-him (Hind-NOM)

'Khalid, she (Hind) was writing to him.'

b. kaan-at/*kaana hind-un $\quad$ y-aktub-u

PERF.be-3FS/PERF.be-3MS Hind-NOM 3-IMPERF.write-MS.IND

to-her (Khalid-NOM)

la-haa (xaalid-un)

'Hind, he (Khalid) was writing to her.' 
According to Demirdache (2013: 301-303), the mismatch in the agreement morphology, in (61a), on the thematic verb taktubu 'she writes' in the lower clause and on the auxiliary verb kaana 'he was' in the higher clause, and, similarly, in (61b), on the thematic verb yaktubu 'he writes' in the lower clause and on the auxiliary verb kaanat 'she was' in the higher clause is evidence that there is an EXPL pro with which the object Topic DP agrees given that, at LF, it is this Topic DP that replaces EXPL pro.

However, these cases of 'weak' subject-verb agreement only arise whenever a DP element, which is not the subject or a subject-related element, intervenes in the anaphoric relation that binds the thematic verb, in the lower clause, to the auxiliary verb, in the higher clause, in such bi-clausal constructions. Given the locality of agreement relations in both A- and A'-dependency relations, it makes sense that the verb picks up the 'closest' DP element in the clause, with which it ultimately agrees in person and gender at the interface. In this case, the subject DP that the auxiliary verb (i.e., kaana in (61a) and kaanat in (61b)) agrees with is the 'intervening' object DP xaalidun in (61a) and hindun in (61b). According to the system of assumptions for the derivation of VSO sentences adopted in this thesis, this 'intervening' DP would be merged in [Spec, TP] in the higher clause of a structure like (59b) above to satisfy the EPP in that position.

Whenever no problem of intervention in agreement relations arises in VSO structural configurations in SA, the subject-verb agreement configuration, in which the partially agreeing verb is involved, will show no restrictions on either encoding third person masculine singular agreement features or third person feminine singular agreement features. This account can be upheld by reference to evidence provided in Fassi Fehri (1993: 38-39), (see examples (55a-b) in section 3.3.4):
(62) a. Pinna-hu
zaara-nii
thalath-u
shaaৎ iraat-in
that-EXPL.3MS visited.3MS-me three.M-NOM poets.F-GEN
Literally: 'It visited me three poets'
b. Pinna-ha zaarat-nii thalath-u shaa9iraat-in that-EXPL.3FS visited.3FS-me three.M-NOM poets.F-GEN Literally: 'It visited me three poets'

In these sentences, the verb can either agree in third person masculine singular (i.e. zaara 'they(M) visited) or third person feminine singular (i.e. zaarat 'they(F) visited). The same is true of the EXPL pronominal clitic $-h u /-h a$ attached to the 
complementizer Pinna 'that'. Other cases of such 'freedom' of subject-verb agreement in SA VSO word order have been provided in section 3.3.4.

In Chapter Two and Chapter Three, I have developed an analysis whereby EXPL pro, as an empty category, is assumed to be absent in the syntax of VSO sentences in SA (vs. its presence in the syntax of VSO sentences in TA or MA, as developed in this thesis). This analysis has been based on the corollary that the microparametric difference between TA, or MA, and SA in the derivation of subject-verb agreement configurations in VSO sentences is linked to the non-projection of the $\operatorname{Agr}_{S}(\mathrm{P})$ projection in $\mathrm{SA}$ as opposed to the necessity of the projection of $\operatorname{Agr}_{\mathrm{S}}(\mathrm{P})$ in TA and MA.

In Chapter Two and Chapter Three, I also followed Chomsky (1995b, 2001, 2004) and Roberts (2010a,b) in the assumption that pro elements (referential or expletive) are D categories (see also Demirdache 2013: 287-289 for a similar assumption within the framework of her incorporation analysis of subject agreement in SA). In my analysis of subject-verb agreement configurations, these elements can only be syntactically represented at PF in the Spec position of an $\mathrm{Agr}_{S}$ node (either by first Merge as in the case of EXPL pro, or by second Merge as in the case of referential pro). Nevertheless, although both referential pro and EXPL pro are D heads merged in a Spec position, referential pro differs from EXPL pro in that referential pro only has agreement features of its own, much the same as lexical DPs. As for EXPL pro, it is devoid of $\varphi$-/Agree-features, but has a D-feature (which is also its categorial label) - a property that makes it similar to EXPL there (Chomsky 1995a,c; Roberts 2010b) save for the difference in the phonetic realization of the two items - i.e., phonetically not realized in the case of EXPL pro in a null subject language (e.g. TA or MA in the analysis adopted in this thesis), but the mandatory phonetic realization of EXPL there in the subject position of the clause of English, as a non-null subject language.

Combining the assumption, adopted here, that EXPL pro is not represented in the IP domain in the sentence structure of VSO constructions in SA and Demirdache's (2013) assumptions as to the incorporation of agreement morphemes onto the head that precedes them, EXPL pro, as a D element, would still have some kind of raison d'être in the sentence structure of VSO constructions in SA since it can be realized as a $3^{\text {rd }}$ clitic pronoun in such instances where it can 'incorporate' on some lexical 
element (such as complementizer Pinna 'that' in (63a-b) below) phonetically realizing a higher functional head projection potentially merged within the structure of matrix sentences in SA above IP (see section 3.3.4):

(63) a. Pinna-hu kaan-a hunaaka Taalib-un

that-EXPL PERF.be-3MS there student.INDEF-NOM

fii l-Hadiiqat-i

in the-garden-GEN

'It is indeed the case that there was a student in the garden.'

b. Pinna-hu kaan-a hunaaka muSallimuuna

that-EXPL PERF.be-3MS there teachers.INDEF-NOM

yudrarrisuuna n-naHwa

3-IMPERF.teach-MP the-syntax-ACC

'It is indeed the case that there were (some) teachers teaching syntax.'

In (63a,b) and, likewise, in (59a) and (60a) above, the assumption I have adopted in this thesis is that EXPL pro is not merged at the highest level of the IP domain since the higher clause exemplifies the VSO word order, in which, as I have assumed, the $\mathrm{Agr}_{\mathrm{S}}$ node and its maximal projection are not derived. As the structures given in (59b) and (60b) show, the verb is higher than the IP domain, which projects as a simple TP structure where [Spec, TP] is occupied by the locative pro-form hunaaka 'there'. As discussed in relation to structure (59b) and (60b) above, in (59b), the DP muYallimuuna 'teachers' is in [Spec, $\left.\mathrm{Agr}_{\mathrm{S}} \mathrm{P}\right]$ as the structural subject position in the lower clause, which $\mathrm{V}$, in the higher clause of the bi-clausal construction (59a), selects. As for the DP Taalibun 'a student' in (60b), it is in the Spec position of the PP small clause selected by V.

On the new proviso (defended in this thesis especially in light of such evidence as provided in (59) and (60) above) that the (auxiliary) verb, in the structure of VSO sentences, is higher than the highest functional projection within the IP domain, I have entertained the idea that the EXPL element $-h u$, which cliticizes onto a matrix complementizer like Pinna, as shown in sentences (62a-b) and (63a-b), would be some form of phonetic realization of the otherwise non-merged EXPL pro within the structure of these sentences.

The assumption that the D-feature of some functional head element (like EXPL pro in the present analysis) can get some form of phonetic realization (i.e., third person masculine/feminine morphology in the case at hand) is not new. Citing 
Borg \& Azzopardi-Alexander (1997), Benmamoun (2000b: 79) accounts for the phonetic realization of negative $m a(a)$ as $m h u x$ in the Maltese Arabic negative sentence in (64) below, as follows:

(64) Hutek mhux sejrin ghalissa

(Maltese Arabic)

Siblings.2S NEG.3MS going for-now

'Your brothers and sisters are not leaving.'

"mhux is composed of the discontinuous $m a-x$ and the pronominal $-h u$, which carries the third masculine singular features. The latter features are exactly the features that realize expletive pronouns in Arabic and are plausibly a realization of the $[+\mathrm{D}]$ feature of negation."

Such a process of the phonetic realization of some D-feature as $3^{\text {rd }}$ person singular features in Benmamoun's (2000b) account of the feature structure of functional elements in Arabic is also exemplified by evidence coming from Arabic dialects in the Gulf region where the NEG element $m a(a)$ can vary according to gender: mu/mi (Benmamoun 2000b: 79-80, citing Holes 1990: 73):

(65) haadhi mi zoojt-i

(Gulf Arabic)

this NEG.3FS wife-my

'This is not my wife.'

Benmamoun (2000b: 80) comments on such a sample example as follows:

Holes (1990) ... states that the negative $m u$ is made up of $m a$ and the third masculine singular pronoun $h u$ and $m i$ is made up of $m a$ and the third feminine singular pronoun $h i$. Within the present analysis, the difference between dialects where negation varies according to gender has to do with the feature structure of negation. In the former dialects negation is not specified for gender while in the latter it is specified to gender. This is exactly what we expect if negation carries categorial nominal features.

According to the feature-based analysis adopted in this thesis, the variation in question is not to be understood merely in terms of Benmamoun's (2000b) [+D] feature. This variation seems to be of a more general character within the sentence structure of Arabic taking the form of a more general $[i \mathrm{~F}]$-feature not exclusively linked to 'focus' (i.e., the $[i \mathrm{~F}]$ feature, which I have assumed is a property of 
functional head elements in the so-called 'focus' field of the split-CP domain (Poletto 2000b and Benincà \& Poletto 2004) in particular) and to the (negative) assertiveness force, which has the property of linking $[i \mathrm{~F}]$ to clause type and ultimately to the $[i \mathrm{~T}]$ feature of Fin at the lower end of the split-CP domain (cf. R\&R's account of the $[+$ Fin $]$ feature of Fin). The $[i F]$ feature would also have a bearing on 'topicalization' processes as in the case of the EXPL clitic head $-h u$ in [Spec, TopP], but in such 'topicalization' cases, it would take the more specific form of an $[i \mathrm{D}]$ feature at the highest level of the structure of VSO sentences with a matrix complementizer like in SA (according to the present analysis). As noted in Chapter Three (see section 3.3.4), it could be the case that some dialects of Arabic have retained some of the properties that are somewhat special to the standard variety, such as, in particular, the subjectverb agreement asymmetry in VSO vs. SVO sentences, but still showing evidence of the agreement patterns that are common in the dialects, as against the standard variety.

However, the common characteristic of such processes is that they basically involve head-head Agree relations in terms of interpretable features that need only be 'identified' within the structure where they are being merged. In the system of assumptions developed in this thesis about the identification of features on the functional heads, neither the varying $[i F]$ feature I have assumed is involved in the 'identification' of features in the 'focus' field of the split-CP domain, nor the $[i \mathrm{D}]$ feature of the Top head and the associated $[i \mathrm{D}]$ feature of the EXPL clitic element $-h u$ is related to any Case-requirement or to the satisfaction of the EPP.

In the Agree feature-based account of the data presented above, the raison d'être of the element endowed with such an $[i \mathrm{~F}]$ feature is to phonetically realize some $\mathrm{X}$ head within the split-CP domain of the relevant varieties of Arabic for the expression of some information-related property of the element, such as 'focus' or (negative) assertiveness. As far as the EXPL clitic element - $h u$ is concerned, the process by which EXPL pro can be phonetically realized in the split-CP domain, to which the verb in VSO subject-verb configurations has raised, would be a special case of a more general process of the phonetic realization of such functional elements within this domain of sentence structure. On this account, the variation in question would concern some additional functional feature of a more general character, either linked to 'focus' or to the proposition being asserted, as in the case of the $[i F]$ feature 
of negation, or linked to some $[i \mathrm{D}]$ feature, as in the case of the EXPL clitic head $-h u$ in [Spec, TopP], under the assumptions of the present analysis.

Ultimately, whether the licensing of the relevant features on functional elements (with the varying interpretability values of these features, as for example [iD] on Top vs. [ $u \mathrm{D}]$ on $\operatorname{Agr}_{\mathrm{S}}$ as developed in this thesis) involves identification or valuation within the relevant domains where they are being merged, this licensing process is conducive to the general purpose of the derivation of sentences in the grammar of a particular language, i.e., the generation of agreement configurations in the broad sense of the term (subject-verb agreement being the prototype).

Returning to the claim of a bi-clausal structure for the sentences in (51a) and (51b) - as represented in (52a) and (52b), repectively -, Akkal \& Gonegai (2000: 1314) maintain that, in constructions containing both a main verb, in a lower clause, and an auxiliary verb kaana (which they refer to as a 'temporaliser verb' inserted under T), in a higher clause, the tense of the auxiliary is 'referential', while the tense of the main verb is 'anaphoric': "Hence, the anaphoric tense on the main verb draws its temporal content from the referential tense of the temporaliser" (Akkal \& Gonegai 2000: 14). The argument that applies to auxiliary kaana should logically also apply to the 'negative auxiliary' laysa (and by extension to the NEG element maa, save for the Merge of maa in Foc). As the structures in (52a) above, and (66b) below show, the same head-raising processes apply in the derivation of both kinds of VSO sentences involving laysa raised to Fin in (52a) and kaana raised to that same node in the split$\mathrm{CP}$ domain in (66b):

$\begin{array}{lll}\text { (66) a. Pinna-hu kaana-t } & \begin{array}{l}\text { Pal-Pumahaat-u } \\ \text { ya-Drib-na }\end{array} \\ \text { that-it } & \text { PERF.be-3FS } & \text { the-mothers-NOM }\end{array}$

Pawlaad-a-hunna boys-ACC-their 'The fact is that mothers used to hit their children.' 
b.

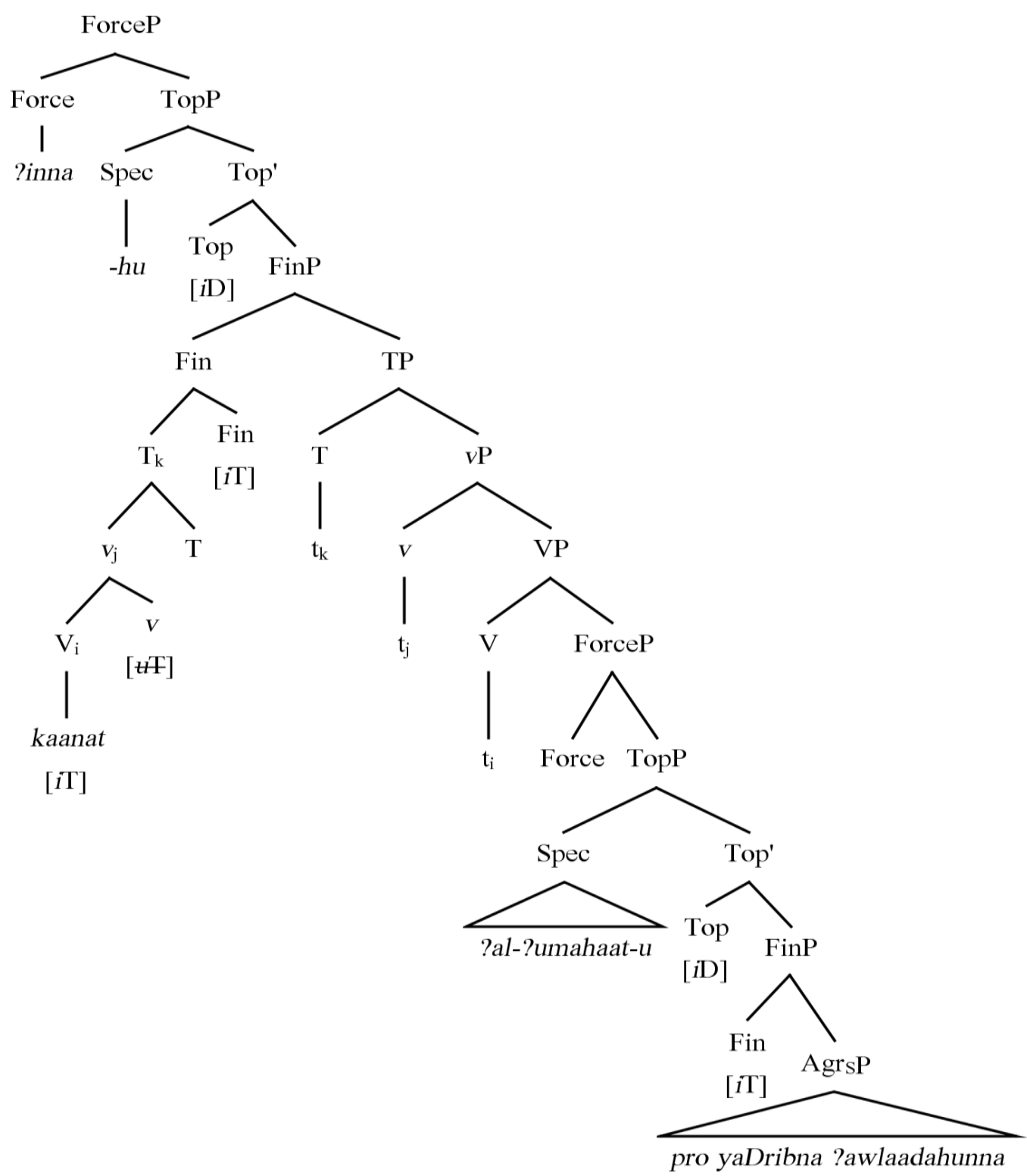

In (66a), the auxiliary used is kaana merged in $\mathrm{V}$ in the higher clause. It raises to attach to $v$, and the complex [ $\mathrm{V}-v]$ raises to T. As discussed in section 3.3.2.3, auxiliary kaana has a certain $[+\mathrm{F}]$ feature that is involved in the dependency relations and in predication as auxiliaries link to $\mathrm{T}$ for interpretation at the interface. Roberts (2010a: 162) characterizes such a $[+F]$ feature as an $[i \mathrm{~T}]$ feature. According to Roberts (2010a: 162), auxiliaries lack V-features but bear interpretable T features, which trigger them to raise to T. In the probe-goal-Agree system proposed here, the auxiliary kaana first attaches to $v$ and subsequently raises further up to T. Then, the 
inflectional complex [V-v-T] ends up attached to Fin, partially inflected for third person feminine agreement at Spell Out (see the discussion of (51a) and (53) above).

As was the case in (51a), The DP Pal-Pumahaat-u, in (66a), has the status of Topic in [Spec, TopP] and is co-referential with pro base-generated in [Spec, $\left.\operatorname{Agr}_{S} \mathrm{P}\right]$. Given the Merge of the DP Pal-Pumahaat- $u$ as a Topic in the specifier of TopP, the complementizer Pallaatii cannot be merged in a sentence like (66a) with auxiliary kaana without giving rise to an ungrammatical result (*Pinna-hu kaana-t PalPumahaat-u Pallaatii yaDrib-na Pawlaad-a-hunna). On this account, the minimal contrast between structure (66b) with auxiliary kaana and structure (52a) with the NEG auxiliary element laysa, in relation to the possibility of merging complementizer Pallaatii, may boil down to a difference in the feature structure of laysa as against the feature structure of kaana. That is, whereas laysa in (52a) (and likewise for maa in (52b)) is generated with a focus feature denoting 'contrastive focus' (Ouhalla 1993: 296-297; Benmamoun 2000b: 109 in relation to $\mathrm{maa}$ ), as the translation of sentence (66a) shows, auxiliary kaana does not have such a feature.

A similar analysis as has been argued for sentences (51a) and (66a) applies to the corresponding sentences (67a) and (68a) below, respectively, where the DP PalPumahaat- $u$ is merged as a Topic DP in [Spec, TopP] in the higher clause ((67b) and (68b) are the structural representions for (67a) and (68a), respectively):

(67) a. Pinna Pal-Pumahaat-a las-na ya-Drib-na that-it the-mothers-ACC NEG-3FP 3-IMPERF.hit-FP Pawlaad-a-hunna boys-ACC-their

'The fact is that mothers do not hit their children.' 
b.

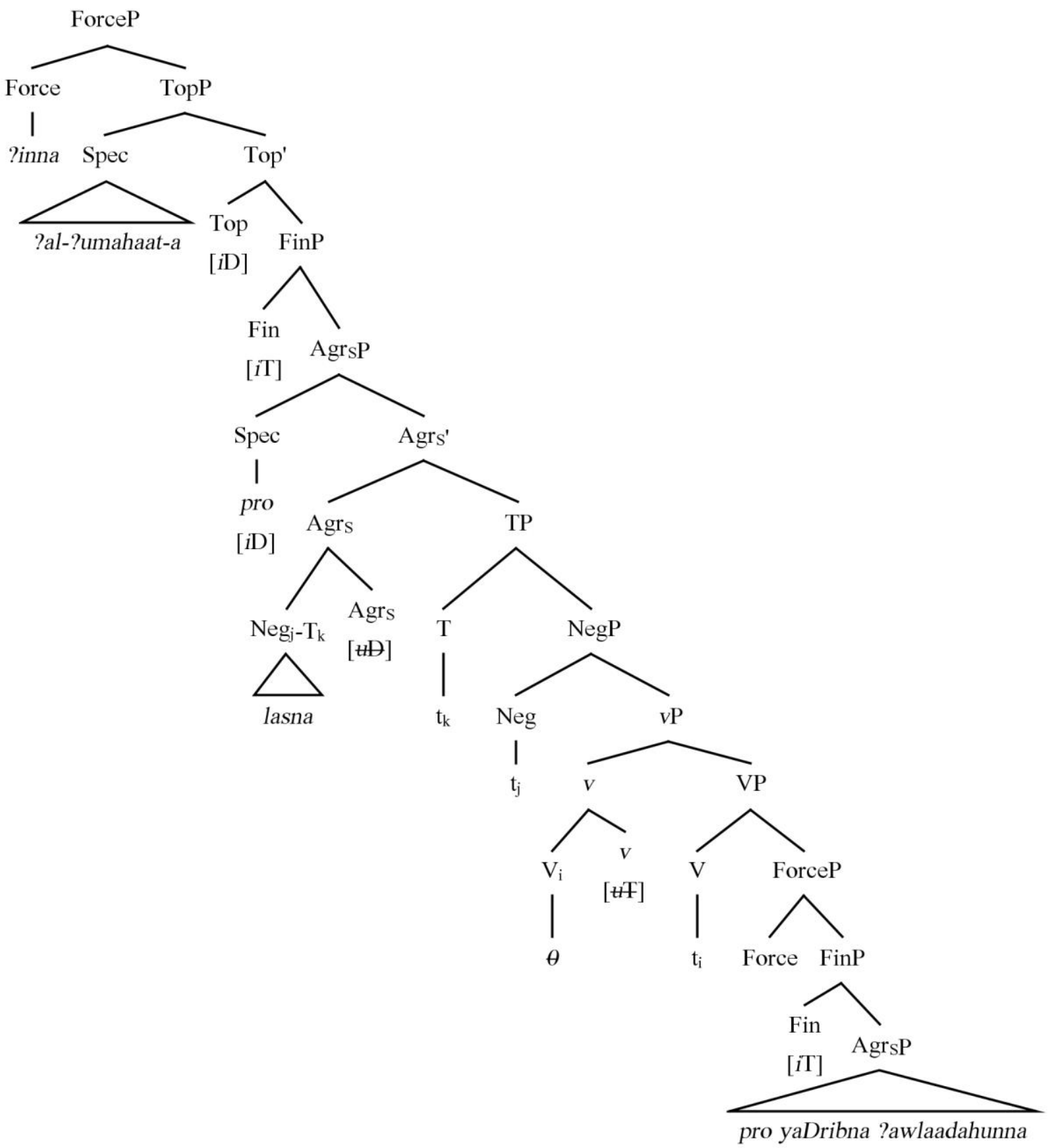

Sentence (67a) is similar to sentence (51a). However, the main difference between the two sentences is that, in sentence (67a), the contrastive focus interpretation for sentence (51a) (with the NEG element laysa-t raised to Fin) disappears. The difference in interpretation between (51a) and (67a) makes sense if what is primarily relevant in the derivation of sentence (67a) is the topicalization process of the DP PalPumahaat-a in the Spec position of a TopP projection in the higher clause at Spell Out as represented in (67b). In this case, the auxiliary verb laysa is fully inflected for subject-verb agreement (i.e., lasna) and it does not leave the IP domain of the higher 
clause (projected as $\operatorname{Agr}_{S} \mathrm{P}$ ). Sentence (67a) above would thus be the present tense 'neutral' negative counterpart of sentence (68a) with auxiliary kaana: ${ }^{40}$

(68) a. Pinna Pal-Pumahaat-a kun-na ya-Drib-na that the-mothers-ACC PERF.be-3FP 3-IMPERF.hit-FP Pawlaad-a-hunna boys-ACC-their

'The fact is that mothers used to hit their children.'

b.

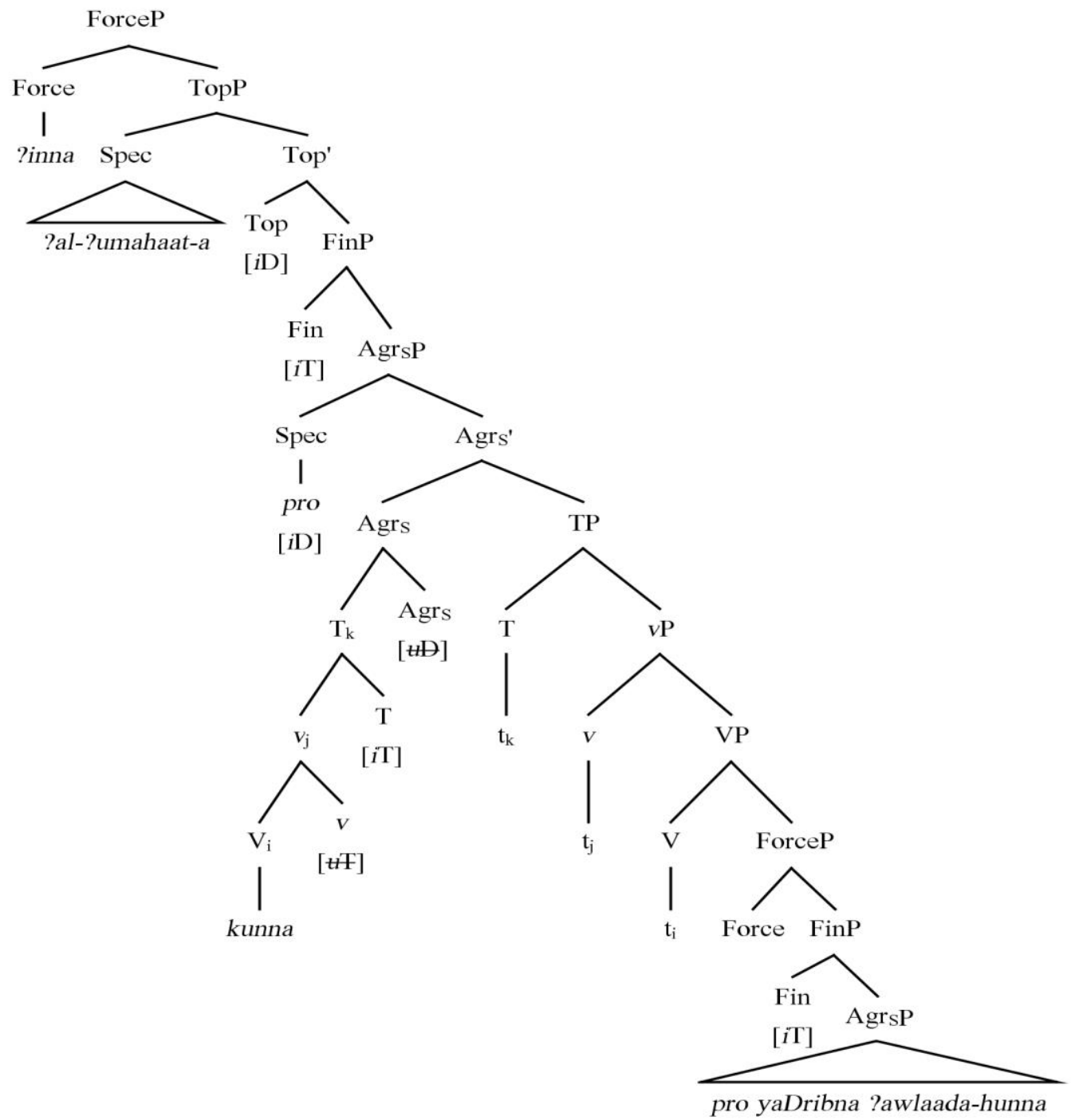

In (68a), the auxiliary used is kaana merged in $\mathrm{V}$ in the higher clause. It raises to attach to $v$, and the complex [V-v] raises to T. The inflectional complex [V-v-T] ends

\footnotetext{
${ }^{40}$ The word 'neutral' here is meant to express the idea that no contrastive focus is implied in (67a) or (68a).
} 
up attached to $\operatorname{Agr}_{S}$ where the auxiliary kaana is realized as kunna at Spell Out since it is fully inflected for subject-verb agreement.

The negative counterpart of (68a) (kept in the past tense) could either yield a sentence like (69a) or like (69b):

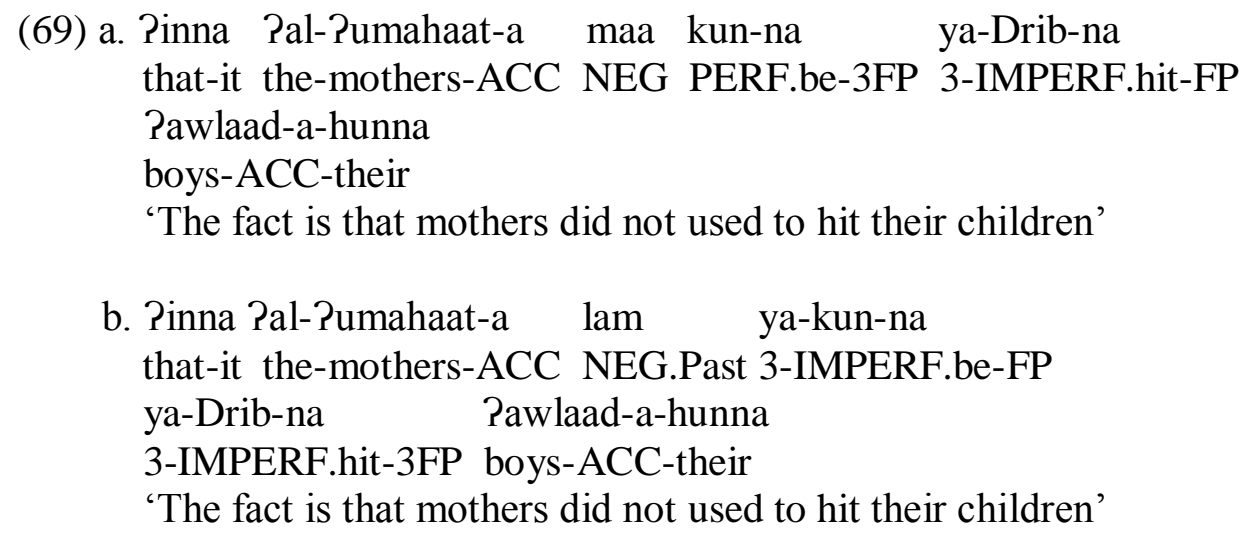

Whereas sentence (69a), with maa as the element used to negate sentence (68a), can have the 'negative contrastive focus' interpretation, sentence (69b), with lam as the NEG element, cannot have that interpretation (cf. Benmamoun 2000b: 109). Thus, according to the analysis provided in sections 4.1.2.5.2 and 4.1.2.5.3, the 'focus' $[i F]$ feature that I have assumed to be a characteristic of all NEG elements in SA is, nevertheless, a feature that can be associated with a contrastive interpretation of the verbal event or situation it is associated with.

In section 4.1.2.6 below, I continue with the discussion of the interaction of tense and modality in SA matrix sentences in such instances where the first element in the sentence is a subject-related Topic DP directly merged in [Spec, TopP].

\subsubsection{The interaction of tense and modality in the derivation of SA SVO sentences}

Putting aside the discussion of negation in SA sentences (irrespective of VSO or SVO word ordering), contrary to the derivation of VSO sentences in SA, as discussed in section 4.1.2.2, in a SVO structure as represented in (70b) below, the fully-inflected verb does not raise further than the $\mathrm{Agr}_{S}$ node, the specifier position of which is occupied by a referential pro:

\section{(70) SA SVO}

a. Pal-?awlaad-u qad jaa?-uu the-boys-NOM MOD PERF.come-3MP Lit.: 'As for the boys, they indeed came.' 
b.

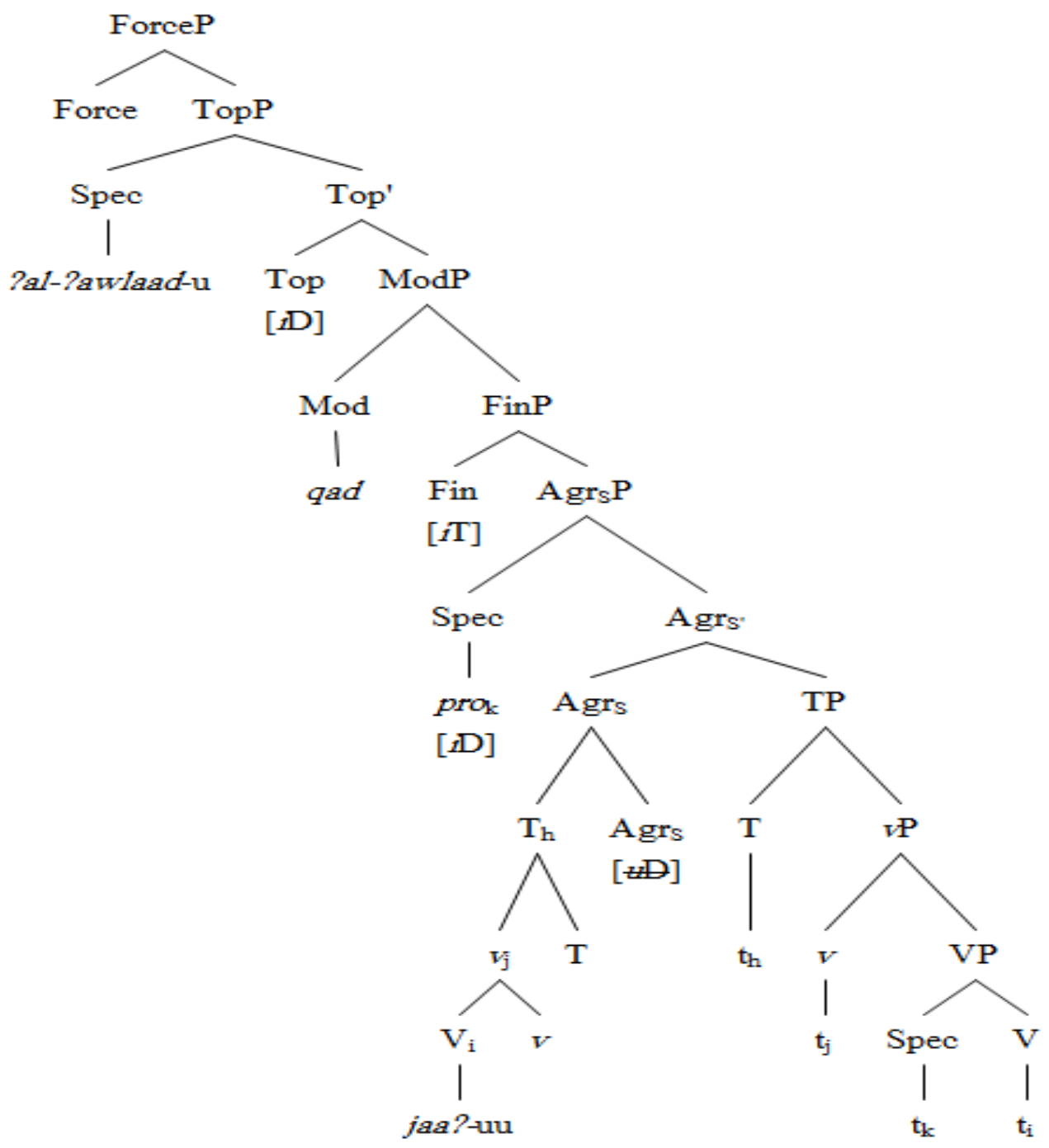

Recall from Chapter Two (sections 2.1.2.3.2 and 2.1.2.3.3) that I am adopting an analysis of SVO sentences in SA whereby the initial DP ?al-Pawlaad- $u$ 'the boys' in such sentences is a Topic DP base-generated in [Spec, TopP] in the split-CP domain, as represented in (70b). The Top head projection in the split-CP domain is endowed with an $[i \mathrm{D}]$ feature that allows the Top node to function as the repository of a 'higher predication' relation in connection with subject-verb agreement and the EPP in the IP domain (cf. Rizzi 1997: 287).

In addition to the essential link between the Topic DP ?al-Pawlaad-u and the subject inflection - $и u$ on the verb jaa?-uи 'they came', which resumes the Topic DP, in (70b), it is by virtue of its intrinsic $[i \mathrm{D}]$ feature that the 'resumptive' pro in [Spec, $\left.\mathrm{Agr}_{S} \mathrm{P}\right]$ functions as an agreement link, within the IP domain proper, between $[u \mathrm{D}]$ on $\operatorname{Agr}_{S}$ and $\varphi$-feature valuation/EPP satisfaction within the $\mathrm{V}-v$-T-Agr ${ }_{S}$ verbal inflectional complex. 


\subsubsection{Summary}

In this section, I have dealt with the derivation of VSO and SVO structural configurations in SA whereby Focus and Topic elements interact with tense/modality properties of sentences for the satisfaction of the $\mathrm{C}-\left(\mathrm{Agr}_{\mathrm{S}}\right)-\mathrm{T}$ dependency and the identification of the relevant features. In the derivation of the sentences in question, the morphological manifestation of Tense and subject-verb agreement in SA is thus determined via the head-head and/or Spec-head agreement relations involved in the Merge and raising processes linking the IP domain to the split-CP domain.

In this respect, I have assumed that the derivation of VSO sentences, in SA, involves the raising of the verb to the Fin node projecting at the lower part of the split-CP domain. In SA VSO word order, Fin selects a TP that does not further project to $\mathrm{Agr}_{S} \mathrm{P}$. In the absence of the functional $\mathrm{Agr}_{\mathrm{S}}$ node in SA VSO word order, the verb, raised to $\mathrm{T}$ and partially agreeing with the subject $\mathrm{DP}$ raised out of $[\mathrm{Spec}, v \mathrm{P} / \mathrm{VP}]$ to [Spec, TP], further raises to Fin. I have assumed, following $R \& R$, that this raising process is in response to the identification of some [+Fin] feature (which I reinterpreted in terms of an $[i \mathrm{~T}]$ feature on Fin) similar to the interpretable T-feature of $\mathrm{T}$ in the IP domain. The head-head agreement configuration, which arises as the outcome of the selectional relation that links $\mathrm{T}$ to Fin, satisfies the C-T dependency in such VSO instances. The possibility of merging tense/modal particles such as the modal qad (as discussed in section 4.1.2.3) or the NEG elements lam and lan (as discussed in section 4.1.2.5. above) under the relevant functional nodes that project above FinP in these configurations is further evidence that the verb does not raise further than the Fin head projection in the structure of the split-CP domain (contra Aoun et al. 1994, 2010).

As for the derivation of SVO structural configurations in SA, I have assumed that it involves the base-generation of 'subject' DPs as Topics in the left-periphery. In this case, full subject-verb agreement and the satisfaction of the EPP in the IP domain follow from the presence of a referential pro with an intrinsic $[i \mathrm{D}]$ feature in $[\mathrm{Spec}$, $\left.\operatorname{Agr}_{S} \mathrm{P}\right]$, which not only links the uninterpretable D-feature on $\operatorname{Agr}_{S}($ i.e., $[u \mathrm{D}]$ on $\left.\operatorname{Agr}_{S}\right)$ to $\varphi$-feature valuation and the satisfaction of the EPP at the IP domain, but also satisfies the C- Agr $_{S}-\mathrm{T}$ dependency at Spell Out by linking $[i \mathrm{D}]$ on the Top node projected in the split-CP domain to the $\mathrm{V}-\mathrm{v}$-T-Agr $\mathrm{S}$ complex in IP. 
The projection of Force and Fin, at opposite sides of each other in the split-CP domain in matrix sentences in SA, is a necessary condition for the derivation of such sentences. It accounts for the default [+declarative] interpretation these sentences get at the interface irrespective of whether the matrix complementizer Pinna is inserted under Force or not. In the presence of an interrogative yes/no marker (i.e., SA $2 a$ or hal), Force - signalling the [+interrogative] clause type - links to the T-features in Fin for the well-formedness the C-T dependency.

A similar account has been shown to hold of sentences where a NEG element is merged in the split-CP domain whereby the Merge process of the marker of negation not only accounts for clause type (i.e., interpreting the sentence as [+negative]), but also for negative assertiveness as the sentence is interpreted at the interface. An additional contrastive focus interpretation is brought about in sentence structure by the NEG element maa, merged in Foc, and the NEG element lays $a$ - in the cases where the latter is raised to the CP domain.

In section 4.2 below, I discuss the derivation of wh-dependencies in the split$\mathrm{CP}$ domain of SA involving Topics and Focused elements and the differences between SA and TA in the derivation of such dependencies.

\subsection{The derivation of wh-dependencies in Arabic: SA vs. TA}

\subsubsection{Introduction}

The evidence discussed in this section suggests that the derivation of whdependencies in SA exploits the different specifier and head positions provided by the splitting of the CP domain (Rizzi 1997). In contrast, TA does not resort to specific specifier or head C-positions. Rather, TA makes use of adjunction of A'-moved elements to the left of IP/Agr ${ }_{S} \mathrm{P}$.

As far as the derivation of wh-dependencies in Arabic is concerned, I reinterpret Ouhalla's (1993) 'morphological' identification of the features involved in such wh-dependencies capitalizing on the assumption that only one feature needs to be structurally licensed on Foc - namely the [WH]-feature (represented as an interpretable $[i \mathrm{WH}]$ feature on the moved wh-word, but as an $[u \mathrm{~F}]$ uninterpretable feature on Foc). This $[u \mathrm{~F}]$ feature corresponds to Chomsky's (2008) 'edge' feature on C, which Chomsky (2008: 148) characterizes as different from the Agree-feature (or $\varphi$-features) that originates on $\mathrm{C}$ and is passed down to $\mathrm{T}$ in relation to the satisfaction 
of the EPP at the IP level in English. Nevertheless, this Spec-head agreement mode of licensing, in which the $[u \mathrm{~F}]$ feature on Foc gets valued, represents one of two modes of licensing co-existing in the derivation of wh-dependencies. The second mode of licensing applies between Foc and the Fin head in terms of a head-head agreement mode of licensing, which generalizes to any node that projects within the 'focus sublayer' of the SA split-CP domain relating Fin to the nodes that project above it for the well-formedness of the C-T dependency. In section 4.2.2, I deal with whdependencies in SA where Topic DPs have to be higher than focused/wh-elements. In 4.2.3, I have a look at the absence of the strict ordering between a left-dislocated element and a wh-element in TA.

\subsubsection{Wh-dependencies in SA and the strict Topic DP, wh-element word order}

In this section, I deal with the derivation of wh-dependencies in SA. The evidence discussed here suggests that wh-elements are internally merged in [Spec, FocP] (in the cases where wh-movement occurs) or base-generated in [Spec, ForceP] (in some cases of wh-question formation and in relativization where a complementizer element has to morphologically realize the Force head). The derivation of such whdependencies shows that a wh-word has to follow a Topic DP in SA.

Thus, in the SA wh-questions in (71) below, only (71a) (structurally represented as in (72)) is grammatical: ${ }^{41}$

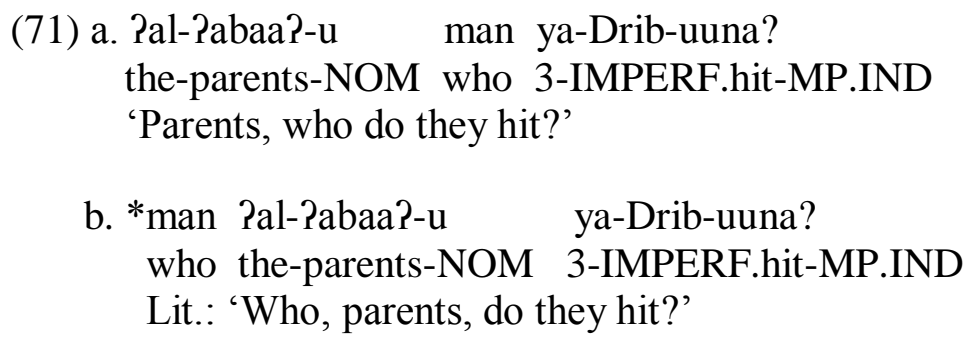

\footnotetext{
${ }^{41}$ I come back to a discussion of (71b) repeated as (73b) below.
} 
(72)

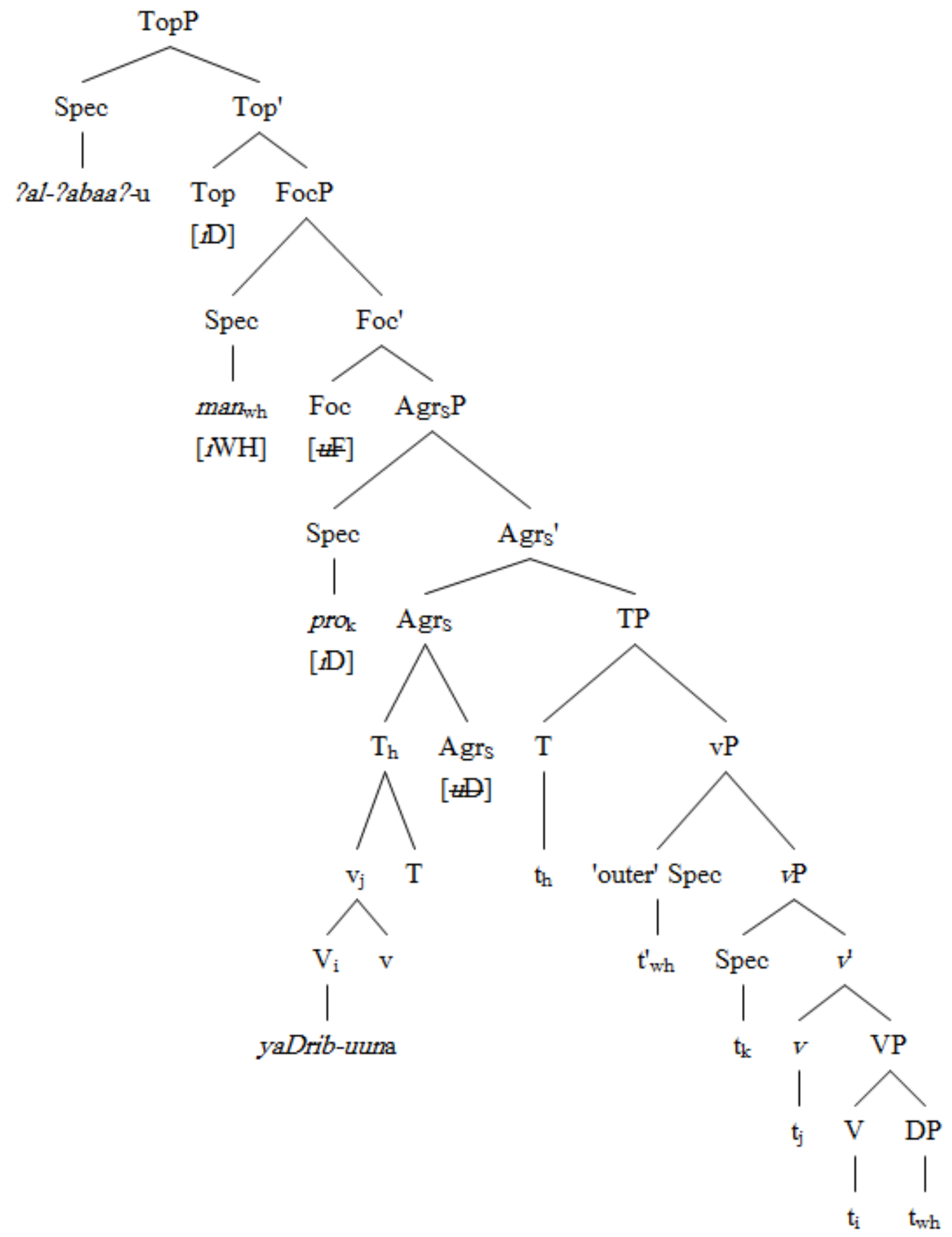

In structure (72), the initial DP ?al-Pabaa?- $u$ 'parents' is base-generated in [Spec,

TopP] in the split-CP domain, resumed in the IP domain by the subject inflection on the verb yaDrib-uuna 'they hit'. The Topic DP is co-referential with a 'resumptive' pro raised out of the subject position in $[\mathrm{Spec}, v \mathrm{P}]$ for EPP satisfaction at the IP/Agr $\mathrm{P}$ 
level. The structure also shows the wh-movement of an object DP. ${ }^{42}$ According to Rizzi (1997: 298), wh-elements (question operators, but not relative operators) occupy the Spec of FocP in between Rizzi's (1997) two TopP projections. ${ }^{43}$

As far as a representation like (72) is concerned, I follow Ouhalla (1993) in the assumption that feature identification applies in terms of a certain set of features on a head F, maximally projecting to FP (= FinP in R\&R's account and FocP in Aoun et al.'s 2010 analysis), that interact for an adequate construal, at the interface, of the particular structure (i.e., clause type: wh-question, yes/no question, negative sentence, declarative sentence, and so on) being derived. In Ouhalla's (1993: 282-283) own terms, the head $\mathrm{F}$ is "specified for both the feature $[+\mathrm{wh}]$ which characterizes whphrases (and wh-questions), and the feature $[+\mathrm{F}]$ which characterizes f-phrases (and sentences with focus)". ${ }^{44}$ In this sense, the licensing conditions that Ouhalla (1993: 297) assumes to be operable in the derivation of sentences in SA in terms of the Identification Requirement are generalizable to all structural configurations involving the interaction of some element merged into the structure with the modal/tense properties of the sentences in question. This is true of particles inserted into the structure of the sentence to signal some focus property of the sentence, as in negation, or of a wh-word moved to some peripheral position to satisfy what Rizzi (1996, 2006) calls Criteria.

However, Ouhalla's (1993) $[+\mathrm{F}]$ feature would be an intrinsic property of a functional head different from the head Fin (not represented in (72) for ease of exposition, see footnote 42). As discussed in section 4.1.2.4, in relation to the feature structure of functional projections within the split-CP domain, the label $[+\mathrm{F}]$ could be characterized as some additional property of the nodes that project in the 'Focus sublayer' of the split-CP domain of SA which relates to the 'inflectional' feature(s) on Fin, as the structure is being derived, for the well-formedness of the C-T dependency

\footnotetext{
${ }^{42}$ For ease of exposition, I do not represent the FinP and ForceP projections in (72) in the split-CP domain of the structure (the same applies to (77) below). As discussed in section 4.1.2.4, Ouhalla's (1993) $[+\mathrm{F}]$ feature is structurally related to Fin, which, in the present analysis, is the repository of the [iT] features or R\&R's [+Fin] feature.

${ }^{43}$ As Rizzi (1997: 298) points out, relative operators in English/Italian occupy [Spec, ForceP]. I discuss relativization in $\mathrm{SA}$ in this section below

${ }^{44}$ Ouhalla's (1993) assumption of the interaction of two types of features in the derivation of whdependencies or focus structures is inherent to Chomsky's (1995b: 289-290) account of wh-movement whereby wh-moved elements are assumed to have intrinsic wh-features that are comparable to the intrinsic $\varphi$-features of DPs. At the same time, wh-elements are assumed to have an interpretable Qfeature (comparable to the D-feature of DPs) that matches an uninterpretable Q-feature on C.
} 
at the interface. When Focus projects maximally as FocusP with a Spec position in the derivation of wh-dependencies, as discussed here, it seems that Ouhalla's $[+\mathrm{F}]$ and $[+\mathrm{WH}]$ features on the head $\mathrm{F}$ (for the Merge of focused and wh-elements in his analysis) are actually one and the same 'focus' feature on the Foc head (contra Ouhalla 1993: 282-283).

This 'focus' $[+\mathrm{F}]$ feature is uninterpretable on Foc (i.e., $[u \mathrm{~F}])$ in whdependencies between interrogative wh-words and the Foc head. However, as discussed in section 4.1.2.5, this 'focus' feature is interpretable on Neg (i.e., [iF]) (see also footnote 47 below). On this account, the $[u F]$ feature on the Foc head, seems to be involved in two separate but, nonetheless, interrelated licensing processes: a Spechead agreement process for the 'checking' or valuation of the uninterpretable $[u \mathrm{~F}]$ feature on Foc in relation to the interpretable wh-feature of the raised wh-element (which values it), and a head-head agreement process for the identification of the C-T dependency linking Foc to the finiteness/tense features of the Fin head (i.e., [iT] on T and Fin), projecting in the lower part of the split-CP domain (cf. R\&R: 141).

The licensing conditions on these nodes are, first and foremost, conceived of in terms of head-head agreement configurations on the basis of the feature structure of these nodes. Nevertheless, these licensing conditions apply somewhat differently in the representation of wh-dependencies at the interface in that an additional Spec-head agreement process has to apply for the valuation of a certain uninterpretable feature on the Focus head that is characteristically involved in the representation of such dependencies. $^{45}$

On the basis of such assumptions, the identification process for the licensing of wh-features, in terms of a $[u \mathrm{~F}]$ feature on Foc, cannot simply be conceived of in terms of Rizzi's $(1996,2006)$ notion of 'Criterial satisfaction' as a form of feature identification at the interface. According to Rizzi (1996, 1997, 2006), whdependencies are subject to an interface licensing requirement or a process of Criterial satisfaction. Criterial satisfaction establishes a Spec-head relation between the whword moved to [Spec, CP] ([Spec, FocP] in Rizzi 1997) and the C head (the Foc head node in Rizzi 1997). Rizzi (1996, 1997, 2006) has it that the relationship between the two elements is actually not conceived of in terms of the morpho-syntactic

\footnotetext{
${ }^{45}$ Similar assumptions apply to the subject-verb agreement configurations common in declarative V2 sentences in a V2 language like German (see section 4.3).
} 
'checking'/valuation of features, but in terms of identification of those features for interpretability at the interface.

Rather, as my discussion of Ouhalla's (1993) feature-based analysis of whdependencies above points out, the derivation of such dependencies in the grammar of languages involves both the identification of features on the respective head projections via head-head agreement relations (i.e., in the present analysis, the interpretable $[i \mathrm{~T}]$-feature on Fin/T and the uninterpretable $[u \mathrm{~F}]$-feature on Foc, which gets valued by the matching $[i \mathrm{WH}]$-feature on the wh-element) and the licensing of wh-features via the Spec-head agreement mechanism of feature-valuation (i.e., the $[u \mathrm{~F}]$-feature on Focus in relation to $[i \mathrm{WH}]$ on the wh-element), as conceived of in Chomsky's (1995b) traditional feature-checking analysis of wh-dependencies and subject-verb agreement configurations.

As far as wh-dependencies are concerned, the $[u \mathrm{~F}]$ feature on Foc in $(72)$ is structurally licensed (in the sense of 'checked'/'valued') via a basic Spec-head agreement relation, in which the interpretable feature $[i \mathrm{WH}]$ in $(72)$ on the wh-moved element man 'who' in [Spec, FocP] is responsible for eliminating $[u \mathrm{~F}]$ on Foc. This feature licensing process is similar to the process whereby the $[u \mathrm{D}]$ feature of $\mathrm{Agr}_{S}$ in the IP domain is valued. ${ }^{46}$ Thus, in the case of wh-dependencies, licensing involves an additional feature (namely, the $[u \mathrm{~F}]$ feature on Foc) which would have to be checked/valued at Spell Out. In addition to this feature-valuation process, the licensing mechanism involved in wh-dependencies would also require the identification of the relevant 'focus' feature (in such wh-dependencies) in relation to the $[i \mathrm{~T}]$ features on the Fin head. As discussed earlier, this process exclusively relies on a strict head-head agreement dependency relation that does not rely on any morphological feature-checking. ${ }^{47}$ On such an account, Criterial satisfaction at the

\footnotetext{
${ }^{46}$ In section 4.3, I discuss the cases where the valuation of the $[u \mathrm{D}]$ feature (in combination with the $\mathrm{EPP}$ ) is subject to parameterization with reference to two language types - namely, VSO as in SA and V2 as in German.

${ }^{47}$ This formulation of the licensing conditions operable in the derivation of wh-dependencies is reminiscent of Rizzi's (1990) Relativized Minimality account of dependency relations in A, A', and X chain formation whereby two modes of licensing seem to be operable. First, some functional heads (namely, T and Agr in English-type languages) act as 'head-governors' in relation to lexical heads (mainly V, but also A, N, and P). Second, dependency relations also rely on some notion of 'antecedent government' where coindexation between links, which result from chain formation in A-movement and A'-movement, is essential for licensing the coindexed elements in Spec positions. Since Spec-head agreement applies, the dependency relations are extended to involve head positions (i.e., establishing generalized Spec-head/head-head agreement relations as in the Barriers framework (Chomsky 1986a)). As far as A'-dependencies are concerned, Rizzi's (1990) attempt to unify both modes of dependency
} 
interface would be the result of the interaction of both modes of licensing conditions (cf. Rizzi \& Shlonsky 2007). The system of head-head and Spec-head agreement relationships established in sentence structure for the licensing of the relevant features in subject-verb agreement configurations and wh-dependencies would be an essential ingredient in the establishment of the structural dependency relations that link the $\mathrm{CP}$ domain to the IP domain.

This account of the wh-movement process of man 'who' to [Spec, FocP] in (72) strengthens the structural similarity between the T-head in the split-IP domain and the Foc-head in the Split-CP domain, save for the difference between the two agreement relationships that arise in each case in terms of interpretability and identification and/or valuation of features. Raised DPs in the IP/Agr ${ }_{S} \mathrm{P}$ domain check/value an uninterpretable counterpart of their $\varphi$-features on the T-node that probes for them. Similarly, the Spec-head agreement relation between the Foc head and the wh-word man in [Spec, FocP] in (72) is established in terms of some counterpart of the interpretable $[i \mathrm{WH}]$-features on the wh-word man (in this case, the $[u \mathrm{~F}]$-feature on Foc), which satisfies Rizzi's $(1996,2006)$ Wh-Criterion (reinterpreted in terms of the Phase-theoretic process of valuation, as discussed in the two previous paragraphs) or Ouhalla's (1994) and Aoun et al.'s (2010) 'morphological identification' analysis of dependency formation (see section 4.1.2.4). In addition to this essential valuation process in the derivation of wh-dependencies in SA, the identification requirement of features in terms of which the C-T dependency is established at the interface involves the $[i T]$ feature on Fin in relation to both the $T$ node and the Foc node. ${ }^{48}$

However, the main difference between subject-verb agreement configurations and the agreement relations involved in wh-dependencies (involving the projection of FocP in the split-CP domain) is the type of 'edge' feature involved in each

\footnotetext{
licensing seems to point to the legitimacy, in the current terms of probe-goal-Agree relations, of a morphological Spec-head agreement licensing mechanism, for the valuation of uninterpretable features on the functional head by the matching of interpretable features of wh-elements, co-existing with a head-head agreement licensing mechanism that operates strictly in terms of 'identification' of some $[+\mathrm{F}]$ 'focus' feature on functional heads within the C-T dependency (either $[u \mathrm{~F}]$, as in whdependencies, or $[i \mathrm{~F}]$ in strictly head-head agreement relationships as those that link Neg or Mod to Fin and $\mathrm{T}$ in the $\mathrm{C}-\mathrm{T}$ dependency at the interface).

${ }^{48}$ In wh-dependencies involving relativization where a Topic DP is involved, the Foc node does not project (Rizzi 1997: 297-298; see section 4.3.1 below). In this case, identification of the C-T dependency proceeds with the Force head signalling [+declarative] force as the default clause type.
} 
dependency relation. In subject-verb agreement configurations, the relevant 'edge' feature is the uninterpretable $[u \mathrm{D}]$ feature on $\mathrm{Agr}_{S}$, which combines with the EPP feature of T. ${ }^{49}$ In wh-dependencies, the relevant 'edge' feature is the uninterpretable $[u \mathrm{~F}]$ feature that moved wh-elements in [Spec, FocP] value on Foc.

As already discussed above, the Top head that projects in the split-CP domain of SA is endowed with a D-feature conceived to be an interpretable feature on Top (i.e., [iD] on Top) essentially dissociated from the subject-verb agreement that arises in the IP domain under the T-Agr complex node (but still related to it in terms of syntactic predication, which does not rely on $\varphi$-agreement relations). Thus, the Topiccomment configuration, as represented in a structure like (72) above, has to satisfy the kind of 'higher predication' that Rizzi (1997: 287) links to $\operatorname{Agr}_{S}$ in the IP domain. In these terms, both Top and $\mathrm{Agr}_{\mathrm{S}}$ (in fact, the $\mathrm{T}-\mathrm{Agr}_{\mathrm{S}}$ inflectional complex) configurationally connect a DP to sentential predication much like V-v does within $\nu \mathrm{P} / \mathrm{VP}$ (Rothstein 1983, 1995). According to such assumptions, the D-feature of Top, in the structures where the Top node projects, does not need to be valued, but it still needs to satisfy identification within the $\mathrm{C}-\mathrm{Agr}_{\mathrm{S}} \mathrm{T}$ dependency for tense interpretation at the interface (cf. R\&R: 137-141).

Irrespective of the $\mathrm{A} / \mathrm{A}$ ' dichotomy that differentiates between a 'topic DP' (in an A'-specifier) and a 'subject DP' (in an A-specifier), the two DPs ?al-Pabaa?- $u$ 'parents' and pro, in (72), are connected by the Spec-head/head-head configurational agreement relations that link the two Spec positions they occupy - i.e., [Spec, TopP] and $\left[\mathrm{Spec}, \mathrm{Agr}_{\mathrm{S}} \mathrm{P}\right]$ - and the head nodes they are agreeing with - the two functional heads Top and $\mathrm{Agr}_{\mathrm{s}}$. As discussed above, both Top and $\mathrm{Agr}_{\mathrm{S}}$ are assumed to be endowed with a D-feature (interpretable on Top, but uninterpretable on $\mathrm{Agr}_{\mathrm{S}}$ ). Thus, in (74), the D-feature of $\mathrm{Agr}_{S}$ - having already been satisfied via the licensing of pro in $\left[\mathrm{Spec}, \operatorname{Agr}_{S} \mathrm{P}\right]$ - is the necessary link in the head-head dependency relation between $\operatorname{Agr}_{S}$ and Top.

Under such assumptions, the presence of pro (with its interpretable inherent D-feature) in [Spec, $\operatorname{Agr}_{S} \mathrm{P}$ ], in an SA SVO structure like (72), determines subjectverb agreement at the 'strong Phase' in the sense of Chomsky (2001, 2004). Merging

\footnotetext{
${ }^{49}$ The valuation of $[u \mathrm{D}]$ features in subject-verb agreement configurations is subject to parameterization according to the structure being derived in a particular language. As will be dealt with in section 4.3, in the parametric account of the derivation of sentence structure in such structural configurations as SA VSO vs. SVO word ordering possibilities and German V2 phenomenology, subject-verb agreement and the satisfaction of the EPP operate in terms of the availability or unavailability of uninterpretable $[u \mathrm{D}]$ features.
} 
pro in $\left[\mathrm{Spec}, \operatorname{Agr}_{\mathrm{S}} \mathrm{P}\right]$ is a necessary derivational step in such structural configurations not only to link D on $\operatorname{Agr}_{S}$ to $\varphi$-feature specification (for the licensing of agreement on the inflected verb, under T-Agrs, and the satisfaction of the EPP; cf. Biberauer 2010), but also to link the interpretable D-feature on Top to the $\mathrm{V}-v$-T-Agr $\mathrm{S}$ complex in relation to subject-verb agreement. By that token, the processes involved in the derivation of SA SVO sentences satisfy identification of the C-Agr $-\mathrm{T}$ dependency at Spell Out at the point in the derivation where pro is merged in [Spec, $\left.\operatorname{Agr}_{S} \mathrm{P}\right]$. In these terms, the assumption that the DP is base-generated in [Spec, TopP] at the highest part of the split-CP domain and not moved to that position may be justification for the claim, made above, that no checking/valuation is needed at the level of TopP.

As already pointed out with reference to sentences (71a-b) (repeated below as (73a-b)), reversing the order of the Topic DP ?al-?abaa?- $u$ and the wh-moved word man leads to ungrammaticality:

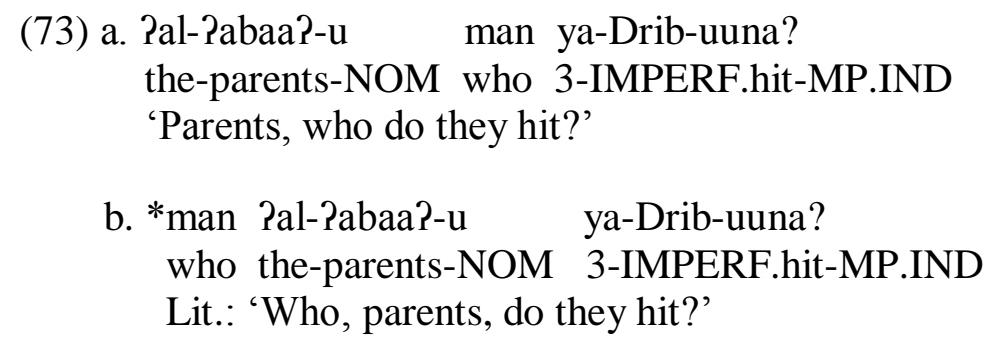

The ungrammaticality of Focus > Topic DP constituent ordering in SA also obtains with such wh-words as ?ayna 'where' and the wh-word Q marker hal (see the discussion in relation to example (34a) above), as in (74a-d) below, (adapted from Shlonsky 2000: 330-331):

(74) a. zayd-un Payna qaabal-tu-hu?

Zayd-NOM where PERF.meet-1S-him

'Zayd, where did I meet him?'

b. zayd-un hal qaabal-tu-hu?

Zayd-NOM Q PERF.meet-1S-him

'Zayd, did I meet him?'

c. *2ayna zayd-un qaabal-tu-hu?

where Zayd-NOM PERF.meet-1S-him

'Zayd, where did I meet him?'

d. *hal zayd-un qaabal-tu-hu?

Q Zayd-NOM PERF.meet-1S-him

'Zayd, did I meet him?. 
Other cases of the same constituent ordering restriction involve Focalized DPs preceding Topic DPs, as in the ungrammatical sentence (75b). The converse constituent ordering is well formed, as in (75a) (examples adapted from Bakir 1980, quoted in Shlonsky 2000: 330):
(75) a. faatimat-u wardat-an
PaSTaa-haa
saalim-un (SA)
Fatima-NOM flower.INDEF-ACC PERF.give.3MS-it(F) Salim-NOM
'It is a flower that to Fatima, Salim gave.'
b. *wardat-an faatimat-u PaiTaa-haa saalim-un flower.INDEF-ACC Fatima-NOM PERF.give.3MS-it(F) Salim-NOM 'It is a flower that to Fatima, Salim gave.'

Wh-extraction from an embedded clause does not pose a problem for the relative order of a focused element and Topic DP. Sentence (76) below, for example, is a case of successive-cyclic wh-movement of a wh-word out of the embedded $\operatorname{Agr}_{S} \mathrm{P}$ to the $[\mathrm{Spec}, \mathrm{FocP}]$ position in the matrix clause (as partially represented in the bracketed structure (77)):
(76) man qult-a Pinna Pal-Pabaa?-a ya-Drib-uuna? whoPERF.say-2MS that the-parents-ACC 3-IMPERF.hit-MP.IND 'Who did you say that parents hit?'
(77) [ForceP [FocP $\operatorname{man}_{\mathrm{wh}}$ qult-a [ForceP $\mathrm{t}_{\mathrm{wh}}$ [Force Pinna [TopP Pal-Pabaa?-a ... [AgrSP ya-Drib-uuna ... [ [v $\left.\left.\left.\left.\left.\left.\left.\mathrm{v}_{\mathrm{wh}}\right]\right]\right]\right]\right]\right]\right]$ ?

In (77), the wh-word man 'who' in [Spec, FocP] and the Topic DP ?al-Pabaa?-a in [Spec, TopP] occur in two different split-CP domains, in which case the restriction on Foci preceding Topics does not apply.

However, there are cases of wh-dependencies within a matrix clause in SA where the order of constituents [wh-word] ... [Topic DP], which is shown to be ungrammatical in (73b) and (74c-d) is allowed. These cases involve a phonetically realized Force head, as in (78a) below. In (78b), I provide the VSO counterpart of (78a).

(78) a. man *(Palladhii) Pal-Pabaa?-u yaDrib-uuna(-hu)? who (that.MS) the-parents-NOM 3-PERF.hit-MP.IND(-him) Lit.: 'Who is it that parents are hitting (him)?'
b. man (?alladhii) ya-Drib-u(-hu) Pal-Pabaa?-u? who (that.MS) 3-IMPERF.hit-3MS.IND(-him) the-fathers-NOM 'Who is it that the fathers hit him?'


The obligatory presence of the complementizer ?alladhii in (78a) suggests that the structure of the sentence is different from that of the ungrammatical wh-question in (71b)/(73b). In (78b), the DP ?al-Pabaa?- $u$ is postverbal and does not occur in TopP (see section 4.1.2.1 for the derivation of VSO sentences in SA).

In the VSO sentence (79) below, the verb ya-Drib- $u$ cannot co-occur with a Topic DP in initial position:

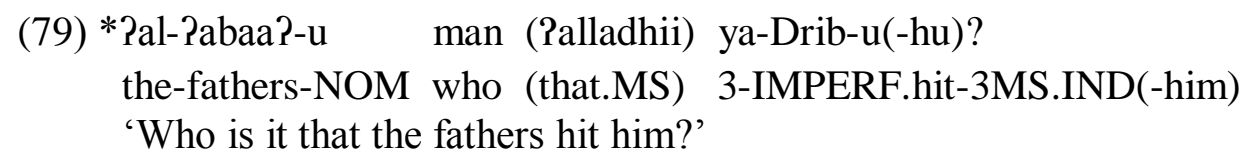

The ungrammaticality of (79) is predicted on the assumption that a Topic DP like ?al?abaa?- $u$ in (79) will have to be resumed by a referential pro element in the IP domain that will determine full subject-verb agreement with the verbal inflectional complex. This is, however, not the case in (79) where the verb is only 'partially' inflected for $3^{\text {rd }}$ person singular agreement as a characteristic of the VSO construction type.

Before analysing the structure of (78a) and (78b) and the difference in the obligatoriness or optionality of the complementizer Palladhii (see the discussion of (83b) and (84b) below), I will focus on the structure of the relative clauses in (80), which is comparable to that of the wh-question in (78a):

(80) a. Pal-walad-u *(Palladhii) Pal-Pabaa?-u ya-Drib-uuna(-hu) ... $\quad$ (SA) the-boy-NOM (that.MS) the-parents-NOM 3-IMPERF.hit-MP.IND(-him) 'The boy that parents are hitting him ...'

b. Pal-bint-u *(Pallatii) Pal-Pabaa?-u ya-Drib-uuna(-haa) ... the-girl-NOM (that.FS) the-parents-NOM 3-IMPERF.hit-MP.IND(-her) 'The girl that parents are hitting her ...'

c. Pal-Pawlaad-u *(Palladhiina) Ral-?abaa?-u the-boys-NOM (that.MP) the-parents-NOM ya-Drib-uuna(-hum) ... 3-IMPERF.hit-MP.IND(-them.M) 'The boys that parents are hitting them ...'

d. Pal-banaat-u *(Pallaatii) Pal-Pabaa?-u ya-Drib-uuna(-hunna) ... the-girls-NOM (that.FP) the-parents-NOM 3-IMPERF.hit-MP.IND(-them.F) 'The girl that parents are hitting her ...' 
As the relative clauses in (80a-d) show, agreement between the relative complementizer, under Force, and the NP, in [Spec, ForceP], is in number and gender. The fact that the complementizer in Force agrees in number/gender via Spec-head agreement with the element in the specifier position of the node they are inserted into suggests that the NPs walad- $u$, bint- $u$, ?awlaad- $u$, banaat- $u$ in (80a-d) are in [Spec, ForceP]. As Rizzi (1997: 297-298) observes, relative operators precede Topic DPs and, thus, "occupy the specifier of Force". Rizzi (1997: 298) contrasts (81a) with (81b) from Italian:

(81) a. Un uomo a cui, il premio Nobel, lo daranno senz'altro ... (Italian) 'A man to whom, the Nobel Prize, they will give it undoubtedly ...'

b. *Un uomo, il premio Nobel, a cui lo daranno senz'altro ... 'A man, the Nobel Prize, to whom they will give it undoubtedly ...'

In (81a), the relative operator 'a cui' occupies [Spec, ForceP] and precedes the topicalized DP 'il premio Nobel' in [Spec, TopP]. Reversing the order of these constituents gives rise to an ungrammatical output, as shown in (81b).

Rizzi's account as to the order of relatives and Topics gives the right configuration for the representation of the DPs in (80a-d) to the left of the Topic DP ?al-?abaa?-u, as structurally represented in (82) below for sentence (80a): ${ }^{.0}$

\footnotetext{
${ }^{50}$ For ease of exposition, I do not represent the FinP projection in (82) and (83b).
} 
$(82)$

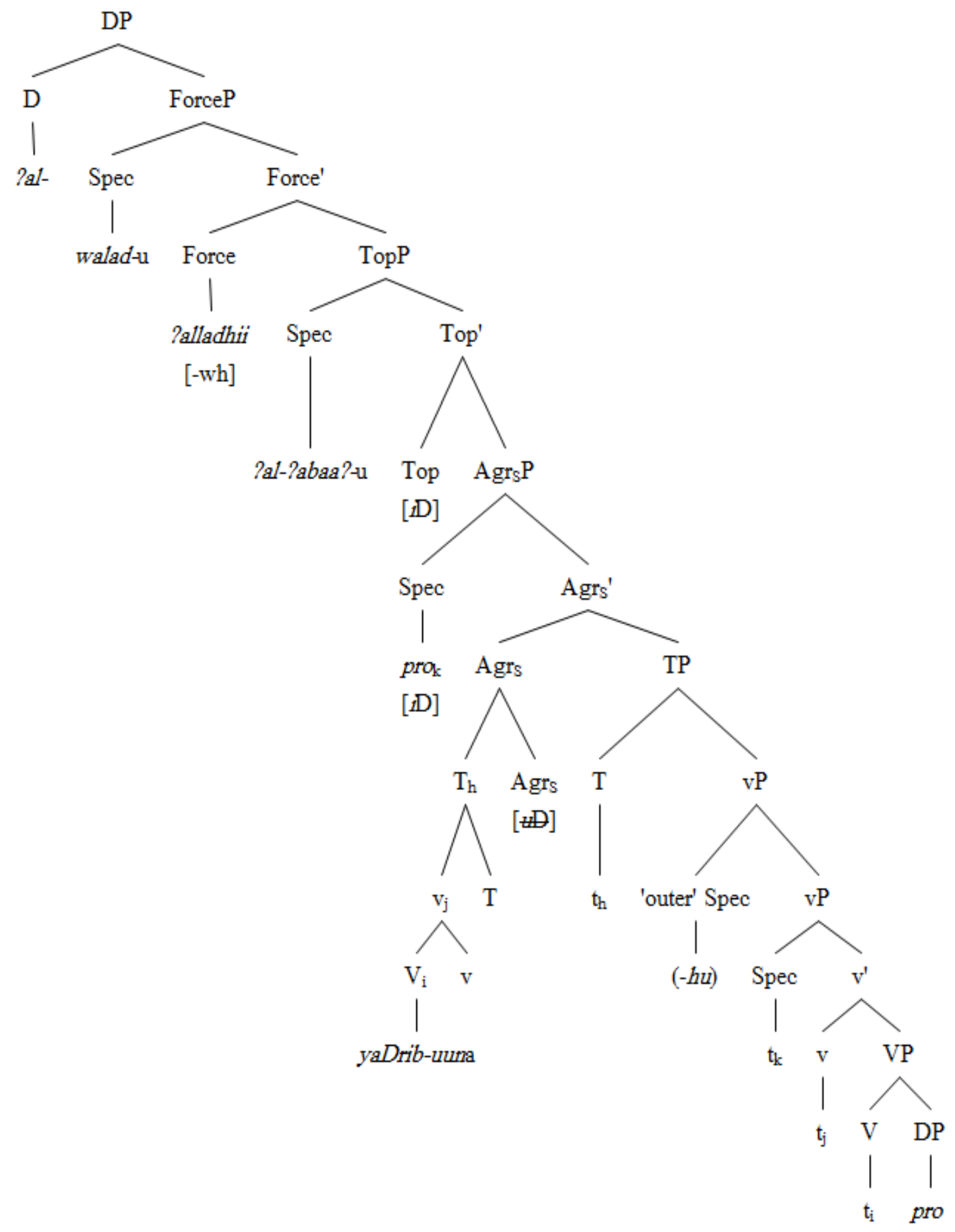

In structure (82), Force is realized as a relative complementizer Palladhii 'who', but no actual wh-movement takes place. Instead, a resumptive pro is inserted in object position to account for the optionality of the object clitic - $h u$ in 'outer' [Spec, vP] from where the clitic attaches to the raised verb under the inflectional complex V-v-T- 


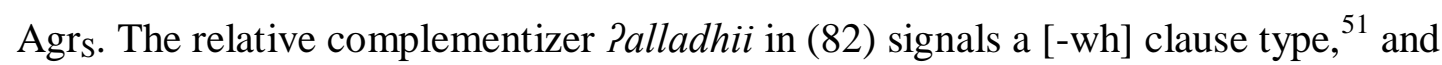
the function of the D element selecting ForceP ensures that this is the interpretation the structure gets at the interface.

Coming back to the derivation of (78a) and (78b), the structure of (78a) (repeated below as (83a)) is as in (83b), where the structure of $\operatorname{Agr}_{S} \mathrm{P}$ is the same as the structure of $\operatorname{Agr}_{S} \mathrm{P}$ in (82):
(83) a. man *(Palladhii) Pal-Pabaa?-u yaDrib-uuna(-hu)? who (that.MS) the-parents-NOM 3-PERF.hit-MP.IND(-him)
'Who is it that parents are hitting (him)?

b.

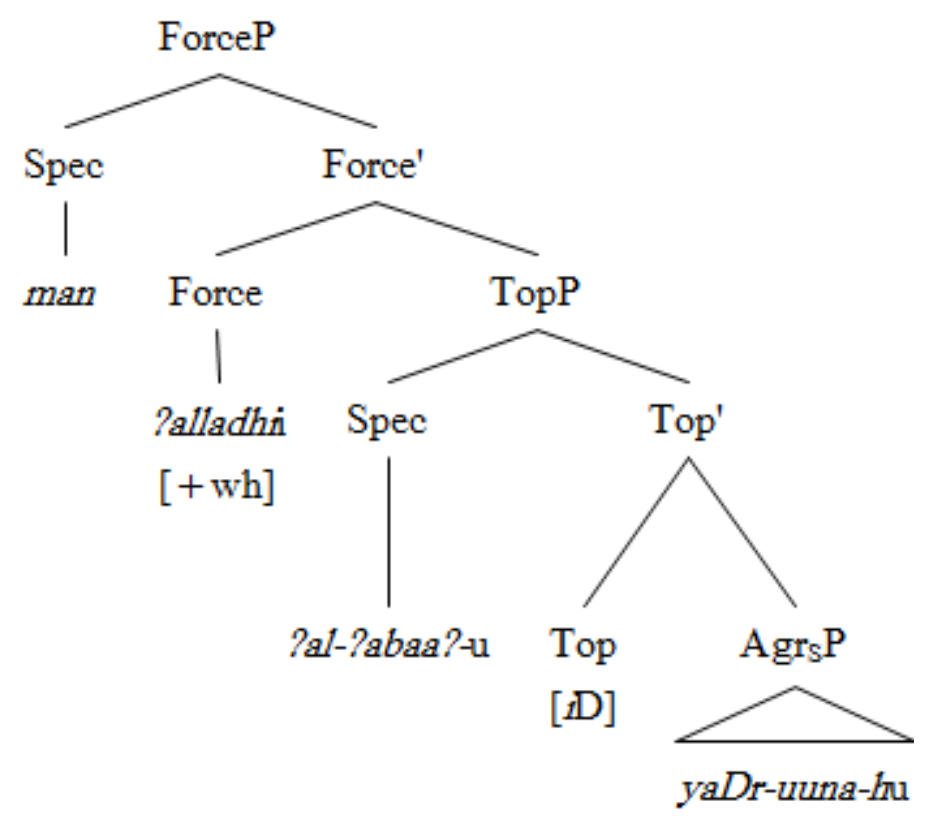

As far as representation ( $83 \mathrm{~b}$ ) is concerned, the wh-word man 'who' is assumed to be base-generated in [Spec, ForceP], similarly to the base-generation of the relative NP walad- $u$ in the structural representation (82). Structures (82) and (83b) show the same characteristics in terms of the merge of the wh-word man 'who', in (83b), and of the head of the relative clause i.e., the relativized NP walad- $u$ 'boy' in (82). Both elements are assumed to be base-generated in [Spec, ForceP], suggesting that no whmovement is involved in structure (83b). Thus, the wh-dependency is formed on the basis of a resumptive link between the relativized NP walad- $u$ in (82) and the object resumptive pronominal pro complement to $\mathrm{V}$ (which is also resumed by the optional

\footnotetext{
${ }^{51}$ As I will be discussing in relation to Table 4.2 below, the Force node in (82) would also be marked as [+predicative] feature, as against structures (83b) and (84b), which would be marked as [-predicative].
} 
pronominal clitic $-h u$ in 'outer' $[\mathrm{Spec}, v \mathrm{P}]$ in (82)). The same is true of the whdependency in (83b) involving the wh-word man, the object resumptive pronominal pro and the optional pronominal clitic $-h u$. My assumption is that there is no $[u \mathrm{~F}]$ feature on the Force head that would trigger wh-movement in the first place in such structures.

As for the structure of (78b) (repeated below as (84a)), it is represented as in (84b):

(84) a. man (?alladhii) ya-Drib-u(-hu)

who (that.MS) 3-IMPERF.hit-3MS.IND(-him) the-fathers-NOM 'Who is it that the fathers hit him?'

b.

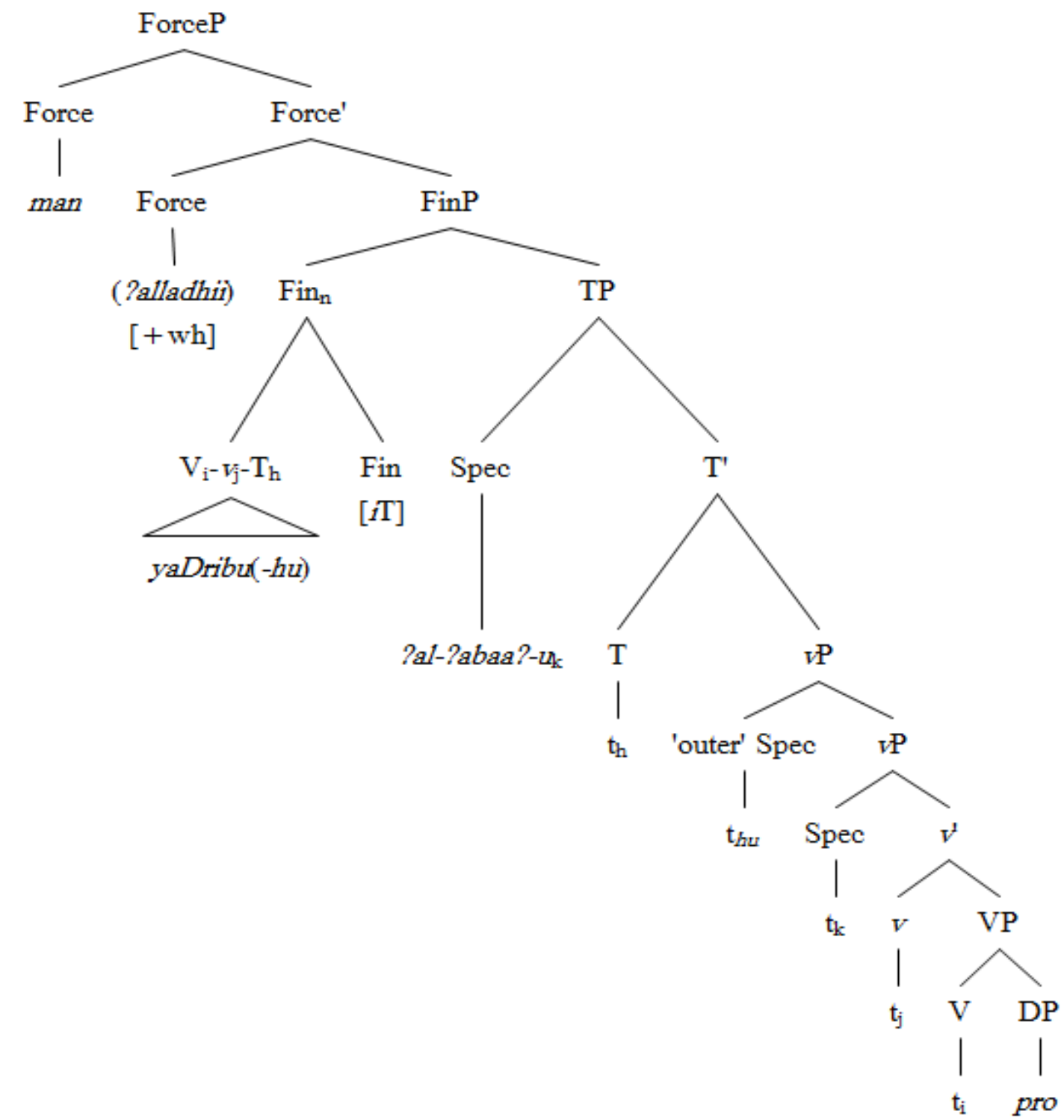


The optionality of the complementizer Palladhii realizing the head Force in structure (84b) is concomitant with the fact that no Topic DP is generated in such a sentence on the assumption adopted in this thesis maintaining that, whenever the raising process of the inflectional verbal complex V-v-T to Fin applies in VSO structural configurations in SA, the 'subject' DP cannot be a Topic, but is a subject DP in [Spec, TP] in the IP domain. On such assumptions, no topic DP is merged in the split-CP domain of a wh-construction like (84a) in contrast to the ungrammatical whconstruction in (73b) above where ?al-Pabaa?- $u$ functions as a Topic DP following the wh-word man in [Spec, ForceP].

The grammar of SA provides two other alternatives for (78a)/(83a). One alternative is to have the type of wh-constructions shown in (85a-d) below:

(85) a. man huwa (Pal-walad-u) Palladhii Pal-Pabaa?-u who he (the-boy-NOM) that.MS the-parents-NOM ya-Drib-uuna(-hu)? 3-IMPERF.hit-MP.IND(-him)

Lit: 'Who is he (the boy) that parents are hitting (him)?'

b. man hum (Pal-Pawlaad-u) Palladhiina Pal-Pabaa?-u who they(M) (the-boys-NOM ) that.MP the-parents-NOM ya-Drib-uuna(-hum)? 3-IMPERF.hit-MP.IND(-them)

Lit: 'Who are they (the boys) that parents are hitting (them)?'

c. man hiya (Pal-bint-u) Pallatii Pal-Pabaa?-u who she (the-girl-NOM) that.FS the-parents-NOM ya-Drib-uuna(-haa)?

3-IMPERF.hit-MP.IND(-her)

Lit: 'Who is she (the girl) that parents are hitting (her)?'

d. man hunna (Pal-banaat-u) Pallaatii Pal-Pabaa?-u who they $(\mathrm{F})$ (the-girls-NOM) that.FP the-parents-NOM ya-Drib-uuna(-hunna)? 3-IMPERF.hit-MP.IND(-them.F)

Lit: 'Who are they (the girls) that parents are hitting (them)?'

The wh-constructions (85a-d) show the sequence of a wh-word, a pronoun and a relative clause, in which the DP Pal-walad- $u$.., coming before the complementizer Palladhii or one of its variants is optional. These constructions are reminiscent of what Ouhalla (1999: 336, 341) designates as the counterpart of English clefts in SA. Thus, 
for example, substituting the wh-word man in (85c) with a proper noun like Zaynab yields a sentence like (86) below (adapted from Ouhalla 1999: 341):

(86) Zaynab-u hiya (Pal-bint-u) Pallatii Pal-Pabaa?-u Zaynab-NOM she (the-girl-NOM) that.FS the-parents-NOM ya-Drib-uuna-haa.

3-IMPERF.hit-3MP.IND-her

Lit: 'Zaynab is she (the girl) that parents are hitting.'

'It is Zayab who parents are hitting (her).'

According to Ouhalla (1999: 341), the structure of such sentences as (86) in SA is [F(ocus)-DP PRON RC], which "involves a structure close, but not identical, to the structure of clefts in English".

Exploiting Rizzi’s (1990) [ $\pm \mathrm{wh}]$; [ \pm predicative] feature system, as given in

Table 4.2 below, clause type in the wh-structures (83b) and (84b) above can be distinguished from clause type in a relative clause like (82) on the basis of the [ \pm predicative] feature:

Table 4.2. The feature structure of complementizer elements (adapted from Rizzi 1990: $67-68)^{52}$

\begin{tabular}{|l|l|l|}
\hline $\begin{array}{c}\text { Complementizer types } \\
\text { in English }\end{array}$ & \multicolumn{1}{|c|}{ Feature structure } & \multicolumn{1}{|c|}{ Example } \\
\hline Declarative C & {$[-\mathrm{wh}] ;$ [-predicative] } & 'I know that you saw it' \\
\hline Interrogative C & {$[+\mathrm{wh}] ;$ [-predicative] } & 'I wonder what you saw' \\
\hline Relative C 1 & {$[+\mathrm{wh}] ;$ [+predicative] } & 'The thing which you saw' \\
\hline Relative C 2 & $[-\mathrm{wh}] ;$ [+predicative $]$ & 'The thing that you saw' \\
\hline
\end{tabular}

Thus, on the basis of the feature structure of complementizer types in Table 4.2, what seems to be important in the derivation of (83a) and (84a) is not only the value given to clause type in terms of the feature system [ $\pm \mathrm{wh}]$ per se, but also its

\footnotetext{
${ }^{52}$ The difference between Relative C1 and Relative C2 in English is that only Relative C2 is phonetically realized as that. As for the Spec position of these two kinds of relatives in English, both are assumed to be filled up by some operator element. While the Spec of Relative $\mathrm{C} 1$ is occupied by an overt operator, i.e., a wh element like which in the sample example given above, the Spec of Relative $\mathrm{C} 2$ is occupied by a covert operator $O p$. Thus, a partial structure for these relatives would be as in (ia) and ib):
}

(i) a. The thing [CP which [c $\varnothing \quad$ [IP you saw]]] b. The thing [CP Op [c that [IP you saw]]] 
characterization in terms of the [ \pm predicative] feature system. According to Rizzi (1990), the [+predicative] feature specification is a distinctive property of relatives (in conjunction with the $[ \pm w h]$ feature specification) as against both declarative clauses, which are [-predicative] (and [-wh]), and interrogatives, which are [-predicative] (and [+wh]). Thus, if Rizzi's characterization of the feature structure of relatives crosslinguistically is correct, whereas the complementizer Palladhii in the interrogative sentence (83a) (as represented in (83b)) and in sentence (84a) (as represented in (84b)) is [+wh] and, essentially, [-predicative] - namely, a wh-question, the complementizer Palladhii in the relative construction (80a) (as represented in (82) above) is [-wh] and [+predicative] - namely a relative clause. ${ }^{53}$

Another alternative to the wh-question in $(78 a) /(83 a)$ is to use a wh-phrase as in English which boy, which girl and so on. In the structural representation in (88) for the wh-question in (87a), I represent the wh-phrase as a QP, the head position of which realizes the Q-head element Payyu/Payyatu: ${ }^{54}$

(87) a. Payyu walad-in (*? Palladhii) Pal-Pabaa?-u which(M) boy-GEN that.MS the-parents-NOM yaDrib-uuna(-hu)? 3-IMPERF.hit-MP.IND(-him) 'Which boy do parents hit?'

b. Payyatu bint-in (*? ?allatii) Pal-?abaa?-u which(F) girl-GEN that.FS the-parents-NOM yaDrib-uuna(-ha)? 3- IMPERF-hit-MP.IND(-her)

'Which girl do parents hit?'

\footnotetext{
${ }^{53}$ In his analysis of constituent questions in Palestinian Arabic, Shlonsky (2002: 143) also refers to Rizzi's (1990: 67-68) typology of complementizer types to pin down the characteristics of the C head illi (which is the counterpart of SA Palladhii) in interrogatives and relatives, but he considers the Palestinian Arabic complementizer illi to be [+predicational] in both interrogative and restrictive relative clauses. However, as shown in Table 4.2, the [+predicative] feature is a characteristic feature of relatives, but not of interrogatives. This characterization should apply both to Palladhii and to its counterpart illi in Palestinian Arabic.

${ }^{54}$ I had qualms about the sentences in (87a-d) with Palladhii in Force. I thank Dr. Hassan Makhad for confirming my grammaticality judgement. According to Dr. Makhad, some Moroccan professional teachers of Arabic, who were specifically asked about the grammaticality of such sentences, do not consider them ungrammatical. According to them, the best guess is that something seems to be wrong with the presence of the lexicalized complementizer in (87a-d).
} 
c. Payyu Pawlaad-in (*? Palladhiina) Pal-Pabaa?-u

which(M) boys-GEN that.MP the-parents-NOM

ya-Drib-uuna(-hum)?

3-IMPERF.hit-MP.IND(-them.M)

'Which boys do parents hit?'

d. Payyatu banaat-in (*? Pallaatii) Pal-Pabaa?-u

which $(\mathrm{F})$ girls-GEN that.FP the-parents-NOM

ya-Drib-uuna(-hunna)?

3-IMPERF.hit-MP.IND(-them.F)

'Which girls do parents hit?'

(88)

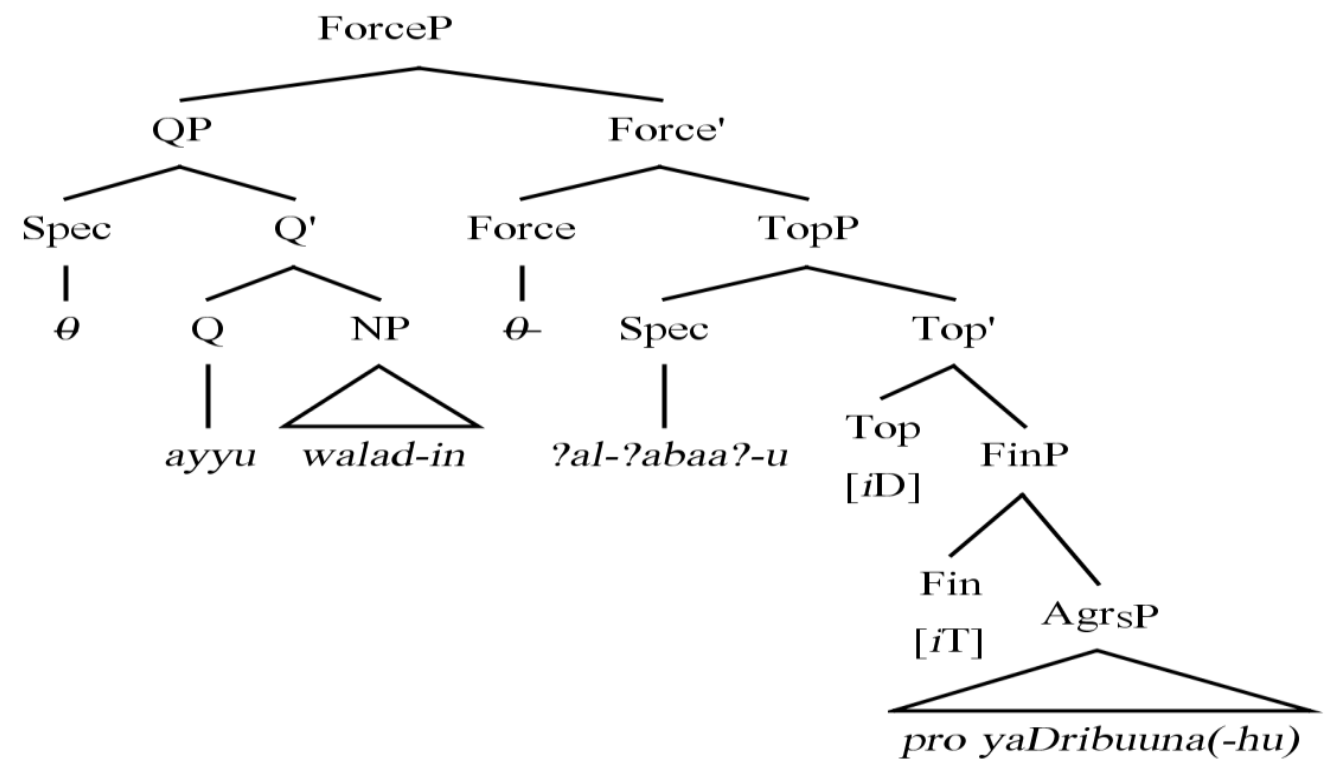

As (88) shows, in this second alternative construction to (78a)/(83a), Palladhii cannot be merged in Force. Thus, the structure of the wh-constructions in (87a-d) is more like the structure of the ungrammatical wh-construction (73b) above than like the structure of (78a), where the topicalized DP ?al-Pabaa?-u 'parents' cannot follow the wh-word man without having the complementizer ?alladhii follow the wh-element.

Nevertheless, the wh-constructions in (87a-d) are 'grammatical' with no need of merging the complementizer Palladhii (or one of its variants) in Force.

The non-realization of the complementizer Palladhii in a structure like (88) can be accounted for by assuming that some kind of agreement (morphologically encoded on the head Payyu/ayyatu in (87a-d)) has already been established within the QP Payyu walad-in in [Spec, ForceP]. The head status of the element Payyu/Payyatu is suggested by the morphological composition of this element - namely, an initial 
syllable $? a$ that is synonymous with the Q-head marker $? a$ (inserted under Force in a structure like (32b) above) and some gender morpheme $-y u /-y a t u .{ }^{55}$ Thus, in (89), the Q-head marker Payyu agrees in either masculine or feminine gender with its sister NP. The agreement relation established within the QP [Q NP] in [Spec, ForceP] in (89) is akin to the relation of a head D and its complement NP [D NP]. The only difference between the Q-NP head-complement relation in (88) and the relation of D to its NP complement is that, whereas the Q-head Payyu/Payyatu in (88) 'assigns' Case namely, GEN(itive) Case - to its complement, no such Case-marking relation exists between D and its complement NP. Thus, it might be the case that this morphologically marked head-complement agreement relationship (marked for gender agreement on the Q-head and for GEN Case on the complement NP) between the head Payyu/Payyatu and its complement NP waladin in (88) is a sufficient licensing condition for the wh-phrase ?ayyu waladin with no need of lexically realizing Force as ?alladhii in such a case. ${ }^{56}$

In (87a), the reverse ordering of the Topic DP ?al-?abaa?- $u$ 'parents' and the QP Payyu walad-in is also allowed yielding a sentence similar in structure to (71a)(73a) above (compare structure (89b) below to structure (60) above). Thus, sentence (89a) would have a structure like (89b):

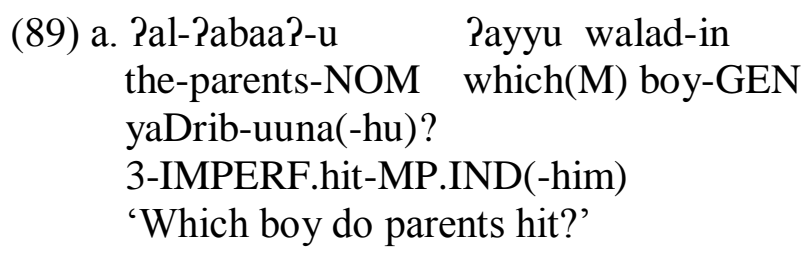

\footnotetext{
${ }^{55}$ The characteristic of a head taking a gender morpheme is reminiscent of 'partially' inflected verbs in SA VSO word order as discussed in section 3.3.4. This is also the characteristic of the verb-like element laysa discussed in section 4.1.2.5 above. Both the NEG element laysa and the Q-element ?ayyu inflect for gender in agreement with the DP to their right in sentence structure.

56 There are also such SA interrogative sentences as in (i) below where the ayyu element is morphophonologically spelled out as one constituent substitutable by the wh-word man, as the sentences in (i) below show. Not surprisingly, in this case the insertion of the complementizer ?alladhi under the Force head position becomes optional:
}

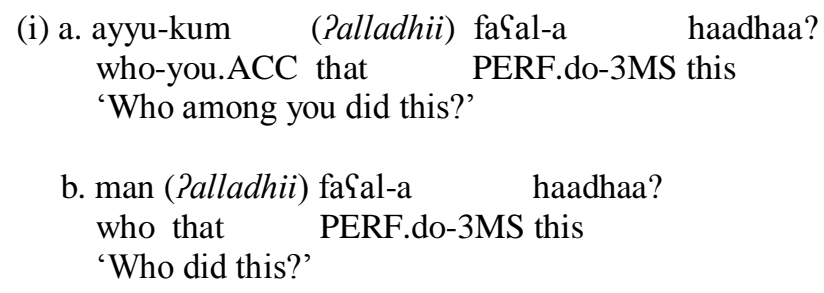


b.

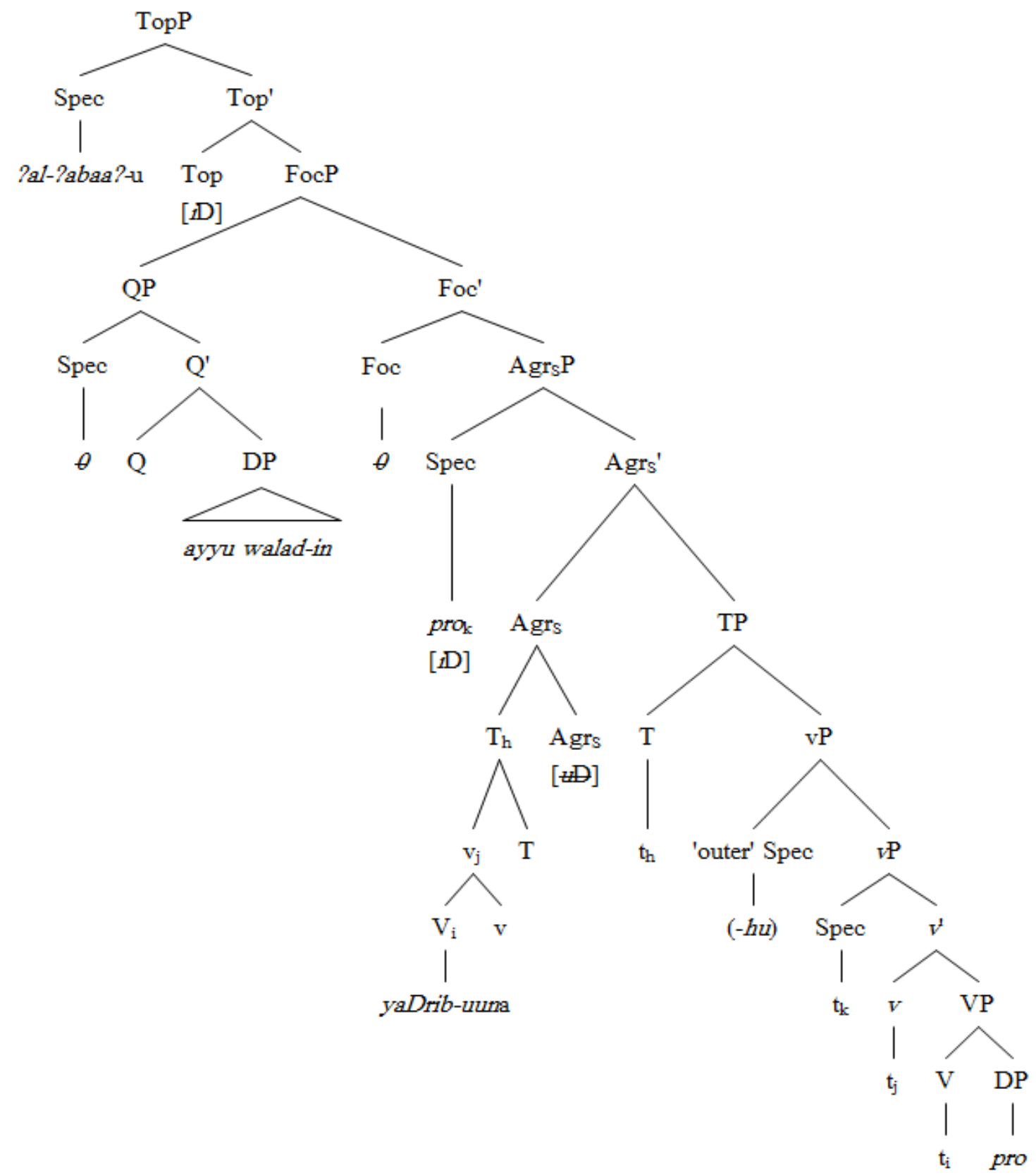

In (89b), the Topic DP Pal-Pabaa?- $u$ base-generated in [Spec, TopP], and resumed by a resumptive pro in $\left[\mathrm{Spec}, \mathrm{Agr}_{\mathrm{S}} \mathrm{P}\right]$, precedes the $\mathrm{QP}$ ?ayyu walad-in wh-moved to [Spec, FocP]. As in (76), Force remains empty in (89b).

This section has shown that a Focused element, which can either be a wh-word or a focused DP, can only be preceded by a Topic and cannot be followed by it. As far as wh-interrogatives are concerned, the derivation of such dependencies in SA is not necessarily a process of wh-extraction. In the cases where wh-extraction takes place, the trigger of wh-movement is assumed to be a $[u \mathrm{~F}]$ feature of the Foc head, valued by the moved wh-element in [Spec, FocP]. In the cases where a resumptive strategy 
replaces wh-extraction, the wh-element is spelled out in [Spec, ForceP], with the complementizer Palladhii merged in Force (i.e., the head that determines clause type). Only in the cases where a Topic DP is not involved in such wh-dependencies is the Merge of Palladhii optional. In the cases where Palladhii is obligatory, what differentiates a relative complementizer type from an interrogative complementizer type is the $[ \pm$ predicative] feature in conjunction with the $[ \pm w h]$ feature. My assumption has been that, irrespective of whether the Force head is realized or left empty in such instances of interrogative sentences, no $[u \mathrm{~F}]$ feature needs to be valued. In the next section I deal with wh-dependencies in TA.

\subsubsection{The absence of word ordering restrictions on 'topicalized' DPs and wh- elements in wh-dependencies in TA}

In this section, I first show that, unlike the derivation of wh-dependencies in SA which allows the base generation of wh-elements when a Topic DP is also derived in the structure, wh-dependencies in TA are formed via movement of the wh-word and allow different orderings of a wh-word and a 'topicalized' DP. Then, on the basis of sample wh-constructions in TA, I propose that TA does not resort to the expansion of $\mathrm{CP}$ in the form of a ForceP-FinP split system. Rather, judging from the absence of the strict word ordering of a Topic DP and a wh-element in TA, the language makes use of adjunction of A'-moved elements to the left of IP/Agr $\mathrm{A}$.

The TA example in (90a) is the corresponding wh-question to the SA whconstruction $(78 a) /(83 a)$ repeated below as $(90 b)$ :

(90) a. shkuun (illi) buu-k u um-ək $\quad \mathrm{y}-\mathrm{Drb}-\mathrm{uu}(*-\mathrm{h})$ ? who (that) father-your and mother-your 3-IMPERF.hit-MP(*-him) 'Who are your parents hitting?'

b. man *(?alladhii) Pal-?abaa?-u yaDrib-uuna(-hu)? who (that.MS) the-parents-NOM 3-PERF.hit-MP.IND(-him) Lit.: 'Who is it that parents are hitting (him)?'

In contrast to SA (90b), the TA wh-construction in (90a) allows a wh-word to precede a DP element whether the complementizer illi (which is the equivalent of SA Palladhii) is present or not. I propose that the structure of (90a) is as in (91) below: 


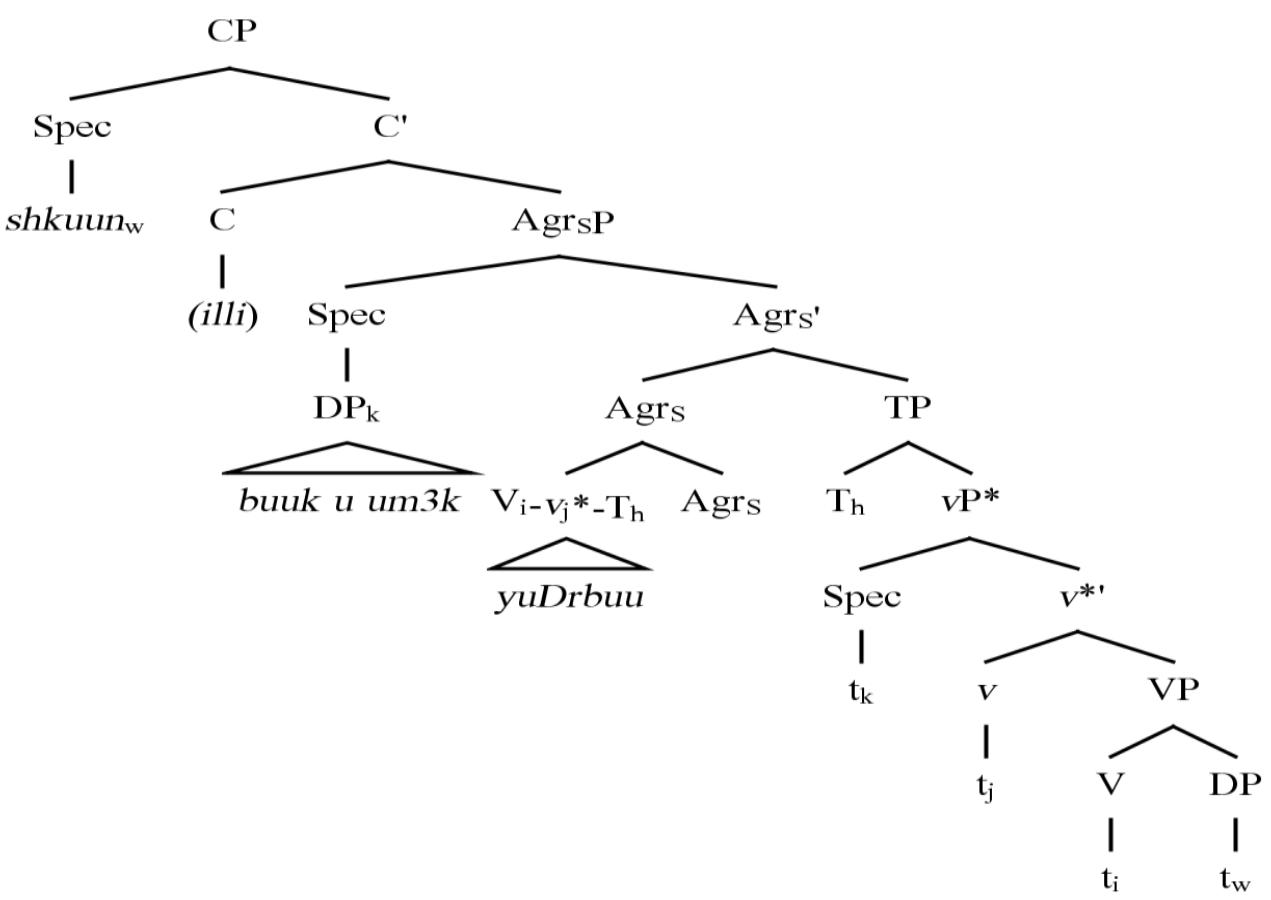

Structure (91) shows that, unlike the ungrammaticality of the word ordering of a whword and a Topic DP in SA wh-dependencies without there being a phonologically realized complementizer element (?alladhii in (90b) above), TA wh-dependencies do not exemplify the sequence of a wh-word and a Topic DP but the sequence of a whword in [Spec, CP] and a subject DP in [Spec, IP/Agr $\left.{ }_{S} \mathrm{P}\right]$.

However, as the relative clauses (92a) and (92b) show, both TA and SA require the presence of a relative complementizer:

(92) a. l-ulid *(illi) buu-k u um-ək y-Drb-uu(-h) ... the-boy (that) father-your and mother-your 3-IMPERF.hit-MP(-him) 'The boy who your parents are hitting him ...'

b. Pal-walad-u *(?alladhii) Pal-?abaa?-u ya-Drib-uuna(-hu) ... (SA) the-boy-NOM (that.MS) the-parents-NOM 3-IMPERF.hit-MP.IND(-him) 'The boy that parents are hitting him ...'

Apart from the word ordering difference that differentiates TA wh-questions from SA wh-questions and the presence or absence of a phonetically realized complementizer as discussed above, the spelling out of resumptive pronoun - $h$ (the TA equivalent of SA - $h u$ ) yields an ungrammatical result in (90a) - in contrast with (90b) -, irrespective of the presence or absence of the complementizer illi. This 
account suggests that wh-movement has actually occurred in the TA example leaving a wh-trace in the vacated object position, as represented in (91). These structural differences in the derivation of the wh-questions (90a) and (90b) in TA and SA, respectively, may be an indication that TA, unlike SA, does not resort to the projection of CP as ForceP-FinP à la Rizzi (1997) with a TopP projection following the Force head. Rather, TA makes use of adjunction structures to derive these A'dependencies.

Thus, this difference in the formation of wh-questions between TA and SA accounts for why, in the case where a Topic DP is merged in [Spec, TopP] as in SA (90b) above, the preceding wh-element in [Spec, ForceP] has to co-occur with the complementizer Palladhii merged in Force allowing the base-generation of the whelement in [Spec, ForceP] instead of having it moved from within $v$ P/VP. On such an account, since, in SA, initial 'subject' DPs are Topics base-generated in [Spec, TopP], wh-elements would have to be moved to [Spec, FocP] below the Topic DP (see structure (72) above) or, alternatively, be generated in [Spec, ForceP] with the complementizer Palladhii realizing the Force head, as shown in (90b).

Along with structure (91), structure (93b), for the wh-question in (93a) below, is also grammatical in TA:

(93) a. buu-k u um-ək shkuun (illi) y-Drb-uu(*-h)? father-your and mother-your who (that) 3-IMPERF.hit-MP(*-him) 'Who is it that your father and mother are hitting? 
b.

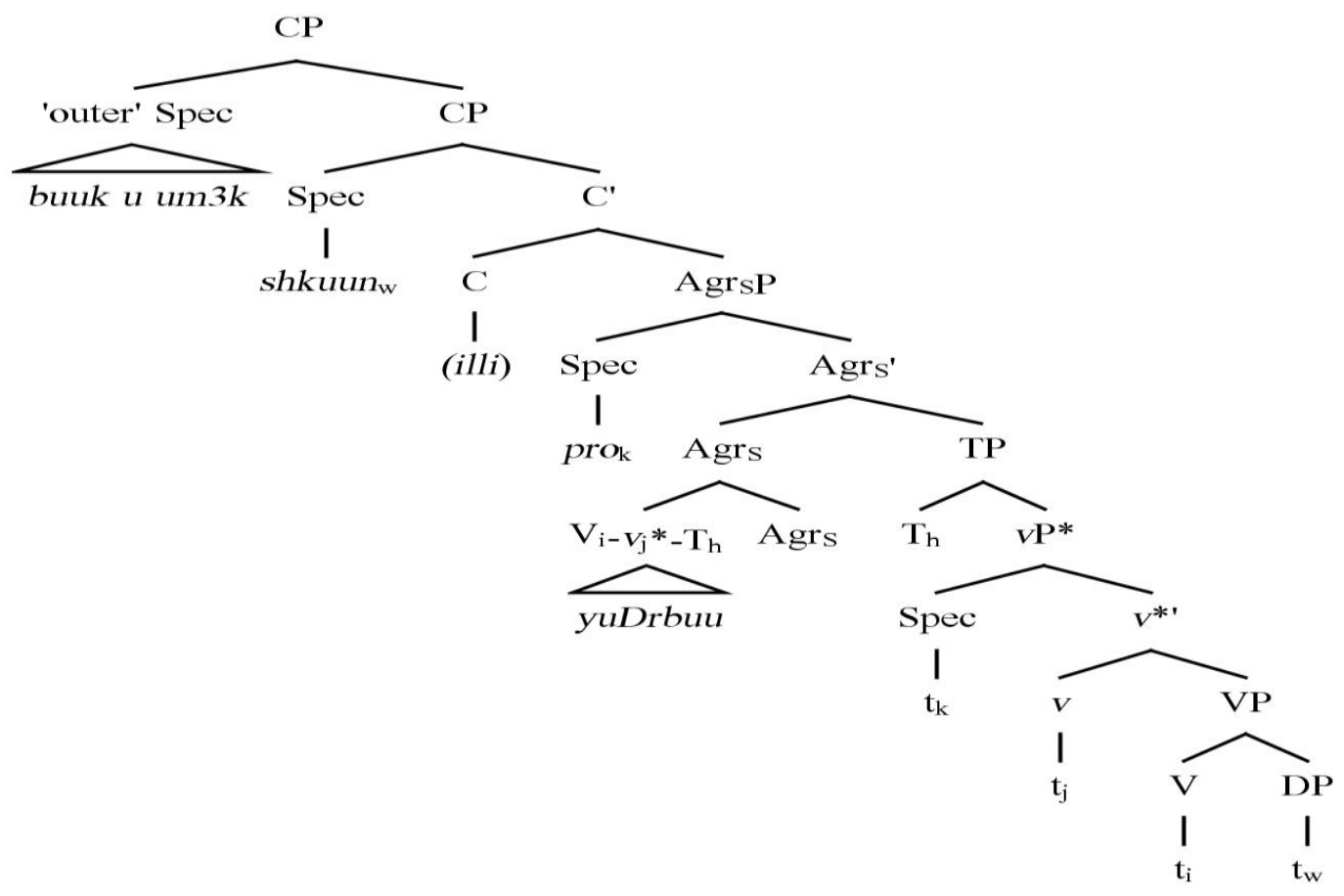

Unlike the subject DP buu-k u um-ək internally merged in [Spec, $\left.\operatorname{Agr}_{S} \mathrm{P}\right]$ in (91), the left-dislocated DP buu- $k$ u um-ək to the left of the wh-word shkuun 'who' in (93b) is Left-adjoined to CP. As in (93b), the wh-word shkuun, is raised from within $v \mathrm{P} / \mathrm{VP}$ and internally merged in [Spec, CP]. As for the [Spec, IP/Agr $\mathrm{PP}]$ position in (93b), it is occupied by a resumptive pro element raised out of [Spec, $v \mathrm{P}]$, which resumes the left-dislocated DP buu- $k$ u um-ək.

If the proposed analysis for the derivation of wh-dependencies in TA vs. SA in terms of a difference in how the $\mathrm{CP}$ domain projects is reasonable enough, this analysis would account for the fact that wh-movement in the instance of topicalization in TA does not obey any ordering constraint between the left-dislocated element and the wh-moved element, as shown in the TA examples in (94), which are the counterpart of Bakir's (2011: 193) original examples from Iraqi Arabic as shown in (95 a,b):

(94) a. 1-kursi ween khalleet-u the-chair where put.2MS-it 'Where did you put the chair?' 
b. ween 1-kursi khalleet-u where the-chair put.2MS-it

'Where did you put the chair?'

(95) a. l-qanafa ween khalleet-ha the-chair where put.2MS-it

(Iraqi Arabic)

'Where did you put the chair?'

b. ween l-qanafa khalleet-ha

where the-chair put.2MS-it

'Where did you put the chair?'

As Bakir (2011) notes, the strict order of Topic DPs and focused elements is not observed in Iraqi Arabic. Thus, in these instances of wh-dependency formation by movement, Bakir (2011: 200) opts for "adjunction of left-dislocated elements in the $\mathrm{CP}$ domain" and assumes that the left-dislocated object DP l-qanafa 'the chair' in (95a) and (95b) is an adjoined position to the left of IP/CP. Bakir (2011: 200) adds that: "... if recursion in the CP structure is allowed, it will create the necessary loci for the moved/base-generated elements without any need to specify the individual Cpositions with unique functions".

Thus, the proposed analysis of the derivation of wh-dependencies in SA vs. TA in this section, along with Bakir's (2011) claims about A'-dependencies in Iraqi Arabic may suggest that, whereas SA resorts to the expansion of the CP domain as a ForceP-FinP split system for the topicalization and focalization of constituents, modern spoken dialects like TA and Iraqi Arabic make use of adjunction structures for the derivation of such dependencies.

The differences in the constituent ordering possibilities of a wh-word and a DP element in SA vs. TA do not extend to the cases where both the focused element and the 'topicalized' element are DPs. As already discussed in relation to the SA examples (75a,b) (repeated below as $(96 a, b)$, adapted from Bakir 1980), whether the focused element is a wh-element or a DP, its ordering in relation to the 'topicalized' element is strictly Topic > Focus:
(96) a. faatimat-u wardat-an
PaiTaa-haa
saalim-un (SA)
Fatima-NOM flower.INDEF-ACC PERF.give.3MS-it(F) Salim-NOM
'It is a flower that to Fatima, Salim gave.'
b. *wardat-an
faatimat-u
PaSTaa-haa
saalim-un
flower.INDEF-ACC Fatima-NOM PERF.give.3MS-it(F) Salim-NOM
'It is a flower that to Fatima, Salim gave.' 
As (97) below shows, the equivalent TA examples to the SA sentences in (97) are both ungrammatical:

$$
\begin{aligned}
& \text { (97) a. *faatima warda PafTaa-haa salim } \\
& \text { Fatima flower.INDF PERF.give.3MS-it(F) Salim } \\
& \text { 'It is a flower that to Fatima, Salim gave.' } \\
& \text { b. *warda faatima PaiTaa-haa saalim } \\
& \text { flower.INDEF Fatima PERF.give.3MS-it(F) Salim } \\
& \text { 'It is a flower that to Fatima, Salim gave.' }
\end{aligned}
$$

The ungrammaticality of both (97a) and (97b) is accounted for on the assumption that TA does not allow the preposing of a DP element for the sake of (contrastively) focusing it. Thus, the TA sentence in (98b) below is ungrammatical if the focused DP is not kept in situ as in (98a) (which is the equivalent of Ouhalla's (1999: 338) original MA sentence (99) below):
(98) a. nadia shr-at KTAB (ma-hu-sh majalla) Nadia PERF.buy-3FS book.INDEF (NEG-it-NEG magazine)
'Nadia bought a BOOK (not a magazine).'
b. *? KTAB nadia shr-at (ma-hu-sh majalla) (TA) book.INDEF Nadia PERF.buy-3FS (NEG-it-NEG magazine) 'Nadia bought a BOOK (not a magazine).'
(99) nadia shr-at KTAB (mashi majalla) Nadia PERF.buy-3FS book.INDEF (NEG magazine)
'Nadia bought a book (not a magazine).'

As shown in (100), the equivalent of (98b) in SA is grammatical (Ouhalla 1999: 338):

$$
\begin{aligned}
& \text { (100) KITAAB-AN ishtar-at nadia (laa majallat-an) } \\
& \text { Book.INDEF-ACC PERF.buy-3FS Nadia (NEG magazine) } \\
& \text { 'It was a BOOK that Nadia bought (not a magazine).' }
\end{aligned}
$$


To sum up, this section has shown that the derivation of wh-dependencies in TA is exclusively a process of wh-extraction, via which wh-elements in interrogatives are wh-moved (vs. SA where wh-elements are sometimes base-generated, as discussed in relation to example (83a) above). As for focused DPs, the sample examples discussed in this section have shown that they are kept in situ. Whdependencies in TA are formed via movement of the wh-word and allow different orderings of a wh-word and a 'topicalized' DP. Such characteristics have been claimed to follow from the assumption that the language makes use of adjunction of A'-moved elements to the left of IP/Agr $\mathrm{P}$.

In the next section, I have a closer look at the V2 phenomenology and how this phenomenology compares to the 'basic' VSO word ordering of a VSO language like SA. As discussed in Chapter Three, the valuation of D-features at the level where T-features are represented is essential for the derivation of rather 'rich' subject-verb agreement configurations as well as the Merge of expletive elements.

\subsection{Subject-verb agreement configurations in 'basic' VSO and V2 languages}

In this section, I deal with the derivation of the V2 phenomenology and how this phenomenology compares to the derivation of the 'basic' VSO word order of a VSO language like SA. This analysis proposes that it is the availability or non-availability of D-features in conjunction with the T-features of $\mathrm{T}$ and Fin at the highest level of functional syntactic structure that determines the derivation of subject-verb agreement configurations in sentences exhibiting the basic VSO phenomenology and the V2 phenomenology. Under such a premise, at the 'edge' of constructions in the functional field linking the IP domain to the CP domain in languages with 'basic' VSO word ordering (e.g., SA) and languages exhibiting the declarative matrix V2 phenomenon (e.g., German), T-features and D-features on functional head projections interact in the derivation of subject-verb agreement configurations.

In section 4.3.1, I develop a feature-based analysis of the V2/'weak' V2 phenomenology. In section 4.3.2, I attempt to show how the assumption of the interdependency of T-features and D-features provides an alternative account for some peculiar subject-verb agreement configurations in the variety of English studied by Kimball \& Aissen (1971) and by Kayne (1989b, 2000) (briefly referred to in section 3.1.1.1), and for cases of complementizer agreement in west Flemish, as studied by Haegeman \& Koppen (2012). Section 4.3.3 is a conclusion. 


\subsubsection{A feature-based analysis of the V2/'weak' V2 phenomenology}

The feature structure of the higher functional field, in languages with 'basic' VSO word ordering (e.g, SA) and languages exhibiting the declarative matrix V2 phenomenon (e.g., German), configures a parametrically available uninterpretable $[u \mathrm{D}]$ feature that interacts with the $[i T]$ features of $\mathrm{T}$ and Fin. In such a feature-based parameterized system of subject-verb agreement configurations, three language types and/or construction types are actually posited to occur cross-linguistically:

(i) Language/construction type A: This type concerns declarative matrix V2 sentences in Germanic configuring a lexical or EXPL subject (e.g., es in German) in initial position. The sentences in (101a) and (101b) are adapted from Schwartz \& Vikner (1996: 20), structurally represented as in (102a) and (102b), respectively:

(101) a. Ein Junge ist gekommen
A boy is come

(German)

b. Es ist ein Junge gekommen

There is a boy come

(102) a.

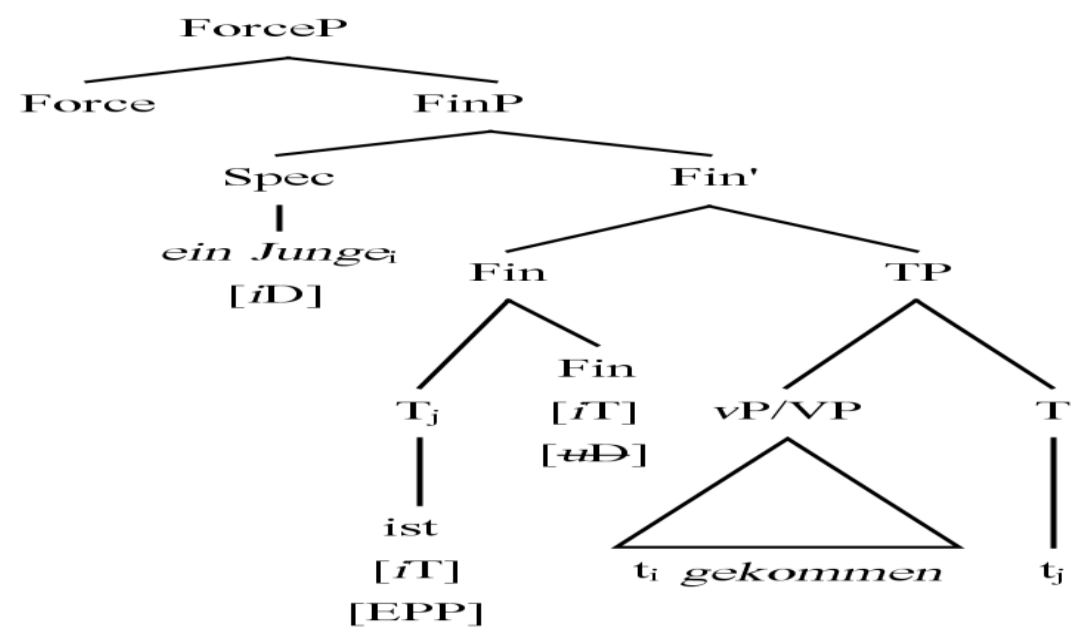


b.

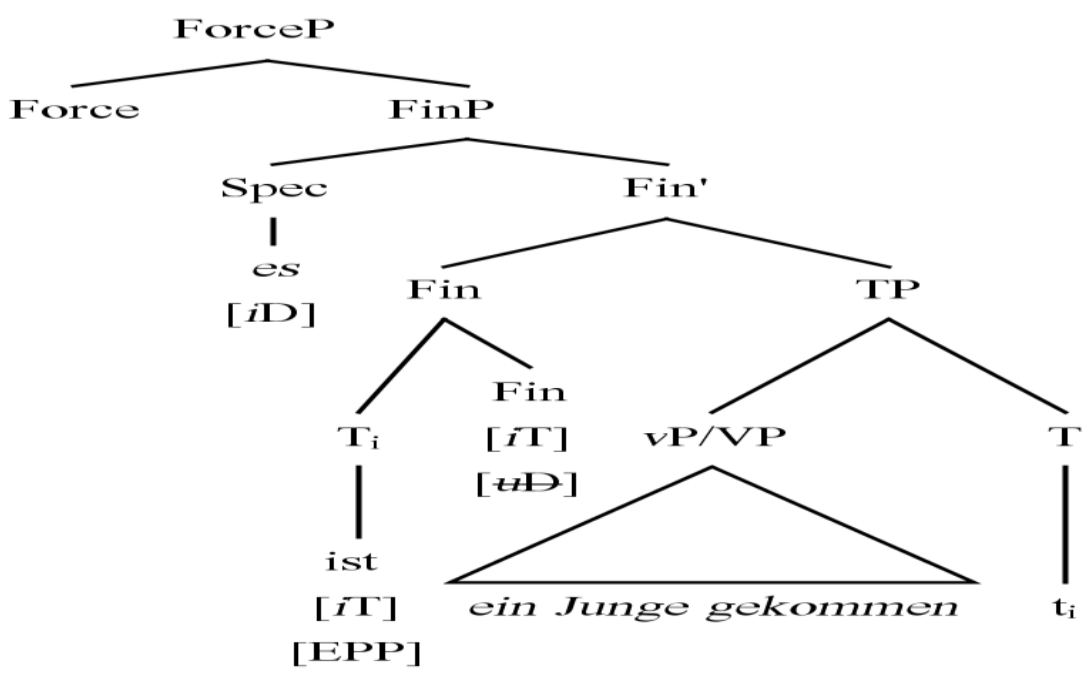

As represented in (102b) for sentence (101b), the subject DP is assumed not to leave the $v \mathrm{P} / \mathrm{VP}$ domain (this is also true of structure (105b) for German construction type $\mathrm{B}$ below). In both (102a) and (102b), T raises to Fin and a $[u \mathrm{D}]$-feature is generated on Fin where the valuation of $[u \mathrm{D}]$ coincides with the valuation of the EPP via a Spechead agreement relation with the subject DP (ein Junge 'a boy' in (102a) and es 'there' in (102b)) in [Spec, FinP].

The assumption that the subject remains in situ in $v \mathrm{P} / \mathrm{VP}$ is based on Haider's (2010: 156) argument that the subject in OV structures is licensed within the VP, just like other arguments. According to Haider's (2010) analysis, one example that shows that the subject remains lower than a shifted object (in 'outer' [Spec, $v \mathrm{P}]$ ) is a structure where only part of the shifted object is scrambled to initial position within a $\mathrm{V} 2$ configuration, as represented in (103) (clause bracketing mine):

(103) $\left[{ }_{C P}[\text { Von Mozart }]_{\mathrm{i}} \text { hat ja }{ }_{\nu * \mathrm{P}} \text { [die ersten Symphonien } \mathrm{t}_{\mathrm{i}}\right]_{\mathrm{j}}\left[v^{* \mathrm{P}}\right.$ kaum einer $\left[\mathrm{vp}_{\mathrm{p}} \mathrm{t}_{\mathrm{j}}\right.$ of Mozart has Part the first symphonies hardly anyone

[v' auf CD [v aufgenommen]]]]]]

(German)

on $\mathrm{CD}$ recorded

'Hardly anyone has recorded the first symphonies of Mozart on CD.'

A similar suggestion is provided by Rouveret (2010: 235) on the basis of such examples as in (104) below:

(104) a. Es kam gestern ein Junge

(German)

There came yesterday a boy 


\section{b. Gestern kam (*es) ein Junge \\ Yesterday came there a boy}

In (104), the subject DP ein Junge remains lower than the temporal adverb gestern.

On the assumption that the verb is in C (= Fin) and the subject kept in situ in [Spec, $\nu$ P] in such V2 contexts, Rouveret (2010: 235) maintains that: “... there is no evidence that the SpecTP position is projected/filled".

\section{(ii) Language/construction type B: In the derivation of Germanic V2}

declarative sentences with 'topicalized' XPs, the $[u \mathrm{D}]$-feature is generated on an $\operatorname{Agr}_{S}$ node in the IP domain to which $\mathrm{T}$ raises. Thus, this construction type is different from both construction type $\mathrm{A}$ in (i) and construction type $\mathrm{C}$ in (iii) below. ${ }^{57}$ Sentence (105a) (adapted from Schwartz \& Vikner 1996: 20) is an instance of the topicalization of an adverbial, structurally represented as in (105b): ${ }^{.5}$

(105) a. Gestern pro ist ein Junge gekommen

(German) Yesterday is a boy come

\footnotetext{
${ }^{57}$ This difference between V2 structural configurations that involves a 'topicalized XP' and V2 sentences that involve a subject DP (lexical or expletive) is reminiscent of Travis's (1991) asymmetrical account of V2 sentences, where the asymmetry is between subject elements that are merged in [Spec, IP] and non-subject elements that are merged in [Spec, CP]. In my account, the asymmetry is between subject DPs or an EXPL element like es merged in [Spec, FinP], and nonsubject elements including adverbials and object DPs merged in [Spec, TopP]. In construction type B, I take 'topicalization' of a temporal adverbial as a representative example of this second type of V2 configurations.

${ }^{58}$ Construction type $\mathrm{B}$ is the only matrix V2 declarative construction that projects an $\mathrm{Agr}_{\mathrm{S}}(\mathrm{P})$ in the Spec position of which EXPL pro is merged. In contrast to the analysis adopted here, the V2-outsideIP analysis of Schwarts \& Vikner (1996: 20) configures an EXPL pro in some position in IP (presumably, in [Spec, IP/TP] according to Schwarts \& Vikner's 1996 analysis):
}

(i) Gestern ist pro ein Junge gekommen Yesterday is a boy come

(German) 
b.

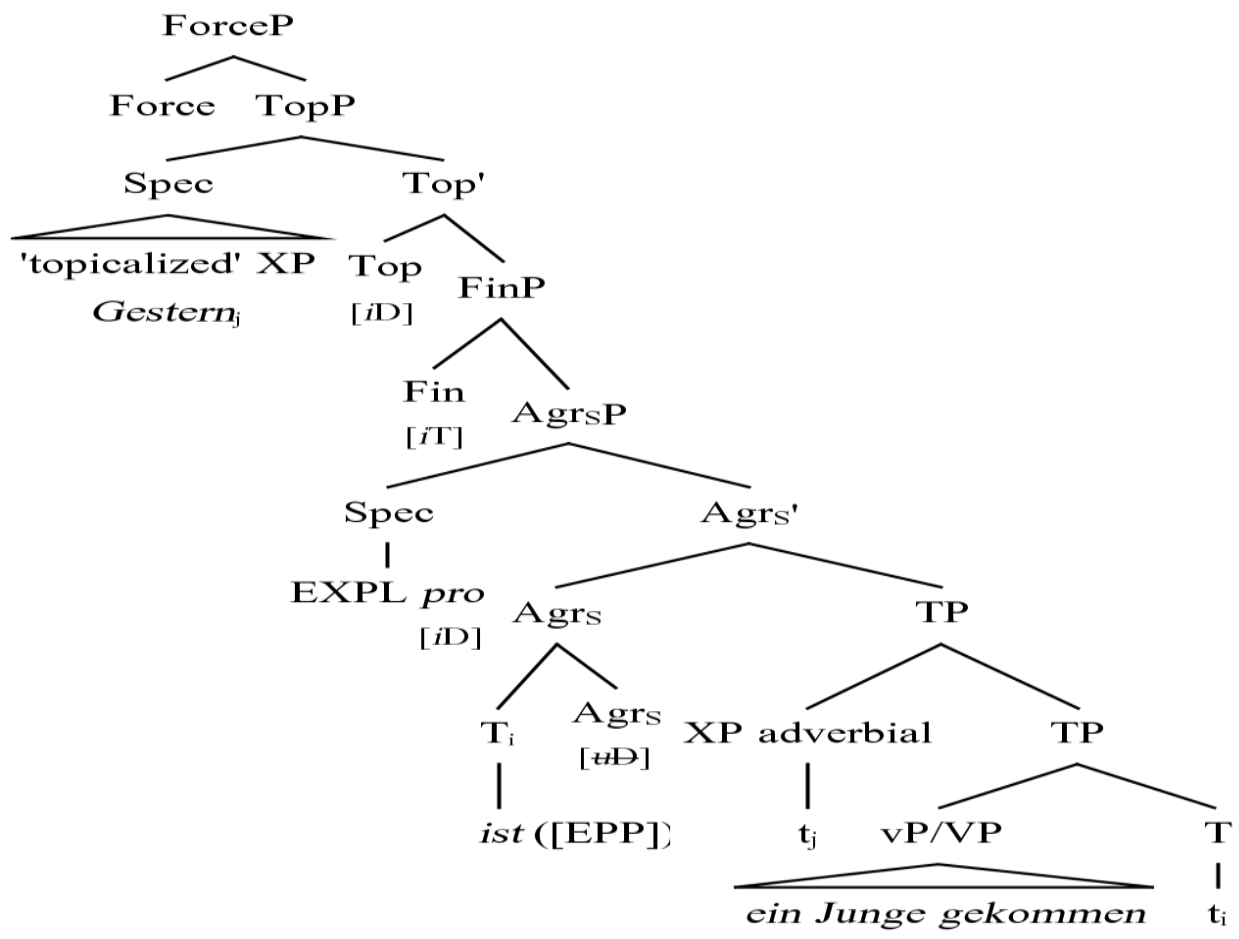

In $(105 b), \operatorname{Agr}_{S}(\mathrm{P})$ projects and EXPL pro is merged in $\left[\mathrm{Spec}, \operatorname{Agr}_{S} \mathrm{P}\right]$. The 'topicalized' temporal adverb gestern 'yesterday' is raised from some adjoined position to $\mathrm{T}$ and merged in the specifier position of a TopP projection above FinP. In these XP topicalization cases in V2 declaratives in German, pro at the highest level in the IP domain (i.e., in [Spec, $\left.\mathrm{Agr}_{S} \mathrm{P}\right]$ ) deletes after $\varphi$-agreement has applied (see section 3.1.2.2 in relation to footnote 7, and section 3.2.1.2 for a discussion of Roberts' 2010a,b copy deletion theory).

According to Roberts (2010b: 84), feature(s) of pro (consisting of a D-feature only for expletive pro, and D- and Agree features for argumental pro), are marked for deletion at PF on the assumption that they are 'defective' elements with only a subset of the features of the probe $\mathrm{T}$ (or, under the present analysis, the inflectional complex $\mathrm{T}-\mathrm{Agr}_{\mathrm{S}}$ after the raising of $\mathrm{T}$ to attach to $\mathrm{Agr}_{\mathrm{S}}$ ). Thus, in XP topicalization cases in matrix V2, the feature(s) of pro are 'copied' onto T(-Agr $)$ and pro deletes at PF.

The combination of the valuation of $[u \mathrm{D}]$ on $\mathrm{Agr}_{S}$ and the satisfaction of the EPP requirement in $\left[\mathrm{Spec}, \mathrm{Agr}_{\mathrm{S}} \mathrm{P}\right]$ is also a characteristic of $\mathrm{SVO}$ sentences in $\mathrm{SA}$ where the initial DP element in sentence structure is a Topic DP (see (109) below).

The complementary distribution of EXPL-insertion in German, either in [Spec, FinP], as in (102b), or [Spec, $\left.\operatorname{Agr}_{S} \mathrm{P}\right]$, as in $(105 \mathrm{~b})$ is accounted for in light of 
grammaticality contrasts as in (106a-b) and (107a-b) (Schwartz \& Vikner 1996: 20; similar contrasts obtain in Yiddish and Icelandic): ${ }^{59}$

(106) a. Gestern pro ist ein Junge gekommen Yesterday EXPL is a boy come

(German)

b. *pro ist ein Junge gekommen

EXPL is a boy come

(107) a. *Gestern ist es ein Junge gekommen

Yesterday is there a boy come

b. Es ist ein Junge gekommen

There is a boy come

In (106a), the V2 effect obtains at Spell Out as the EPP is satisfied in [Spec, $\operatorname{Agr}_{S} \mathrm{P}$ ], after $\mathrm{T}$ raises to $\mathrm{Agr}_{\mathrm{S}}$, in conjunction with the valuation of $[u \mathrm{D}]$ on $\mathrm{Agr}_{S}$. By contrast, the V2 effect does not obtain in (106b), as no lexicalized element occupies the first position of the clause at PF. Thus, (106b) is essentially not a V2 configuration. In this case, the only grammatical output is to merge the lexicalized subject counterpart of EXPL pro - i.e., EXPL $e s-$ as in (107b).

In (107a), the Merge of EXPL es in [Spec, TP] satisfies the EPP. However, as T raises to Fin, the EPP should have obtained in the Spec position of the superordinate head node to which $\mathrm{T}$ is attached (i.e., Fin). In (107a), the adverbial gestern cannot satisfy the EPP as it is in [Spec, TopP], which is not an EPP position, nor, for that matter, a position associated with a $[u \mathrm{D}]$ feature. By contrast, in $(107 \mathrm{~b})$, the EXPL es is merged in [Spec, FinP] where the EPP is satisfied and the additional 'edge' feature $[u \mathrm{D}]$ valued (see the discussion of structure (102b) above).

As noted above (see footnote 57), the present analysis is in parallel with Travis's (1991) asymmetrical account of V2 sentences, where the asymmetry is between subject elements that are merged in [Spec, IP] and non-subject elements that are merged in [Spec, CP]. In the present analysis of matrix declarative V2 sentences, the asymmetry is between XP-topicalization cases involving EXPL pro-insertion for EPP satisfaction in the IP domain and the Merge of subject elements (EXPL es or lexical subjects) for EPP satisfaction in [Spec, FinP].

${ }^{59}$ See footnote 58 above in relation to my adaptation of sentence (105a). 
(iii) Language/construction type C: This type concerns VSO structural configurations in languages with 'basic' VSO phenomenology. As discussed in section 4.1.2.1 above, Carnie et al. (1994: 87) and Carnie et al. (2000: 41) refer to the phenomenon exhibited by this type of construction as the 'weak V2 phenomenon' or the 'weak V2 effect' (in Carnie et al. 2000) according to which the verbal complex V$v$-T is in Fin at Spell Out. In the probe-goal-Agree system assumed here, $\operatorname{Agr}_{S}$ fails to project in the derivation of 'basic' VSO sentences. I exemplify such a language/construction type with the SA VSO sentence in (108a), structurally represented as in (108b):

$\begin{array}{ll}\text { (108) a. Pinna-hu jaa?-a } & \text { Pal-Pawlaad-u } \\ \text { that-EXPL PERF.come-3MS } & \text { the-boys-NOM }\end{array}$

b.

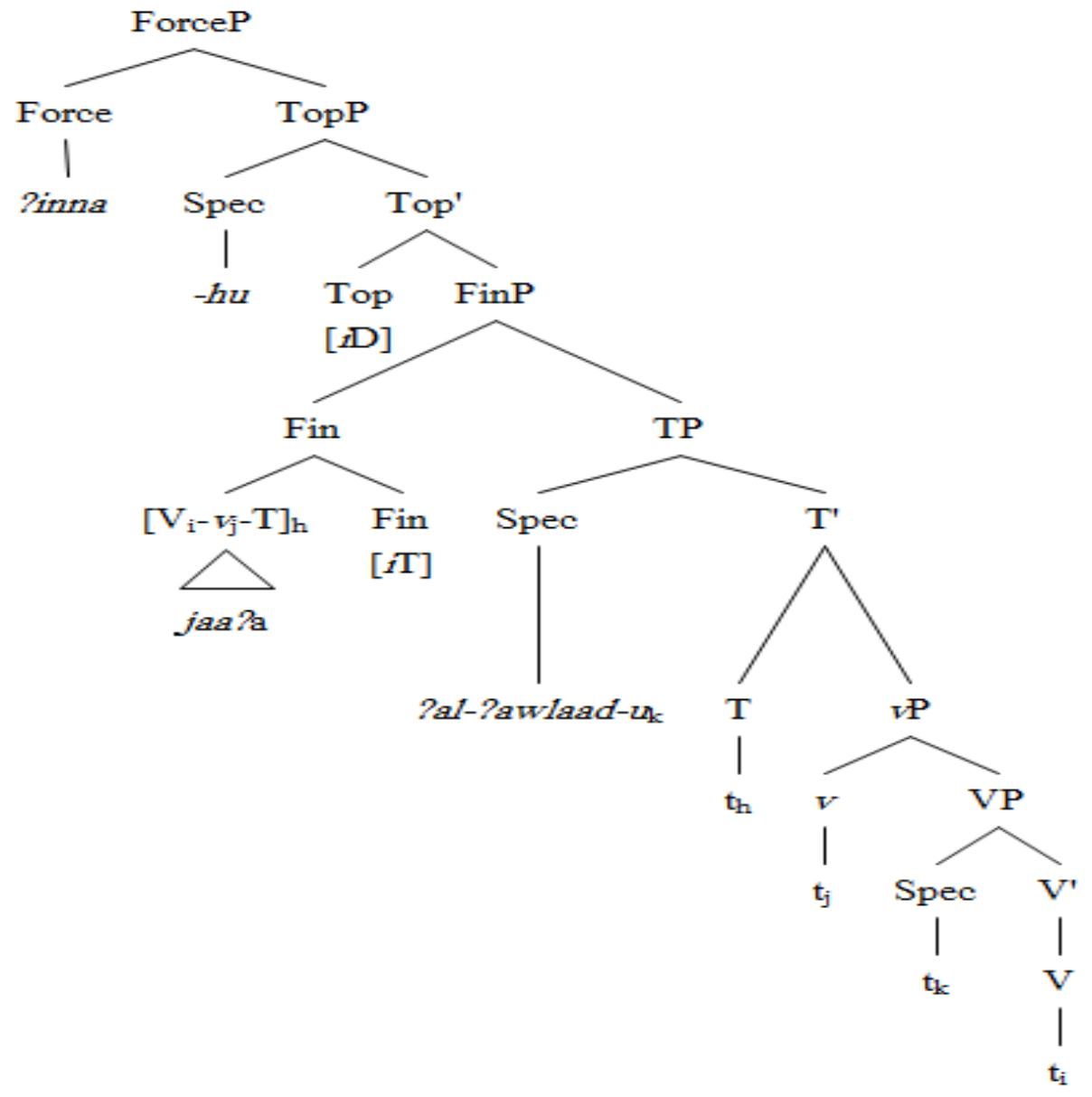

In structure (108b), the verb jaa?a 'came' raises to Fin past the subject DP ?al-

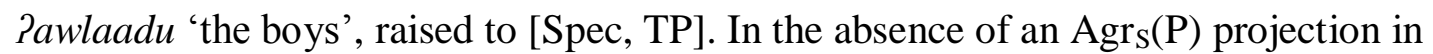
the 'basic' VSO phenomenology, the $[u \mathrm{D}]$ feature is not generated. Thus, the 
satisfaction of the EPP at the IP level in such structural configurations is dissociated from the valuation of $\mathrm{a}[u \mathrm{D}]$ feature. Neither is the $[u \mathrm{D}]$-feature valuation triggered at the level where the verbal complex $\mathrm{V}-v$-T is raised to the Fin node due to the absence of the right Spec-head agreement relation under which $[u \mathrm{D}]$-feature valuation and EPP satisfaction obtain.

As discussed in the previous chapters, SA allows the alternative 'basic' SVO word ordering (in conformity with Greenberg's 1963 Universal 6). In the derivation of SVO sentences in SA, an $\mathrm{Agr}_{\mathrm{S}}$ node projects to allow a referential pro to be merged in $\left[\mathrm{Spec}, \operatorname{Agr}_{S} \mathrm{P}\right]$ as a resumptive strategy for the identification of the Topic DP in [Spec, TopP]. On this account, the SVO sentence in (109a) below has the representation in (109b):

(109) a. Pinna Pal-Pawlaad-a pro jaa?-uu

$$
\text { that the-boys-ACC PERF.come-3MP }
$$

b.

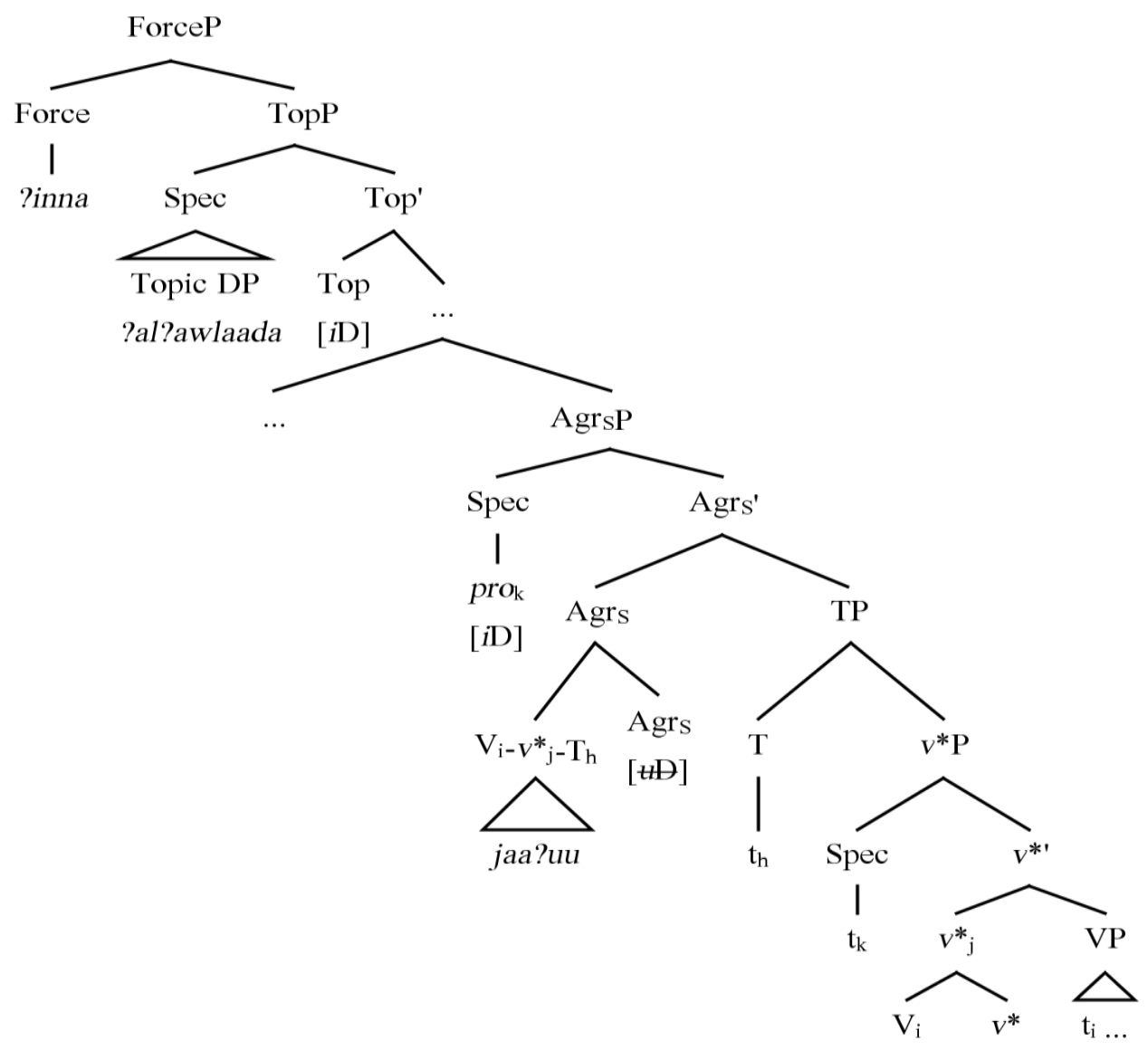


In (109b), the T-to-Agr raising process is triggered by a $[u \mathrm{D}]$-feature on $\mathrm{Agr}_{\mathrm{S}}$. As T raises to $\mathrm{Agr}_{S}$, the $[u \mathrm{D}]$-feature on $\mathrm{Agr}_{S}$ gets valued in the process of valuing the EPP and $\varphi$-features of $\mathrm{T}$ in relation to the resumptive pro raised out of $\nu \mathrm{P} / \mathrm{VP}$ to [Spec, $\left.\operatorname{Agr}_{S} \mathrm{P}\right]$.

On the basis of the three language/construction types described in relation to structures (102a,b), (105b) and (108b) above three main claims can be made:

(110) The parametric availability of $[u \mathrm{D}]$ as an 'edge' feature

A. What would account for the V2 phenomenon is an uninterpretable 'edge' feature $[u \mathrm{D}]$ on $\mathrm{Agr}_{\mathrm{S}}$ or Fin in the relevant subject-verb agreement V2 configurations? In contrast to these V2 configurations, in subject-verb agreement configurations in the 'weak' V2 case in 'basic' SA VSO word ordering, $[u \mathrm{D}]$ on $\mathrm{Agr}_{\mathrm{S}}$ or Fin is not generated. Language type $\mathrm{C}$ is the only instance where the valuation of $[u \mathrm{D}]$ does not coincide with the identification of $[i \mathrm{~T}]$ features at the interface, hence my reinterpretation of Carnie et al.'s (1994) claim about the 'weak' V2 phenomenon in 'basic' VSO word ordering. In such a context, no $[u \mathrm{D}]$-feature is generated.

B. [iT]-features (cf. R\&R's [+Fin] feature), which are generated on Fin and passed down to T, play an important role in the 'identification' of the C$\left(\mathrm{Agr}_{S}\right)-\mathrm{T}$ dependency along with the valuation of the EPP. The 'identification' of $[i \mathrm{~T}]$-features is largely a question of head-head agreement relations, which may coincide with the valuation of $[u \mathrm{D}]$ and EPP features.

C. In structural contexts where a Top node projects above FinP, the identification process involves an $[i \mathrm{D}]$-feature on Top. The identification of such a feature on Top does not involve EPP-feature valuation. Similar considerations arise in the context of relative clause formation.

As the tree representations in (102a,b), (105b) and (108b) show, an important property of the parameterized feature-based system I am adopting in terms of a language-specific/construction-specific $[u \mathrm{D}]$-feature is that when the $[u \mathrm{D}]$-feature is generated on $\mathrm{Agr}_{S}$ or Fin, it coincides with the valuation of the EPP-feature. This is the case of the language/construction type A (i.e., structures (102a) and (102b)) and type B (i.e., structure (105b)). It is in the context of such a combination of features on functional nodes that V2 configurations arise. When $[u \mathrm{D}]$ is not generated, as in 
language type C (structure (108b)), the EPP feature does not coincide with the process of T-to-Fin (nor with the process of T-to-Agr , for the absence of $\mathrm{Agr}_{\mathrm{S}}$ ) giving rise to a 'weak' V2 configuration.

Within the feature-based system introduced above, the projection of $\operatorname{Agr}_{\mathrm{S}}$ regulates the subject-verb agreement configurations that arise as the inflectional field is merged in clause structure. The projection of $\mathrm{Agr}_{\mathrm{S}}$ in the higher functional field in the IP domain makes the status of the $\mathrm{Agr}_{\mathrm{S}}$ node look similar to the functional nodes that project within the left periphery of clause structure. The only difference is that the $\operatorname{Agr}_{S}$ node is intrinsically linked to $\varphi$-feature specification and EPP valuation in the languages/structures where 'richer' instances of subject-verb agreement arise at the level of Spell Out. The same is true of Fin in V2 matrix declarative structures in Germanic where a lexical subject or an overt EXPL has to be merged in [Spec, FinP] in satisfaction of the EPP at Spell-Out.

\subsubsection{Feature inheritance (Chomsky 2008) as $\varphi$-feature sharing}

\subsubsection{Introduction}

In this section, I extend on claims of the presence of an 'edge' feature (EF) in C that interacts with the $\varphi$-features (Agree features) that $\mathrm{C}$ passes down to $\mathrm{T}$ (Chomsky 2008: 149-150). ${ }^{60}$ Thus, in sections 4.3.2.2 and 4.3.2.3, I attempt to show how the assumption of the interdependency of $[i \mathrm{~T}]$-features and $[u \mathrm{D}]$-features provides an alternative account for the derivation of some peculiar subject-verb agreement configurations in the variety of English studied by Kimball \& Aissen (1971) and by Kayne (1989b, 2000) and in West Flemish (Haegeman \& Koppen 2012).

In this context, I propose the working hypothesis that languages parametrically allow a particular feature (in the case at hand, the $[u \mathrm{D}]$-feature) to be generated in relation to particular structural configurations arising out of feature dependency relations between $\mathrm{C}$ and $\mathrm{T}$ conceived of as feature sharing.

\footnotetext{
${ }^{60}$ In Chomsky $(2001,2004)$, the 'edge' feature was conceived of as an EPP feature that the Phase heads $\mathrm{C}$ and $v^{*}$ could acquire in languages that allow scrambling such as German and Dutch.
} 


\subsubsection{Kayne's (1989b, 2000) AGR-raising analysis revisited: A feature sharing alternative}

In section 3.1.1.1, I relied on the account provided by Kayne (1989b, 2000) for the subject-verb agreement configurations in a variety of English (previously studied by Kimball \& Aissen 1971) to support my claim of the legitimacy of linking cases of parametric variation in the derivation of subject-verb agreement configurations crosslinguistically to the availability or non-availability of some $\operatorname{Agr}(\mathrm{s})$ node in sentence structure. The relevant English sentences are repeated in (111a,b) below:

(111) a. Which girls do the boy think should be invited? (Kayne's 2000 (18): 193)

b. *Which girl does the boys think should be invited? (Kayne's 2000 (19): 193) According to Kayne's (1989b, 2000) analysis, there is an agreement element in C raised from within the IP domain in (111a), but not in (111b). On the basis of this assumption about the availability of an agreement element in C, Kayne (1989b, 2000) was able to account for the kind of subject-verb agreement observed in $\mathrm{CP}$ between the wh-phrase Which girls and the auxiliary do in (112a) where whatever features are present on $\mathrm{C}$ allow this kind of subject-verb agreement.

However, the contrast between (111a) and (111b) may be reformulated in terms of the availability of a $[u \mathrm{D}]$ feature in sentence structure relying on a $\varphi$-feature sharing conception of Chomsky's (2008) feature-inheritance mechanism. Let us provide a feature structure to the sentences in $(111 \mathrm{a}, \mathrm{b})$, as in $(112 \mathrm{a}, \mathrm{b})$ below:

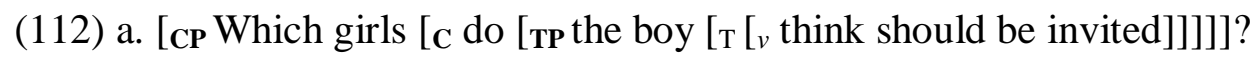

$[u \mathrm{D}]$

$[i \mathrm{~T}] \quad[i \mathrm{~T}]$

b. ${ }^{*}\left[\mathbf{C P}\right.$ Which girl [C does [Tт the boys ${ }_{\mathrm{T}}\left[{ }_{v}\right.$ think should be invited] $\left.\left.\left.]\right]\right]\right]$ ? $[u \mathrm{D}]$

$[i \mathrm{~T}]$

$[i \mathrm{~T}]$

In (112a,b), I suggest a (partial) feature structure in terms of the core assumption I am proposing in relation to the parametric availability of a $[u \mathrm{D}]$ feature on some functional head as an 'edge' feature. The generation of the $[u \mathrm{D}]$ feature on the relevant functional node $(\mathrm{C}$ in $(112 \mathrm{a}, \mathrm{b}))$ in conjunction with $[i \mathrm{~T}]$ gives rise to $\varphi$ agreement at Spell-Out. Nevertheless, satisfaction of the EPP only applies at the IP level within the C-T dependency in English. The identification of the C-T dependency 
is also established in terms of tense interpretation at the interface (i.e., $[i T]$ has to be 'identified' on both $\mathrm{T}$ and $\mathrm{C}$ as the structure is transferred to PF and LF for interpretation).

According to a $\varphi$-feature sharing conception of Chomsky's (2008) featureinheritance mechanism, the complex of $\varphi$-feature(s) (namely, number in English) that is generated in $\mathrm{C}$ has to be shared with $\mathrm{T}$ in the process of the identification of the $\mathrm{C}-\mathrm{T}$ dependency at Spell Out after the 'edge' feature $[u \mathrm{D}]$ and the tense feature $[i T]$ combine and establish a $\varphi$-agreement relation between which girl in [Spec, $\mathrm{CP}]$ and does in C. In $(112 \mathrm{a}, \mathrm{b})$, the $\varphi$-agreement relation at the level of CP in terms of [ $u \mathrm{D}]$ and $[i \mathrm{~T}]$ is established between the wh-phrase in $[\mathrm{Spec}, \mathrm{CP}]$ and the auxiliary in $\mathrm{C}$. This $\varphi$-feature sharing mechanism yields the feature structure in $(113 a, b)$ below:

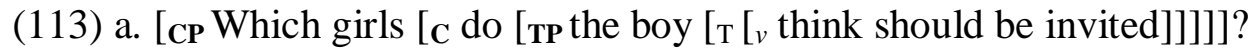

$$
\begin{aligned}
& {[u \mathrm{D}]} \\
& {[i \mathrm{~T}]} \\
& {[+\mathrm{P}] \leftrightarrow[+\mathrm{P}]=[+\mathrm{P}]}
\end{aligned}
$$

b. ${ }^{*}\left[\mathbf{C P}\right.$ Which girl [c does [тр the boys $\left[{ }_{\mathrm{T}}\left[{ }_{v}\right.\right.$ think should be invited $\left.\left.\left.]\right]\right]\right]$ ?

$$
\begin{aligned}
& {[u \mathrm{D}]} \\
& {[i \mathrm{~T}] \quad[i \mathrm{~T}](\mathrm{EPP})} \\
& {[-\mathrm{P}] \quad \leftrightarrow \quad[-\mathrm{P}] \neq[+\mathrm{P}]}
\end{aligned}
$$

In (113a), the $\varphi$-feature (i.e., $[+\mathrm{P}]$ for the number feature [+Plural]) that is shared between $\mathrm{C}$ and $\mathrm{T}$ establishes a Spec-head agreement relationship that is realized as 'Plural' at the 'edge' level within CP. Since T has to 'Agree' in $\varphi$-features with the head $\mathrm{C}$ that selects it in sentence structure, the Spec-head-/head-head feature-sharing mechanism applies and C 'passes down' agreement in number (i.e., [+P]) to $\mathrm{T}$ in the IP domain. [iT] features also match.

In contrast, in (113b), a mismatch in $\varphi$-agreement arises within the IP domain as the structure is handed over to the interface for interpretation. The $\varphi$-feature ([-P] for the number feature [-Plural]) that is shared between $\mathrm{C}$ and T establishes a Spechead agreement relationship, at Spell Out, that is realized as [-P] at the 'edge' level within CP. Given the C-T dependency, T 'shares' the same $\varphi$-features with $\mathrm{C}$ (i.e., [-P]) as they are 'passed down' from the 'edge' head C to T. Similarly, both instances of $[i \mathrm{~T}]$ features (i.e., $[i \mathrm{~T}]$ in $\mathrm{C}$ and $[i \mathrm{~T}]$ in $\mathrm{T}$ ) have to match as they combine with $\varphi$ agreement in their respective domains. However, as the EPP is ultimately satisfied 
after subject-verb agreement is established within the IP domain, a $\varphi$-agreement realization on the verbal inflectional complex different from the $\varphi$-agreement realization established in $\mathrm{CP}$ arises. As can be seen in the feature structure provided in (113b), $\varphi$-agreement within IP is realized as $[+\mathrm{P}]$ (irrespective of the $\left(3^{\text {rd }}\right.$ person) plural DP boys); hence, the mismatch in $\varphi$-agreement between $\mathrm{T}$ and $\mathrm{C}$ at Spell Out. ${ }^{61}$

What the analysis in terms of an additional 'edge' $[u \mathrm{D}]$ feature suggests is that the association of $[u \mathrm{D}]$ and $[i \mathrm{~T}]$ in the $\mathrm{CP}$ domain, in such sentences as in $(112 \mathrm{a}, \mathrm{b}) / 113 \mathrm{a}, \mathrm{b})$ in the relevant English dialect, gives rise to a $\varphi$-agreement configuration established in terms of the Spec-head/head-head agreement relation. However, this agreement configuration between $[u \mathrm{D}]$ and $[i \mathrm{~T}]$ in $\mathrm{CP}$ happens to be dissociated from the domain of EPP feature valuation, which, in English, has to be satisfied within IP as the effect of subject-verb agreement. The result is a mismatch in the realization of $\varphi$-agreement within the two domains CP and IP in this English dialect in (112b)/113b) vs. (112a)/113a).

The idea of a basic dissociation between the Spec-head agreement relation established in $\mathrm{CP}$ in terms of an additional $[u \mathrm{D}]$ feature in $\mathrm{C}$ and the domain of EPP satisfaction within the IP in such a variety of English in the examples in (112)/(113) can be better understood in light of relative clause constructions where the same agreement configuration witnessed in (112)/(113) also obtains. Nevertheless, in the relative clause construction, as Kimball \& Aissen (1971: 244) note "agreement may occur between the main verb of the clause and the head of the relative clause as well as between the verb and the subject of the clause". The optionality of agreement in such a case is exemplified in (114) below (from Kimball \& Aissen 1971: 241-242):

(114) a. Mark knows the people who Clark thinks are in the garden

b. Mark knows the people who Clark think are in the garden

\footnotetext{
${ }^{61}$ I represent 'do/does', in (112a,b) and (113a,b), as being merged in $\mathrm{C}$ and not in some lower position below C (as Kayne 1989b/2000 assumes) in such a residual V2 configuration in this variety of English. Judging from the fact that in the grammatical (112a)/(113a) both the verb and the auxiliary are 'plural' (irrespective of the singular DP boy), it might be the case that it is by virtue of each instance of intrinsic $[i \mathrm{~T}]$ features, i.e., $[i \mathrm{~T}]$ on the auxiliary, as a functional element associated with 'tense', and $[i \mathrm{~T}]$ on the $\mathrm{C}$-node, that $\varphi$-agreement in CP in this variety of English is determined as $[+\mathrm{P}]$ in $(112 \mathrm{a}) /(113 \mathrm{a})$, and as $[-\mathrm{P}]$ in $(112 \mathrm{~b}) /(113 \mathrm{~b})$. Both instances of $[i \mathrm{~T}]$ combine with $[u \mathrm{D}]$ on $\mathrm{C}$ as an 'edge' feature and they conjointly determine $\varphi$-agreement at Spell Out. However, only in the derivation of $(112 b) / 113 b)$ does $\varphi$-agreement lead to a mismatch, as discussed above. In this case, whether the subject DP in [Spec, TP] is singular or plural would thus not count in the computation of the $\mathrm{C}$ - $\mathrm{T} \varphi$-agreement dependency at Spell Out. Ultimately, at Spell Out, the satisfaction of the EPP would follow from $\varphi$-agreement determined by the $\mathrm{C}-\mathrm{T}$ dependency in the derivation of sentence structure.
} 
The question that poses itself in relation to (114) is the following: Where does the optionality of agreement in the derivation of the relative clause in (114) come from? $?^{62}$ My assumption is that the optionality of agreement in such a configuration arises out of the establishment of two domains of 'predication' within the structure of relative clauses. The first is the domain of 'syntactic predication' within the IP. The other is the domain of 'higher predication' that obtains between the relative clause and the $\mathrm{D}(\mathrm{P})$ element it is predicated of. No such 'higher predication' exists in the derivation of interrogative and declarative clauses, as already discussed in relation to Table 4.2 in section 4.2.2 above.

The same reasoning applies to the Topic-comment structures in (115) below (Kimball \& Aissen 1971: 244):

(115) a. These houses my uncle buys and sells

b. These houses my uncle buy and sell

In (115), the DP of which the tensed IP is predicated sets its own structural conditions for the licensing of subject-verb agreement within IP irrespective of the topicalized object DP these houses.

However, to account for why there is optional subject-verb agreement in the derivation of the sentences in (114) and (115) as either linking the main verb and the DP/NP in the left periphery of the clause or linking the main verb and the 'subject of predication' within IP, it might be the case that since two domains of syntactic predication seem to interact within the derivation of these structural configurations, the verb is 'free' to pick either one. ${ }^{63}$

\footnotetext{
${ }^{62}$ The same is true of Topic-comment structures, as will be dealt with below.

${ }^{63}$ The possibility of the agreement relationship between the DP (the) people (who) and the main verb in (114), and between the DP the houses and the main verb in (115) might be a consequence of the clausal scope relationship that links between the external D-head the, which selects the $\mathrm{CP}$ wh-relative clause, and the locus of agreement within IP (i.e. the T-node) in (114), and between the Top head of the Topiccomment structure and the T-node in (115). In (114), it is by virtue of [ $i \mathrm{D}]$ on $\mathrm{D}$ (and presumably, as well, by virtue of an $[i \mathrm{~T}]$ feature allowing the D-head to select the $\mathrm{CP}$ ) that the scope relationship is established at LF. In (115), the scope relation is established in terms of the intrinsic interpretable Dfeature of the Top head (i.e., [iD] on Top) in addition to an $[i \mathrm{~T}]$ feature that links the Top head to both Fin and T. In contrast to the scope relationship that links $[i \mathrm{D}]$ and $[i \mathrm{~T}]$ at the higher part of the split $\mathrm{CP}$ domain in (114) and (115) to $\mathrm{T}$ and the verb, no clausal scope relationship links between the subject of predication in [Spec, IP/TP] and the verb. In this case, the relationship between the subject DP and the verb is strictly predicational, but this relationship ultimately also follows from the EPP requirement as a requirement that is tightly linked to the valuation of uninterpretable $\varphi$-features via the specific agreement pattern of a head (i.e., the head of IP) and its Spec. As already discussed in the previous chapters, no EPP requirement ever arises in the derivation of Topic-comment structures at the level
} 
In light of this account, it might be possible now to give a more plausible account of the grammaticality contrast between $(112 b) /(113 b)$ and (112a)/(113a) above. As discussed above, the possibility of generating an additional $[u \mathrm{D}]$ feature on $\mathrm{C}$ in such wh-constructions in the relevant variety of English, as a parametric option, follows from the assumption that there are $\varphi$-/Agree features that are universally generated on C to start with (as proposed by Chomsky 2001, 2004, 2008). Within the present framework of assumptions that parameterizes the probe-goal Agree system in terms of the possibility of generating an additional $[u \mathrm{D}]$ feature on Phase heads at the 'edge', the 'edge' $[u \mathrm{D}]$ feature is responsible for establishing a special Spechead/head-head agreement configuration at the level of $\mathrm{CP}$ or $\operatorname{Agr}_{\mathrm{S}} \mathrm{P}$, according to the language or the particular structure being derived within the language in question. ${ }^{64}$

Thus, under the Phase-theoretic assumption that Agree-features (i.e., number features in the present context), within the $\varphi$-set of functional nodes, start out in $\mathrm{C}$ and are 'passed down' to $\mathrm{T}$, the existence of a parametrically available $[u \mathrm{D}]$ feature in $\mathrm{C}$ determines an agreement configuration in terms of which the $\mathrm{C}-\left(\mathrm{Agr}_{\mathrm{S}}\right)-\mathrm{T}$ dependency is established at Spell Out. It follows that, at the level where $\varphi$-agreement is realized and the EPP satisfied in clause structure within IP in English, whatever agreement specification has been established via the special Spec-head/head-head agreement configuration within $\mathrm{CP}$, in the variety of English in question, will have to be preserved as the structure is handed over to the interface for interpretation. As discussed above in relation to the grammaticality contrast between (112a/113a) and $(112 b / 113 b)$, any mismatch in agreement features that arises at the level where the EPP is satisfied in English (i.e., within IP), inevitably leads to a deviant structure that does not converge at the interface.

In the following section, I discuss Haegeman \& Koppen's (2012) assumptions about $\varphi$-agreement within the clause structure of Dutch/West Flemish in relation to the phenomenon of Complementizer Agreement (CA) in such a language variety.

where the Topic DP is merged in the left periphery. In the derivation of these structures, no actual valuation of features is under way (cf. R\&R).

${ }^{64}$ As discussed in section 3.2.1.2, Roberts (2010b) and Biberauer \& Roberts (2010) account for parametric variation within closely-related Romance and/or null-subject systems in terms of an additional D-feature as an intrinsic property of the T-node. In Roberts' (2010b) and Biberauer \& Roberts' (2010) account of the 'richer' cases of subject-verb agreement witnessed within some nullsubject systems, it is the additional D-feature on T, as an uninterpretable feature, which establishes the special agreement environment at the level of IP. In the probe-goal-Agree system I am adopting, the parametrically available additional $[u \mathrm{D}]$ feature is a property of 'Phase' heads, i.e., Agr $_{S}$ (when it projects) or C. 


\subsubsection{Complementizer Agreement in Dutch External Possessor Agreement configurations}

In the following discussion, I suggest (contra Haegeman \& Koppen 2012) that 'feature inheritance' as $\varphi$-feature sharing is relevant to the derivation of non-V2 CA configurations in embedded clauses in Dutch /West Flemish. Thus, as proposed by Chomsky (2008), $\varphi$-features start out in C, but they are passed down to $\mathrm{T}$ in the process of the derivation of the $\mathrm{C}-\left(\mathrm{Agr}_{\mathrm{S}}\right)-\mathrm{T}$ dependency in clause structure. As for the other features involved in the C-(Agr $)$-T dependency - namely, the EPP feature along with the parametrically available $[u \mathrm{D}]$ feature, and $[i \mathrm{~T}]$ features - , my proposal is that these features are not subject to 'inheritance'. As discussed above, the EPP feature is an exclusive property of the T-node. The $[u \mathrm{D}]$ feature is a feature inherent to separate functional head different from $\mathrm{T}$. [iT] features obtain on both Fin and $\mathrm{T}$ as these two nodes form the basis of the probe-goal-Agree dependency relationships linking the IP domain to the $\mathrm{CP}$ domain.

Haegeman \& Koppen (2012: 448-451) discuss External Possessor Agreement (EPA) configurations as a special case of CA in West Flemish where a mismatch arises between the CA agreement configuration established in the CP domain and the subject-verb agreement configuration established in the IP domain. According to Haegeman \& Koppen (2012: 448-451), EPA does not show the usual characteristics of CA in Dutch in that the $\varphi$-agreement configuration that is established in CP is different from the $\varphi$-agreement configuration that arises in IP in contravention of Chomsky's feature- inheritance theory based on the premise of an essential C-T $\varphi$ agreement dependency.

A representative example of EPA is the case where the subject possessor DP and the subject possessee DP are separated by a focused adjunct element (i.e., toen juste 'just then' shown in bold in (116a)) signalling the projection of a FocP in between the two subject DPs, as represented in (116b) for the EPA case in (104a): ${ }^{65}$

\footnotetext{
${ }^{65}$ Haegeman \& Koppen (2012: 450) do not represent the FinP projection, but I assume that FinP should be represented to account for, first, the essential link between IP and the CP (= FinP) in terms of finiteness in that it is by virtue of intrinsic $[i \mathrm{~T}]$ features that the selectional relation between Fin and $\mathrm{T}$ can be represented in finite clauses. Second, the projection of FinP will also account for the two separate feature inheritance processes that such cases of EPA allow (Haegeman \& Koppen 2012: 450; see the discussion of such EPA instances below). In the alternative analysis I am seeking in terms of $[u \mathrm{D}]$ features, [Spec, AlphaP] might be [Spec, TopP] - an A'-position. The valuation of the $[u \mathrm{D}]$ feature at the higher $\mathrm{C}$ position Force is conceived of as a Subject Criterion established on the basis of a head-head Agree relation between a head and another head (cf. Rizzi \& Shlonsky 2007: 139). Thus,
} 
(116) a. ... omda-n/*omdat André en Valère toen juste underen computer kapot was because-P/because A. and V. then just their computer broken was '... because André and Valère's computer broke just then'

(West Flemish, adapted from Haegeman \& Koppen 2012: 449-450)

b.

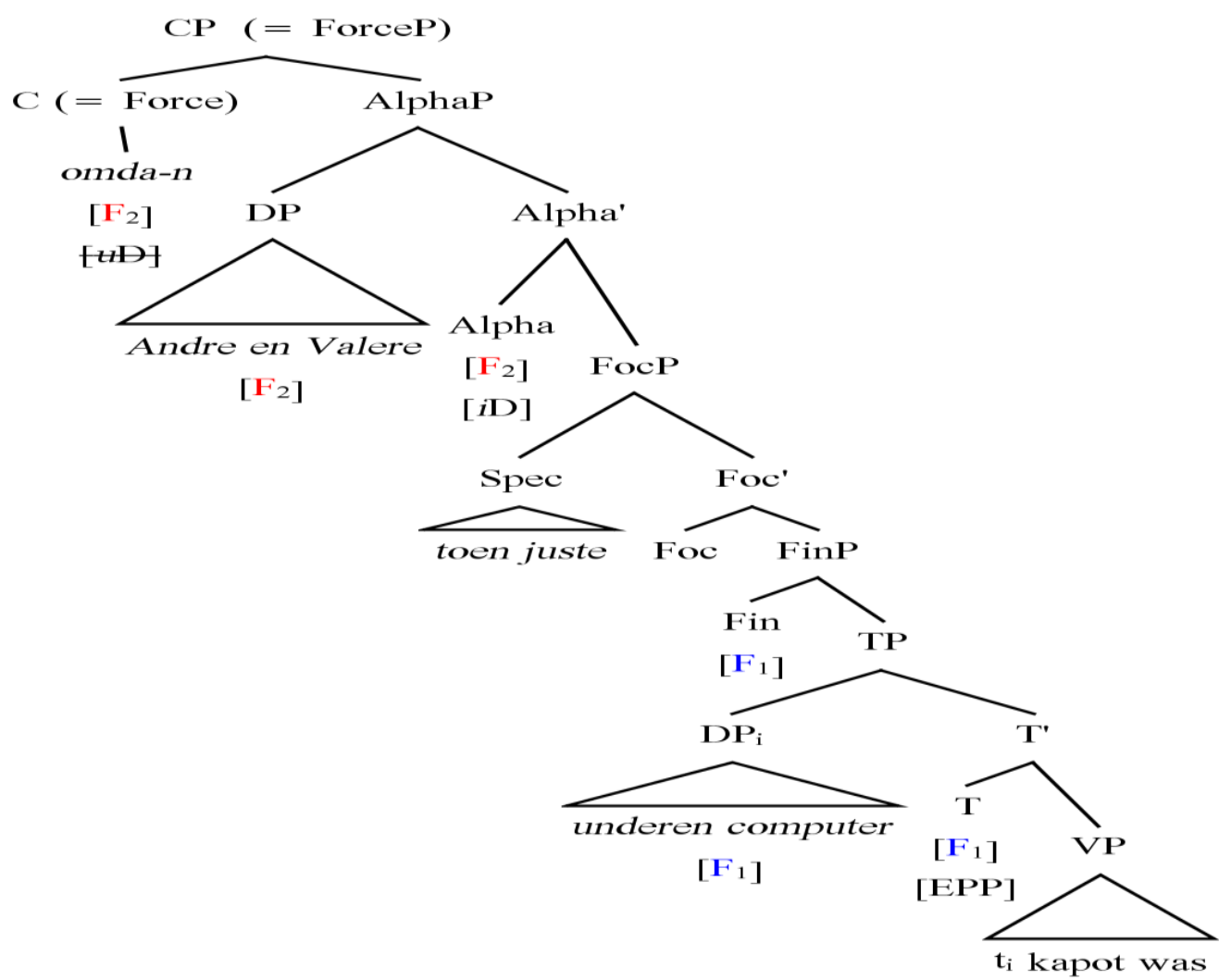

As the tree representation (116b) shows, the $\varphi$-agreement configuration that is established in the higher part of CP (i.e., Force and its maximal projection ForceP) is different from the $\varphi$-agreement configuration that arises in the lower part of CP (i.e., Fin) in relation to IP since the domain of $\varphi$-agreement at the level of ForceP between the features encoded on the complementizer omdan 'because' in Force and the plural possessor DP André en Valère is dissociated from the $\varphi$-agreement relation that links the features encoded on Fin and the singular possessee DP underen computer 'their computer' in IP.

Force and Top engage in an extra subject-related Agree relation over and above the subject-verb agreement relation in the IP domain for EPP satisfaction. The extra subject-related Agree relation between Force and Top is established in terms of an additional $[u \mathrm{D}]$ feature on Force, not related to the EPP (contra Haegeman \& Koppen's 2012: 450 claim of an additional EPP feature on Alpha valued by the subject element in [Spec, Alpha] - an A-position according to Haegeman \& Koppen 2012: 450). 
In the probe-goal-Agree system I am assuming here, making the assumption that an additional 'edge' $[u \mathrm{D}]$ feature is generated on Force in an EPA case like (104) accounts for the $\varphi$-agreement relationship that is established between Force, Alpha and the possessor DP in [Spec, AlphaP] as being dissociated from the $\varphi$-agreement relationship that is established between Fin, T and the singular possessee DP in [Spec, TP]. Thus, in (104), feature-sharing establishes a head-head agreement relationship in $\varphi$-features (i.e., $\left[\mathrm{F}_{2}\right]$ in (116)) applying between $\mathrm{C}(=$ Force) and Alpha whereby $[u \mathrm{D}]$ on Force is valued. This head-head agreement relationship, in turn, triggers Spec-head agreement between Alpha and [Spec, AlphaP]. The plural possessor DP André en Valère now shares the $\left[F_{2}\right] \varphi$-feature with Force and Alpha. This head-head/Spechead agreement relation at the highest level of the split-CP domain sets this $\varphi$ agreement configuration apart from the other $\varphi$-agreement configuration established in the lower part of the split-CP domain between Fin and IP, as Fin agrees with I/T and with the specifier position of its complement IP. In this case, the head-head/Spechead agreement relation between Fin, I/T and the singular possessee DP underen computer 'their computer' in [Spec, IP/TP] is established in terms of the featuresharing of the $\left[\mathrm{F}_{1}\right] \varphi$-feature. The possessee DP underen computer satisfies the EPP in [Spec, IP/TP], as well.

As Haegeman \& Koppen (2012: 449) note, "a focused temporal adjunct ... is a crucial ingredient in licensing EPA: without it EPA is not possible", as the ungrammatical EPA case in (117) shows:

(117) ...omdat/*omda-n André en Valère underen computer kapot was because/because-PL André and Valère their computer broken was '...because André and Valère's computer broke'

(West Flemish, Haegeman \& Koppen 2012: 449)

(117) is ungrammatical if, in the absence of a focused temporal adjunct, $\varphi$-agreement on $\mathrm{C}$ (= Fin) does not match $\varphi$-agreement established in IP in conjunction with EPP satisfaction in [Spec, TP].

As Haegeman \& Koppen (2012: 449) observe, only one subject position is available in a sentence like (117) - namely, [Spec, TP]. This suggestion conforms to the analysis I am adopting whereby the $\varphi$-agreement relationship that is established at Spell Out, at the level where the C-T dependency is interpreted, is determined both within CP and IP/TP as feature inheritance/feature sharing applies. Thus, in (117), the 
EPP is satisfied within [Spec, TP] where the subject André en Valère underen computer 'André en Valère's computer' is finally merged. On this account, the $\varphi$ agreement that is manifested on the lexical complementizer omdat has to be the same as the $\varphi$-agreement that is manifested within IP between the subject of predication and the verb on the assumption that the subject-verb $\varphi$-agreement configuration that shows up in IP is actually a reflection of the essential $\varphi$-feature dependency that binds $\mathrm{T}$ to $\mathrm{C}$ (= Fin).

Under such an account of EPA, it might be the case that two sets of $\varphi$-features are actually generated in $\mathrm{CP}$ in such constructions in West Flemish: one set of $\varphi$ features is generated on the higher C-node (= Force in Rizzi’s 1997 split-CP system) and another set of $\varphi$-features is generated on Fin in the lower part of the CP domain selecting TP as complement in sentence structure. In this case, the C-T $\varphi$-feature dependency still holds between Fin and $\mathrm{T}$ (each of which is also generated, as an intrinsic property, with the essential $[i \mathrm{~T}]$ feature for tense interpretation at Spell Out at the C-T Phase, and for EPP satisfaction within IP). This alternative account of the EPA configuration is in line with the assumption Haegeman \& Koppen (2012: 451) entertain that an additional (Case) feature is introduced in the structure where EPA occurs, which determines the special $\varphi$-agreement configuration. In the account I am proposing in terms of an additional 'edge' $[u \mathrm{D}]$ feature, it is this 'edge' feature that, presumably, triggers the special agreement in the higher part of the $\mathrm{CP}$ where the possessor DP is merged, and by that token determines EPA. Thus, EPA arises out of a 'matching' relationship, parametrically available in the Dutch dialect under consideration, between an 'edge' $[u \mathrm{D}]$ feature in the higher part of the split functional $\mathrm{CP}$ domain of the language and a lower $[i \mathrm{D}]$ feature base-generated on the Alpha head Haegeman \& Koppen (2012: 449-450) are postulating. ${ }^{66}$

As Haegeman \& Koppen (2012: 451, footnote 12) note, the EPA configuration is different from other cases of $\mathrm{CA}$ in Dutch where "both $\mathrm{C}^{0}$ and $\mathrm{T}^{0}$ have uninterpretable $\varphi$-features and probe for the subject" in line with Chomsky's featureinheritance framework. In the alternative account I am proposing, what distinguishes EPA is the parametrically available $[u \mathrm{D}]$ feature, which, as an 'edge' feature, is generated higher in the derived structure. In such an agreement configuration, as

\footnotetext{
${ }^{66}$ Under such an alternative, the 'extra' EPP feature, which Haegeman \& Koppen (2012: 450, see their tree structure in (16)) postulate on Alpha, would rather be the intrinsic interpretable [iD] feature that, in my account, is generated on Top (Top being essentially dissociated from the EPP, see also note 63 above).
} 
suggested by Haegeman \& Koppen (2012: 451, footnote 12), the mechanism of feature inheritance would operate according to a 'multiple phase' feature inheritance theory. In the alternative account I am proposing, two separate $\varphi$-agreement domains are established: The first $\varphi$-agreement domain is established between the higher $\mathrm{C}$ node (i.e., Force) and Haegeman \& Koppen's (2012) head node Alpha. The second $\varphi$ agreement domain is established between a lower $\mathrm{C}$ node (i.e., Fin, in the split-CP system I am adopting) and the T-node. Each $\varphi$-agreement domain would set its own licensing conditions as the structure is handed over to the interface for interpretability at Spell Out. This account fits well with the assumption that the derivation of such language-specific CA configurations actually goes through 'multiple phases' or two separate $\varphi$-agreement domains, as discussed above.

This account confirms the assumption of the present analysis that there is a kind of feature-sharing mechanism that applies between $\mathrm{C}$ (i.e., Fin or both Force and Fin as in EPA in West Flemish,) and the head (and the specifier of that head) that C selects in clause structure. The feature-sharing relationship between the highest head in the CP domain in EPA configurations in West Flemish is established in terms of an additional $[u \mathrm{D}]$ feature on Force that sets the domain of $\varphi$-agreement in $\mathrm{CP}$ apart from the domain of $\varphi$-agreement in IP.

In sum, these CA cases seem to favour an account where the generation of an additional $[u \mathrm{D}]$ feature at the 'edge' systematically gives rise to cases of subject-verb agreement that are 'richer' than other cases according to the specific character of these agreement configurations in the language in question.

\subsection{Conclusion}

In conclusion, the analysis of the different structural configurations in this chapter provides confirmation for the view that there are $\varphi$-/Agree features that are universally generated on $\mathrm{C}$ (i.e., Force and/or Fin, in the present framework) to start with (as proposed by Chomsky 2001, 2004, 2008). The probe-goal-Agree system adopted here has centred on the assumption of an additional 'edge' $[u \mathrm{D}]$-feature generated either on $\mathrm{Agr}_{\mathrm{S}}$ or on Fin, which is a parametric option available to typologically similar languages (or varieties of the same language).

In particular, in relation to V-initial sentences in VSO languages (e.g., SA), I have assumed that no $[u \mathrm{D}]$-feature is generated on Fin or on $\mathrm{Agr}_{S}$ for the absence of an $\operatorname{Agr}_{S}(\mathrm{P})$ projection in the derivation of such sentences. Unlike in $\mathrm{V} 2$ 
configurations with a lexical subject DP or EXPL DP in initial position in Germanic, the EPP, in SA VSO sentences, exclusively applies in the IP domain in [Spec, TP], and the T-to-Fin raising process in such sentences is not EPP-related.

More generally, the analysis of the VSO/V2 phenomenology in section 4.3 has shown that 'richer' Spec-head/head-head agreement configurations are established at the level of $\mathrm{CP}(=\mathrm{FinP})$ or $\operatorname{Agr}_{\mathrm{S}} \mathrm{P}$, according to the language or the particular structure being derived.

In all such contexts, the derivation of 'Subjects' and 'Topics' follows from the distribution of D-features: $[u \mathrm{D}]$-features (on Fin or $\mathrm{Agr}_{\mathrm{S}}$ ) vs. $[i \mathrm{D}]$-features on D of relatives or on Top.

Similarly, the language-specific $\varphi$-agreement configurations within $\mathrm{CP}$ in the residual V2 case in a variety of English and in the EPA case in West Flemish have been accounted for in terms of an additional 'edge' $[u \mathrm{D}]$-feature on the highest functional node in the $\mathrm{CP}$ domain (= Force in EPA cases). The feature-sharing interpretation of feature inheritance accounts for a possible mismatch between $\varphi$ agreement within $\mathrm{CP}$ and $\varphi$-agreement within IP in the process of feature inheritance between Force and/or Fin and the head (and the complement of that head) Force/Fin selects in sentence structure. In the variety of English, no feature mismatch arises in relatives or with Topic DPs where $[u \mathrm{D}]$ is absent. Likewise, $[u \mathrm{D}]$ is absent in West Flemish when the EPA pattern is not triggered, thus failing to establish a separate $\varphi$ agreement configuration within $\mathrm{CP}$. 


\section{CHAPTER FIVE}

\section{SUMMARY AND CONCLUSIONS}

In this dissertation, we have been concerned, in particular, with the derivation of the subject-verb agreement configurations in Arabic (the standard variety and the dialects) and, more generally, with the representation of syntactic dependency relations cross-linguistically.

The proposed feature-based analysis of parametric and micro-parametric variation shows that variation between typologically similar and even typologically different languages is minimal. This variation is limited to the interaction of functional feature combinations (namely, D-features and T-features) in the derivation of sentences.

According to a Cartographic conception of dependency relations on the basis of which subject-verb agreement configurations are established, my proposal has been that the EPP feature (or the Extended Projection Principle, as it was called at first in the early 1980s) remains a universal property of sentence structure associated with $\mathrm{T}$. In the Phase-theoretic framework (Chomsky 2001, 2004) adopted here, the EPP feature is an intrinsic property of the T node. However, the present analysis, although arguing for the position that the EPP feature and $\varphi$-features (or, according to Chomsky 2008, Agree-features) are essentially tied together, they remain different in that the EPP feature is not passed down from C (= Fin) along with Agree-features (pace Chomsky's 2008: 157 suggestion that this could possibly be the case).

In this light, the derivation of VSO and SVO sentences in the standard variety of Arabic and the dialects has been proposed to vary in terms of the Merge processes (including movement) that the elements selected for the Numeration within the course of the derivation are subject to. The presence or absence of a single categorial functional feature (i.e., the parametrically available or non-available $[u \mathrm{D}]$-feature of $\mathrm{Agr}_{S}$ ) has the effect of setting parametric differences in the probe-goal-Agree relations that drive the derivation of the sentences in question.

Thus, in relation to the VSO word ordering in Arabic (see, in particular, Chapter Two and Chapter Three for such varieties of Arabic as SA, TA and MA and 
Chapter Four for SA, basically), the structure in (1) below would be the representation of SA VSO sentences at the interface:

(1) SA VSO word order

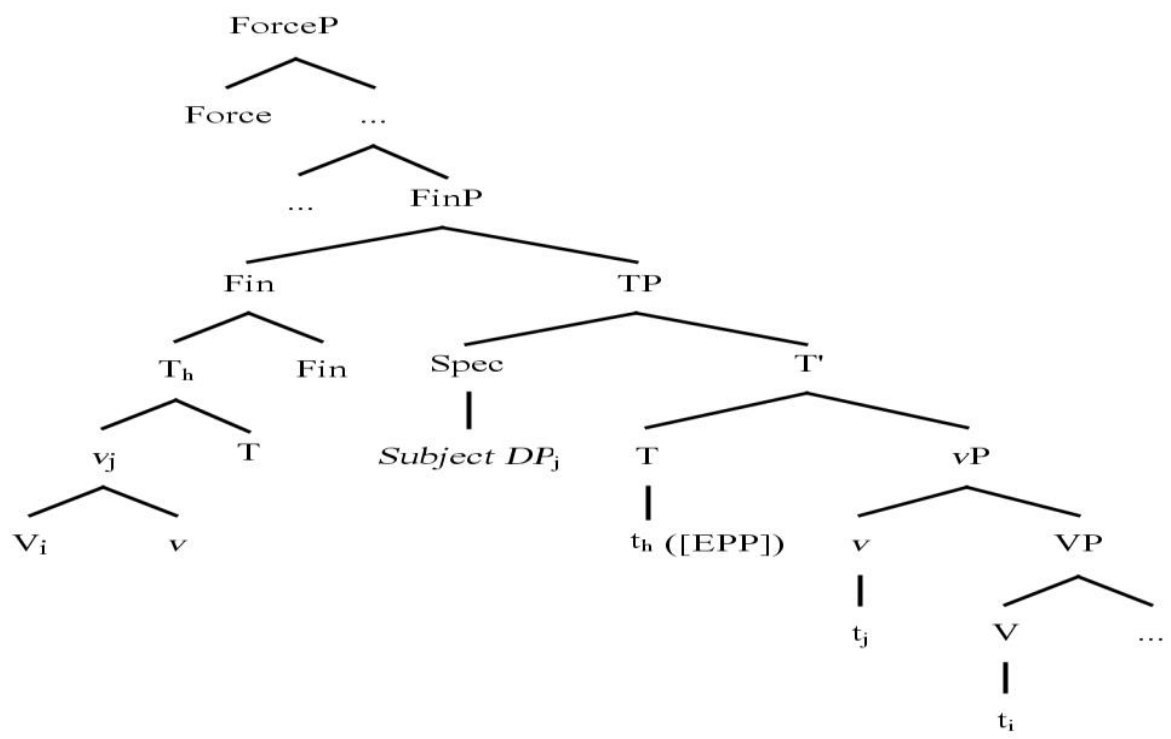

In (1), the V-v-T complex raises to Fin and the subject DP is raised to [Spec, TP] where it satisfies the EPP (mainly, extending Aoun et al.'s 2010 proposal; see section 4.1.2.1). In the analysis adopted in this thesis, the raising of the subject DP to [Spec, $\mathrm{TP}]$ is also correlated by the absence of $\mathrm{Agr}_{\mathrm{S}}$ and its Spec position (in contrast to the modern spoken dialects - for example, TA - where $\mathrm{Agr}_{S}$ necessarily projects; see (3) below).

In between FinP and ForceP, the structure of SA allows for the projection of different functional projections, not limited to Focus elements, which are merged under a ForceP projection, or to Topic elements, which are merged under a TopP projection, but also to such categories that would allow for the Merge of such functional elements as the SA modal particle qad and the NEG elements laa/lam/lan. Thus, the ellipsis in the SA structure (1) can include a NegP projection and a ModP projection. As for the NEG element maa in SA, I have followed Bahloul (2008) in the assumption that it is base-generated in the Foc head in the split-CP domain. Structures (2a) and (2b) below show the distribution of NEG elements in the split-CP domain of sentences in SA: 
(2) a.

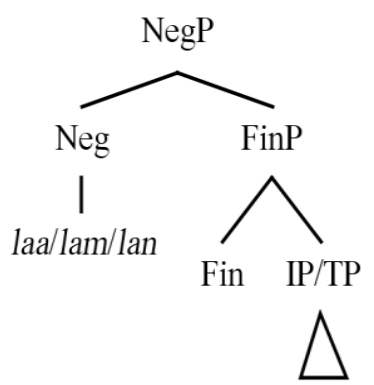

b.

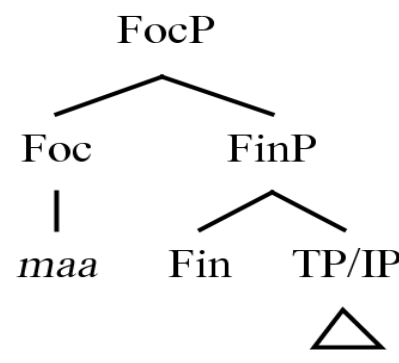

In contrast to SA, the verb, in the TA VSO word ordering, is raised to the inflectional domain (projecting as $\operatorname{Agr}_{S} \mathrm{P}$ ) and stops in the $\operatorname{Agr}_{S}$ node above the Tnode of sentence structure. The subject DP remains in situ in [Spec, $\left.\mathrm{v}^{*} \mathrm{P} / \mathrm{VP}\right]$ in a post-verbal position, and an EXPL pro occupies the [Spec, IP/Agr $\mathrm{P}]$ position, as represented in (3) below:

(3) TA VSO word order

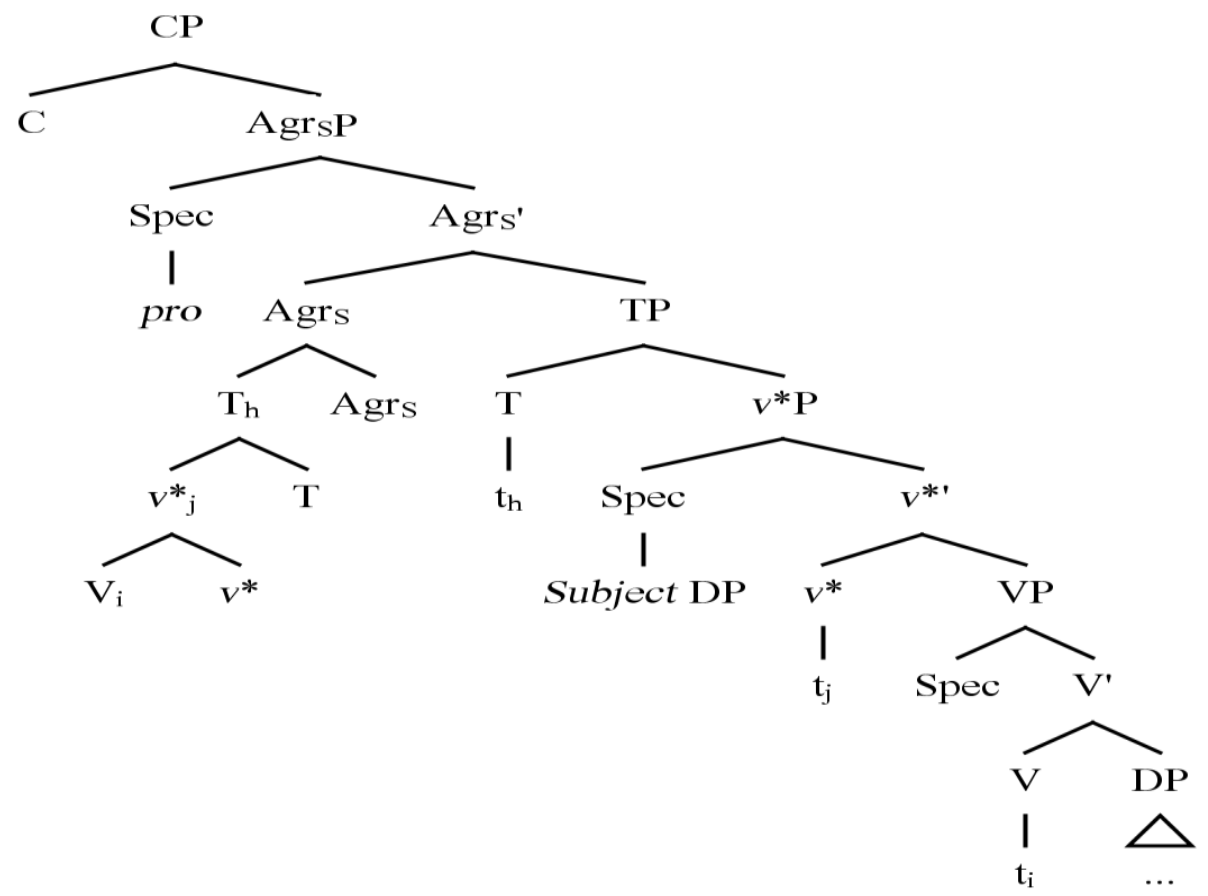


In relation to the TA VSO structure (3), my assumption has been that, whereas in SA VSO sentences expletive pro is not instantiated in the highest Spec position in the IP domain for EPP satisfaction (i.e., [Spec, TP] in (1) above), TA VSO sentences require the projection of $\operatorname{Agr}_{S} \mathrm{P}$ and its Spec position for expletive pro insertion and EPP satisfaction. As (2) shows, the verbal complex $\mathrm{V}-v$-T is raised to the $\mathrm{Agr}_{\mathrm{S}}$ node and does not raise further up.

As for Negation in TA (where only the NEG element maa is instantiated in the language), I have assumed that it is projected within the IP domain below T(P) (cf. Benmamoun 2000b for SA and the modern spoken dialects and Halila 1992 for TA): (4)

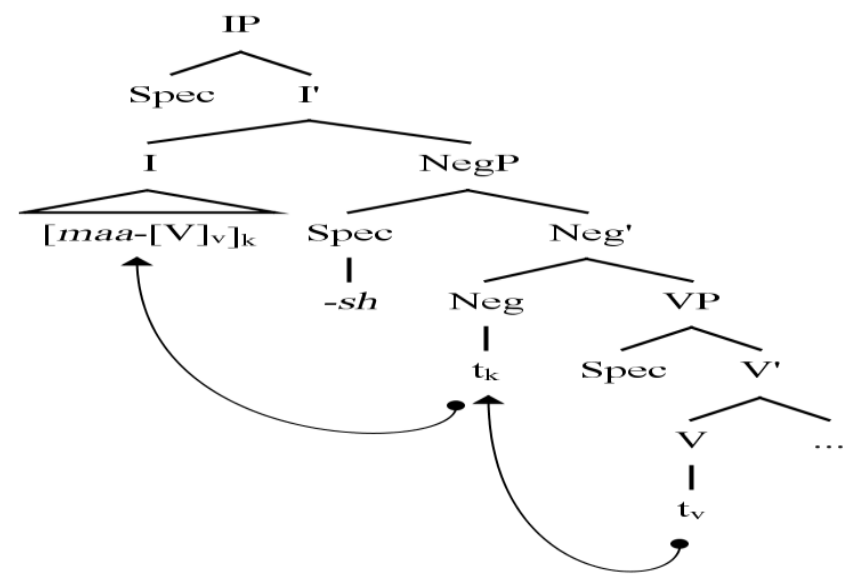

As discussed in Chapter Two, in particular, in the derivation of Arabic SVO word ordering, I have assumed the micro-variation that the standard variety and the dialects of Arabic are subject to whether the subject-related DP is a Topic DP basegenerated in the left periphery of sentences, which is the case in SA SVO configurations, or is raised out of $\left[\mathrm{Spec}, v^{*} \mathrm{P} / \mathrm{VP}\right]$ to $\left[\mathrm{Spec}, \operatorname{Agr}_{S} \mathrm{P}\right]$, which is the case for a spoken dialect like TA. This derivational difference gives rise to the two representations in (5) and (6) for SA and TA SVO word ordering, respectively: 
(5) SA SVO word order

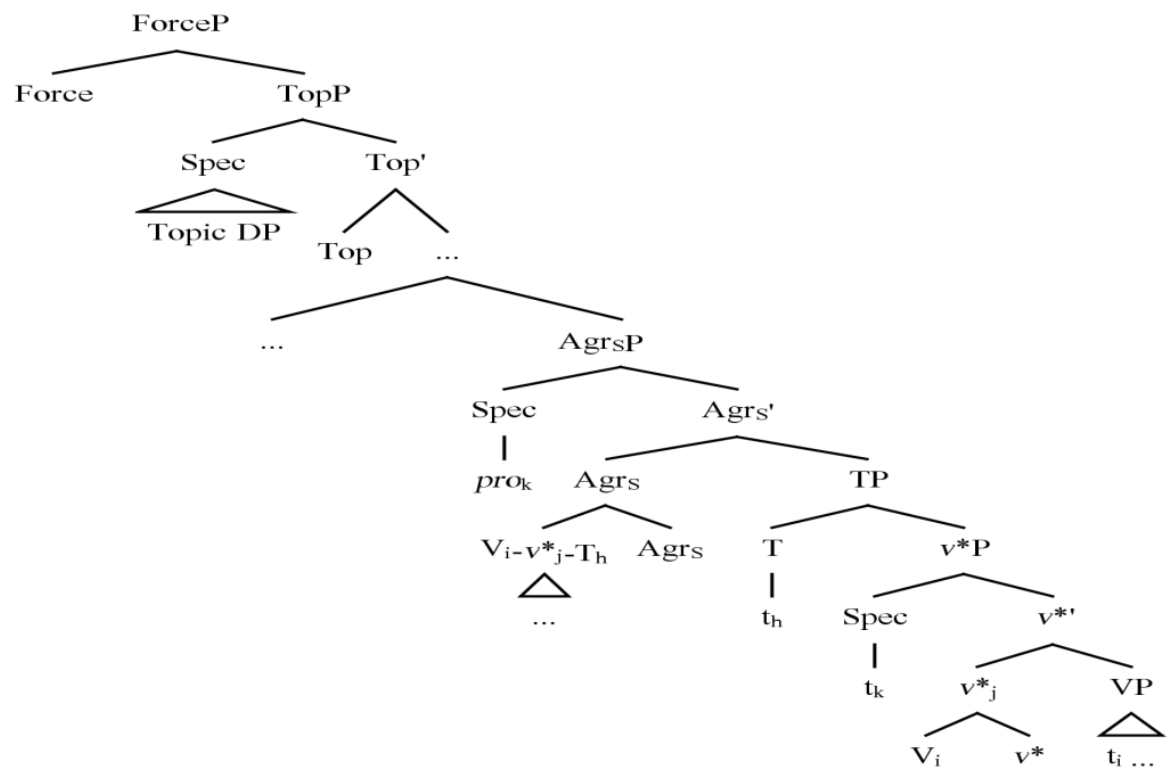

(6) TA SVO word order

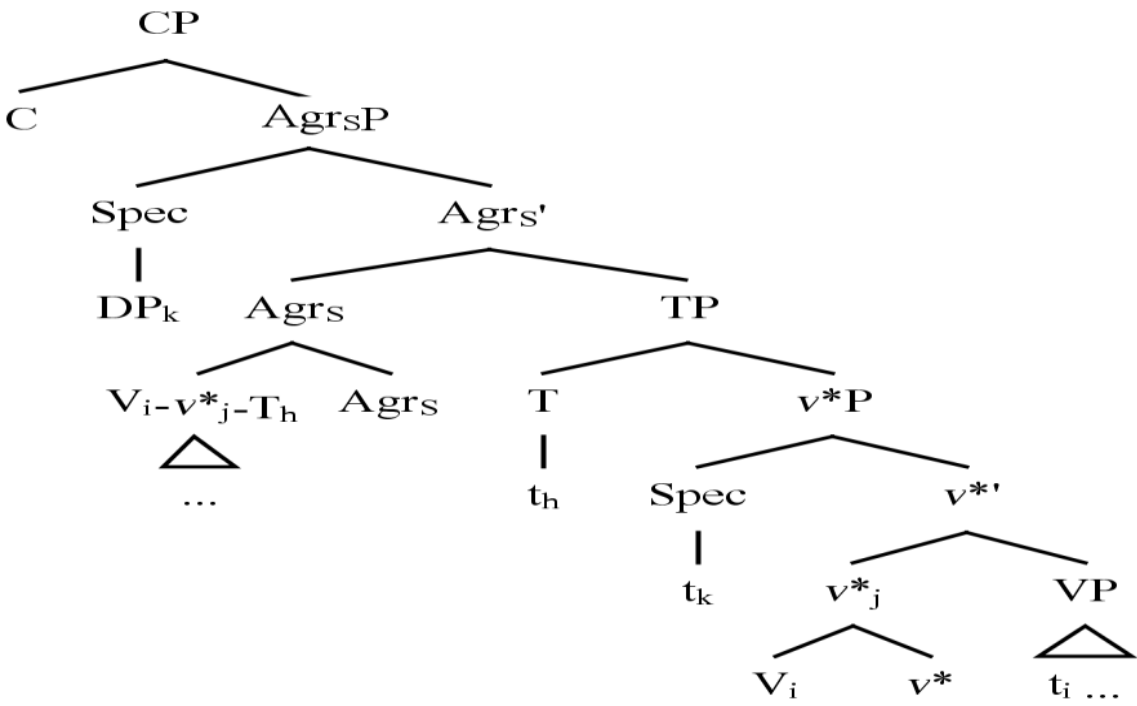

As (5) proposes, SA SVO word ordering allows the CP domain to split up (as it does in SA VSO word ordering) to allow for the projection of a TopP projection, which is essential for the representation of the subject-properties of SVO sentences in SA. Again, the ellipsis in the SA SVO structure (5) signals the potential projection of functional nodes for the Merge of elements linked to 'focus' and/or to 'modality'. In the TA SVO structure in (6), $\operatorname{Agr}_{S} \mathrm{P}$ projects as the highest maximal projection within 
IP (as in SA SVO word ordering). However, unlike the derivation of SA SVO word ordering, $\mathrm{Agr}_{\mathrm{S}} \mathrm{P}$ in TA is selected as complement to a $\mathrm{C}$ node, which maximally projects as a non-split $\mathrm{CP}$.

The proposed analysis of subject-verb agreement in Arabic (with some crosslinguistic comparisons) is basically similar to R\&R's (2002) T-dependency approach, which links the rather 'rich' subject-verb agreement configurations familiar in a V2 language like German to the raising of $\mathrm{T}$ to a superordinate node (Fin in German) for the satisfaction of the EPP. Thus, the probe-goal-Agree analysis argued for here takes it that the EPP is not a 'Topic' feature (contra Rouveret 2010). Unlike R\&R, I assume that 'topicalized' XP elements are not EPP 'checkers', contrary to subject DPs, which are the only elements (EXPL or referential) that can satisfy the EPP (or the Subject Criterion, Rizzi 2006).

The proposed analysis also conforms to Chomsky's (2008) Phase-theoretic framework (vs. Chomsky 2001, 2004) in dissociating the EPP from the so-called 'edge' uninterpretable feature of C. Nevertheless, unlike Chomsky (2008), I assume that this 'edge' feature (i.e., $[u \mathrm{D}]$ in the present analysis) is a property of a number of superordinate nodes, which relates to $\varphi$-agreement (and, ultimately, to the EPP as an exclusive property of the T-node). This is mainly the case with subject-verb agreement configurations in SVO sentences in Arabic at the IP level projecting as $\mathrm{Agr}_{S} \mathrm{P}$ (with T raising to $\mathrm{Agr}_{\mathrm{S}}$, as shown in structure (5) above) and in $\mathrm{V} 2$ matrix declarative clauses in Germanic (e.g., German), which show an asymmetry between matrix V2 cases involving XP-topicalization (with T raising to $\mathrm{Agr}_{\mathrm{S}}$ ) and matrix V2 cases involving subject DPs (with T raising to Fin).

The presence of a $[u \mathrm{D}]$ feature, as a property of superordinate nodes projecting in the higher functional field in the left periphery, has also been proposed to be a characteristic of the subject-verb agreement configurations involving the $\mathrm{CP}$ level in the variety of English studied by Kimball \& Aissen (1971) and by Kayne (1989, 2000), and the special cases of complementizer agreement in West Flemish studied by Haegeman \& Koppen 2012. In both cases, [ $u \mathrm{D}]$ combines with $\varphi$-agreement, to the exclusion of the EPP in the relevant constructions. In the case of the variety of English, the valuation of $[u \mathrm{D}]$ at the $\mathrm{CP}$ level in wh-constructions (vs. topic-comment constructions and relative clauses where $[u \mathrm{D}]$ is absent) triggers a special case of subject-verb agreement, which has to conform to the $\varphi$-agreement established at the 
IP level where the EPP applies. In the West Flemish case, two $\varphi$-agreement relations are established: one $\varphi$-agreement relation (dissociated from the EPP contra Haegeman $\&$ Koppen 2012) is established at the level of Force in relation to a Topic-like node (Haegeman \& Koppen's 2012 Alpha node) and a second $\varphi$-agreement relation is established at the level of IP in relation to the EPP. Thus, this special combination of $[u \mathrm{D}]$ and $\varphi$-agreement (to the exclusion of the EPP) in the higher functional field in the cases at hand is responsible for the rather unique character of the agreement configurations witnessed in these constructions.

The parameterized probe-goal-Agree feature-based analysis of functional categories in sentence structure has also been of major significance in pinning down the two modes of dependency relation formation involving the interaction of $\mathrm{T}$ features and D-features (uninterpretable or interpretable) or between $\mathrm{T}$-features and a certain array of F-features (uninterpretable or interpretable). The first mode of licensing is the basic head-head agreement relation. This agreement relation is the basis for verb-movement to the functional field above $v \mathrm{P} / \mathrm{VP}$ in $\mathrm{V}$-raising languages in relation to the 'identification' of T-features on the functional heads $\mathrm{T}$ and Fin in VSO configurations in SA. This basic agreement relation also involves a certain array of F-features in relation to the tense, modal and/or focus properties of sentences, as in the case of the derivation of negative sentences in SA (cf. Ouhalla 1993, R\&R 2002). The second mode of licensing is the Spec-head agreement relation. This agreement relation is brought about by the Merge (internal or external) of $\mathrm{D}(\mathrm{P})$ elements, as in subject-verb agreement configurations at the level of IP in SVO configurations in SA or at the level of CP in declarative matrix V2 configurations, and the Merge of whelements in interrogative constructions. This Spec-head agreement relation is the basis for the valuation of uninterpretable features in the relevant constructions: $[u \mathrm{D}]$ on $\mathrm{Agr}_{S}$ in relation to the Merge of EXPL pro in [Spec $\left.\mathrm{Agr}_{S} \mathrm{P}\right]$ in 'topicalization' cases in SA or German, and on Fin in relation to the Merge of subject elements in the relevant V2 matrix clauses.

Moreover, this conception of feature-based dependency formation reinforces a key assumption of this thesis - namely, the dissociation between Topics and subjects. In other terms, similar to the head-head dependency relations that are involved in the identification of features in the split-CP domain, the only condition that applies to Topics in [Spec, TopP] is the identification of an $[i \mathrm{D}]$ feature on the Top node in 
relation to the $[i \mathrm{~T}]$ features on Fin and $\mathrm{T}$, and ultimately, in relation to $\varphi$-agreement and the EPP at the IP level. 


\section{REFERENCES}

Aarts, Bas. 1992. Small Clauses in English: The Nonverbal Types. Berlin: de Gruyter.

Ackema, Peter \& Ad Neeleman. 2003. Context-Sensitive Spell-Out. Natural Language and Linguistic Theory 21: 681-735.

Akkal Ahmed \& Abdel-kader Gonegai. 2000. On the status of AgrS in some Null Subject Languages. In Research in Afroasiatic Grammar, ed. by Jacqueline Lecarme, Jean Lowenstamm and Ur Shlonsky, 1-22. Amsterdam: John Benjamins.

Al-Aswad, Mohamed k. 1983. Contrastive Analysis of Arabic and English Verbs in Tense, Aspect and Structure. Unpublished PhD diss., University of Michigan. Alexiadou, Artemis \& Elena Anagnostopoulou. 1998. Parametrizing AGR: Word Order, V-movement and EPP-checking. Natural Language and Linguistic Theory 16: 491-539.

Alexiadou, Artemis \& Elena Anagnostopoulou. 2001. The Subject-in-situ Generalization and the Role of Case in Driving Computations. Linguistic Inquiry 32: 193-231.

Alharbi, Abdallah H.M. 2000. The NEG elements in clause structure. Syntaxis 3: 4573.

Aoun, Joseph \& Elabbas Benmamoun. 1999. Gapping, PF merger, and patterns of partial agreement. In Fragments: Studies in Ellipsis and Gapping, ed. By Shalom Lappin and Elabbas Benmamoun, 175-192. New York: Oxford University Press.

Aoun, Joseph, Elabbas Benmamoun \& Dominique Sportiche. 1994. Agreement, Word Order and Conjunction in Some Varieties of Arabic. Linguistic Inquiry 25: $195-220$.

Aoun, Joseph, Elabbas Benmamoun \& Lina Choueiri. 2010. The Syntax of Arabic. Cambridge: Cambridge University Press.

Ayoub, Georgine. 1981. Structure de la phrase verbale en Arabe Standard. Thèse de doctorat de troisième cycle. Université de Paris VII.

Bahloul, Maher. 2008. Structure and Function of the Arabic Verb. New York: Routledge.

Bakir, Murtadha J. 1980. Aspects of Clause Structure in Arabic. PhD diss., Indiana University. 
Bakir, Murtadha J. 2011. Against the split-CP hypothesis. Evidence from Iraqi Arabic. In Perspectives on Arabic Linguistics: Papers from the Annual Symposia on Arabic Linguistics. Volume XXII-XXIII, ed. by Ellen Broselow \& Hamid Ouali, 187-202. College Park, Maryland, 2008 and Milwaukee, Wisconsin, 2009. Amsterdam: John Benjamins.

Baltin, Mark R. \& Chris Collins (eds.). 2001. The Handbook of Contemporary Syntactic Theory. Malden, MA: Blackwell.

Barlow, Michael \& Ferguson, Charles A. (eds.). 1988. Agreement in Natural Language: Approaches, Theories, Descriptions. Stanford, Center for the Study of Language and Information, Stanford University.

Belletti, Adriana \& Luigi Rizzi (eds.). 1996. Parameters and Functional Heads. Essays in Comparative Syntax. Oxford: Oxford University Press.

Belletti, Adriana. 2001. Agreement Projections. In The Handbook of Contemporary Syntactic Theory, ed. by Baltin, Mark R. \& Chris Collins, 483-510. Malden, MA: Blackwell.

Belletti, Adriana (ed.). 2004. Structures and Beyond: The Cartography of Syntactic Structure, Vol. 3. Oxford: Oxford University Press.

Benincà, Paola \& Cecilia Poletto 2004. Topic, Focus and V2: Defining the CP sublayers. In The Structure of CP and IP, The Cartography of Syntactic Structures, vol.2 Luigi Rizzi (ed.), 52-75. New York: Oxford University Press.

Benmamoun, Elabbas. 1992a. Structural conditions on agreement. Proceedings of NELS 22: 17-32. Amherst, MA: University of Massachusetts, Graduate Linguistic Student Publications.

Benmamoun, Elabbas. 1992b. Functional and Inflectional Morphology: Problems of Projection, Representation and Derivation, PhD diss., University of Southern California.

Benmamoun, Elabbas. 1995. The conditions on pro and the ECP. Proceedings of WCCFL 13, ed. by Raul Aranovich, William Byrne, Susanne Preuss, \& Martha Senturia, 73-88. Stanford: CSLI Publications.

Benmamoun, Elabbas. 1997. Agreement in Arabic and the PF interface. Proceedings of WCCFL 15, ed. by Brian Agbayani and Sze-Wing Tang, 33-47. Stanford: CSLI Publications. 
Benmamoun, Elabbas. 2000a. Agreement Asymmetries and the PF interface. In Lecarme et al. 2000, 23-40. Amsterdam: John Benjamins.

Benmamoun, Elabbas. 2000b. The Feature Structure of Functional Categories: a Comparative Study of Arabic Dialects, Oxford: Oxford University Press.

Benmamoun, Elabbas. 2008. Clause structure and the syntax of verbless sentences. In Foundational Issues in Linguistic Theory, ed. by Robert Freidin, Carlos Otero, \& Maria Luisa Zubizarreta, 105-131. Cambridge, MA: MIT Press.

Biberauer, Theresa, 2010. Semi null-subject languages, expletives and expletive pro reconsidered. In Theresa Biberauer et al., 153-199. Cambridge: Cambridge University Press.

Biberauer, Theresa, Anders Holmberg, Ian Roberts \& Michelle Sheehan. 2010. Parametric Variation: Null Subjects in Minimalist Theory. Cambridge: Cambridge University Press.

Biberauer, Theresa \& Ian Roberts. 2010. Subjects, Tense and Verb-movement. In Theresa Biberauer et al., 263-302. Cambridge: Cambridge University Press.

Black, James \& Virginia Motapanyane (eds.) 1996. Microparametric Syntax and Dialect Variation. Amsterdam: John Benjamins.

Bobaljik, Jonathan D. 1994. What Does Adjacency Do? MITWPL 22: 1-31.

Bobaljik Johnathan D. \& Dianne Jonas. 1996. Subject Positions and the Roles of TP. Linguistic Inquiry 27: 195-236.

Bobaljik Johnathan D. \& Andrew Carnie. 1996. A minimalist approach to some problems of Irish word order. In The Syntax of the Celtic Languages, ed. by Robert D. Borsley \& Ian Roberts, 223-240. Cambridge: Cambridge University Press.

Bobaljik, Johnothan D. 2002. A-chains at the PF-interface: Copies and 'covert' movement. Natural Language and Linguistic Theory 20: 197-267.

Borsley Robert D. \& Ian Roberts (eds.) 1996. The Syntax of the Celtic Languages. Cambridge: Cambridge University Press.

Borg, Albert \& Marie Azzopardi-Alexander, 1997. Maltese. Routledge: London.

Bulos, Afif A.1965. The Arabic Trilateral Verb. A Comparative Study of Grammatical Concepts and Processes. Beirut: KHAYATS. 
Cardinaletti Anna \& Michal Starke. 1999. The typology of structural deficiency: A case study of the three classes of pronouns. In Clitics in the Languages of Europe, ed. by Henk van Riemsdijk, 145-235. Berlin: de Gruyter.

Carnie, Andrew, Heidi Harley \& Elisabeth Pyatt. 1994. The resurrection: Raising to Comp? Some evidence from Old Irish. Studies in the Linguistic Sciences 24: 85-100.

Carnie, Andrew, Heidi Harley \& Elisabeth Pyatt. 2000. VSO order as raising out of IP? Some evidence from Old Irish. In The Syntax of Verb-initial Languages, ed. by Andrew Carnie \& Eithne Guilfoyle, 39-59. Oxford: Oxford University Press.

Cheng, Lisa. 1991. On the Typology of Wh-questions. PhD diss., MIT.

Chomsky, Noam. 1981. Lectures on Government and Binding. Dordrecht: Foris.

Chomsky, Noam. 1982. Some Concepts and Consequences of the Theory of Government and Binding. Cambridge, MA: MIT Press.

Chomsky, Noam. 1986a. Barriers. Cambridge, MA: MIT Press.

Chomsky, Noam. 1986b. Knowledge of Language: Its Nature, Origins and Use. New York: Praeger.

Chomsky, Noam. 1989. Some notes on economy of derivation and representation. In Noam Chomsky. 1995c (Pp. 129-166). First published in 1989 in MIT Working Papers in Linguistics 10: 43-74. Reprinted in Chomsky, Noam. 1991. Principles and Parameters in Comparative Grammar, ed. by Robert Freidin, 417-455. Cambridge, MA: MIT Press.

Chomsky, Noam. 1993. A minimalist program for linguistic theory. In Noam Chomsky. 1995c (Pp. 167-217). First published in The View from Building 20: Essays in Linguistics in Honor of Sylvain Bromberger, ed. by Kenneth L. Hale \& S. Jay Keyser. 1991, 1-52. Cambridge, MA: MIT Press.

Chomsky. 1995a. Bare phrase structure. In Government and Binding Theory and the Minimalist Program, ed. by Gert Webelhuth, 383-439. Oxford: Basil Blackwell.

Chomsky, Noam. 1995b. Categories and transformations. In Noam Chomsky 1995c, (Pp. 219-394).

Chomsky, Noam. 1995c. The Minimalist Program, Cambridge, MA: MIT Press. 
Chomsky, Noam . 1998. Minimalist Inquiries: the Framework. In Step by step: Essays on minimalist syntax in honor of Howard Lasnik, ed. by Roger Martin, David Michael \& Juan Uriagereka, 89-155. Cambridge, Mass.: MIT Press.

Chomsky, Noam. 2001. Derivation by Phase. In Ken Hale: A Life in Language, ed. by Michael Kenstowicz, 1-52. Cambridge, MA: MIT Press.

Chomsky, Noam. 2004. Beyond Explanatory Adequacy. In Structures and Beyond: The Cartography of Syntactic Structure, Vol. 3, ed. by Adriana Belletti, 104131. New York: Oxford University Press.

Chomsky, Noam. 2008. On phases. In Foundational Issues in Linguistic Theory, ed. by Robert Freidin, Carlos Otero, \& Maria Luisa Zubizarreta, 133-166. Cambridge, MA: MIT Press.

Chomsky, Noam \& Howard Lasnik.1993. The theory of principles and parameters. In Noam Chomsky 1995c, 13-127. First published as 'Principles and parameters theory'. In Syntax: An International Handbook of Contemporary Research, ed. by Joachim Jacobs, Armin Von Stechow, Wolfgang Sternefeld \& Theo Vennemann, 506-569. Berlin: de Gruyter.

Cinque, Guglielmo. 1999. Adverbs and Functional Heads: A Cross-linguistic Perspective. Oxford: Oxford University Press.

Coopmans, Peter, Martin B.H. Everaert \& Jane Grimshaw (eds.) 2000. Lexical Specification and Insertion. Amsterdam: John Benjamins.

Cowell, Mark W. 1964. A Reference Grammar of Syrian Arabic. Washington D.C.: Georgetown University Press.

Dahl, Östen. 1985. Tense and Aspect Systems. Oxford: Blackwell.

Dahl, Östen \& Fathi Talmoudhi. 1979.Qad and Laqad - tense/aspect and pragmatics in Arabic. In Aspectology: Workshop at the Fifth Scandinavian Conference of Linguistics, ed. by Thore Petterson, 51-68. Stockholm: Almqvist \& Wiksell.

Davies, William D. \& Stanley Dubinsky (eds.) 2006. Objects and Other Subjects: Grammatical Function, Functional Categories and Configurationality. Dordrecht: Kluwer Academic Publishers.

Demirdache Hamida. 1989. Nominative NPs in Modern Standard Arabic. Ms. Massachusetts Institute of Technology. 
Demirdache, Hamida. 2013. Nominative NPs in Modern Standard Arabic. In Phonologie, Morphologie, Syntaxe. Mélanges offerts à Jean-Pierre Angoujard. ed. by Ali Tifrit, 277-325. Collection Rivages Linguistiques, Presse Universitaire de Rennes.

Er-Rayyan, Mohammad. 1986. Toward the construction of a temporal system for natural language in the light of the Arabic and English languages. Unpublished PhD diss., University of Pennsylvania.

Fassi Fehri, Abdelkader. 1982. Linguistique arabe: forme et interprétation. Rabat: Faculté des Lettres et Sciences Humaines.

Fassi Fehri, Abdelkader. 1992. Sous-spécification, accord et pronoms en arabe. Revue québécoise de linguistique, vol. 22 (1): 117-143.

Fassi Fehri, Abdelkader. 1993. Issues in the Structure of Arabic Clauses and Words. Dordrecht: Kluwer Academic Publishers.

Fassi Fehri, Abdelkader. 2000. Distributing Features and Affixes in Arabic Subject Verb Agreement Paradigms. In Research in Afroasiatic Grammar, ed. by JacquelineLecarme, Jean Lowenstamm \& Ur Shlonsky, 79-100. Amsterdam: John Benjamins.

Fassi Fehri, Abderkader. 2004. Temporal / Aspectual Interaction and Variation across Arabic Heights. In The Syntax of Time ed. by Jacqueline Guéron \& Jacqueline Lacarme, 235-258. Cambrige, MA: MIT Press.

Fassi Fehri, Abderkader. 2012. Key Features and Parameters in Arabic Grammar. Amsterdam: John Benjamins.

Felser, Claudia. 2003. Wh-copying, phases, and successive cyclicity. Lingua 114: 543-574.

Givón, Talmy. 1976. Topic, pronoun and grammatical agreement. In Subject and Topic, ed. by Charles N. Li, 149-188. London: Academic Press.

Greenberg Joseph H. 1963/1966. Some universals of grammar with particular reference to the order of meaningful elements. Universals of grammar, ed. by Joseph H. Greenberg, 73-113. Mass.: MIT Press.

Grimshaw, Jane. 2000. Locality and extended projection. In Lexical Specification and Insertion, ed. by Peter Coopmans, Martin B. H. Everaert \& Jane Grimshaw, 115-133. Amsterdam: John Benjamins. 
Guasti, Maria T. \& Luigi Rizzi. 2002. Agreement and Tense as distinct syntactic positions: Evidence from acquisition. In The Structure of DP and IP The Cartography of Syntactic Structures, vol. 1, ed. by Cinque Guglielmo, 167194. NewYork: Oxford University Press.

Haegeman, Liliane \& Marjo van Koppen. 2012. Complementizer Agreement and the relation between $\mathrm{C}^{\mathrm{o}}$ and $\mathrm{T}^{\mathrm{o}}$. Linguistic Inquiry 43:441-454.

Haegeman, Liliane \& Raffaella Zanuttini. 1991. Negative heads and the Neg Criterion. The Linguistic Review 8: 233-251.

Haider, Hubert. 2010. The Syntax of German. Cambridge: Cambridge University Press.

Halila, Hafedh. 1992. Subject Specificity Effects in Tunisian Arabic. Unpublished PhD diss., University of Southern California.

Harbert, Wayne \& Maher Bahloul. 2002. Postverbal subjects in Arabic and the theory of agreement. In Themes in Arabic and Hebrew Syntax, ed. by Jamal Ouhalla and Ur Shlonsky, 45-70. Dordrecht: Kluwer Academic Publishers.

Harris, Zellig S. 1947. Methods in Structural Linguistics. Chicago: University of Chicago Press.

Hassan, M. Hassan. 1990. A contrastive study of tense and aspect in English and Arabic with special reference to translation. Unpublished $\mathrm{PhD}$ diss., University of Bath, England.

Holes, Clive. 1990. Gulf Arabic. Routledge: London.

Holmberg, Anders. 1999. Remarks on Holmberg's Generalization. Studia Linguistica 53: $1-39$.

Holmberg, Anders. 2000. Scandinavian Stylistic Fronting: How any category can become an expletive. Linguistic Inquiry 31: 445-83.

Holmberg, Anders \& Platzack, Christer. 1995. The Role of Inflection in Scandinavian Syntax. New York: Oxford University Press.

Horvath, Julia. 1995. Structural Focus, structural Case, and the notion of featureassignment. In Discourse Configurational Languages, ed. by Katalin É Kiss, 28-64. Oxford: Oxford University Press.

Hoyt, Frederick. 2009. Verbal Clause. In The Encyclopedia of Arabic Language and Linguistics, vol. IV: Q-Z. ed. by Mushira Eid, Alla Elgibali, Manfred Woidich \& Andrzej Zaborski, 653-659. Brill: Leiden and Boston. 
Jaeggli, Osvaldo and Kenneth J. Safir. 1989. The null subject parameter and parametric theory. In The Null Subject Parameter, ed. by Osvaldo Jaeggli \& Kenneth J. Safir, 1-44. Dordrecht: Kluwer Academic Publishers.

Ibn-Hishaam, Sabd-Allah. 1359; edn 1964. Mughni Al-labiib San Lughat AlPiSraab 'The indispensable reference for the (wise) learner of the syntax of the (Arabic) language', ed. by Mohammad Mubarak et al. Damascus: Dar al-Fikr.

Jouini, Kemel. 2012. Dependency relations in the syntactic structure of Tunisian Arabic. Arab World English Journal 3(4): 36-57.

Jouitteau, Mélanie. 2005. La Syntaxe Comparée du Breton, PhD diss., Université de Naoned/Nantes.

Kayne, S. Richard. 1989a. Facets of Romance past participle agreement. In Dialect Variation and the Theory of Grammar, ed. by Paola Beninca, 85-104. Dordrecht: Foris.

Kayne, S. Richard. 1989b. Notes on English agreement. Central Institute of English and Foreign Languages Bulletin 1: 41-67. Hyderabad, India. Reprinted in Richard S. Kayne. 2000. (Pp. 187-205).

Kayne, S. Richard. 2000. Parameters and Universals, Oxford: Oxford University Press.

Kayne, S. Richard. 2008. Expletives, datives, and the tension between morphology and syntax. In The limits of Syntactic Variation, ed. by Theresa Biberauer, 175-218. Amsterdam: John Benjamins.

Kimball, Judith \& John Aissen. 1971. I think, you think, he think. Linguistic Inquiry 2: $242-246$.

Kiss, Katalin É. 1995. Discourse Configurational Languages. Oxford: Oxford University Press.

Koopman, Hilda \& Dominique Sportiche. 1991. The position of subjects. Lingua 85: 211-258.

Laka, Miren I. M. 1990. Negation in Syntax: on the Nature of Functional Categories and Projections. Cambridge, MA: MIT.

Larson, Richard K. 1988. On the double object construction. Linguistic Inquiry 19: 335-391. 
Lasnik, Howard. 1995. Case and expletives revisited: On Greed and other human failings. Linguistic Inquiry 26: 615-33.

Lasnik, Howard. 1999. Minimalist Analysis. Malden, MA: Blackwell.

Lasnik Howard. 2001. A note on the EPP. Linguistic Inquiry 32: 356-362.

Lecarme, Jacqueline, Jean Lowenstamm \& Ur Shlonsky (eds.) 2000. Research in Afroasiatic Grammar. Amsterdam: John Benjamins.

Makhoukh, Ahmed. 1998. Agreement Phenomena in Standard Arabic, PhD diss., Faculté des Lettres, Meknes, Morocco.

Massam, Diane. 2000. VSO and VOS: Aspects of Niuean Word Order. In The Syntax of Verb-Initial Languages, ed. by Andrew Carnie \& Eithne Guilfoyle, 97116. Oxford: Oxford University Press.

McCloskey, James. 1997. Subjecthood and subject positions. In Elements of Grammar, ed. by Liliane Haegeman, 197-235. Dordrecht: Kluwer Academic Publishers.

McCloskey, James. 2001. The distribution of subject properties in Irish. In Objects and Other Subjects: Grammatical Function, Functional Categories and Configurationality, ed. by William D. Davies \& Stanley Dubinsky, 157-192. Dordrecht: Kluwer Academic Publishers.

Miyagawa, Shiguru. 2010. Why Agree? Why Move? Unifying Agreement-Based and Discourse Configurational Languages. Cambridge, MA: MIT Press.

Mohammad, Mohammad A. 1989. The Sentence Structure of Arabic. PhD diss., USC Los Angeles, California.

Moutaouakil. Ahmed. 1989. Pragmatic Functions in a Functional Grammar of Arabic. Dordrecht: Foris.

Müller, Gereon. 2006. Pro-drop and impoverishment. In Form, structure and grammar. A Festschrift presented to Günther Grewendorf on the occasion of his 60th birthday, ed. by Patrick Brandt \& Eric Fuss, 93-115. Berlin: Akademie Verlag.

Müller, Gereon, \& Wolfgang Stemefeld. 1993. Improper movement and unambiguous Binding. Linguistic Inquiry 24, 461-507.

Ouhalla, Jamal. 1991. Functional Categories and Parametric Variation. London: Routledge.

Ouhalla, Jamal. 1993. Negation focus and tense: the Arabic maa and laa. Rivista di Linguistica 5: 275-298. 
Ouhalla, Jamal. 1994. Focus in Standard Arabic. Linguistics in Potsdam 1: 65-92.

Ouhalla, Jamal. 1999. Focus and Arabic clefts. In The Grammar of Focus, ed. by

Georges Rebuschi and Laurice Tuller, 335-359. Amsterdam: John Benjamins.

Pesetsky, David \& Ester Torrego. 2001. T-to-C movement: causes and

consequences. In Ken Hale: A Life in Language, ed. by Michael Kenstowicz, 355-426. Cambridge, MA: MIT Press.

Pollock, Jean-Yves. 1989. Verb movement, Universal Grammar, and the structure of IP. Linguistic Inquiry 20: 365-424.

Poletto, Cecilia. 2000a. The Higher Functional Field in the Northern Italian Dialects. Oxford: Oxford University Press.

Poletto, Cecilia. 2000b. The left periphery of V2- Rhaetoromance Dialects: a new view on V2 and V3. In Syntactic Microvariation, ed. by Sjef Barbiers, Leonie Cornips \& Susanne van der Kleij, 214-242. Amsterdam, Meertens Instituut: electronic publication.

Radford, Andrew. 1988. Small children's Small Clauses. Transactions of the Philological Society $86: 1-46$

Rizzi, Luigi. 1982. Issues in Italian Syntax. Dordrecht: Foris.

Rizzi, Luigi. 1986. Null objects and the theory of pro. Linguistic Inquiry 17: 501-57

Rizzi, Luigi. 1990. Relativized Minimality. Cambridge, MA: MIT Press.

Rizzi, Luigi. 1996. Residual Verb Second and the Wh-Criterion. In Adriana Belletti and Luigi Rizzi (eds.), 63-90. Oxford: Oxford University Press.

Rizzi, Luigi. 1997. The fine structure of the left periphery. In Elements of Grammar, ed. by Liliane Haegeman, 281-337. Dordrecht: Kluwer Academic Publishers.

Rizzi, Luigi (ed.). 2004a. The Structure of CP and IP, The Cartography of Syntactic Structures, vol.2. New York: Oxford University Press.

Rizzi, Luigi. 2004b. Locality and the left periphery. In Structures and Beyond: The Cartography of Syntactic Structure, Vol. 3, ed. by Belletti Adriana, 223-251. New York: Oxford University Press.

Rizzi, Luigi. 2006. On the form of Chains: Criterial positions and ECP effects. In Wh-Movement: Moving On, ed. by Lisa Lai-Shen Cheng \& Norbert Corver, 97-133. Cambridge, MA: MIT Press.

Rizzi, Luigi \& Ur Shlonsky. 2007. Strategies of subject extraction. In Interfaces + Recursion = Language $?$ Chomsky's Minimalism and the View from Syntax- 
Semantics, ed. by Uli Sauerland \& Hans-Martin Gärtner, 115-160. Berlin: Mouton de Gruyter.

Roberts, Ian. 2010a. Agreement and Head Movement : Clitics, Incorporation, and Defective Goals. Cambridge, MA: MIT Press.

Roberts, Ian. 2010b. A deletion analysis of null subjects. In Theresa Biberauer et al., 58-87. Cambridge: Cambridge University Press.

Roberts, Ian \& Anders Holmberg. 2010. Introduction: parameters in Minimalist theory. In Theresa Biberauer et al., 1-57. Cambridge: Cambridge University Press.

Roberts, Ian \& Anna Roussou. 2002. The Extended Projection Principle as a condition on the tense dependency. In Subjects, Expletives, and the EPP, ed. by Peter Svenonius, 125-155. Oxford: Oxford University Press.

Rohrbacher, Bernhard Wolfgang. 1994. The Germanic VO languages and the full paradigm: a theory of $V$ to I raising. $\mathrm{PhD}$ diss., University of Massachusetts, Amherst.

Roman, André. 1991. De l'accord et du pseudo-accord du féminin en arabe. Annales Islamologiques 25: 27-56.

Rothstein, Susan D. 1983. The Syntactic Forms of Predication. PhD diss., MIT.

Rothstein, Susan D. 1995. Pleonastics and the interpretation of pronouns. Linguistic Inquiry 26: 499-529.

Rouveret, Alain. 2010. On Subject-verb languages. Lingua 120: 232-263.

Ryding, Karin . 2005. A Reference Grammar of Modern Standard Arabic. New York: Cambridge University Press.

Safir, Ken. 1985. Syntactic Chains. Cambridge: Cambridge University Press.

Schwartz, Bonnie D. \& Sten Vikner. 1996. The verb always leaves IP in V2 clauses. In Adriana Belletti \& Luigi Rizzi (eds.), 11-62. Oxford: Oxford University Press.

Sibawayh, Amr ibn Qanbar . 796; edn 1881. Al-kitaab, according to the manuscripts of Cairo, the Escurial, Oxford, Paris, Saint-Petersburg and Vienna. Published by Hartwig Derenbourg. Paris: Imprimerie Nationale.

Siewierska, Anna. 1993. On the interplay of factors in the determination of word order. In Syntax: An International Handbook of Contemporary Research, ed. by Joachim Jacobs, Arnim von Stechow, Wolfgang Sternefeld, \&Theo Vennemann, 826-847. Berlin: de Gruyter. 
Shlonsky, Ur. 1997. Clause Structure and Word Order in Hebrew and Arabic: An Essay in Comparative Semitic Syntax. New York: Oxford University Press.

Shlonsky, Ur. 2000. Remarks on the complementizer layer of Standard Arabic. In Reasearch in Afroasiatic Grammar, ed. by Jacqueline Lecarme, Jean Lowenstamm \& Ur Shlonsky, 325-343. Amsterdam: John Benjamins.

Shlonsky, Ur. 2002. Constituent Questions in Palestinian Arabic. In Themes in Arabic and Hebrew Syntax, ed. by Jamal Ouhalla \& Ur Shlonsky, 137-160. Dordrecht: Kluwer Academic Publishers.

Soltan, Usama. 2006. Standard Arabic subject-verb agreement asymmetry revised in an Agree-based minimalist syntax. In Agreement Systems, ed. by Cedric Boeckx, 239-265. Amsterdam: John Benjamins.

Sportiche, Dominique. 1989. Movement, Agreement, and Case. Ms. UCLA.

Sportiche, Dominique. 1998. Partitions and Atoms of Clause Structure: Subjects, Agreement, Case, and Clitics. London; New York: Routledge.

Stowell, Timothy. 1981. Origins of Phrase Structure. PhD diss., MIT.

Stowell, Timothy. 1996. The phrase structure of Tense. In Phrase Structure and the Lexicon, ed. by Johan Rooryck and Laurie Zaring, 271-286. Dordrecht: Kluwer Academic Publishers.

Stowell, Timothy. 2007. The syntactic expression of Tense. Lingua 117: 437-463.

Svenonius, Peter (ed.) 2002. Subjects, Expletives, and the EPP. Oxford: Oxford University Press.

Tallerman, Maggie. 1997. Celtic word order. In Constituent Order in the Languages of Europe, ed. by Anna Siewierska, 599-648. Berlin: de Gruyter.

Travis, Lisa de Mena. 1991. Parameters of phrase structure and verb-second phenomena. In Principles and Parameters in Comparative Grammar, ed. by Robert Freidin, 339-364. Cambridge, MA: MIT Press.

Van Gelderen, Elly. 1993. The Rise of Functional Categories. Amsterdam: John Benjamins.

Vangsnes, Øystein A. 2002. Icelandic expletive constructions and the distribution of subject types. In Subjects, Expletives, and the EPP, ed. by Peter Svenonius, 43-70. Oxford: Oxford University Press.

Watanabe, Akira. 1991. Wh-in-situ, Subjacency, and chain formation. Ms., MIT. Webelhuth, Gert (ed.) 1995. Government and Binding Theory and the Minimalist Program. Oxford: Blackwell. 
Wright, William. (n.d.). A Grammar of the Arabic Language. Third edition. 1996.

Librairie du Liban: Beirut. 